\title{
Contribution to the knowledge of beetles (Insecta Coleoptera) of some protected areas of Apulia, Basilicata and Calabria (Italy)
}

\author{
Fernando Angelini
}

Via De Reggio 142, 72021 Francavilla Fontana, Brindisi, Italy; email: angelini_fernando@libero.it

The lists of Coleoptera published by the author between 1986 and 1996 concerning the following protected areas from southern Italy are updated: Gargano Promontory, Policoro Wood, Pantano Lake of Pignola, Pollino Massif and Sila Plateau. Data is integrated with subsequent collections of the writer and with bibliographic citations of other authors. With the same criteria the species of further three areas are also listed: Mount Vulture-Monticchio Lakes, Central Lucan Apennines and Aspromonte. A total of 5,130 taxa were recorded for the eight surveyed National or Regional Parks, which are located in Apulia, Basilicata and Calabria regions (Italy). Of these species, 472 are endemic to the Italian territory. The saproxylic species amount to 1,115 , their related IUCN category is referred to and an interpretation of the emerging data is suggested. A chronology of the entomological researches carried out in the past has been reconstructed, moreover description of main features and geographical boundaries of the treated sites are provided. All taxa subject of this study are listed in taxonomic order with indication of the areas where their presence occurs and specifying the data source. The total number of species for each family is evaluated area by area and the data on the most representative of Coleoptera families are briefly commented, with remarks on the current state of research.

KEY WORDS Biodiversity; Coleoptera; National Parks; southern Italy; species.

Received 10.10.2019; accepted 21.02.2020; published online 16.03.2020

\section{INTRODUCTION}

The realization of this work stems from the need to update, in the taxonomy and nomenclature, several catalogues on southern Italy Coleoptera published by the author in the decade 1986-1996: Coleotterofauna del Massiccio del Pollino (Angelini, 1986), Coleotterofauna del bosco di Policoro (Angelini \& Montemurro, 1986), Coleotterofauna del Promontorio del Gargano (Angelini, 1987), Coleotterofauna dell'Altopiano della Sila (Angelini, 1991) and Coleotterofauna della Riserva Naturale WWF Lago Pantano di Pignola (Angelini, 1996).
The research carried out in the following years by the author has besides produced a significant amount of unpublished data, which here go to integrate those previously published. Likewise, to offer an overview as accurate and complete as possible of the current state of knowledge on the Coleoptera in these areas, all the species and data reported by other authors in the vast bibliography (annexed) have been added.

It has been decided to expand the portion of territory examined in the previous catalogues to include the Park of the Mount Vulture-Monticchio Lakes, the Central Lucan Apennines and the Aspro- 
monte since for these areas the amount of data collected over the years is significant and at the same time there is a body of literature that dates back to the first entomological explorations of Southern Italy, going back to the last quarter of XVIII century. Map of figure 1 shows the general location of all the study areas.

For the Gargano Promontory, data from Holdhaus (1911), previously excluded by Angelini (1987), are also included.

It is necessary to present the unpublished or bibliographic data in the form of a table rather than provide a full list with accurate information on the collection localities since this solution, although more appropriate, would require a remarkably more voluminous contribution; a similar update is underway, with comprehensive detail of all the localities, bibliographic references and status changes that have occurred over time, to be published exclusively online.

\section{The coleopterological researches in Apulia, Basilicata and Calabria}

The extreme south of Italy is a reservoir of great biodiversity and of a fauna with a rather different composition than the rest of the Peninsula. Entomo-

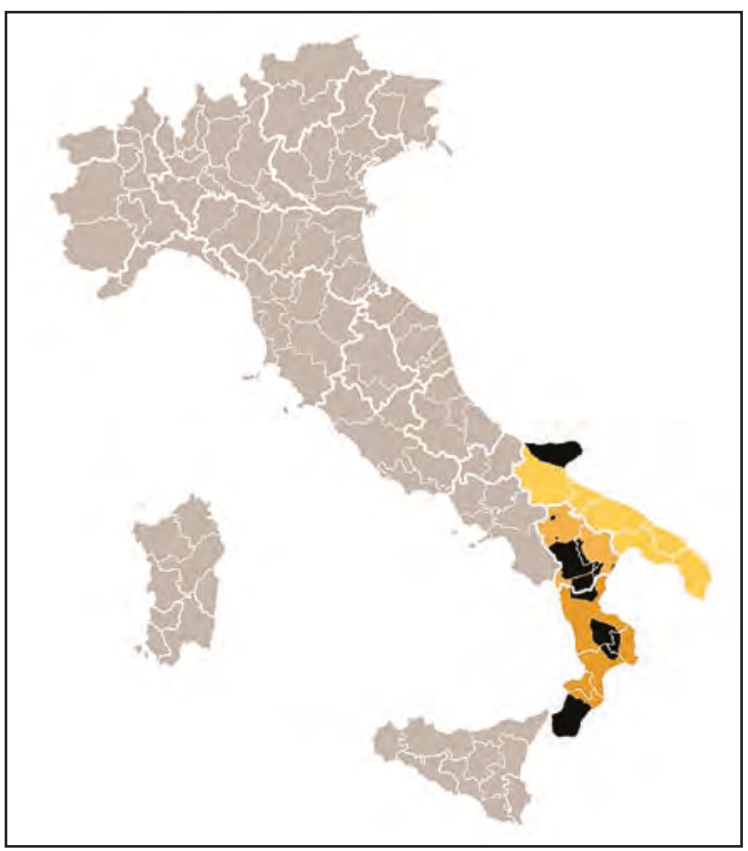

Figure 1. Study areas: in black, the location of the eight sites in southern Italy covered by this study. logically, this territory suffered greatly during the 19 th century due to the substantial lack of naturalists native to these regions and for the difficulty of connections by land (Salfi, 1963: 4) because of the local wilderness; it is not a coincidence that the history that will be exposed is clearly different from that of neighboring Sicily (Romano, 2006).

Only in the 20th century, studies on entomofauna have become more systematic, sometimes promoted by Italian universities and museums to acquire valuable study material.

A substantial contribution came from the author's personal researches, being himself favored by residing in Apulia and thus being able to more easily and frequently explore the target regions of this work, and from the many colleagues and friends entomologists with whom he shared fruitful hikes over the years.

The following is a chronicle of the explorations that involved these areas.

1783. The earlier research of which we know about in southern Italy are conducted in Aspromonte (Calabria ulteriore) in 1783 by Giulio Candida and Giuseppe Stefanelli and published by Vincenzo Petagna (1787), which lists 239 species of collected insects. It should be emphasized that this publication was a great success at the time so that the first print, which took place in 1786 and has not been possible to obtain, was followed by others including that of 1787 mentioned in the bibliography and most frequently cited.

1826. Luigi Petagna, Giovanni Terrone and Michele Tenore from 3 to 16 July make a botanical expedition from Naples to Cosenza, visit Monte Sirino and Pollino and mention 4 species of Coleoptera.

1827. Oronzo Gabriele Costa visits the Aspromonte together with Giovanni Gussone in the month of July (Costa A., 1863) and the list of vertebrates and arthropods they found is published 12 years later (Costa O.G., 1839), although the related report is read at the Royal Academy of Sciences the following year.

1851. Christian Suffrian (1851: 79) describes Chrysolina interstincta based on two specimens coming respectively from Sicily and Calabria and collected by Ludwig Parreyss (Daccordi \& Ruffo, 2005: 121, teste Bourdonné); no other data was 
found concerning the research carried out by Parreyss in Calabria.

1859. Achille Costa visits Sila and Aspromonte (Costa A., 1881: 1). Four years later he publishes the data about Aspromonte (Costa A., 1863) listing 577 Coleoptera species, which include those previously mentioned by Petagna (1787).

1870-1875. Giovanni Battista Adami, A. Steffanini, Filippo Fanzago, Guelfo Cavanna and L. Panato presumably perform entomological research in Calabria in these years and a first list (devoid of collecting sites) of 300 species of Coleoptera is published by Bertolini (1876).

1876. Achille Costa from July 11th to September 12th of 1876 visits Sila, the surroundings of Tiriolo and Ciro and makes known the results of his research five years later (Costa A., 1881: 45-51).

1876. Guelfo Cavanna and other naturalists visit the Aspromonte in the months of May and June and their material is studied by various specialists, but the results are not divulged.

1878. Flaminio Baudi di Selve conducts researches in Calabria: Omoglymmius germari (Ganglbauer, 1891) in Mazzei et al. (2019).

1880. Antonio Biondi, Cesare Caroti and Guelfo Cavanna between the 8th and 16th of July 1880 carry out research in Rionero and Mount Vulture and between 24th and 28th of July the same year on Pollino. The results are released by various specialists and the whole list of collected Coleoptera is published by Baudi, Piccioli \& Cavanna (1882). Cavanna (1882) also publishes a detailed report about this mission.

1881. (April), 1883 (September), 1884 (May), 1887 (August) Andrea Fiori is in Calabria (in 1884 and 1887 with Flaminio Baudi di Selve); dates of the last three trips are taken from one of his works (Fiori, 1894: 87); many data are disclosed in various papers both by Fiori and Baudi. Most of the citations found in Luigioni (1929) sub "Cal" are taken from these research, and secondarily from the publications mentioned above and a few others that follow.

1887. Andrea Fiori in August visits Sila (Fiori, 1894) and Gargano (Fiori, 1914b); only some of the data are made known in scattered works.

1896. Achille Costa visits Pollino Massif(Salfi,
1963: 19 and Ruffo \& Vigna Taglianti, 1988: 13) but does not publish the relevant data.

1905. Gustav Paganetti-Hummler visits Aspromonte for a long time; from one of his papers (Paganetti-Hummler, 1910) can be deduced that he collected in the following periods and localities: April-May in Gerace, Antonimina and Ciminà, from May to the beginning of August in Santa Cristina, Sant'Eufemia and Aspromonte. It is known that a manuscript exists which lists, with collecting localities, the species found in Aspromonte by Paganetti-Hummler (Magistretti, 1960: 111), but unfortunately it was not possible to trace it.

1906. Giacomo Cecconi visits Tremiti Islands between 15 and 25 of February and from 25 of May for a few days. The material collected is published by him two years later (Cecconi, 1908).

1906-1907. Karl Holdhaus visits Gargano from 10 to 30 of May in 1906 accompanied by Hamilkar Stolz and later from 3 to 18 April 1907 together with Alfred Kniž and Moritz Hilf. The latter stayed until the 20th of June to collect Coleoptera on behalf of Otto Leonhard. The results of these researches are published four years later (Holdhaus, 1911).

1909 and 1910. Alfredo Andreini carries out long researches in Basilicata in the surroundings of Latronico and Maratea; from examination of his finds, mentioned in many publications as well as from the direct study of specimens he collected and are now kept in the Dodero collection at Genoa (Poggi i.l.), it is possible to establish that he stayed there from April 21 to September 20 of 1909; instead for the year 1910 the timespan of its permanence is not possible to ascertain.

1911, 1912 and 1913. Andrea Fiori visits Mount Vulture in the month of May 1911 (Ruffo \& Vigna Taglianti, 1988: 15). From 15th of April to 4th of June 1912 he makes researches in Calabria and Sicily (Fiori 1915a and 1915b). In 1913 he goes on Gargano (Fiori, 1914b, 1915a). Only a few individual findings are published in the above mentioned works.

1913. Adolfo Falzoni explores Sila in the month of June (Fiori, 1914a: 18).

1921. Andrea Fiori in August visits Sila: Clinidium canaliculatum (O.G. Costa, 1839) in Mazzei et al., 2019). 
1923. Giuseppe Müller visits Mount Vulture (Ruffo \& Vigna Taglianti, 1988: 15).

1925. Arturo Schatzmayr visits Mount Vulture in May (Schatzmayr, 1926: 145, sub Oedemera subulata Olivier, $1790=$ Oedemera pthysica (Scopoli, 1763).

1926. Jaroslav Štorkán searches the Gargano in June. A list of species not previously mentioned by Holdhaus (1911) is published by Roubal (1932).

1933. An examination of Zombori's work (1984) shows that Agostino Dodero collected beetles in Sila at Camigliatello from 27 May to $23 \mathrm{Au}-$ gust 1933; further data related to Cosenza and San Pietro in Guarano are deduced from the publications of Poggi (2009) and Liberti \& Poggi (2018). Reference to his findings is also present in papers by other authors.

1933. Arturo Schatzmayr and Carl Koch visit Pollino in July (Tassi, 1966a: 1 and Magistretti 1955: 1); only three contributions are published on this mission (Koch, 1933a, Koch, 1933 b and Wittmer, 1935)

1934, 1937 and 1940. Alessandro Ghigi explores Gargano in August 1934, then in 1937 (unknown period) and again, together with Francesco Pio Pomini, in the second half of April. Between the end of May and 5 June 1940 both of them, besides Gargano, also visit the Tremiti Islands. The results are published by Gridelli (1949b).

1939. Lars Brundin \& Thure Palm visit Pollino in June and in a short note several species of Coleoptera are reported (Palm, 1939). The Curculionidae collected in this expedition are treated by Magnano (1957a), and Cerambycidae by Tassi (1966a).Various data are disclosed in works by other authors, including the description of some new species (Nyholm, 1948).

1939. Edgardo Moltoni from June 25 to July 14 collects in Sila and results are published by Schatzmayr (1941 and 1943).

1941-1945. Alessandro Focarile in July 1941 and, with Giovanni Mariani, in April 1942 is on Mount Vulture; between 5 September 1943 and 10 April 1945 he carries out research in various places in Apulia and Basilicata including Mount Vulture and Pollino Massif (Focarile, 1959: 18). The results of these researches were only published in small part by different authors.

1948. Giovanni Binaghi visits Gargano in the spring; only a few of these data are published (Binaghi, 1948).

1948, 1949, 1950 and 1957. Sandro Ruffo, Edoardo Gridelli, Antonio Giordani Soika, Mario Magistretti and Angelo Pasa go to Gargano, Tremiti Islands, Murge and Salento as part of a research on Apulian fauna promoted by Umberto D'Ancona of the Institute of Adriatic Studies (Gridelli, 1949b: 148; Magistretti, 1966: 8 and Ruffo \& Vigna Taglianti, 1988: 21). The results are published by Gridelli (1949a, 1949b and 1950), by Ruffo (1955) as well as by about fifteen Italian and foreign specialists in the journal "Memorie di Biogeografia Adriatica" (19501962) (Ruffo \& Vigna Taglianti, 1988: 21).

1950. Giovanni Binaghi in May is in Sila and in Aspromonte, only some data is published in various works.

1950, 1951 and 1953. Marcello La Greca, Michele Sarà and R. Capaldo (7 July-10 August 1950), Sandro Ruffo, Angelo Pasa and Livio Tamanini (10-23 June 1951 and 12-24 June 1953) carry out a series of research campaigns on Pollino promoted by Naples University. The results are published between 1950 and 1966 in thirty-two memoirs (Salfi, 1951; Ruffo \& Vigna Taglianti, 1988: 21) seventeen of which dedicated to Coleoptera.

1955. Guido Grandi promotes a research campaign for the Institute of Entomology of Bologna University at the Umbra Forest (Gargano). Searches begin on July the 4th and last for about one month; in addition to Grandi himself, Maria Matilde Principi, Giorgio Fiori, Egidio Mellini and Dante Faggioli also take part. The first results are published by Faggioli (1955); the researches conducted are illustrated by Grandi (1956).

1955. Marcello La Greca visits the Tremiti Islands as part of a research project promoted by the Institute of Zoology and Comparative Anatomy of Bari University.

1956-1958. Alessandro Focarile and Giovanni Mariani in these years undertake coleopterological research in 23 sites along the Ionian shorelines of Apulia, Basilicata and Calabria; they do sampling in Policoro Wood on May 27-29 of 1956, June 9 of 
1957 and May 24 of 1958. The outcomes of these researches are illustrated in a series of eighteen works released between 1959 and 1979.

1957, 1958, 1963 and 1966. As part of a research project on Apennine fauna promoted by the Civic Museum of Natural History of Verona and coordinated by Sandro Ruffo (started in 1954 with a mission on the Sibillini Mountains), in Aspromonte six expeditions made up of a total of nine members are performed: in May and July 1957, June and August 1958, August 1963, October 1966 (Magistretti $\&$ Ruffo, 1969: 388). The results for 16 families are illustrated in eight papers.

1957. Anna and Mario Magistretti, Angelo Pasa, Sandro Ruffo and Livio Tamanini visit Policoro Wood in May (Magistretti \& Ruffo, 1969: 387); individual data are published in many papers.

1960. Anna and Mario Magistretti, Angelo Pasa, Sandro Ruffo and Livio Tamanini visit Mount Vulture and the Monticchio Lakes in June (Magistretti \& Ruffo, 1969: 387); only single data are published in several works.

1960. Some Roman entomologists, stimulated by Frederick Hartig, go exploring Mount Vulture (Ruffo \& Vigna Taglianti, 1988: 15).

1960, 1961, 1962 and 1967. As part of the research on Apennine fauna promoted by the Civic $\mathrm{Mu}-$ seum of Natural History of Verona, five missions carried out by a total of seven members visit Sila, the Coastal Chain and Serre Calabre: in June and August 1960, August 1961, August 1962, July 1967 (Magistretti \& Ruffo, 1969: 388). The results concerning 12 families of Coleoptera are published in five papers.

1960, 1961, 1962 and 1963. Giorgio Marcuzzi conducts his research in Apulia (comprehensive of Gargano and the Tremiti islands, including Pianosa) and in Basilicata's province of Matera. The results are shown in the works of Marcuzzi (1962) and Marcuzzi \& Lafisca Turchetto (1977).

1961. Walter Liebmann visits Aspromonte (Magnano, 1964: 107) but the results do not appear to have been published.

1962. Giovanni Sichel and his colleagues go to Aspromonte (Tassi, 1966: 622) but the outcome of their research is not known.
1963, 1964 and 1967. As part of the research on Apennine fauna promoted by the Civic Museum of Natural History of Verona, four missions composed by a total of five members visit Apulia (Gargano, Murge, Terra d'Otranto) and Basilicata (Matera): in September 1963, June 1964, May and September 1967. Results are partially published in many papers. Just Magistretti (1966) releases data about Carabidae on the basis of all the specimens collected in Apulia in the overall expedition period.

1963. Gunnar Dahlgren from June 27th to July 7th visits Lorica in Sila by himself (from June 9th to June 26th he explores other Calabrian sites: Paola, Rogliano, Cosenza and Castigione) and the results are published (Dahlgren, 1964).

1964. A series of researches on the Central Lucan Apennines, Mount Vulture, Pollino and Policoro is begun by numerous members of the Roman Association of Entomology (Fabio and Mario Cassola, Franco Tassi, Silvio Bruno, Vincenzo Vomero, Gianni Gobbi, Giuseppe Maria Carpaneto, Franco Marozzini, Mario and Giuseppe Pinzari, Francesco Sacco, Paolo Marcello Brignoli, Walter Rossi, Paolo Audisio, Enzo Colonnelli) (Gobbi, 1971a: 28) but only a small part of the enormous amount of data collected is published (Gobbi, 1971a, 1973a, 2018; Colonnelli, 1974a; Gobbi \& Piattella, 2008).

1965, 1968, 1970 e 1972. Giovanni Binaghi, Italo Bucciarelli, Alessandro Focarile, Giovanni Mariani, Giuseppe Meggiolaro and Carlalberto Ravizza visit Mount Sirino in the Central Lucan Apennines; only a contribution on the Carabids is presented by Ravizza (1973) as a whole, while the remaining data are individually published in scattered works.

1965-1967. Michele Sarà and Giovanni Salamanna make a series of explorations of the Tremiti Islands in the context of a research on island populations promoted and financed by the National Research Council, in this specific case entrusted to the Institute of Zoology and Comparative Anatomy of Bari University. Data regarding Scarabaeidae family are published by Salamanna (1968).

1968-1976. Helio Pierotti visits Pollino, Sila and Aspromonte on several occasions and announces the results regarding genus Aphodius in 1977. Between 1969 and 1973 even Giovanni Dellacasa 
repeatedly goes to Sila and Aspromonte and the author's results are included in Pierotti's work (1977).

1968. In the month of July the author's personal searches in southern Italy begin, assiduously lasting for over 40 years for periods varying from 1 to over 20 days. The results of this research have been published only very partially in a series of twentyeight works, some of which are dedicated to specific areas: Policoro (1986, with F. Montemurro), Pollino (1986), Gargano (1987), Sila (1991), Lago Pantano di Pignola (1996), light traps in Laghi Pantano di Pignola and San Giuliano (1998); other data were recorded in works dedicated to specific families: Hydradephaga (1972-1984), Hydrophilidae (1973 and 1974 with G. Ferro), Ptiliidae (1997, with M. Sörensson), Carabidae (1997, with V. Monzini), Curculionoidea (1997, with R. Caldara), Latridiidae (1999, with W. Rücker), Leiodinae (1985-1995), Cryptophagidae (1981, 1984 and 1996 with J.C. Otero), Malachinii (2001, with C. Pasqual), Phalacridae (1995, with Z. Švec) and Pselaphinae (2001, with G. Sabella). More than 500,000 specimens were collected overall, all studied by specialists (except for several Staphylinidae, Ptiliidae, Nitidulidae, Scydmaeninae, Mordellidae, Scraptidae, Bruchinae, Phalacridae, Corylophidae, Alexidae) and belonging to over 4,500 species. Data also appear in publications of many other authors, including descriptions of about one hundred new species.

1973. As part of the Verona Museum's Programs, Roberto Pace undertakes a series of research in Central southern Apennines and visits in particular Gargano, Pollino Massif, Orsomarso Mountains, Serre Calabre, Sambiase and Aspromonte. He describes many hypogeous new species in a total of thirteen publications (1974b-2002).

It is deemed of no use to report the researches undertaken after the 1970s, when connections became easier and many were the entomologists who visited the southern regions. Related data are dispersed in an endless series of works (well over 300 , with descriptions of more than 140 endemic species) while there are no coordinated research campaigns with overall publication of the data.

From the late 1980s to date, Pietro Brandmayr and his collaborators have been a reference point for entomological research mainly in Sila, but also in other territories from Aspromonte to Pollino. Their research, though essentially aimed at ecological studies, in particular about Carabidae used as environmental indicators, has not left out the other Coleoptera families, especially the saproxylic beetles. With their results they have produced dozens of works, both of divulgative and scientific nature, also including descriptions of new species.

\section{MATERIAL AND METHODS}

\section{Study areas}

Only in the last thirty years the eight areas covered by this study have been gradually placed under special protection through the establishment of several National and Regional Parks. Entomological research, on the other hand, as described above, has a much longer history and the writer himself has done most of his research before such protected areas were created.

It is not always easy to give an interpretation of the borders that entomologists, especially in the past, have attributed to these area. Their specimens were often labeled only with generic toponyms such as "Gargano", "Sila", "Aspromonte", etc., and usually the meaning was in a geographical sense, the one marked by natural boundaries. We must nevertheless bear in mind that in some circumstances these natural elements (like woods, lakes, marshes, lagoons, etc.) may have undergone drastic changes, both in extension and in quality, due to the anthropic impact in the last few centuries, a phenomenon much accelerated in the most recent decades. This caused the "contraction" of several natural boundaries compared to how they were in the past.

Another factor to be considered, on the opposite side, is that the newly defined Parks perimeters in some cases excluded shorelines, wet areas, or portions of forests of considerable interest, environments which were often included in the areas of activity of entomological expeditions.

From this it follows that only in specific circumstances the delimitation of the areas under study coincides quite faithfully with the administrative borders of the respective newly established protected areas (such as in Policoro Wood, Mount Vulture-Monticchio Lakes, Pantano Lake of Pignola) 
while this does not happen in other areas such as Gargano Promontory, Pollino Massif, Sila Plateau and Aspromonte. For this reason, it is here considered appropriate to briefly explain the boundaries as they were historically meant and which ones are adopted in this work, they are those generally meant in the "entomological" sense.

The study areas located in Apulia, Basilicata and Calabria are respectively indicated in figures 2, 3 and 4.

1. Gargano promontory (Apulia: Foggia province, Fig. 2) is a karst plateau that stretches out towards the Adriatic Sea, almost devoid of a superficial river network. It includes a great variety of peculiar environments ranging from the millenary Foresta Umbra to the Mediterranean scrub, from coastal lagoons to pre-desert hills and plains.

All records to the east of an ideal line linking the mouth of Fortore river with Poggio Imperiale, Apricena, San Marco in Lamis, San Giovanni Rotondo and Manfredonia have been included. The Gargano National Park (established by Law No. 394 of 6.12.1991) is part of this territory, but excludes the south-eastern coast between Vieste and Torre del Segnale, wide areas between Lake Varano and Carpino-Vico del Gargano as well as all the territories south of Lesina Lake.

Gargano Promontory was explored, from an entomological point of view, since 1887 but initially just a few finds were disclosed. Almost 20 years

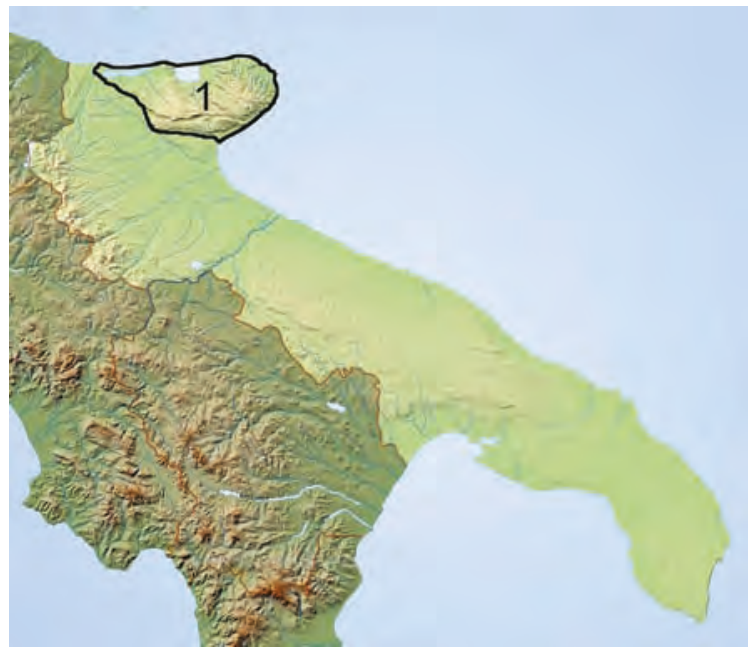

Figure 2. Apulia (Italy): 1. Gargano Promontory. later, in 1906-1907, Holdhaus and his collaborators carried out long and thorough researches and the overall results were announced by Holdhaus himself (1911). In the following years the territory has been the subject of several researches promoted both by individual entomologists and by public institutions and many general works or dedicated to single families have been published.

The writer undertook research in this area in the years 1970-1984 and published the overall results in a general catalogue in 1987; after this date he carried out further significant research aimed mainly at the investigation of the soil fauna.

2. Policoro Wood (Basilicata: Matera province, Fig. 3). The Riserva regionale Naturale Orientata Bosco Pantano di Policoro was established with L.R. n. 28 of 08.09.1999.

On the Ionian coast, historically, there was one of the southern most important floodplain forests, partially marshy, comprising the nuclei of Pantano soprano and Pantano sottano. In the XVIII century the extent of the forest was already much reduced but it was still described by travellers as an enchanted, luxuriant and uncontaminated place devoid even of viable trails. Nowadays only a very small portion of the old and renowned wood remains, with a limited naturalness degree, along the course of the river Sinni downstream of the Metaponto-Sibari railway and to the southeast of the interpoderal road that runs parallel to the railway at about 300 meters. An area of reforestation, mainly of Pinus halepensis, Eucalyptus camaldulensis and E. globulosus, has been included in the Reserve, east of the municipal road that leads from Policoro Wood to the dewatering system. The occasional floods of Sinni river pour debris on its outfall that sometimes carry an exceptional quantity of invertebrates (for example, on 17.IX.2000 sifting the beached debris, over 300 Coleoptera species were found).

The first entomological researches carried out on Policoro Wood are quite recent as they date back to 1956-1958 and the results were announced in a series of works that appeared between 1959 and 1979; the site gained notoriety and in the following years was the object of many explorations by entomologists from all over Italy but only a small number of the results was published in an organized manner, while many data were scattered in several papers. 
The writer, together with his friend Fernando Montemurro, has done research here in the years 1976-1984 and published the overall results in a general catalogue in 1986. After this publication he carried out further research whose data are reported with the abbreviation " $\mathrm{N}$ " in the respective column of Table 1.

Parks of Lucanian Apennines (sites 3, 4, 5 and 6). The Lucan Apennines is a section of the southern Apennines that stretches as an arc from the Sella di Conza (between Campania and Basilicata) to Passo dello Scalone (in Calabria). It is bordered by the Ofanto river to the north, Sele to the west, Bradano to the east, from the Tyrrhenian to the south-west, from the plain of Sibari and the Gulf of Taranto to the south-east and also includes the mountains of $\mathrm{Ci}$ lento in Campania and Orsomarso in Calabria.

The author gathered data all across this wide sector of the Apennines. For some areas he got a significant number of records on the coleopterological fauna (over a thousand species) and each of these areas will be separately treated: 3) Parks of Mount Vulture-Monticchio Lakes, 4) Regional Reserve of Pantano Lake of Pignola, 5) the complex Central Lucan Apennines-Sellata-Volturino-Viggiano-The Lagonegrese and Sirino massif, 6) Pollino National Park (partially). Below is a brief description of these sites, whose location is shown in figure 3 , and respective National and Regional Parks exact designations based on the establishment acts.

On other areas of the Lucan Apennines, namely Cilento and Orsomarso Mountains, particularly interesting data are known as well as records referred to new species, but unfortunately not numerically significant, which is why it was preferred to omit them in this study.

3. Mount Vulture-Monticchio Lakes (Basilicata: Potenza province, Fig. 3) includes both the Parco Naturale Regionale del Vulture (established with L.R. n. 28 of 20.11.2017) and the Riserva Regionale Lago Piccolo di Monticchio (established with D.P.G.R. n. 1183 of 30.08.1984).

The woods of Mount Vulture, an ancient inactive volcano, surround two lakes of volcanic origin and are characterized by the remarkable variety of habitats and by very special microclimatic conditions which allow the presence of a low altitude beech forest (650 $\mathrm{m}$ a.s.l.). The site still has a suffi- cient degree of naturalness despite the massive tourist exploitation.

The first entomological researches on Mount Vulture and its lakes date back to 1880 and were made known two years later in a list including species collected also in other sites. In XX century the place was visited by numerous researchers with data published in several works. The entomological findings deduced from these contributions come from a small area around Mount Vulture: the summit, the territories adjacent the two lakes of Monticchio and the road that links the town of Rionero in Vulture to Mount Vulture.

The writer began occasional explorations in this area in 1970. A particularly intense period of research followed in the years 2000-2003, after which he drafted a report and a detailed list of collected species that he delivered to Basilicata Region.

4. Pantano Lake of Pignola (Basilicata, Potenza province, Fig. 3) was established as Riserva Regionale Lago Pantano di Pignola with D.P.G.R. n. 795 of 19.06.1984.

The lake, which occupies a piedmont depression at $750 \mathrm{~m}$ a.s.l. bounded to the north by Serra Stantiere, to the west by the ridges of Petrucco relief and to the south by the watershed of La Rocca, is managed by WWF and has a fair degree of naturality, although before being fenced it was subject to considerable upheavals. The reduced slope of the banks allows diversified aquatic vegetation, while the meadows and the sparse arboreal vegetation in contact with this ancient wetland are rich in ecological niches that make it an area of high biodiversity.

The only entomological researches on Pantano Lake of Pignola were conducted by the writer in the years 1991-1994 and were carried out along the entire perimeter of the lake, already fenced at the time. The results were released in a special publication by the WWF in 1996. After this publication further research was undertaken, the data of which are shown with the symbol "B" or " $\mathrm{N}$ " in the respective column.

5. Central Lucan Apennines-Central area Province of Potenza (Basilicata: Potenza and Matera provinces, Fig. 3) here refers to the "Sellata-Monte Croccia-Monte Volturino-Viggiano-LagonegreseSirino massif" complex. 
It is a complicated mountain system that constitutes the central backbone of Lucan Apennines, which varies in landscape from the harsh and almost dolomitic aspect of the northern peaks to the more gentle mountains of the Maddalena, to return to the grandeur of Mount Sirino massif and Mount Alpi in the southernmost offshoots. The whole territory is rich in woods that in the lower altitudinal zone are constituted by evergreen oaks and thermophilous broad-leaved trees and in the upper zone by Quercus cerris and mixed beech-woods, as well as some isolated chestnut groves and the residual forest of Abetina di Laurenzana, which hosts a local variety of Abies alba.

The entomological data shown come from places located between the middle and upper course of Basento and Sinni rivers; the north-western border lies between the sources of the Basento river (Fossa Cupa) and the villages of Brienza and Atena Lucana; the south-western boundary runs along the border with Campania region up to Lagonegro and, near Lauria, joins the Sinni river which represents its southern border thus including Mount Sirino and Mount Alpi; the southeast boundary is an ideal line connecting Salandra with Stigliano, Sant'Angelo and Valsinni.

In this area we find the following Reserves and National Parks:

a. Riserva Nazionale Naturale Antropologica Monte Croccia (established with D.M.A.F. of 11.09.1971);

b. Parco Regionale Gallipoli Cognato e Piccole Dolomiti Lucane (established with L.R. n. 47 of 24.11.1997);

c. Riserva Regionale Abetina di Laurenzana (established with D.P.G.R. n. 2 of 4.01.1988);

d. Riserva Regionale Lago Laudemio (established with L.R. n. 42 of 22.05 .1980 and D.P.G.R. n. 426 of 19.04.1985);

e. Parco Nazionale dell'Appennino Lucano Val d'Agri Lagonegrese (established with D.P.R. of 8.12.2007);

f. A small part of Pollino National Park that protrude to the north of Sinni river and which, of significance, includes Mount Alpi.

News of the first research in Central Lucan Apennines dates back only to 1909 and there is no work of synthesis on the Coleoptera fauna; rather, there are many data dispersed in a myriad of works even with description of new species which refer to the second half of the last century.

The writer has carried out several researches, generally of short duration (1-5 days), between 1980 and 2000, but so far he had communicated only single data in contributions dedicated to specific families despite having found over 1,000 Coleoptera species.

6. Pollino Massif (Basilicata: Potenza and Matera provinces, Calabria: Cosenza province, Fig. 3). Parco Nazionale del Pollino was established with Law $n$. 67 of 11.03.1988; with subsequent acts and decrees the perimeter has been extended (Law n. 305 of 28.8.1989, D.M. of 31.12.1990, D.P.R. of 15.11.1993 and D.P.R. of 2.12.1997) so that it currently represents the largest national park in Italy. In it we find Riserva Naturale Biogenetica Statale Rubbio and Riserva Naturale Orientata Gole del Raganello in Basilicata, Riserva Naturale Statale Valle del Fiume Lao and Riserva Naturale Orientata Valle del Fiume Argentino in Calabria (Orsomarso mountains).

The Pollino Massif includes all the highest peaks of southern Apennines, whose forms have been shaped by the ancient glaciations and which for part of the year are covered by snowfields. The alternation of karstic phenomena and morainic deposits makes the landscape very evocative. Some

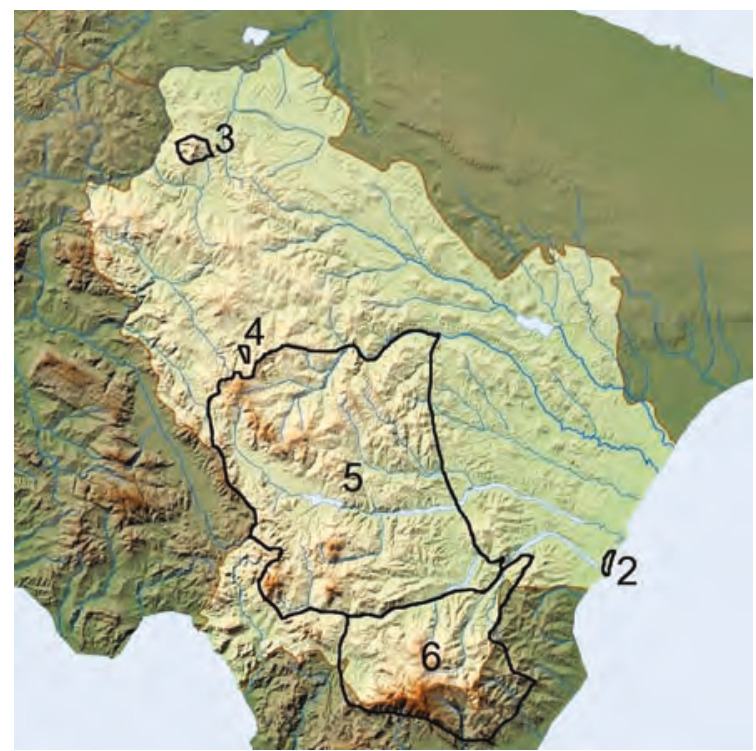

Figure 3. Basilicata (Italy): 2. Policoro Wood; 3. Mount Vulture-Monticchio Lakes; 4. Pantano Lake of Pignola; 5. Central Lucan Apennines; 6. Pollino Massif. 
plant associations are unique to this area, where for example at the highest altitudes rare relict populations of Pinus leucodermis can be found. Also chestnut groves, oak woods and beech woods show noteworthy characteristics.

Considering the origin of most of the data, here it was intended for "Pollino" the whole area that includes the peaks of Monte Pollino, Serra del Prete, Dolcedorme, Serre di Crispo and Ciavole, Coppola di Paola and the neighboring territories; very different is the current perimeter of Pollino National Park extending from the Tyrrhenian to the Ionian.

Boundaries of Pollino Massif pass therefore to the north along the course of Sinni river from Episcopia (Potenza province) to Valsinni (Matera province), to the east and west follow the border with Calabria region but protrude widely from it at Alessandria del Carretto up to Francavilla Marittima where the southern border follows the course of Raganello stream and from here the localities of Frascineto, Morano Calabro, Campotenese and Mormanno. Pollino Massif shows a barely acceptable state of naturalness, although the anthropic impact is definitely excessive and favored both by the construction of new roads and by poor surveillance.

The first research on Pollino Massif dates back to 1880 and was published in 1882 . Later it was the subject of studies whose results were only partially published; starting from the middle of last century wider research campaign were promoted by Naples University and finally the results were published in 17 works dedicated to Coleoptera. Several other researches have been carried out by single entomologist whose data were dispersed in many papers or made known in contributions dedicated to single families.

The writer used to explore Pollino Massif in the years 1969-1985 and he published the overall results in a general catalogue in 1986. Later he further investigated the area until around 2000, collecting nearly 500 further species.

7. Sila Plateau (Calabria: Cosenza, Catanzaro and Crotone provinces, Fig. 4). Parco Nazionale della Sila was established with Law n. 344 of 8.10.1997 and D.P.R. of 14.11.2002. In 2014 the site was included in the UNESCO Man and Biosphere Program.

It is one of the areas that are historically less populated and with the highest percentage of wooded surface of Italy. The plateau is made up of igneous and metamorphic rocks that clearly differentiate it from the calcareous bulk of the Apennines and that influence the form of the landscape, as well as that of the vegetation. In fact, at high altitudes, Pinus nigra laricio dominates and, even higher, Fagus sylvatica, frequently interspersed with Abies alba.

The data considered in this work and often reported by the authors as "Sila" concern an area that extends far beyond the boundaries of Sila National Park, as it includes wide wooded areas to the north and east of Cecita Lake and both east and west of Ampollino Lake as well as to south of the latter. As far as man's impact is concerned, the same is true as for what was said about Pollino Massif, though the situation is slightly better.

The first researches in Sila Plateau date back to 1859, but these results were never disclosed. Later it was the subject of further explorations, of which only some were published in their entirey in 1881, 1941 and 1943, while other data were dispersed in a myriad of contributions. In the 1960s the territory was the subject of a research project promoted by Verona Museum and the results relating to some of the families were announced in several papers. Meanwhile and subsequently the Pollino Massif had become the destination of a large number of entomologists whose results were only partially communicated in numerous contributions.

The writer in the years 1969-1988 visited Sila Plateau several times for periods of 7-15 days and therefore disclosed the results of its collections in a general catalogue in 1991.

8. Aspromonte (Calabria: Reggio Calabria province, Fig. 4). Parco Nazionale dell'Aspromonte was established with Law n. 305 of 28.08.1989, D.P.R. of 14.01.1994 and D.P.R. of 19.07.2008).

This is a mountainous massif with a jagged topography occupying the southernmost tip of the Stivale (the Boot: in a figurative sense, par excellence, Italy, so called because of its geographical shape that resembles that of a boot) and whose flora and fauna are extremely heterogeneous due to multiple factors: the low latitude, the elevation height differences, the presence of tectonic terraces as well as the proximity of the sea and the different exposure of mountainsides respect to this. Like Sila Plateau, Aspromonte consists of rocks more similar to Alpine ones than those of the Apennines, and also here in the woods the Pinus nigra laricio is predominant above $800 \mathrm{~m}$, 
while from the $1,200 \mathrm{~m}$ and at higher altitudes the prevailing tree cover is Fagus sylvatica and Abies alba. A peculiar feature of superficial hydrography is the "fiumara", a characteristic type of watercourse with a very wide pebbly bed and for most of the year dry, but seasonally subject to flash floods.

In this work, Aspromonte is meant in a geographical sense and therefore is that area located south of Petrace river and of "fiumare" of Platì and $\mathrm{Ca}$ reri; it is thus considered a portion of territory that is more than double in area compared to that of the National Park. This choice was made both to include the many data reported over almost two centuries of entomological investigations and to not exclude the fauna of the coasts, which has particularly interesting but different species to that of the mountainous areas.

It should be noted here that mainly the fauna of south-eastern territories, riparian and not, between Locri and Ionian Saline is particularly interesting also because of the different geology, nature of the soil, and the degree of man's influence compared to that of the territory located to the west between Rosarno and Reggio Calabria. The general impact of man's activities is more or less analogous to that of other National Parks of southern Italy, conside-

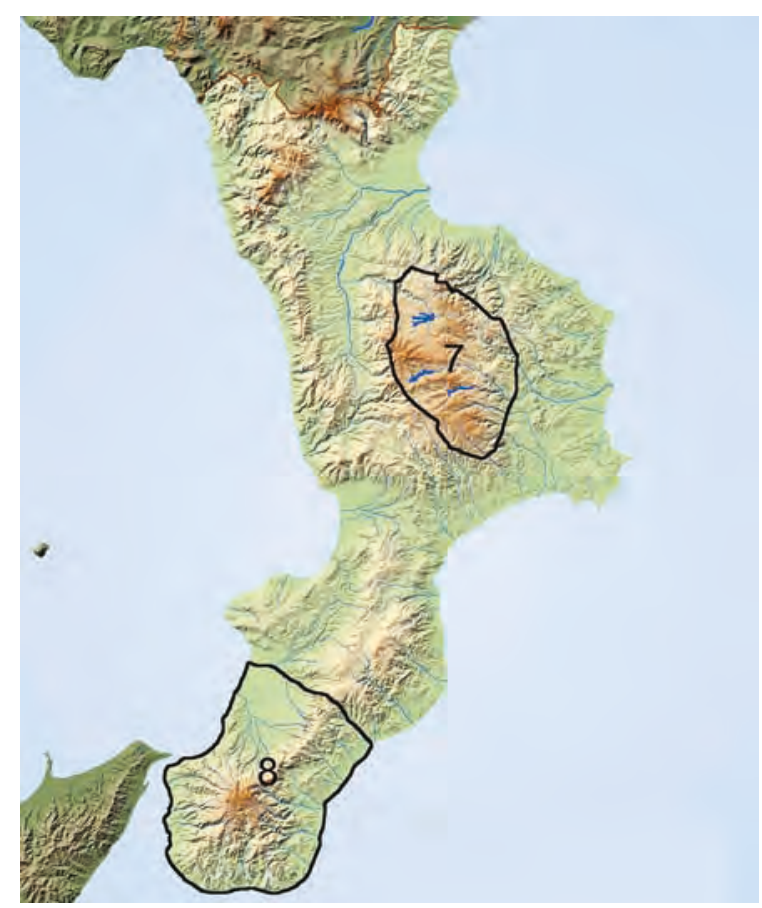

Figure 4. Calabria (Italy): 7. Sila Plateau; 8. Aspromonte. ring that "Park" in this region often means uncontrolled tourism and illegal building rather than carefully safeguarding the natural environments that remain.

Of the eight sites here treated, Aspromonte, approached by sea from Sicily, is the one that was first investigated with research dating back to 1783 , the results being announced three years later. Further studies bear dates of the first half of XIX century demonstrating the attraction that this site had already gained among entomologists two centuries ago. The research continued discontinuously and with data published only partially until the midtwentieth century, when Aspromonte was the object of accurate research by members of the expeditions promoted by Verona Museum and the results concerning some families were announced in various papers. In the following years further short journeys were made by several entomologists, some of whom did not publish any results and other who did so sporadically in various works.

The writer visited Piani d'Aspromonte for the first time around 1975 and until 2000 undertook many and accurate researches, though the results until now were released only minimally in various publications dedicated to single families.

\section{Samples}

In order to integrate and update the previous catalogues, all the publications that could be found were consulted. Every paper bearing data on southern Italy has been carefully examined, and possible synonymies and taxonomic status changes have been checked.

Furthermore, the new records resulting from the research carried out by the author himself after the publication of the aforementioned catalogues are reported.

For field research, a wide range of collecting techniques has been used, mainly on sight, with collecting nets, entomological umbrella, sifting (of leaf litter, rotten wood, hay, mushrooms, dung, soil under stones in meadows or woods, etc.) combined with the use of Winkler/Moczarski extractors, light traps, collecting net for aquatic beetles, aerial and pitfall traps both with and without attractive baits.

All the study material has been identified by specialists but substantial quantities of specimens are still undetermined (17,000 Staphylinidae, 
10,000 Ptiliidae, 7,500 Nitidulidae, 3,700 Scydmaeninae, over 3,000 Mordellidae and Scraptiidae, 2,100 Bruchinae, 1,700 Phalacridae, 1,700 Corylophidae, 1,300 Alexiidae, 600 Clambidae).

Most of the specimens collected by the writer are kept in the author's personal collection, currently deposited at the Museum of Natural History, Section of Zoology, of Florence University.

The results are shown in Table 1, in which the families are listed according to the taxonomic order adopted by Bouchard et al. (2011).

For the nomenclature, the status and the sequence of subfamilies and tribes within of families, reference was made to volumes $1-8$ of the "Catalogue of Palaearctic Coleoptera", with some adjustments according to the works listed below:

Ptiliidae: Sörensson \& Delgado, 2019

Elateroidea: Kundrata et al., 2014

Elateridae: Kundrata et al., 2018

Drilini: Kundrata \& Bocak, 2011

Cebrionini: Kundrata \& Bocak, 2011

Peltidae: Gimmel et al., 2019

Lophocateridae: Gimmel et al., 2019

Trogossitidae: Gimmel et al., 2019

Thymalidae: Gimmel et al., 2019

Rhadalidae: Bocakova et al., 2011; Gimmel et al., 2019

Malachiinae: Gimmel et al., 2019

Dasytinae: Gimmel et al., 2019

Cucujoidea - Coccinelloidea: Robertson et al., 2015

Nitidulidae: Cline et al., 2014

Cybocephalidae: Cline et al., 2014

Teredidae: Robertson et al., 2015

Anamorphidae: Robertson et al., 2015

Mycetaeidae: Robertson et al., 2015

Curculionoidea: Alonso-Zarazaga et al., 2017

Also consulted were the works of Żyła \& Solodovnikov (2019), Vasilikopoulos et al. (2019) and Zhang et al. (2018) but it was considered more appropriate not to take them into account here.

Names, initials and diacritical marks of the various authors have been verified on Bousquet (2016).

Species, contrariwise, are listed in alphabetical order for the simple fact that names of eventual subgenus have been omitted to make the "Species" column more manageable.

The species lists, subdivided by family, were subjected to a critical examination by the colleagues referred to in the "Acknowledgments" chapter, who reviewed the taxonomic status and added unpublished data eventually in their possession.

Taxonomic status of species and of superior categories is updated to 31.12.2019.

Symbols used in Table 1:

$\mathrm{X}$ : species previously reported in the author's catalogues of Gargano Promontory, Policoro Wood, Pantano Lake of Pignola, Pollino Massif and Sila Plateau;

B: new bibliographic data, relating to the areas already mentioned as well as to Mount VultureMonticchio Lakes, Central Lucan Apennines and Aspromonte. In cases where the presence of a species in a given area is documented both in the bibliography and in CKmap, it was preferred to report the record with the symbol "B";

CK: data resulting from "CKmap" (national database) if not otherwise published;

H: Holdhaus citations (1911) concerning Gargano Promontory and omitted in the author's previous contribution to this promontory (Angelini, 1987);

$\mathrm{N}$ : new data from the author, if not already mentioned in other publications and then reported with the symbol "B";

!: records added by specialists who have reviewed the families of their competence: Paolo Audisio (Hydraenidae and Nitidulidae), Cesare Bellò (Curculionidae), Mauro Daccordi (Chrysomelidae), Jiř́ Háva (Dermestidae), Francesco Izzillo (Buprestidae and Cerambycidae), Leonardo Latella (Carabidae), Carlo Leonardi (Alticinae), Gianfranco Liberti (Rhadalidae and Melyridae), Andrea Liberto (Oedemeridae), Gianluca Magnani (Buprestidae), Alessandro Mascagni (Elmidae and Dryopidae), Antonio Mazzei (Carabidae), Giuseppe Platia (Elateridae), Roberto Poggi (Pselaphinae), Saverio Rocchi (Helophoridae), Davide Sassi (Chrysomelidae), Pierpaolo Vienna (Histeridae), Adriano Zanetti (Staphylinidae), Iuri Zappi (Cleridae).

[E]: endemic species.

The "IUCN Category" column shows the IUCN (International Union for the Conservation of Nature and Natural Resources) Categories, as adopted in the Italian Red Lists of saproxylic Coleoptera referred to, compiled by Audisio et al. (2014) and Carpaneto et al. (2015), and the same abbreviations are used. 
Table 1. THE LIST OF SPECIES found in the eight study sites.

\begin{tabular}{|c|c|c|c|c|c|c|c|}
\hline Species & 焉 & $\begin{array}{l}\overline{0} \\
0 \\
\vdots \\
0 \\
\vdots \\
\frac{0}{0} \\
\vdots\end{array}$ & 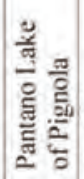 & 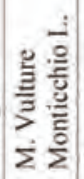 & 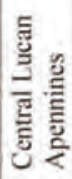 & $\begin{array}{l}\bar{y} \\
\sum_{0}^{0} \\
0 \\
\frac{0}{0}\end{array}$ & \\
\hline
\end{tabular}

COLEOPTERA Linnaeus, 1758

MYXOPHAGA Crowson, 1955

SPHAERIUSOIDEA Erichson, 1845

SPHAERIUSIDAE Erichson, 1845

Hájek in Cat. Pal. Col., vol. 1, 2017

Microsporus acaroides Walt, 1838 N
ADEPHAGA Schellenberg, 1806
GYRINIDAE Latreille, 1810
Gyrininae Latreille, 1810
Gyrinini Latreille, 1810
Hajek \& Fery in Cat. Pal. Col., vol. 1, 2017

Aulonogyrus concinnus (Klug, 1834)

Aulonogyrus striatus (Fabricius, 1792)

Gyrinus caspius Ménétriés, 1832

Gyrimus dejeani Brullé, 1832

Gyrimus distinctus Aubé, 1838

Gyrinus substriatus Stephens, 1828

Gyrinus suffriani W. Scriba, 1855

Gyrinus urinator Illiger, 1807

\begin{tabular}{|l|l|}
\hline & $\mathrm{X}$ \\
\hline & $\mathrm{X}$ \\
\hline $\mathrm{H}$ & $\mathrm{X}$ \\
\hline $\mathrm{H}$ & $\mathrm{X}$ \\
\hline & \\
$\mathrm{X}$ & $\mathrm{X}$ \\
\hline & $\mathrm{X}$ \\
$\mathrm{X}$ & $\mathrm{X}$ \\
\hline
\end{tabular}

\begin{tabular}{|l|l|l|l|l|l|l|l|}
\hline & & N & B & X & X & B & \\
\hline & & N & B & & X & B & \\
\hline & & B & B & & & \\
\hline B & & & & & & \\
\hline X & B & N & X & X & B & \\
\hline & & & & & & \\
\hline
\end{tabular}

Orectochilus villosus villosus (O.F. Müller, 1776)

RHYSODIDAE Laporte, 1840

Clinidiini R.T. Bell \& J.R. Bell, 1978

Huber, Marggi \& Löbl in Cat. Pal. Col., vol. 1, 2017

Clinidium canaliculatum (O.G. Costa, 1839)

Omoglymmiini R.T. Bell \& J.R. Bell, 1978

Omoglymmius germari (Ganglbauer, 1891)

Rhysodini Laporte, 1840

\begin{tabular}{|l|l|l|l|l|l|l|l|}
$\mathrm{X}$ & $\mathrm{X}$ & $\mathrm{B}$ & $\mathrm{B}$ & $\mathrm{B}$ & & $\mathrm{N}$ & $\mathrm{CR}$ \\
\hline
\end{tabular}

Rhysodes sulcatus (Fabricius, 1787)

$\mathrm{B}$

B $\quad$ B

\begin{tabular}{|l|l|l|l|}
\hline & & \\
\hline
\end{tabular}

CARABIDAE Latreille, 1802

Nebriinae Laporte, 1834

Nebriini Laporte, 1834

Huber in Cat. Pal. Col., vol. 1, 2017 (Genus Leistus: Farkač)

Eurynebria complanata (Linnaeus, 1767)

Leistus ferrugineus (Linnaeus, 1758)

Leistus fulvibarbis fulvibarbis Dejean, 1826

Leistus parvicollis Chaudoir, 1869

Leistus rufomarginatus (Duftschmid, 1812)

Leistus spinibarbis fiorii Lutshnik, 1913 [E]

Nebria andalusia Rambur, 1837

Nebria brevicollis (Fabricius, 1792)

Nebria kratteri kratteri Dejean, 1831 [E]

Nebria pennisii Magrini, 1987 [E]

Nebria psammodes (P. Rossi, 1792)

Notiophilini Motschulsky, 1850

\begin{tabular}{|c|c|c|c|c|c|c|c|c|}
\hline X & & & & & & & B \\
\hline H & X & X & B & B & X & X & B & \\
\hline H & & & & & & & & \\
\hline H & & & & N & X & & B & \\
\hline B? & & X & B & B & X & X & B & \\
\hline & & & & & & & B & \\
\hline H & X & & B & B & X & X & B & \\
\hline & & & B & B & X & X & B & \\
\hline & & & & B & & & & \\
\hline H & X & & B & N & X & X & B & \\
\hline
\end{tabular}

Bousquet in Cat. Pal. Col., vol. 1, 2017

Notiophilus aestuans Dejean, 1826

Notiophilus aquaticus (Linnaeus, 1758)

Notiophilus biguttatus (Fabricius, 1779)

\begin{tabular}{|l|l|l|l|l|l|l|l|l|}
\hline & N & & N & & X & X & B & \\
\hline & & & & & X & & B & \\
\hline X & & & B & N & X & X & B & \\
\hline
\end{tabular}


Species

Notiophilus geminatus Dejean, 1831

Notiophilus germinyi Fauvel, 1863

Notiophilus palustris (Duftschmid, 1812)

Notiophilus quadripuncratus Dejean, 1826

Notiophilus rufipes Curtis, 1829

Notiophilus substriatus G.R. Waterhouse, 1833

Carabinae Latreille, 1802

\begin{tabular}{|c|c|c|c|c|c|c|c|c|}
\hline 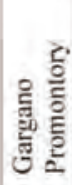 & $\begin{array}{l}\square \\
0 \\
0 \\
0 \\
0 \\
\frac{.0}{0} \\
2\end{array}$ & 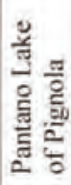 & 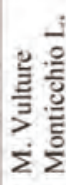 & 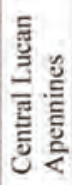 & 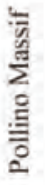 & 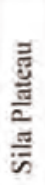 & 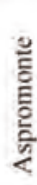 & 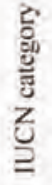 \\
\hline \multirow[t]{3}{*}{$\mathrm{X}$} & $\mathrm{X}$ & $X$ & B & & $\mathrm{X}$ & $\mathrm{X}$ & B & \\
\hline & & & & & & & $\mathrm{N}$ & \\
\hline & & & B & B & & & & \\
\hline $\mathrm{H}$ & & & & & & B & B & \\
\hline $\mathrm{H}$ & $\mathrm{X}$ & & B & B & $\mathrm{X}$ & $\mathrm{X}$ & B & \\
\hline $\mathrm{H}$ & $\mathrm{X}$ & $\mathrm{X}$ & B & & $X$ & $\mathrm{X}$ & B & \\
\hline
\end{tabular}

Carabini Latreille, 1802

Calosomatina Lapouge, 1927

Hăckel in Cat. Pal. Col., vol. 1, 2017

Calosoma inquisitor inquisitor (Linnaeus, 1758)

Calosoma maderae maderae (Fabricius, 1775)

Calosoma sycophanta sycophanta (Linnaeus, 1758)

\begin{tabular}{|l|l|l|l|l|l|l|l|l|}
\hline H & X & & N & & X & X & & \\
\hline X & X & & & & & & B & \\
\hline N & X & & N & B & X & B & B & \\
\hline
\end{tabular}

Carabina Latreille, 1802

Brezina, Huber \& Marggi in Cat. Pal. Col., vol. I, 2017

Carabus convexus convexus Fabricius, 1775

Carabus coriaceus coriaceus Linnaeus, 1758

Carabus coriaceus bruttianus Born, 1906 [E]

Carabus gramulatus aetolicus Schaum, 1857

Carabus gramulatus calabricus Spettoli \& Vigna Taglianti, 2001

[E]

Carabus lefebvrei bayardi Solier, 1835 [E]

Carabus lefebvrei lefebvrei Dejean, 1826 [E]

Carabus morbillosus alternans Palliardi, 1825 [E]

Carabus preslii neumeyeri Schaum, 1856

Carabus rossii Dejean, 1826 [E]

Carabus violacens picenus A. Villa \& G.B. Villa, 1839

\begin{tabular}{|c|c|c|c|c|c|c|c|c|}
\hline H & & & B & CK & X & CK & B & \\
\hline H & X & X & N & CK & X & & & \\
\hline & & & & & & B & B & \\
\hline & & & & & N & & & \\
\hline H & X & & CK & B & X & X & & \\
\hline & & & & & & & B & \\
\hline & & & B & B & X & X & B & \\
\hline H & & & B & N & CK & X & B & \\
\hline X & & X & B & CK & X & X & B \\
\hline
\end{tabular}

Cychrini Perty, 1830

Cychrina Perty, 1830

Häckel in Cat. Pal. Col., vol. 1, 2017

Cychrus attenuatus latialis Luigioni, 1922 [E]

Cychrus italicus Bonelli, 1810

Cicindelinae Latreille, 1802

Cicindelini Latreille, 1802

Cicindelina Latreille, 1802

Putchkov \& Matalin in Cat. Pal. Col., vol. 1, 2017

Calomera littoralis nemoralis (A.G. Olivier, 1790)

Cassolaia maura cupreothoracica Korell \& Cassola, 1987

Cephalota circumdata circumdata (Dejean, 1822)

Cicindela campestris campestris Linnaeus, 1758

Cicindela majalis Mandl, 1935 [E]

Cylindera arenaria arenaria Fuessly, 1775

Cylindera germanica muelleri (Magistretti, 1966)

Cylindera trisignata trisignata (Dejean, 1822)

Myriochila melancholica melancholica (Fabricius, 1798)

\begin{tabular}{|c|c|c|c|c|c|c|c|c|}
\hline $\mathrm{H}$ & $\mathrm{X}$ & & & & & & $\mathrm{B}$ & \\
\hline & & & & & & & $\mathrm{B}$ & \\
\hline & $\mathrm{B}$ & & & & & & & \\
$\mathrm{X}$ & $\mathrm{X}$ & $\mathrm{X}$ & & $\mathrm{B}$ & $\mathrm{X}$ & $\mathrm{X}$ & $\mathrm{B}$ & \\
\hline & $\mathrm{X}$ & & & $\mathrm{B}$ & & $\mathrm{X}$ & $\mathrm{N}$ & \\
\hline & $\mathrm{X}$ & & & & & & & \\
\hline $\mathrm{X}$ & $\mathrm{X}$ & & & & & & & \\
\hline $\mathrm{X}$ & $\mathrm{X}$ & & & & & & & \\
\hline $\mathrm{X}$ & & & & & & & & \\
\hline
\end{tabular}

Loricerinae Bonelli, 1810

Loricerini Bonelli, 1810

Bousquet in Cat. Pal. Col., vol. 1, 2017

Loricera pilicornis pilicornis (Fabricius, 1775)

Elaphrinae Latreille, 1802

Elaphrini Latreille, 1802

Goulet in Cat. Pal. Col., vol. 1, 2017 


\begin{tabular}{|c|c|c|c|c|c|c|c|c|c|}
\hline Species & 总产 & 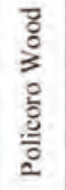 & 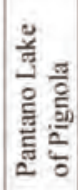 & 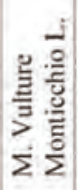 & 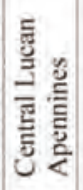 & 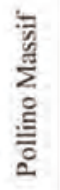 & 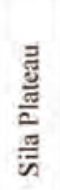 & 总 & 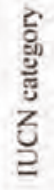 \\
\hline Elaphrus uliginosus Fabricius, 1792 & & & & & $\mathrm{~N}$ & $\mathrm{~N}$ & $\mathrm{X}$ & & \\
\hline \multicolumn{10}{|c|}{$\begin{array}{c}\text { Omophroninae Bonelli, } 1810 \\
\text { Omophronini Bonelli, } 1810 \\
\text { Iuber, Marggi \& Valainis in Cat. Pal. Col., vol }\end{array}$} \\
\hline Omophron limbatum (Fabricius, 1777) & $\mathrm{X}$ & $\mathrm{X}$ & & & $\mathrm{N}$ & $\mathrm{N}$ & B & B & \\
\hline \multicolumn{10}{|c|}{$\begin{array}{l}\text { Scaritinae Bonelli, } 1810 \\
\text { Clivinini Rafinesque, } 1815 \\
\text { Clivinina Rafinesque, } 1815 \\
\text { Balkenhol in Cat. Pal. Col., vol. 1, } 2017\end{array}$} \\
\hline Clivina collaris (Herbst, 1784) & & $\mathrm{N}$ & & & & $\mathrm{N}$ & B & $\mathrm{N}$ & \\
\hline Clivina fossor fossor (Linnaeus, 1758) & $\mathrm{X}$ & $\mathrm{X}$ & $X$ & $\mathrm{~N}$ & $\mathrm{~N}$ & $\mathrm{X}$ & & B & \\
\hline Clivina laevifrons Chaudoir, 1842 & & $\mathrm{~N}$ & & & & & & & \\
\hline Reicheia italica Holdhaus, 1924 [E] & & & & $\mathrm{N}$ & B & $\mathrm{N}$ & $\mathrm{N}$ & B & \\
\hline Typhloreicheia usslaubi (Saulcy, 1870) [E] & $\mathrm{H}$ & & & & B & & & & \\
\hline \multicolumn{10}{|c|}{ Dyschiriini H.J. Kolbe, 1880} \\
\hline Dyschirius apicalis Putzeys, 1846 & $\mathrm{X}$ & $\mathrm{X}$ & & & & & & B & \\
\hline Dyschirius auriculatus auriculatus Wollaston, 1867 & & & & & & & & B & \\
\hline Dyschirius chalybeus chalybeus Putzeys, 1846 & & $\mathrm{X}$ & $\mathrm{N}$ & & & & & $\mathrm{N}$ & \\
\hline Dyschirius cylindricus hauseri A. Fleischer, 1898 & B & & & & & & & & \\
\hline Dyschirius gracilis gracilis (Heer, 1837) & & $\mathrm{N}$ & & & & & $\mathrm{X}$ & & \\
\hline Dyschirius importumus importumus Schaum, 1857 & $\mathrm{X}$ & $\mathrm{X}$ & & & $\mathrm{N}$ & & & B & \\
\hline Dyschirius intermedius Putzeys, 1846 & & $\mathrm{X}$ & & & & & & & \\
\hline Dyschirius laeviusculus Putzeys, 1846 & & $\mathrm{~N}$ & & & & & & & \\
\hline Dyschirius luticola luticola Chaudoir, 1850 & $\mathrm{X}$ & $\mathrm{X}$ & & & & & & & \\
\hline Dyschirius minutus mimutus (Dejean, 1825) & & & & & & & & $\mathrm{B}$ & \\
\hline Dyschirius nitidus nitidus (Dejean, 1825) & & $\mathrm{X}$ & & & & & & & \\
\hline Dyschirius numidicus numidicus Putzeys, 1846 & $\mathrm{X}$ & $\mathrm{X}$ & & & & & & & \\
\hline Dyschirius parallelus ruficornis Putzeys, 1846 & & $\mathrm{X}$ & & & & & & & \\
\hline Dyschirius punctatus (Dejean, 1825 ) & & $\mathrm{X}$ & & & & & & B & \\
\hline Dyschirius salimus salimus Schaum, 1843 & & $\mathrm{X}$ & & & & & & & \\
\hline Dyschirius strumosus Erichson, 1837 & & $\mathrm{~N}$ & & & & & & & \\
\hline Dyschirius substriatus substriatus (Duftschmid, 1812) & & $\mathrm{X}$ & & & & & & & \\
\hline \multicolumn{10}{|c|}{$\begin{array}{l}\text { Scaritini Bonelli, } 1810 \\
\text { Scaritina Bonelli, } 1810\end{array}$} \\
\hline Distichus planus (Bonelli, 1813) & $\mathrm{X}$ & & & & & & & B & \\
\hline Scariles buparius (Forster, 1771) & $\mathrm{X}$ & $\mathrm{X}$ & & & & & & B & \\
\hline Scarites laevigatus Fabricius, 1792 & $\mathrm{X}$ & $\mathrm{X}$ & & & & & & B & \\
\hline Scarites terricola terricola Bonelli, 1813 & $\mathrm{X}$ & $\mathrm{X}$ & & & & & & $\mathrm{B}$ & \\
\hline \multicolumn{10}{|c|}{$\begin{array}{l}\text { Apotominae LeConte, } 1853 \\
\text { Wrase in Cat. Pal. Col., vol. 1, } 2017\end{array}$} \\
\hline Apotomus nifithorax Pecchioli, 1837 & & $\mathrm{~N}$ & B & & & & & & \\
\hline Apotomus rufus (P. Rossi, 1790) & & & & & & & & B & \\
\hline $\begin{array}{r}\text { Siagonin } \\
\text { Siagon } \\
\text { Huber \& Marggi i }\end{array}$ & $\begin{array}{l}\text { i, } 181 \\
1813 \\
\text { col., vo }\end{array}$ & 1. 1,2 & 2017 & & & & & & \\
\hline Siagona europaea europaea Dejean, 1826 & & $\mathrm{~B}$ & & $\mathrm{~N}$ & $\mathrm{~N}$ & $\mathrm{X}$ & $\mathrm{B}$ & B & \\
\hline $\begin{array}{r}\begin{array}{r}\text { Trechin } \\
\text { Anillir }\end{array} \\
\text { Zaballos, Pérezgonzáles, Andújar Fen }\end{array}$ & $\begin{array}{l}181 \\
1937 \\
\text { achino }\end{array}$ & in $\mathrm{Ca}$ & at. Pal. & $\mathrm{Col}_{\text {, }}$ & vol. 1 & 201 & & & \\
\hline Anillus bordonii Magrini \& Vanni, 1993 [E] & & & & & B & & & $\mathrm{B}$ & \\
\hline Rhegmatobius fiorii (Ganglbauer, 1900) [E] & & & & & B & & & & \\
\hline
\end{tabular}




\section{Species}

Rhegmatobius solarii Magrini \& Sciaky, 1995 [E]

\section{Bembidiini Stephens, 1827}

Bembidiina Stephens, 1827

Marggi, Toledano \& Neri in Cat. Pal. Col., vol. 1, 2017

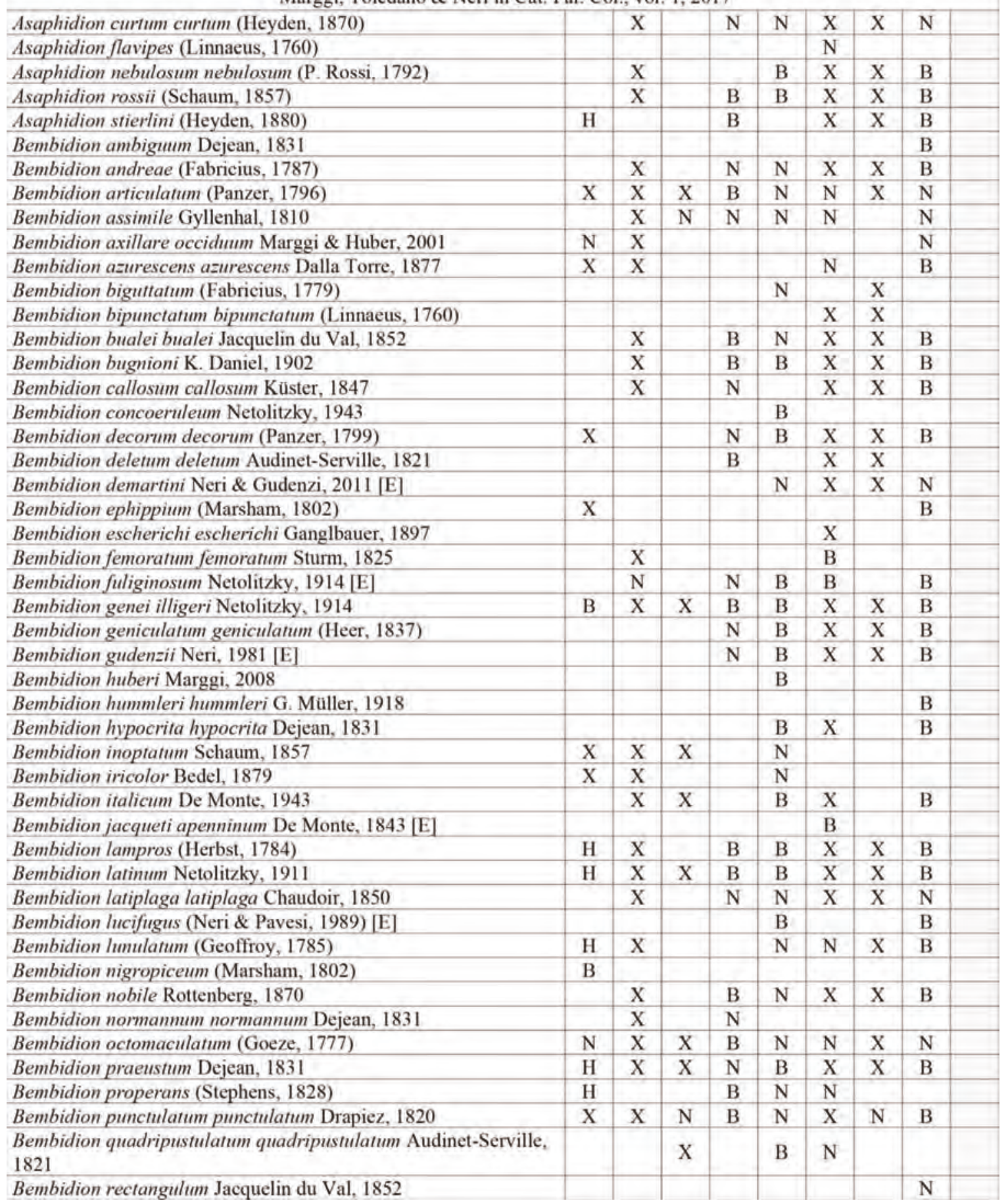




\begin{tabular}{|c|c|c|c|c|c|c|c|c|c|}
\hline Species & 吾 & $\begin{array}{l}\overrightarrow{0} \\
\vdots \\
3 \\
0 \\
\frac{0}{0} \\
\vdots \\
0\end{array}$ & 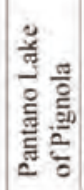 & 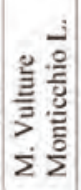 & 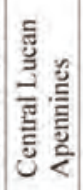 & 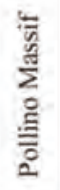 & 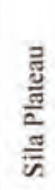 & 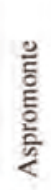 & 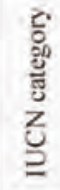 \\
\hline Bembidion scapulare scapulare Dejean, 1831 & & $\mathrm{X}$ & & & & $\mathrm{N}$ & & B & \\
\hline Bembidion semipunctatum (Donovan, 1806 ) & & & & & $\mathrm{N}$ & & & & \\
\hline Bembidion siculum siculum Dejean, 1831 [E] & & & & & B & & & B & \\
\hline Bembidion steimbuehleri steinbuehleri Ganglbauer, 1891 & B & & & & & & & & \\
\hline Bembidion subfasciatum Chaudoir, 1850 & $\mathrm{X}$ & $\mathrm{B}$ & & & & & & & \\
\hline Bembidion tenellum tenellum Erichson, 1837 & $\mathrm{X}$ & $\mathrm{N}$ & $\mathrm{X}$ & $\mathrm{N}$ & $\mathrm{N}$ & $\mathrm{N}$ & & $\mathrm{B}$ & \\
\hline Bembidion testaceum testaceum (Duftschmid, 1812) & & $\mathrm{X}$ & & & & & & & \\
\hline Bembidion tethys Netolitzky, 1926 & $\mathrm{X}$ & $\mathrm{N}$ & & & B & $\mathrm{N}$ & & & \\
\hline Bembidion tetracolum tetracolum Say, 1823 & & $\mathrm{~N}$ & & & $\mathrm{~N}$ & $\mathrm{~N}$ & & $\mathrm{~N}$ & \\
\hline Bembidion varicolor varicolor (Fabricius, 1803 ) & & & & & & $\mathrm{N}$ & $\mathrm{N}$ & $\mathrm{B}$ & \\
\hline Bembidion varium (A.G. Olivier, 1795) & & $\mathrm{N}$ & $\mathrm{X}$ & & $\mathrm{N}$ & $\mathrm{N}$ & & $\mathrm{B}$ & \\
\hline Bembidion vseteckai dissimile G. Müller, 1943 & & & & & & & & $\mathrm{~B}$ & \\
\hline Ocys harpaloides (Audinet-Serville, 1821) & & $\mathrm{X}$ & $\mathrm{N}$ & $\mathrm{N}$ & B & & B & B & \\
\hline Sinechostictus cribrum stenacrus (De Monte, 1947) [E] & & & & & & & & $\mathrm{B}$ & \\
\hline Sinechostictus dahlii (Dejean, 1831) & & $\mathrm{X}$ & $\mathrm{X}$ & & B & $\mathrm{N}$ & $\mathrm{X}$ & B & \\
\hline Sinechostictus doderoi (Ganglbauer, 1891) & & & & & B & & & & \\
\hline Sinechostictus elongatus (Dejean, 1831) & & & & & $\mathrm{N}$ & & & $\mathrm{B}$ & \\
\hline Sinechostictus imustus (Jacquelin du Val, 1857) & & & & & B & & & & \\
\hline Sinechostictus solarii (G. Müller, 1918) [E] & & & & & B & & & & \\
\hline
\end{tabular}

Tachyina Motschulsky, 1862

Kopencký in Cat. Pal. Col., vol. 1, 2017

Polyderis algiricus (P.H. Lucas, 1846)

Polyderis brevicornis (Chaudoir, 1846)

Porotachys bisulcatus (Nicolai, 1822)

Tachys bistriatus bistriatus (Duftschmid, 1812)

Tachys fulvicollis (Dejean, 1831)

Tachys micros (Fischer von Waldheim, 1828)

Tachys scutellaris scutellaris Stephens, 1828

Tachyta nana nana (Gyllenhal, 1810)

Tachyura diabrachys (Kolenati, 1845)

Tachyura haemorrhoidalis (Ponza, 1805)

Tachyura parvula (Dejean, 1831)

Tachyura sexstriata (Duftschmid, 1812)

Tachyura thoracica (Kolenati, 1845)

\begin{tabular}{|c|c|c|c|c|c|c|c|c|}
\hline H & X & & & & & & B & \\
\hline & N & & & & & & & \\
N & X & N & & N & N & X & B & \\
\hline H & X & X & N & N & N & & N & \\
\hline & X & X & B & & & & & \\
X & N & & & & & & B & \\
\hline X & X & & N & N & & & B & \\
\hline H & & & & & X & B & & \\
\hline X & X & X & B & N & N & X & B & \\
\hline X & X & X & & N & & & B & \\
\hline & N & X & & & & & B & \\
\hline & & X & & N & X & X & B & \\
\hline & & & & N & & & N & \\
\hline
\end{tabular}

Pogonini Laporte, 1834

Bousquet in Cat. Pal. Col., vol. 1, 2017

Pogonistes gracilis (Dejean, 1828)

Pogonus chalceus chalceus (Marsham, 1802)

Pogonus gilvipes Dejean, 1828

Pogonus littoralis (Duftschmid, 1812)

Pogonus riparius Dejean, 1828

\begin{tabular}{|c|c|c|c|c|c|c|c|c|}
\hline & $\mathrm{B}$ & & & & & & & \\
\hline $\mathrm{X}$ & & & & & & & $\mathrm{B}$ & \\
\hline & $\mathrm{X}$ & & & & & & & \\
\hline $\mathrm{X}$ & $\mathrm{X}$ & & & & & & & \\
\hline
\end{tabular}

Trechini Bonelli, 1810

Perileptina Sloane, 1903

Belousov in Cat. Pal. Col., vol. 1, 2017

Perileptus areolatus areolatus (Creutzer, 1799)

Trechina Bonelli, 1810

Duvalius caoduroi Pace, 1986 [E]

Trechus angelae Magrini, 1984 [E]

Trechus arthuri P. Moravec \& Lompe, 2003 [E]

Trechus binotatus Putzeys, 1870

Trechus obtusus obtusus Erichson, 1837

Trechus quadristriatus (Schrank, 1781)

Trechus subnotatus Dejean, 1831

\begin{tabular}{|l|l|l|l|l|l|l|l|}
$\mathrm{N}$ & $\mathrm{X}$ & $\mathrm{X}$ & $\mathrm{B}$ & $\mathrm{N}$ & $\mathrm{X}$ & & $\mathrm{B}$
\end{tabular}


Species

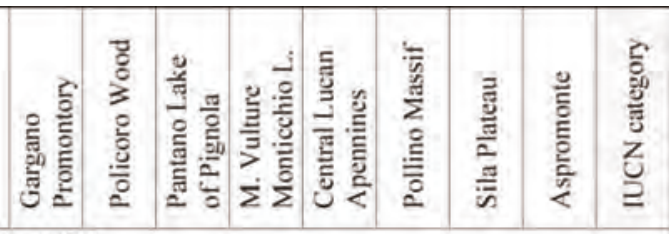

Trechodina Jeannel, 1926

Thalassophilus longicornis (Sturm, 1825)

\section{Brachininae Bonelli, 1810 \\ Brachinini Bonelli, 1810 \\ Brachinina Bonelli, 1810}

Hrdlička in Cat. Pal. Col., vol. 1, 2017

Brachinus bayardi Dejean, 1831

Brachinus brevicollis Motschulsky, 1844

Brachimus crepitans (Linnaeus, 1758)

Brachinus elegans Chaudoir, 1842

Brachimus exhalans (P. Rossi, 1792)

Brachinus explodens Duftschmid, 1812

Brachinus immaculicornis immaculicornis Dejean, 1826

Brachinus italicus (Dejean, 1831) [E]

Brachinus nigricornis Gebler, 1830

Brachinus plagiatus Reiche, 1868

Brachinus psophia Audinet-Serville, 1821

Brachinus sclopeta (Fabricius, 1792)

\begin{tabular}{|c|c|c|c|c|c|c|c|}
\hline & $X$ & & & & & & \\
\hline & & & & & & B & \\
\hline H & $X$ & X & $\mathrm{N}$ & $\mathrm{N}$ & X & B & B \\
\hline $\mathrm{H}$ & & & & & & & \\
\hline X & $X$ & & & & & & \\
\hline $\mathrm{H}$ & & & 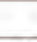 & B & X & X & $\mathrm{N}$ \\
\hline & X & & B & & & B & B \\
\hline & $X$ & X & $\mathrm{N}$ & $\mathrm{N}$ & $\mathrm{N}$ & $\mathrm{X}$ & B \\
\hline $\mathrm{N}$ & X & & $\mathrm{N}$ & & & & \\
\hline $\mathrm{H}$ & $\mathrm{X}$ & $X$ & & & & & \\
\hline & $\mathrm{N}$ & & & & $\mathrm{N}$ & B & B \\
\hline $\mathrm{H}$ & $\mathrm{X}$ & & $\mathrm{N}$ & $\mathrm{N}$ & $\mathrm{X}$ & B & \\
\hline
\end{tabular}

\section{Harpalinae Bonelli, 1810 \\ Chlaeniini Brullé, 1834 \\ Callistina Laporte, 1834}

Kirschenhofer in Cat. Pal. Col., vol. 1, 2017

Callistus lumatus lunatus (Fabricius, 1775)

Chlaenius chrysocephalus (P. Rossi, 1790)

Chlaenius circumscriptus (Duftschmid, 1812)

Chlaenius decipiens (L. Dufour, 1820)

Chlaenius festivus (Panzer, 1796)

Chlaenius nigricornis (Fabricius, 1787)

Chlaenius nitidulus (Schrank, 1781)

Chlaenius olivieri Crotch, 1871

Chlaenitus spoliatus spoliatus (P. Rossi, 1792)

Chlaenius terminatus Dejean, I826

Chlaenius tristis tristis (Schaller, 1783)

Chlaenius velutimus (Duftschmid, 1812)

Chlaenius vestitus (Paykull, 1790)

Drypta dentata (P. Rossi, 1790)

Drypta distincta (P. Rossi, 1792)

Harpalini Bonelli, 1810

Chlaeniina Brullé, 1834

Amblystomina Fauvel, 1889

Wrase in Cat. Pal. Col., vol. I, 2017

Amblystomus metallescens (Dejean, 1829)

Amblystomus niger (Heer, 1841)

Amblystomus solsky Reiche, 1864

\begin{tabular}{|l|l|l|l|l|l|l|l|l|}
\hline $\mathrm{X}$ & $\mathrm{X}$ & & $\mathrm{N}$ & $\mathrm{N}$ & $\mathrm{X}$ & & $\mathrm{B}$ & \\
\hline $\mathrm{X}$ & $\mathrm{X}$ & $\mathrm{X}$ & & & & & & \\
\hline & $\mathrm{X}$ & & & & & & $\mathrm{B}$ & \\
\hline
\end{tabular}

Anisodactylina Lacordaire, 1854

Kataev \& Wrase in Cat. Pal. Col., vol. 1, 2017

\begin{tabular}{|l|c|c|c|c|c|c|c|c|}
\hline Anisodactylus binotatus (Fabricius, 1787) & H & X & & B & B & X & X & B \\
\hline Anisodactylus intermedius Dejean, 1829 & & X & & & & & \\
\hline Anisodactylus poeciloides poeciloides (Stephens, 1828) & X & N & & & & & \\
\hline Anisodactylus pueli bucciarellii Sciaky, 1979 [E] & B & & & B & & & \\
\hline Anisodactylus virens virens Dejean, 1829 & X & X & & & & \\
\hline Diachromus germamus (Linnaeus, 1758) & H & X & N & B & N & & X & N \\
\hline
\end{tabular}


Gvnandromorphus etruscus (Quensel, 1806)

Scybalicus oblongiusculus (Dejean, 1829)

Ditomina Bonelli, 1810

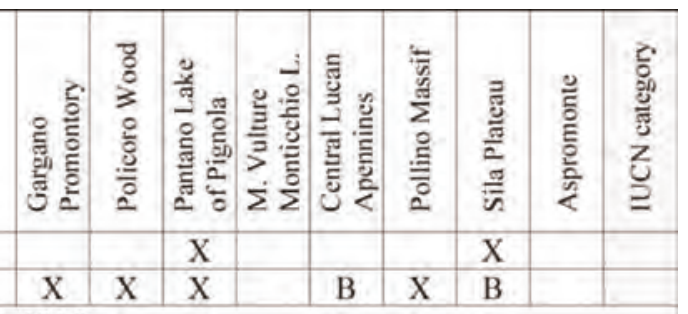

Wrase \& Kataev in Cat. Pal. Col., vol. 1, 2017

Carterus dama (P. Rossi, 1792)

Carterus fulvipes (Latreille, 1817)

Carterus gilvipes (Piochard de la Brûlerie, 1873)

Carterus rotundicollis Rambur, 1837

Ditomus calydonius calydonius (P. Rossi, 1790)

Ditomus tricuspidatus (Fabricius, 1792)

Dixus clypeatus (P+Rossi, 1790)

Dixus obscurus (Dejean, 1825)

Graniger femoralis (Coquerel, 1859)

Oedesis caucasicus (Dejean, 1831)

Oedesis villosulus (Reiche, 1860)

Tschitscherinellus cordatus cordatus (Dejean, 1825)

\begin{tabular}{|c|c|c|c|c|c|c|}
\hline \multirow{3}{*}{$\mathrm{H}$} & & & & & & \\
\hline & & & & & B & B \\
\hline & & & $\mathrm{N}$ & & & B \\
\hline & & & & B & & B \\
\hline $\mathrm{N}$ & $X$ & $\mathrm{~N}$ & & $\mathrm{X}$ & B & B \\
\hline $\mathrm{H}$ & $\mathrm{X}$ & & B & & B & B \\
\hline $\mathrm{H}$ & & & & & $\mathrm{X}$ & B \\
\hline $\mathrm{H}$ & $\mathrm{N}$ & & & $\mathrm{X}$ & $\mathrm{X}$ & B \\
\hline \multirow[t]{5}{*}{$\mathrm{X}$} & $\mathrm{N}$ & & & & & \\
\hline & & & B & & & \\
\hline & B & & & & & \\
\hline & & & & & & B \\
\hline & & & & & B & \\
\hline
\end{tabular}

Harpalina Bonelli, 1810

Kataev \& Wrase in Cat. Pal. Col., vol. 1, 2017

Acinopus ammophilus Dejean, 1829

Acinopus baudii baudii A. Fiori, 1913 [E]

Acinopus megacephalus (P. Rossi, 1794)

Acinopus picipes (A.G. Olivier, 1795)

Daptus vittahis Fischer von Waldheim, 1823

Harpalus affinis (Schrank, 1781)

Harpalus anxius (Duftschmid, 1812)

Harpalus atratus Latreille, 1804

Harpalus attemuatus Stephens, 1828

Harpalus calceatus Duftschmid, 1812

Harpalus cupreus cupreus Dejean, 1829

Harpalus decipiens Dejean, 1829

Harpalus dimidiatus (P. Rossi, 1790)

Harpalus distinguendus distinguendus (Duftschmid, 1812)

Harpalus flavicornis flavicornis Dejean, 1829

Harpalus griseus (Panzer, 1796)

Harpalus honestus (Duftschmid, 1812)

Harpalus italus Schaum, 1860

Harpalus litigiosus Dejean, 1829

Harpalus melancholicus reicheianus Kataev, 2012

Harpalus oblitus oblitus Dejean, 1829

Harpalus pumilus Sturm, 1818

Harpalus punctatostriatus Dejean, 1829

Harpalus pygmaeus Dejean, 1829

Harpalus rubripes (Duftschmid, 1812)

Harpalus rufipalpis rufipalpis Sturm, 1818

Harpalus rufipes (DeGeer, 1774)

Harpalus serripes serripes (Quensel, 1806)

Harpalus siculus Dejean, 1829

Harpalus smaragdimus (Duftschmid, 1812)

Harpalus sulphuripes sulphuripes Germar, 1823

Harpalus tardus (Panzer, 1796)

Harpalus tenebrosus tenebrosus Dejean, 1829

Ophomus ardosiacus (Lutshnik, 1922)

Ophomus azureus (Fabricius, 1775)

\begin{tabular}{|c|c|c|c|c|c|c|c|c|}
\hline & $X$ & & & & & B & $\mathrm{N}$ & \\
\hline & & & & & & & B & \\
\hline X & & & $\mathrm{N}$ & & $\mathrm{X}$ & $X$ & B & \\
\hline $\mathrm{H}$ & $\mathrm{X}$ & & $\mathrm{N}$ & & $\mathrm{X}$ & $\mathrm{X}$ & & \\
\hline & B & & & & & & & \\
\hline X & $\mathrm{N}$ & & B & B & $\mathrm{X}$ & $X$ & B & \\
\hline $\mathrm{H}$ & & & B & $\mathrm{N}$ & & & & \\
\hline X & & & B & B & $\mathrm{N}$ & $X$ & B & \\
\hline X & $X$ & & $\mathrm{~N}$ & & $\mathrm{X}$ & $\mathrm{X}$ & & \\
\hline B & & & & & & & & \\
\hline $\mathrm{H}$ & & $\mathrm{X}$ & $\mathrm{N}$ & B & $\mathrm{N}$ & B & B & \\
\hline X & & & B & & X & $X$ & B & \\
\hline $\mathrm{H}$ & X & $\mathrm{X}$ & $\mathrm{N}$ & B & $\mathrm{X}$ & & $\mathrm{N}$ & \\
\hline $\mathrm{H}$ & $X$ & & $\mathrm{~N}$ & B & $\mathrm{X}$ & $X$ & B & \\
\hline $\mathrm{H}$ & & $\mathrm{X}$ & B & $\mathrm{N}$ & & & $\mathrm{N}$ & \\
\hline X & $X$ & $\mathrm{X}$ & & & $\mathrm{N}$ & & B & \\
\hline $\mathrm{H}$ & & & $\mathrm{N}$ & B & $\mathrm{X}$ & & $\mathrm{N}$ & \\
\hline $\mathrm{H}$ & & $\mathrm{X}$ & & & & & & \\
\hline $\mathrm{X}$ & & & & & & & & \\
\hline X & $X$ & & & & X & & B & \\
\hline $\mathrm{H}$ & & & $\mathrm{N}$ & B & X & $\mathrm{X}$ & & \\
\hline & & & & & & $X$ & B & \\
\hline & & & & $\mathrm{N}$ & & & & \\
\hline X & & & & & & & & \\
\hline$X$ & $X$ & $\mathrm{X}$ & B & $\mathrm{N}$ & $\mathrm{X}$ & $\mathrm{X}$ & B & \\
\hline & & & & & & B & & \\
\hline $\mathrm{H}$ & $X$ & $\mathrm{X}$ & B & $\mathrm{N}$ & $\mathrm{X}$ & $X$ & B & \\
\hline $\mathrm{H}$ & $X$ & & B & B & $\mathrm{X}$ & $\mathrm{X}$ & B & \\
\hline & & & & & & & B & \\
\hline & $\mathrm{N}$ & & $\mathrm{N}$ & $\mathrm{N}$ & & & & \\
\hline $\mathrm{H}$ & $\mathrm{N}$ & & B & B & B & $\mathrm{X}$ & B & \\
\hline $\mathrm{H}$ & & & B & B & $\mathrm{X}$ & $\mathrm{X}$ & B & \\
\hline $\mathrm{H}$ & $\mathrm{N}$ & $\mathrm{X}$ & B & & & & & \\
\hline & $\mathrm{N}$ & $\mathrm{X}$ & & & & B & & \\
\hline $\mathrm{H}$ & & & $\mathrm{N}$ & B & $\mathrm{X}$ & & B & \\
\hline
\end{tabular}


Species

Ophomus cordatus (Duftschmid, 1812)

Ophomus cribricollis (Dejean, 1829)

Ophonus diffinis (Dejean, 1829)

Ophomus ferrugatus Reitter, 1902

Ophonus franzinionum Sciaky, 1987

Ophonus incisus (Dejean, 1829)

Ophomus jeanneli Sciaky, 1987

Ophomus melletii melletii (Heer, 1837)

Ophomus parallelus (Dejean, 1829)

Ophomus pumcticeps Stephens, 1828

Ophonus puncticollis (Paykull, 1798)

Ophomus rotundatus (Dejean, 1829)

Ophonus rufibarbis (Fabricius, 1792)

Ophomus rupicola (Sturm, 1818)

Ophonus sabulicola (Panzer, 1796)

Ophonus schaubergerianus (Puel, 1937)

Ophomus similis (Dejean, 1829)

Ophonus subquadratus (Dejean, 1829)

Parophonus hespericus Jeanne, 1985

Parophonus hispamus (Rambur, 1838)

Parophonus maculicornis (Duftschmid, 1812)

Parophonus mendax (P. Rossi, 1790)

Parophonus planicollis (Dejean, 1829)

Trichotichmus nitens (Heer, 1837)

Stenolophina Kirby, 1837

\begin{tabular}{|c|c|c|c|c|c|c|c|c|}
\hline 䌒 & $\begin{array}{l}\overrightarrow{8} \\
0 \\
0 \\
0 \\
\frac{0}{0} \\
\frac{0}{0} \\
0\end{array}$ & 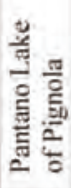 & 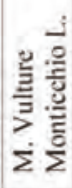 & 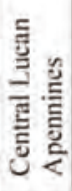 & 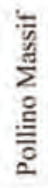 & $\begin{array}{l}\frac{\overrightarrow{\mathrm{g}}}{\mathrm{E}} \\
\frac{\mathrm{w}}{\mathrm{a}} \\
\frac{\mathrm{m}}{\mathrm{n}}\end{array}$ & 总 & 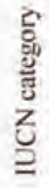 \\
\hline$X$ & & & & & $\mathrm{X}$ & & & \\
\hline$X$ & & & & & & & & \\
\hline \multirow[t]{3}{*}{$\mathrm{X}$} & $\mathrm{N}$ & & & & & & & \\
\hline & & & & & & & $\mathrm{N}$ & \\
\hline & $\mathrm{N}$ & & & & & & B & \\
\hline $\mathrm{X}$ & & & & B & & B & B & \\
\hline \multirow[t]{3}{*}{ B } & & & & $\mathrm{N}$ & & $\mathrm{N}$ & & \\
\hline & & $\mathrm{N}$ & B & & B & & & \\
\hline & & & & & $\mathrm{N}$ & & & \\
\hline $\mathrm{X}$ & $\mathrm{N}$ & $\mathrm{N}$ & & $\mathrm{N}$ & & $\mathrm{X}$ & B & \\
\hline \multirow[t]{2}{*}{ B } & & & B & & & & & \\
\hline & $X$ & $\mathrm{X}$ & & $\mathrm{N}$ & & & B & \\
\hline B & & $X$ & B & B & & B & $\mathrm{N}$ & \\
\hline $\mathrm{X}$ & $\mathrm{N}$ & $\mathrm{X}$ & & B & $\mathrm{X}$ & B & & \\
\hline $\mathrm{H}$ & & $X$ & $\mathrm{~N}$ & & $\mathrm{X}$ & B & B & \\
\hline $\mathrm{H}$ & & & B & & & & B & \\
\hline B & $X$ & & & & & & B & \\
\hline $\mathrm{H}$ & $\mathrm{X}$ & & & & & & B & \\
\hline \multirow[t]{2}{*}{$\mathrm{N}$} & & & $\mathrm{N}$ & & & & $\mathrm{N}$ & \\
\hline & $\mathrm{X}$ & $\mathrm{N}$ & $\mathrm{N}$ & & & B & B & \\
\hline $\mathrm{H}$ & $\mathrm{N}$ & & B & & $\mathrm{X}$ & B & B & \\
\hline $\mathrm{H}$ & $\mathrm{X}$ & $X$ & & & & B & & \\
\hline \multirow[t]{2}{*}{$\mathrm{H}$} & $\mathrm{X}$ & & & & & & B & \\
\hline & $X$ & $N$ & B & B & $\mathrm{X}$ & $\mathrm{X}$ & B & \\
\hline
\end{tabular}

Jager \& Kataev in Cat. Pal. Col., vol. 1, 2017

Acupalpus brunnipes (Sturm, 1825)

Acupalpus dubius Schilsky, 1888

Acupalpus elegans (Dejean, 1829)

Acupalpus exiguus Dejean, 1829

Acupalpus interstitialis Reitter, 1884

Acupalpus luteatus (Duftschmid, 1812)

Acupalpus maculatus (Schaum, 1860)

Acupalpus mediterraneus Csiki, 1932

Acupalpus meridiamus (Linnaeus, 1760)

Acupalpus notatus Mulsant \& Rey, 1861

Acupalpus parvulus (Sturm, 1825)

Acupalpus puncticollis (Coquerel, 1859)

Anthracus consputus (Duftschmid, 1812)

Anthracus quarnerensis (Reitter, 1884)

Anthracus transversalis (Schaum, 1862)

Bradycellus caucasicus (Chaudoir, 1846)

Bradycellus lusitanicus (Dejean, 1829)

Bradycellus verbasci (Duftschmid, 1812)

Dicheirotrichus chloroticus (Dejean, 1829)

Stenolophus abdominalis persicus Mannerheim, 1844

Stenolophus discophorus (Fischer von Waldheim, 1823)

Stenolophus marginatus Dejean, I829

Stenolophus mixtus (Herbst, 1784)

Stenolophus proximus Dejean, 1829

Stenolophus skrimshiramus Stephens, 1828

Stenolophus teutomus (Schrank, 1781)

\begin{tabular}{|c|c|c|c|c|c|c|c|}
\hline & $\mathrm{N}$ & & & & & & B \\
\hline & & $\mathrm{X}$ & & & & & $\mathrm{N}$ \\
\hline $\mathrm{H}$ & $X$ & & & $\mathrm{~N}$ & $\mathrm{~N}$ & & $\mathrm{~N}$ \\
\hline $\mathrm{X}$ & & $\mathrm{X}$ & $\mathrm{N}$ & $\mathrm{N}$ & $\mathrm{N}$ & & $\mathrm{N}$ \\
\hline & & & & & & & $\mathrm{N}$ \\
\hline$X$ & $\mathrm{~N}$ & $X$ & & & & & \\
\hline $\mathrm{X}$ & $\mathrm{X}$ & $X$ & B & $\mathrm{N}$ & $\mathrm{N}$ & $\mathrm{X}$ & $\mathrm{N}$ \\
\hline & & & & B & & & \\
\hline $\mathrm{H}$ & $\mathrm{X}$ & $\mathrm{X}$ & & B & $\mathrm{N}$ & $\mathrm{X}$ & B \\
\hline $\mathrm{X}$ & $\mathrm{X}$ & & & $\mathrm{N}$ & $\mathrm{N}$ & $\mathrm{X}$ & B \\
\hline & & & & & $\mathrm{X}$ & $\mathrm{X}$ & B \\
\hline$X$ & & & & $\mathrm{~N}$ & & & \\
\hline & & & & & B & & B \\
\hline $\mathrm{H}$ & $\mathrm{X}$ & & & & & & \\
\hline & & & & & & & B \\
\hline & & & & & & $\mathrm{X}$ & B \\
\hline & & $\mathrm{N}$ & & & & & B \\
\hline$X$ & B & $\mathrm{X}$ & & B & $\mathrm{N}$ & & B \\
\hline & & & & & & B & B \\
\hline & $\mathrm{N}$ & $\mathrm{X}$ & & $\mathrm{N}$ & $\mathrm{X}$ & & B \\
\hline & $\mathrm{X}$ & $\mathrm{X}$ & & $\mathrm{N}$ & & & \\
\hline & $X$ & & & $\mathrm{~N}$ & $\mathrm{X}$ & & B \\
\hline $\mathrm{H}$ & $\mathrm{N}$ & $\mathrm{X}$ & B & $\mathrm{N}$ & $\mathrm{N}$ & & $\mathrm{N}$ \\
\hline $\mathrm{X}$ & $\mathrm{X}$ & $\mathrm{X}$ & & & & & \\
\hline $\mathrm{X}$ & $\mathrm{X}$ & $\mathrm{X}$ & & & & & $\mathrm{N}$ \\
\hline $\mathrm{H}$ & X & & B & $\mathrm{N}$ & $\mathrm{X}$ & X & B \\
\hline
\end{tabular}


Species

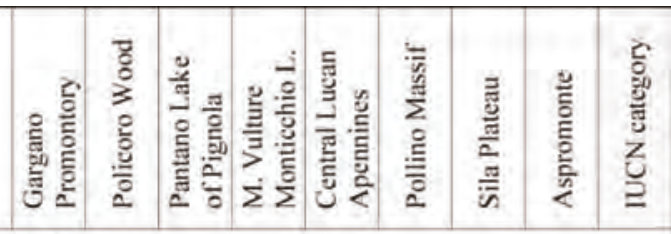

Lebiini Bonelli, 1810

Apenina G.E. Ball, 1983

Kabak in Cat. Pal. Col., vol. I, 2017

Platytarus faminii faminii (Dejean, 1826)

Cymindidina Laporte, 1834

Cymindis axillaris axillaris (Fabricius, 1794)

Cymindis etrusca etrusca Bassi, 1834

Cymindis humeralis (Geoffroy, 1785)

Cymindis miliaris (Fabricius, 1801)

Pseudomasoreus canigoulensis (Fairmaire \& Laboulbène, 1854)

\begin{tabular}{|c|c|c|c|c|c|c|}
\hline $\mathrm{H}$ & & & B & $\mathrm{X}$ & $X$ & B \\
\hline & & $\mathrm{N}$ & & X & $\mathrm{X}$ & $\mathrm{N}$ \\
\hline & & B & $\mathrm{N}$ & & & \\
\hline $\mathrm{H}$ & & B & & B & $X$ & B \\
\hline $\mathrm{H}$ & $\mathrm{N}$ & & & & B & B \\
\hline
\end{tabular}

Demetriadina Bates, 1886

Demetrias atricapillus (Linnaeus, 1758)

Demetrias imperialis (Germar, 1823)

Apristus europaeus Mateu, 1980

Apristus reticulatus Schaum, 1857

Apristus subaeneus Chaudoir, 1846

Calodromins spilotus (Illiger, 1798)

Dromius agilis (Fabricius, 1787)

Dromius fenestratus (Fabricius, 1794)

Dromius meridionalis Dejean, 1825

Dromius quadrimaculatus (Linnaeus, 1758)

Lionychus maritimus Fairmaire, 1862

Lionychus quadrillum (Duftschmid, 1812)

Metadromius nanus (A. Fiori, 1914) [E]

Microlestes abeillei abeillei (C.N.F. Brisout de Barneville, 1885)

Microlestes corticalis (L. Dufour, 1820)

Microlestes fissuralis (Reitter, 1901)

Microlestes fulvibasis (Reitter, 1901)

Microlestes levipennis levipennis P.H. Lucas, 1846

Microlestes luctuosus luctuosus Holdhaus, 1904

Microlestes mauritanicus (P.H. Lucas, 1846)

Microlestes maurus maurus (Sturm, 1827)

Microlestes minutulus (Goeze, 1777)

Microlestes negrita negrita (Wollaston, 1854)

Microlestes plagiatus (Duftschmid, 1812)

Paradromius linearis (A.G. Olivier, 1795)

Paradromius longiceps (Dejean, 1826)

Philorhizus crucifer confusus Sciaky, 1991

Philorhizus crucifer crucifer (P.H. Lucas, 1846)

Philorhizus melanocephalus (Dejean, 1825)

Philorhizus quadrisignatus (Dejean, 1825)

Syntomus foveatus (Geoffroy, 1785)

Syntomus impressus impressus (Dejean, 1825)

Syntomus obscuroguttatus (Duftschmid, 1812)

Syntomus silensis (A. Fiori, 1899) [E]

Syntomus truncatellus (Linnaeus, 1760)

Dromiusina Bonelli, 1810

\begin{tabular}{|l|l|l|l|l|l|l|l|l|}
\hline $\mathrm{H}$ & $\mathrm{X}$ & $\mathrm{X}$ & $\mathrm{N}$ & $\mathrm{N}$ & $\mathrm{X}$ & $\mathrm{X}$ & $\mathrm{B}$ & \\
\hline & $\mathrm{X}$ & & & & & & & \\
\hline
\end{tabular}

\begin{tabular}{|c|c|c|c|c|c|c|c|}
\hline & & & & $\mathrm{N}$ & & & $\mathrm{N}$ \\
\hline & X & & & & & & \\
\hline & & & & & & & B \\
\hline & $\mathrm{N}$ & & & $\mathrm{N}$ & & $\mathrm{X}$ & \\
\hline & & & $\mathrm{N}$ & $\mathrm{N}$ & X & $\mathrm{X}$ & \\
\hline & & & & $\mathrm{N}$ & $\mathrm{N}$ & & \\
\hline & $\mathrm{X}$ & & & & & & \\
\hline $\mathrm{X}$ & & $\mathrm{X}$ & & $\mathrm{N}$ & $\mathrm{X}$ & $\mathrm{X}$ & $\mathrm{N}$ \\
\hline & & & & & & & $\mathrm{N}$ \\
\hline & & & B & $\mathrm{N}$ & $\mathrm{X}$ & $\mathrm{X}$ & B \\
\hline & $\mathrm{N}$ & $X$ & & & & B & \\
\hline $\mathrm{H}$ & & & & & & & \\
\hline & $\mathrm{N}$ & X & & & & & $\mathrm{N}$ \\
\hline $\mathrm{H}$ & $\mathrm{N}$ & $X$ & $\mathrm{~N}$ & $\mathrm{~N}$ & $\mathrm{~N}$ & B & B \\
\hline $\mathrm{H}$ & $\mathrm{X}$ & $\mathrm{X}$ & $\mathrm{N}$ & $\mathrm{N}$ & & & B \\
\hline & & & $\mathrm{N}$ & & & $\mathrm{X}$ & B \\
\hline $\mathrm{H}$ & $\mathrm{X}$ & $\mathrm{X}$ & $\mathrm{N}$ & B & $\mathrm{N}$ & $\mathrm{X}$ & B \\
\hline & & $X$ & & & & & \\
\hline $\mathrm{X}$ & $\mathrm{X}$ & & $\mathrm{N}$ & B & $\mathrm{X}$ & $X$ & $\mathrm{~N}$ \\
\hline & $\mathrm{N}$ & $\mathrm{X}$ & B & & & & \\
\hline $\mathrm{H}$ & $\mathrm{X}$ & & $\mathrm{N}$ & & & & $\mathrm{N}$ \\
\hline & X & & $\mathrm{N}$ & & & & $\mathrm{N}$ \\
\hline $\mathrm{H}$ & $X$ & $X$ & B & B & $\mathrm{X}$ & $\mathrm{X}$ & B \\
\hline $\mathrm{N}$ & B & & & & & & \\
\hline B & & $\mathrm{X}$ & B & B & & & \\
\hline & & & & & $\mathrm{X}$ & $\mathrm{X}$ & B \\
\hline $\mathrm{H}$ & $\mathrm{X}$ & $\mathrm{X}$ & B & $\mathrm{N}$ & $\mathrm{X}$ & $\mathrm{X}$ & B \\
\hline & B & & & & $\mathrm{X}$ & & B \\
\hline & & & & B & & & \\
\hline $\mathrm{X}$ & $\mathrm{N}$ & & & & & B & B \\
\hline $\mathrm{X}$ & $\mathrm{X}$ & $\mathrm{X}$ & $\mathrm{N}$ & $\mathrm{N}$ & $\mathrm{N}$ & B & B \\
\hline & & & & & & $\mathrm{X}$ & B \\
\hline & & & & B & & & \\
\hline
\end{tabular}

Lebiina Bonelli, 1810

Lebia cruxminor cruxminor (Linnaeus, 1758)

Lebia cyanocephala cyanocephala (Linnaeus, 1758)

Lebia fulvicollis thoracica Hoppe \& Hornschuh, 1825

Lebia humeralis Dejean, 1825

\begin{tabular}{|c|c|c|c|c|c|c|c|c|}
\hline H & & X & & B & X & X & B & \\
\hline H & X & X & B & N & X & X & B & \\
\hline & X & & & & & B & B & \\
\hline H & X & & N & N & X & X & N & \\
\hline
\end{tabular}


Species

Lebia lepida Brullé, 1834

Lebia marginata (Geoffroy, 1785)

Lebia scapularis scapularis (Geoffroy, 1785)

Lebia Irimaculata (Villers, 1789)

\begin{tabular}{|c|c|c|c|c|c|c|c|c|}
\hline 总 & $\begin{array}{l}\overline{6} \\
0 \\
3 \\
0 \\
\frac{0}{0} \\
2 \\
2\end{array}$ & 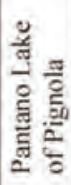 & 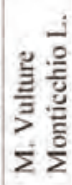 & 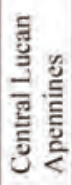 & 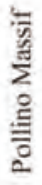 & 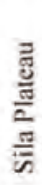 & 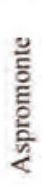 & 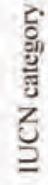 \\
\hline & $\mathrm{X}$ & & & & & & & \\
\hline & & & $\mathrm{N}$ & $\mathrm{N}$ & $X$ & X & B & \\
\hline$X$ & $\mathrm{X}$ & & & B & $\mathrm{X}$ & $\mathrm{X}$ & B & \\
\hline $\mathrm{H}$ & & & & & $X$ & $\mathrm{X}$ & & \\
\hline
\end{tabular}

Licinini Bonelli, 1810

Licinina Bonelli, 1810

Huber \& Marggi in Cat. Pal. Col., vol. 1, 2017

Badister bullatus (Schrank, 1798)

Badister collaris Motschulsky, 1844

Badister meridionalis Puel, 1925

Licimus cassideus cassideus (Fabricius, 1792)

Licinus italicus Puel, 1925 [E]

Licimus punctatulus gramulatus Dejean, 1826

Licimus silphoides (P. Rossi, 1790)

\begin{tabular}{|l|l|l|l|l|l|l|l|l|}
\hline & & & & & N & B & & \\
\hline B & & X & & & B & & & \\
\hline H & & & & & X & & & \\
\hline & & & & B & X & & & \\
\hline & & & & & & & B & \\
\hline B & X & & B & N & X & B & B & \\
\hline
\end{tabular}

Masoreini Chaudoir, 1871

Bousquet in Cat. Pal. Col., vol. 1, 2017

Masoreus wetterhallii wetterhallii (Gyllenhal, 1813)

$$
\mathrm{X}
$$

Odacanthini Laporte, 1834

Bousquet in Cat. Pal. Col., vol. 1,2017

Odacantha melamura (Linnaeus, 1766)

Oodini LaFerté-Sénectère, 1851

Bousquet in Cat. Pal. Col., vol. 1, 2017

Oodes gracilis A. Villa \& G. B. Villa, 1833

Oodes helopioides helopioides (Fabricius, 1792)

Panagaeini Bonelli, 1810

Häckel \& Kirschenhofer in Cat. Pal. Col., vol. 1, 2017

Panagaeus bipustulatus (Fabricius, 1775)

Panagaeus cruxmajor (Linnaeus, 1758)

\begin{tabular}{|l|l|l|l|l|l|l|l|l|}
\hline $\mathrm{H}$ & & & & $\mathrm{X}$ & & $\mathrm{B}$ & \\
\hline & $\mathrm{X}$ & $\mathrm{X}$ & & & & & & \\
\hline
\end{tabular}

Platynini Bonelli, 1810

Schmidt in Cat. Pal. Col., vol. 1, 2017

\begin{tabular}{|c|c|c|c|c|c|c|c|c|c|}
\hline Agonum duftschmidi J. Schmidt, 1994 & & $\mathrm{X}$ & $\mathrm{X}$ & B & $\mathrm{N}$ & & $\mathrm{X}$ & & \\
\hline Agomum emarginatum (Gyllenhal, 1827) & & & & $\mathrm{N}$ & $\mathrm{N}$ & & & & \\
\hline Agomum longicorne Chaudoir, 1846 & $\mathrm{X}$ & & & $\mathrm{N}$ & & & & & \\
\hline Agomum lugens (Duftschmid, 1812) & & $\mathrm{N}$ & $\mathrm{X}$ & & & $\mathrm{N}$ & $\mathrm{X}$ & & \\
\hline Agonum marginatum (Linnaeus, 1758) & $\mathrm{X}$ & $\mathrm{X}$ & & $\mathrm{N}$ & & $\mathrm{X}$ & $\mathrm{X}$ & $\mathrm{N}$ & \\
\hline Agonum muelleri (Herbst, 1784) & & & & B & $\mathrm{N}$ & $\mathrm{X}$ & $\mathrm{X}$ & & \\
\hline Agomum nigrum Dejean, 1828 & $\mathrm{X}$ & $\mathrm{X}$ & $\mathrm{X}$ & & & & $\mathrm{X}$ & B & \\
\hline Agomum mumidicum (P.H. Lucas, 1846) & $\mathrm{X}$ & $\mathrm{X}$ & & & & & & $\mathrm{N}$ & \\
\hline Agonum permoestum Puel, 1938 & & $\mathrm{~N}$ & & $\mathrm{~N}$ & $\mathrm{~N}$ & $\mathrm{~N}$ & $\mathrm{~B}$ & & \\
\hline Agomum sexpunctatum (Linnaeus, 1758) & & & & & & & $\mathrm{X}$ & & \\
\hline Agonum sordidum Dejean, 1828 & $\mathrm{H}$ & $\mathrm{B}$ & & & $\mathrm{N}$ & & & B & \\
\hline Agonum thoreyi Dejean, 1828 & & $\mathrm{X}$ & & & $\mathrm{N}$ & & & $\mathrm{N}$ & \\
\hline Agomum tulliae Mazzei \& Brandmayr, 2017 & & & & & & & $\mathrm{~B}$ & & \\
\hline Agomum versutum Sturm, 1824 & & & & $\mathrm{~N}$ & $\mathrm{~N}$ & B & & & \\
\hline Agomum viridicupreum viridicupreum (Goeze, 1777) & $\mathrm{X}$ & & & B & $\mathrm{B}$ & $\mathrm{X}$ & $\mathrm{X}$ & & \\
\hline Anchomenus dorsalis dorsalis (Pontoppidan, 1763) & $\mathrm{H}$ & & $\mathrm{X}$ & B & $\mathrm{N}$ & $\mathrm{X}$ & $\mathrm{X}$ & B & \\
\hline Atranus ruficollis (Gautier des Cottes, 1858) & & $\mathrm{X}$ & & & & & & & \\
\hline Limodromus assimilis (Paykull, 1790) & & & & & & & $\mathrm{X}$ & & \\
\hline Olisthopus fuscatus Dejean, 1828 & $\mathrm{H}$ & $\mathrm{X}$ & & $\mathrm{N}$ & $\mathrm{N}$ & & & B & \\
\hline Olisthopus glabricollis (Germar, 1817) & $\mathrm{H}$ & & & & & $\mathrm{X}$ & & & \\
\hline Oxypselaphus obscurus (Herbst, 1784) & & & $\mathrm{X}$ & & & & & & \\
\hline Paranchus albipes (Fabricius, 1796) & $\mathrm{X}$ & $\mathrm{X}$ & $\mathrm{N}$ & $\mathrm{N}$ & $\mathrm{N}$ & $\mathrm{X}$ & $\mathrm{X}$ & B & \\
\hline
\end{tabular}


Pterostichini Bonelli, 1810

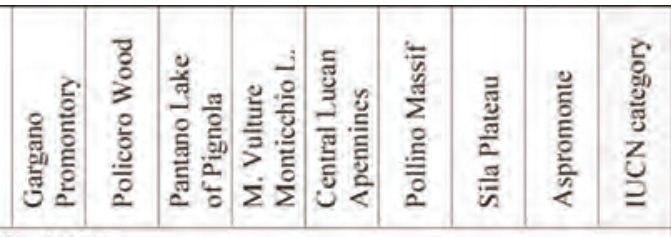

Bousquet in Cat. Pal. Col., vol. 1, 2017

\begin{tabular}{|c|c|c|c|c|c|c|c|c|}
\hline Abax parallelepipedus curtulus (Fairmaire, 1856) [E] & $\mathrm{X}$ & $\mathrm{X}$ & & B & B & $\mathrm{X}$ & $\mathrm{X}$ & B \\
\hline Pedius imquinatus (Sturm, 1824) & & $\mathrm{X}$ & & & & & & \\
\hline Percus bilineatus (Dejean, 1828) [E] & $\mathrm{H}$ & $\mathrm{X}$ & & CK & B & $\mathrm{X}$ & $\mathrm{X}$ & $\mathrm{N}$ \\
\hline Poecilus cupreus cupreus (Linnaeus, 1758) & $\mathrm{H}$ & $\mathrm{X}$ & B & B & $\mathrm{N}$ & $\mathrm{X}$ & $\mathrm{X}$ & B \\
\hline Poecilus cursorius cursorius (Dejean, 1828) & $\mathrm{H}$ & $\mathrm{X}$ & & & & & & \\
\hline Poecilus gisellae gisellae (Csiki, 1930) & & & & & & & & B \\
\hline Poecilus kovi viaticus (Dejean, 1828) & & & & & & CK & & \\
\hline Poecilus nitidus (Dejean, 1828) & $\mathrm{H}$ & B & & & & & & \\
\hline Poecilus pantanellii (A. Fiori, 1903) [E] & & B & & B & & & B & $\mathrm{B}$ \\
\hline Poecilus versicolor (Sturm, 1824) & & & $\mathrm{N}$ & $\mathrm{N}$ & & & & \\
\hline Plerostichus anthracimus biimpressus (Küster, 1853) & & $\mathrm{X}$ & & & & $\mathrm{N}$ & & \\
\hline Pterostichus apenninus (Dejean, 1831) & $\mathrm{X}$ & $\mathrm{X}$ & & $\mathrm{N}$ & $\mathrm{N}$ & $\mathrm{X}$ & $\mathrm{X}$ & B \\
\hline Pterostichus cursor (Dejean, 1828) & $\mathrm{H}$ & $\mathrm{X}$ & $\mathrm{X}$ & B & $\mathrm{N}$ & $\mathrm{N}$ & & \\
\hline Pterostichus dubius amorei Ganglbauer, 1907 [E] & & & & $\mathrm{N}$ & $\mathrm{N}$ & $\mathrm{X}$ & $\mathrm{X}$ & \\
\hline Pterostichus elongatus (Duftschmid, 1812) & $\mathrm{H}$ & $\mathrm{N}$ & & & & & & \\
\hline Pterostichus focarilei Casale \& Giachino, 1985 [E] & & & & & B & $\mathrm{X}$ & & \\
\hline $\begin{array}{l}\text { Pterostichus gracilis giordanii Bucciarelli \& Sopracordevole, } \\
1958\end{array}$ & $\mathrm{H}$ & $\mathrm{X}$ & $\mathrm{X}$ & & & & & \\
\hline Pterostichus impressicollis (Fairmaire \& Laboulbène, 1854) & & & & & B & & $\mathrm{X}$ & \\
\hline Pterostichus macer macer (Marsham, 1802) & & & & B & & & & \\
\hline Pterostichus melas italicus (Dejean, 1828) & $\mathrm{H}$ & $\mathrm{X}$ & $\mathrm{X}$ & B & B & $\mathrm{X}$ & $\mathrm{X}$ & $\mathrm{B}$ \\
\hline Pterostichus micans Heer, 1838 & & & & B & B & $\mathrm{X}$ & & \\
\hline Pterostichus niger niger (Schaller, 1783) & $\mathrm{H}$ & $\mathrm{X}$ & & $\mathrm{N}$ & $\mathrm{N}$ & $\mathrm{X}$ & $\mathrm{X}$ & $\mathrm{B}$ \\
\hline Plerostichus nigrita nigrita (Paykull, 1790) & $\mathrm{X}$ & $\mathrm{X}$ & $\mathrm{X}$ & B & B & $\mathrm{X}$ & $\mathrm{X}$ & B \\
\hline Pterostichus ovoideus mainardii Straneo, 1934 [E] & & & & & & B & & \\
\hline Pterostichus quadrifoveolatus Letzner, 1852 & & & & & & $\mathrm{X}$ & $\mathrm{X}$ & B \\
\hline Pterostichus ruffoi Sciaky, 1984 [E] & & & & & & & $\mathrm{X}$ & B \\
\hline Pterostichus strenuиs (Panzer, 1797) & & & & $\mathrm{N}$ & $\mathrm{N}$ & $\mathrm{X}$ & $\mathrm{X}$ & \\
\hline Pterostichus vernalis (Panzer, 1796 ) & & $\mathrm{N}$ & $\mathrm{X}$ & & $\mathrm{N}$ & & $\mathrm{CK}$ & $\mathrm{N}$ \\
\hline Stomis pumicatus pumicatus (Panzer, 1796) & $\mathrm{H}$ & & $\mathrm{X}$ & & B & $\mathrm{X}$ & & B \\
\hline
\end{tabular}

Sphodrini Laporte, 1834

Atranopsina Baehr, 1982

Hovorka in Cat. Pal. Col., vol. 1, 2017

Platyderus neapolitamus (Reiche, 1855) [E]

Platyderus paganettii Guéorguiev, 2009 [E]

Platyderus vignai Guéorguiev, 2009 [E]

\begin{tabular}{|c|c|c|c|c|c|c|c|c|}
\hline H & X & & B & B & X & X & B & \\
\hline & & & & & & & B & \\
\hline B & & & & & & & N & \\
\hline
\end{tabular}

Calathina Laporte, 1834

Hovorka in Cat. Pal. Col., vol. 1, 2017

Calathus ambiginus ambiguus (Paykull, 1790)

Calathus cinctus Motschulsky, 1850

Calathus circumseptus Germar, 1823

Calathus erratus erratus (C.R. Sahlberg, 1827)

Calathus focarilei Schatzmayr, 1947 [E]

Calathus fracassii fracassii Heyden, 1908 [E]

Calathus fuscipes punctipennis Germar, 1823

Calathus melanocephalus melanocephalus (Linnaeus, 1758)

Calathus micropterus (Duftschmid, 1812)

Calathus montivagus Dejean, 1831 [E]

Calathis pirazzolii Putzeys, 1873 [E]

Calathus rotundicollis Dejean, 1828

Calathus sirentensis D'Amore Fracassi, 1908 [E]

\begin{tabular}{|c|c|c|c|c|c|c|c|c|}
\hline & N & & & & & & & \\
\hline H & X & & B & N & X & X & B & \\
\hline & X & & & & & & & \\
\hline & & & & N & & & & \\
\hline H & X & X & B & N & X & X & B & \\
\hline H & & & N & B & X & X & N & \\
\hline & & & B & & & B & B & \\
\hline H & X & & N & B & X & X & B & \\
\hline & & & & & N & & & \\
\hline H & X & X & B & B & X & X & B & \\
\hline & & & & B & X & X & & \\
\hline
\end{tabular}


Species

Sphodrina Laporte, 1834

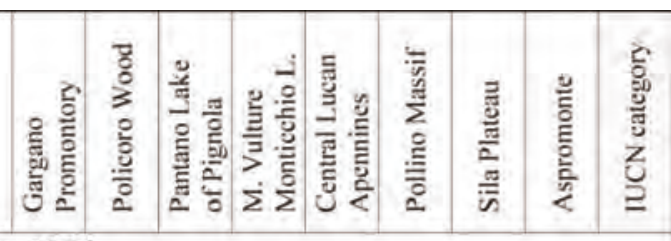

Casale in Cat. Pal. Col., vol. 1, 2017

Laemostenus acutangulus (L.W. Schaufuss, 1862) [E]

Laemostemus algerinus algerinus (Gory, 1833)

Laemostenus barbarus (P.H. Lucas, 1846)

Laemostenus cimmerius cimmerius (Fischer von Waldheim, 1823)

Laemostenus complanatus (Dejean, 1828)

Laemostenus vemustus (Dejean, 1828)

Sphodrus leticophthalmus (Linnaeus, 1758)

Synuchina Lindroth, 1956

Hovorka in Cat. Pal. Col., vol. 1, 2017

Symuchus vivalis vivalis (Illiger, 1798)

$$
\mathrm{X}
$$

Zabrini Bonelli, 1810

Amarina C. Zimmermann, 1832

Hieke in Cat. Pal. Col., vol. 1, 2017

Amara aenea (DeGeer, 1774)

Amara anthobia A. Villa \& G.B. Villa, 1833

Amara apricaria (Paykull, 1790)

Amara aulica (Panzer, 1796)

Amara concinna C. Zimmermann, 1832

Amara consularis (Duftschmid, 1812)

Amara cursitans C. Zimmermann, 1832

Amara curta Dejean, 1828

Amara eturynota (Panzer, 1796)

Amara famelica C. Zimmermann, 1832

Amara familiaris (Duftschmid, 1812)

Amara filvipes (Audinet-Serville, 1821)

Amara kulti Fassati, 1947

Amara lucida (Duftschmid, 1812)

Amara lunicollis Schiödte, 1837

Amara montana Dejean, 1828

Amara nitida Sturm, 1825

Amara ovata (Fabricius, 1792)

Amara praetermissa (C.R. Sahlberg, 1827)

Amara mufipes Dejean, 1828

Amara sicula Dejean, 1831 [E]

Amara similata (Gyllenhal, 1810)

\begin{tabular}{|c|c|c|c|c|c|c|c|}
\hline $\mathrm{H}$ & $X$ & & B & B & $\mathrm{X}$ & $X$ & B \\
\hline $\mathrm{H}$ & $\mathrm{X}$ & & B & $\mathrm{N}$ & $\mathrm{X}$ & $\mathrm{X}$ & B \\
\hline \multirow[t]{3}{*}{$\mathrm{H}$} & $\mathrm{N}$ & $X$ & B & $\mathrm{N}$ & $\mathrm{X}$ & B & B \\
\hline & & & & B & $\mathrm{X}$ & B & \\
\hline & & & & B & & & \\
\hline \multirow[t]{3}{*}{$\mathrm{H}$} & & & & & & & \\
\hline & $\mathrm{N}$ & & & & & & \\
\hline & & & & B & & & B \\
\hline \multirow[t]{2}{*}{$\mathrm{H}$} & $\mathrm{X}$ & & B & B & $\mathrm{X}$ & $\mathrm{X}$ & B \\
\hline & & & & B & & & B \\
\hline \multirow[t]{3}{*}{$\mathrm{X}$} & $\mathrm{X}$ & & B & $\mathrm{N}$ & $\mathrm{X}$ & $\mathrm{X}$ & B \\
\hline & & & & & & $X$ & \\
\hline & & & & & & & B \\
\hline \multirow[t]{4}{*}{$\mathrm{H}$} & & & B & & $\mathrm{X}$ & & \\
\hline & & & & & & $\mathrm{X}$ & \\
\hline & $\mathrm{X}$ & & & $\mathrm{N}$ & & & B \\
\hline & & & & $\mathrm{N}$ & & $\mathrm{X}$ & \\
\hline \multirow[t]{2}{*}{$\mathrm{H}$} & $\mathrm{X}$ & & B & B & $\mathrm{X}$ & & B \\
\hline & & & & & $\mathrm{X}$ & & \\
\hline B & & & & & & $\mathrm{X}$ & \\
\hline $\mathrm{X}$ & & & & B & $\mathrm{X}$ & $\mathrm{X}$ & B \\
\hline $\mathrm{X}$ & $\mathrm{X}$ & & & & & $\mathrm{X}$ & $\mathrm{N}$ \\
\hline
\end{tabular}

Zabrina Bonelli, 1810

Serrano \& Andújar in Cat. Pal. Col., vol. 1, 2017

Zabrus costae Heyden, 1891 [E]

Zabrus ignavus ignavus Csiki, 1907

Zabrus orsinii Dejean, 1831 [E]

Zabrus tenebrioides tenebrioides (Goeze, 1777)

\begin{tabular}{|l|l|l|l|l|l|l|l|l|}
\hline & & N & B & X & & & \\
\hline & & B & & & & & \\
\hline & & & N & & X & & & \\
\hline X & X & & & & X & & & \\
\hline
\end{tabular}

Zuphiini Bonelli, 1810

Zuphiina Bonelli, 1810

Huber \& Marggi in Cat. Pal. Col., vol. 1, 2017

Parazuphium chevrolatii chevrolatii (Laporte, 1833)

Polisticus connexus (Geoffroy, 1785)

Zuphium olens (P, Rossi, 1790)

HALIPLIDAE Aubé, 1836

van Vondel in Cat. Pal. Col., vol. 1, 2017

Haliplus flavicollis Sturm, 1834 


\begin{tabular}{|c|c|c|c|c|c|c|c|c|c|}
\hline Species & 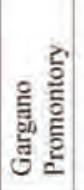 & $\begin{array}{l}7 \\
0 \\
0 \\
0 \\
0 \\
0 \\
\frac{0}{0} \\
2\end{array}$ & 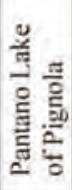 & 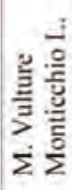 & 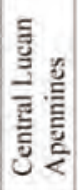 & 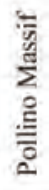 & 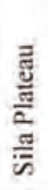 & 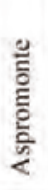 & 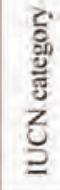 \\
\hline Haliplus guttatus Aubé, 1836 & $\mathrm{X}$ & $\mathrm{X}$ & & & B & & $\mathrm{X}$ & $\mathrm{N}$ & \\
\hline Haliplus lineatocollis (Marsham, 1802) & $\mathrm{H}$ & $\mathrm{X}$ & $\mathrm{X}$ & B & B & $\mathrm{N}$ & $\mathrm{X}$ & B & \\
\hline Haliplus mucronatus Stephens, 1828 & $\mathrm{H}$ & $\mathrm{X}$ & & & B & B & & $\mathrm{N}$ & \\
\hline Haliplus obliquus (Fabricius, 1787) & $\mathrm{H}$ & $\mathrm{X}$ & & $\mathrm{N}$ & B & $\mathrm{X}$ & $\mathrm{X}$ & & \\
\hline Haliplus ruficollis (DeGeer, 1774) & $\mathrm{H}$ & $\mathrm{X}$ & $\mathrm{X}$ & $\mathrm{N}$ & B & $\mathrm{X}$ & $\mathrm{X}$ & & \\
\hline Haliphus variegatus Sturm, 1834 & $\mathrm{X}$ & $\mathrm{X}$ & & & & & & $\mathrm{N}$ & \\
\hline Peltodytes caesus (Duftschmid, 1805) & B & $\mathrm{X}$ & & $\mathrm{N}$ & & & & $\mathrm{N}$ & \\
\hline Peltodytes rotumdatus (Aubé, 1836) & $\mathrm{X}$ & $\mathrm{X}$ & $\mathrm{X}$ & & B & $\mathrm{N}$ & & & \\
\hline
\end{tabular}

Peltodytes rotundatus (Aubé, 1836 )

NOTERIDAE C.G. Thomson, 1860

Noterinae C.G. Thomson, 1860

Noterini C.G. Thomson, 1860

Hájek in Cat. Pal. Col., vol. 1, 2017

Noterzis clavicornis (DeGeer, 1774)

Noterus crassicornis (O.F. Müller, 1776)

\begin{tabular}{|l|l|l|l|l|l|l|l|l|}
\hline $\mathrm{H}$ & $\mathrm{X}$ & $\mathrm{X}$ & $\mathrm{N}$ & $\mathrm{B}$ & $\mathrm{X}$ & $\mathrm{X}$ & $\mathrm{N}$ & \\
\hline & $\mathrm{X}$ & & & & & $\mathrm{X}$ & & \\
\hline
\end{tabular}

HYGROBIIDAE Régimbart, 1879

Hajek in Cat. Pal. Col., vol. 1, 2017

\begin{tabular}{|c|c|c|c|c|}
\hline Hygrobia hermanni (Fabricius, 1775) & $\mathrm{X} / \mathrm{B}$ & $\mathrm{B}$ & $\mathrm{X}$ & \\
\hline & DYTISCIDAE Leach, 1815 \\
& Agabinae J. Thomson, 1867 \\
& Agabini J. Thomson, 1867 \\
& Hajjek in Cat. Pal. Col., vol. 1, 2017
\end{tabular}

\begin{tabular}{|c|c|c|c|c|c|c|c|c|}
\hline Agabus biguttatus (A.G. Olivier, 1795) & $\mathrm{X}$ & $\mathrm{X}$ & & & B & $\mathrm{X}$ & $\mathrm{X}$ & B \\
\hline Agabus bipustulatus (Linnaeus, 1767) & $\mathrm{H}$ & $\mathrm{X}$ & $\mathrm{X}$ & B & B & $\mathrm{X}$ & $\mathrm{X}$ & B \\
\hline Agabus brumneus (Fabricius, 1798) & $\mathrm{H}$ & $\mathrm{X}$ & & B & B & & $\mathrm{X}$ & \\
\hline Agabus congener (Thunberg, 1794) & & & & & & & $\mathrm{X}$ & \\
\hline Agabus conspersus (Marsham, 1802) & $\mathrm{H}$ & $\mathrm{X}$ & & & B & & $\mathrm{X}$ & $\mathrm{N}$ \\
\hline Agabus didymus (A.G. Olivier, 1795) & $\mathrm{H}$ & $\mathrm{X}$ & $\mathrm{X}$ & $\mathrm{N}$ & B & $\mathrm{X}$ & $\mathrm{X}$ & B \\
\hline Agabus dilatatus (Brullé, 1832) & & & & & B & & & B \\
\hline Agabus guitatus gnittatus (Paykull, 1798) & & & & & B & $\mathrm{X}$ & $\mathrm{X}$ & B \\
\hline Agabus nebulosus (Forster, 1771) & $\mathrm{X}$ & $\mathrm{X}$ & $\mathrm{X}$ & $\mathrm{N}$ & B & $\mathrm{X}$ & $\mathrm{X}$ & $\mathrm{N}$ \\
\hline Agabus paludosus (Fabricius, 1801) & $\mathrm{X}$ & & & $\mathrm{N}$ & B & $\mathrm{X}$ & $\mathrm{X}$ & \\
\hline Ilybius ater (DeGeer, 1774) & $\mathrm{X}$ & & & & & $\mathrm{X}$ & $\mathrm{X}$ & \\
\hline Ilybius chalconatus (Panzer, 1796) & $\mathrm{B}$ & & $\mathrm{X}$ & & B & $\mathrm{X}$ & $\mathrm{X}$ & B \\
\hline Mybius fuliginosus fuliginosus (Fabricius, 1792) & & $\mathrm{X}$ & $\mathrm{X}$ & $\mathrm{N}$ & B & $\mathrm{X}$ & $\mathrm{X}$ & \\
\hline Ilybius lagabrumensis Schizzerotto \& Fery, 1990 [E] & & & & & B & B & & \\
\hline Ilybius pederzanii Fery \& Nilsson, 1993 [E] & & B & $\mathrm{N}$ & & B & & & B \\
\hline Ilvbius pseudoneglectus Franciscolo, 1972 & B & B & & & & & & \\
\hline Ilybius quadriguttatus Lacordaire, 1835 & & $\mathrm{X}$ & & & & & & \\
\hline
\end{tabular}

Ilvbius quadriguttatus Lacordaire, 1835

Colymbetinae Erichson, 1837

Colymbetini Erichson, 1837

Colymbetes fiuscus (Linnaeus, 1758)

Meladema coriacea Laporte, 1835

Melanodytes pustulatus (P. Rossi, 1792)

Rhantus suturalis (W.S. Macleay, 1825)

\section{Copelatinae Branden, 1885}

Copelatini Branden, 1885

Liopterus haemorrhoidalis (Fabricius, 1787

Cybistrinae Sharp, 1880

Cybistrini Sharp, 1880

Cybister lateralimarginalis lateralimarginalis (DeGeer, 1774)

\begin{tabular}{|l|l|l|l|l|l|l|}
$\mathrm{X}$ & $\mathrm{X}$ & & & & & $\mathrm{B}$ \\
\hline
\end{tabular}

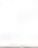




\section{Species}

Cybister tripunctatus africanus Laporte, 1834

Dytiscinae Leach, 1815

\begin{tabular}{|c|c|c|c|c|c|c|c|c|}
\hline 总 & $\begin{array}{l}\overrightarrow{0} \\
\frac{0}{0} \\
0 \\
\frac{0}{0} \\
\frac{.0}{0} \\
2\end{array}$ & 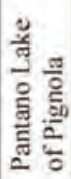 & 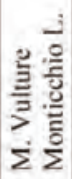 & 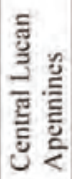 & 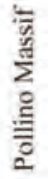 & 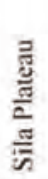 & 总 & 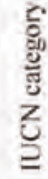 \\
\hline & $X$ & & & & B & & B & \\
\hline
\end{tabular}

Aciliini J. Thomson, 1867

Acilius sulcatus (Linnaeus, 1758)

Graphoderus cinereus (Linnaeus, 1758)

$$
\mathrm{X}
$$

Dytiscini Leach, 1815

Dytiscus circumflexus Fabricius, 1801

Dytiscus marginalis marginalis Linnaeus, 1758

Dytiscus mutinensis Branden, 1885

\begin{tabular}{|c|c|c|c|c|c|c|c|c|}
\hline $\mathrm{X}$ & $\mathrm{X}$ & & & $\mathrm{B}$ & & $\mathrm{X}$ & $\mathrm{B}$ & \\
\hline & & $\mathrm{X}$ & $\mathrm{B}$ & $\mathrm{B}$ & $\mathrm{X}$ & $\mathrm{X}$ & $\mathrm{N}$ & \\
\hline & $\mathrm{X}$ & & & & & & & \\
\hline
\end{tabular}

Eretini Crotch, 1873

Eretes griseus (Fabricius, 1781)

Hydaticini Sharp, 1882

Hydaticus leander (P. Rossi, 1790)

Hydaticus seminiger (DeGeer, 1774)

\begin{tabular}{|c|c|c|c|c|c|c|c|c|}
\hline $\mathrm{X}$ & $\mathrm{X}$ & & & & & & $\mathrm{N}$ & \\
\hline & $\mathrm{X}$ & & & & & & & \\
\hline
\end{tabular}

Hydroporinae Aubé, 1836

Bidessini Sharp, 1882

Bidessus calabricus Guignot, 1957

Bidessus delicatulus (Schaum, 1844)

Bidessus goudotii (Laporte, 1835)

Bidessus muelleri Zimmermann. 1927

Bidessus pumilus (Aubé, 1838)

Bidessus unistriatus (Goeze, 1777)

Hydroglyphus geminus (Fabricius, 1792)

Hydroglyphus signatellus (Klug, 1834)

Yola bicarinata bicarinata (Latreille, 1804)

\begin{tabular}{|c|c|c|c|c|c|c|c|c|}
\hline X & X & & & B & X & & B & \\
\hline & & X & & B & N & & B & \\
\hline & & & & & & & B & \\
\hline & X & & & & & & & \\
\hline & & & & & & & & \\
\hline H & X & X & N & B & X & X & B & \\
\hline H & & & & & & & B & \\
\hline X & X & & & B & & & N & \\
\hline
\end{tabular}

Hydroporini Aubé, 1836

Deronectina Galewski, 1994

Boreonectes griseostriatus griseostriatus (DeGeer, 1774)

Deronectes angelinii Fery \& Brancucci, 1997 [E]

Deronectes aubei aubei (Mulsant, 1843)

Deronectes moestus inconspectus (Leprieur, 1876)

Deronectes semirufius (Germar, 1844)

Nebrioporus canaliculatus (Lacordaire, 1835)

Nebrioporus ceresyi (Aubé, 1838)

Nebrioporus fenestratus (Germar, 1838) [E]

Nebrioporus luctuosus (Aubé, 1838)

Nebrioporus sansii (Aubé, 1838)

Nectoporus sammarkii sammarkii (C.R. Sahlberg, 1826)

Oreodytes meridionalis Binaghi \& Sanfilippo, 1971 [E]

Scarodytes halensis (Fabricius, 1787)

Scarodytes pederzanii Angelini, 1973

Scarodytes ruffoi Franciscolo, 1961 [E]

Stictotarsus procerus (Aubé, 1838)

Graptodytes bilineatus (Sturm, 1835)

Graptodytes flavipes (A.G. Olivier, 1795)

Graptodytes fractus (Sharp, 1882)

Graptodytes ignotus (Mulsant \& Rey, 1861)

Graptodytes varius (Aubé, 1838)

Graptodytes veterator veterator (Zimmermann, 1918)

Hydroporus analis Aubé, I838

Hydroporus apenninus Pederzani \& Rocchi, 2005 [E]

Hydroporina Aubé, 1836

\begin{tabular}{|c|c|c|c|c|c|c|}
\hline & & & B & $\mathrm{X}$ & $\mathrm{X}$ & \\
\hline & & & & & B & \\
\hline & & & & & & B \\
\hline \multirow[t]{3}{*}{ B } & & & B & $\mathrm{X}$ & $\mathrm{X}$ & B \\
\hline & & & B & B & B & B \\
\hline & $\mathrm{X}$ & & B & B & B & \\
\hline \multirow[t]{6}{*}{ B } & $X$ & & & & & $\mathrm{~N}$ \\
\hline & & & & & & B \\
\hline & $\mathrm{X}$ & & B & B & $\mathrm{X}$ & \\
\hline & $\mathrm{X}$ & & B & $\mathrm{X}$ & & B \\
\hline & & & & & $\mathrm{X}$ & \\
\hline & & & & & $\mathrm{X}$ & \\
\hline \multirow[t]{4}{*}{$\mathrm{X}$} & B & $\mathrm{X}$ & B & $\mathrm{X}$ & B & B \\
\hline & & & & B & $X$ & \\
\hline & & & B & X & & \\
\hline & & & B & & & \\
\hline
\end{tabular}

\begin{tabular}{|l|l|l|l|l|l|l|l|l|l|}
\hline & X & & & & N & X & X & & \\
\hline & & & & & B & B & & & \\
\hline & & & & & & & & B & \\
\hline & & X & & & B & X & B & B & \\
\hline & & X & X & & & & & B & \\
\hline inn, 1918) & & & & & B & X & X & & \\
\hline
\end{tabular}




\begin{tabular}{|c|c|c|c|c|c|c|c|c|c|}
\hline Species & 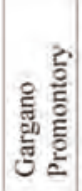 & 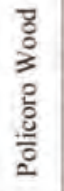 & 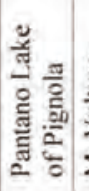 & 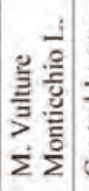 & 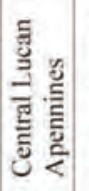 & 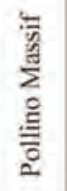 & 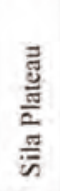 & 总 & 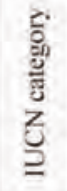 \\
\hline Hydroporus discretus discretus Fairmaire \& C.N.F. Brisout, 1859 & & & & & B & $\mathrm{x}$ & $\mathrm{x}$ & B & \\
\hline Hydroporus jonicus jonicus L. Miller, 1862 & $\mathrm{H}$ & $\mathrm{X}$ & & & & & & & \\
\hline Hydroporus limbatus Aubé, 1838 & $\mathrm{x}$ & & & & & & & B & \\
\hline Hydroportus marginatus (Duftschmid, 1805) & & & & & B & & & B & \\
\hline Hydroporis memnonius Nicolai, 1822 & $\mathrm{H}$ & $\mathrm{X}$ & $\mathrm{X}$ & & B & $\mathrm{x}$ & $\mathrm{X}$ & $\mathrm{N}$ & \\
\hline Hydroporus nigrita (Fabricius, 1792) & & & & & B & $\mathrm{X}$ & $\mathrm{X}$ & & \\
\hline Hydroporus obsoletus Aubé, 1838 & & & & & B & $\mathrm{x}$ & $\mathrm{X}$ & B & \\
\hline Hydroporus palustris (Linnaeus, 1760) & & & $\mathrm{X}$ & & B & $\mathrm{X}$ & $\mathrm{X}$ & & \\
\hline Hydroporus plamus (Fabricius, 1782) & $\mathrm{X}$ & $\mathrm{X}$ & & & B & $\mathrm{x}$ & & B & \\
\hline Hydroporus pubescens (Gyllenhal, 1808) & $\mathrm{X}$ & $\mathrm{X}$ & $\mathrm{X}$ & $\mathrm{N}$ & B & $\mathrm{x}$ & $\mathrm{X}$ & B & \\
\hline Hydroporus tessellatus (Drapiez, 1819) & $\mathrm{X}$ & $\mathrm{X}$ & $\mathrm{X}$ & $\mathrm{N}$ & B & $\mathrm{x}$ & $\mathrm{X}$ & B & \\
\hline Porhydrus lineatus (Fabricius, 1775) & & $\mathrm{X}$ & & & & $\mathrm{x}$ & & B & \\
\hline Porhydrus obliquesignatus (Bielz, 1852) & B & & & & B & & $\mathrm{X}$ & & \\
\hline Stictonectes optatus (Seidlitz, 1887) & & & & & B & & & B & \\
\hline \multicolumn{10}{|c|}{ Hydrovatini Sharp, 1880} \\
\hline Hydrovatus cuspidatus (Kunze, 1818) & $\mathrm{H}$ & $\mathrm{X}$ & & & & & & B & \\
\hline \multicolumn{10}{|c|}{ Hygrotini Portevin, 1929} \\
\hline Hygrotus confluens (Fabricius, 1787) & $\mathrm{X}$ & $\mathrm{X}$ & & & B & & & $\mathrm{N}$ & \\
\hline Hygrotus impressopunctatus (Schaller, 1783) & & $\mathrm{N}$ & & & & & & & \\
\hline Hygrotus inaequalis (Fabricius, 1777) & B & $\mathrm{X}$ & $\mathrm{X}$ & $\mathrm{N}$ & B & $\mathrm{x}$ & $\mathrm{x}$ & $\mathrm{N}$ & \\
\hline Hygrotus parallellogrammus (Ahrens, 1812) & B & $\mathrm{X}$ & & & & & & & \\
\hline \multicolumn{10}{|l|}{ Hyphydrini Gistel, 1848} \\
\hline Hyphydrus anatolicus Guignot, 1957 & & $\mathrm{X}$ & & & & & & & \\
\hline Hyphydrus aubei Ganglbauer, 1891 & B & $\mathrm{X}$ & $\mathrm{X}$ & & B & & $\mathrm{x}$ & $\mathrm{N}$ & \\
\hline \multicolumn{10}{|c|}{$\begin{array}{l}\text { Laccophilinae Gistel, } 1848 \\
\text { Laccophilini Gistel, } 1848\end{array}$} \\
\hline Laccophilus hyalimus (DeGeer, 1774) & $\mathrm{X}$ & $\mathrm{X}$ & $\mathrm{N}$ & & B & & B & B & \\
\hline Laccophilus mimutus (Linnaeus, 1758) & $\mathrm{x}$ & $\mathrm{X}$ & $\mathrm{X}$ & B & & $\mathrm{x}$ & $\mathrm{x}$ & $\mathrm{N}$ & \\
\hline Laccophilus poecilus Klug, 1834 & $\mathrm{H}$ & $\mathrm{x}$ & & B & & $\mathrm{N}$ & $\mathrm{N}$ & $\mathrm{N}$ & \\
\hline $\begin{array}{l}\text { POLYPHAGA En } \\
\text { STAPHYLINIFORMIA } \\
\text { HYDROPHILOIDEA }\end{array}$ & \multicolumn{9}{|c|}{$\begin{array}{l}\text { STAPHYLINIFORMIA Lameere, } 1900 \\
\text { HYDROPHILOIDEA Latreille, } 1802\end{array}$} \\
\hline $\begin{array}{r}\text { HYDROPHILIDAE L } \\
\text { Hydrophilinae Latr } \\
\text { Berosini Mulsant } \\
\text { Prokin, Przewoźny \& Ryndevich in }\end{array}$ & $\begin{array}{l}\text { atreil } \\
\text { ille, } 1 \\
1844 \\
\text { t. Pal. }\end{array}$ & $\begin{array}{l}\text { le, } 1 \\
802 \\
\text { Col. }\end{array}$ & 802 & 2015 & & & & & \\
\hline Berosus affinis Brullé, 1835 & $\mathrm{H}$ & $\mathrm{x}$ & $\mathrm{x}$ & $\mathrm{N}$ & CK & $\mathrm{N}$ & & B & \\
\hline Berosus hispanicus Küster, 1847 & $\mathrm{X}$ & $\mathrm{X}$ & & & & & & B & \\
\hline Berasus jaechi Schödl, 1991 & B & $\mathrm{X}$ & & & & & & & \\
\hline Berosus luridus (Linnaeus, 1760) & & $\mathrm{X}$ & & & & $\mathrm{N}$ & & & \\
\hline Berosus signaticollis (Charpentier, 1825) & $\mathrm{x}$ & $\mathrm{x}$ & & & & $\mathrm{X}$ & $\mathrm{x}$ & & \\
\hline \multicolumn{10}{|c|}{ Laccobiini Houlbert, 1922} \\
\hline Laccobius albescens Rottenberg, 1874 & & B & & B & & $\mathrm{N}$ & $\mathrm{X}$ & B & \\
\hline Laccobius alternus Motschulsky, 1855 & & $\mathrm{X}$ & & & $\mathrm{CK}$ & & $\mathrm{X}$ & B & \\
\hline Laccobius bipunctatus (Fabricius, 1775) & $\mathrm{H}$ & & & & & & $\mathrm{X}$ & & \\
\hline Laccobius gracilis gracilis Motschulsky, 1855 & & $\mathrm{X}$ & & & & CK & $\mathrm{X}$ & B & \\
\hline Laccobius minutus (Linnaeus, 1758) & & & & B & & & $\mathrm{x}$ & & \\
\hline Laccobius neapolitamus Rottenberg, 1874 & & $\mathrm{X}$ & & $\mathrm{N}$ & $\mathrm{CK}$ & $\mathrm{X}$ & $\mathrm{x}$ & B & \\
\hline Laccobius obscuratus Rottenberg, 1874 & $\mathrm{X}$ & & & & B & $\mathrm{X}$ & $\mathrm{x}$ & B & \\
\hline Laccobius revelierei Perris, 1864 & & $\mathrm{~N}$ & & & & & $\mathrm{X}$ & & \\
\hline Laccobius simulatrix d'Orchymont, 1932 & & $\mathrm{x}$ & & & $\mathrm{CK}$ & & $\mathrm{x}$ & B & \\
\hline
\end{tabular}


Species

Laccobius sinuatus simuatus Motschulsky, 1849

Paracymus aeneus (Germar, 1824)

Paracymus scutellaris (Rosenhauer, 1856)

Hydrobiusini Mulsant, I844

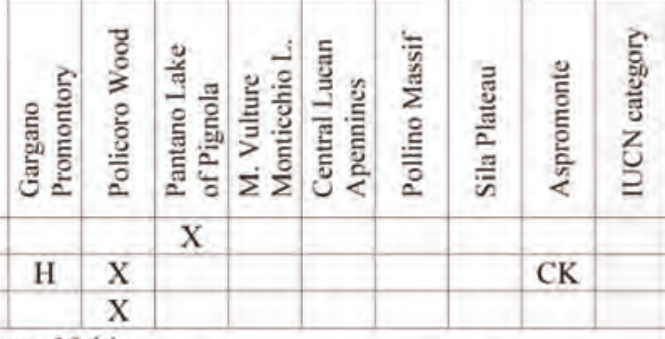

Hydrobius fuscipes (Linnaeus, 1758)

Limnoxemus niger (Gmelin, 1790)

Hydrophilus piceus (Linnaeus, 1758)

Hydrophilini Latreille, 1802

\begin{tabular}{|l|l|l|l|l|l|l|l}
$\mathrm{X}$ & $\mathrm{X}$ & & & $\mathrm{X}$ & $\mathrm{X}$ & & \\
\hline $\mathrm{X}$ & $\mathrm{X}$ & $\mathrm{N}$ & & $\mathrm{N}$ & & & \\
\hline
\end{tabular}

\begin{tabular}{|c|c|c|c|c|c|c|c|c|}
\hline \multicolumn{9}{|c|}{ Hydrophilini Latreille, 1802} \\
\hline Hydrochara caraboides (Linnaeus, 1758) & $\mathrm{N}$ & $\mathrm{X}$ & $\mathrm{X}$ & & CK & $\mathrm{N}$ & & \\
\hline Hydrophilus piceus (Linnaeus, 1758) & $\mathrm{H}$ & $\mathrm{X}$ & $\mathrm{X}$ & & & & & $\mathrm{N}$ \\
\hline \multicolumn{9}{|c|}{$\begin{array}{c}\text { Chaetarthriinae Bedel, } 1881 \\
\text { Anacaenini M. Hansen, } 1991\end{array}$} \\
\hline Anacaena bipustulata (Marsham, 1802) & & $\mathrm{X}$ & & $\mathrm{N}$ & & $\mathrm{X}$ & $\mathrm{X}$ & B \\
\hline Anacaena globulus (Paykull, 1798) & & & & $\mathrm{N}$ & & $\mathrm{X}$ & $\mathrm{X}$ & $\mathrm{N}$ \\
\hline Anacaena limbata (Fabricius, 1792) & $\mathrm{X}$ & $\mathrm{X}$ & $\mathrm{X}$ & $\mathrm{N}$ & $\mathrm{N}$ & $\mathrm{X}$ & $\mathrm{X}$ & $\mathrm{N}$ \\
\hline Chaetarthria seminulum (Herbst, 1797) & & & & & & & & $\mathrm{N}$ \\
\hline
\end{tabular}

Enochrinae Short \& Fikáček, 2013

Cymbiodyta marginella (Fabricius, 1792)

Enochrus affinis (Thunberg, 1794)

Enochrus ater (Kuwert, 1888)

Enochrus bicolor (Fabricius, 1792)

Enochrus calabricus Ferro, 1986 [E]

Enochrus fuscipennis (C.G. Thomson, 1884)

Enochrus halophilus (Bedel, 1879)

Enochrus melanocephalus (A.G. Olivier, 1793)

Enochrus ochropterus (Marsham, 1802)

Enochrus politus (Küster, 1849)

Enochrus quadripunctatus (Herbst, 1797)

Enochrus testaceus (Fabricius, 1801)

Acidocerinae Zaitzev, 1908

Chasmogenus livornicus (Kuwert, 1890)

Helochares lividus (Forster, 1771)

Helochares obscurus (O.F. Müller, 1776)

Sphaeridiinae Latreille, 1802

\begin{tabular}{r|r}
$X$ & $X$ \\
$X$ & $X$ \\
& \\
& \\
den, 1802 \\
\end{tabular}

Coelostoma hispanicum (Küster, 1848)

Coelostoma orbiculare (Fabricius, 1775)

Dactylosternum abdominale (Fabricius, 1792)

Megasternini Mulsant, 1844

Cercyon granarius Erichson, 1837

Cercyon haemorrhoidalis (Fabricius, 1775)

Cercyon impressus (Sturm, 1807)

Cercyon laminatus Sharp, 1873

Cercyon marimus C.G. Thomson, 1853

Cercyon nigriceps (Marsham, 1802)

Cercyon obsoletus (Gyllenhal, 1808)

Cercyon pygmaeus (Illiger, 1801)

Cercyon quisquilius (Linnaeus, 1760)

Cercyon subsulcatus Rey, 1885

Cercyon terminatus (Marsham, 1802)

Cercyon tristis (Illiger, 1801)

Cercyon unipunclatus (Linnaeus, 1758)

Cercyon ustulatus (Preyssler, 1790)

Coelostomatini L. Heyden, 189

\begin{tabular}{|l|l|l|l|l|l|l|l|}
\hline & & N & & & & \\
\hline & & & & & & B & \\
\hline & X & & & & & CK \\
\hline & & & & & X & & \\
\hline X & & & & & & & \\
\hline & & & & & & & \\
\hline X & & & & B & & \\
\hline & X & & N & X & X & & \\
\hline & X & & & & & & \\
\hline
\end{tabular}

\begin{tabular}{|c|c|c|c|c|c|c|c|}
\hline $\mathrm{X}$ & & & & & & & \\
\hline $\mathrm{X}$ & $\mathrm{X}$ & $\mathrm{B}$ & $\mathrm{N}$ & $\mathrm{X}$ & $\mathrm{X}$ & $\mathrm{CK}$ & \\
\hline & $\mathrm{X}$ & & $\mathrm{CK}$ & & & & \\
\hline
\end{tabular}

$$
891
$$




\begin{tabular}{|c|c|c|c|c|c|c|c|c|c|}
\hline Species & 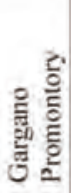 & $\begin{array}{l}\overrightarrow{0} \\
\vdots \\
0 \\
0 \\
\frac{0}{0} \\
\frac{.0}{0} \\
0\end{array}$ & 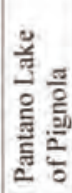 & 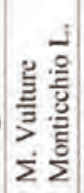 & 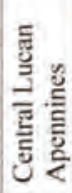 & 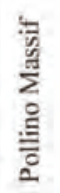 & 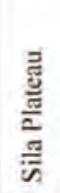 & 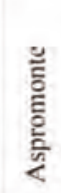 & 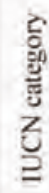 \\
\hline Cryptopleurum crenatum (Kugelann, 1794) & & & $\mathrm{X}$ & & & & & & \\
\hline Cryptopleurum minutum (Fabricius, 1775) & $\mathrm{X}$ & $\mathrm{X}$ & $\mathrm{X}$ & $\mathrm{N}$ & $\mathrm{N}$ & $\mathrm{X}$ & $\mathrm{X}$ & CK & \\
\hline Cryptopleurum subtile Sharp, 1884 & & & CK & & & & & & \\
\hline Megasternum concinnum (Marsham, 1802) & $\mathrm{H}$ & B & $\mathrm{X}$ & $\mathrm{N}$ & CK & $\mathrm{X}$ & $\mathrm{X}$ & CK & \\
\hline \multicolumn{10}{|c|}{ Sphaeridiini Latreille, 1802} \\
\hline Sphaeridium bipustulatum Fabricius, 1781 & $\mathrm{H}$ & $\mathrm{X}$ & $\mathrm{X}$ & & $\mathrm{N}$ & $\mathrm{X}$ & $\mathrm{X}$ & CK & \\
\hline Sphaeridium lunatum Fabricius, 1792 & $\mathrm{X}$ & $\mathrm{N}$ & & & & CK & CK & CK & \\
\hline Sphaeridium scarabaeoides (Linnaeus, 1758) & $\mathrm{H}$ & $\mathrm{X}$ & $\mathrm{X}$ & $\mathrm{N}$ & $\mathrm{N}$ & $\mathrm{X}$ & $\mathrm{X}$ & CK & \\
\hline Sphaeridium substriatum Faldermann, 1838 & B & $\mathrm{X}$ & $\mathrm{X}$ & $\mathrm{N}$ & $\mathrm{N}$ & $\mathrm{X}$ & $\mathrm{X}$ & & \\
\hline
\end{tabular}

HELOPHORIDAE Leach, 1815

Fikáček, Angus, Gentili, Jia \& Minoshima in Cat. Pal. Col., vol. 2, 2015

\begin{tabular}{|c|c|c|c|c|c|c|c|c|}
\hline Helophorus aequalis J. Thomson, 1868 & & & & & & & B & \\
\hline Helophorus alternans Gené, 1836 & $\mathrm{H}$ & CK & & $\mathrm{N}$ & $\mathrm{N}$ & & & CK \\
\hline Helophorus aquaticus (Linnaeus, 1758) & $\mathrm{H}$ & $\mathrm{X}$ & $\mathrm{N}$ & $\mathrm{N}$ & B & $\mathrm{X}$ & $\mathrm{X}$ & $\mathrm{N}$ \\
\hline Helophorus asperatus Rey, 1885 & & & & & & $\mathrm{X}$ & & \\
\hline Helophorus brevipalpis brevipalpis Bedel, 1881 & $\mathrm{H}$ & $\mathrm{X}$ & $\mathrm{X}$ & $\mathrm{N}$ & $\mathrm{N}$ & $\mathrm{X}$ & $\mathrm{X}$ & $\mathrm{N}$ \\
\hline Helophorus discrepans Rey, 1885 & & & & & & $\mathrm{X}$ & B & \\
\hline Helophorus dorsalis (Marsham, 1802) & $\mathrm{H}$ & $\mathrm{X}$ & $\mathrm{X}$ & & & $\mathrm{N}$ & & \\
\hline Helophorus flavipes Fabricius, 1792 & CK & $\mathrm{X}$ & $\mathrm{X}$ & $\mathrm{N}$ & $\mathrm{N}$ & $\mathrm{X}$ & $\mathrm{X}$ & CK \\
\hline Helophorus fulgidicollis Motschulsky, 1860 & B & & & & & & & \\
\hline Helophorus glacialis A. Villa \& G.B. Villa, 1833 & & & & & & $\mathrm{X}$ & & \\
\hline Helophorus gramularis (Linnaeus, 1760) & $\mathrm{X}$ & CK & & & & $\mathrm{X}$ & $\mathrm{X}$ & \\
\hline Helophorus grisens Herbst, 1793 & CK & & & & & $\mathrm{X}$ & & \\
\hline Helophorus illustris Sharp, 1916 & CK & $\mathrm{X}$ & & & & & & \\
\hline Helophorus longitarsis Wollaston, 1864 & $\mathrm{X}$ & B & & & & $\mathrm{X}$ & $\mathrm{X}$ & \\
\hline Helophorus milleri Kuwert, 1886 & CK & $\mathrm{X}$ & $\mathrm{X}$ & $\mathrm{N}$ & & $\mathrm{X}$ & $\mathrm{X}$ & CK \\
\hline Helophortus minutus Fabricius, 1775 & & CK & & $\mathrm{N}$ & & $\mathrm{X}$ & $\mathrm{X}$ & \\
\hline Helophorus porculus Bedel, 1881 & & $\mathrm{~N}$ & $\mathrm{X}$ & & & & & $!$ \\
\hline Helophorus rufipes (Bosc, 1791) & $\mathrm{H}$ & CK & & $\mathrm{N}$ & & $\mathrm{X}$ & & \\
\hline
\end{tabular}

HYDROCHIDAE C.G. Thomson, 1859

Fikáček, Angus, Gentili, Jia \& Minoshima in Cat. Pal. Col., vol. 2, 2015

Hydrochus angustatus Germar, 1824

Hydrochus flavipennis Küster, 1852

Hydrochus grandicollis Kiesenwetter, 1870

\begin{tabular}{|l|l|l|l|l|l|l|l|l|} 
& $\mathrm{X}$ & & & $\mathrm{X}$ & & & \\
\hline $\mathrm{CK}$ & $\mathrm{B}$ & $\mathrm{X}$ & $\mathrm{N}$ & $\mathrm{N}$ & $\mathrm{X}$ & $\mathrm{X}$ & $\mathrm{N}$ & \\
\hline & & & & & $\mathrm{X}$ & $\mathrm{X}$ & & \\
\hline
\end{tabular}

GEORISSIDAE Laporte, 1840

Fikáček \& Przewoźny in Cat. Pal. Col., vol. 2, 2015

Georissus costatus Laporte, 1840

Georissus crenulatus (P. Rossi, 1794)

Georissus laesicollis Germar, 1832

HISTERIDAE Gyllenhal, 1808

\begin{tabular}{|c|c|c|c|c|c|}
\hline $\mathrm{X}$ & & $\mathrm{CK}$ & & & $\mathrm{CK}$ \\
\hline $\mathrm{CK}$ & & & $\mathrm{B}$ & & $\mathrm{CK}$ \\
\hline
\end{tabular}

Abraeinae W.S. Macleay, 1819

Abraeini W.S. Macleay, 1819

Lackner, Mazur \& Newton in Cat. Pal. Col, vol. 2, 2015

Abraeus granulum Erichson, 1839

Abraens perpusillus (Marsham, 1802)

Chaetabraeus globulus (Creutzer, 1799)

Chaetabraeus lucidus (Peyerimhoff, 1917)

\begin{tabular}{|c|c|c|c|c|c|c|c|c|}
\hline CK & & & & CK & X & & CK & LC \\
\hline B & X & & N & CK & X & & CK & LC \\
\hline H & X & & & N & & & & \\
\hline & & & & & N & & B & \\
\hline
\end{tabular}

Acritini Wenzel, 1944

Acritus homoeopathicus Wollaston, 1857

\begin{tabular}{|c|c|c|c|c|c|c|c|c|}
\hline $\mathrm{H}$ & & & & & & & $\mathrm{CK}$ & $\mathrm{NT}$ \\
\hline $\mathrm{H}$ & $\mathrm{CK}$ & $\mathrm{X}$ & $\mathrm{CK}$ & $\mathrm{CK}$ & $\mathrm{CK}$ & $\mathrm{X}$ & $\mathrm{B}$ & \\
\hline $\mathrm{H}$ & $\mathrm{CK}$ & & & $\mathrm{CK}$ & & & & \\
\hline
\end{tabular}

Acritus italicus Reitter, 1904

Acritus mimutus (Herbst, 1791) 
Species

Acritus nigricornis (Hoffmann, 1803)

Aeletes atomarius (Aubé, 1842)

Halacritus punctum (Aubé, 1842)

Eubrachium hispidulum (Bremi-Wolf, 1855)

Plegaderus dissectus Erichson, 1839

Plegaderus otti Marseul, 1856

Plegaderus sanatus gobanzi G. Müller, 1903

Plegaderus saucius Erichson, 1834

Dendrophilinae Reitter, 1909

Anapleini Olexa, 1982

Bacanimus consobrimus (Aubé, 1850)

Dendrophilini Reitter, 1909

Plegaderini Portevin, 1929

\begin{tabular}{|c|c|c|c|c|c|c|c|c|}
\hline 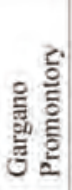 & $\begin{array}{l}\bar{\delta} \\
\vdots \\
\vdots \\
0 \\
0 \\
0 \\
0\end{array}$ & 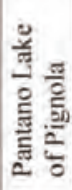 & 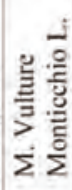 & 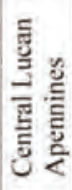 & 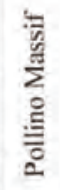 & 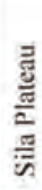 & हू & 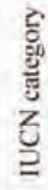 \\
\hline $\mathrm{X}$ & CK & & $\mathrm{N}$ & & CK & $\mathrm{X}$ & CK & \\
\hline & $\mathrm{X}$ & & CK & & CK & & CK & LC \\
\hline $\mathrm{K}$ & & & CK & & & & & \\
\hline
\end{tabular}

\begin{tabular}{|c|c|c|c|c|c|c|c|c|}
\hline $\mathrm{B}$ & & & & $\mathrm{CK}$ & & & & \\
\hline $\mathrm{X}$ & $\mathrm{X}$ & & $\mathrm{CK}$ & $\mathrm{X}$ & $\mathrm{CK}$ & $\mathrm{CK}$ & $\mathrm{LC}$ \\
\hline $\mathrm{X}$ & $\mathrm{X}$ & & & $\mathrm{N}$ & & $\mathrm{X}$ & & $\mathrm{VU}$ \\
\hline & & & & & & $\mathrm{CK}$ & & $\mathrm{CR}$ \\
\hline & & & & & & $\mathrm{X}$ & $\mathrm{CK}$ & $\mathrm{VU}$ \\
\hline
\end{tabular}

Dendrophilus punctatus punctatus (Herbst, 1791

Kissister minimus (Laporte, 1840)

Paromalini Reitter, 1909

\begin{tabular}{|c|c|c|c|c|c|c|c|c|}
\hline & CK & & & & & & B & LC \\
\hline & X & X & N & CK & X & X & CK & \\
\hline
\end{tabular}

Carcinops pumilio (Erichson, 1834)

Paromalus filum Reitter, 1884

Paromalus flavicormis (Herbst, 1791)

Paromalus parallelepipedus (Herbst, 1791)

Platylomalus complanatus (Panzer, 1797)

Haeteriinae Marseul, 1857

Haetaerius ferrugineus (A.G. Olivier, 1789) $\mathrm{H}$

\begin{tabular}{|c|c|c|c|c|c|c|c|c|}
\hline X & X & & & N & X & X & & \\
\hline CK & CK & & & CK & CK & & & VU \\
\hline H & X & X & N & CK & X & X & B & LC \\
\hline CK & CK & & N & CK & X & X & CK & LC \\
\hline CK & X & & & B & X & X & B & LC \\
\hline
\end{tabular}

Histerinae Gyllenhal, 1808

Histerini Gyllenhal, 1808

\begin{tabular}{|c|c|c|c|c|c|c|c|c|c|}
\hline Atholus bimaculatus (Linnaeus, 1758) & $\mathrm{H}$ & $\mathrm{X}$ & & $\mathrm{N}$ & CK & & CK & CK & \\
\hline Atholus corvinus (Germar, 1817) & $\mathrm{X}$ & & & & & & $\mathrm{X}$ & & \\
\hline Atholus duodecimstriatus duodecimstriatus (Schrank, 1781) & $\mathrm{H}$ & $\mathrm{X}$ & $\mathrm{X}$ & $\mathrm{N}$ & CK & $\mathrm{X}$ & $\mathrm{X}$ & B & \\
\hline Atholus scutellaris (Erichson, 1834) & B & & & & & & & & \\
\hline Atholus siculus (Tournier, 1869) & CK & & & & & & & & \\
\hline Hister bissexstriatus Fabricius, 1801 & & & & & B & & & CK & \\
\hline Hister capsirensis Auzat, 1922 & & & & & CK & & $\mathrm{X}$ & CK & \\
\hline Hister fimestes Erichson, 1834 & CK & & & & & & CK & & \\
\hline Hister helluo Truqui, 1852 & & $\mathrm{X}$ & & & & CK & & B & \\
\hline Hister illigeri illigeri Duftschmid, 1805 & $\mathrm{H}$ & $\mathrm{X}$ & $\mathrm{X}$ & $\mathrm{N}$ & CK & $\mathrm{X}$ & $\mathrm{X}$ & B & \\
\hline Hister lugubris Truqui, 1852 & & & CK & & & & & & \\
\hline Hister mediterraneus Lundgren, 1991 & $\mathrm{~N}$ & & & & & & & & \\
\hline Hister moerens Erichson, 1834 & B & & & & CK & CK & & CK & \\
\hline Hister quadrimaculatus Linnaeus, 1758 & $\mathrm{H}$ & $\mathrm{X}$ & $\mathrm{X}$ & $\mathrm{N}$ & CK & $\mathrm{X}$ & $\mathrm{X}$ & CK & \\
\hline Hister quadrinotatus quadrinotatus L.G. Scriba, 1790 & $\mathrm{H}$ & & & & & & & & \\
\hline Hister teter Truqui, 1852 & $\mathrm{X}$ & CK & & CK & CK & $\mathrm{X}$ & $\mathrm{X}$ & & \\
\hline Hister unicolor umicolor Linnaeus, 1758 & $\mathrm{X}$ & $\mathrm{X}$ & & & CK & CK & $\mathrm{X}$ & CK & \\
\hline Margarinotus brumneus (Fabricius, 1775) & $\mathrm{X}$ & $\mathrm{X}$ & $\mathrm{X}$ & & CK & & CK & CK & \\
\hline Margarinotus carbonarius carbonarius (Hoffmann, 1803) & $\mathrm{X}$ & & & & & $\mathrm{X}$ & & B & \\
\hline Margarinotus ignobilis (Marseul, 1854) & $\mathrm{X}$ & B & & & $\mathrm{N}$ & $\mathrm{X}$ & & & \\
\hline Margarinotus merdarius (Hoffmann, 1803) & & & & & & CK & & & LC \\
\hline Margarinotus neglectus (Germar, 1813) & $\mathrm{X}$ & & & & CK & $\mathrm{X}$ & $\mathrm{X}$ & CK & \\
\hline Margarinotus obscurus (Kugelann, 1792) & $\mathrm{H}$ & & & B & CK & & $\mathrm{X}$ & $\mathrm{CK}$ & \\
\hline Margarinotus punctiventer (Marseul, 1854) & $\mathrm{X}$ & & & CK & CK & $\mathrm{X}$ & $\mathrm{X}$ & CK & \\
\hline Margarinotus purpurascens (Herbst, 1791) & $\mathrm{H}$ & $\mathrm{X}$ & & $\mathrm{CK}$ & CK & $\mathrm{X}$ & & & \\
\hline Margarinotus striola succicola (C.G. Thomson, 1862) & & & & & CK & $\mathrm{X}$ & $\mathrm{X}$ & $\mathrm{B}$ & NT \\
\hline Margarinotus ventralis (Marseul, 1854) & B & & & $\mathrm{N}$ & CK & $\mathrm{X}$ & $\mathrm{X}$ & CK & \\
\hline
\end{tabular}


Merohister ariasi (Marseul, 1864

Pachylister inaequalis (A.G. Olivier, 1789)

Pactolimus major (Linnaeus, 1767)

\begin{tabular}{|c|c|c|c|c|c|c|c|c|}
\hline 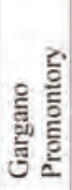 & $\begin{array}{l}\vec{c} \\
0 \\
\vdots \\
0 \\
\frac{3}{0} \\
\frac{3}{0} \\
2\end{array}$ & 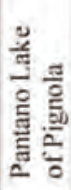 & 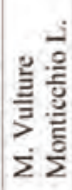 & 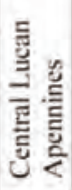 & 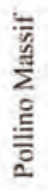 & 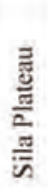 & 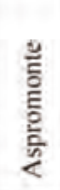 & 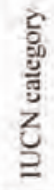 \\
\hline CK & & & & CK & & & & EN \\
\hline$X$ & CK & & $\mathrm{N}$ & CK & $X$ & $X$ & CK & \\
\hline $\mathrm{X}$ & $\mathrm{X}$ & & & & & & B & \\
\hline
\end{tabular}

Platysomatini Bickhardt, 1914

Eurosomides minor (P. Rossi, 1792)

Platysoma angustatum (Hoffmann, 1803)

Platysoma compressum (Herbst, 1783)

Platysoma elongatum elongatum (Thunberg, 1787)

Platysoma filiforme Erichson, 1834

Platysoma lineare Erichson, 1834

Onthophilinae W.S. Macleay, 1819

Onthophilus affinis L. Redtenbacher, 1847

Onthophilus punctatus cicatricosus Reitter, 1884

Onthophilus punctatus punctatus (O.F. Müller, 1776)

Onthophilus striatus striatus (Forster, 1771)

\begin{tabular}{|c|c|c|c|c|c|c|c|}
\hline $\mathrm{H}$ & $\mathrm{X}$ & & CK & $\mathrm{X}$ & $\mathrm{X}$ & & \\
\hline & & & & & CK & & VU \\
\hline $\mathrm{H}$ & & & CK & $\mathrm{X}$ & CK & B & LC \\
\hline B & $\mathrm{X}$ & $\mathrm{N}$ & & $\mathrm{X}$ & $\mathrm{X}$ & B & LC \\
\hline $\mathrm{X}$ & & & & & $X$ & & NT \\
\hline CK & & & & & $X$ & CK & VU \\
\hline
\end{tabular}

Saprininae C.É. Blanchard, 1845

\begin{tabular}{|c|c|c|c|c|c|c|c|c|}
\hline X & & & & & & & & \\
\hline & CK & & & & & & \\
\hline H & X & & & CK & N & X & CK \\
\hline H & X & X & CK & CK & X & B & B \\
\hline
\end{tabular}

Chalcionellus aemulus (Illiger, 1807)

Chalcionellus decemstriatus decemstriatus (P. Rossi, 1792)

Gnathoncus buyssoni Auzat, 1917

Gnathoncus communis (Marseul, 1862)

Gnathoncus nannetensis (Marseul, 1862)

Gnathoncus rotundatus (Kugelann, 1792)

Hypocacculus metallescens (Erichson, 1834)

Hypocacculus spretulus (Erichson, 1834)

Hypocaccus brasiliensis (Paykull, 1811)

Hypocaccus dimidiatus dimidiatus (Illiger, 1807)

Hypocaccus rubripes (Erichson, 1834)

Hypocaccus rugifions (Paykull, 1798)

Pholioxemus schatzmayri (G. Múller, 1910)

Saprinus acuminatus actminatus (Fabricius, 1798)

Saprinus aegialins Reitter, 1884

Saprimus aeneus (Fabricius, 1775)

Saprinus caerulescens caerulescens (Hoffmann, 1803)

Saprinus calatravensis Fuente, 1899

Saprinus chalcites (Illiger, 1807)

Saprinus detersus (Illiger, 1807)

Saprimus furvus Erichson, 1834

Saprimus georgicus Marseul, 1862

Saprinus godet (Brullé, 1832)

Saprinus immundus (Gyllenhal, 1827)

Saprimus lautus lautus Erichson, 1839

Saprimus maculatus (P. Rossi, 1792)

Saprinus planiusculus Motschulsky, 1849

Saprinus polinus politus (Brahm, 1790)

Saprinus semistriatus (L.G. Scriba, 1790

Saprinus subnitescens Bickhardt, 1909

Saprinus tenuistritus sparsutus Solsky, 1876

Tribalinae Bickhardt, 1914

Epierus comptus Erichson, 1834

Psendepierus italicus (Paykull, 1811)

Tribalus scaphidiformis (Illiger, 1807)

\begin{tabular}{|c|c|c|c|c|c|c|c|c|}
\hline CK & X & & & CK & & & CK & \\
\hline H & X & X & & CK & CK & CK & B & \\
\hline & & & & CK & & & ! & \\
\hline & CK & & & B & CK & & & \\
\hline & CK & & & & & & & LC \\
\hline X & & & & & & & CK & \\
\hline X & X & & & & & X & CK & \\
\hline X & X & & & CK & & & CK & \\
\hline CK & X & & & CK & CK & X & CK & \\
\hline H & & & & & & & & \\
\hline H & X & & & CK & CK & X & CK & \\
\hline X & X & & CK & CK & X & X & B & \\
\hline & & & & & & CK & & \\
\hline X & X & & & CK & & & & \\
\hline CK & CK & & & CK & X & CK & & \\
\hline H & X & & & CK & & & & \\
\hline CK & X & & N & CK & X & X & & \\
\hline CK & X & & N & CK & X & & & \\
\hline X & X & & & & & & CK & \\
\hline CK & & & & CK & & & & \\
\hline & & & CK & & X? & & & \\
\hline & & & & N & B & & & \\
\hline B & & & & CK & & & & \\
\hline & & & & CK & N & CK & & \\
\hline H & X & & N & CK & X & X & CK & \\
\hline H & & & & CK & X & X & & \\
\hline & & X & N & CK & X & X & CK & \\
\hline & & & CK & X & B & & \\
\hline
\end{tabular}

\begin{tabular}{|c|c|c|c|c|c|c|c|c|}
\hline $\mathrm{H}$ & $\mathrm{X}$ & & $\mathrm{N}$ & $\mathrm{CK}$ & $\mathrm{X}$ & $\mathrm{X}$ & $\mathrm{CK}$ & $\mathrm{LC}$ \\
\hline $\mathrm{X}$ & $\mathrm{X}$ & & $\mathrm{N}$ & $\mathrm{CK}$ & $\mathrm{X}$ & $\mathrm{X}$ & $\mathrm{CK}$ & $\mathrm{LC}$ \\
\hline & & & & & $\mathrm{CK}$ & & $\mathrm{CK}$ & \\
\hline
\end{tabular}




\section{Species}

\begin{tabular}{|c|c|c|c|c|c|c|c|c|}
\hline 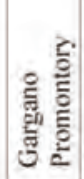 & $\begin{array}{l}\overline{0} \\
0 \\
3 \\
0 \\
\frac{2}{0} \\
\frac{3}{0} \\
2\end{array}$ & 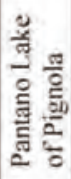 & 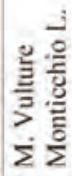 & 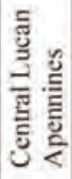 & 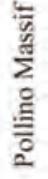 & $\frac{\frac{\overrightarrow{\mathrm{E}}}{\frac{\mathrm{m}}{2}}}{\frac{\mathrm{m}}{\mathrm{n}}}$ & 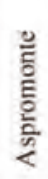 & 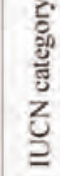 \\
\hline
\end{tabular}

STAPHYLINOIDEA Latreille, 1802

\section{HYDRAENIDAE Mulsant, 1844}

Hydraeninae Mulsant, 1844

Hydraenini Mulsant, 1844

Jäch \& Skale in Cat. Pal. Col., vol. 2, 2015

Hydraena alia d'Orchymont, 1934 [E]

Hydraena angulosa Mulsant, 1844

Hydraena assimilis Rey, 1885

Hydraena devillei Ganglbauer, 1901

Hydraena heterogyna Bedel, 1898

Hydraena imperatrix Kniz, 1919 [E]

Hydraena minutissima Stephens, 1829

Hydraena pygmaea pygmaea G.R. Waterhouse, 1833

Hydraena sicula Kiesenwetter, 1849 [E]

Hydraena similis d'Orchymont, 1930 [E]

Hydraena solarii Pretner, 1930 [E]

Hydraena sp.cfr. sanfilippoi Audisio \& De Biase, 1995 [E]

Hydraena spinipes Baudi di Selve, 1882 [E]

Hydraena subimpressa Rey, 1885

Hydraena subirregularis Pic, 1918 [E]

Hydraena truncata Rey, 1885

\begin{tabular}{|l|c|c|c|c|c|c|c|c|}
\hline & & & CK & & X & & \\
\hline & & & B & & & & \\
\hline & & & & X & X & CK & \\
\hline & & & N & X & X & B & \\
\hline & & & CK & X & X & B & \\
\hline & & & & CK & CK & CK & \\
\hline & & & & & X & CK & \\
\hline & & & & & & B & \\
\hline & & & CK & CK & X & B & \\
\hline & & & B & & & & \\
\hline & & CK & CK & CK & X & N & \\
\hline & & & CK & CK & X & B & \\
\hline & & & & & X & B & \\
\hline & & & B & X & X & B & \\
\hline
\end{tabular}

Limnebiini Mulsant, 1844

Limnebius calabricus Jäch, 1993 [E]

Limnebius furcatus Baudi di Selve, 1872

Limnebius nitiduloides Baudi di Selve, 1872

Limnebius nitidus (Marsham, 1802)

Limnebius oblongus Rey, 1883

Limnebius papposus Mulsant, 1844

Limnebius perparvulus Rey, 1884

\begin{tabular}{|c|c|c|c|c|c|c|c|c|}
\hline $\mathrm{X}$ & & & & & $\mathrm{CK}$ & $\mathrm{X}$ & $\mathrm{CK}$ & \\
\hline $\mathrm{X}$ & $\mathrm{X}$ & $\mathrm{N}$ & & & $\mathrm{X}$ & $\mathrm{X}$ & & \\
& & & & $\mathrm{CK}$ & $\mathrm{X}$ & $\mathrm{X}$ & & \\
\hline & & & & & $\mathrm{X}$ & & & \\
\hline & & & & $\mathrm{N}$ & & & & \\
\hline & & & & & $\mathrm{X}$ & $\mathrm{X}$ & & \\
\hline
\end{tabular}

Ochthebiinae C.G. Thomson, 1859

Aulacochthebius exaratus Mulsant, 1844

Ochthebius adriaticus adriaticus Reitter, 1886

Ochthebius basilicatus A. Fiori, 1915 [E]

Ochthebius brevicollis Baudi di Selve, 1864

Ochthebius corcyraeus Jäch, 1990

Ochthebius cremulatus Mulsant \& Rey, 1850

Ochthebius dentifer Rey, 1885

Ochthebius difficilis Mulsant, 1844

Ochthebius dilatatus Stephens, 1829

Ochthebius gagliardii d'Orchymont, 1940 [E]

Ochthebius impressipennis Rey, 1886

Ochthebius lamuginosus Reiche \& Saulcy, 1856

Ochthebius latinorum (leniştea, 1988) [E]

Ochthebius mediterraneus (Ieniștea, 1988)

Ochthebius minimus (Fabricius, 1792)

Ochthebius nobilis A. Villa \& G.B. Villa, 1835

Ochthebius opacus Baudi di Selve, 1882

Ochthebius pilosus Waltl, 1835

Ochthebius punctatus Stephens, 1829

Ochihebius quadricollis Mulsant, 1844

Ochthebius quadrifossulatus Waltl, 1835

\begin{tabular}{|c|c|c|c|c|c|c|c|c|c|}
\hline & N & X & & & & & & \\
\hline B & & & & & & & & \\
\hline B & CK & & CK & B & & & & \\
\hline & & & N & B & CK & & B & \\
\hline H & CK & & X & & CK & X & X & N & \\
\hline B & X & & & & & & & \\
\hline B & & & & CK & & B & \\
\hline & & & & & & & B & \\
\hline CK & & & & & CK & & B & \\
\hline & & & & B & N & & B & \\
\hline H & X & & & & & & & \\
\hline & & & & & & & B & \\
\hline & & & & B & & & B & \\
\hline
\end{tabular}


Ochthebius ragusae Kuwert, 1887

Ochthebius siculus Kuwert, 1887

Ochthebius subinteger Mulsant \& Rey, 1861

Ochthebius subpictus subpictus Wollaston, 1857

Ochthebius tacapasensis tacapasensis Ferro, 1983

Ochthebius urbanelliae Audisio, Trizzino \& De Biase, 2010

Ochthebius virgula Ferro, 1986 [E]

Ochthebius viridescens Ieniștea, 1988

Ochthebius viridis viridis Peyron, 1858

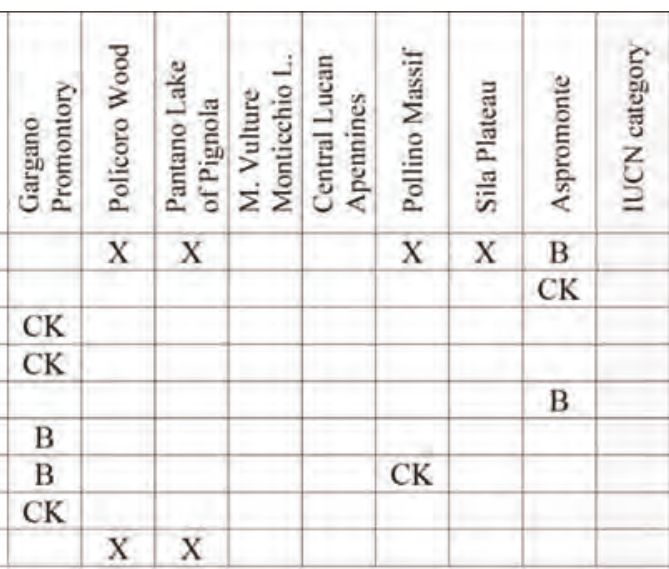

PTILIIDAE Erichson, 1845

Nossidiinae Sörensson \& Delgado, 2019

Sörenson in Cat. Pal. Col. Vol. 2, 2015; Sörenson \& Delgado, 2019

Nossidium flachi Ganglbauer, 1898

Nossidium pilosellum (Marsham, 1802)

\begin{tabular}{|l|l|l|l|l|l|l|l|l|}
\hline B & & & & B & & & & DD \\
\hline B & $\mathrm{X}$ & & N & B & B & N & B & DD \\
\hline
\end{tabular}

Ptiliinae Erichson, 1845

Actidiini Portevin, 1929

Actidium coarctatum (Haliday, 1855)

Ptiliini Erichson, 1845

Millidium minutissimum (Weber \& Mohr, 1804)

Ptiliola kunzei (Heer, 1841)

Ptiliolum africanum Peyerimhoff, 1917

Ptiliolum caledonicum (Sharp, 1871)

Ptiliolum fuscum (Erichson, 1845)

Ptiliolum hopffgarteni (Flach, 1888)

Ptiliolum marginatum (Aubé, 1850)

Priliolum oedipus (Flach, 1886)

Ptiliolum schwarzi (Flach, 1887)

Ptiliolum spencei (Allibert, 1844)

Ptilium caesum Erichson, 1845

Ptilium exaratum (Allibert, 1844)

Ptilium tenue Kraatz, 1858

\begin{tabular}{|c|c|c|c|c|c|c|c|}
\hline & $\mathrm{N}$ & & & & & & DD \\
\hline \multicolumn{8}{|c|}{845} \\
\hline & $\mathrm{X}$ & & & & & & \\
\hline \multirow[t]{2}{*}{$\mathrm{X}$} & $X$ & & & & & $\mathrm{~N}$ & DD \\
\hline & & & B & B & & B & \\
\hline \multirow[t]{3}{*}{$\mathrm{H}$} & & & & & & & DD \\
\hline & $X$ & & & & & $\mathrm{~N}$ & DD \\
\hline & & & $\mathrm{N}$ & B & & B & DD \\
\hline \multirow[t]{4}{*}{$\mathrm{X}$} & & $\mathrm{X}$ & & B & $\mathrm{X}$ & $\mathrm{N}$ & DD \\
\hline & & & & B & & B & DD \\
\hline & & & B & B & B & B & DD \\
\hline & & & $\mathrm{N}$ & B & & & DD \\
\hline $\mathrm{H}$ & & & & & & & DD \\
\hline $\mathrm{X}$ & & & & & & & DD \\
\hline $\mathrm{X}$ & & B & & & & & DD \\
\hline
\end{tabular}

Ptenidium brenskei Flach, 1887

Ptenidium fuscicorne Erichson, 1845

Ptenidium intermedium Wankowicz, 1869

Ptenidium laevigatum Erichson, 1845

Ptenidium longicorne Fuss, 1868

Plenidium nitidum (Heer, 1841)

Plenidium pusillum (Gyllenhal, 1808)

Ptenidium turgidum C.G. Thomson, 1855

Ptenidiini Flach, 1889

\section{Ptinellini Reitter, 1906}

Microptilium pulchellum (Allibert, 1844)

Pteryx ganglbaueri Erichson, 1909 [E]

Pteryx suturalis (Heer, 1841)

Ptinella aptera (Guérin-Méneville, 1839)

Ptinella denticollis (Fairmaire, 1858)

Ptinella limbata (Heer, 1841)

Ptinella mekura Kubota, 1943

\begin{tabular}{|c|c|c|c|c|c|c|c|c|}
\hline & & & & & & & B & DD \\
\hline & B & & & & & & B & DD \\
\hline & & B & & & & & B & DD \\
\hline & X & & & N & X & X & N & DD \\
\hline & X & & & & & & B & DD \\
\hline H & X & B & N & & N & N & B & DD \\
\hline X & X & & & & X & & N & DD \\
\hline
\end{tabular}

\begin{tabular}{|c|c|c|c|c|c|c|c|c|}
\hline & & B & & & & & & \\
\hline & & & & & & & B & NT \\
\hline & & & N & B & X & X & B & DD \\
\hline & & & & & & & DD \\
\hline & & & & & & & & DD \\
\hline & & & B & & & & DD \\
\hline
\end{tabular}

Acrotrichini Reitter, 1909

Nephanina Portevin, 1929

Nephanes titan (Newman, 1834) 
Species

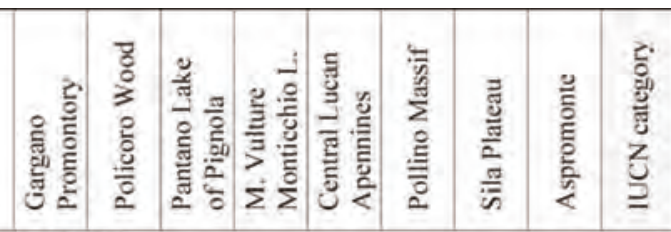

Acrotrichina Reitter, 1909

\begin{tabular}{|c|c|c|c|c|c|c|c|c|}
\hline Acrotrichis arnoldi Rosskothen, 1935 & & & & & & & & B \\
\hline Acrotrichis atomaria (DeGeer, 1774) & $\mathrm{H}$ & B & $\mathrm{B}$ & $\mathrm{N}$ & B & B & $\mathrm{N}$ & B \\
\hline Acrotrichis danica Sundt, 1958 & & & & & & & & B \\
\hline Acrotrichis dispar (A. Matthews, 1865) & & & & & & B & & \\
\hline Acrotrichis fascicularis (Herbst, 1793) & B & & $\mathrm{X}$ & B & B & B & $\mathrm{N}$ & B \\
\hline Acrotrichis grandicollis (Mannerheim, 1844) & B & $\mathrm{X}$ & $\mathrm{X}$ & B & B & B & & $\mathrm{N}$ \\
\hline Acrotrichis intermedia (Gillmeister, 1845) & $\mathrm{H}$ & B & $\mathrm{X}$ & B & B & B & $\mathrm{B}$ & B \\
\hline Acrotrichis montandonii (Allibert, 1844) & $\mathrm{X}$ & $=$ & & 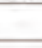 & B & $\mathrm{X}$ & $\mathrm{X}$ & B \\
\hline Acrotrichis pumila (Erichson, 1845) & & $\mathrm{N}$ & & & & & & \\
\hline Acrotrichis rosskotheni Sundt, 1971 & B & B & & B & B & B & B & B \\
\hline Acrotrichis sericans (Heer, 1841) & & & $\mathrm{X}$ & & B & $\mathrm{N}$ & $\mathrm{N}$ & $\mathrm{N}$ \\
\hline Acrotrichis sitkaensis (Motschulsky, 1845) & & & & & $\mathrm{N}$ & $\mathrm{X}$ & & \\
\hline Acrotrichis thoracica (Waltl, 1838) & & B & $\mathrm{X}$ & & & & & \\
\hline
\end{tabular}

AGYRTIDAE C,G. Thomson, 1859

Agyrtinae C.G. Thomson, 1859

Rúžička in Cat. Pal. Col., vol. 2, 2015

Agyrtes bicolor Laporte, 1840

LEIODIDAE Fleming, 1821

Cholevinae Kirby, 1837

Anemadini Hatch, 1928

Anemadina Hatch, 1928

Perreau in Cat. Pal. Col., vol. 2, 2015

\begin{tabular}{|l|c|c|c|c|c|c|c|c|c|}
\hline Anemadus acicularis (Kraatz, 1852) & $\mathrm{H}$ & $\mathrm{X}$ & $\mathrm{CK}$ & $\mathrm{B}$ & $\mathrm{B}$ & $\mathrm{CK}$ & & & \\
\hline Anemadus italicus (Zoia, 1990) [E] & & $\mathrm{CK}$ & $\mathrm{X}$ & $\mathrm{B}$ & $\mathrm{B}$ & $\mathrm{B}$ & $\mathrm{B}$ & $\mathrm{B}$ & \\
\hline Anemadus strigosus strigosus (Kraatz, 1852) & & $\mathrm{X}$ & & $\mathrm{CK}$ & $\mathrm{B}$ & $\mathrm{X}$ & $\mathrm{X}$ & $\mathrm{CK}$ & \\
\hline Speonemadus orchesioides (Fairmaire, 1879) & & & $\mathrm{CK}$ & & & & $\mathrm{B}$ & & \\
\hline
\end{tabular}

Speonemadus orchesioides (Fairmaire, 1879)

Cholevini Kirby, 1837

Catopina Chaudoir, 1845

Catops coracimus Kellner, 1846

Catops fuliginosus Erichson, 1837

Catops fuscus fuscoides Reitter, 1909

Catops grandicollis Erichson, 1837

Catops kirbyi kirbyi (Spence, 1815)

Catops nigricans (Spence, 1813)

Catops nigriclavis Gerhardt, 1900

Catops nitidicollis Kraatz, 1856

Catops picipes (Fabricius, 1787)

Catops subfiuscus subfuscus Kellner, 1846

Catops tristis tristis (Panzer, 1794)

Catops ventricosus rotundatus Szymezakowski, 1963

Dreposcia umbrina (Erichson, 1837)

Sciodrepoides watsoni watsoni (Spence, 1815)

Cholevina Kirby, 1837

\begin{tabular}{|c|c|c|c|c|c|c|c|c|}
\hline $\mathrm{X}$ & $\mathrm{CK}$ & & & $\mathrm{CK}$ & & & & \\
\hline $\mathrm{CK}$ & & & $\mathrm{CK}$ & $\mathrm{CK}$ & $\mathrm{X}$ & $\mathrm{X}$ & $\mathrm{N}$ & \\
\hline $\mathrm{X}$ & $\mathrm{X}$ & & & CK & & & & \\
\hline & & $\mathrm{CK}$ & $\mathrm{N}$ & $\mathrm{B}$ & $\mathrm{N}$ & & & \\
\hline & & & $\mathrm{B}$ & $\mathrm{B}$ & $\mathrm{X}$ & $\mathrm{X}$ & $\mathrm{B}$ & \\
\hline & & $\mathrm{CK}$ & $\mathrm{N}$ & $\mathrm{CK}$ & $\mathrm{X}$ & $\mathrm{X}$ & $\mathrm{B}$ & \\
\hline $\mathrm{X}$ & & & & $\mathrm{N}$ & & & & \\
\hline & & & $\mathrm{CK}$ & & $\mathrm{N}$ & & & \\
\hline & & $\mathrm{X}$ & $\mathrm{B}$ & $\mathrm{B}$ & $\mathrm{N}$ & & $\mathrm{CK}$ & \\
\hline & & $\mathrm{X}$ & $\mathrm{CK}$ & $\mathrm{CK}$ & $\mathrm{X}$ & $\mathrm{X}$ & $\mathrm{CK}$ & \\
\hline & & & & $\mathrm{CK}$ & & & $\mathrm{CK}$ & \\
\hline $\mathrm{X}$ & $\mathrm{X}$ & & & $\mathrm{CK}$ & $\mathrm{CK}$ & & & \\
\hline
\end{tabular}

\begin{tabular}{|l|c|c|c|c|c|c|c|c|c|}
\hline Catopsimorphus orientalis orientalis Aubé, 1850 & $\mathrm{H}$ & $\mathrm{X}$ & & $\mathrm{CK}$ & & & $\mathrm{N}$ & \\
\hline Choleva cisteloides cisteloides (Frölich, 1799) & $\mathrm{H}$ & & CK & CK & $\mathrm{X}$ & $\mathrm{X}$ & CK & \\
\hline Choleva garganona Reitter, 1913 [E] & $\mathrm{X}$ & & $\mathrm{CK}$ & $\mathrm{CK}$ & $\mathrm{CK}$ & & CK & \\
\hline Choleva oblonga menozzii Jeannel, 1923 [E] & $\mathrm{H}$ & & & & CK & & & \\
\hline Choleva reitteri Petri, 1915 & & & CK & CK & & & B & \\
\hline Choleva sturmi C.N.F. Brisout de Barneville, 1863 & H & X & CK & CK & N & & CK & \\
\hline Nargus badius badius (Sturm, 1839) & H & X & X & CK & CK & X & X & B & \\
\hline
\end{tabular}




\begin{tabular}{|c|c|c|c|c|c|c|c|c|c|}
\hline Species & 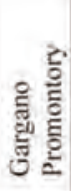 & $\begin{array}{l}\bar{\zeta} \\
\vdots \\
3 \\
0 \\
\frac{2}{3} \\
\frac{0}{0} \\
=\end{array}$ & 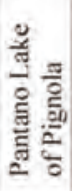 & 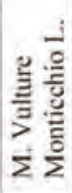 & 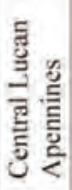 & 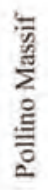 & $\begin{array}{l}\frac{\overrightarrow{\mathrm{e}}}{\mathrm{w}} \\
\frac{\mathrm{m}}{\mathrm{a}} \\
\frac{\mathrm{g}}{\mathrm{n}}\end{array}$ & 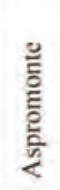 & 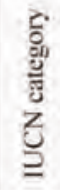 \\
\hline Nargus brunneus (Sturm, 1839) & & & & & CK & & & CK & \\
\hline Nargus velox velox (Spence, 1813) & $\mathrm{X}$ & $\mathrm{X}$ & $\mathrm{X}$ & & CK & $\mathrm{X}$ & $\mathrm{X}$ & CK & \\
\hline Nargus wilkini (Spence, 1813) & & & $\mathrm{CK}$ & CK & $\mathrm{CK}$ & $\mathrm{X}$ & $\mathrm{CK}$ & B & \\
\hline
\end{tabular}

Nargus wilkini (Spence, 1813)

Leptodirini Lacordaire, 1854

Bathysciina Horn, 1880

Phaneropella lesinae (Reitter, 1881) Pholeuina Reitter, 1886

Bathysciola ruffoi Tamanini, 1955 [E] B
1886

Ptomaphagini Jeannel, 1911

Ptomaphagina Jeannel, 1911

Ptomophagus divaricatus Jeannel, 1934

Ptomophagus pius pius (Seidlitz, 1887) [E]

Coloninae Horn, 1880

Colon affine Sturm, 1839

Colon bidentatum (C.R. Sahlberg, 1822)

Colon fuscicorne Kraatz, 1852

Colon griseum Czwalina, 1881

\begin{tabular}{|l|l|l|l|l|l|l|l|l|}
\hline $\mathrm{H}$ & & & & & & & & \\
\hline $\mathrm{H}$ & & & & & & & & \\
\hline & & & & $\mathrm{N}$ & $\mathrm{X}$ & & $\mathrm{N}$ & \\
\hline
\end{tabular}

Leiodinae Fleming, 1821

Agathidiini Westwood, 1838

\begin{tabular}{|c|c|c|c|c|c|c|c|c|c|}
\hline Agathidium badium Erichson, 1845 & $\mathrm{H}$ & $\mathrm{X}$ & $\mathrm{X}$ & $\mathrm{N}$ & $\mathrm{N}$ & $\mathrm{X}$ & $\mathrm{X}$ & B & LC \\
\hline Agathidium brisouti Reitter, 1884 & $\mathrm{X}$ & $\mathrm{N}$ & & $\mathrm{N}$ & & $\mathrm{N}$ & $\mathrm{X}$ & B & DD \\
\hline Agathidium dentatum dentatum Mulsant \& Rey, 1861 & & $\mathrm{~N}$ & & & $\mathrm{~N}$ & $\mathrm{~N}$ & & B & LC \\
\hline Agathidium laevigatulum Reitter, 1904 & & $\mathrm{~N}$ & & $\mathrm{~N}$ & $\mathrm{~N}$ & & & & LC \\
\hline Agathidium laevigatum laevigatum Erichson, 1845 & $\mathrm{H}$ & & & $\mathrm{N}$ & $\mathrm{N}$ & $\mathrm{N}$ & & & LC \\
\hline Agathidium mandibulare Sturm, 1807 & $\mathrm{X}$ & & & & & $\mathrm{X}$ & $\mathrm{X}$ & $\mathrm{N}$ & LC \\
\hline Agathidium marginatum Sturm, 1807 & & & & & $\mathrm{~N}$ & $\mathrm{X}$ & & & LC \\
\hline Agathiditum montemurroi Angelini \& De Marzo, 1985 & & $\mathrm{X}$ & & & & $\mathrm{X}$ & $\mathrm{X}$ & B & VU \\
\hline Agathidium nigrimum Sturm, 1807 & $\mathrm{H}$ & $\mathrm{N}$ & & $\mathrm{N}$ & $\mathrm{N}$ & $\mathrm{X}$ & $\mathrm{X}$ & B & LC \\
\hline Agathidium nigripenne (Fabricius, 1792) & $\mathrm{H}$ & & & & $\mathrm{N}$ & $\mathrm{X}$ & & B & LC \\
\hline Agathidium nudum C. Hampe, 1870 & $\mathrm{X}$ & $\mathrm{B}$ & & & & $\mathrm{X}$ & $\mathrm{X}$ & B & DD \\
\hline Agathidium obenbergeri Hlisnikovsky், 1964 [E] & & $\mathrm{X}$ & & $\mathrm{N}$ & $\mathrm{N}$ & $\mathrm{X}$ & $\mathrm{X}$ & B & LC \\
\hline Agathidium paganettianum Hlisnikovský, 1964 [E] & B & & & & & $\mathrm{N}$ & $\mathrm{X}$ & B & LC \\
\hline Agathidium pisanum C.N.F. Brisout de Barneville, 1872 & & $\mathrm{~N}$ & $\mathrm{X}$ & & $\mathrm{N}$ & $\mathrm{X}$ & $\mathrm{X}$ & B & LC \\
\hline Agathidium plagiatum (Gyllenhal, 1810) & $\mathrm{X}$ & $\mathrm{X}$ & & & & $\mathrm{X}$ & $\mathrm{X}$ & B & $\mathrm{LC}$ \\
\hline Agathidium rotundatum paganettii Reitter, 1908 [E] & & $\mathrm{N}$ & & & $\mathrm{N}$ & $\mathrm{X}$ & $\mathrm{X}$ & B & LC \\
\hline Agathidium seminulum (Linnaeus, 1758) & $\mathrm{x}$ & & & & $\mathrm{N}$ & $\mathrm{X}$ & & B & LC \\
\hline Agathidium varians varians Beck, 1817 & $\mathrm{X}$ & $\mathrm{X}$ & & $\mathrm{N}$ & & & & $\mathrm{B}$ & LC \\
\hline Amphicyllis globiformis (C.R. Sahlberg, 1833) & $\mathrm{H}$ & $\mathrm{N}$ & & & & & $\mathrm{X}$ & & LC \\
\hline Amphicyllis globus (Fabricius, 1792) & & $\mathrm{N}$ & & $\mathrm{N}$ & $\mathrm{N}$ & $\mathrm{X}$ & $\mathrm{X}$ & $\mathrm{N}$ & LC \\
\hline Anisotoma castanea castanea (Herbst, 1791) & $\mathrm{X}$ & & & & $\mathrm{N}$ & $\mathrm{X}$ & $\mathrm{X}$ & $\mathrm{N}$ & LC \\
\hline Anisoloma glabra (Fabricius, 1787) & $\mathrm{X}$ & & & & & $\mathrm{X}$ & $\mathrm{X}$ & $\mathrm{N}$ & VU \\
\hline Anisotoma humeralis (Herbst, 1791) & $\mathrm{X}$ & $\mathrm{X}$ & & & $\mathrm{N}$ & $\mathrm{X}$ & $\mathrm{X}$ & $\mathrm{N}$ & LC \\
\hline Anisotoma orbicularis (Herbst, 1791) & $\mathrm{H}$ & & & $\mathrm{N}$ & & $\mathrm{X}$ & $\mathrm{X}$ & & LC \\
\hline \multicolumn{10}{|c|}{ Leiodini Fleming, 1821} \\
\hline Leiodes badia (Sturm, 1807) & & $\mathrm{X}$ & & $\mathrm{N}$ & & $\mathrm{X}$ & $\mathrm{X}$ & $\mathrm{N}$ & \\
\hline Leiodes brumnea (Sturm, 1807) & $\mathrm{H}$ & & & & CK & & & & \\
\hline Leiodes calcarata (Erichson, 1845) & $\mathrm{H}$ & & $\mathrm{X}$ & & & $\mathrm{X}$ & & & \\
\hline Leiodes cinnamomea (Panzer, 1793) & & & & & $\mathrm{N}$ & & & & \\
\hline Leiodes flavicornis (C.N.F. Brisout de Barneville, 1884) & & $\mathrm{X}$ & & & & & & B & \\
\hline Leiodes litura Stephens, 1835 & & & & & & & & B & \\
\hline Leiodes longipes (W.L.E. Schmidt, 1841) & & & & & & $\mathrm{X}$ & & & \\
\hline Leiodes macropus (Rye, 1873) & & & & & $\mathrm{N}$ & & & & \\
\hline
\end{tabular}


Species

Leiodes oblonga (Erichson, 1845)

Leiodes obscura (Fairmaire \& Coquerel, 1859)

Pseudoliodini Portevin, 1926

\begin{tabular}{|c|c|c|c|c|c|c|c|c|}
\hline हू & $\begin{array}{l}\overline{3} \\
\vdots \\
3 \\
\vdots \\
\frac{0}{0} \\
\frac{3}{0} \\
=\end{array}$ & 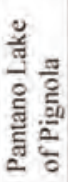 & 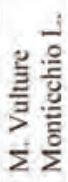 & 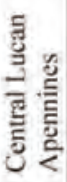 & 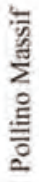 & 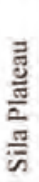 & 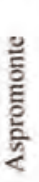 & 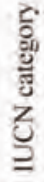 \\
\hline $\mathrm{H}$ & & & & & & & & \\
\hline B & & & & & & & & \\
\hline
\end{tabular}

Agaricophagus cephalotes (W.L.E. Schmidt, 1841)

Agaricophagus italicus Hlisnikovský, 1964

Colenis immunda (Sturm, 1807)

Sogdini Lopatin, 1961

\begin{tabular}{|l|l|l|l|l|l|l|l|l|}
\hline & & $\mathrm{N}$ & & $\mathrm{N}$ & $\mathrm{X}$ & & & \\
\hline $\mathrm{H}$ & $\mathrm{X}$ & $\mathrm{X}$ & $\mathrm{N}$ & $\mathrm{N}$ & & $\mathrm{X}$ & $\mathrm{N}$ & \\
\hline
\end{tabular}

Hydnobius punctatus (Sturm, 1807)

$\mathrm{H}$

Platypsyllinae Ritsema, 1869

Leptimus testaceus P.W.J. Müller, 1817

\section{SILPHIDAE Latreille, 1806}

\begin{tabular}{|l|l|l|l|l|l|l|}
$\mathrm{H}$ & $\mathrm{X}$ & & $\mathrm{N}$ & $\mathrm{X}$ & $\mathrm{X}$ & $\mathrm{CK}$ \\
\hline
\end{tabular}

Silphinae Latreille, 1806

Rủžička in Cat. Pal. Col., vol. 2, 2015

\begin{tabular}{|l|c|c|c|c|c|c|c|c|c|}
\hline Ablattaria laevigata (Fabricius, 1775) & H & X & X & N & N & X & X & N & \\
\hline Aclypea undata (O.F. Müller, 1776) & H & & & & & X & X & & \\
\hline Dendroxena quadrimaculata (Scopoli, 1771) & & & X & N & N & X & X & N & \\
\hline Necrodes littoralis (Linnaeus, 1758) & X & X & X & & & N & & N & \\
\hline Phosphuga atrata atrata (Limnaeus, 1758) & & & & & & & & B & \\
\hline Silpha olivieri Bedel, 1887 & H & X & X & & N & X & X & N & \\
\hline Silpha tristis Illiger, 1798 & & X & & N & N & X & X & N & \\
\hline Thanatophilus rugosus (Linnaeus, 1758) & & X & & & & & X & N & \\
\hline Thanatophilus simuatus (Fabricius, 1775) & & & \\
\hline
\end{tabular}

Thanatophilus simuatus (Fabricius, 1775)

Nicrophorinae Kirby, 1837

\begin{tabular}{|c|c|c|c|c|c|c|c|}
\hline Nicrophorus humator (Gleditsch, 1767) & & & & $\mathrm{N}$ & B & $\mathrm{X}$ & \\
\hline Nicrophorus interruptus Stephens, 1830 & $\mathrm{X}$ & $\mathrm{X}$ & $\mathrm{N}$ & & $\mathrm{X}$ & $\mathrm{X}$ & $\mathrm{N}$ \\
\hline Nicrophorus investigator Zetterstedt, 1824 & $\mathrm{X}$ & & & & & & \\
\hline Nicrophorus vespilloides Herbst, 1783 & & $\mathrm{~N}$ & $\mathrm{~N}$ & B & $\mathrm{N}$ & & \\
\hline
\end{tabular}

STAPHYLINIDAE Latreille, 1802

Omaliinae W.S. Macleay, 1825

Anthophagini C.G. Thomson, 1859

Smetana in Cat. Pal. Col., vol. 2, 2015

\begin{tabular}{|c|c|c|c|c|c|c|c|c|}
\hline Acidota cruentata Mannerheim, 1830 & & & & & CK & & & B \\
\hline Amphichroum canaliculatum (Erichson, 1840) & & & $\mathrm{X}$ & & B & $\mathrm{X}$ & $\mathrm{X}$ & \\
\hline Anthobium atrocephalum (Gyllenhal, 1827) & $\mathrm{H}$ & & CK & CK & $\mathrm{CK}$ & $\mathrm{X}$ & $\mathrm{X}$ & B \\
\hline Anthobium melanocephalum (Illiger, 1794) & & & & & B & $\mathrm{X}$ & $\mathrm{X}$ & B \\
\hline Anthophagus alpestris kochi Bernhauer, 1935 [E] & & & & & & B & & \\
\hline Anthophagus bicornis (Block, 1799) & & & & & $\mathrm{CK}$ & $\mathrm{X}$ & & \\
\hline Anthophagus fauveli caprai Koch, 1933 [E] & CK & CK & & CK & $\mathrm{CK}$ & $\mathrm{X}$ & $\mathrm{X}$ & B \\
\hline Anthophagus torretassoi pollinensis Koch, 1934 [E] & & & & CK & CK & $\mathrm{X}$ & & \\
\hline Deliphrosoma platyophthalmum (Koch, 1937) [E] & & & & & & $\mathrm{X}$ & & \\
\hline Geodromicus pedemontanus Fagel, 1958 & & & & & & & $\mathrm{X}$ & \\
\hline Lesteva corsica Perris, 1869 & & & & & & & & CK \\
\hline Lesteva lepontia Baudi di Selve, 1870 & & & & CK & & & CK & \\
\hline Lesteva longoelytrata longoelytrata (Goeze, 1777) & & & & & CK & CK & CK & B \\
\hline Lesteva lictuosa Fauvel, 1871 & & & & & & $\mathrm{X}$ & & \\
\hline Lesteva monticola Kiesenwetter, 1847 & & & & $\mathrm{~N}$ & $\mathrm{CK}$ & $\mathrm{X}$ & CK & $\mathrm{CK}$ \\
\hline Lesteva omissa omissa Mulsant \& Rey, 1880 & & & & & CK & & CK & \\
\hline Lesteva sicula sicula Erichson, 1840 & $\mathrm{X}$ & & CK & CK & B & CK & CK & B \\
\hline Orochares angustatus (Erichson, 1840) & & & & & CK & & & \\
\hline Orochares calaber calaber (Zanetti, 1983) [E] & & & & & & & & B \\
\hline Philorimum sordidum (Stephens, I834) & $\mathrm{X}$ & & & & & & & \\
\hline
\end{tabular}




\begin{tabular}{|c|c|c|c|c|c|c|c|c|c|}
\hline Species & 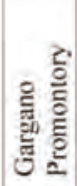 & $\begin{array}{l}7 \\
0 \\
\vdots \\
0 \\
0 \\
0 \\
0 \\
0\end{array}$ & 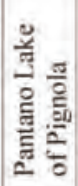 & 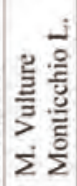 & 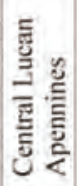 & 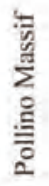 & 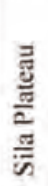 & $\begin{array}{l}\text { Eั } \\
\frac{\mathrm{E}}{2} \\
\frac{5}{2}\end{array}$ & 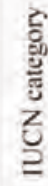 \\
\hline Phyllodrepoidea crenata (Ganglbauer, 1895) & $\mathrm{X}$ & & & & CK & & & & NT \\
\hline
\end{tabular}

Coryphiini Jakobson, 1908

Boreaphilus velox (Heer, 1839)

Coryphium angusticolle Stephens, 1834

\begin{tabular}{|l|l|l|l|l|l|l|l|}
\hline $\mathrm{H}$ & & $\mathrm{N}$ & $\mathrm{CK}$ & $\mathrm{B}$ & $\mathrm{CK}$ & & $\mathrm{B}$ \\
\hline
\end{tabular}

Eusphalerini Hatch, 1957

Eusphalerum atrum (Heer, 1839)

Eusphalerum bargaglii (Luze, 1910)

Eusphalerum baudii (A. Fiori, 1894) [E]

Eusphalerum bivittatum (Eppelsheim, 1887) [E]

Eusphalerum calabrum Zanetti, 1980 [E]

Eusphalerum cribrellum (Fauvel, 1900) [E]

Eusphalerum italicum italicum (Koch, 1938) [E]

Eusphalerum martinae Zanetti, 2004 [E]

Eusphalerum primulae (Stephens, 1834)

Eusphalertum rectangulum (Baudi di Selve, 1870)

Eusphalerum ruffoi (Scheerpeltz, 1956) [E]

Eusphalerum schatzmayri (Koch, 1938) [E]

Eusphalerum signatum angulatum (Luze, 1911) [E]

Eusphalerum stussineri (Bernhauer, I909) [E]

Eusphalerum viertli (Ganglbauer, 1895)

Omaliini W.S. Macleay, 1825

\begin{tabular}{|c|c|c|c|c|c|c|c|c|c|}
\hline Acrolocha amabilis (Heer, 1841) & & & & & CK & $\mathrm{X}$ & & B & \\
\hline Acrolocha daccordii Zanetti, 1979 [E] & & & CK & & B & CK & CK & & \\
\hline Acrolocha sulcula (Stephens, 1834) & & & & & B & CK & & & \\
\hline Dropephylla ioptera (Stephens, 1834) & & & & & B & $\mathrm{N}$ & & & $\mathrm{LC}$ \\
\hline Dropephylla vilis (Erichson, 1840) & & & & & & & CK & CK & NT \\
\hline Hapalaraea pygmaea (Paykull, 1800) & & $\mathrm{X}$ & & & & CK & & & VU \\
\hline Omalium caesum Gravenhorst, 1806 & $\mathrm{H}$ & & CK & CK & CK & $\mathrm{N}$ & CK & B & \\
\hline Omalium cinnamomeum Kraatz, 1857 & $\mathrm{H}$ & $\mathrm{X}$ & & CK & $\mathrm{CK}$ & $\mathrm{N}$ & CK & B & \\
\hline Omalium excavatum Stephens, 1834 & & & & $\mathrm{~N}$ & CK & $\mathrm{X}$ & CK & B & \\
\hline Omalium italictim Bernhauer, 1902 & $\mathrm{H}$ & CK & & CK & CK & CK & CK & B & \\
\hline Omalium rivulare (Paykull, 1789) & $\mathrm{H}$ & & & CK & CK & $\mathrm{X}$ & $\mathrm{X}$ & CK & \\
\hline Omalium rugatum Mulsant \& Rey, 1880 & CK & & CK & CK & $\mathrm{CK}$ & CK & CK & CK & \\
\hline Paraphloeostiba gayndahensis (W.S. Macleay, 1873) & $\mathrm{N}$ & CK & $\mathrm{X}$ & B & CK & CK & CK & CK & \\
\hline Phloeonomus minimus (Erichson, 1839) & & & & & CK & CK & & & VU \\
\hline Phloeonomus pumctipenmis J. Thomson, 1867 & CK & & & & CK & CK & CK & & LC \\
\hline Phloeonomus pusillus (Gravenhorst, 1806) & $\mathrm{x}$ & CK & & & CK & $\mathrm{X}$ & $\mathrm{X}$ & & LC \\
\hline Phloeostiba plana (Paykull, 1792) & & & $\mathrm{N}$ & & CK & CK & & & LC \\
\hline Phyllodrepa angelinii Zanetti, 2009 [E] & & & & & B & & & & \\
\hline Phyllodrepa floralis (Paykull, 1789) & & & & CK & CK & $\mathrm{X}$ & $\mathrm{X}$ & & \\
\hline Phyllodrepa melanocephala pollinensis Scheerpeltz, 1956 [E] & & & & & CK & $\mathrm{X}$ & $\mathrm{X}$ & & VU \\
\hline Phyllodrepa nigra (Gravenhorst, 1806) & $\mathrm{X}$ & $\mathrm{X}$ & & & CK & B & & & VU \\
\hline Phyllodrepa salicis (Gyllenhal, 1810) & $\mathrm{X}$ & & & & & & $\mathrm{X}$ & & VU \\
\hline Xylodromus affinis (Gerhardt, 1877) & $\mathrm{H}$ & $\mathrm{X}$ & $\mathrm{X}$ & CK & $\mathrm{CK}$ & $\mathrm{X}$ & & & \\
\hline Xylodromus depressus (Gravenhorst, 1802) & & & $\mathrm{N}$ & & $\mathrm{N}$ & $\mathrm{X}$ & & & \\
\hline Xylodromus testaceus (Erichson, 1840) & CK & & & CK & CK & CK & $\mathrm{N}$ & CK & \\
\hline Xylostiba bosnica (Bernhauer, 1902) & & $\mathrm{X}$ & & & CK & & & & $\mathrm{VU}$ \\
\hline
\end{tabular}

Xylostiba bosnica (Bernhauer, 1902)

Proteininae Erichson, 1839

Anepiini Steel, 1966

\begin{tabular}{|l|l|l|l|l|l|l|l|l|}
\hline Megarthrus bellevoyei Saulcy, 1862 & X & X & & N & N & & N & \\
\hline Megarthrus depressus (Paykull, 1789) & H & & & & & & \\
\hline Megarthrus prosseni Schatzmayr, 1904 & & & & & N & N & & \\
\hline
\end{tabular}


Species

Metopsia similis Zerche, 1998

Proteinus angelinii Dauphin, 1999 [E]

Proteinus atomarius Erichson, 1840

Proteinus brachypterus (Fabricius, 1792)

Proteimus cremulatus Pandellé, 1867

Proteinus ovalis Stephens, 1834

\begin{tabular}{|c|c|c|c|c|c|c|c|c|}
\hline 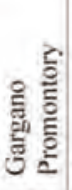 & $\begin{array}{l}\overrightarrow{0} \\
\vdots \\
3 \\
0 \\
\frac{0}{0} \\
\frac{.0}{0} \\
2\end{array}$ & 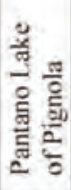 & 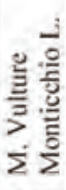 & 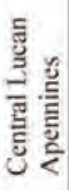 & 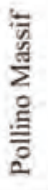 & 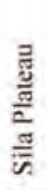 & 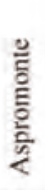 & 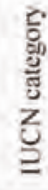 \\
\hline $\mathrm{H}$ & $\mathrm{N}$ & $\mathrm{N}$ & $\mathrm{N}$ & $\mathrm{N}$ & $\mathrm{X}$ & $\mathrm{X}$ & B & \\
\hline & & & & B & & & & \\
\hline & $X$ & $\mathrm{~N}$ & & & $X$ & $X$ & $\mathrm{~N}$ & \\
\hline $\mathrm{H}$ & $\mathrm{X}$ & $\mathrm{N}$ & & $\mathrm{N}$ & $X$ & $X$ & $\mathrm{~N}$ & \\
\hline & & & & & & $X$ & & \\
\hline & & & & $\mathrm{N}$ & & & & \\
\hline
\end{tabular}

Micropeplinae Leach, 1815

Micropeplus calabricus Reitter, 1907 [E]

Micropeplus fulvus fulvus Erichson, 1840

Micropeplus latus C. Hampe, 1861

Micropeplus marietti Jacquelin du Val, 1857

Micropeplus porcatus (Paykull, 1789)

Micropeplus staphylinoides (Marsham, 1802)

Dasycerinae Reitter, 1887

Dasycerus sulcatus Brongniart, 1800

Pselaphinae Latreille, 1802

Batrisitae Reitter, 1882

Batrisini Reitter, 1882

Batrisina Reitter, 1882

Löbl \& Besuchet in Cat. Pal. Col., vol. 2, 2015

Batrisodes adnexus (C. Hampe, 1863)

Batrisodes delaporti (Aubé, 1833)

Batrisodes oculatus (Aubé, 1833)

Batrisodes venustus (Reichenbach, 1816)

Batrisus formicarius Aubé, 1833

Clavigerini Leach, 1815

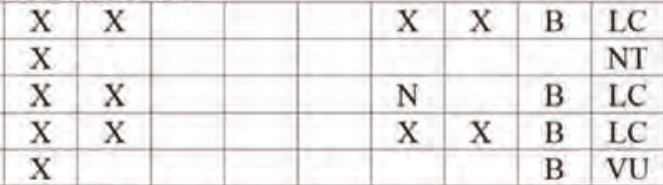

Apoderigerina Jeannel, 1954

\begin{tabular}{c|c|} 
Claviger apenninus Baudi di Selve, $1870[\mathrm{E}]$ & $\mathrm{X}$ \\
\hline \multicolumn{3}{|c|}{ Euplectini Streubel, 1839}
\end{tabular}

Euplectus bonvouloiri narentimus Reitter, 1882

Euplectus bonvouloiri rosae Raffray, 1910

Euplectus brunneus Grimmer, 1841

Euplectus corsicus Guillebeau, 1888

Euplectus karstenii (Reichenbach, 1816)

Euplectus kirbyi hummleri Reitter, 1906

Euplectus piceus lucanus Meggiolaro, 1966 [E]

Euplectus theryi Guillebeau, 1894

Euplectus tholini Guillebeau, 1888

Euplectus verticalis Reitter, 1884

Mayetiini Winkler, 1925

\begin{tabular}{|c|c|c|c|c|c|c|c|c|}
\hline CK & X & & & CK & CK & & B & LC \\
\hline & B & & & & & ! & & LC \\
\hline X & X & & N & & X & X & B & NT \\
\hline H & & & & & & & B & LC \\
\hline X & X & & CK & & X & & & LC \\
\hline H & X & & N & CK & X & X & B & LC \\
\hline & & & & B & & & & CR \\
\hline & CK & & CK & CK & & B & NT \\
\hline X & X & & & & & B & VU \\
\hline
\end{tabular}

Mayetia garganica Pace, 1975 [E]

Trichonychini Reitter, 1882

Bibloporina O. Park, 1951

\begin{tabular}{|c|c|c|c|c|c|c|}
\hline Amauronyx maerkelii (Aubé, 1844) & $\mathrm{X}$ & & & & B & NT \\
\hline Aphilia crassipes (Raffray. 1908) & & & B & & B & \\
\hline Bibloplectus ambiguns (Reichenbach, 1816) & & & & & B & \\
\hline Bibloplectus delhermi Guillebeau, 1888 & CK & CK & & & & \\
\hline Bibloplectus limatus Normand, 1939 & CK & & & & B & \\
\hline Bibloplectus obtusus Guillebeau, 1888 & & $\mathrm{X}$ & & & & \\
\hline Bibloplectus pusillus (Denny, 1825) & CK & & & & & \\
\hline Bibloplectus tenebrosus (Reitter, 1880) & & & & & B & \\
\hline Bibloporus bicolor devillei Jeannel, 1950 & & & $\mathrm{CK}$ & $\mathrm{X}$ & B & NT \\
\hline
\end{tabular}




\begin{tabular}{|c|c|c|c|c|c|c|c|c|c|}
\hline Species & 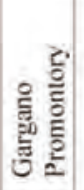 & $\begin{array}{l}\overline{0} \\
0 \\
3 \\
0 \\
0 \\
\frac{0}{0} \\
0 \\
2\end{array}$ & 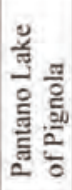 & 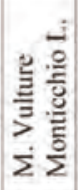 & 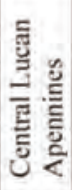 & 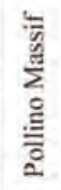 & 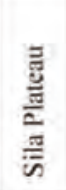 & 参 & 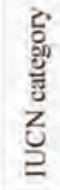 \\
\hline Bibloporus mayeti Guillebeau, 1888 & $!$ & & & & CK & & & & NT \\
\hline Bibloporus mimutus Raffray, 1914 & CK & & & & & & & B & NT \\
\hline Bibloporus ultimus Guillebeau, 1892 & $!$ & & & & CK & $\mathrm{X}$ & & B & NT \\
\hline Pseudoplectus perplexus (Jacquelin du Val, 1854) & & CK & & & & & & $\mathrm{B}$ & \\
\hline Trichonyx sulcicollis (Reichenbach, 1816) & $\mathrm{H}$ & $\mathrm{N}$ & & & & & $\mathrm{X}$ & & NT \\
\hline Trimium besucheti Sabella, 1989 [E] & & & & & & & & $\mathrm{B}$ & VU \\
\hline Trimium brevicorne (Reichenbach, 1816) & & & & CK & CK & $!$ & & & LC \\
\hline Trimium minimum Dodero, 1900 & & $\mathrm{X}$ & & & & & & B & NT \\
\hline Trimium paganettii Reitter, 1906 [E] & & & & & CK & $!$ & & B & VU \\
\hline Trimium zoufali Krauss, 1900 [E] & & & & & CK & & & B & $\mathrm{LC}$ \\
\hline \multicolumn{10}{|c|}{ Trichonychini taxa incertae sedis } \\
\hline Meliceria sulciventris (Guillebeau, 1888) & CK & B & & & CK & & & B & VU \\
\hline Plectophloeus fischeri (Aubé, 1833) & & & & & & CK & CK & B & LC \\
\hline Plectophloeus nitidus (Fairmaire, 1858) & & & & & & & & B & LC \\
\hline Plectophloeus nubigena bosnicus Besuchet, 1964 & & & & & CK & $\mathrm{N}$ & CK & B & VU \\
\hline
\end{tabular}

Faromus aubei P.H. Lucas, 1854

Faronini Reitter, 1882

Faronus lafertei Aubé, 1844

Faromus stolzi W. Blattný \& C. Blattný, 1914 [E]

\begin{tabular}{|l|l|l|l|l|l|l|l|}
\hline $\mathrm{H}$ & & $\mathrm{CK}$ & & & & $\mathrm{B}$ \\
\hline
\end{tabular}

Brachyglutini Raffray, 1904

Brachyglutina Raffray, 1904

Brachygluta abrupta Dodero, 1919

Brachyghuta angelinii Sabella, 1997 [E]

Brachygluta appennina (Saulcy, 1876)

Brachygluta aubei (Tournier, 1868)

Brachygluta fossulata (Reichenbach, 1816)

Brachygluta foveola foveola (Motschulsky, 1840)

Brachygluta furcata (Motschulsky, 1835)

Brachygluta galathea (Saulcy, 1876) [E]

Brachygluta guillemardi (Saulcy, 1876)

Brachygluta helferi helferi (Schmidt-Göbel, 1836)

Brachygluta hipponensis (Saulcy, 1876)

Brachygluta lefebvrei lefebvrei (Aubé, 1833)

Brachygluta numidica (Saulcy, 1876)

Brachygluta pandellei (Saulcy, 1876)

Brachygluta perforata (Aubé, 1833)

Brachygluta ragusae (Saulcy, 1876)

Brachygluta tibialis (Aubé, 1844)

Brachygluta trigonoprocta (Ganglbauer, 1895)

Fagniezia impressa (Panzer, 1803)

Reichenbachia chevrieri (Aubé, 1844)

Reichenbachia nigriventris (Schaum, 1859)

Rybaxis longicornis (Leach, 1817)

Trissemus antennatus antennatus (Aubé, 1833)

Trissemus olivieri (Raffray, 1871)

\begin{tabular}{|c|c|c|c|c|c|c|c|c|c|}
\hline & $X$ & $\mathrm{X}$ & & & & & & & \\
\hline & & & B & & & & & & \\
\hline & & CK & $\mathrm{X}$ & & CK & $\mathrm{X}$ & $X$ & B & \\
\hline & & & & & & & & B & \\
\hline & & $!$ & & CK & CK & CK & & & \\
\hline \multirow[t]{4}{*}{ 0) } & $\mathrm{X}$ & $\mathrm{X}$ & $\mathrm{X}$ & $\mathrm{N}$ & & $X$ & $\mathrm{X}$ & B & \\
\hline & & & $\mathrm{X}$ & & & & & & \\
\hline & & $\mathrm{X}$ & & & & & & B & \\
\hline & & & $\mathrm{X}$ & & & & & & \\
\hline \multirow[b]{15}{*}{ 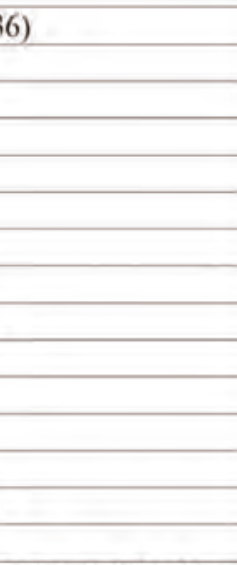 } & $X$ & $\mathrm{X}$ & ! & $\mathrm{N}$ & & $\mathrm{X}$ & $X$ & B & \\
\hline & & CK & & & & & & B & \\
\hline & CK & & $\mathrm{X}$ & & & & & & \\
\hline & & & & & & & CK & & \\
\hline & & & & & & & & B & \\
\hline & $\mathrm{X}$ & $\mathrm{X}$ & $\mathrm{X}$ & & CK & $\mathrm{N}$ & & & \\
\hline & $\mathrm{H}$ & $\mathrm{X}$ & & $\mathrm{N}$ & CK & $\mathrm{X}$ & & B & \\
\hline & B & & & & & & & & \\
\hline & & $\mathrm{X}$ & $\mathrm{X}$ & & & CK & & & \\
\hline & & $\mathrm{X}$ & & & & & & & \\
\hline & $X$ & & & & & $!$ & & B & \\
\hline & $X$ & $X$ & & & & & & B & \\
\hline & X & $\mathrm{X}$ & $X$ & CK & CK & $\mathrm{N}$ & & B & \\
\hline & & $\mathrm{X}$ & CK & CK & CK & & & B & \\
\hline & $\mathrm{N}$ & $\mathrm{N}$ & $\mathrm{X}$ & $\mathrm{N}$ & $\mathrm{N}$ & & & B & \\
\hline \multicolumn{10}{|c|}{ yythinini Raffray, 1890} \\
\hline & & CK & & & CK & & & & \\
\hline & & & & & $\mathrm{B}$ & $\mathrm{X}$ & $\mathrm{X}$ & B & VU \\
\hline & $\mathrm{H}$ & $\mathrm{X}$ & $\mathrm{X}$ & $\mathrm{N}$ & $\mathrm{N}$ & $\mathrm{X}$ & $\mathrm{X}$ & B & \\
\hline \multirow[t]{2}{*}{ 14) $[\mathrm{E}]$} & & & & $\mathrm{N}$ & B & $\mathrm{X}$ & $\mathrm{X}$ & $\mathrm{B}$ & \\
\hline & & $\mathrm{X}$ & $\mathrm{X}$ & B & B & $\mathrm{X}$ & $\mathrm{X}$ & B & \\
\hline E] & & $\mathrm{X}$ & & $\mathrm{N}$ & CK & $\mathrm{X}$ & $\mathrm{X}$ & B & \\
\hline & & & & & $\mathrm{B}$ & ! & & & \\
\hline
\end{tabular}

Bryaxis chevrolati (Aubé, 1833)

Bryaxis curtisi orientalis (Karaman, 1952)

Bryaxis italicus (Baudi di Selve, 1870)

Bryaxis paganettii (W. Blattný \& C. Blattný, 1914) [E]

Bryaxis pedator (Reitter, 1882) [E]

Bryaxis picteti meridionalis (Machulka, 1932) [E]

Bryaxis porzenna (Reitter, 1882)

Bythinini Raffray, 1890 
Species

Bryaxis samniticus W. Blattný \& C. Blattný, 1914 [E]

Tychobythimus andreinii (Dodero, 1919) [E]

Tychobythinus glabratus (Rye, 1870)

Tychobythinus gularis (Dodero, 1919) [E]

Tychini Raffray, 1904

\begin{tabular}{|c|c|c|c|c|c|c|c|c|}
\hline 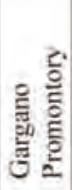 & $\begin{array}{l}\overline{3} \\
\vdots \\
3 \\
0 \\
\frac{0}{3} \\
\frac{3}{0} \\
0\end{array}$ & 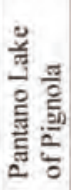 & 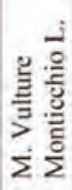 & 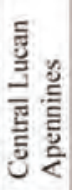 & 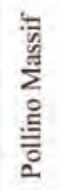 & 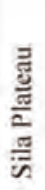 & 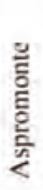 & 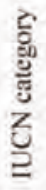 \\
\hline X & X & & $\mathrm{N}$ & B & $\mathrm{X}$ & $\mathrm{X}$ & & \\
\hline & & & & B & & & & \\
\hline $\mathrm{H}$ & B & & & B & CK & & B & \\
\hline & & $X$ & & B & & & & \\
\hline
\end{tabular}

Tychus angelinii Sabella \& Poggi, 1997 [E]

Tychus corsicus spissatus Rey, 1888

Tychus dalmatimus Reitter, 1880

Tychus florentimus Reitter, 1884 [E]

Tychus jacquelinii Boieldieu, 1859

Tychus lucanus Sabella \& Poggi, 1997 [E]

Tychus normandi Jeannel, 1950

Tychus pullus Kiesenwetter, 1858

Ctenistes palpalis Reichenbach, 1816

Ctenistini C.É. Blanchard, 1845

\begin{tabular}{|l|l|l|l|l|l|l|l|l|}
\hline & & B & & CK & & & B & \\
\hline & & X & & CK & & & & \\
\hline & & & & & & & & \\
\hline & & & & & & & B & \\
\hline & & & & CK & & & & \\
\hline & & & CK & CK & CK & & B & \\
\hline
\end{tabular}

Pselaphini Latreille, 1802

Pselaphogenius aspromontanis (Reitter, 1910) [E]

Pselaphogenius calabrus (Reitter, 1910) [E]

Pselaphogenius lucanicus Besuchet, 1964 [E]

Pselaphogenius neapolitanus (Besuchet, 1964) [E]

Pselaphus parvis Karaman, 1940

Tyrini Reitter, 1882

\begin{tabular}{|c|c|c|c|c|c|c|c|c|}
\hline & & & & & & & B & \\
\hline & & & & & & & B & \\
\hline & & & & B & & & & \\
\hline & X & X & B & CK & CK & & & \\
\hline
\end{tabular}

Tyrina Reitter, 1882

Tyrus mucronatus mucronatus (Panzer, 1805)

Phloeocharinae Erichson, 1839

Phloeocharis subtilissima Mannerheim, 1830

Tachyporinae W.S. Macleay, 1825

Mycetoporini C.G. Thomson, 1859

Bolitobius cingulatus Mannerheim, 1830

Bryophacis rufus (Erichson, 1839)

Bryoporus cernuus (Gravenhorst, 1806)

Bryoporus multipunctus C. Hampe, 1867

Carphacis striatus (A.G. Olivier, 1795)

Ischnosoma longicorne (Mäklin, 1847)

Ischnosoma splendidum (Gravenhorst, 1806)

Lordithon exoletus (Erichson, 1839)

Lordithon lumulatus (Linnaeus, 1760)

Lordithon thoracicus thoracicus (Fabricius, 1777)

Lordithon trimaculatus (Fabricius, 1792)

Mycetoporns altaicus Luze, 1901

Mycetoporus angularis Mulsant \& Rey, 1853

Mycetoporus baudueri Mulsant \& Rey, 1875

Mycetoporus brucki (Pandellé, 1869)

Mycetoporus confinis Rey, 1883

Mycetoporus eppelsheimiamus Fagel, 1968

Mycetoporus forticornis Fauvel, 1875

Mycetoporus glaber glaber (Sperk, 1835)

Mycetoporus longulus Mannerheim, 1830

Mycetoporns macrocephalus Bernhauer, 1917

Mycetoporus mulsanti Ganglbauer, 1895

Mycetoporus nigricollis Stephens, 1835

Mycetoporus piceolus Rey, 1883

\begin{tabular}{|l|l|l|l|l|l|l|l|l|l|}
\hline H & & & & & N & & & \\
\hline & & & & N & X & & & \\
\hline X & & & & N & N & N & N & \\
\hline H & X & X & N & N & N & & N & \\
\hline H & X & & & N & X & X & B & \\
\hline H & & & N & N & X & X & N & \\
\hline N & X & X & N & N & X & X & B & \\
\hline & & & & N & & & \\
\hline H & & & & & & & & \\
\hline H & N & & & & & & & \\
\hline & & & & & X & & & \\
\hline & & & & N & & & N & \\
\hline H & & & & & & & & \\
\hline & & & & & & & B & \\
\hline & & & & & X & & & \\
\hline H & & & & N & X & & B & \\
\hline H & & & & N & X & & & \\
\hline H & X & X & & & N & & N & \\
\hline H & & & & N & & & & \\
\hline
\end{tabular}




\begin{tabular}{|c|c|c|c|c|c|c|c|c|c|}
\hline Species & 总 & 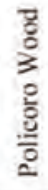 & 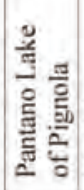 & 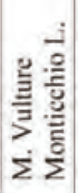 & 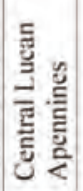 & 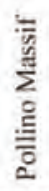 & $\frac{\text { 总 }}{\frac{m}{a}}$ & 竎 & 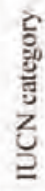 \\
\hline Mycetoporus punctus (Gravenhorst, 1806) & & & & $\mathrm{N}$ & $\mathrm{N}$ & $\mathrm{X}$ & & $\mathrm{N}$ & \\
\hline Mycetoporus reichei (Pandellé, 1869) & $\mathrm{H}$ & & & & $\mathrm{N}$ & & & $\mathrm{N}$ & \\
\hline Mycetoporus rufescens (Stephens, 1832) & $\mathrm{H}$ & & & $\mathrm{N}$ & $\mathrm{N}$ & $\mathrm{X}$ & $\mathrm{X}$ & B & \\
\hline Mycetoporis silvaticus Jablokoff-Khnzorian, 1962 & & & & & B & & & $\mathrm{B}$ & \\
\hline Mycetoporus solidicornis Wollaston, 1864 & & $\mathrm{X}$ & & $\mathrm{N}$ & & & & & \\
\hline Parabolitobius inclinans (Gravenhorst, 1806) & & & & & $\mathrm{N}$ & $\mathrm{X}$ & $\mathrm{X}$ & & \\
\hline
\end{tabular}

Tachyporini W.S. Macleay, 1825

Cilea silphoides (Linnaeus, 1767)

Sepedophilus bipunctatus (Gravenhorst, 1802)

Sepedophilus bipustulatus (Gravenhorst, 1802)

Sepedophilus immaculatus (Stephens, 1832)

Sepedophilus littoreus (Linnaeus, 1758)

Sepedophilus hisitanicus Hammond, 1973

Sepedophilus marshami (Stephens, 1832)

Sepedophilus nigripennis (Stephens, 1832)

Sepedophilus testaceus (Fabricius, 1792)

Tachimus flavolimbatus Pandellé, 1869

Tachinus humeralis humeralis Gravenhorst, 1802

Tachimus marginellus angelinii Schulke, 1996 [E]

Tachimus rufipes (Linnaeus, 1758)

Tachinus subterraneus (Linnaeus, 1758)

Tachyporis abner Saulcy, 1865

Tachyporus atriceps Stephens, 1832

Tachyporus caucasicus Kolenati, 1846

Tachyporus chrysomelinus (Linnaeus, 1758)

Tachyporus dispar (Paykull, 1789)

Tachyporus formosus A. Matthew, 1838

Tachyporus hypnorum (Fabricius, 1775)

Tachyporus nitidulus (Fabricius, 1781)

Tachyporus pusillus Gravenhorst, 1806

Tachyporus scitulus Erichson, 1839

Tachyporus solutus Erichson, 1839

Trichophyinae J. Thomson, 1858

\begin{tabular}{|c|c|c|c|c|c|c|c|c|}
\hline & $\mathrm{X}$ & $\mathrm{X}$ & $\mathrm{N}$ & & $\mathrm{X}$ & $\mathrm{X}$ & & \\
\hline $\mathrm{N}$ & $\mathrm{N}$ & & & & & & & NT \\
\hline \multirow[t]{5}{*}{ X } & $\mathrm{X}$ & & & & $\mathrm{X}$ & X & & NT \\
\hline & $\mathrm{X}$ & $\mathrm{X}$ & $\mathrm{N}$ & & & & & LC \\
\hline & & & & $\mathrm{N}$ & & & & \\
\hline & $\mathrm{X}$ & $\mathrm{X}$ & & & $\mathrm{X}$ & $\mathrm{X}$ & & NT \\
\hline & & $\mathrm{X}$ & & $\mathrm{N}$ & & & & LC \\
\hline \multirow[t]{2}{*}{$\mathrm{H}$} & $X$ & $\mathrm{X}$ & & $\mathrm{N}$ & & & B & \\
\hline & $\mathrm{X}$ & & & $\mathrm{N}$ & $\mathrm{N}$ & $\mathrm{N}$ & $\mathrm{N}$ & LC \\
\hline $\mathrm{H}$ & $\mathrm{X}$ & & & & & & $\mathrm{N}$ & \\
\hline $\mathrm{X}$ & & $\mathrm{N}$ & $\mathrm{N}$ & $\mathrm{N}$ & $\mathrm{X}$ & $\mathrm{X}$ & $\mathrm{N}$ & \\
\hline \multirow[t]{2}{*}{$\mathrm{X}$} & $\mathrm{X}$ & & $\mathrm{N}$ & B & $\mathrm{X}$ & $\mathrm{X}$ & B & \\
\hline & & & & $\mathrm{N}$ & $\mathrm{N}$ & & & \\
\hline \multirow[t]{2}{*}{$\mathrm{X}$} & & $\mathrm{X}$ & $\mathrm{N}$ & $\mathrm{N}$ & $\mathrm{X}$ & $\mathrm{X}$ & & \\
\hline & & & & $\mathrm{N}$ & & $\mathrm{X}$ & $\mathrm{N}$ & \\
\hline \multirow[t]{2}{*}{$\mathrm{X}$} & & & & & & & & \\
\hline & & & & $\mathrm{N}$ & & & $\mathrm{N}$ & \\
\hline \multirow[t]{3}{*}{$\mathrm{X}$} & $\mathrm{X}$ & $\mathrm{N}$ & $\mathrm{N}$ & & $\mathrm{X}$ & $\mathrm{X}$ & & \\
\hline & & & & $\mathrm{N}$ & & & & \\
\hline & $\mathrm{X}$ & & & & & & B & \\
\hline $\mathrm{X}$ & $\mathrm{X}$ & $\mathrm{X}$ & B & $\mathrm{N}$ & $\mathrm{X}$ & $\mathrm{X}$ & B & \\
\hline $\mathrm{H}$ & $\mathrm{X}$ & $X$ & $\mathrm{~N}$ & $\mathrm{~N}$ & $\mathrm{X}$ & $\mathrm{X}$ & B & \\
\hline \multirow[t]{2}{*}{$\mathrm{H}$} & & $\mathrm{X}$ & $\mathrm{N}$ & & $\mathrm{X}$ & $\mathrm{X}$ & B & \\
\hline & & & & $\mathrm{N}$ & & & & \\
\hline $\mathrm{H}$ & $\mathrm{X}$ & X & $\mathrm{N}$ & $\mathrm{N}$ & $\mathrm{X}$ & & B & \\
\hline
\end{tabular}

Trichophya pilicornis (Gyllenhal, 1810)

$\mathrm{H}$

Habrocerinae Mulsant \& Rey, 1876

Habrocerus capillaricornis (Gravenhorst, 1806)

Habrocerus pisidicus Korge, 1971

Aleocharinae Fleming, 1821

\begin{tabular}{c|} 
H $\quad X$ \\
B \\
g, 1821 \\
g, 1821 \\
1 1821
\end{tabular}

Aleocharina Fleming, 1821

\begin{tabular}{|c|c|c|c|c|c|c|c|c|}
\hline Aleochara bilineata Gyllenhal, 1810 & & $\mathrm{X}$ & & & & & & B \\
\hline Aleochara binotata Kraatz, 1856 & & & & & $\mathrm{~N}$ & & & \\
\hline Aleochara bipustulata (Linnaeus, 1760 ) & $\mathrm{H}$ & $\mathrm{X}$ & $\mathrm{N}$ & $\mathrm{N}$ & $\mathrm{N}$ & $\mathrm{X}$ & $\mathrm{X}$ & B \\
\hline Aleochara brevipennis Gravenhorst, 1806 & & & $\mathrm{~N}$ & & & & & \\
\hline Aleochara crassa Baudi di Selve, 1848 & & & & & & & & $\mathrm{~B}$ \\
\hline Aleochara curtula (Goeze, 1777) & $\mathrm{X}$ & $\mathrm{X}$ & & $\mathrm{N}$ & & $\mathrm{X}$ & $\mathrm{X}$ & \\
\hline Aleochara diversa (J. Sahlberg, 1876) & & & & & & & & B? \\
\hline Aleochara funebris Wollaston, 1864 & & & & & & $\mathrm{X}$ & $\mathrm{X}$ & $\mathrm{N}$ \\
\hline Aleochara grisea Kraatz, 1856 & & $\mathrm{X}$ & & & & & & \\
\hline Aleochara haemoptera Kraatz, 1858 & & & $\mathrm{X}$ & $\mathrm{N}$ & $\mathrm{N}$ & $\mathrm{X}$ & $\mathrm{X}$ & $\mathrm{N}$ \\
\hline Aleochara hummleri Bernhauer, 1914 [E] & & & & & & & $\mathrm{N}$ & \\
\hline Aleochara intricata Mannerheim, 1830 & $\mathrm{H}$ & & $\mathrm{X}$ & $\mathrm{N}$ & $\mathrm{N}$ & $\mathrm{X}$ & $\mathrm{X}$ & B \\
\hline
\end{tabular}


Species

Aleochara lanuginosa Gravenhorst, 1802

Aleochara lata Gravenhorst, 1802

Aleochara laticornis Kraatz, 1856

Aleochara maculata C.N.F. Brisout de Barneville, 1863

Aleochara milleri Kraatz, 1862

Aleochara moesta Gravenhorst, 1802

Aleochara puberula Klug, 1832

Aleochara sparsa Heer, 1839

Aleochara spissicornis Erichson, 1839

Aleochara stichai Likovský, 1965

Aleochara Iristis Gravenhorst, 1806

Aleochara verna Say, 1833

Amarochara bonnairei (Fauvel, 1865)

Tinotus morion (Gravenhorst, 1802)

\begin{tabular}{|c|c|c|c|c|c|c|c|c|}
\hline 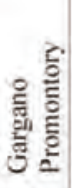 & $\begin{array}{l}70 \\
0 \\
3 \\
0 \\
\frac{0}{0} \\
\frac{.0}{0} \\
2 \\
2\end{array}$ & 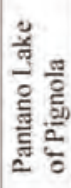 & 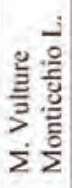 & 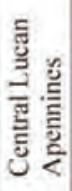 & 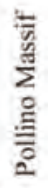 & 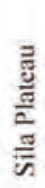 & 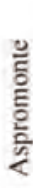 & 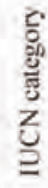 \\
\hline \multirow[t]{2}{*}{$\mathrm{H}$} & & & & $\mathrm{N}$ & $\mathrm{N}$ & & B & \\
\hline & & & & $\mathrm{N}$ & & & & \\
\hline $\mathrm{H}$ & & & & & & & $\mathrm{N}$ & \\
\hline \multirow[t]{4}{*}{$\mathrm{H}$} & & & & & & & & \\
\hline & & & & $\mathrm{N}$ & & & & \\
\hline & $\mathrm{X}$ & & & & & & & \\
\hline & & & & & & & $\mathrm{N}$ & \\
\hline $\mathrm{X}$ & & & $\mathrm{N}$ & $\mathrm{N}$ & $\mathrm{X}$ & $\mathrm{X}$ & B & \\
\hline \multirow[t]{2}{*}{$\mathrm{H}$} & & & & & & & & \\
\hline & & & & $\mathrm{N}$ & $\mathrm{N}$ & & & \\
\hline \multirow[t]{2}{*}{$\mathrm{H}$} & $X$ & & $\mathrm{~N}$ & $\mathrm{~N}$ & $\mathrm{X}$ & $\mathrm{X}$ & B & \\
\hline & & & & & & & $\mathrm{N}$ & \\
\hline $\mathrm{H}$ & & & & B & & & & \\
\hline & $X$ & & & & & $\mathrm{~N}$ & $\mathrm{~N}$ & \\
\hline
\end{tabular}

Athetini Casey, 1910

Athetina Casey, 1910

Acrotona aterrima (Gravenhorst, 1802)

Acrotona benicki (Allen, 1940)

Acrotona muscorrm (C.N.F. Brisout de Barneville, 1860)

Acrotona nigerrima (Aubé, 1850)

Acrotona parens (Mulsant \& Rey, 1852)

Acrotona parvila (Mannerheim, 1830)

Acrotona piceorufa (Mulsant \& Rey, I873)

Acrotona pygmaea (Gravenhorst, 1802)

Alianta incana (Erichson, 1837)

Amischa analis (Gravenhorst, 1802)

Amischa forcipata Mulsant \& Rey, 1873

Atheta aeneicollis (Sharp, 1869) $\mathrm{ef}$.

Atheta amicula (Stephens, 1832)

Atheta atramentaria (Gyllenhal, 1810)

Atheta benickiella Brundin, 1948

Atheta britanniae Bernhauer \& Scheerpeltz, 1926

Atheta burlei Tronquet, 1999

Atheta castanoptera (Mannerheim, 1830)

Atheta celata (Erichson, 1837)

Atheta clientula (Erichson, 1839)

Atheta coriaria (Kraatz, 1856)

Atheta corvina (J. Thomson, 1856)

Atheta crassicornis (Fabricius, 1792)

Atheta dadopora J. Thomson, 1867

Atheta elongatula (Gravenhorst, 1802)

Atheta fungi (Gravenhorst, 1806)

Athera gagatina (Baudi di Selve, 1848)

Atheta harwoodi B.S. Williams, 1930

Atheta hybrida Sharp, 1869

Atheta hygrotopora (Kraatz, 1856)

Atheta hypnorum (Kiesenwetter, 1850)

Atheta incognita (Sharp, 1869)

Atheta intermedia (J. Thomson, 1852)

Atheta laevana (Mulsant \& Rey, 1852)

Atheta laevicauda J. Sahlberg, 1876

Atheta laticollis (Stephens, 1832)

Atheta leonhardi Bernhauer, 1911

\begin{tabular}{|c|c|c|c|c|c|c|c|c|}
\hline & & & & & & & $\mathrm{N}$ & \\
\hline & $\mathrm{N}$ & & & & & & & \\
\hline \multirow[t]{2}{*}{$\mathrm{X}$} & & & & $\mathrm{N}$ & & & B & \\
\hline & & & & $\mathrm{N}$ & & & & \\
\hline $\mathrm{H}$ & & & & & & & B & \\
\hline \multirow[t]{4}{*}{ H } & & & & & & & & \\
\hline & & & & & $\mathrm{N}$ & & $\mathrm{N}$ & \\
\hline & & $\mathrm{N}$ & & & & & B & \\
\hline & $\mathrm{N}$ & & & & & & & \\
\hline \multirow[t]{3}{*}{$\mathrm{H}$} & & $X$ & & $\mathrm{~N}$ & & & & \\
\hline & & & & & & & $\mathrm{N}$ & \\
\hline & & $X$ & & $\mathrm{~N}$ & $\mathrm{~N}$ & & $\mathrm{~N}$ & \\
\hline $\mathrm{H}$ & $X$ & & & & & & & \\
\hline \multirow[t]{4}{*}{$\mathrm{H}$} & & & & $\mathrm{N}$ & & & & \\
\hline & & & & & & & $\mathrm{N}$ & \\
\hline & & & $\mathrm{N}$ & $\mathrm{N}$ & $\mathrm{X}$ & $\mathrm{X}$ & B & \\
\hline & & & & & & & B & \\
\hline \multirow[t]{2}{*}{$\mathrm{H}$} & $\mathrm{N}$ & & $\mathrm{N}$ & & $X$ & & B & \\
\hline & & & & & & & B & \\
\hline \multirow[t]{2}{*}{$\mathrm{H}$} & $X$ & & & & & & B & \\
\hline & & & & $\mathrm{N}$ & & & & \\
\hline \multicolumn{9}{|l|}{$\mathrm{N}$} \\
\hline \multirow[t]{3}{*}{$\mathrm{H}$} & & & $\mathrm{N}$ & $\mathrm{N}$ & & & B & \\
\hline & & & & & $\mathrm{N}$ & & $\mathrm{N}$ & \\
\hline & $\mathrm{X}$ & & & & & & & \\
\hline $\mathrm{H}$ & & & & & $\mathrm{N}$ & & & \\
\hline \multirow[t]{7}{*}{$\mathrm{H}$} & $X$ & & & $\mathrm{~N}$ & $\mathrm{~N}$ & & & \\
\hline & & & & & $\mathrm{N}$ & & B & \\
\hline & & & & $\mathrm{N}$ & $\mathrm{N}$ & & & \\
\hline & & & & & $\mathrm{X}$ & $\mathrm{N}$ & B & \\
\hline & & & & & & & $\mathrm{N}$ & \\
\hline & $\mathrm{N}$ & & & $\mathrm{N}$ & $\mathrm{N}$ & & & \\
\hline & & & & & & $\mathrm{X}$ & & \\
\hline \multirow[t]{4}{*}{$\mathrm{H}$} & & & & & & & & \\
\hline & & & & $\mathrm{N}$ & & & $\mathrm{N}$ & \\
\hline & & & & $\mathrm{N}$ & $\mathrm{N}$ & & & \\
\hline & & & $\mathrm{N}$ & & $\mathrm{X}$ & & & \\
\hline
\end{tabular}




\section{Species}

Atheta liliputana C.N.F. Brisout de Barneville, 1860

Atheta longicornis (Gravenhorst, 1802)

Atheta marcida (Erichson, 1837)

Atheta melanaria (Mannerheim, 1830)

Atheta melanocera (J. Thomson, 1856)

Atheta nigra (Kraatz, 1856)

Atheta nigritula (Gravenhorst, 1802)

Atheta oblita (Erichson, 1839)

Atheta orbata (Erichson, 1837)

Atheta orphana (Erichson, 1837)

Atheta palleola (Erichson, 1837)

Atheta pallidicornis (J. Thomson, 1856)

Atheta palustris (Kiesenwetter, 1844)

Atheta parca (Mulsant \& Rey, 1873)

Atheta picipes (J. Thomson, 1856)

Atheta pilicornis (J. Thomson, 1852)

Atheta putrida (Kraatz, 1856)

Atheta ravilla (Erichson, 1839)

Atheta scapularis (C.R. Sahlberg. 1831)

Atheta taxiceroides Munster, 1932

Atheta testaceipes (Heer, 1839)

Atheta triangulum (Kraatz, 1856)

Atheta trinotata (Kraatz, 1856)

Atheta vaga (Heer, 1839)

Atheta volans (W. Scriba, 1859)

Dinaraea linearis (Gravenhorst, 1802)

Hydrosmecta delicatissima (Bernhauer, 1908)

Liogluta alpestris (Heer, 1839)

Liogluta longiuscula (Gravenhorst, 1802)

Liogluta wuesthoffi (G. Benick, 1938)

Nehemitropia lividipennis (Mannerheim, 1830)

Ousipalia caesula (Erichson, 1839)

Thamiaraeina Fenyes, 1921

\begin{tabular}{|c|c|c|c|c|c|c|c|c|}
\hline 总 & 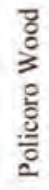 & 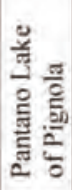 & 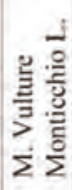 & 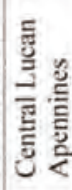 & 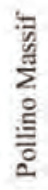 & 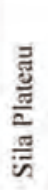 & है & 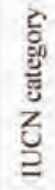 \\
\hline & & & & & $\mathrm{N}$ & & & \\
\hline \multirow[t]{2}{*}{$\mathrm{H}$} & $X$ & $\mathrm{~N}$ & $\mathrm{~N}$ & $\mathrm{~N}$ & $\mathrm{~N}$ & & B & \\
\hline & & & & $\mathrm{N}$ & & $X$ & & \\
\hline \multicolumn{9}{|l|}{$\mathrm{H}$} \\
\hline \multirow[t]{2}{*}{$\mathrm{H}$} & $X$ & & & & & & & \\
\hline & & & & & $\mathrm{N}$ & & & \\
\hline \multirow[t]{6}{*}{$\mathrm{H}$} & & & & $\mathrm{N}$ & $\mathrm{N}$ & $\mathrm{N}$ & $\mathrm{N}$ & \\
\hline & $\mathrm{N}$ & $\mathrm{N}$ & & $\mathrm{N}$ & & & $\mathrm{N}$ & \\
\hline & $\mathrm{N}$ & X & $\mathrm{N}$ & & & & B & \\
\hline & $\mathrm{X}$ & & & & & & B & \\
\hline & & & & $\mathrm{N}$ & & & & \\
\hline & & & & & $X$ & & & LC \\
\hline \multirow[t]{2}{*}{$\mathrm{H}$} & & & & $\mathrm{N}$ & & & B & \\
\hline & & & & & & & $\mathrm{N}$ & \\
\hline \multirow[t]{3}{*}{$\mathrm{H}$} & & & & $\mathrm{N}$ & & $X$ & $\mathrm{~N}$ & LC \\
\hline & & & & $\mathrm{N}$ & & & & \\
\hline & & & & $\mathrm{N}$ & & & & \\
\hline \multirow[t]{3}{*}{$\mathrm{H}$} & & $\mathrm{N}$ & & $\mathrm{N}$ & & & $\mathrm{N}$ & \\
\hline & & $\mathrm{X}$ & & & & & $\mathrm{N}$ & \\
\hline & & & & $\mathrm{N}$ & & & & \\
\hline $\mathrm{H}$ & $\mathrm{X}$ & $\mathrm{N}$ & & & & & & \\
\hline $\mathrm{H}$ & $\mathrm{X}$ & & $\mathrm{N}$ & $\mathrm{N}$ & $\mathrm{N}$ & $\mathrm{X}$ & $\mathrm{N}$ & \\
\hline \multirow[t]{3}{*}{$\mathrm{H}$} & & & & $\mathrm{N}$ & $\mathrm{N}$ & $X$ & B & \\
\hline & & & & $\mathrm{N}$ & & & & \\
\hline & & $\mathrm{N}$ & & & & & & \\
\hline \multirow[t]{3}{*}{$\mathrm{X}$} & & & & & & & & NT \\
\hline & & & & & & & B & \\
\hline & & & & & $\mathrm{X}$ & $\mathrm{N}$ & $\mathrm{N}$ & \\
\hline \multirow[t]{4}{*}{$\mathrm{H}$} & & $X$ & B & $\mathrm{N}$ & $\mathrm{X}$ & $\mathrm{N}$ & B & \\
\hline & & & & $\mathrm{N}$ & $\mathrm{N}$ & & & \\
\hline & $\mathrm{X}$ & X & $\mathrm{N}$ & & $\mathrm{X}$ & & B & \\
\hline & $X$ & & & & & & & \\
\hline \multicolumn{9}{|c|}{ es, 1921} \\
\hline & $\mathrm{X}$ & $\mathrm{N}$ & & $\mathrm{N}$ & & & B & LC \\
\hline & $x$ & & & $\mathrm{~N}$ & $\mathrm{~N}$ & & & LC \\
\hline
\end{tabular}

Thamiaraea cinnamomea (Gravenhorst, 1802)

Thamiaraea hospita (Märkel, 1844)

Autaliini C.G. Thomson, 1859

\begin{tabular}{|c|c|c|c|c|c|c|c|c|}
\hline Autalia impressa (A.G. Olivier, 1795) & $\mathrm{H}$ & $\mathrm{X}$ & & $\mathrm{N}$ & $\mathrm{N}$ & $\mathrm{N}$ & & B \\
\hline Autalia longicornis Scheerpeltz, 1947 & $\mathrm{~N}$ & $\mathrm{X}$ & $\mathrm{N}$ & & $\mathrm{N}$ & $\mathrm{N}$ & $\mathrm{N}$ & B \\
\hline Autalia pumcticollis Sharp, 1864 & & & & & & $\mathrm{~N}$ & & \\
\hline Autalia rivularis (Gravenhorst, 1802) & $\mathrm{H}$ & & & & & $\mathrm{N}$ & $\mathrm{N}$ & \\
\hline \multicolumn{9}{|c|}{ Falagriini Mulsant \& Rey, 1873} \\
\hline Cordalia obscura (Gravenhorst, 1802) & $\mathrm{H}$ & $\mathrm{X}$ & $\mathrm{N}$ & $\mathrm{N}$ & $\mathrm{N}$ & $\mathrm{N}$ & $\mathrm{N}$ & $\mathrm{N}$ \\
\hline Falagria splendens Kraatz, 1858 & & $\mathrm{X}$ & $\mathrm{X}$ & & & $\mathrm{N}$ & & \\
\hline Falagria sulcatula (Gravenhorst, 1806) & & $\mathrm{X}$ & $\mathrm{X}$ & & & & & \\
\hline Falagrioma thoracica (Stephens, 1832) & & & $\mathrm{N}$ & $\mathrm{N}$ & $\mathrm{N}$ & $\mathrm{N}$ & & B \\
\hline
\end{tabular}

Falagrioma thoracica (Stephens, 1832)

Alevonota crypticola (Pace, 1977)

Alevonota egregia (Rye, 1876)

Alevonota gracilenta (Erichson, 1839)

Alevonota rufotestacea (Kraatz, 1856)

Aloconota gregaria (Erichson, 1839)

Aloconota insecta (J. Thomson, 1856)

Aloconota planifrons (G.R. Waterhouse, 1863)

Geostibini Seevers, 1978

\begin{tabular}{|l|l|l|l|l|l|l|l|l|}
\hline & & & & B & & & & \\
\hline H & B & & & & & & & \\
\hline B & & & & N & & & B & \\
\hline & X & N & & N & N & N & N & \\
\hline H & & & & & & & N & \\
\hline & & & & N & & & & \\
\hline
\end{tabular}


Species

Aloconota sulcifrons (Stephens, 1832)

Callicerus atricollis (Aubé, 1850)

Callicerus rigidicornis (Erichson, 1839)

Earola reyi (Kiesenwetter, 1850)

Geostiba aspromontana Pace, 1977

Geostiba focarilei (Scheerpeltz, 1956) [E]

Geostiba garganica Pace, 2002 [E]

Geostiba leonhardi (Bernhauer, 1908) [E]

Geostiba lucana Pace, 2002 [E]

Geostiba tamaninii (Scheerpeltz, 1956) [E]

Homalotini Heer, 1839

Bolitocharina C.G. Thomson, 1859

Bolitochara bella Märkel, 1844

Bolitochara obliqua Erichson, 1837

Bolitochara tecta Assing, 2014

Caloderina hierosolymitana (Saulcy, 1865)

Euryusa optabilis Heer, 1839

Leptusa fuliginosa (Aubé, 1850)

Leptusa fumida (Erichson, 1839)

Leptusa kochiana aspromontana Pace, 1977 [E]

Leptusa kochiana kochiana Bernhauer, 1936 [E]

Leptusa pulchella (Mannerheim, 1830)

Leptusa ruficollis (Erichson, 1839)

Leptusa salernitana Pace, 1979 [E]

Leptusa secreta Bernhauer, 1900

\begin{tabular}{|c|c|c|c|c|c|c|c|c|}
\hline 善产 & $\begin{array}{l}\frac{7}{8} \\
\frac{0}{3} \\
\frac{0}{0} \\
\frac{.0}{0} \\
0\end{array}$ & 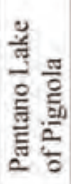 & 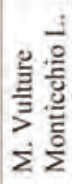 & 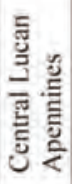 & 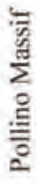 & 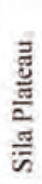 & 总 & 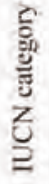 \\
\hline & & & $\mathrm{N}$ & $\mathrm{N}$ & $\mathrm{X}$ & & B & \\
\hline $\mathrm{H}$ & $\mathrm{X}$ & $\mathrm{N}$ & & & & & & \\
\hline & & & & & & & $\mathrm{N}$ & \\
\hline & & & & $\mathrm{N}$ & $\mathrm{X}$ & & B & \\
\hline & & & & & & & B & \\
\hline & & & & & X & & & \\
\hline B & & & & & & & & \\
\hline $\mathrm{H}$ & & & & & & & & \\
\hline & & & B & & & & & \\
\hline & & & & & X & & & \\
\hline
\end{tabular}

Agaricochara latissima (Stephens, 1832)

Gyrophaena affinis Mannerheim, 1830

Gyrophaena bihamata J. Thomson, 1867

Gyrophaena congrua Erichson, 1837

Gyrophaena joyioides Wüsthoff, 1937

Gyrophaena lucidula Erichson, 1837

Gyrophaena manca Erichson, 1839

Gyrophaena muensteri A. Strand, 1935

Gyrophaena nitidula (Gyllenhal, 1810)

Gyrophaena poweri Crotch, 1867

Anomognathus cuspidatus (Erichson, 1839)

Homalota plana (Gyllenhal, 1810)

\section{Gyrophaenina Kraatz, 1856}

\begin{tabular}{|c|c|c|c|c|c|c|c|}
\hline $\mathrm{H}$ & $X$ & $\mathrm{~N}$ & $\mathrm{~N}$ & B & & B & \\
\hline $\mathrm{H}$ & & & & X & X & & LC \\
\hline & & $\mathrm{N}$ & B & X & & B & CR \\
\hline $\mathrm{H}$ & & & & X & & $\mathrm{N}$ & \\
\hline$X$ & & & & X & X & & LC \\
\hline & & & & $\mathrm{N}$ & & & VU \\
\hline & & & $\mathrm{N}$ & CK & & & LC \\
\hline & & & & & & B & \\
\hline & & & CK & X & & & \\
\hline & & & $\mathrm{N}$ & X & & B & LC \\
\hline $\mathrm{H}$ & & $\mathrm{N}$ & $\mathrm{N}$ & B & B & $\mathrm{N}$ & LC \\
\hline & & & B & & & & \\
\hline & & & & CK & & B & \\
\hline
\end{tabular}

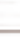

\begin{tabular}{|c|c|c|c|c|c|c|c|c|}
\hline H & & & & & & & & \\
\hline X & X & & & & & & & \\
\hline H & & & & & & & & \\
\hline & N & N & & & X & & & \\
\hline & X & N & & & & & & \\
\hline X & & & & & & & & \\
\hline & & & & N & & & & \\
\hline
\end{tabular}

Homalotina Heer, 1839

\section{Hypocyphtini Laporte, 1835}

Cypha longicornis (Paykull, 1800)

Cypha ovulum (Heer, 1839)

Cypha semimulum (Erichson, 1839)

Cypha spathulata Assing, 2007

Cypha tarsalis (Luze, 1902)

Holobus apicatus (Erichson, 1837)

Holobus flavicornis (Lacordaire, 1835)

Oligota inflata (Mannerheim, 1830)

Oligota muensteri latior Kapp, 2019

Oligota pumilio Kiesenwetter, 1858

Oligota pumctulata Heer, 1839

Oligota pusillima (Gravenhorst, 1806)

\begin{tabular}{|c|c|c|c|c|c|c|c|c|c|}
\hline H & X & X & N & N & N & & N & \\
\hline & & & & & & & N & \\
\hline I & & ! & & & & & ! & \\
\hline & & & & & N & & & \\
\hline H & & & & & & & N & \\
\hline & X & & & & & & & \\
\hline & B & & & B & & & B & \\
\hline & & N & & & & & & \\
\hline & & X & & & N & & & \\
\hline
\end{tabular}


Species

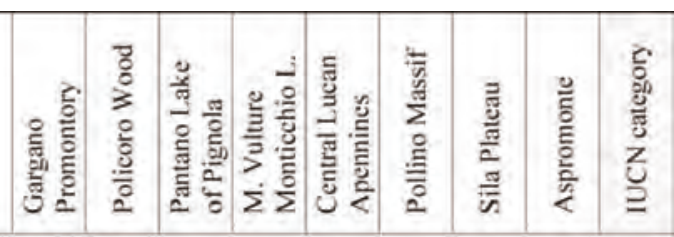

Lomechusini Fleming, 1821

Myrmedoniina J. Thomson, 1867

Drusilla canaliculata (Fabricius, 1787)

Drusilla italica (Bernhauer, 1903) [E]

Myrmoecia rigida (Erichson, 1839)

Pella cognata (Märkel, 1842)

Pella fimesta (Gravenhorst, 1806)

Pella humeralis (Gravenhorst, 1802)

Pella laticollis (Märkel, 1844)

Pella ruficollis (Grimm, 1845)

Pella similis (Märkel, 1844)

Zyras collaris (Paykull, 1800)

Zyras fulgidus (Gravenhorst, 1806)

\begin{tabular}{|c|c|c|c|c|c|c|}
\hline & & $\mathrm{X}$ & & & $\mathrm{N}$ & \\
\hline & $\mathrm{X}$ & & $\mathrm{N}$ & $\mathrm{N}$ & $X$ & B \\
\hline & & $\mathrm{X}$ & & & & \\
\hline \multirow[t]{3}{*}{$\mathrm{H}$} & & & & & & \\
\hline & & & & $\mathrm{N}$ & & \\
\hline & & & & $\mathrm{N}$ & & $\mathrm{N}$ \\
\hline $\mathrm{H}$ & & $X$ & & & & \\
\hline $\mathrm{X}$ & $\mathrm{X}$ & & & & & \\
\hline $\mathrm{H}$ & & & & & & \\
\hline $\mathrm{H}$ & & & & & & $\mathrm{N}$ \\
\hline $\mathrm{H}$ & & & & $\mathrm{N}$ & & \\
\hline
\end{tabular}

Myllaena brevicornis (A.H. Matthews, 1838)

Myllaena intermedia Erichson, 1837

Myllaenini Ganglbauer, 1895

Oxypodini C.G. Thomson, 1859

Dinardina Mulsant \& Rey, 1873

Homoeusa acuminata (Märkel, 1842)

Meoticina Seevers, 1978

Meotica exilis (Gravenhorst, 1806 )

$$
\begin{array}{l|l}
\text { H } & \mathrm{N}
\end{array}
$$

1978

Crataraea suturalis (Mannerheim, 1830)

Haploglossa gentilis (Märkel, 1844)

Haploglossa marginalis (Gravenhorst, 1806)

Haploglossa picipennis (Gyllenhal, 1827)

Haploglossa villosila (Stephens, 1832)

Calodera aethiops (Gravenhorst, 1802)

Calodera ligula Assing, 1996

Calodera nigrita Mannerheim, 1830

Cousya defecta (Mulsant \& Rey, 1875)

Cousya longitarsis (J. Thomson, 1867)

Cousya nigrata (Fairmaire \& Laboulbène, 1856)

Dexiogya corticina (Erichson, 1837)

Euryalaea murina (Erichson, 1839)

Ocalea badia Erichson, 1837

Ocalea pollinensis Scheerpeltz, 1956

Oxypoda abdominalis (Mannerheim, 1830)

Oxypoda alternans (Gravenhorst, 1802)

Oxypoda anmularis (Mannerheim, 1830)

Oxypoda brevicornis (Stephens, 1832)

Oxypoda carbonaria (Heer, 1841)

Oxypoda doderoi Bernhauer, 1902

Oxypoda ferruginea Erichson, 1839

Oxypoda formosa Kraatz, 1856

Oxypoda fusina Mulsant \& Rey, 1875

Oxypoda haemorrhoa (Mannerheim, 1830)

Oxypoda induta Mulsant \& Rey, 1861

Oxypoda lurida Wollaston, 1857

Oxypoda nova Bernhauer, 1902

Oxypoda opaca (Gravenhorst, 1802)

\section{Oxypodina C.G. Thomson, 1859}

\begin{tabular}{l|l|l|l|l|l|l|l|}
\hline Xes, 1918 \\
\hline X & & & & X & X & & \\
\hline & & & N & & & & \\
\hline & & & N & & & & \\
\hline & & & & & & N & \\
\hline
\end{tabular}

\begin{tabular}{|c|c|c|c|c|c|c|c|c|}
\hline & & X & & & & & N & \\
\hline H & & & & & & & & \\
\hline H & X & & & & & & & \\
\hline & X & & & & & & N & LC \\
\hline H & & & & & & & & \\
\hline & & & & & X & & & \\
\hline X & X & N & N & N & X & X & B & \\
\hline N & & X? & N & N & N & & N & \\
\hline & & N & & & & & & \\
\hline H & & & & & & & B & \\
\hline X & & N & & N & X & & B & \\
\hline X & & & & B & B & & & \\
\hline H & & & & & & & N & \\
\hline H & & & & & & & & \\
\hline H & & & & & & & & \\
\hline
\end{tabular}


Species

Oxypoda togata Erichson, 1837

Oxypoda vittata Märkel, 1842

Tetralaucopora longitarsis (Erichson, 1839)

Phloeoporina C.G. Thomson, 1859

\begin{tabular}{|c|c|c|c|c|c|c|c|c|}
\hline 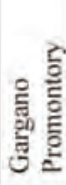 & $\begin{array}{l}\bar{g} \\
\frac{0}{3} \\
0 \\
\frac{0}{0} \\
\frac{.0}{0} \\
0\end{array}$ & 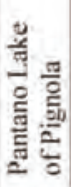 & 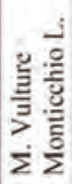 & 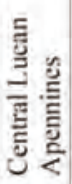 & 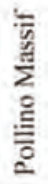 & 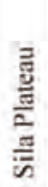 & 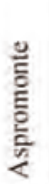 & 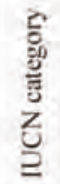 \\
\hline & & $\mathrm{N}$ & & & & & & \\
\hline $\mathrm{H}$ & & & $N$ & & $\mathrm{~N}$ & & B & \\
\hline & $\mathrm{N}$ & & & $\mathrm{N}$ & & & B & \\
\hline
\end{tabular}

Phloeopora teres (Gravenhorst, 1802)

Phloeopora testacea (Mannerheim, 1830)

Phytosini J. Thomson, 1867

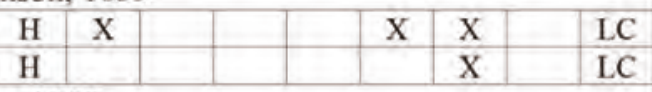

Phytosus spinifer Curtis, 1838

$$
\mathrm{X}
$$

Placusini Mulsant \& Rey, 1871

Placusa adscita Erichson, 1839

Placisa atrata (Mannerheim, 1830)

Placusa complanata Erichson, 1839

Placusa pumilio (Gravenhorst, 1802)

Pronomaea picea Heer, 1841

\begin{tabular}{|l|l|l|l|l|l|l|l|}
\hline H & & & N & & & & NT \\
\hline & & & & & & & LC \\
\hline & & & & & X & & LC \\
\hline
\end{tabular}

Pronomaeini Mulsant \& Rey, 1873

Tachyusini C.G. Thomson, 1859

Dilacra luteipes (Erichson, 1837)

Gnypeta carbonaria (Mannerheim, 1830)

Gnypeta rubrior Tottenham, 1939

Ischnopoda umbratica (Erichson, 1837)

Tachyusa balteata Erichson, 1839

Tachyusa coarctata Erichson, 1837

Tachyusa objecta Mulsant \& Rey, 1870

Taxicerini Lohse, 1989

Taxicera deplanata (Gravenhorst, 1802)

\section{Scaphidiinae Latreille, 1806 \\ Scaphiini Achard, 1924}

Löbl in Cat. Pal. Col., vol. 2, 2015

Scaphium immaculatum (A.G. Olivier, 1790)

Scaphidiini Latreille, 1806

Scaphidium quadrimaculatum A.G. Olivier, 1790

Scaphisomatini Casey, 1894

Baeocera nobilis nobilis Reitter, 1884

Scaphisoma agaricinum (Linnaeus, 1758)

Scaphisoma boleti boleti (Panzer, 1793)

Scaphisoma boreale Lundblad, 1952

Scaphisoma flavonotatum Pic, 1905

Scaphisoma italicum Tamanini, 1955

Scaphisoma loebli Tamanini, 1969

Scaphisoma obenbergeri Löbl, 1963

Scaphisoma subalpinum subalpinum Reitter, 1880

\begin{tabular}{|c|c|c|c|c|c|c|c|}
\hline $\mathrm{X}$ & & & & & & & \\
\hline $\mathrm{N}$ & & & & & & $\mathrm{B}$ & \\
\hline & & & & & & $\mathrm{B}$ & \\
\hline $\mathrm{X}$ & & & & $\mathrm{N}$ & & & \\
\hline
\end{tabular}

\begin{tabular}{|l|l|l|}
\hline & & B \\
\hline
\end{tabular}

B

\begin{tabular}{|c|c|c|c|c|c|c|c|}
\hline $\mathrm{N}$ & & & & $\mathrm{N}$ & & $\mathrm{N}$ & $\mathrm{VU}$ \\
\hline $\mathrm{H}$ & $\mathrm{X}$ & & & & & & LC \\
\hline $\mathrm{N}$ & $X$ & $\mathrm{~N}$ & $\mathrm{~N}$ & $\mathrm{X}$ & $\mathrm{N}$ & $\mathrm{N}$ & \\
\hline $\mathrm{X}$ & & & & $\mathrm{X}$ & $\mathrm{X}$ & $\mathrm{N}$ & NT \\
\hline $\mathrm{N}$ & $\mathrm{N}$ & & & $\mathrm{N}$ & & & \\
\hline$X$ & $X$ & $\mathrm{~N}$ & $\mathrm{~N}$ & $X$ & $\mathrm{X}$ & B & LC \\
\hline & $\mathrm{X}$ & & & $\mathrm{N}$ & & & NT \\
\hline & & & & $\mathrm{N}$ & & & NT \\
\hline $\mathrm{X}$ & & $\mathrm{N}$ & & B & & & LC \\
\hline
\end{tabular}

Piestinae Erichson, 1839

Siagonium humerale Germar, 1836 $\mathrm{H}$

Thoracophorina Reitter, 1909

Thoracophorus corticinus Motschulsky, 1837 $\mathrm{B}$

\section{Oxytelinae Fleming, 1821}

Blediini Adam, 2001

Bledius atricapillus (Germar, 1825)

Bledius cribricollis Heer, 1839

Bledius fossor Heer, 1839

Bledius frater Kraatz, 1857

\begin{tabular}{|c|c|c|c|c|c|c|c|}
\hline $\mathrm{X}$ & & & & & & & \\
\hline $\mathrm{N}$ & & & & & & & \\
\hline & & & & $\mathrm{X}$ & & $\mathrm{B}$ & \\
\hline $\mathrm{X}$ & & & & & & & \\
\hline
\end{tabular}




\begin{tabular}{|c|c|c|c|c|c|c|c|c|c|}
\hline Species & 总 & 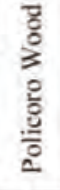 & 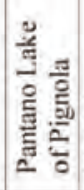 & 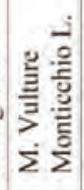 & 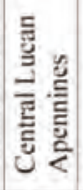 & 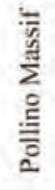 & 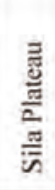 & है & 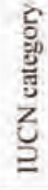 \\
\hline Bledius furcatus (A.G. Olivier, 18I1) & & $\mathrm{X}$ & & & $\mathrm{N}$ & & & $\mathrm{B}$ & \\
\hline Bledius gallicus (Gravenhorst, 1806) & & & $\mathrm{N}$ & & & & & & \\
\hline Bledius graellsi graellsi Fauvel, 1865 & & $\mathrm{X}$ & & & & & & & \\
\hline Bleditus heterocerus Eppelsheim, 1889 & & $\mathrm{~N}$ & & & & & & & \\
\hline Bledius lohsei Schülke, 2011 & & $\mathrm{X}$ & & & & & & & \\
\hline Bledius minor doderoi Bondroit, 1912 & $\mathrm{X}$ & & & & & & & & \\
\hline Bledius minor minor Mulsant \&. Rey, 1878 & & $\mathrm{X}$ & & & $\mathrm{N}$ & & & & \\
\hline Bledius occidentalis Bondroit, 1907 & & & & & & & & $\mathrm{~N}$ & \\
\hline Bledius tricornis (Herbst, 1784) & & $\mathrm{X}$ & & & & & & & \\
\hline Bledius tristis Erichson, 1840 & $\mathrm{X}$ & & & & & & & & \\
\hline Bledius unicornis (Germar, 1825) & & $\mathrm{X}$ & & & & & & $\mathrm{N}$ & \\
\hline Bledius verres Erichson, 1840 & & $\mathrm{X}$ & & & & & & B & \\
\hline \multicolumn{10}{|c|}{ Deleasteriini Reitter, 1909} \\
\hline Deleaster dichrous (Gravenhorst, 1802) & & & $\mathrm{X}$ & & $\mathrm{N}$ & $\mathrm{X}$ & & $\mathrm{N}$ & \\
\hline \multicolumn{10}{|c|}{ Oxytelini Fleming, 1821} \\
\hline Anotylus complanatus (Erichson, 1839) & $\mathrm{X}$ & $\mathrm{X}$ & & & & $\mathrm{N}$ & & B & \\
\hline Anotylus intricatus (Erichson, 1840) & $\mathrm{X}$ & & $\mathrm{X}$ & & $\mathrm{N}$ & & & $\mathrm{N}$ & \\
\hline Anotylus imustus (Gravenhorst, 1806) & $\mathrm{H}$ & $\mathrm{X}$ & $\mathrm{X}$ & $\mathrm{N}$ & $\mathrm{N}$ & $\mathrm{X}$ & $\mathrm{N}$ & $\mathrm{B}$ & \\
\hline Anotylus nitidulus (Gravenhorst, 1802) & $\mathrm{X}$ & $\mathrm{X}$ & $\mathrm{X}$ & & $\mathrm{N}$ & $\mathrm{X}$ & $\mathrm{X}$ & $\mathrm{B}$ & \\
\hline Anotylus pumilus (Erichson, 1839) & & & $\mathrm{X}$ & & & & & $\mathrm{B}$ & \\
\hline Anotylus rugosus (Fabricius, 1775) & $\mathrm{X}$ & $\mathrm{X}$ & $\mathrm{X}$ & & $\mathrm{N}$ & $\mathrm{X}$ & $\mathrm{X}$ & $\mathrm{N}$ & \\
\hline Anotylus sculpturatus (Gravenhorst, 1806) & $\mathrm{H}$ & $\mathrm{X}$ & $\mathrm{X}$ & $\mathrm{N}$ & $\mathrm{N}$ & $\mathrm{X}$ & $\mathrm{X}$ & B & \\
\hline Anotylus speculifrons (Kraatz, 1857) & $\mathrm{H}$ & $\mathrm{N}$ & & & $\mathrm{N}$ & & & $\mathrm{N}$ & \\
\hline Anotyhis tetracarinatus (Block, 1799) & $\mathrm{H}$ & & $\mathrm{X}$ & & $\mathrm{N}$ & $\mathrm{N}$ & & B & \\
\hline Oxytelus laqueatus (Marsham, 1802) & & $\mathrm{X}$ & & & $\mathrm{N}$ & & & & \\
\hline Oxytelus migrator Fauvel, 1904 & & & & & $\mathrm{~N}$ & & & & \\
\hline Oxytelus piceus (Linnaeus, 1767) & $\mathrm{H}$ & $\mathrm{X}$ & $\mathrm{X}$ & $\mathrm{N}$ & & $\mathrm{X}$ & $\mathrm{N}$ & $\mathrm{N}$ & \\
\hline Oxytelus sculptus Gravenhorst, 1806 & & $\mathrm{X}$ & $\mathrm{X}$ & & & & & & \\
\hline Platystethus alutaceus J. Thomson, 1861 & & & $\mathrm{~N}$ & & & $\mathrm{~N}$ & & & \\
\hline Platystethus arenarius (Geoffroy, 1785) & & & & & $\mathrm{N}$ & & & $\mathrm{B}$ & \\
\hline Platystethus burlei C.N.F. Brisout de Barneville, 1862 & & & $\mathrm{~N}$ & & & & & & \\
\hline Plarystethus cornutus cormutus (Gravenhorst, 1802) & & $\mathrm{X}$ & $\mathrm{X}$ & & & & & B & \\
\hline Platystethus degener Mulsant \& Rey, 1878 & & & $\mathrm{~N}$ & $\mathrm{~N}$ & & $\mathrm{~N}$ & & $\mathrm{~N}$ & \\
\hline Platystethus nitens (C.R. Sahlberg, 1832) & $\mathrm{H}$ & $\mathrm{X}$ & $\mathrm{N}$ & $\mathrm{N}$ & $\mathrm{N}$ & $\mathrm{X}$ & $\mathrm{X}$ & $\mathrm{N}$ & \\
\hline Platystethus spinosus Erichson, 1840 & $\mathrm{H}$ & $\mathrm{X}$ & $\mathrm{X}$ & & & & & & \\
\hline
\end{tabular}

Planeustomini Jacquelin du Val, 1857

Planeustomus cephalotes (Erichson, 1840)

Planeustomus elegantulus (Kraatz, 1857)

Thinobiini J. Sahlberg, 1876

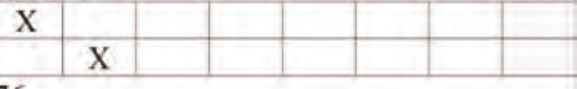

Carpelimus bilineatus Stephens, 1834

Carpelimus corticimus (Gravenhorst, 1806)

Carpelimus despectus (Baudi di Selve, 1870)

Carpelimus exiguus (Erichson, 1839)

Carpelimus foveolatus (C.R. Sahlberg, 1832)

Carpelimus gracilis (Mannerheim, 1830)

Carpelimus nigrita anthracinus (Mulsant \& Rey, 1861)

Carpelimus nitidus (Baudi di Selve, 1848)

Carpelimus obesus (Kiesenwetter, 1844)

Carpelimus politus politus (Kiesenwetter, 1850)

Carpelimus pusillus (Gravenhorst, 1802)

Carpelimus rivularis (Motschulsky, 1860)

Thinodromus arcuatus (Stephens, 1834)

Thinodromus hirticollis Mulsant \& Rey, 1878

\begin{tabular}{|l|l|l|l|l|l|l|l|l|}
\hline & X & X & & & & & & \\
\hline & X & X & N & N & & & B & \\
\hline & X & & & N & & & & \\
\hline & X & N & & & & & N & \\
\hline & X & & & & & & & \\
\hline & X & X & & N & & & & \\
\hline & & X & & & & & & \\
\hline & X & X & N & & & & N & \\
\hline & & X & & & & & B & \\
\hline & X & & N & N & & & B & \\
\hline & & & & & N & & N & \\
\hline & X & & & & & & & \\
\hline
\end{tabular}


Species

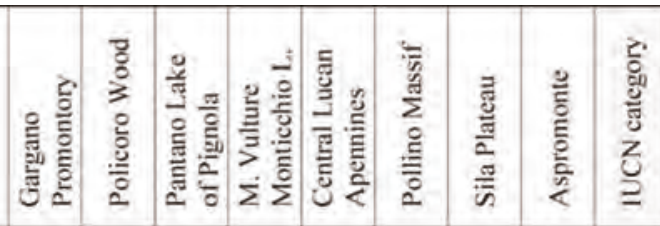

Oxyporinae Fleming, 1821

Oxyporus rufus rufus (Linnaeus, 1758)

$\mathrm{X} \quad \mathrm{X}$

Steninae W.S. Macleay, 1825

Stenus aceris Stephens, 1833

\begin{tabular}{|c|c|c|c|c|c|c|c|}
\hline H & X & & N & N & X & X & B \\
\hline & & & & & X & X & B \\
\hline & & N & N & N & X & & B \\
\hline H & X & & & N & & & B \\
\hline X & & & & & N & X & B \\
\hline & & & & & & & N \\
\hline
\end{tabular}

Stenus asphaltinus Erichson, 1840

Stenus assequens assequens Rey, 1884

Stenus ater Mannerheim, 1830

Stenus atratulus Erichson, 1839

Stenus bimaculatoides Scheerpeltz, 1969 [E]

Stenus bimaculatus Gyllenhal, 1810

Stenus binaghii Puthz, 1970 [E]

Stenus brumnipes brunnipes Stephens, 1833

Stemus butrintensis Smetana, 1959

Stenus caligicola Puthz, 1970 [E]

Stenus chobauti L. Benick, 1927

Stenus cicindeloides (Schaller, 1783)

Stenus comma peroculatus Puthz, 1986 [E]

Stemus cordatus Gravenhorst, 1802

Stemis cribratus Kiesenwetter, 1850

Stenus elegans Rosenhauer, 1856

Stenus flavipes flavipes Stephens, 1833

Stenus fossulatus Erichson, 1840

Stenus fuscicornis Erichson, 1840

Stemus guttula gutrula P.W.J. Müller, 1821

Stenus hospes Erichson, 1840

Stenus impressus Germar, 1824

Stenus incanus Erichson, 1839

Stemus intricatus zoufali A. Fleischer, 1909

Stenus juno (Paykull, 1789)

Stenus longipes Heer, 1839

Stenus longitarsis edentatus Puthz, 1986

Stenus longitarsis longitarsis J. Thomson, 1851

Stenus melanopus (Marsham, 1802)

Stenus mendicus Erichson, 1840

Stemus namus Stephens, 1833

Stemus ochropus Kiesenwetter, 1858

Stenus ossium Stephens, 1833

Stemus pallitarsis pallitarsis Stephens, 1833

Stenus picipennis Erichson, 1840

Stenus picipes picipes Stephens, 1833

Stemus planifrons planifrons Rey, 1884

Stenus scaber Fauvel, 1871

Stemus similis (Herbst, 1784)

Stemus solutus Erichson, 1840

Stenus subaeneus Erichson, 1840

Stenus trivialis Kraatz, 1857

Stenus umbricus Baudi di Selve, 1870

Stenus vitalei Bernhauer, 1935

Euaesthetinae C.G. Thomson, 1859

Euaesthetini C.G. Thomson, 1859

Octavius vitalei vitalei Bernhauer, 1908 


\section{Scydmaeninae Leach, 1815 \\ Cephenniitae Reitter, 1882 \\ Cephenniini Reitter, 1882}

Vit \& Besuchet in Cat. Pal. Col, vol. 2, 2015

Cephennium apicale apicale Reitter, 1882 [E]

Cephennium romanum W. Blattný \& C. Blattný, 1914 [E]

Cephennium garganicum Pace, 1977 [E]

Eutheiini Casey, 1897

Vit in Cat. PaI. Col., vol. 2, 2015

Eutheia formicetorum Reitter, 1882

Eutheia schaumi Kiesenwetter, 1858

Euthiconus conicicollis (Fairmaire, 1855)

\section{Scydmaenitae Leach, 1815 \\ Chevrolatiini Reitter, 1882 \\ Vit in Cat. Pal. Col., vol. 2, 2015}

Chevrolatia insignis Jacquelin du Val, 1850

\begin{tabular}{l|l|l|}
$\mathrm{H}$ & $\mathrm{X}$ & $\mathrm{X}$
\end{tabular}

Cyrtoscydmini L.W. Schaufuss, 1889

Davies in Cat. Pal. Col., vol. 2, 2015

Euconnus campestris L.W. Schaufuss, 1866

Euconnus claviger claviger (P.W.J. Müller \& Kunze, 1822)

Euconmus denticornis (P.W.J. Müller \& Kunze, 1822)

Euconmus fimetarius (Chaudoir, 1845)

Euconmus hirticollis (Illiger, 1798)

Euconnus intrusus intrusus (Schaum, 1844)

Euconmus italicus Reitter, 1911 [E]

Euconmus maklinii (Mannerheim, 1844)

Euconnus oblongus oblongus (Sturm, 1838)

Euconnus pubicollis (P.W.J. Müller \& Kunze, 1822)

Euconnus rutilipennis (P.W.J. Müller \& Kunze, 1822)

Euconnus wetterhalli (Gyllenhal, 1813)

Microscydmus minimus (Chaudoir, 1845)

Microscydmus namus (Schaum, 1844)

Neuraphes angulatus (P.W.J. Müller \& Kunze, 1822)

Neuraphes fiorii Reitter, 1887

Neuraphes planiceps Reitter, 1884 [E]

Neuraphes plicicollis plicicollis (Reitter, 1880)

Neuraphes rubicundis (Schaum, 1841)

Neuraphes ruthenus Machulka, 1925

Neuraphes stussineri Reitter, 1891 [E]

Neuraphes talparum Lokay, 1920

Scydmoraphes angelinii Castellini, 1987 [E]

Scydmoraphes diutius Castellini, 1987 [E]

Scydmoraphes geticus (Saulcy, 1877)

Scydmoraphes helvolus (Schaum, 1844)

Scydmoraphes leptocerus (Reitter, 1882)

Scydmoraphes panormitanus (Ragusa, 1892) [E]

Scydmoraphes romamis Franz, 1961 [E]

Scydmoraphes sparshalli (Denny, 1825)

Scydmoraphes tarsalis Machulka, 1930 [E]

Stenichnus apulicus (Pic, 1902) [E]

Stenichmus damryi hilfi Holdhaus, 1915

Stenichnus egregius Holdhaus, 1915 [E]

\begin{tabular}{|c|c|c|c|c|c|c|c|}
\hline & B & & & & & & $\mathrm{N}$ \\
\hline & X & & & & & & \\
\hline & B & & & & & & $\mathrm{N}$ \\
\hline X & $\mathrm{X}$ & & & & & & $\mathrm{N}$ \\
\hline \multirow[t]{7}{*}{$\mathrm{X}$} & X & & & & & & \\
\hline & B & & & & & & \\
\hline & & & & & $\mathrm{N}$ & & \\
\hline & $\mathrm{X}$ & & & & & & \\
\hline & & & $\mathrm{N}$ & $\mathrm{N}$ & $\mathrm{N}$ & & $\mathrm{N}$ \\
\hline & & & $\mathrm{N}$ & $\mathrm{N}$ & $\mathrm{N}$ & & \\
\hline & & & & $\mathrm{N}$ & & & \\
\hline \multirow[t]{2}{*}{$\mathrm{X}$} & $\mathrm{X}$ & & & & & & \\
\hline & & & & $\mathrm{N}$ & & & \\
\hline B & $\mathrm{X}$ & & & & $\mathrm{X}$ & B & B \\
\hline $\mathrm{H}$ & & $X$ & & & $\mathrm{X}$ & $\mathrm{X}$ & $\mathrm{N}$ \\
\hline \multirow[t]{3}{*}{$\mathrm{X}$} & $\mathrm{N}$ & & $\mathrm{N}$ & $\mathrm{N}$ & $\mathrm{N}$ & & \\
\hline & & & $\mathrm{N}$ & $\mathrm{N}$ & $\mathrm{X}$ & $\mathrm{X}$ & $\mathrm{N}$ \\
\hline & & & & $\mathrm{N}$ & & & \\
\hline \multirow[t]{11}{*}{$\mathrm{H}$ ? } & & & & & & & \\
\hline & & & & B & & & \\
\hline & & & & & & $\mathrm{X}$ & \\
\hline & & & & & & & $\mathrm{N}$ \\
\hline & & & & B & $\mathrm{N}$ & $\mathrm{N}$ & \\
\hline & & B & B & B & B & B & B \\
\hline & $\mathrm{X}$ & & & $\mathrm{N}$ & & & \\
\hline & & & & $\mathrm{N}$ & $\mathrm{N}$ & $\mathrm{X}$ & $\mathrm{N}$ \\
\hline & & & B & B & & $\mathrm{N}$ & \\
\hline & & & & & & & $\mathrm{N}$ \\
\hline & & & & & & & $\mathrm{N}$ \\
\hline \multirow[t]{3}{*}{$\mathrm{X}$} & $X$ & & & B & B & B & B \\
\hline & $\mathrm{N}$ & & & $\mathrm{N}$ & $\mathrm{N}$ & & B \\
\hline & & $\mathrm{N}$ & & $\mathrm{N}$ & $\mathrm{N}$ & & B \\
\hline \multirow[t]{2}{*}{$\mathrm{H}$} & & & & $\mathrm{N}$ & $\mathrm{N}$ & $\mathrm{N}$ & \\
\hline & & & & $\mathrm{N}$ & $\mathrm{X}$ & & B \\
\hline
\end{tabular}


Species

Stenichmus helferi helferi (Schaum, 184I)

Stenichnus paganettii Franz, 1960

Stenichnus pelliceus Holdhaus, 1908

Stenichmus pusillus (P.W.J. Müller \& Kunze, 1822)

Stenichnus scutellaris (P.W.J. Müller \& Kunze, 1822)

Scydmaenini Leach, 1815

\begin{tabular}{|c|c|c|c|c|c|c|c|c|}
\hline 总产 & $\begin{array}{l}\vec{c} \\
0 \\
3 \\
0 \\
\frac{0}{0} \\
\frac{.3}{0} \\
0\end{array}$ & 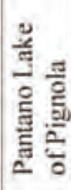 & 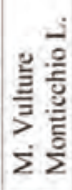 & 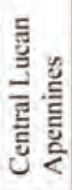 & $\begin{array}{l}\overline{\bar{v}} \\
\frac{8}{8} \\
0 \\
\overline{\bar{E}} \\
0\end{array}$ & 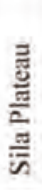 & 产 & 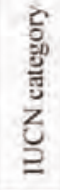 \\
\hline $\mathrm{H}$ & $\mathrm{X}$ & & $\mathrm{N}$ & $\mathrm{N}$ & $\mathrm{N}$ & & $\mathrm{N}$ & \\
\hline $\mathrm{N}$ & & $\mathrm{N}$ & $\mathrm{N}$ & $\mathrm{N}$ & $\mathrm{N}$ & X & B & \\
\hline & & & & & & & $\mathrm{N}$ & \\
\hline X & $X$ & $X$ & & $\mathrm{~N}$ & & & & \\
\hline$X$ & $\mathrm{X}$ & & & & & & & \\
\hline
\end{tabular}

Vit in Cat. Pal. Col., vol. 2, 2015

Scydmaemus antidotus Schaum, 1841 [E]

Scydmaenus cormutus Motschulsky, 1844

Scydmaemus hellwigii (Herbst, 1791)

Scydmaenus rufus P.W.J. Müller \& Kunze, 1822

Scydmaenus tarsatus P.W.J. Müller \& Kunze, 1822

\begin{tabular}{|l|l|l|l|l|l|l|l|l|}
\hline & X & & & N & N & & N & \\
\hline X & X & & N & N & X & & & LC \\
\hline X & X & & & & N & & & VU \\
\hline H & X & X & N & N & N & & N & \\
\hline
\end{tabular}

Mastiginae Fleming, 1821

Mastigitae Fleming, 1821

Leptomastacini Casey, 1897

Vit in Cat. Pal. Col., vol. 2, 2015

Leptomastax hypogea Pirazzoli, 1855

Leptomastax emeryi Simon, 1881

Mastigini Fleming. 1821

\begin{tabular}{|l|l|l|l|l|l|l|l|l|}
\hline H & & & N & & N & & N & \\
\hline H & N & & & & & & & \\
\hline
\end{tabular}

Palaeostigus heydenii (Rottenberg, 1870) [E]

Palaeostigus pilifer (Kraatz, 1879) [E]

Palaeostigus ruficornis neapolitamus (Ganglbauer, 1899) [E]

\begin{tabular}{|l|l|l|l|l|l|l|l|l|}
\hline H & X & & N & B & X & X & B & \\
\hline X & X & X & & B & X & X & B & \\
\hline & & & B & B & & X & & \\
\hline
\end{tabular}

Leptotyphlinae Fauvel, 1874

Entomoculiini Coiffait, 1957

Allotyphlus garganicus Pace, 1975 [E]

Allotyphlus sipontinus Pace, 1985 [E]

Allotyphlus transadriaticus Pace, 1977 [E]

Neocyrtotyphlus apulus (Pace, 1975) [E]

Pseudopsinae Ganglbauer, 1895

Pseudopsis sulcata Newman, 1834

\section{Paederinae Fleming, 1821 \\ Paederini Fleming, 1821}

Astenina Hatch, 1957

Astemus bimaculatus bimaculatus (Erichson, 1840)

Astemus gracilis (Paykull, 1789)

Astenus immaculatus Stephens, 1833

Astenus knischi Bernhauer, 1928 [E]

Astenus lyonessius (Joy, 1908)

Astenus melanurus (Küster, 1853)

Astenus thoracicus thoracicus (Baudi di Selve, 1857)

\begin{tabular}{|l|l|l|l|l|l|l|l|l|}
\hline $\mathrm{H}$ & & & $\mathrm{N}$ & & & & $\mathrm{N}$ & \\
\hline $\mathrm{H}$ & & & & & & & & \\
\hline $\mathrm{X}$ & $\mathrm{X}$ & $\mathrm{X}$ & & & $\mathrm{N}$ & & & \\
\hline $\mathrm{X}$ & & & & & & & & \\
\hline $\mathrm{X}$ & & $\mathrm{X}$ & & $\mathrm{N}$ & & $\mathrm{X}$ & $\mathrm{B}$ & \\
\hline $\mathrm{X}$ & & & & & & & $\mathrm{N}$ & \\
\hline & $\mathrm{X}$ & $\mathrm{X}$ & & $\mathrm{N}$ & & & $\mathrm{N}$ & \\
\hline
\end{tabular}

Cryptobiina Casey, 1905

Ochthephilum brevipenne (Mulsant \& Rey, 1861)

Ochthephilum collare (Reitter, 1884)

Leptobium gracile (Gravenhorst, 1802)

Leptobitum pominii (Gridelli, 1949) [E]

Scotonomus andreinii Binaghi, 1970 [E]

Dolicaonina Casey, 1905

\begin{tabular}{|l|l|l|l|l|l|l|l|l|}
\hline B & X & & & & & & & \\
\hline & X & X & B & & & & & \\
\hline
\end{tabular}

\begin{tabular}{|l|l|l|l|l|l|l|l|l|}
\hline H & X & X & & N & & & N & \\
\hline X & & & & & & & & \\
\hline & & & & B & & & & \\
\hline
\end{tabular}

Lathrobiina Laporte, 1835

Achenium depressum (Gravenhorst, 1802)

Achenium italicum Koch, 1937 [E]

Achenium striatum (Latreille, 1804)

\begin{tabular}{|l|l|l|l|l|l|l|l|l|}
$\mathrm{X}$ & $\mathrm{X}$ & $\mathrm{X}$ & & $\mathrm{N}$ & & & & \\
\hline $\mathrm{X}$ & & & & & & $\mathrm{N}$ & \\
\hline
\end{tabular}


Species

Achenium tenellum Erichson, 1840

Domene stilicina (Erichson, 1840)

Lathrobium crassipes Mulsant \& Rey, 1878

Lathrobium garganicum Koch, 1939 [E]

Lathrobium longulum Gravenhorst, 1802

Lobrathium multipumctum (Gravenhorst, 1802)

Micrillus testaceus (Erichson, 1840)

Platydomene picipes picipes (Erichson, 1840)

Pseudobium gridellii Jarrige, 1949

Pseudolathra lusitanica (Erichson, 1840)

Scimbalium anale (Nordmann, 1837)

Tetartopeus angustatus angustatus (Lacordaire, 1835)

Tetartopeus paeneinsularum A. Bordoni, 1982

Medonina Casey, 1905

\begin{tabular}{|c|c|c|c|c|c|c|c|}
\hline Hypomedon debilicornis (Wollaston, 1857) & & $\mathrm{X}$ & & & & & $\mathrm{N}$ \\
\hline Lithocharis ochracea (Gravenhorst, 1802) & & $\mathrm{X}$ & & & $\mathrm{N}$ & & \\
\hline Luzea nigritula (Erichson, 1840) & $\mathrm{N}$ & $\mathrm{X}$ & $\mathrm{X}$ & & $\mathrm{N}$ & & $\mathrm{N}$ \\
\hline Medon brunneus (Erichson, 1839) & $\mathrm{H}$ & & $\mathrm{X}$ & $\mathrm{N}$ & $\mathrm{N}$ & $\mathrm{N}$ & \\
\hline Medon perniger Coiffait, 1978 & $\mathrm{X}$ & & $\mathrm{X}$ & & B & $\mathrm{X}$ & $\mathrm{B}$ \\
\hline Medon ripicola (Kraatz, 1854) & $\mathrm{H}$ & & & & & & \\
\hline Pseudomedon obsoletus (Nordmann, 1837) & $\mathrm{X}$ & $\mathrm{N}$ & & & & & \\
\hline Sunius algiricus (Coiffait, 1970) & & & & & & & B \\
\hline Sumius italicus (Coiffait, 1961) [E] & & $\mathrm{X}$ & $\mathrm{X}$ & & & B & $\mathrm{N}$ \\
\hline Sunius pugliamus (Coiffait, 1961) [E] & & & & & $\mathrm{N}$ & & \\
\hline
\end{tabular}

Sunius pugliamus (Coiffait, 1961) [E]

Paederina Fleming, 1821

Paederidus rubrothoracicus (Goeze, 1777)

Paederidus ruficollis (Fabricius, 1777)

Paederus balcanicus Koch, 1938

Paederus baudii Fairmaire, 1860

Paederus fuscipes fuscipes Curtis, 1826

Paederus littoralis littoralis Gravenhorst, 1802

Paederus riparius (Linnaeus, 1758)

\begin{tabular}{|c|c|c|c|c|c|c|c|c|} 
& X & & N & N & X & X & B & \\
\hline & X & & & N & N & N & B & \\
\hline X & & X & N & N & N & & N & \\
\hline & & & N & & X & X & B & \\
\hline H & X & X & & N & N & N & B & \\
\hline X & X & X & & N & N & & B & \\
\hline H & & & & & & & & \\
\hline
\end{tabular}

Scopaeina Mulsant \& Rey, 1878

Micranops pilicornis (Baudi di Selve, 1870)

Scopaeus bicolor Baudi di Selve, 1848

Scopaens debilis Hochhuth, 1851

Scopaeus gracilis (Sperk, 1835)

Scopaeus laevigatus (Gyllenhal, 1827)

Scopaeus mitratus Binaghi, 1935

Scopaeus portai Luze, 1910

Scopaens siculus Binaghi, 1935 [E]

Scopaeus sulcicollis (Stephens, 1833)

\begin{tabular}{|l|l|l|l|l|l|l|l|l|}
\hline & & & & N & & & & \\
\hline & & X & & & & & N & \\
\hline & N & & & & & & & \\
\hline & X & X & & & & & & \\
\hline & X & X & & N & & & B & \\
\hline & X & X & & & & & & \\
\hline
\end{tabular}

Stilicina Casey, 1905

Rugilus angustatus (Geoffroy, 1785)

Rugilus orbiculatus (Paykull, 1789)

Rugilus rossii (Zanetti, 1977) [E]

Rugilus similis (Erichson, 1839)

Rugilus subtilis (Erichson, 1840)

Procirrina Bernhauer \& Schubert, 1912

\begin{tabular}{|c|c|c|c|c|c|c|c|c|}
\hline$H$ & & & & N & & & & \\
\hline$H$ & $\mathrm{X}$ & $\mathrm{X}$ & $\mathrm{N}$ & $\mathrm{N}$ & $\mathrm{N}$ & $\mathrm{N}$ & $\mathrm{B}$ & \\
\hline $\mathrm{H}$ & $\mathrm{X}$ & & $\mathrm{N}$ & $\mathrm{N}$ & $\mathrm{N}$ & & $\mathrm{B}$ & \\
\hline $\mathrm{H}$ & & $\mathrm{X}$ & & & $\mathrm{N}$ & & & \\
\hline & & & & N & & & & \\
\hline
\end{tabular}

Oedichinus paederinus Erichson, 1840

Procirrus lefebvrei lefebvrei Latreille, 1829

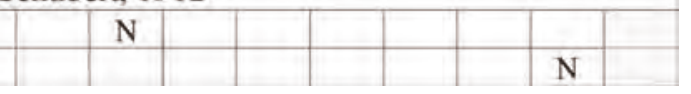


Species

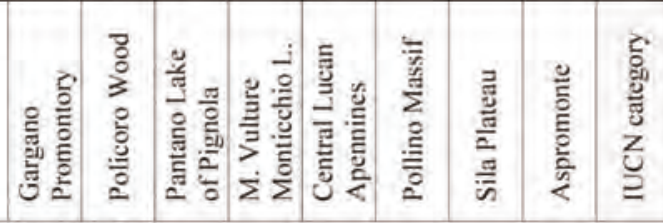

Staphylininae Latreille, 1802

Othiini C.G. Thomson, 1859

\begin{tabular}{|c|c|c|c|c|c|c|c|c|c|}
\hline Atrecus affinis (Paykull, 1789) & & & & $\mathrm{N}$ & $\mathrm{N}$ & $\mathrm{X}$ & $\mathrm{X}$ & B & LC \\
\hline Atrecus ardeamus Ciceroni, 1990 [E] & & & & & $\mathrm{B}$ & B & B & & EN \\
\hline Atrecus longiceps (Fauvel, 1873) & & & & & & $\mathrm{N}$ & & & LC \\
\hline Othius laeviusculus Stephens, 1833 & $\mathrm{H}$ & $\mathrm{X}$ & $\mathrm{X}$ & $\mathrm{N}$ & $\mathrm{N}$ & $\mathrm{X}$ & $\mathrm{X}$ & $\mathrm{N}$ & \\
\hline Othius lapidicola Mårkel \& Kiesenwetter, 1848 & $\mathrm{~N}$ & & & $\mathrm{~N}$ & $\mathrm{~N}$ & $\mathrm{X}$ & $\mathrm{X}$ & $\mathrm{N}$ & \\
\hline Othius punchilatus (Goeze, 1777) & $\mathrm{H}$ & & & $\mathrm{N}$ & $\mathrm{N}$ & $\mathrm{N}$ & & & \\
\hline
\end{tabular}

Othius punctulatus (Goeze, 1777)

Staphylinini Latreille, 1802

Amblyopinina Seevers, 1944

Heterothops binotatus (Gravenhorst, 1802)

Heterothops minutus Wollaston, 1860

Heterothops praevius Erichson, 1839

\begin{tabular}{|l|l|l|l|l|l|l|l|l|}
$\mathrm{X}$ & & & & & & & & \\
\hline $\mathrm{H}$ & & & & & $\mathrm{X}$ & $\mathrm{X}$ & & \\
& $\mathrm{X}$ & $\mathrm{X}$ & & & $\mathrm{N}$ & & $\mathrm{N}$ & \\
\hline
\end{tabular}

Bisnius fimetarius (Gravenhorst, 1802)

Bisnitus sordidus (Gravenhorst, 1802)

Cafius xantholoma (Gravenhorst, 1806)

Erichsonius cinerascens (Gravenhorst, 1802)

Erichsonius signaticornis (Mulsant \& Rey, 1853)

Erichsonius subopacus (Hochhuth, 1851)

Gabrius breviventer (Sperk, 1835)

Gabritus doderoi Gridelli, 1920

Gabrius femoralis (Hochhuth, 1851)

Gabrius nigritulus (Gravenhorst, 1802)

Gabrius osseticus (Kolenati, 1846)

Gabrius sexualis Smetana, 1954

Gabrius splendidulus (Gravenhorst, 1802)

Gabronthus maritimus (Motschulsky, 1858)

Neobisnius lathrobioides (Baudi di Selve, 1848)

Neobisnius procerulus procerulus (Gravenhorst, 1806)

Orthidus cribratus (Erichson, 1840)

Philonthus albipes (Gravenhorst, 1802)

Philonthus atratus (Gravenhorst, 1802)

Philonthus carbonarius (Gravenhorst, 1802)

Philonthus cognatus Stephens, 1832

Philonthus concinnus (Gravenhorst, 1802)

Philonthus confinis A. Strand, 1941

Philonthus coprophilus Jarrige, 1949

Philonthus corruscus (Gravenhorst, 1802)

Philonthus cruentatus (Gmelin, 1790)

Philonthus debilis (Gravenhorst, 1802)

Philonthus decortus (Gravenhorst, 1802)

Philonthus dimidiatipennis Erichson, 1840

Philonthus ebenimus (Gravenhorst, 1802)

Philonthus fenestratus Fauvel, 1872

Philonthus frigidus Märkel \& Kiesenwetter, 1848

Philonthus fimarius (Gravenhorst, 1806)

Philonthus gagates Mulsant \& Rey, 1876

Philonthus intermedius (Lacordaire, 1835)

Philonthus jurgans Tottenham, 1937

Philonthus laminatus (Creutzer, 1799)

Philonthus longicornis Stephens, 1832

Philonthus micans (Gravenhorst, 1802)

Philonthina Kirby, 1837

\begin{tabular}{|c|c|c|c|c|c|c|c|c|}
\hline $\mathrm{X}$ & & $\mathrm{X}$ & $\mathrm{N}$ & $\mathrm{N}$ & $\mathrm{X}$ & $\mathrm{X}$ & B & \\
\hline $\mathrm{X}$ & X & & & & X & & B & \\
\hline \multirow[t]{5}{*}{$\mathrm{X}$} & $\mathrm{X}$ & & & & & & & \\
\hline & & & B & & & & & \\
\hline & & & & & $\mathrm{N}$ & & & \\
\hline & $\mathrm{X}$ & & & & & & & \\
\hline & $\mathrm{X}$ & & & & & & & \\
\hline B & $\mathrm{X}$ & & $\mathrm{N}$ & & $\mathrm{X}$ & $\mathrm{X}$ & B & \\
\hline $\mathrm{H}$ & & & $\mathrm{N}$ & $\mathrm{N}$ & $\mathrm{N}$ & & & \\
\hline $\mathrm{H}$ & $\mathrm{X}$ & $\mathrm{X}$ & B & $\mathrm{N}$ & $\mathrm{X}$ & $\mathrm{X}$ & B & \\
\hline \multirow[t]{3}{*}{$\mathrm{X}$} & & & & & & $X$ & & \\
\hline & & $\mathrm{X}$ & & $\mathrm{N}$ & $\mathrm{X}$ & & $\mathrm{N}$ & \\
\hline & & & & & $\mathrm{X}$ & & & LC \\
\hline \multirow[t]{3}{*}{$\mathrm{X}$} & $\mathrm{X}$ & & & $\mathrm{N}$ & $\mathrm{N}$ & & $\mathrm{N}$ & \\
\hline & & $\mathrm{X}$ & B & & & & B & \\
\hline & & $\mathrm{X}$ & & & & & & \\
\hline \multirow[t]{3}{*}{$\mathrm{X}$} & $\mathrm{X}$ & & & & & & & \\
\hline & & $\mathrm{X}$ & & & & & & \\
\hline & & $\mathrm{X}$ & & & $\mathrm{X}$ & & B & \\
\hline $\mathrm{H}$ & $\mathrm{N}$ & & & & & & B & \\
\hline $\mathrm{H}$ & $X$ & & B & $\mathrm{N}$ & $\mathrm{X}$ & $X$ & B & \\
\hline \multirow[t]{3}{*}{$\mathrm{H}$} & $X$ & $\mathrm{X}$ & $\mathrm{N}$ & $\mathrm{N}$ & $\mathrm{X}$ & $\mathrm{X}$ & B & \\
\hline & & $\mathrm{X}$ & & $\mathrm{N}$ & & & $\mathrm{N}$ & \\
\hline & & $\mathrm{X}$ & & & $\mathrm{X}$ & $\mathrm{X}$ & & \\
\hline \multirow[t]{2}{*}{$\mathrm{H}$} & $\mathrm{X}$ & $\mathrm{X}$ & & & $X$ & & & \\
\hline & $X$ & & & & & $\mathrm{X}$ & & \\
\hline \multirow[t]{3}{*}{$\mathrm{H}$} & $\mathrm{X}$ & & $\mathrm{N}$ & $\mathrm{N}$ & $\mathrm{N}$ & X & B & \\
\hline & & & $\mathrm{N}$ & $\mathrm{N}$ & $X$ & $\mathrm{X}$ & & \\
\hline & & & & $\mathrm{N}$ & & & & \\
\hline $\mathrm{X}$ & & & & & $X$ & & & \\
\hline \multirow[t]{4}{*}{$\mathrm{x}$} & $\mathrm{X}$ & & & & $\mathrm{X}$ & $X$ & & \\
\hline & & & & & $X$ & & & \\
\hline & & $\mathrm{X}$ & & & $X$ & & & \\
\hline & & & & & & $\mathrm{X}$ & & \\
\hline \multirow[t]{2}{*}{$\mathrm{X}$} & $\mathrm{X}$ & $\mathrm{X}$ & $\mathrm{N}$ & $\mathrm{N}$ & $X$ & $\mathrm{X}$ & B & \\
\hline & $\mathrm{X}$ & & $\mathrm{N}$ & & $X$ & $X$ & & \\
\hline \multirow[t]{2}{*}{$\mathrm{X}$} & & & & $\mathrm{N}$ & $\mathrm{X}$ & & B & \\
\hline & $\mathrm{X}$ & & & & & & & \\
\hline $\mathrm{H}$ & $\mathrm{X}$ & $\mathrm{X}$ & $\mathrm{N}$ & $\mathrm{N}$ & & & & \\
\hline
\end{tabular}




\begin{tabular}{|c|c|c|c|c|c|c|c|c|c|}
\hline Species & 吾 & $\begin{array}{l}\vec{b} \\
0 \\
j \\
0 \\
\frac{2}{0} \\
\frac{.}{0} \\
2\end{array}$ & 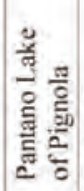 & 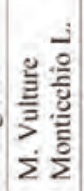 & 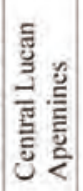 & 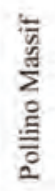 & $\begin{array}{l}\frac{\overrightarrow{\mathrm{e}}}{\frac{\mathrm{e}}{2}} \\
\frac{\mathrm{m}}{\bar{b}}\end{array}$ & 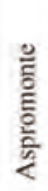 & 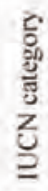 \\
\hline Philonthus nitidicollis (Lacordaire, 1835) & & & & & & $\mathrm{X}$ & $\mathrm{X}$ & B & \\
\hline Philonthus oblitus Jarrige, 1951 & & B & & & & & & & \\
\hline Philonthus paganettii Linke, 1915 [E] & & & & & & & $\mathrm{X}$ & B & \\
\hline Philonthus parvicornis (Gravenhorst, 1802) & $\mathrm{X}$ & $\mathrm{X}$ & $\mathrm{X}$ & & $\mathrm{N}$ & $\mathrm{N}$ & & & \\
\hline Philonthus politus (Linnaeus, 1758) & $\mathrm{H}$ & $\mathrm{X}$ & & & & $\mathrm{X}$ & $\mathrm{X}$ & & \\
\hline Philonthus punctus punctus (Gravenhorst, 1802) & & & $\mathrm{X}$ & $\mathrm{N}$ & & & & & \\
\hline Philonthus quisquiliarius quisquiliarius (Gyllenhal, 1810) & & $\mathrm{N}$ & $\mathrm{X}$ & $\mathrm{B}$ & $\mathrm{N}$ & $\mathrm{N}$ & $\mathrm{X}$ & $\mathrm{N}$ & \\
\hline Philonthus rectangulus Sharp, 1874 & & & $\mathrm{X}$ & $\mathrm{N}$ & & $\mathrm{X}$ & $\mathrm{X}$ & B & \\
\hline Philonthus rubripennis Stephens, 1832 & & & & & $\mathrm{~N}$ & & & & \\
\hline Philonthus rufimanus rufimamus Erichson, 1840 & & & & & $\mathrm{~N}$ & & $\mathrm{X}$ & B & \\
\hline Philonthus sanguinolentus (Gravenhorst, 1802) & & & $\mathrm{X}$ & & & & & & \\
\hline Philonthus spinipes kabardensis (Bolov \& Kryzhanovskij, 1969) & & & & & $\mathrm{N}$ & & & & \\
\hline Philonthus splendens splendens (Fabricius, 1792) & $\mathrm{X}$ & & & & & & & & \\
\hline Philonthus succicola C.G. Thomson, 1860 & & $\mathrm{X}$ & & $\mathrm{N}$ & $\mathrm{N}$ & & & B & \\
\hline Philonthus temuicornis Mulsant \& Rey, 1853 & & $\mathrm{X}$ & & & & & & B & \\
\hline Philonthus umbratilis (Gravenhorst, 1802) & $\mathrm{X}$ & & $\mathrm{X}$ & & $\mathrm{N}$ & $\mathrm{N}$ & & B & \\
\hline Philonthus varians (Paykull, 1789) & & & & & & $\mathrm{X}$ & $\mathrm{X}$ & & \\
\hline Philonthus ventralis (Gravenhorst, 1802) & $\mathrm{H}$ & $\mathrm{N}$ & & & & & & & \\
\hline
\end{tabular}

Philonthus ventralis (Gravenhorst, 1802)

Quediina Kraatz, 1857

\begin{tabular}{|c|c|c|c|c|c|c|c|c|c|}
\hline Acylophorus glaberrimus (Herbst, 1784) & & & $\mathrm{X}$ & & & & & & \\
\hline Euryporus aeneiventris P.H. Lucas, 1849 & & & & & & & & B & \\
\hline Euryporus picipes (Paykull, 1800) & $\mathrm{X}$ & & & & & & & & \\
\hline Quedius abietum Kiesenwetter, 1858 & & & & & $\mathrm{~B}$ & $\mathrm{X}$ & $\mathrm{X}$ & B & VU \\
\hline Quedius aetolicus Kraatz, 1858 & & & & & & $\mathrm{~N}$ & & & VU \\
\hline Quedius aspromontanus Bernhauer, 1908 & $\mathrm{X}$ & & & $\mathrm{B}$ & B & $\mathrm{X}$ & $\mathrm{X}$ & B & \\
\hline Quedius boops boops (Gravenhorst, 1802) & $\mathrm{H}$ & & $\mathrm{X}$ & $\mathrm{N}$ & $\mathrm{N}$ & $\mathrm{X}$ & $\mathrm{X}$ & $\mathrm{N}$ & \\
\hline Quedius bruttius Zanetti, 1977 [E] & & & & & & $\mathrm{X}$ & $\mathrm{X}$ & B & \\
\hline Quedius cinctus (Paykull, 1790) & $\mathrm{H}$ & $\mathrm{X}$ & $\mathrm{X}$ & B & $\mathrm{N}$ & $\mathrm{X}$ & $\mathrm{X}$ & B & \\
\hline Quedius crassus Fairmaire, 1860 & & & & & $\mathrm{~N}$ & & & & \\
\hline Quedius cruentus (A.G. Olivier, 1795) & $\mathrm{X}$ & & & $\mathrm{N}$ & $\mathrm{N}$ & $\mathrm{X}$ & $\mathrm{X}$ & B & LC \\
\hline Quedius curtipennis Bernhauer, 1908 & B & & $\mathrm{X}$ & $\mathrm{N}$ & $\mathrm{N}$ & & & & \\
\hline Quedius curtus Erichson, 1840 & & & & & & $\mathrm{X}$ & & & \\
\hline Quedius cyanescens Mulsant \& Rey, 1876 & & & & & & & & $\mathrm{~N}$ & \\
\hline Quedius dilatatus (Fabricius, 1787) & & $\mathrm{X}$ & & & & & & & \\
\hline Quedius dubius dubius (Heer, 1839) & & & & & & $\mathrm{X}$ & $\mathrm{X}$ & & \\
\hline Quedius fulgidus fulgidus (Fabricius, 1792) & & & & B & B & $\mathrm{X}$ & & & \\
\hline Quedius fumatus (Stephens, 1833) & $\mathrm{H}$ & $\mathrm{X}$ & & $\mathrm{N}$ & $\mathrm{N}$ & $\mathrm{N}$ & $\mathrm{N}$ & B & \\
\hline Quedius humeralis Stephens, 1832 & $\mathrm{~B}$ & & & & $\mathrm{~N}$ & $\mathrm{X}$ & & $\mathrm{N}$ & \\
\hline Quedius invreae Gridelli, 1924 & & & $\mathrm{X}$ & & $\mathrm{N}$ & & & & \\
\hline Quedius italicus Gridelli, 1925 & & & & & & $\mathrm{X}$ & & $\mathrm{N}$ & \\
\hline Quedius latialis Gridelli, I924 [E] & $\mathrm{X}$ & & & & $\mathrm{N}$ & $\mathrm{X}$ & $\mathrm{B}$ & & \\
\hline Quedius latinus Gridelli, 1938 & $\mathrm{X}$ & $\mathrm{X}$ & $\mathrm{X}$ & $\mathrm{N}$ & $\mathrm{N}$ & $\mathrm{X}$ & $\mathrm{X}$ & B & \\
\hline Quedius levicollis (Brullé, 1832) & $\mathrm{H}$ & $\mathrm{X}$ & $\mathrm{X}$ & B & $\mathrm{N}$ & $\mathrm{X}$ & $\mathrm{X}$ & B & \\
\hline Quedius lucidulus Erichson, 1839 & & & & & & & & B & \\
\hline Quedius masoni Zanetti, 1992 & B & B & & B & B & B & B & B & \\
\hline Quedius meridiocarpathicus Smetana, 1958 & & & $\mathrm{~N}$ & & & & & & \\
\hline Quedius mesomelimus silensis Fiori, 1894 [E] & $\mathrm{H}$ & & & & $\mathrm{N}$ & $\mathrm{X}$ & $\mathrm{X}$ & $\mathrm{B}$ & \\
\hline Quedius molochimus (Gravenhorst, 1806) & $\mathrm{X}$ & & & & & & & & \\
\hline Quedius nemoralis nemoralis Baudi di Selve, 1848 & & $\mathrm{X}$ & & & & $\mathrm{X}$ & $\mathrm{X}$ & B & \\
\hline Quedius nigriceps Kraatz, 1857 & & & & & $\mathrm{~N}$ & $\mathrm{~N}$ & $\mathrm{X}$ ? & & \\
\hline Quedius nigrocaeruleus Fauvel, 1876 & & & $\mathrm{~N}$ & B & $\mathrm{N}$ & $\mathrm{X}$ & $\mathrm{X}$ & $\mathrm{N}$ & \\
\hline Quedius nitipennis (Stephens, 1833) & & $\mathrm{X}$ & $\mathrm{X}$ & & $\mathrm{N}$ & $\mathrm{N}$ & 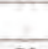 & $\mathrm{N}$ & \\
\hline Quedius obscuripennis Bernhauer, 1901 & $\mathrm{X}$ & & & & $\mathrm{N}$ & $\mathrm{X}$ & $\mathrm{X}$ & & \\
\hline
\end{tabular}


Species

Quedius ochripennis (Ménétriés, 1832)

Quedius pallipes P.H. Lucas, 1846

Quedius paradisianus (Heer, 1839)

Quedius picipes (Mannerheim, 1830)

Quedius reitteri Gridelli, 1925

Quedius satanas Bernhauer, 1899

Quedius scintillans (Gravenhorst, 1806)

Quedius semiaeneus (Stephens, 1833)

Quedius semiobscurus (Marsham, 1802)

Quedius suturalis suturalis Kiesenwetter, 1845

Quedius trumcicola Fairmaire \& Laboulbène, 1856

Quedius umbrinus Erichson, 1839

Staphylinina Latreille, 1802

Creophilus maxillosus maxillosus (Linnaeus, 1758)

Dinothenarus flavocephalus flavocephalus (Goeze, 1777)

Dinothenarus fossor (Scopoli, 1771)

Dinothenarus pubescens pubescens (DeGeer, 1774)

Emus hirtus (Linnaeus, 1758)

Ocypus fortunatarum Wollaston, 1871

Ocypus fulvipennis Erichson, 1840

Ocypus italicus (Aragona, 1830) [E]

Ocypus mus (Brullè, 1832)

Ocypus nitens nitens (Schrank, 1781)

Ocypus olens (O.F. Müller, 1764)

Ocypus ophthalmicus ophthalmicus (Scopoli, 1763)

Ocypus picipennis picipennis (Fabricius, 1792)

Ocypus sericeicollis (Ménétriés, 1832)

Ontholestes murinus (Linnaeus, 1758)

Platydracus flavopunctatus (Latreille, 1804)

Platydracus fulvipes (Scopoli, 1763)

Platydracus stercorarius stercorarius (A.G. Olivier, 1795)

Staphylimus caesareus Cederhjelm, 1798

Staphylimus dimidiaticornis Gemminger, 1851

Tasgius falcifer falcifer (Nordmann, 1837)

Tasgius globulifer globulifer (Geoffroy, 1785)

Tasgius morsitans (P. Rossi, 1790)

Tasgius pedator pedator (Gravenhorst, 1802)

Tasgius winkleri (Bernhauer, 1906)

\section{Tanygnathinina Reitter, 1909}

\begin{tabular}{|c|c|c|c|c|c|c|c|c|c|}
\hline X & X & & & N & X & X & N & \\
\hline & & & B & B & B & & & \\
\hline H & & & & B & N & B & B & \\
\hline B & & & & & & & B & \\
\hline B & & & & B & X & B & B & \\
\hline H & X & N & B & B & X & X & B & \\
\hline B & & & & B & N & X & B & \\
\hline B & X & & N & N & B & B & B & \\
\hline H & X & N & N & B & X & X & B & \\
\hline H & X & & N & B & X & CK & B & \\
\hline H & & & & CK & X & & & \\
\hline H & N & & B & B & B & B & B & \\
\hline H & X & X & B & B & X & X & N & \\
\hline & & & & CK & B & & & \\
\hline & B & X & & & & & & \\
\hline X & CK & & & N & B & & & \\
\hline X & X & & & N & & & B & \\
\hline & B & X & & & B & B & B & \\
\hline & X & & N & B & N & & B & \\
\hline H & X & & & & X & X & & \\
\hline X & B & & N & N & X & B & B & \\
\hline X & & & & & B & & B & \\
\hline B & X & & B & & N & & B & \\
\hline , & & & & & & \\
\hline
\end{tabular}

Astrapaeus ulmi (P. Rossi, 1790)

Quedionuchus plagiatus (Mannerheim, 1843)

Xantholinini Erichson, 1839

Gauropterus fulgidus (Fabricius, 1787)

Gyrohypmus angustatus Stephens, 1833

Gyrohypmus fracticornis (O.F. Müller, 1776)

Gyrohypnus punctulatus (Paykull, 1789)

Hypnogyra angularis (Ganglbauer, 1895)

Leptacinus batychrus (Gyllenhal, 1827)

Leptacinus intermedius Donisthorpe, 1936

Leptacinus othioides Baudi di Selve, 1870

Leptacinus pusillus (Stephens, 1833)

Megalimus glabratus (Gravenhorst, 1802)

Nudobins collaris (Erichson, 1839)

\begin{tabular}{|c|c|c|c|c|c|c|c|c|}
\hline $\mathrm{X}$ & $\mathrm{X}$ & & & $\mathrm{N}$ & & & $\mathrm{N}$ & \\
\hline
\end{tabular}

\begin{tabular}{|l|l|l|l|l|l|l|l|l|l|}
\hline \multicolumn{1}{|c|}{ Xantholinini Erichson, } & H & X & N & & N & N & & B & \\
\hline & H & & X & N & N & X & X & N & \\
\hline & & X & X & N & & X & X & & \\
\hline & H & & & & & X & & B & \\
\hline 6 & & N & & & & & X & & LC \\
\hline & & X & X & & B & & & N & \\
\hline & & N & X & & N & N & & N & \\
\hline & X & & X & & & & & & \\
\hline & & & B & & & & & B & \\
\hline & & X & & & N & N & & B & \\
\hline
\end{tabular}




\begin{tabular}{|c|c|c|c|c|c|c|c|c|c|}
\hline Species & 总 & $\begin{array}{l}7 \\
\vdots \\
3 \\
\vdots \\
\frac{0}{0} \\
\frac{0}{0}\end{array}$ & 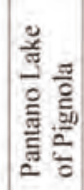 & 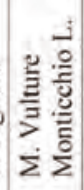 & 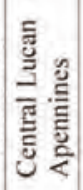 & 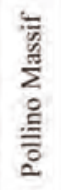 & $\frac{\vec{z}}{\frac{\vec{g}}{2}}$ & हैं & 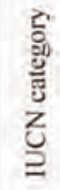 \\
\hline Phacophallus parumpunctatus (Gyllenhal, 1827) & $\mathrm{X}$ & $\mathrm{X}$ & $\mathrm{X}$ & $\mathrm{N}$ & & $\mathrm{N}$ & & B & \\
\hline Stenistoderus cephalotes cephalotes (Kraatz, 1858) & & & & & & & B & & \\
\hline Stenistoderus nothus (Erichson, 1839) & $\mathrm{H}$ & $\mathrm{X}$ & $\mathrm{X}$ & & & & & $\mathrm{N}$ & \\
\hline Vulda garganica (Bernhauer, 1908) [E] & $\mathrm{H}$ & & & & & & & & \\
\hline Vulda sbordonii Pace, 1979 [E] & $\mathrm{X}$ & & & & & & & & \\
\hline Xantholinus appenninicola Steel, 1946 & & $\mathrm{X}$ & & $\mathrm{N}$ & $\mathrm{N}$ & $\mathrm{X}$ & $\mathrm{X}$ & $\mathrm{B}$ & \\
\hline Xantholimus audrasi Coiffait, 1956 & & & $\mathrm{X}$ & & & & & & \\
\hline Xantholinus cerrutii Coiffait, 1964 [E] & & & & & & B & & & \\
\hline Xantholinus elegans (A.G. Olivier, 1795) & & & & & & $\mathrm{X}$ & & & \\
\hline Xantholimus graecus Kraatz, 1858 & & & & & & & & $\mathrm{~B}$ & \\
\hline Xantholimus linearis linearis (A.G. Olivier, 1795) & $\mathrm{H}$ & & $\mathrm{X}$ & & $\mathrm{N}$ & $\mathrm{N}$ & & $\mathrm{B}$ & \\
\hline Xantholinus longiventris Heer, 1839 & & & $\mathrm{X}$ & B & & & & & \\
\hline Xantholinus proceroides Coiffait, 1971 [E] & $\mathrm{X}$ & $\mathrm{N}$ & $\mathrm{X}$ & $\mathrm{N}$ & $\mathrm{N}$ & $\mathrm{N}$ & & $\mathrm{B}$ & \\
\hline Xantholinus rufipennis Erichson, 1839 & $\mathrm{~B}$ & & & & & & & B & \\
\hline \multicolumn{10}{|c|}{$\begin{array}{l}\text { SCARABAEIFORMIA Crowson, } 1960 \\
\text { SCARABAEOIDEA Latreille, } 1802\end{array}$} \\
\hline \multicolumn{10}{|c|}{$\begin{array}{l}\text { GEOTRUPIDAE Latreille, } 1802 \\
\text { Bolboceratinae Mulsant, } 1842 \\
\text { Odonteini Shokhin, } 2007 \\
\text { Král, Löbl \& Nikolajev in Cat. Pal. Col., vol. 3, } 2016\end{array}$} \\
\hline Odonteus armiger (Scopoli, 1772) & & B & & & & & & & \\
\hline \multicolumn{9}{|c|}{ Enoplotrupini Paulian, 1945} & \\
\hline Typhaeus typhoeus (Linnaeus, 1758) & $\mathrm{H}$ & $\mathrm{X}$ & $\mathrm{X}$ & & & $\mathrm{X}$ & $\mathrm{X}$ & $\mathrm{B}$ & \\
\hline \multicolumn{10}{|c|}{ Geotrupini Latreille, 1802} \\
\hline Anoplotrupes stercorosus (L.G. Scriba, 1791) & $\mathrm{X}$ & & & $\mathrm{N}$ & & & $\mathrm{X}$ & $\mathrm{B}$ & \\
\hline Geotrupes mutator (Marsham, 1802) & & & & & & & & $\mathrm{N}$ & \\
\hline Geotrupes spiniger (Marsham, 1802) & $\mathrm{H}$ & $\mathrm{X}$ & $\mathrm{X}$ & & $\mathrm{N}$ & $\mathrm{X}$ & $\mathrm{X}$ & $\mathrm{N}$ & \\
\hline Jekelius brullei brullei (Jekel, 1866) & $\mathrm{H}$ & & & & & & & & \\
\hline Jekelius intermedius (O.G. Costa, 1839) & B & $\mathrm{X}$ & & $\mathrm{N}$ & $\mathrm{N}$ & $\mathrm{N}$ & & $\mathrm{B}$ & \\
\hline Sericotrupes niger (Marsham, 1802) & $\mathrm{H}$ & $\mathrm{B}$ & $\mathrm{X}$ & $\mathrm{N}$ & $\mathrm{N}$ & $\mathrm{X}$ & $\mathrm{X}$ & $\mathrm{B}$ & \\
\hline Trypocopris pyrenaeus cyanicolor (Capra, 1930) [E] & & & & B & $\mathrm{N}$ & $\mathrm{N}$ & $\mathrm{X}$ & & \\
\hline Trypocopris pyrenaeus splendens (Heer, 1841) & $\mathrm{H}$ & & & & & & & & \\
\hline Trypocopris vernalis apenninicus Mariani, 1958 [E] & & & & $\mathrm{N}$ & $\mathrm{N}$ & $\mathrm{X}$ & $\mathrm{X}$ & B & \\
\hline \multicolumn{10}{|c|}{$\begin{array}{l}\text { TROGIDAE W.S. Macleay, } 1819 \\
\text { Troginae W.S. Macleay, } 1819 \\
\text { Pittino \& Bezděk in Cat. Pal. Col., vol. 3, } 2016\end{array}$} \\
\hline Trox litoralis Pittino, 1991 & & $\mathrm{~B}$ & & & & & & & \\
\hline Trox niger P. Rossi, 1792 & $\mathrm{H}$ & $\mathrm{X}$ & & & $\mathrm{N}$ & $\mathrm{X}$ & & $\mathrm{N}$ & \\
\hline $\begin{array}{r}\text { LUCANIDA } \\
\text { Aesalinae W. } \\
\text { Aesalini W.S } \\
\text { Bartolozzi, Sprecher-Uebersax \& }\end{array}$ & $\begin{array}{l}\text { ille, } \\
\text { eay, } \\
\text { ay, } 18 \\
\text { in Cat. }\end{array}$ & $\begin{array}{l}180 \\
1819 \\
19 \\
\text { Pal. }\end{array}$ & Col., v & vol. 3 , & 2016 & & & & \\
\hline Aesalus scarabaeoides meridionalis Bartolozzi, 1989 [E] & & $\mathrm{B}$ & & & & & & & CR \\
\hline \multicolumn{10}{|c|}{ Sinodendrini LeConte, 1861} \\
\hline Sinodendron cylindricum (Linnaeus, 1758) & $\mathrm{B}$ & $\mathrm{X}$ & & $\mathrm{N}$ & B & $\mathrm{X}$ & $\mathrm{X}$ & $\mathrm{B}$ & LC \\
\hline \multicolumn{10}{|c|}{$\begin{array}{c}\text { Lucaninae Latreille, } 1804 \\
\text { Dorcini Parry, } 1864\end{array}$} \\
\hline Dorcus parallelipipedus (Linnaeus, 1758) & $\mathrm{X}$ & $\mathrm{X}$ & & B & CK & $\mathrm{X}$ & $\mathrm{X}$ & $\mathrm{B}$ & LC \\
\hline
\end{tabular}


Species

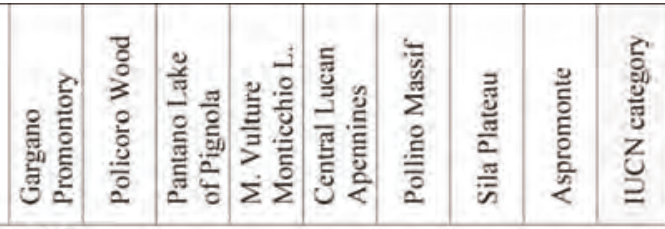

Lucanini Latreille, 1804

\begin{tabular}{|c|c|c|c|c|c|c|c|c|}
\hline Lucamus tetraodon tetraodon Thunberg, 1806 & $\mathrm{X}$ & $\mathrm{X}$ & $\mathrm{N}$ & B & $\mathrm{X}$ & $\mathrm{X}$ & B & LC \\
\hline \multicolumn{9}{|c|}{ Platycerini Mulsant, 1842} \\
\hline Platycerus caprea (DeGeer, 1774) & & & B & B & $\mathrm{X}$ & B & & LC \\
\hline Platycertus caraboides (Linnaeus, 1758) & $\mathrm{N}$ & & & $\mathrm{CK}$ & $\mathrm{X}$ & $\mathrm{X}$ & B & LC \\
\hline
\end{tabular}

OCHODAEIDAE Mulsant \& Rey, 1871

Ochodaeinae Mulsant \& Rey, 1871

Ochodaeini Mulsant \& Rey, 1871

Nikolajev \& Bezděk in Cat. Pal. Col., vol. 3, 2016

Ochodaeus chrysomeloides (Schrank, 1781)

Ochodaeus cychramoides Reitter, 1892 [E]

\begin{tabular}{|l|l|l|l|l|l|l|l|}
\hline $\mathrm{X}$ & & & & & & \\
\hline $\mathrm{B}$ & & & & & & & \\
\hline
\end{tabular}

HYBOSORIDAE Erichson, 1847

Hybosorinae Erichson, 1847

Ballerio \& Bezdẽk in Cat. Pal. Col., vol. 3, 2016

Hybosorus illigeri Reiche, 1853 B $\mid$ B $\mid \mathrm{X}$

Aphodiinae Leach, 1815

Aphodiini Leach, 1815

Aphodiina Leach, 1815

M. \& G. Dellacasa, Král \& Bezděk in Cat. Pal. Col., vol. 3, 2016

\begin{tabular}{|c|c|c|c|c|c|c|c|c|}
\hline Acanthobodilus immundus (Creutzer, 1799) & $\mathrm{H}$ & $\mathrm{X}$ & $\mathrm{X}$ & & & $\mathrm{X}$ & & B \\
\hline Acrossus depressus (Kugelann, 1792) & $\mathrm{X}$ & & & $\mathrm{N}$ & $\mathrm{N}$ & $\mathrm{X}$ & & $\mathrm{B}$ \\
\hline Acrossus laticollis (Baudi di Selve, 1870) & & & & $\mathrm{N}$ & $\mathrm{N}$ & $\mathrm{X}$ & $\mathrm{X}$ & B \\
\hline Acrossus luridus (Fabricius, 1775) & $\mathrm{H}$ & $\mathrm{X}$ & $\mathrm{X}$ & $\mathrm{N}$ & & $\mathrm{N}$ & $\mathrm{X}$ & $\mathrm{B}$ \\
\hline Acrossus rufipes (Linnaeus, 1758) & $\mathrm{X}$ & & & $\mathrm{N}$ & $\mathrm{N}$ & $\mathrm{X}$ & $\mathrm{X}$ & $\mathrm{B}$ \\
\hline Acrossus siculus buturensis (G. Dellacasa, 1983) [E] & & & & & & $\mathrm{X}$ & $\mathrm{X}$ & $\mathrm{B}$ \\
\hline Agoliinus ragusae (Reitter, 1892) [E] & & & & & $\mathrm{B}$ & $\mathrm{X}$ & & \\
\hline Agrilimus ater (DeGeer, 1774) & & & & B & & & & $\mathrm{B}$ \\
\hline Agrilinus constans (Duftschmid, 1805) & $\mathrm{X}$ & & & & & & & $\mathrm{B}$ \\
\hline Agrilinus convexus (Erichson, 1848) & & & & B & B & & B & B \\
\hline Agrilinus ibericus basilicatus (A. Fiori, 1907) & & & & B & B & & & \\
\hline Alocoderus hydrochaeris (Fabricius, 1798) & & & & & & & & $\mathrm{B}$ \\
\hline Amidorus obscurus obscurus (Fabricius, 1792) & & & & B & & $\mathrm{X}$ & $\mathrm{X}$ & \\
\hline Amidorus thermicola (Sturm, 1800) & & & & & B & & & \\
\hline Aphodius coniugatus (Panzer, 1795) & & & & B & $\mathrm{N}$ & $\mathrm{X}$ & $\mathrm{X}$ & B \\
\hline Aphodius fimetarius (Linnaeus, 1758) s.1. & $\mathrm{H}$ & $\mathrm{X}$ & $\mathrm{X}$ & $\mathrm{N}$ & $\mathrm{N}$ & $\mathrm{X}$ & $\mathrm{X}$ & B \\
\hline Aphodius foetidus (Herbst, 1783) & $\mathrm{H}$ & & $\mathrm{X}$ & $\mathrm{N}$ & $\mathrm{N}$ & $\mathrm{X}$ & $\mathrm{X}$ & $\mathrm{B}$ \\
\hline Biralus mahunkaorum Ádám, 1983 & $\mathrm{X}$ & & & & & $\mathrm{N}$ & & B \\
\hline Bodiloides ictericus ghardimaouensis (Balthasar, 1929) & $\mathrm{H}$ & $\mathrm{X}$ & $X$ & & $\mathrm{~N}$ & $\mathrm{X}$ & $\mathrm{X}$ & B \\
\hline Bodilopsis rufa (Moll, 1782) & & $\mathrm{B}$ & $\mathrm{X}$ & $\mathrm{N}$ & & $\mathrm{X}$ & $\mathrm{X}$ & $\mathrm{N}$ \\
\hline Bodilus beduimus (Reitter, 1892) & & $\mathrm{X}$ & & & & $\mathrm{X}$ & $\mathrm{X}$ & \\
\hline Bodilus lugens (Creutzer, 1799) & $\mathrm{H}$ & & & & B & $\mathrm{X}$ & $\mathrm{X}$ & B \\
\hline Calamosternus granarius (Linnaeus, 1767) & $\mathrm{H}$ & $\mathrm{X}$ & & $\mathrm{N}$ & $\mathrm{N}$ & $\mathrm{X}$ & $\mathrm{X}$ & $\mathrm{N}$ \\
\hline Calamostermus mayeri (Pilleri, 1953) & B & $\mathrm{X}$ & & & & & & \\
\hline Chilothorax conspurcatus (Linnaeus, 1758) & & & & & & $\mathrm{X}$ & & \\
\hline Chilothorax distinctus distinctus (O.F. Müller, 1776) & & & & & & & $\mathrm{X}$ & \\
\hline Chilothorax lineolatus (Illiger, 1803) & $\mathrm{H}$ & $\mathrm{X}$ & $\mathrm{N}$ & $\mathrm{N}$ & & $\mathrm{N}$ & & B \\
\hline Chilothorax paykulli (Bedel, 1908) & & & & & & & $\mathrm{X}$ & \\
\hline Colobopteris erraticus (Linnaeus, 1758 ) & $\mathrm{H}$ & $\mathrm{X}$ & $\mathrm{X}$ & $\mathrm{N}$ & $\mathrm{N}$ & $\mathrm{X}$ & $\mathrm{X}$ & B \\
\hline Coprimorphus scrutator (Herbst, 1789) & & & & $\mathrm{N}$ & $\mathrm{N}$ & $\mathrm{X}$ & $\mathrm{X}$ & B \\
\hline Erytus aequalis (A. Schmidt, 1907) & & & & & & & & B \\
\hline
\end{tabular}




\begin{tabular}{|c|c|c|c|c|c|c|c|c|c|}
\hline Species & 施 & $\begin{array}{l}\overrightarrow{0} \\
0 \\
0 \\
0 \\
\frac{0}{0} \\
\frac{.0}{0} \\
2\end{array}$ & 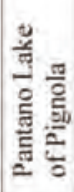 & 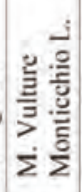 & 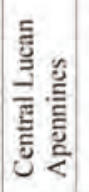 & 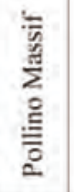 & $\frac{\frac{\vec{g}}{\frac{g}{3}}}{\frac{\vec{g}}{2}}$ & 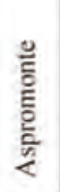 & 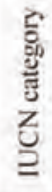 \\
\hline Esymus merdarius (Fabricius, 1775) & $\mathrm{H}$ & & & B & $\mathrm{N}$ & B & $\mathrm{x}$ & B & \\
\hline Esymus pusillus pusillus (Herbst, 1789) & $\mathrm{H}$ & $\mathrm{X}$ & & $\mathrm{N}$ & B & $\mathrm{X}$ & $\mathrm{X}$ & B & \\
\hline Eudolus quadriguttatus (Herbst, 1783) & B & $\mathrm{X}$ & & & & $\mathrm{N}$ & & B & \\
\hline Euhheptaulacus carinatus carinatus (Germar, 1824) & $\mathrm{X}$ & & & & $\mathrm{N}$ & $\mathrm{X}$ & $\mathrm{X}$ & B & \\
\hline Euorodalus paracoenosus (Balthasar \& Hrubant, 1960) & $\mathrm{x}$ & & & & & & $\mathrm{X}$ & B & \\
\hline Euorodalus tersus (Erichson, 1848) & & $\mathrm{X}$ & & & & & & B & \\
\hline Labarrus lividus (A.G. Olivier, 1789) & $\mathrm{X}$ & $\mathrm{X}$ & & & & CK & & B & \\
\hline Limarus zenkeri (Germar, 1813) & $\mathrm{x}$ & & & B & & $\mathrm{x}$ & $\mathrm{x}$ & B & \\
\hline Liothorax niger (Illiger, 1798) & & & & & B & $\mathrm{X}$ & $\mathrm{X}$ & $\mathrm{N}$ & \\
\hline Loraphodius suarius (Faldermann, 1835) & & & & & & $\mathrm{N}$ & & & \\
\hline Mecynodes striatulus (Waltl, 1835) & $\mathrm{N}$ & & & & & & & B & \\
\hline Melinopterns consputus (Creutzer, 1799) & & & & $\mathrm{N}$ & $\mathrm{N}$ & $\mathrm{X}$ & $\mathrm{x}$ & B & \\
\hline Melinopterns prodromus (Brahm, 1790) & $\mathrm{H}$ & & $\mathrm{N}$ & $\mathrm{N}$ & $\mathrm{N}$ & $\mathrm{N}$ & $\mathrm{X}$ & B & \\
\hline Melinopterus reyi (Reitter, 1892) & & & & & B & B & & B & \\
\hline Melinopterus sphacelatus sphacelatus (Panzer, 1798) & & & & & $\mathrm{N}$ & $\mathrm{X}$ & $\mathrm{X}$ & B & \\
\hline Melinopterus stolzi (Reitter, 1906) & & & & B & $\mathrm{N}$ & & & & \\
\hline Neagolins schlumbergeri sammiticus (J. Daniel, 1902) [E] & & & & & & $\mathrm{X}$ & & & \\
\hline Nialus varians (Duftschmid, 1805) & $\mathrm{H}$ & $\mathrm{X}$ & $\mathrm{X}$ & $\mathrm{N}$ & & & & B & \\
\hline Nimbus contaminatus (Herbst, 1783) & & & & $\mathrm{N}$ & & $\mathrm{X}$ & $\mathrm{X}$ & & \\
\hline Nimbus johnsoni (Baraud, 1976) & & & & & $\mathrm{N}$ & & & $\mathrm{N}$ & \\
\hline Nimbus obliteratus (Panzer, 1823) & $\mathrm{H}$ & & & $\mathrm{N}$ & $\mathrm{N}$ & $\mathrm{N}$ & $\mathrm{X}$ & B & \\
\hline Otophorus haemorrhoidalis (Linnaeus, 1758) & $\mathrm{H}$ & $\mathrm{X}$ & & $\mathrm{N}$ & $\mathrm{N}$ & $\mathrm{X}$ & $\mathrm{X}$ & B & \\
\hline Oxyomus sylvestris (Scopoli, 1763) & & $\mathrm{N}$ & & & & & & B & \\
\hline Parammoecius corvinus (Erichson, 1848) & & & & $\mathrm{N}$ & & $\mathrm{X}$ & $\mathrm{x}$ & $\mathrm{N}$ & \\
\hline Phalacronothus biguttatus (Germar, 1824) & & B & & B & & & & B & \\
\hline Phalacronothus quadrimaculatus (Linnaeus, 1760) & $\mathrm{X}$ & & & B & B & & $\mathrm{x}$ & B & \\
\hline Plagiogonus arenarius (A.G. Olivier, 1789) & B & & & & & & & B & \\
\hline Planolinoides borealis (Gyllenhal, 1827) & $\mathrm{H}$ & $\mathrm{X}$ & & B & B & $\mathrm{X}$ & $\mathrm{X}$ & B & \\
\hline Planolinus fasciatus (A.G. Olivier, 1789) & $\mathrm{H}$ & & & B & & $\mathrm{X}$ & $\mathrm{X}$ & & \\
\hline Pseudacrossus sufferhus (A. Schmidt, 1916) & B & & & & B & & $\mathrm{x}$ & B & \\
\hline Sigorus porcus (Fabricius, 1792) & & & & & $\mathrm{N}$ & $\mathrm{X}$ & $\mathrm{x}$ & B & \\
\hline Subrimus sturmi (Harold, 1870) & $\mathrm{H}$ & $\mathrm{X}$ & & $\mathrm{N}$ & $\mathrm{N}$ & $\mathrm{x}$ & $\mathrm{X}$ & $\mathrm{N}$ & \\
\hline Teuchestes fossor (Linnaeus, 1758) & & & & & $\mathrm{N}$ & $\mathrm{X}$ & $\mathrm{x}$ & & \\
\hline Trichonotulus scrofa (Fabricius, 1787) & $\mathrm{H}$ & $\mathrm{X}$ & & & $\mathrm{N}$ & & & B & \\
\hline Volinus sticticus (Panzer, 1798) & $\mathrm{H}$ & & & B & $\mathrm{N}$ & $\mathrm{X}$ & $\mathrm{N}$ & B & \\
\hline
\end{tabular}

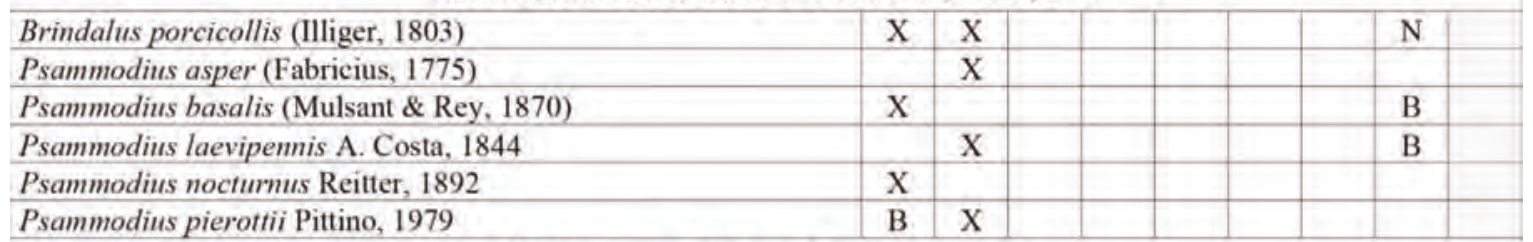

Psammodius pierottii Pittino, 1979

Rhyssemina Pittino \& Mariani, 1986

\begin{tabular}{|l|c|c|c|c|c|c|c|c|c|}
\hline Pleurophortus caesus (Panzer, 1796) & H & X & X & B & N & X & X & B \\
\hline Pleurophorus mediterranicus Pittino \& Mariani, 1986 & & B & & & & & \\
\hline Rhyssemus annaedicatus Pierotti, 1980 & & B & & B & & & N \\
\hline Rhyssemus germamus (Linnaeus, 1767) s.l. & X & X & & & & & N & \\
\hline Rhyssemus parallelus Reitter, 1892 & B & & & & & & & \\
\hline Rhyssemus sulcatus (A.G. Olivier, 1789) & & B & & & & & \\
\hline Trichiorhyssemus dalmatimus Petrovitz, 1967 & & & \\
\hline
\end{tabular}


Species

Scarabaeinae Latreille, 1802

Coprini Leach, 1815

Král \& Bezdẽk in Cat. Pal. Col., vol. 3, 2016

Copris hispanus cavolinii (V. Petagna, 1792)

Copris lunaris (Linnaeus, 1758)

Copris umbilicatus Abeille de Perrin, 1901

\begin{tabular}{|c|c|c|c|c|c|c|c|c|}
$\mathrm{X}$ & $\mathrm{X}$ & $\mathrm{X}$ & & $\mathrm{N}$ & & & $\mathrm{N}$ & \\
\hline $\mathrm{H}$ & $\mathrm{X}$ & & $\mathrm{N}$ & $\mathrm{N}$ & $\mathrm{X}$ & & $\mathrm{N}$ & \\
\hline $\mathrm{B}$ & & & & $\mathrm{N}$ & $\mathrm{X}$ & & & \\
\hline
\end{tabular}

Gymnopleurini Lacordaire, 1856

Bezděk in Cat. Pal. Col., vol. 3, 2016

Gymnopleurus flagellatus flagellatus (Fabricius, 1787)

Gymnopleurus geoffroyi (Fuessly, 1775)

Gymnopleurus mopsus mopsus (Pallas, 1781)

Gymnopleurus sturmii (W.S. Macleay, 1821)

\begin{tabular}{|c|c|c|c|c|c|c|c|c|}
\hline & B & & & & & X & B & \\
\hline X & X & & N & & X & & & \\
\hline X & X & & & N & X & & B & \\
\hline H & X & N & & N & X & & B & \\
\hline
\end{tabular}

Oniticellini H.J. Kolbe, 1905

Oniticellina H.J. Kolbe, 1905

Bezděk in Cat. Pal. Col., vol. 3, 2016

Euoniticellus fulvus (Goeze, 1777)

Euoniticellus intermedius (Reiche, 1850)

Euoniticellus pallipes (Fabricius, 1781)

Onitini Laporte, 1840

Bezděk in Cat, Pal. Col, vol. 3, 2016

Bubas bison (Linnaeus, 1767)

Cheironitis fircifer (P. Rossi, 1792)

Cheironitis irroratus (P. Rossi, 1790)

Onthophagini Burmeister, 1846

\begin{tabular}{|c|c|c|c|c|c|c|c|c|}
\hline H & X & X & & & & & N & \\
\hline B & B & & N & N & X & & B & \\
\hline & X & & & & & B & B & \\
\hline
\end{tabular}

Ziani \& Bezděk in Cat. Pal. Col., vol. 3, 2016

Caccobius schreberi (Linnaeus, 1767)

Euonthophagus amyntas amyntas (A.G. Olivier, 1789)

Onthophagus andalusicus andalusicus Waltl, 1835

Onthophagus coenobita (Herbst, 1783)

Onthophagus fracticornis (Preyssler, 1790)

Onthophagus furcatus (Fabricius, 1781)

Onthophagus grossepunctatus Reitter, 1905

Onthophagus illyricus (Scopoli, 1763)

Onthophagus joannae Goljan, 1953

Onthophagus lemur (Fabricius, 1781)

Onthophagus maki (Illiger, 1803)

Onthophagus opacicollis Reitter, 1892

Onthophagus ovatus (Linnaeus, 1767)

Onthophagus ruficapillus Brullé, 1832

Onthophagus semicornis (Panzer, 1798)

Onthophagus taurus (Schreber, 1759)

Onthophagus vacca (Linnaeus, 1767)

Onthophaguis verticicornis (Laicharting, 1781)

\begin{tabular}{|c|c|c|c|c|c|c|c|}
\hline $\mathrm{H}$ & $\mathrm{X}$ & $\mathrm{X}$ & $\mathrm{N}$ & $\mathrm{N}$ & $\mathrm{X}$ & $\mathrm{X}$ & B \\
\hline $\mathrm{H}$ & & & & $\mathrm{N}$ & $\mathrm{X}$ & & B \\
\hline B & B & & & & & & B \\
\hline B & X & & & $\mathrm{N}$ & $\mathrm{N}$ & & \\
\hline $\mathrm{H}$ & & & $\mathrm{N}$ & $\mathrm{N}$ & X & $\mathrm{X}$ & B \\
\hline B & X & & $\mathrm{N}$ & & X & X & B \\
\hline $\mathrm{H}$ & $X$ & & B & $\mathrm{N}$ & $X$ & $X$ & B \\
\hline & B & & & & X & X & B \\
\hline & $X$ & & B & $\mathrm{N}$ & $X$ & $X$ & \\
\hline $\mathrm{H}$ & & & & $\mathrm{N}$ & X & X & B \\
\hline$X$ & & & & $\mathrm{~N}$ & $\mathrm{X}$ & & B \\
\hline B & X & & $\mathrm{N}$ & $\mathrm{N}$ & $\mathrm{X}$ & $\mathrm{X}$ & CK \\
\hline $\mathrm{H}$ & & & & & & & B \\
\hline$X$ & $\mathrm{X}$ & X & $\mathrm{N}$ & $\mathrm{N}$ & $X$ & $\mathrm{X}$ & $\mathrm{N}$ \\
\hline & & & & B & & & \\
\hline B & $X$ & $\mathrm{X}$ & $\mathrm{N}$ & $\mathrm{N}$ & $\mathrm{X}$ & $X$ & B \\
\hline $\mathrm{H}$ & $X$ & $\mathrm{X}$ & $\mathrm{N}$ & $\mathrm{N}$ & $\mathrm{X}$ & $X$ & B \\
\hline $\mathrm{H}$ & X & & $\mathrm{N}$ & & X & X & \\
\hline
\end{tabular}

Scarabaeini Latreille, 1802

Král \& Bezdēk in Cat. Pal. Col., vol. 3, 2016

Ateuchetus semipunclatus (Fabricius, 1792)

Atenchetus variolosus (Fabricius, 1787)

Scarabaeus sacer Linnaeus, 1758

Scarabaeus typhon (Fischer von Waldheim, 1823)

\begin{tabular}{|c|c|c|c|c|c|c|}
\hline$X$ & $X$ & & & $\mathrm{X}$ & $X$ & B \\
\hline $\mathrm{H}$ & X & $\mathrm{N}$ & $\mathrm{N}$ & $\mathrm{X}$ & $\mathrm{X}$ & B \\
\hline $\mathrm{H}$ & $X$ & & & & & B \\
\hline $\mathrm{H}$ & X & $\mathrm{N}$ & B & $\mathrm{X}$ & $\mathrm{X}$ & B \\
\hline
\end{tabular}

Sisyphini Mulsant, 1842

Bezděk in Cat. Pal. Col., vol. 3, 2016

Sisyphus schaefferi schaefferi (Linnaeus, 1758)

\begin{tabular}{|c|c|c|c|c|c|c|}
\hline $\mathrm{H} \mid \mathrm{X}$ & $\mathrm{N}$ & $\mathrm{N}$ & $\mathrm{X}$ & $\mathrm{X}$ & $\mathrm{B}$ \\
\hline
\end{tabular}


Species

Orphninae Erichson, 1847

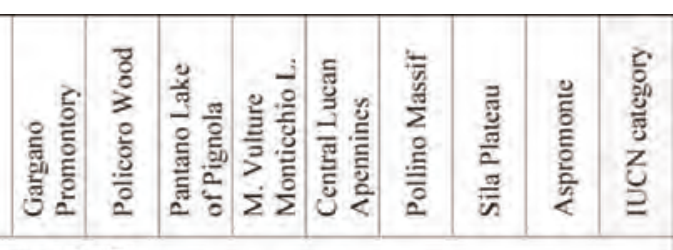

Orphnini Erichson, 1847

Bezdẽk in Cat. Pal. Col., vol. 3, 2016

Chaetonyx robustus italicus Mariani, $1946[\mathrm{E}]$
Melolonthinae Leach, 1819
Hopliini Latreille, 1829

Bezdêk in Cat. Pal. Col., vol. 3, 2016

\begin{tabular}{|l|c|c|c|c|c|c|c|c|}
\hline Hoplia argentea (Poda von Neuhaus, 1761) & $\mathrm{X}$ & & $\mathrm{N}$ & $\mathrm{X}$ & $\mathrm{X}$ & & \\
\hline Hoplia meridiana Uliana, Liberto \& Leo, 2017 [E] & & $\mathrm{X}$ & & & & & \\
\hline Hoplia mimuta (Panzer, 1789) [E] & & $\mathrm{X}$ & & $\mathrm{N}$ & $\mathrm{N}$ & $\mathrm{X}$ & & $\mathrm{B}$ \\
\hline Hoplia paganettii G. Müller, 1907 [E] & & & & & $\mathrm{B}$ & \\
\hline
\end{tabular}

Hoplia paganettii G. Müller, 1907 [E]

Melolonthini Leach, 1819

Bezděk in Cat. Pal. Col., vol. 3, 2016

\begin{tabular}{|l|c|c|c|c|c|c|c|c|c|}
\hline Anoxia matutinalis matutinalis Laporte, 1832 & $\mathrm{X}$ & $\mathrm{X}$ & $\mathrm{X}$ & & $\mathrm{N}$ & $\mathrm{X}$ & $\mathrm{B}$ & \\
\hline Anoxia orientalis (Krynicki, 1832) & & & & & & & $\mathrm{B}$ \\
\hline Anoxia scutellaris scutellaris Mulsant, 1842 & & $\mathrm{B}$ & & & $\mathrm{B}$ & $\mathrm{X}$ & & \\
\hline Melolontha melolontha (Linnaeus, 1758) & $\mathrm{H}$ & & $\mathrm{X}$ & $\mathrm{B}$ & & $\mathrm{N}$ & $\mathrm{X}$ & $\mathrm{B}$ & \\
\hline Melolontha pectoralis Megerle von Mühlfeld, 1812 & $\mathrm{X}$ & $\mathrm{X}$ & & & $\mathrm{B}$ & $\mathrm{X}$ & & $\mathrm{B}$ & \\
\hline Polyphylla fullo fullo (Linnaeus, 1758)
\end{tabular}

Polyphylla fullo fullo (Linnaeus, 1758)

Pachypodini Erichson, 1840

Bezděk in Cat. Pal. Col, vol. 3, 2016

\begin{tabular}{l|r|r|}
\hline Pachypus candidae (V. Petagna, 1787) & $\mathrm{X}$ \\
\hline Rhizotrogini Burmeister, 1855
\end{tabular}

Bezděk in Cat. Pal. Col., vol. 3, 2016

\begin{tabular}{|c|c|c|c|c|c|c|c|c|}
\hline Amadotrogus quercanum (Burmeister, 1855) & & B & & & & $\mathrm{X}$ & & \\
\hline Amphimallon assimile (Herbst, 1790) & $\mathrm{H}$ & $\mathrm{X}$ & & & & $\mathrm{X}$ & $\mathrm{N}$ & \\
\hline Amphimallon fiscum (Scopoli, 1786) & & & & B & $\mathrm{X}$ & $\mathrm{X}$ & & \\
\hline Amphimallon javeti Stierlin, 1864 [E] & & & & & & & B & \\
\hline Amphimallon ochraceum Knoch, 1801 & & & & & $\mathrm{~N}$ & B & & \\
\hline Amphimallon psendomajale Sabatinelli, 1977 [E] & & & & & & $\mathrm{X}$ & & \\
\hline Amphimallon solstitiale solstitiale (Linnaeus, 1758) & & & $\mathrm{N}$ & $\mathrm{N}$ & $\mathrm{X}$ & $\mathrm{X}$ & B & \\
\hline Amphimallon vitalei Luigioni, 1932 [E] & & & & & & & B & \\
\hline Firmimus ciliatus vexillis (Reitter, 1902) [E] & $\mathrm{H}$ & $\mathrm{X}$ & & & & $\mathrm{X}$ & & \\
\hline Haplidia etrusca (Kraatz, 1882) [E] & $\mathrm{X}$ & $\mathrm{X}$ & & & & & & \\
\hline Haplidia transversa transversa (Fabricius, 1801) & $\mathrm{X}$ & & & & & & & \\
\hline Holochelus fraxinicola (Hagenbach, 1825) & $\mathrm{H}$ & & & & & & & \\
\hline Rhizotrogus aestivus (A.G. Olivier, 1789) & & & & $\mathrm{N}$ & $\mathrm{X}$ & $X$ & B & \\
\hline
\end{tabular}

Sericini Kirby, 1837

Ahrens \& Bezděk in Cat. Pal. Col., vol. 3, 2016

Omaloplia ruricola nicolasi (Baraud, 1965)

Triodontella nitidula (P. Rossi, 1790)

Rutelinae W.S. Macleay, 1819

Zorn \& Bezděk in Cat. Pal. Col., vol. 3, 2016

\begin{tabular}{|c|c|c|c|c|c|c|c|}
\hline Anisoplia monticola marginata Kraatz, 1883 [E] & & & & & & $\mathrm{X}$ & $\mathrm{B}$ \\
\hline Anisoplia monticola monticola Erichson, 1847 & & B & $\mathrm{N}$ & $\mathrm{N}$ & $\mathrm{X}$ & & \\
\hline Anisoplia sabatinellii Baraud, 1991 [E] & B & & & & & $\mathrm{B}$ & \\
\hline Anisoplia tempestiva Erichson, 1847 & $\mathrm{H}$ & $\mathrm{X}$ & $\mathrm{N}$ & & $\mathrm{X}$ & $\mathrm{X}$ & \\
\hline Anomala ausonia Erichson, 1847 & $\mathrm{X}$ & $\mathrm{X}$ & & $\mathrm{N}$ & & & B \\
\hline Exomala leonii (Luigioni, 1932) [E] & & & & & B & & \\
\hline Mimela junii calabrica Machatschke, 1957 [E] & & $\mathrm{X}$ & B & $\mathrm{N}$ & $\mathrm{X}$ & $\mathrm{X}$ & $\mathrm{B}$ \\
\hline
\end{tabular}


Species

Dynastinae W.S. Macleay, 1819

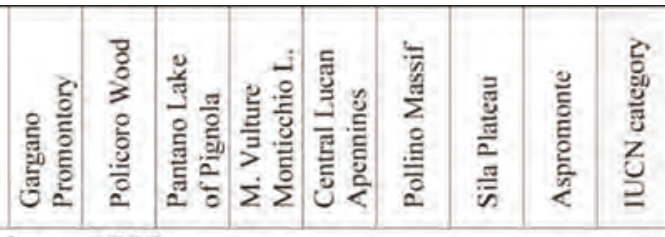

Oryctini Mulsant, 1842

Krell \& Bezděk in Cat. Pal. Col., vol. 3, 2016

\begin{tabular}{|l|l|l|l|l|l|l|l|l|l|}
\hline Oryctes nasicornis corniculatus A. Villa \& G.B. Villa, 1833 & X & X & X & N & N & X & X & N & LC
\end{tabular}

Calicnemis latreillii Laporte, 1832

Pentodon bidens punctatus (Villers, 1789)

Phyllognathus excavatus (Forster, 1771)

\section{Pentodontini Mulsant, 1842}

Cetoniinae Leach, 1815

Cetoniini Leach, 1815

Cetoniina Leach, 1815

Bezděk in Cat. Pal, Col., vol. 3, 2016

Aethiessa floralis squamosa (Gory \& Percheron, 1833) [E]

Cetonia aurata pisana Heer, 1841

Oxythyrea fimesta (Poda von Neuhaus, 1761)

Protaetia affinis affinis (Andersch, 1797)

Protaetia cuprea cuprea (Fabricius, 1775)

Protaetia morio morio (Fabricius, 1781)

Protaetia speciosissima (Scopoli, 1786)

Protaetia squamosa crassicollis Burmeister, 1842 [E]

Tropinota hirta hirta (Poda von Neuhaus, 1761)

Tropinota squalida squalida (Scopoli, 1783)

\begin{tabular}{|c|c|c|c|c|c|c|c|c|}
\hline H & X & X & B & N & X & X & B & LC \\
\hline H & X & X & B & N & X & X & B & \\
\hline X & B & & & & & & & LC \\
\hline H & X & & & N & & X & B & LC \\
\hline H & X & X & N & N & X & & & \\
\hline X & & & & N & & & N & LC \\
\hline B & & & & & & & B & VU \\
\hline H & X & X & N & N & X & X & N & \\
\hline H & X & X & N & N & X & X & B & \\
\hline
\end{tabular}

Osmodermatini Schenkling, 1922

Osmoderma italicum Sparacio, 2001 [E]

Trichiini Fleming, 1821

Trichiina Fleming. 1821

Gnorimus nobilis nobilis (Linnaeus, 1758)

Gnorimus variabilis (Linnaeus, 1758)

Trichitus fascialus (Linnaeus, I758)

Trichius gallicus gallicus Dejean, 1821

Valgus hemipterus hemipterus (Linnaeus, 1758)

Valgini Mulsant, 1842

\section{ELATERIFORMIA Crowson, 1960 \\ SCIRTOIDEA Fleming, 1821}

EUCINETIDAE Lacordaire, 1857

Löbl in Cat. Pal. Col., vol. 3, 2016

Eucinetus haemorrhoidalis (Germar, 1818)

Nycteus hopffgarteni prospector Vit, 1985

\begin{tabular}{|l|l|l|l|l|l|l|}
\hline $\mathrm{X}$ & $\mathrm{X}$ & & & $\mathrm{X}$ & $\mathrm{X}$ & $\mathrm{N}$ \\
\hline
\end{tabular}

CLAMBIDAE Fischer von Waldheim, 1821

Calyptomerinae Crowson, 1955

Löbl in Cat. Pal. Col., vol. 3, 2016

Calyptomerus dubius (Marsham, 1802)

\begin{tabular}{l|l}
$\mathrm{H}$ & $\mathrm{X}$ \\
aldheim, 1821
\end{tabular}

Clambus caucasus Endrödy-Younga, 1960

Clambus dux dux Endrödy-Younga, 1960

Clambus hayekae Endrödy-Younga, 1960

Clambus minutus complicans Wollaston, 1864

Clambus minutus mimutus (Sturm, 1807)

Clambus nigrellus Reitter, 1914

Clambus nigriclavis Stephens, 1835

\begin{tabular}{|c|c|c|c|c|c|c|c|c|}
\hline & X & X & N & & N & & & LC \\
\hline & N & & & & & & & DD \\
\hline & & & & & & & B & LC \\
\hline H & X & X & N & & N & X & & LC \\
\hline & & & & & & & N & DD \\
\hline & & X & & & N & & & DD \\
\hline
\end{tabular}




\begin{tabular}{|c|c|c|c|c|c|c|c|c|c|}
\hline Species & 总 & $\begin{array}{l}3 \\
0 \\
3 \\
3 \\
\frac{0}{0} \\
\frac{0}{0} \\
2\end{array}$ & 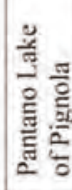 & 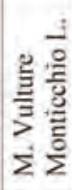 & 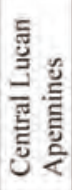 & 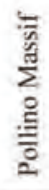 & 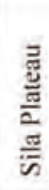 & है & 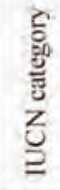 \\
\hline Clambus pallidulus pallidulus Reitter, 1911 & $\mathrm{X}$ & $\mathrm{N}$ & & & $\mathrm{N}$ & $\mathrm{X}$ & & B & LC \\
\hline Clambus pubescens L. Redtenbacher, 1847 & & & $\mathrm{X}$ & & & & & & LC \\
\hline Clambus punctulum (L. Beck, 1817) & $\mathrm{X}$ & $\mathrm{N}$ & & & $\mathrm{N}$ & $\mathrm{N}$ & & & LC \\
\hline Loricaster testaceus pumilus Reitter, 1884 & $\mathrm{X}$ & $\mathrm{N}$ & & $\mathrm{N}$ & $\mathrm{N}$ & $\mathrm{X}$ & $\mathrm{X}$ & $\mathrm{N}$ & LC \\
\hline
\end{tabular}

SCIRTIDAE Fleming, 1821

Scirtinae Fleming, 1821

Klausnitzet in Cat. Pal. Col,, vol. 3, 2016

\begin{tabular}{|c|c|c|c|c|c|c|c|c|c|}
\hline & & & & & & & & & \\
\hline Contacyphon coarctatus (Paykull, 1799) & $\mathrm{H}$ & $\mathrm{X}$ & & $\mathrm{N}$ & $\mathrm{N}$ & $\mathrm{N}$ & $\mathrm{X}$ & $\mathrm{N}$ & \\
\hline Contacyphon laevipennis (Tournier, 1868) & & $\mathrm{X}$ & $\mathrm{N}$ & $\mathrm{N}$ & $\mathrm{N}$ & & & & \\
\hline Contacyphon ochraceus ochraceus (Stephens, 1830) & & $\mathrm{N}$ & & $\mathrm{N}$ & & $\mathrm{N}$ & & $\mathrm{N}$ & \\
\hline Contacyphon padi (Linnaeus, 1758) & $\mathrm{H}$ & $\mathrm{N}$ & $\mathrm{X}$ & & & & & & \\
\hline Contacyphon palmi (Nyholm, 1948) & & & & & $\mathrm{N}$ & $\mathrm{X}$ & & $\mathrm{N}$ & \\
\hline Contacyphon palustris (C.G. Thomson, 1855) & & $\mathrm{X}$ & $\mathrm{X}$ & $\mathrm{N}$ & $\mathrm{N}$ & $\mathrm{X}$ & $\mathrm{X}$ & $\mathrm{N}$ & \\
\hline Contacyphon unguiculatus Nyholm, 1949 [E] & & & $\mathrm{X}$ & & & $\mathrm{X}$ & $\mathrm{X}$ & & \\
\hline Contacyphon variabilis (Thunberg, 1787) & $\mathrm{H}$ & & & & & & & & \\
\hline Elodes angelinii (Klausnitzer, 1987) [E] & & & & & B & $\mathrm{B}$ & $\mathrm{X}$ & & \\
\hline Elodes denticulatus (Klausnitzer, 1973) [E] & & & & $\mathrm{N}$ & $\mathrm{N}$ & $\mathrm{N}$ & $\mathrm{X}$ & $\mathrm{N}$ & \\
\hline Elodes minutus (Linnaeus, 1767) & & $\mathrm{X}$ & & & & & & & \\
\hline Hydrocyphon deflexicollis (P.W.J. Müller, I821) & & & & & & & & $\mathrm{N}$ & \\
\hline Hydrocyphon hydrocyphonoides (Tournier, 1868) & & $\mathrm{N}$ & & & & & & B & \\
\hline Microcara pilosula Reitter, 1911 [E] & & & $\mathrm{X}$ & $\mathrm{N}$ & $\mathrm{N}$ & $\mathrm{X}$ & $\mathrm{X}$ & $\mathrm{N}$ & \\
\hline Odeles marginata (Fabricius, 1798) & & & & & & & $\mathrm{X}$ & & \\
\hline Prionocyphon serricornis (P.W.J. Müller, 1821) & & $\mathrm{N}$ & & & $\mathrm{N}$ & & & & NT \\
\hline Scirtes hemisphaericus (Linnaeus, 1758) & & & & & $\mathrm{N}$ & & & & \\
\hline Scirtes orbicularis (Panzer, 1793) & $\mathrm{H}$ & & & & & & & & \\
\hline
\end{tabular}

Scirtes orbicularis (Panzer, 1793)

DASCILLOIDEA Guérin-Méneville, 1843

DASCILLIDAE Guérin-Méneville, 1843

Dascillinae Guérin-Méneville, 1843

Dascillini Guérin-Méneville, 1843

Hájek in Cat. Pal. Col., vol. 3, 2016

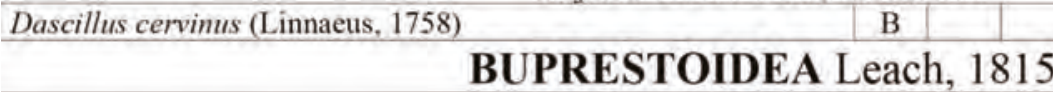 \\ BUPRESTIDAE Leach, 1815 \\ Polycestinae Lacordaire, 1857 \\ Acmaeoderini Kerremans, 1893 \\ Volkovitsh in Cat. Pal. Col., vol 3, 2016}

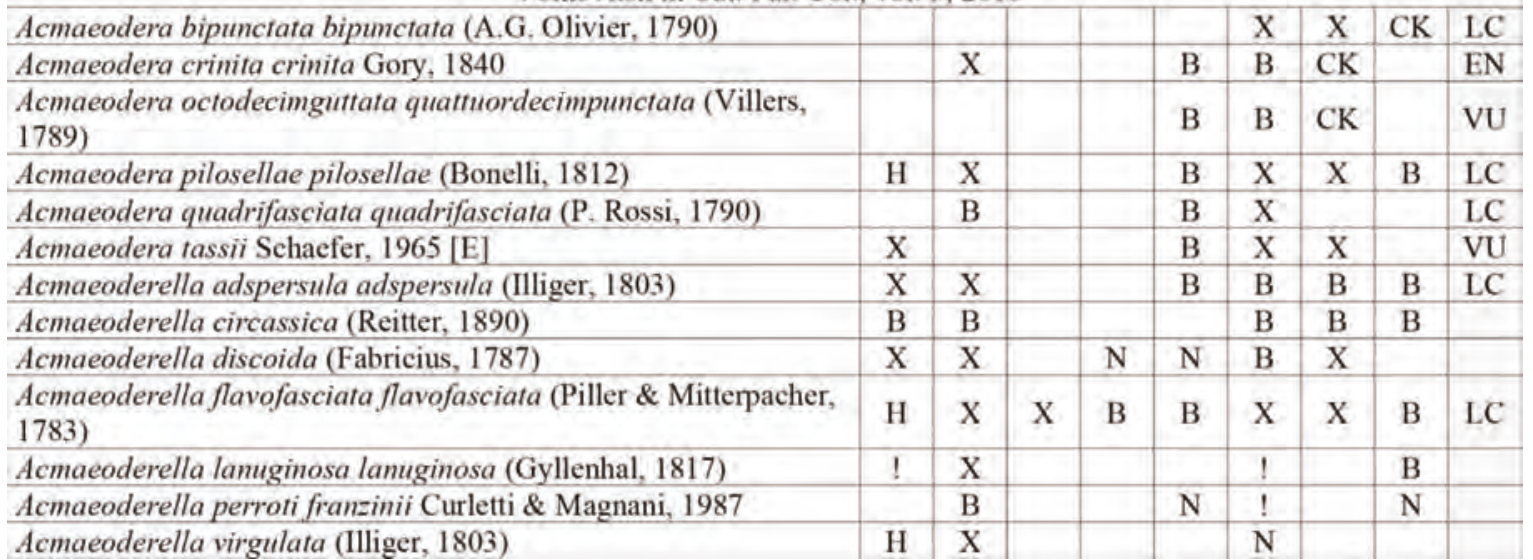


Species

Ptosimini Kerremans, 1903

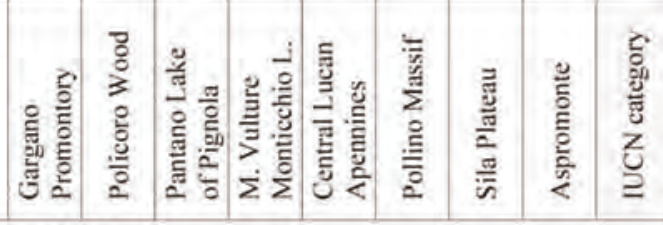

Ptosima undecimmaculata undecimmaculata (Herbst, 1784)

H $\mathrm{X}$

\begin{tabular}{l|l|l|}
\hline B & B & X \\
\hline
\end{tabular}

\begin{tabular}{l|l} 
B & LC
\end{tabular}

Chrysochroinae Laporte, 1835

Chalcophorini Lacordaire, 1857

Kubáñ in Cat. Pal. Col., vol. 3, 2016

Chalcophora intermedia intermedia (Rey, 1890)

Chalcophora mariana (Linnaeus, 1758)

Chalcophorella fabricii (P. Rossi, 1794)

\begin{tabular}{|l|l|l|l|l|l|l|l|l|}
\hline & & & & & & X & & EN \\
\hline & & & & & & X & & LC \\
\hline
\end{tabular}

Dicercini Gistel, 1848

Kubáñ in Cat, Pal. Col., vol. 3, 2016

Capnodis cariosa cariosa (Pallas, 1776

Capnodis miliaris miliaris (Klug. 1829)

Capnodis tenebricosa tenebricosa (A.G. Olivier, 1790)

Capnodis tenebrionis (Linnaeus, 1760)

Cyphosoma euphraticum insulare (Kiesenwetter, 1858)

Dicerca aenea aenea (Linnaeus, 1760)

Dicerca alni (Fischer von Waldheim, 1824)

Dicerca berolinensis (Herbst, 1779)

Perotis lugubris lugubris (Fabricius, 1777)

Perotis lugubris meridionalis Izzillo \& Sparacio, 2011 [E]

Poecilonotini Jakobson, 1913

\begin{tabular}{|c|c|c|c|c|c|c|c|c|}
\hline $\mathrm{H}$ & $\mathrm{X}$ & & & & $\mathrm{B}$ & & $\mathrm{B}$ & $\mathrm{LC}$ \\
\hline & $\mathrm{X}$ & & & & $\mathrm{B}$ & $\mathrm{B}$ & $\mathrm{B}$ & $\mathrm{VU}$ \\
\hline $\mathrm{H}$ & $\mathrm{X}$ & & & $\mathrm{B}$ & $\mathrm{B}$ & & $\mathrm{N}$ & \\
\hline $\mathrm{H}$ & $\mathrm{X}$ & $\mathrm{X}$ & $\mathrm{N}$ & $\mathrm{B}$ & $\mathrm{B}$ & & $\mathrm{N}$ & LC \\
\hline & $\mathrm{B}$ & & & & & & & NT \\
\hline $\mathrm{X}$ & $\mathrm{X}$ & & & $\mathrm{B}$ & $\mathrm{N}$ & $\mathrm{B}$ & $\mathrm{B}$ & $\mathrm{LC}$ \\
\hline $\mathrm{X}$ & $\mathrm{B}$ & & $\mathrm{B}$ & ! & $\mathrm{X}$ & $\mathrm{X}$ & $\mathrm{B}$ & $\mathrm{NT}$ \\
\hline & $\mathrm{B}$ & & & & & & & NT \\
\hline $\mathrm{H}$ & & & & & & & & LC \\
\hline & $\mathrm{B}$ & & & & & & & NT \\
\hline
\end{tabular}

Kubáñ in Cat. Pal. Col., vol. 3, 2016

Lamprodila decipiens decipiens (Gebler, 1847

Lamprodila festiva festiva (Linnaeus, 1767)

Lamprodila mirifica mirifica (Mulsant, 1855)

Lamprodila solieri (Laporte \& Gory, 1837)

Poecilonota variolosa variolosa (Paykull, 1799)

\begin{tabular}{|c|c|c|c|c|c|}
\hline B & B & B & & & LC \\
\hline B & & & & B & LC \\
\hline$X$ & B & & & B & LC \\
\hline $\mathrm{X}$ & & & & & CR \\
\hline $\mathrm{X}$ & & B & $\mathrm{X}$ & & LC \\
\hline
\end{tabular}

Sphenopterini Lacordaire, 1857

Kalashian in Cat. Pal. Col., vol, 3, 2016

Sphenoptera antiqua antiqua (Illiger, 1803)

Sphenoptera barbarica (Gmelin, 1790)

Sphenoptera gemmata gemmata (A.G. Olivier, 1790

Sphenoptera laportei E. Saunders, 1871

Sphenoptera rauca (Fabricius, 1787)

\begin{tabular}{|c|c|c|c|c|c|c|}
\hline $\mathrm{B}$ & & $\mathrm{I}$ & $\mathrm{X}$ & $\mathrm{B}$ & & \\
\hline $\mathrm{B}$ & & & $\mathrm{B}$ & $\mathrm{B}$ & $\mathrm{B}$ & \\
\hline $\mathrm{B}$ & & $\mathrm{B}$ & $\mathrm{B}$ & & & \\
\hline & & & $\mathrm{X}$ & $\mathrm{X}$ & $\mathrm{B}$ & \\
\hline $\mathrm{B}$ & & & $\mathrm{B}$ & & $\mathrm{B}$ & \\
\hline
\end{tabular}

Buprestinae Leach, 1815

Anthaxiini Gory \& Laporte, 1839

Kubáń in Cat. Pal. Col., vol. 3, 2016

\begin{tabular}{|c|c|c|c|c|c|c|c|c|c|}
\hline Anthaxia anatolica anatolica Chevrolat, 1838 & $\mathrm{X}$ & $\mathrm{X}$ & & & & $\mathrm{N}$ & & & \\
\hline Anthaxia cichorii (A.G. Olivier, 1790) & & $\mathrm{B}$ & $\mathrm{X}$ & B & B & $\mathrm{X}$ & $\mathrm{X}$ & B & LC \\
\hline Anthaxia confusa confusa Gory, 1841 & B & $\mathrm{B}$ & & & B & & $\mathrm{X}$ & $\mathrm{N}$ & LC \\
\hline Anthaxia croesus (Villers, 1789) & $\mathrm{H}$ & B & & & B & $\mathrm{X}$ & & B & LC \\
\hline Anthaxia cyanescens Gory, 1841 & B & & & & B & $\mathrm{X}$ & & & LC \\
\hline Anthaxia dimidiata (Thunberg, 1789) & $\mathrm{H}$ & $\mathrm{X}$ & & & & $\mathrm{N}$ & & B & LC \\
\hline Anthaxia fulgurans (Schrank, 1789) & & B & & $\mathrm{B}$ & B & $\mathrm{X}$ & $\mathrm{X}$ & $\mathrm{N}$ & LC \\
\hline Anthaxia godeti Gory \& Laporte, 1839 & & & & $\mathrm{~N}$ & $\mathrm{~N}$ & $\mathrm{X}$ & $\mathrm{X}$ & B & LC \\
\hline Anthaxia hackeri J. Frivaldszky, 1884 & & $\mathrm{~B}$ & & & B & & & & CR \\
\hline Anthaxia helvetica apennina Obenberger, 1938 [E] & & & & & B & $\mathrm{X}$ & $\mathrm{X}$ & B & LC \\
\hline Anthaxia hungarica hungarica (Scopoli, 1772) & $\mathrm{H}$ & $\mathrm{X}$ & $\mathrm{X}$ & $\mathrm{N}$ & B & $\mathrm{X}$ & $\mathrm{X}$ & B & LC \\
\hline Anthaxia hypomelaena (Illiger, 1803) & $\mathrm{X}$ & $\mathrm{B}$ & $\mathrm{N}$ & $\mathrm{B}$ & B & $\mathrm{X}$ & & $\mathrm{N}$ & \\
\hline Anthaxia istriana Rosenhauer, 1847 & & & & & & B & $\mathrm{X}$ & & LC \\
\hline Anthaxia kochi Obenberger, 1938 [E] & & & & & B & $\mathrm{X}$ & $\mathrm{X}$ & B & VU \\
\hline Anthaxia liae Gobbi, 1983 [E] & & & & & $!$ & $\mathrm{B}$ & $\mathrm{X}$ & B & $\mathrm{LC}$ \\
\hline
\end{tabular}


Species

Anthaxia lucens Küster, 1852

Anthaxia manca (Linnaeus, 1767)

Anthaxia mendizabali Cobos, 1965

Anthaxia millefolii polychloros Abeille de Perrin, 1894

Anthaxia nitidula (Linnaeus, 1758)

Anthaxia oberthuri Schaefer, 1938

Anthaxia praeclara praeclara Mannerheim, 1837

Anthaxia salicis salicis (Fabricius, 1777)

Anthaxia scylla Levey, 1985

Anthaxia semicuprea Küster, 1851

Anthaxia senicula senicula (Schrank, 1789)

Anthaxia sepulchralis sepulchralis (Fabricius, 1801)

Anthaxia suzannae Théry, 1942

Anthaxia thalassophila thalassophila Abeille de Perrin, 1900

Anthaxia umbellatarum umbellatarum (Fabricius, 1787)

Buprestini Leach, 1815

Buprestis cupressi Germar, 1836

Buprestis haemorrhoidalis haemorrhoidalis Herbst, 1780

Buprestis humeralis Klug, 1829

Buprestis novemmaculata novemmaculata Linnaeus, 1767

Buprestis octoguttata octoguttata Linnaeus, 1758

Buprestis splendens splendens Fabricius, 1775

Eurythyrea austriaca (Linnaeus, 1767)

Eurythyrea micans (Fabricius, 1792)

Eurythyrea quercus (Herbst, 1780)

Chrysobothrini Gory \& Laporte, 1938

\begin{tabular}{|c|c|c|c|c|c|c|}
\hline B & X & & & & & LC \\
\hline & B & & $\mathrm{X}$ & $\mathrm{X}$ & & LC \\
\hline & B & & & & & EN \\
\hline$X$ & & & $\mathrm{X}$ & $\mathrm{X}$ & B & LC \\
\hline & & B & $\mathrm{X}$ & & & LC \\
\hline & & & $\mathrm{X}$ & & & EN \\
\hline & & $\mathrm{N}$ & $\mathrm{X}$ & $\mathrm{X}$ & B & VU \\
\hline & $X$ & & & B & B & LC \\
\hline & & & & & B & CR \\
\hline
\end{tabular}

Chrysobothris affinis affinis (Fabricius, 1794)

Chrysobothris chrysostigma chrysostigma (Linnaeus, 1758)

Chrysobothris solieri Gory \& Laporte, 1837

Melanophilini Bedel, 1921

Melanophila acuminata (DeGeer, 1774)

Melanophila cuspidata (Klug, 1829)

Phaenops cyanea (Fabricius, 1775)

Phaenops knoteki ochsi Schaefer, 1947

Trachypteris picta decostigma (Fabricius, 1787)

\begin{tabular}{|c|c|c|c|c|c|c|c|c|}
\hline $\mathrm{X}$ & $\mathrm{X}$ & & $\mathrm{B}$ & $\mathrm{B}$ & $\mathrm{X}$ & $\mathrm{X}$ & $\mathrm{B}$ & LC \\
\hline & & & & & $\mathrm{B}$ & & & LC \\
\hline $\mathrm{B}$ & $\mathrm{B}$ & & & & $\mathrm{B}$ & $\mathrm{X}$ & & LC \\
\hline
\end{tabular}

\begin{tabular}{|l|l|l|l|l|l|l|l|l|l|}
\hline & B & X & & & l & & B & & LC \\
\hline & & & & & & & X & & NT \\
\hline & & & & & & X & B & B & VU \\
\hline us, 1787) & & $X$ & & & & & & CK & LC \\
\hline
\end{tabular}

\section{Agrilinae Laporte, 1835 \\ Agrilini Laporte, 1835 \\ Agrilina Laporte, 1835}

Jendek in Cat. Pal. Col., vol. 3, 2016

\begin{tabular}{|c|c|c|c|c|c|c|c|c|c|}
\hline Agrilus albogularis artemisiae C.N.F. Brisout de Barneville, 1863 & & B & & & & $\mathrm{B}$ & $\mathrm{B}$ & & \\
\hline Agrilus albomarginatus A. Fiori, 1906 & B & & & & & $!$ & & B & LC \\
\hline Agrilus angustulus angustulus (Illiger, 1803) [E] & $\mathrm{X}$ & $\mathrm{X}$ & $\mathrm{X}$ & B & B & $\mathrm{X}$ & $\mathrm{X}$ & $\mathrm{N}$ & LC \\
\hline Agrilus antiquus croaticus Abeille de Perrin, 1897 & & & & & & B & $\mathrm{X}$ & & LC \\
\hline Agrilus ater (Linnaeus, 1767) & & $\mathrm{X}$ & & & & $\mathrm{B}$ & $\mathrm{B}$ & & LC \\
\hline Agrilus auricollis Kiesenwetter, 1857 & & B & & & & & & & LC \\
\hline Agrilus biguttatus (Fabricius, 1777) & CK & $\mathrm{N}$ & & & & $\mathrm{B}$ & $\mathrm{X}$ & & LC \\
\hline Agrilus convexicollis L. Redtenbacher, 1847 & $\mathrm{H}$ & $\mathrm{X}$ & $\mathrm{X}$ & $\mathrm{B}$ & $!$ & $\mathrm{X}$ & & & LC \\
\hline Agrilus cuprescens (Ménétriés, 1832) & $\mathrm{X}$ & $\mathrm{B}$ & $\mathrm{X}$ & $\mathrm{N}$ & B & $\mathrm{X}$ & $\mathrm{X}$ & $\mathrm{B}$ & \\
\hline Agrilus cyanescens italicus Obenberger, 1920 [E] & & B & & & B & & & & LC \\
\hline Agrilus derasofasciatus Lacordaire, 1835 & B & $\mathrm{B}$ & & & $\mathrm{B}$ & & & & LC \\
\hline Agrilus graecus Obenberger, 1916 & & & & & $\mathrm{~B}$ & $\mathrm{~B}$ & & & DD \\
\hline Agrilus graminis Kiesenwetter, 1857 & B & $\mathrm{B}$ & & B & $\mathrm{B}$ & $\mathrm{X}$ & $\mathrm{X}$ & B & LC \\
\hline
\end{tabular}


Species

Agrilus hastulifer (Ratzeburg, 1837)

Agrilus hyperici (Creutzer, 1799)

Agrilus integerrimus (Ratzeburg, 1837)

Agrilus laticornis (Illiger, 1803)

Agrilus lineola lineola Kiesenwetter, 1857

Agrilus marozzinii Gobbi, 1974

Agrilus obscuricollis Kiesenwetter, 1857

Agrilus olivicolor Kiesenwetter, 1857

Agrilus paganettii Obenberger, 1913 [E]

Agrilus pratensis (Ratzeburg, 1837)

Agrilus relegatus alexeevi Bellamy, 1998

Agrilus roscidus Kiesenwetter, 1857

Agrilus sinuatus sinuatus (A.G. Olivier, 1790)

Agrilus solieri solieri Gory \& Laporte, 1837

Agrilus suvorovi populneus Schaefer, 1946

Agrilus viridicaerulans rubi Schaefer, 1937

Agrilus viridis (Linnaeus, 1758)

Aphanisticini Jacquelin du Val, 1859

Aphanisticina Jacquelin du Val, 1859

Kalashian \& Kubán in Cat. Pal. Col., vol. 3, 2016

Aphanisticus angustatus P.H. Lucas, 1846 Aphanisticus elongatus elongatus A. Villa \& G.B. Villa, 1835

Aphanisticus emarginatus (A.G. Olivier, 1790)

Aphanisticus pygmaeus P.H. Lucas, 1849

Cylindromorphus dalmatinus Csiki, 1915

Cylindromorphus gallicus angelinii Curletti, 1990 [E]

\begin{tabular}{|c|c|c|c|c|c|c|c|c|}
\hline X & X & X & N & & N & B & B & \\
& & X & N & B & X & & & \\
\hline & X & N & B & B & & & & \\
\hline & X & & & B & & & & \\
\hline B & B & & & B & & & & \\
\hline & B & & & N & B & & & \\
\hline
\end{tabular}

Coraebini Bedel, 1921

Amorphosomatina Majer, 2000

Kubáñ in Cat. Pal. Col., vol. 3, 2016

Coraebus elatus (Fabricius, 1787)

Coraebus fasciatus (Villers, 1789)

Coraebus rubi (Linnaeus, 1767)

Meliboens amethystinus amethystinus (A.G. Olivier, 1790)

Meliboeus episcopalis (Mannerheim, 1837)

Meliboens fulgidicollis (P.H. Lucas, 1846)

Meliboeus graminis graminis (Panzer, 1789)

Meliboenus parvulus (Küster, 1852)

Tracheini Laporte, 1835

\begin{tabular}{|c|c|c|c|c|c|c|c|c|}
\hline H & X & X & B & B & X & X & N & \\
\hline X & B & & & B & & & B & LC \\
\hline H & X & X & B & B & X & X & B & \\
\hline & B & & & B & N & X & B & \\
\hline X & X & & B & & B & & B & \\
\hline X & & & & B & B & X & B & LC \\
& X & & & B & B & X & B & \\
\hline & B & X & N & B & X & X & B & \\
\hline
\end{tabular}

Pachyschelina Böving \& Craighead, 1931

Kubáñ in Cat. Pal. Col., vol. 3, 2016

Habroloma nanum (Paykull, 1799)

Habroloma triangulare (Lacordaire, I835)

Trachys menthae Bedel, 1921

Trachys minutus minutus (Linnaeus, 1758)

Trachys quercicola Marseul, 1871

Trachys troglodytes Gyllenhal, 1817

Trachys troglodytiformis Obenberger, 1918

\begin{tabular}{|c|c|c|c|c|c|c|c|c|}
\hline & B & & & & & & & \\
\hline & B & & & & & & & \\
\hline X & & & B & & X & & B & \\
\hline H & X & X & N & B & X & X & B & \\
\hline & & X & & & B & & & \\
\hline H & X & X & B & B & ! & B & B & \\
\hline B & X & & B & B & B & B & B & \\
\hline
\end{tabular}

BYRRHOIDEA Latreille, 1804

BYRRHIDAE Latreille, 1804

Byrrhinae Latreille, 1804

Simplocariini Mulsant \& Rey, 1869

Jäger \& Pütz in Cat. Pal. Col., vol. 3, 2016 
Species

Simplocaria semistriata (Fabricius, 1794)

Byrrhus arietimus arietinus Steffahny, 1842

Byrrhus numidicus Normand, 1935

Byrrhus pustulatus pustulatus (Forster, 1771)

Byrrhus pilula (Linnaeus, 1758)

Curimus lariensis (A. Villa \& G.B. Villa, 1833)

Cytilus sericeus (Forster, 1771)

Syncalyptinae Mulsant \& Rey, 1869

Byrrhini Latreille, 1804

\begin{tabular}{|c|c|c|c|c|c|c|c|}
\hline 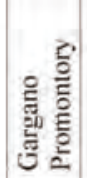 & 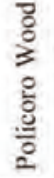 & 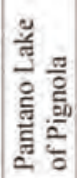 & 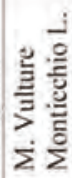 & 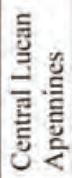 & 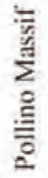 & 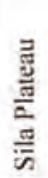 & $\begin{array}{l}\frac{\mathscr{U}}{0} \\
\text { है } \\
\text { के } \\
\text { के }\end{array}$ \\
\hline & & & & $\mathrm{N}$ & & & \\
\hline
\end{tabular}

\begin{tabular}{|l|l|l|l|l|l|l|l|l|l|}
\hline & $\mathrm{X}$ & & & & & & & & \\
\hline & & & & & & B & & B & \\
\hline & & & & & & X & X & & \\
\hline & & & & & & & X & & \\
\hline
\end{tabular}

Syncalyptini Mulsant \& Rey, 1869

Curimopsis maritima maritima (Marsham, 1802)

Curimopsis paleata (Erichson, 1846)

\section{ELMIDAE Curtis, 1830 \\ Elminae Curtis, 1830 \\ EImini Curtis, 1830 \\ EImina Curtis, 1830}

Jäch \& Kodada in Cat. Pal. Col, vol. 3, 2016

Elmis aenea (P.W.J. Müller, 1806)

Elmis mangetii mangetii Latreille, 1802

Elmis rioloides (Kuwert, 1890)

Esolus angustatus (P.W.J. Müller, 1821)

Esolus berthelemyi Olmi, 1975

Esolus parallelepipedus (P.W.J. Müller, 1806)

Limnius intermedius Fairmaire, 1881

Limnius opacus opacus P.W.J. Müller, 1806

Limnius perrisi perrisi (Dufour, 1843)

Oulimnius tuberculatus (P.W.J. Müller, 1806)

Riolus cupreus (P.W.J. Müller, 1806)

Riolus subviolaceus (P.W.J. Müller, 1817)

DRYOPIDAE Billberg, 1820

Kodada \& Jäch in Cat. Pal. Col., vol. 3, 2016

Dryops algiricus (P.H. Lucas, 1846 )

Dryops doderoi Bollow, 1936

Dryops ernesti Gozis, 1886

Dryops luridus (Erichson, 1847)

Dryops lutulentus (Erichson, 1847)

Dryops rufipes (Krynicki, 1832)

Dryops similaris Bollow, 1936

Dryops striatellus (Fairmaire \& C.N.F. Brisout de Barneville,

1859)

Dryops subincamus (Kuwert, 1890)

Dryops sulcipennis (A. Costa, 1883)

Dryops viennensis (Laporte, 1840)

Pomatimus substriatus (P.W.J. Müller, 1806)

\begin{tabular}{|c|c|c|c|c|c|c|c|c|}
\hline $\mathrm{X}$ & $\mathrm{X}$ & $\mathrm{X}$ & $\mathrm{N}$ & $\mathrm{B}$ & & & $\mathrm{CK}$ & \\
\hline $\mathrm{X}$ & $\mathrm{X}$ & & & & & & & \\
\hline & $\mathrm{X}$ & & $\mathrm{N}$ & & $\mathrm{X}$ & $\mathrm{X}$ & & \\
\hline & & $\mathrm{N}$ & & & & $\mathrm{B}$ & $\mathrm{CK}$ & \\
\hline & & I & & & & & \\
\hline $\mathrm{X}$ & & & & & & & \\
\hline $\mathrm{B}$ & $\mathrm{X}$ & & $\mathrm{CK}$ & I & & $\mathrm{B}$ & \\
\hline CK & & & & $\mathrm{X}$ & $\mathrm{X}$ & & \\
\hline & & & & & & & \\
\hline
\end{tabular}

\section{LIMNICHIDAE Erichson, 1846}

Limnichinae Erichson, 1846

Hernando \& Ribera in Cat. Pal. Col., vol. 3, 2016

Bothriophorus atomus Mulsant \& Rey, I852

Limnichus incanuis Kiesenwetter, 1851

Limnichus sericeus (Duftschmid, 1825)

Pelochares versicolor (Waltl, 1838)

\begin{tabular}{|c|c|c|c|c|c|c|c|c|}
\hline & B & & & & B & & B & \\
\hline & B & X & & CK & B & & CK & \\
\hline CK & N & N & & B & & & \\
\hline CK & CK & X & & CK & N & & CK & \\
\hline
\end{tabular}


Species

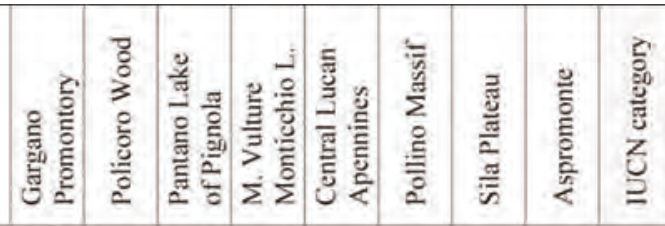

HETEROCERIDAE W.S. Macleay, 1825

Heterocerinae W.S. Macleay, 1825

Augylini Pacheco, 1964

Mascagni in Cat. Pal. Col., vol. 3, 2016

Augvles flavidus (P. Rossi, 1794)

Augyles maritimus (Guérin-Méneville, 1844)

Angyles marmota (Kiesenwetter, 1850)

\section{$\mathbf{H}$}

Heterocerus aragonicus Kiesenwetter, 1850

Heterocernis fenestratus (Thunberg, 1784)

Heterocerus flexuosus Stephens, 1828

Heterocerus holosericeus Rosenhauer, 1856

\section{ELATEROIDEA Leach, 1815}

Kundrata et al., 2014

\begin{tabular}{|c|c|c|c|c|c|c|c|c|}
\hline $\mathrm{X}$ & $\mathrm{X}$ & & $\mathrm{N}$ & $\mathrm{CK}$ & $\mathrm{X}$ & & & \\
\hline $\mathrm{CK}$ & $\mathrm{CK}$ & $\mathrm{X}$ & $\mathrm{N}$ & $\mathrm{CK}$ & $\mathrm{N}$ & $\mathrm{X}$ & $\mathrm{N}$ & \\
\hline & $\mathrm{X}$ & & & & $\mathrm{CK}$ & & $\mathrm{CK}$ & \\
\hline
\end{tabular}

\begin{tabular}{|c|c|c|c|c|c|c|c|c|}
\hline $\mathrm{X}$ & $\mathrm{X}$ & $\mathrm{X}$ & $\mathrm{CK}$ & $\mathrm{N}$ & $\mathrm{X}$ & $\mathrm{X}$ & $\mathrm{B}$ & \\
\hline $\mathrm{X}$ & $\mathrm{X}$ & & & & & & & \\
\hline $\mathrm{X}$ & $\mathrm{X}$ & & & $\mathrm{CK}$ & $\mathrm{CK}$ & & & \\
\hline
\end{tabular}

\begin{tabular}{|c|c|c|c|c|c|c|c|c|}
\hline \multicolumn{9}{|c|}{$\begin{array}{l}\text { ELATEROIDEA Leach, } 1815 \\
\text { Kundrata et al., } 2014\end{array}$} \\
\hline \multicolumn{9}{|c|}{$\begin{array}{l}\text { ARTEMATOPODIDAE Lacordaire, } 1857 \\
\text { Artematopodinae Lacordaire, } 1857 \\
\text { Bocák in Cat. Pal. Col., vol. 4, } 2007\end{array}$} \\
\hline Eurypogon cribratus (Hampe, 1866) [E] & & & & $\mathrm{N}$ & & 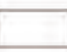 & 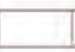 & \\
\hline \multicolumn{9}{|c|}{$\begin{array}{c}\text { EUCNEMIDAE Eschscholtz, } 1829 \\
\text { Melasinae Fleming, 1821 } \\
\text { Dirhagini Reitter, 1911 } \\
\text { Muona in Cat. Pal. Col., vol. 4, } 2007\end{array}$} \\
\hline Farsus dubius (Piller \& Mitterpacher, 1783) & $\mathrm{X}$ & $\mathrm{X}$ & & & $\mathrm{X}$ & $\mathrm{X}$ & $\mathrm{N}$ & LC \\
\hline Microrhagus emyi (Rouget, 1856) & & & & & & $\mathrm{N}$ & $\mathrm{N}$ & VU \\
\hline Microrhagus himmleri (Reitter, 1911) & & $\mathrm{X}$ & & & B & & B & $\mathrm{CR}$ \\
\hline Microrhagus pygmaeus (Fabricius, 1792) & & & & & & $\mathrm{N}$ & & NT \\
\hline \multicolumn{9}{|c|}{ Epiphanini Muona, 1993} \\
\hline Epiphanis cormutus Eschscholtz, 1829 & & & & & & $\mathrm{~N}$ & & Vu \\
\hline \multicolumn{9}{|c|}{ Melasini Fleming, 1821} \\
\hline Isorhipis melasoides (Laporte, 1835) & & & & B & $\mathrm{X}$ & $\mathrm{X}$ & $\mathrm{N}$ & LC \\
\hline Melasis buprestoides (Linnaeus, 1761) & $\mathrm{X}$ & & $\mathrm{N}$ & & $\mathrm{X}$ & & B & LC \\
\hline \multicolumn{9}{|c|}{ Xylobiini Reitter, 1911} \\
\hline Xylophilus corticalis (Paykull, 1800) & & & - & 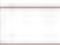 & 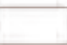 & 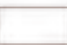 & 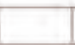 & NT \\
\hline
\end{tabular}

\begin{tabular}{|c|c|c|c|c|c|c|c|c|c|}
\hline Aulonothroscus brevicollis (Bonvouloir, 1859) & & & $\mathrm{N}$ & & & & & & DD \\
\hline Trixagus algiricus (Bonvouloir, 1862) & & & $\mathrm{X}$ & $\mathrm{N}$ & & & $\mathrm{N}$ & $\mathrm{N}$ & DD \\
\hline Trixagus angelinii Leseigneur, 2005 & & & & & $\mathrm{~N}$ & & & $\mathrm{~N}$ & LC \\
\hline Trixagus atticus Reitter, 1921 & & B & $\mathrm{N}$ & & & & & & DD \\
\hline Trixagus carinifrons (Bonvouloir, 1859) & & & & & $\mathrm{N}$ & & & $\mathrm{N}$ & DD \\
\hline Trixagus dermestoides (Linnaeus, 1767) & & & & & $\mathrm{N}$ & $\mathrm{N}$ & & & LC \\
\hline Trixagus duvalii (Bonvouloir, 1859) & & $\mathrm{X}$ & $\mathrm{X}$ & & & & & & DD \\
\hline Trixagus elateroides elateroides (Heer, 1841) & & $\mathrm{N}$ & $\mathrm{X}$ & & $\mathrm{N}$ & $\mathrm{N}$ & $\mathrm{N}$ & $\mathrm{N}$ & LC \\
\hline Trixagus exul (Bonvouloir, 1859) & & & & & $\mathrm{N}$ & & & & \\
\hline Trixagus gracilis Wollaston, 1854 & B & B & B & & $\mathrm{N}$ & $\mathrm{N}$ & $\mathrm{N}$ & $\mathrm{N}$ & LC \\
\hline Trixagus meybohmi Leseigneur, 2005 & & & & & & & & B & NT \\
\hline Trixagus obtusus (Curtis, 1827) & & & $\mathrm{X}$ & & $\mathrm{N}$ & $\mathrm{N}$ & & & LC \\
\hline
\end{tabular}




\section{Species}

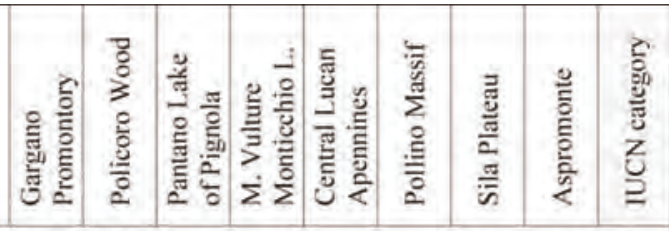

\section{LYCIDAE Laporte, 1838}

Lycinae Laporte, 1838

Erotini LeConte, 1881

Bocáková \& Bocák in Cat. Pal. Col., vol. 4, 2007

Dictyoptera aurora (Herbst, 1784)

Platycis minutus (Fabricius, 1787)

Pyropterus nigroruber (DeGeer, 1774)

\begin{tabular}{|c|c|c|c|c|c|c|}
\hline & $X$ & & & $\mathrm{X}$ & B & $\mathrm{LC}$ \\
\hline & & & $X$ & B & B & LC \\
\hline $\mathrm{X}$ & $\mathrm{X}$ & $\mathrm{N}$ & $\mathrm{X}$ & $X$ & $\mathrm{~N}$ & $\mathrm{LC}$ \\
\hline
\end{tabular}

Calochrominae Lacordaire, 1857

Lygistopterus anorachilus Ragusa, 1883 [E]

Lygistopterus sanguineus (Linnaeus, 1758)

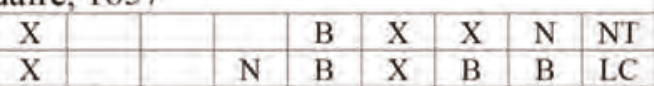

LAMPYRIDAE Latreille, 1817

Lampyrinae Latreille, 1817

Lampyrini Latreille, 1817

Geisthardt \& Satô in Cat. Pal. Col., vol. 4, 2007

Lampyris brutia A. Costa, 1882 [E]

Lampyris noctiluca (Linnaeus, 1767)

Photinini E. Olivier, 1907

Lamprohiza splendidula (Linnaeus, 1767)

\begin{tabular}{|l|l|l|l|l|l|l|l|l|}
\hline & & & $\mathrm{N}$ & & $\mathrm{B}$ & & \\
$\mathrm{X}$ & $\mathrm{X}$ & $\mathrm{X}$ & & & $\mathrm{X}$ & $\mathrm{X}$ & & \\
\hline
\end{tabular}

Luciolinae Lacordaire, 1857

Luciolini Lacordaire, 1857

Luciola italica (Linnaeus, 1767)

Luciola lusitanica (Charpentier, 1825)

CANTHARIDAE Imhoff, 1856

\begin{tabular}{|l|l|l|l|l|l|l|l|l|}
\hline & & $\mathrm{X}$ & & $\mathrm{N}$ & $\mathrm{X}$ & & $\mathrm{N}$ & \\
\hline B & B & & & & & & & \\
\hline
\end{tabular}

Cantharinae Imhoff, 1856

Podabrini LeConte, 1881

Kazantsev \& Brancucci in Cat. Pal. Col., vol, 4, 2007

Malthacus procerulus (Kiesenwetter, 1860)

Ancistronycha abdominalis (Fabricius, 1798)

Ancistronycha lucens Moscardini, 1967

Ancistronycha violacea (Paykull, 1798)

Cantharis corvina Moscardini, 1962 [E]

Cantharis decipiens Baudi di Selve, 1872

Cantharis falzonii A. Fiori, 1914 [E]

Cantharis flavilabris Fallén, 1807

Cantharis fusca Linnaeus, 1758

Cantharis ictaria A. Fiori, I914 [E]

Cantharis italica A. Fiori, 1914 [E]

Cantharis lateralis Linnaeus, 1758

Cantharis liburnica Depoli, 1912

Cantharis livida Linnaeus, 1758

Cantharis merula Moseardini, 1962 [E]

Cantharis monacha Moscardini, 1962 [E]

Cantharis nigricans (O.F. Müller, 1776)

Cantharis obscura Linnaeus, 1758

Cantharis paganettii (Flach, 1907) [E]

Cantharis pallida Goeze, 1777

Cantharis pellucida Fabricius, 1792

Cantharis peninsularis A. Fiori, 1914 [E]

Cantharis rufa Linnaeus, 1758

Cantharis rustica Fallén, 1807

Cantharini Imhoff, 1856 
Species

Cantharis torretassoi Wittmer, 1935 [E]

Cratosilis denticollis (Schummel, 1844)

Cratosilis laeta (Fabricius, 1792)

Cratosilis sicula (Marseul, 1864) [E]

Metacantharis clypeata Illiger, 1798

Metacantharis picciolii (Ragusa, 1870) [E]

Rhagonycha femoralis (Brullé, 1832)

Rhagonycha fulva (Scopoli, 1763)

Rhagonycha fuscitibia Rey, 1891

Rhagonycha lignosa (O.F. Müller, 1764)

Rhagonycha lutea (O.F. Müller, 1764)

Rhagonycha nigriceps (Waltl, 1838)

Rhagonycha nigrosuta A. Fiori, 1899 [E]

Silinae Mulsant, 1862

\begin{tabular}{|c|c|c|c|c|c|c|c|c|}
\hline 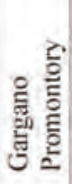 & $\begin{array}{l}\text { 马े } \\
\vdots \\
0 \\
0 \\
\frac{0}{0} \\
\frac{.0}{0} \\
\Omega\end{array}$ & 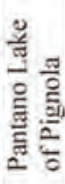 & 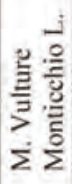 & 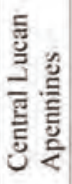 & $\begin{array}{l}\bar{y} \\
\frac{a}{0} \\
0 \\
0 \\
0 \\
0\end{array}$ & 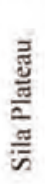 & 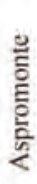 & 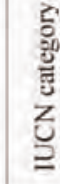 \\
\hline & & & & & X & & & \\
\hline X & $X$ & & $\mathrm{~N}$ & $\mathrm{~N}$ & X & $X$ & B & \\
\hline & & & $\mathrm{N}$ & & X & & B & \\
\hline $\mathrm{H}$ & X & X & $\mathrm{N}$ & $\mathrm{N}$ & X & X & B & \\
\hline $\mathrm{H}$ & $\mathrm{N}$ & X & $\mathrm{N}$ & $\mathrm{N}$ & X & $\mathrm{X}$ & $\mathrm{N}$ & \\
\hline & & & B & $\mathrm{N}$ & & & & \\
\hline $\mathrm{H}$ & $\mathrm{X}$ & & & B & $X$ & X & & \\
\hline $\mathrm{H}$ & $\mathrm{X}$ & X & B & $\mathrm{N}$ & X & $\mathrm{X}$ & B & \\
\hline & $\mathrm{N}$ & & $\mathrm{N}$ & $\mathrm{N}$ & $\mathrm{N}$ & & $\mathrm{N}$ & \\
\hline $\mathrm{H}$ & $\mathrm{N}$ & & B & $\mathrm{N}$ & $X$ & $\mathrm{X}$ & $\mathrm{N}$ & \\
\hline & & & & & $X$ & $\mathrm{X}$ & $\mathrm{N}$ & \\
\hline B & & & & & $\mathrm{N}$ & & & \\
\hline $\mathrm{H}$ & & X & & B & $X$ & $\mathrm{X}$ & B & \\
\hline
\end{tabular}

Silini Mulsant, 1862

Silis ruficollis (Fabricius, 1775)

$\mathrm{X} \mid \mathrm{X}$

Malthininae Kiesenwetter, 1852

Malthinini Kiesenwetter, 1852

Malthinus balteatus Suffrian, 1851

Malthimus deceptor Baudi di Selve, 1893 [E]

Malthinus devillei Abeille de Perrin, 1898

Malthimus fasciatus (A.G. Olivier, 1790)

Malthinus flaveolus (Herbst, 1786)

Malthimus frontalis (Marsham, 1802

Malthimus garganicus A. Fiori, 1915 [E]

Malthinus glabellus Kiesenwetter, 1852

Malthinus insignipes Pic, 1907 [E]

Malthimus neapolitamus Pic, 1905 [E]

Malthimus pseudobiguttatus Constantin, 1975

Malthinus reflexus Wittmer, 1974 [E]

Malthinus scapularis Marseul, 1878

Malthimus scriptus Kiesenwetter, 1852

Malthimus seriepumctatus Kiesenwetter, 1852

Malthimus sordidus sordidus Kiesenwetter, 1871

\begin{tabular}{|c|c|c|c|c|c|c|c|c|}
\hline $\mathrm{X}$ & $\mathrm{X}$ & & & & $\mathrm{X}$ & & $\mathrm{N}$ & \\
\hline $\mathrm{X}$ & & & $\mathrm{N}$ & $\mathrm{N}$ & $\mathrm{N}$ & $\mathrm{X}$ & $\mathrm{N}$ & \\
\hline & & & & & $\mathrm{X}$ & & & \\
\hline & & & & & & & & \\
\hline & & & & & $\mathrm{X}$ & & & \\
\hline X & $\mathrm{N}$ & & $\mathrm{N}$ & $\mathrm{N}$ & $\mathrm{N}$ & $\mathrm{N}$ & $\mathrm{N}$ & \\
\hline & & & & & $\mathrm{B}$ & & $\mathrm{B}$ & \\
\hline & & & & & $\mathrm{X}$ & $\mathrm{N}$ & & \\
\hline & & & & & & & $\mathrm{B}$ & \\
\hline & & & & & $\mathrm{N}$ & & $\mathrm{B}$ & \\
\hline B & & & & & & $\mathrm{X}$ & $\mathrm{B}$ & \\
\hline H & & & $\mathrm{N}$ & $\mathrm{B}$ & & $\mathrm{N}$ & & \\
\hline B & & & & & $\mathrm{N}$ & & & \\
\hline
\end{tabular}

Malthodini Böving \& Craighead, 1930

Malthodes brettius Liberti, 2017 [E]

Malthodes conicus Wittmer, 1970 [E]

Malthodes cordigerioides Pic, 1915 [E]

Malthodes dispar (Germar, 1824)

Malthodes facetus Kiesenwetter, 1863

Malthodes freyi Wittmer, 1939 [E]

Malthodes gratiosus Ganglbauer, 1906 [E]

Malthodes hexacanthus Kiesenwetter, 1852

Malthodes latistylus Liberti, 2017 [E]

Malthodes lobatus Kiesenwetter, 1852

Malthodes mamubriatus Kiesenwetter, 1863 [E]

Malthodes marginatus (Latreille, 1806)

Malthodes murgianus Pic, 1914 [E]

Malthodes parthenias Kiesenwetter, 1871 [E]

Malthodes pinnatus Kiesenwetter, 1871 [E]

Malthodes pratensis Liberti, 2017 [E]

Malthodes rhegimus Liberti, 2017 [E]

\begin{tabular}{|l|l|l|l|l|l|l|l|l|}
\hline & & & & & B & & & \\
\hline & & & & & B & B & & \\
\hline
\end{tabular}


Species

Malthodes siculus Kiesenwetter, 1852

Malthodes solarii Pic, 1912 [E]

Malthodes spectabilis Kiesenwetter, $1871[\mathrm{E}]$

Malthodes spinifer Liberti, 2017 [E]

Malthodes teter A. Fiori, 1908 [E]

Malthodes umbrosus Kiesenwetter, 1871 [E]

OMALISIDAE Lacordaire, 1857

Omalisinae Lacordaire, 1857

Bocák in Cat. Pal. Col., vol. 4, 2007; Kundrata et al., 2015; Bocek et al., 2017

Omalisus fontisbellaquaei Geoffroy, 1785

Thilmaninae Kazantsev, 2005

Phaeopterus unicolor A. Costa, 1857

\section{ELATERIDAE Leach, 1815 \\ Agrypninae Candèze, 1857}

Agrypnini Candèze, 1857

Cate in Cat. Pal. Col., vol. 4, 2007 except Cebrionini and Lissominae; Kundrata et al., 2018

Agrypnus murinus (Linnaeus, 1758)

Compsolacon crenicollis (Ménétriés, 1832)

Lacon lepidopterus (Panzer, 1800)

Lacon punctatus punctatus (Herbst, 1779)

Drilini Blanchard, 1845

\begin{tabular}{|c|c|c|c|c|c|c|c|c|}
\hline $\mathrm{H}$ & $\mathrm{X}$ & & $\mathrm{N}$ & $\mathrm{CK}$ & $\mathrm{X}$ & $\mathrm{X}$ & $\mathrm{B}$ & \\
\hline & $\mathrm{X}$ & & & $\mathrm{CK}$ & $\mathrm{X}$ & & $\mathrm{B}$ & \\
\hline & & & & & & $\mathrm{X}$ & & $\mathrm{EN}$ \\
\hline $\mathrm{H}$ & $\mathrm{X}$ & $\mathrm{X}$ & $\mathrm{N}$ & $\mathrm{CK}$ & $\mathrm{X}$ & $\mathrm{X}$ & $\mathrm{N}$ & $\mathrm{LC}$ \\
\hline
\end{tabular}

Drilus flavescens A.G. Olivier, 1790

Oophorini Gistel, 1848

Aeoloderma crucifer (P. Rossi, 1790)

Drasterius bimaculatus (P. Rossi, 1790)

\begin{tabular}{|l|l|l|l|l|l|l|l|l|}
\hline & $\mathrm{X}$ & & & & & & $\mathrm{B}$ & \\
\hline $\mathrm{H}$ & $\mathrm{X}$ & $\mathrm{X}$ & $\mathrm{CK}$ & $\mathrm{CK}$ & $\mathrm{X}$ & $\mathrm{X}$ & $\mathrm{B}$ & \\
\hline
\end{tabular}

\section{Cardiophorinae Candèze, 1860}

Cardiophorus anticus Erichson, 1840

Cardiophorus castillanus Buysson, 1902

Cardiophorus collaris Erichson, 1840

Cardiophorus elenorae (Gené, 1836)

Cardiophorus exaratus exaratus Enichson, 1840

Cardiophorus goezei Sanchez-Ruiz, 1996)

Cardiophorus gramineus (Scopoli, 1763)

Cardiophorus italicus Platia \& Bartolozzi, 1988 [E]

Cardiophorus nathaliae Platia \& Schimmel, 1988 [E]

Cardiophorus ruficruris (Brullé, 1832)

Cardiophorus ulcerosus (Gené, 1836)

Cardiophorus vestigialis Erichson, 1840

Dicranychus cinereus (Herbst, 1784)

Dicronychus equiseti (Herbst, 1784)

Dicronychus rubripes (Germar, 1824)

Dicronychus versicolor (Mulsant \& Guillebeau, 1856)

Paracardiophorus musculus musculus (Erichson, 1840)

\begin{tabular}{|c|c|c|c|c|c|c|c|c|}
\hline CK & CK & & & & & & CK & NT \\
\hline X & X & X & CK & CK & X & X & B & \\
\hline & & & & & & & B & \\
\hline H & X & & & & & & & \\
\hline CK & X & & CK & CK & X & X & B & \\
\hline B & B & & CK & CK & N & B & & \\
\hline & B & & & & & & & \\
\hline CK & & & & & & & & \\
\hline & X & & & & X & B & CK & \\
\hline X & X & & & CK & X & X & B & \\
\hline H & CK & & CK & CK & X & X & CK & \\
\hline CK & X & & & CK & X & B & & \\
\hline B & & & B & & & & & \\
\hline H & & & CK & CK & X & X & & \\
\hline & X & & & & & X & & \\
\hline
\end{tabular}

Dendrometrinae Gistel, 1848

Dendrometrini Gistel, 1848

Athouina Candèze, 1859

Athous bicolor (Goeze, 1777)

Athous cachecticus Candèze, 1860 [E]

Athous flavipennis Candèze, 1860

Athous haemorrhoidalis (Fabricius, 1801)

Athous ineptus Candèze, 1860

\begin{tabular}{|l|l|l|l|}
\hline & & & \\
\hline & & & \\
\hline & & & $\mathrm{N}$ \\
\hline
\end{tabular}

\begin{tabular}{|c|c|c|c|c|}
\hline B & & & & \\
\hline CK & $\mathrm{B}$ & $\mathrm{X}$ & $\mathrm{B}$ & \\
\hline $\mathrm{B}$ & & & & \\
\hline $\mathrm{CK}$ & $\mathrm{X}$ & $\mathrm{X}$ & $\mathrm{B}$ & \\
\hline CK & $\mathrm{X}$ & $\mathrm{X}$ & $\mathrm{B}$ & \\
\hline
\end{tabular}


Species

Athous limoniiformis Candèze, 1865 [E]

Athous marginicollis Reitter, 1890

Athous puncticollis Kiesenwetter, 1858

Athous subfuscus (O.F. Müller, 1764)

Athous vittatus (Fabricius, 1792)

Athous zappiorum Platia, 1985 [E]

Cidnopus pilosus (Leske, 1785)

Cidnopus psendopilosus Platia \& Gudenzi, 1985

Limonius minutus (Linnaeus, 1758)

Nothodes parvulus (Panzer, 1799)

Pheletes quercus (A.G. Olivier, 1790)

Dendrometrina Gistel, 1848

\begin{tabular}{|c|c|c|c|c|c|c|c|c|}
\hline 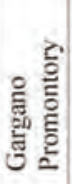 & $\begin{array}{l}3 \\
\vdots \\
3 \\
0 \\
0 \\
0 \\
\frac{0}{0} \\
0\end{array}$ & 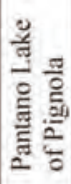 & 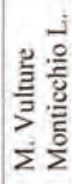 & 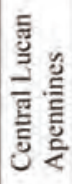 & 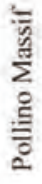 & 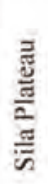 & 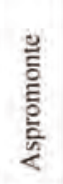 & 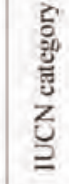 \\
\hline & & & & & & & CK & \\
\hline & & & & & B & $\mathrm{X}$ & B & \\
\hline$!$ & & & & $!$ & $!$ & $!$ & $!$ & \\
\hline$X$ & & & $\mathrm{~N}$ & CK & $X$ & $\mathrm{X}$ & B & \\
\hline $\mathrm{H}$ & $X$ & $X$ & $\mathrm{~N}$ & CK & $X$ & $X$ & B & \\
\hline & & & & B & & & & \\
\hline$!$ & & $\mathrm{X}$ & & CK & B & & & \\
\hline $\mathrm{H}$ & & $\mathrm{X}$ & CK & B & $\mathrm{X}$ & $X$ & & \\
\hline $\mathrm{H}$ & & & CK & CK & $\mathrm{X}$ & $X$ & B & \\
\hline $\mathrm{H}$ & & & $\mathrm{N}$ & & X & $\mathrm{X}$ & B & \\
\hline$X$ & $X$ & & $\mathrm{~N}$ & CK & $\mathrm{X}$ & $\mathrm{X}$ & & \\
\hline
\end{tabular}

Denticollis rubens Piller \& Mitterpacher, 178

Hemicrepidiina Champion, 1894

Harminius florentinus (Desbrochers des Loges, 1870)

Harminius spiniger (Candèze, 1860)

Hemicrepidius hirtus (Herbst, 1784)

Stenagostus rhombeus (A.G. Olivier, 1790)

Prosternini Gistel, 1856

\begin{tabular}{|c|c|c|c|c|c|c|c|c|}
\hline & & & & CK & & $\mathrm{X}$ & & \\
\hline $\mathrm{X}$ & $\mathrm{X}$ & $\mathrm{X}$ & & $\mathrm{N}$ & $\mathrm{X}$ & $!$ & $!$ & \\
\hline $\mathrm{CK}$ & $\mathrm{X}$ & $\mathrm{X}$ & $\mathrm{B}$ & $\mathrm{CK}$ & $\mathrm{X}$ & $\mathrm{X}$ & $\mathrm{B}$ & \\
\hline $\mathrm{X}$ & & & & & $\mathrm{B}$ & ! & ! & $\mathrm{VU}$ \\
\hline
\end{tabular}

Anostirus cerrutii Binaghi, 1940

Aplotarsus incamus (Gyllenhal, 1827)

Calambus bipustulatus (Linnaeus, 1767)

Ctenicera cuprea (Fabricius, 1775)

Ctenicera pectinicornis (Linnaeus, 1758)

Hypoganus inumctus (Lacordaire, 1835)

Mosotalesus impressus impressus (Fabricius, 1792)

Prosternon tessellatum (Linnaeus, 1758)

\begin{tabular}{|c|c|c|c|c|c|c|}
\hline & $\mathrm{N}$ & B & B & $X$ & B & \\
\hline & & & & X & & \\
\hline $\mathrm{X}$ & CK & CK & & CK & & VU \\
\hline & & B & & & & \\
\hline & & & $\mathrm{X}$ & X & & \\
\hline & $\mathrm{CK}$ & B & B & X & & EN \\
\hline & & & $\mathrm{X}$ & & & \\
\hline . & & & X & X & B & \\
\hline
\end{tabular}

Selatosomini Schimmel, Tarnawski, Han \& Platia, 2015

Pristilophus melancholicus melancholicus (Fabricius, 1798)

Selatosomus amplicollis (Germar, 1843)

Selatosomus saginatus (Ménétriés, 1832)

Elaterinae Leach, 1815

Agriotini Champion, 1894

Agriotes acuminatus (Stephens, 1830) Agriotes aequalis Schwarz, 1891 [E]

Agriotes brevis Candèze, 1863

Agriotes corsicus Candèze, 1863

Agriotes infuscatus Desbrochers des Loges, 1870

Agriotes lineatus (Linnaeus, 1767)

Agriotes litigiosus (P. Rossi, 1792)

Agriotes modestus Kiesenwetter, 1858

Agriotes proximus Schwarz, 1891

Agriotes sordidus (Illiger, 1807)

Dalopius marginatus (Linnaeus, 1758)

Ampedini Gistel, 1856

\begin{tabular}{|c|c|c|c|c|c|c|c|}
\hline & & CK & & CK & $\mathrm{X}$ & $X$ & B \\
\hline & & & & B & & & \\
\hline $\mathrm{X}$ & $\mathrm{X}$ & $X$ & CK & CK & $\mathrm{X}$ & $\mathrm{X}$ & $!$ \\
\hline$!$ & & & & CK & $\mathrm{X}$ & $\mathrm{X}$ & B \\
\hline $\mathrm{H}$ & & $X$ & CK & CK & $\mathrm{X}$ & $\mathrm{X}$ & B \\
\hline X & $\mathrm{X}$ & $\mathrm{X}$ & & CK & $\mathrm{X}$ & $\mathrm{X}$ & B \\
\hline$X$ & & $\mathrm{X}$ & & CK & $\mathrm{X}$ & $\mathrm{X}$ & B \\
\hline & $\mathrm{X}$ & & & & & & \\
\hline B & & B & & & & & \\
\hline $\mathrm{H}$ & $\mathrm{X}$ & $X$ & & CK & & & CK \\
\hline $\mathrm{H}$ & & & $\mathrm{N}$ & CK & $\mathrm{X}$ & $\mathrm{X}$ & B \\
\hline
\end{tabular}

Ampedus cardinalis (Schiödte, 1865)

Ampedus cimaberinus (Eschscholtz, 1829)

Ampedus coenobita (A. Costa, 1881)

Ampedus elegantulus (Schönherr, 1817)

Ampedus erythrogonus (P.W.J. Müller, 1821)

Ampedus glycereus (Herbst, 1784)

Ampedus magistrettii Platia \& Schimmel, 1988 [E]

\begin{tabular}{|l|l|l|l|l|l|l|l|l|}
\hline & $\mathrm{B}$ & & & & & $\mathrm{X}$ & & $\mathrm{LC}$ \\
\hline & & & $\mathrm{N}$ & $\mathrm{CK}$ & $\mathrm{X}$ & $\mathrm{X}$ & $\mathrm{B}$ & $\mathrm{LC}$ \\
\hline & & & & & & $\mathrm{X}$ & $\mathrm{B}$ & $\mathrm{NT}$ \\
\hline & & & & & $\mathrm{X}$ & $\mathrm{X}$ & & $\mathrm{VU}$ \\
\hline H & & & & & $\mathrm{B}$ & $\mathrm{X}$ & & $\mathrm{NT}$ \\
\hline B & & & & & $\mathrm{X}$ & & & EN \\
\hline
\end{tabular}


Species

Ampedus melanurus Mulsant \& Guillebeau, 1855

Ampedus nemoralis Bouwer, 1980

Ampedus nigerrimus (Lacordaire, 1835)

Ampedus pomonae (Stephens, 1830)

Ampedus pomorum (Herbst, 1784)

Ampedus praeustus (Fabricius, 1792)

Ampedus quadrisignatus (Gyllenhal, 1817)

Ampedus quercicola (Buysson, 1887)

Ampedus rufipermis (Stephens, 1830)

Ampedus sanguineus (Linnaeus, 1758)

Ampedus sanguinolentus sanguinolentus (Schrank, 1776)

Ampedus simuatus Germar, 1844

Brachygonus megerlei (Lacordaire, 1835)

Reitterelater bouyoni (Chassain, 1992)

Cebrionini Latreille, 1802

\begin{tabular}{|c|c|c|c|c|c|c|c|c|}
\hline & & & & & & & \\
\hline \\
\hline
\end{tabular}

Sảnchez-Ruiz \& Löbl in Cat. Pal. Col., vol. 4, 2007; Kundrata \& Bocák, 2011

Cebrio dubius P. Rossi, 1790 [E]

Cebrio fiorii Leoni, $1906 \mathrm{ef}$. [E]

\begin{tabular}{|l|l|l|l|l|l|l|l|l|}
\hline $\mathrm{X} ?$ & & & & & & & \\
& $\mathrm{~B}$ & $\mathrm{X}$ & $\mathrm{B}$ & & $\mathrm{X}$ & $\mathrm{X}$ ? & & \\
\hline
\end{tabular}

Elaterini Leach, 1815

Elaterina Leach, 1815

Elater ferrugineus ferrugineus Linnaeus, 1758

Mulsanteus guillebeaui (Mulsant \& Godart, 1853)

\begin{tabular}{|l|l|l|l|l|l|l|l|}
\hline & $\mathrm{X}$ & & & $\mathrm{N}$ & & & $\mathrm{VU}$ \\
\hline $\mathrm{X}$ & $\mathrm{N}$ & & & $\mathrm{N}$ & & $\mathrm{B}$ & \\
\hline
\end{tabular}

Megapenthini Gurjeva, 1973

Megapenthes lugens (W. Redtenbacher, 1842)

Procraerus tibialis (Lacordaire, 1835)

\begin{tabular}{|c|c|c|c|c|c|c|c|c|}
\hline H & N & & B & B & & & B & VU \\
\hline & & & B & & & & ! & EN \\
\hline
\end{tabular}

Melanotini Candèze, 1859

\begin{tabular}{|l|c|c|c|c|c|c|c|c|c|}
\hline Melanotus castanipes (Paykull, 1800) & $\mathrm{X}$ & & & $\mathrm{CK}$ & $\mathrm{X}$ & $\mathrm{X}$ & $\mathrm{B}$ & $\mathrm{LC}$ \\
\hline Melanotus crassicollis (Erichson, 1841) & $\mathrm{H}$ & $\mathrm{X}$ & & $\mathrm{N}$ & & $\mathrm{X}$ & $\mathrm{X}$ & $\mathrm{B}$ & \\
\hline Melanotus dichrous (Erichson, 1841) & $\mathrm{X}$ & & $\mathrm{X}$ & & $\mathrm{B}$ & & & $\mathrm{CK}$ & \\
\hline Melanotus fenebrosus (Erichson, 1841) & $\mathrm{H}$ & $\mathrm{X}$ & & $\mathrm{B}$ & $\mathrm{CK}$ & $\mathrm{X}$ & $\mathrm{X}$ & $\mathrm{B}$ & \\
\hline Melanotus villosus (Geoffroy, 1785) & $\mathrm{X}$ & & $\mathrm{X}$ & & $\mathrm{CK}$ & $\mathrm{X}$ & $\mathrm{X}$ & $\mathrm{B}$ & $\mathrm{LC}$ \\
\hline
\end{tabular}

Melanotus villosus (Geoffroy, 1785)

Pomachiliini Candèze, 1859

\begin{tabular}{|l|c|c|c|c|c|c|c|c|}
\hline Betarmon bisbimaculatus (Fabricius, 1803) & $\mathrm{X}$ & $\mathrm{N}$ & $\mathrm{N}$ & $\mathrm{CK}$ & $\mathrm{X}$ & $\mathrm{B}$ & \\
\hline Idiotarmon quadrivittatus (Ragusa, 1893) [E] & $\mathrm{X}$ & & & & $\mathrm{X}$ & $\mathrm{X}$ & $\mathrm{B}$ & \\
\hline Idolus picipennis (Bach, 1852) & & & $\mathrm{CK}$ & $\mathrm{CK}$ & $\mathrm{X}$ & $\mathrm{X}$ & & \\
\hline Isidus moreli Mulsant \& Rey, 1875 & $\mathrm{B}$ & & & & & & $\mathrm{VU}$ \\
\hline
\end{tabular}

Isidus moreli Mulsant \& Rey, 1875

Adrastus axillaris Erichson, 1841

Adrastus limbatus (Fabricius, 1777)

Adrastus rachifer (Geoffroy, 1785)

Adrastus sekerae Reitter, 1910 [E]

Synaptus filiformis (Fabricius, 1781)

Synaptini Gistel, 1856

Lissominae Laporte, 1835

\begin{tabular}{|c|c|c|c|c|c|c|c|c|}
\hline & $\mathrm{X}$ & & & $\mathrm{CK}$ & $\mathrm{CK}$ & $\mathrm{X}$ & & \\
\hline$!$ & & & & ! & & $!$ & & \\
\hline $\mathrm{X}$ & $\mathrm{X}$ & $\mathrm{X}$ & $\mathrm{N}$ & $\mathrm{CK}$ & $\mathrm{X}$ & $\mathrm{X}$ & $\mathrm{B}$ & \\
\hline & $\mathrm{X}$ & & & $\mathrm{CK}$ & & & $\mathrm{B}$ & \\
\hline $\mathrm{X}$ & $\mathrm{X}$ & $\mathrm{CK}$ & $\mathrm{N}$ & $\mathrm{CK}$ & $\mathrm{X}$ & & ! & \\
\hline
\end{tabular}

Lissomini Laporte, 1835

Smetana in Cat. Pal. Col., vol. 4, 2007; Kundrata et al., 2018

Drapetes mordelloides (Host, 1789)

Negastriinae Nakane \& Kishii, 1956

\begin{tabular}{c|c|}
$X|X|$ \\
Kishii, 1956
\end{tabular}

Quasimus liliputamus (Germar, 1844)

Quasimus minutissimus (Germar, 1823)

Zorochros boubersi Leseigneur, 1970

Zorochros curtus (Germar, 1844)

Quasimusini Schimmel \& Tarnawski, 2009 


\begin{tabular}{|c|c|c|c|c|c|c|c|c|c|}
\hline Species & 总 & $\begin{array}{l}\text { zo } \\
30 \\
0 \\
0 \\
\frac{0}{0} \\
\frac{3}{0} \\
2\end{array}$ & 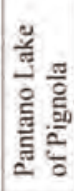 & 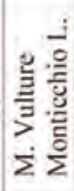 & 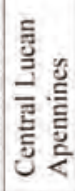 & 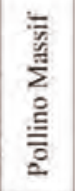 & 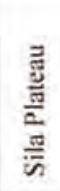 & 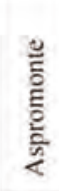 & 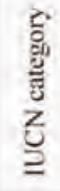 \\
\hline Zorochros dermestoides (Herbst, 1806) & $\mathrm{CK}$ & $\mathrm{CK}$ & & & CK & B & & B & \\
\hline Zorochros meridionalis (Laporte, 1840) & $\mathrm{X}$ & $\mathrm{X}$ & & & & $!$ & & B & \\
\hline Zorochros pumilio (Kiesenwetter, 1858) & & & & & CK & $\mathrm{CK}$ & $\mathrm{X}$ & B & \\
\hline Zorochros trigonochirns (Binaghi, 1933) & & $\mathrm{x}$ & & & $\mathrm{CK}$ & $\mathrm{CK}$ & & B & \\
\hline \multicolumn{10}{|c|}{$\begin{array}{l}\text { DERODONTIFORMIA LeConte, } 1861 \\
\text { DERODONTOIDEA LeConte, } 1861\end{array}$} \\
\hline \multicolumn{10}{|c|}{$\begin{array}{l}\text { DERODONTIDAE LeConte, } 1861 \\
\text { Derodontinae LeConte, } 1861 \\
\text { Háva in Cat. Pal. Col., vol. } 4,2007\end{array}$} \\
\hline $\begin{array}{l}\text { Derodontus raffrayi Grouvelle, } 1917 \text { [E] } \\
\end{array}$ & & B & & & B & B & & & $\mathrm{Vu}$ \\
\hline
\end{tabular}

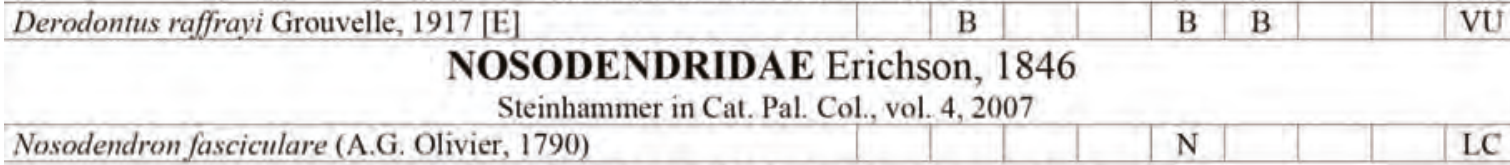

Nosodendron fasciculare (A.G. Olivier, 1790)

BOSTRICHIFORMIA Forbes, 1926

BOSTRICHOIDEA Latreille, 1802

DERMESTIDAE Latreille, 1804

Dermestinae Latreille, 1804

Dermestini Latreille, 1804

Háva in Cat. Pal. Col., vol. 4, 2007






\begin{tabular}{|c|c|c|c|c|c|c|c|c|c|}
\hline Species & 䆖 & $\begin{array}{l}\overline{0} \\
0 \\
0 \\
\frac{0}{2} \\
\frac{0}{0} \\
0\end{array}$ & 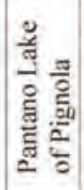 & 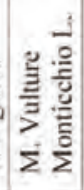 & 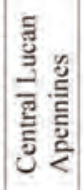 & 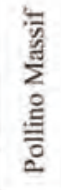 & 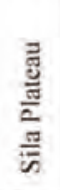 & 䓂 & 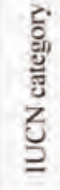 \\
\hline Paranovelsis punctatus (Scopoli, 1772) & & & & & & $\mathrm{X}$ & $\mathrm{X}$ & & \\
\hline \multicolumn{10}{|c|}{$\begin{array}{l}\text { Megatominae Leach, } 1815 \\
\text { Anthrenini Casey, } 1900 \\
\text { Háva in Cat. PaL. Col., vol. 4, } 2007\end{array}$} \\
\hline Anthrenus angustefasciatus Ganglbauer, 1904 & & & & & & $\mathrm{~B}$ & & & \\
\hline Anthremus coloratus Reitter, 1881 & B & & & & & & & & \\
\hline Anthrenus delicatus Kiesenwetter, 1852 & B & & & & & & & & \\
\hline Anthrenus fuscus A.G. Olivier, 1790 & & & & & & $\mathrm{X}$ & $\mathrm{X}$ & & \\
\hline Anthremus munroi Hinton, 1943 & $\mathrm{X}$ & $\mathrm{X}$ & & & & $\mathrm{X}$ & & & \\
\hline Anthrenus pimpinellae pimpinellae (Fabricius, 1775) & $\mathrm{H}$ & $\mathrm{X}$ & & $\mathrm{N}$ & & & & & \\
\hline Anthrenus scrophulariae scrophulariae (Linnaeus, 1758) & & $\mathrm{X}$ & & & & & $\mathrm{X}$ & $\mathrm{N}$ & \\
\hline Anthrenus signatus Erichson, 1846 & $\mathrm{H}$ & $\mathrm{N}$ & B & & & $\mathrm{X}$ & & & \\
\hline Anthrenus verbasci (Linnaeus, 1767) & $\mathrm{H}$ & $\mathrm{X}$ & $\mathrm{X}$ & $\mathrm{N}$ & $\mathrm{N}$ & $\mathrm{X}$ & $\mathrm{X}$ & $\mathrm{N}$ & \\
\hline \multicolumn{10}{|c|}{ Megatomini Leach, 1815} \\
\hline Ctesias serra (Fabricius, 1792) & & & & & & $\mathrm{X}$ & $\mathrm{X}$ & & $\mathrm{LC}$ \\
\hline Globicornis corticalis (Eichhoff, 1863) & & & $\mathrm{N}$ & & $\mathrm{N}$ & $\mathrm{N}$ & & & NT \\
\hline Globicornis emarginata (Gyllenhal, 1808) & $\mathrm{X}$ & $\mathrm{X}$ & & & & $\mathrm{X}$ & $\mathrm{X}$ & & LC \\
\hline $\begin{array}{l}\text { Globicornis fasciata (Fairmaire \& C.N.F. Brisout de Barneville, } \\
\text { 1859) }\end{array}$ & & $\mathrm{N}$ & & & . & B & & & LC \\
\hline Globicornis nigripes (Fabricius, 1792) & & & & & $\mathrm{N}$ & $\mathrm{N}$ & & & LC \\
\hline Globicornis picta (Küster, 1851) & $\mathrm{H}$ & & & & & $\mathrm{X}$ & & & LC \\
\hline Globicornis sulcata (C.N.F. Brisout de Barneville, 1866) & & & & & B & $\mathrm{X}$ & & & NT \\
\hline Globicornis tristis (Reitter, 1881) & & $\mathrm{X}$ & & & & & & & LC \\
\hline Globicornis variegata (Küster, 1851) & & & $\mathrm{X}$ & $\mathrm{N}$ & $\mathrm{N}$ & $\mathrm{X}$ & $\mathrm{X}$ & $\mathrm{N}$ & LC \\
\hline Trogoderma glabrum (Herbst, 1783) & & & & & & $\mathrm{N}$ & & & \\
\hline Trogoderma granarium Everts, 1898 & $\mathrm{X}$ & & & & & & & & \\
\hline Trogoderma inclusum LeConte, 1854 & & $\mathrm{X}$ & & & & & & & \\
\hline
\end{tabular}

BOSTRICHIDAE Latreille, 1802

Bostrichinae Latreille, 1802

Apatini Jacquelin du Val, 1861

Borowski in Cat. Pal. Col., vol. 4, 2007

Apate monachus Fabricius, 1775

Bostrichini Latreille, 1802

\begin{tabular}{|c|c|c|c|c|c|c|c|c|c|}
\hline Amphicerus bimaculatus (A.G. Olivier, 1790) & $\mathrm{X}$ & $\mathrm{X}$ & & & & & & $\mathrm{N}$ & LC \\
\hline Bostrichus capucinus (Linnaeus, 1758) & $\mathrm{X}$ & $\mathrm{X}$ & & $\mathrm{N}$ & $\mathrm{N}$ & $\mathrm{X}$ & $\mathrm{X}$ & B & LC \\
\hline Lichenophanes mumida Lesne, 1899 & & & $\mathrm{~N}$ & & & & & & EN \\
\hline Lichenophanes varius (Illiger, 1801) & $\mathrm{X}$ & B & & & B & & & B & EN \\
\hline Micrapate xyloperthoides (Jacquelin du Val, I859 & $\mathrm{N}$ & & & & & & & & LC \\
\hline
\end{tabular}

Sinoxylonini Lesne, 1899

Sinoxylon muricatum (Linnaeus, 1767)

Sinoxylon perforans (Schrank, 1789)

Xyloperthini Lesne, 1921

Enneadesmus trispinosus (A.G. Olivier, 1795)

Scobicia chevrieri (A. Villa \& G.B. Villa, 1835)

Xylopertha praeusta (Germar, 1817)

Xylopertha retusa (A.G. Olivier, 1790)

Xyloperthella picea picea (A.G. Olivier, 1790)

\begin{tabular}{|l|l|l|l|l|l|l|l|l|}
\hline $\mathrm{X}$ & $\mathrm{X}$ & & & $\mathrm{N}$ & $\mathrm{X}$ & & & LC \\
\hline
\end{tabular}

\begin{tabular}{|l|l|l|l|l|l|l|l|l|}
\hline $\mathrm{X}$ & $\mathrm{X}$ & $\mathrm{X}$ & $\mathrm{N}$ & $\mathrm{N}$ & $\mathrm{X}$ & $\mathrm{X}$ & $\mathrm{N}$ & $\mathrm{LC}$ \\
\hline & & & & & & & $\mathrm{N}$ & LC \\
\hline & & $\mathrm{N}$ & & $\mathrm{N}$ & $\mathrm{X}$ & $\mathrm{N}$ & $\mathrm{VU}$ \\
\hline
\end{tabular}

Dinoderinae C.G. Thomson, 1863

Rhyzopertha dominica (Fabricius, 1792)

Stephanopachys quadricollis (Marseul, 1878)

\begin{tabular}{|l|l|l|l|l|l|l|l|l|}
$\mathrm{X}$ & $\mathrm{X}$ & $\mathrm{X}$ & $\mathrm{N}$ & & $\mathrm{X}$ & $\mathrm{X}$ & $\mathrm{N}$ & \\
& & & & & $\mathrm{X}$ & $\mathrm{X}$ & $\mathrm{N}$ & $\mathrm{VU}$
\end{tabular}


Species

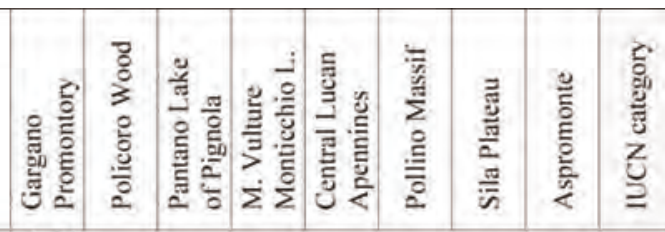

Lyctinae Billberg, 1820

Lyctini Billberg, 1820

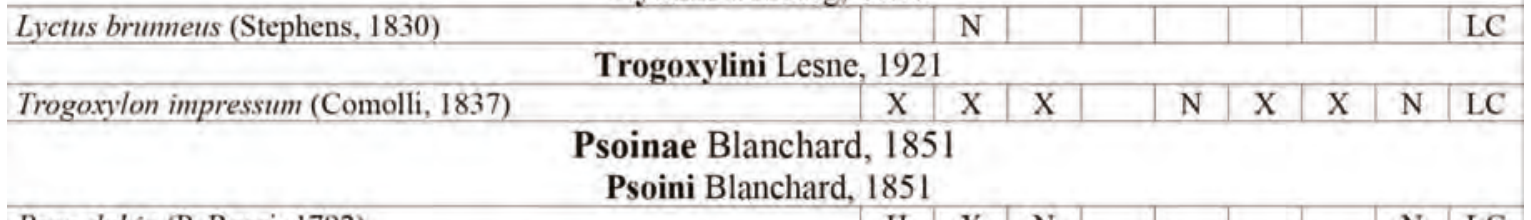

Psoa dubia (P. Rossi, 1792)

PTINIDAE Latreille, 1802

Anobiinae Fleming, 1821

Anobiini Fleming, 1821

Zahradnik in Cat. Pal. Col. Vol. 4, 2007; Zahradnik, 2013

Anobium hederae Ihssen, 1949

Anobium punctatum (DeGeer, 1774)

Cacotemmus thomsoni (Kraatz, 1881)

Hemicoelus canaliculars (C.G. Thomson, 1863)

Hemicoelus costatus (Aragona, 1830)

Hemicoelus fulvicornis (Sturm, 1837)

Microbregma emarginatum (Duftschmid, 1825)

Gastrallini White, 1982

Falsogastrallus unistriatus (Zoufal, 1897)

Gastrallus corsicus Schilsky, 1898

Gastrallus immarginatus (P.W.J. Müller, 1821)

Gastrallus laevigatus (A.G. Olivier, 1790)

Hadrobregmini White, 1982

\begin{tabular}{|c|c|c|c|c|c|c|c|}
\hline $\mathrm{N}$ & $\mathrm{N}$ & & & $\mathrm{N}$ & & & LC \\
\hline$X$ & $X$ & $\mathrm{~N}$ & & $\mathrm{X}$ & $\mathrm{X}$ & $\mathrm{N}$ & LC \\
\hline & $\mathrm{N}$ & & & & & & EN \\
\hline & & & & & & $\mathrm{N}$ & LC \\
\hline $\mathrm{H}$ & & $\mathrm{N}$ & $\mathrm{N}$ & B & $\mathrm{N}$ & $\mathrm{N}$ & LC \\
\hline & $\mathrm{N}$ & $\mathrm{N}$ & & $\mathrm{N}$ & $\mathrm{N}$ & $\mathrm{N}$ & LC \\
\hline H & & & $\mathrm{N}$ & & & & LC \\
\hline
\end{tabular}

Hadrobregmus denticollis (Creutzer, 1796 )

Hadrobregmus pertinax (Linnaeus, 1758)

Nicobiini White, 1982

Nicobium castaneum (A.G. Olivier, 1790)

Stegobiini White, 1982

Oligomerus brumneus (A.G. Olivier, 1790)

Oligomerus ptilinoides (Wollaston, 1854)

Stegobium paniceum (Linnaeus, 1758)

\begin{tabular}{|c|c|c|c|c|}
\hline & $\mathrm{N}$ & & & EN \\
\hline & $\mathrm{N}$ & & $\mathrm{N}$ & LC \\
\hline $\mathrm{N}$ & $\mathrm{X}$ & & $\mathrm{N}$ & LC \\
\hline $\mathrm{H}$ & $\mathrm{N}$ & $\mathrm{N}$ & $\mathrm{N}$ & LC \\
\hline
\end{tabular}

Dorcatominae C.G. Thomson, 1859

Dorcatomini C.G. Thomson, 1859

Caenocara affine (Sturm, 1837)

Caenocara subglobosum (Mulsant \& Rey, 1864)

Dorcatoma chrysomelina Sturm, 1837

Dorcatoma dresdensis Herbst, 1792

Dorcatoma flavicornis (Fabricius, 1792)

Dorcatoma setosella setosella Mulsant \& Rey, 1864

Mizodorcatoma dommeri (Rosenhauer, 1856)

Prothecini White, 1982

\begin{tabular}{|c|c|c|c|c|c|c|}
\hline & $\mathrm{X}$ & & & & $\mathrm{N}$ & LC \\
\hline $\mathrm{X}$ & $X$ & $\mathrm{~N}$ & & X & & LC \\
\hline $\mathrm{N}$ & & & & & $\mathrm{N}$ & LC \\
\hline & & B & $\mathrm{N}$ & & & LC \\
\hline $\mathrm{N}$ & & & & & & LC \\
\hline & & & & & B & LC \\
\hline & $\mathrm{N}$ & & & $\mathrm{N}$ & $\mathrm{N}$ & LC \\
\hline
\end{tabular}

Stagetus andalusiacus cribricollis (Aubẻ, 1861)

Stagetus byrrhoides (Mulsant \& Rey, 1861)

Stagetus calabriensis Toskina, 2012 [E]

Stagetus elongatus (Mulsant \& Rey, 1861)

Stagetus italicus (Reitter, 1885)

Stagetus pilula (Aubé, 1861)

Dryophilinae LeConte, 1861

\begin{tabular}{|c|c|c|c|c|c|c|c|}
\hline & & & & & & B & LC \\
\hline & & & & $\mathrm{N}$ & & $\mathrm{N}$ & LC \\
\hline & & & & & & B & LC \\
\hline & & & & & & $\mathrm{N}$ & LC \\
\hline & & & $\mathrm{N}$ & & $\mathrm{N}$ & $\mathrm{N}$ & LC \\
\hline$X$ & $\mathrm{~N}$ & $X$ & & & & $\mathrm{~N}$ & LC \\
\hline
\end{tabular}

Dryophilini LeConte, 1861 
Species

Dryophilus densipilis Abeille de Perrin, 1872

Dryophilus longicollis (Mulsant \& Rey, 1853)

Dryophilus pusillus (Gyllenhal, 1808)

Grynobius planus (Fabricius, 1787)

Homophthalmus rugicollis (Mulsant \& Rey, 1853)

Ernobiinae Pic, 1912

Ernobiini Pic, 1912

Episernus gentilis (Rosenhauer, 1847)

Episernus granulatus J. Weise, 1887

Episernus striatellus (C.N.F. Brisout de Barneville, 1863)

Ernobius abietimus (Gyllenhal, 1808)

Ernobius angelinii Lohse, 1991 [E]

Ernobius longicornis (Sturm, 1837)

Ernobius mollis mollis (Linnaeus, 1758)

Ermobius nigrinus (Sturm, 1837)

Ochina hirsuta Seidlitz, 1889

Ochina latreillii (Bonelli, 1812)

Ochina ptinoides (Marsham, 1802)

\begin{tabular}{|c|c|c|c|c|c|c|c|c|}
\hline 兽 & $\begin{array}{l}0 \\
0 \\
3 \\
0 \\
0 \\
\frac{0}{0} \\
2\end{array}$ & 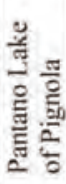 & 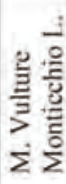 & 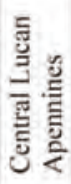 & 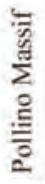 & 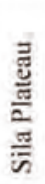 & $\begin{array}{l}\frac{0}{5} \\
\frac{0}{E} \\
\frac{0}{2} \\
\frac{2}{2}\end{array}$ & 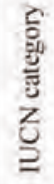 \\
\hline & $\mathrm{N}$ & & & & & $\mathrm{N}$ & $\mathrm{N}$ & LC \\
\hline & & & & & & & $\mathrm{N}$ & LC \\
\hline & $\mathrm{N}$ & & $\mathrm{N}$ & & B & $\mathrm{N}$ & $\mathrm{N}$ & LC \\
\hline & & & & & $\mathrm{N}$ & & & LC \\
\hline $\mathrm{H}$ & $\mathrm{X}$ & & & $\mathrm{N}$ & $X$ & X & $\mathrm{N}$ & LC \\
\hline
\end{tabular}

\section{Ernobini Pic, 1912}

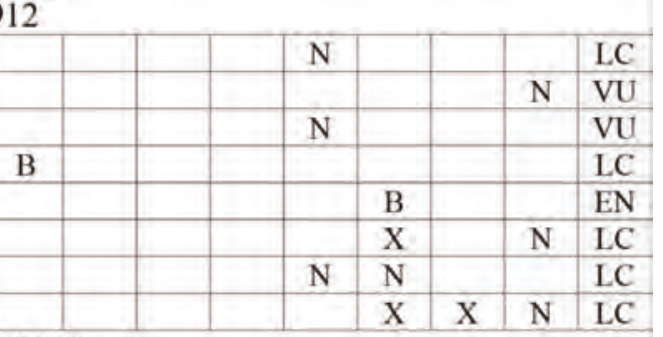

Ochinini Zahradnik, 2013

Hyperisus plumbeum (Illiger, 1801)

Xestobium rufovillosum (DeGeer, 1774)

Xestobium subincantum Reitter, 1878

\begin{tabular}{|c|c|c|c|c|c|c|c|c|}
\hline & X & & & & & & & LC \\
\hline & & & & & X & & N & NT \\
\hline
\end{tabular}

Xestobiini White, 1982

\section{Eucradinae LeConte, 1861 \\ Hedobiini Mulsant \& Rey, 1868}

Hedobia pubescens (A.G. Olivier, 1790)

Ptinomorphus angustatus (C.N.F. Brisout de Barneville, 1862

Ptinomorphus imperialis (Linnaeus, 1767)

Ptinomorphus regalis (Duftschmid, 1825)

\begin{tabular}{|c|c|c|c|c|c|c|c|c|}
\hline H & X & & & & & & N & LC \\
\hline & & & & N & X & X & N & LC \\
\hline & & & & & B & & & EN \\
\hline
\end{tabular}

\section{Gibbiinae Mulsant \& Rey, 1868}

Gibbiini Mulsant \& Rey, 1868

Borowski in Cat. Pal. Col., vol. 4, 2007; Zahradník, 2013

Gibbium psylloides (Czenpinski, 1778)

Mesocoelopodinae Mulsant \& Rey, 1864

Mesocoelopodini Mulsant \& Rey, 1864

Zahradník in Cat. Pal. Col., vol. 4, 2007; Zahradnik, 2013

Mesocoelopus collaris Mulsant \& Rey, 1864

Mesocoelopus creticus Fairmaire, 1880

Mesocoelopus niger (P.W.J. Müller, 1821)

Mesothes ferrugineus (Mulsant \& Rey, 1861)

\begin{tabular}{|c|c|c|c|c|c|c|c|}
\hline $\mathrm{H}$ & $\mathrm{X}$ & & & & & & LC \\
\hline & $\mathrm{N}$ & & & & & & \\
\hline $\mathrm{N}$ & $\mathrm{N}$ & & & $\mathrm{N}$ & $\mathrm{N}$ & & LC \\
\hline $\mathrm{X}$ & & & & $\mathrm{N}$ & & & LC \\
\hline
\end{tabular}

Ptilininae Shuckard, 1840

Ptilinini Shuckard, 1840

Ptilimus fuscus (Geoffroy, 1785)

Ptilimus pectinicornis (Linnaeus, 1758)

Ptininae Latreille, 1802

\begin{tabular}{|l|l|l|l|l|l|l|l|}
\hline $\mathrm{N}$ & & & $\mathrm{N}$ & & & & LC \\
\hline & & & & $\mathrm{X}$ & $\mathrm{X}$ & & LC \\
\hline
\end{tabular}

Ptinini Latreille, 1802

Borowski in Cat. Pal. Col., vol. 4, 2007; Zahradník, 2013

Bruchoptius femoralis Reitter, 1884

Bruchoptius rufipes (A.G. Olivier, 1790)

Dignomus carinatus (P.H. Lucas, 1846)

Dignomus jacqueti (Pic, 1895)

\begin{tabular}{|c|c|c|c|c|c|c|c|c|}
\hline H & X & & N & & N & & \\
\hline & N & & & & & & & \\
\hline X & X & & & & & & N & \\
\hline & & & & & & & N & \\
\hline
\end{tabular}


Species

Niptus hololeucus (Faldermann, 1835)

Pseudeurostus apennimus (Baudi di Selve, [874) [E]

Pseudoptinus lichenum (Marsham, 1802)

Ptimus atricapillus Kiesenwetter, 1877

Ptimus aubei Boieldieu, 1854

Ptimus bidens A.G. Olivier, 1790

Ptimus calcaratus Kiesenwetter, 1877

Ptimus corsicus Kiesenwetter, 1877

Ptinus dubius Sturm, 1837

Ptinus fur (Linnaeus, 1758)

Ptimus latro Fabricius, 1775

Ptinus obesus P.H. Lucas, 1846

Ptinus perrini (Reitter, 1884)

Ptimus pilosus P.W.J. Müller, 1821

Ptinus raptor Sturm, 1837

Ptinus reitteri Pic, 1894

Ptimus sexpunctatus Panzer, 1789

Ptimus spitzyi A. Villa \& G.B. Villa, 1838

Ptinus subpilosus Sturm, 1837

Ptimus tarsalis (Reitter, 1884)

Ptimus variegatus P. Rossi, 1792

\begin{tabular}{|l|l}
\hline 1877 & $\mathrm{H}$ \\
\hline
\end{tabular}

Xyletininae Gistel, 1856

Lasiodermini White, 1982

Zahradnik in Cat. Pal. Col., vol. 4, 2007; Zahradnik, 2013

Lasioderma baudii Schilsky, 1899

Lasioderma bubalus bubalus Fairmaire, 1860

Lasioderma haemorrhoidale (Illiger, 1807)

Lasioderma kiesenwetteri Schilsky, 1899

Lasioderma laeve (Illiger, 1807)

Lasioderma obscurum (Solsky, 1868)

Lasioderma redtenbacheri redtenbacheri (Bach, 1852)

Lasioderma serricorne (Fabricius, 1792)

Xyletinini Gistel, 1856

\begin{tabular}{|l|l|l|l|l|l|l|l|l|}
$\mathrm{X}$ & $\mathrm{X}$ & & $\mathrm{N}$ & & $\mathrm{X}$ & $\mathrm{X}$ & $\mathrm{N}$ & \\
\hline $\mathrm{H}$ & & $\mathrm{N}$ & $\mathrm{N}$ & $\mathrm{N}$ & $\mathrm{X}$ & $\mathrm{X}$ & & \\
\hline $\mathrm{H}$ & & $\mathrm{X}$ & $\mathrm{N}$ & & & & $\mathrm{N}$ & \\
\hline $\mathrm{H}$ & $\mathrm{N}$ & & $\mathrm{N}$ & $\mathrm{N}$ & & & & \\
\hline & $\mathrm{N}$ & $\mathrm{N}$ & & & & & $\mathrm{N}$ & \\
\hline $\mathrm{X}$ & $\mathrm{X}$ & $\mathrm{X}$ & $\mathrm{N}$ & $\mathrm{N}$ & $\mathrm{X}$ & $\mathrm{X}$ & $\mathrm{N}$ & \\
\hline $\mathrm{X}$ & $\mathrm{X}$ & $\mathrm{X}$ & & $\mathrm{N}$ & $\mathrm{N}$ & & $\mathrm{N}$ & \\
\hline & $\mathrm{X}$ & & $\mathrm{N}$ & & $\mathrm{X}$ & & & \\
\hline
\end{tabular}

Xyletimus ater (Creutzer, 1796)

Xyletimus balcanicus Gottwald, 1977

Xyletimus fibyensis Lundblad, 1949

Xyletinus laticollis (Duftschmid, 1825)

Xyletimus longitarsis longitarsis Jansson, 1942

Xyletimus pectinatus pectinatus (Fabricius, 1792)

Xyletinus pectiniferus Fairmaire, 1879

Xyletinus subrotondatus Lareynie, 1852

Xyletinus vaederoeensis Lundberg, 1969

Metholcus phoenicis (Fairmaire, 1859)

Xyletinini genera incertae sedis

\begin{tabular}{|l|l|l|l|l|l|l|l|l|}
\hline & & & & & $\mathrm{N}$ & $\mathrm{N}$ & & LC \\
\hline & & & & $\mathrm{N}$ & & & & VU \\
\hline & $\mathrm{N}$ & & & & & & & VU \\
\hline & & $\mathrm{N}$ & $\mathrm{N}$ & $\mathrm{N}$ & $\mathrm{X}$ & $\mathrm{X}$ & $\mathrm{N}$ & LC \\
\hline & & & $\mathrm{N}$ & & & & & LC \\
\hline & $\mathrm{X}$ & & & & & & & $\mathrm{NT}$ \\
\hline & $\mathrm{N}$ & & & & $\mathrm{X}$ & & & \\
\hline & $\mathrm{N}$ & & & & & & & \\
\hline
\end{tabular}

\section{CUCUJIFORMIA Lameere, 1938 \\ LYMEXYLOIDEA Fleming, 1821 \\ LYMEXYLIDAE Fleming, 1821 \\ Hylecoetinae Gistel, 1856 \\ Cuccodoro in Cat. Pal. Col., vol. 4, 2007}




\begin{tabular}{|c|c|c|c|c|c|c|c|c|c|}
\hline Species & 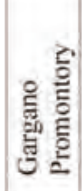 & 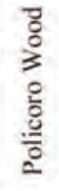 & 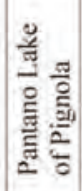 & 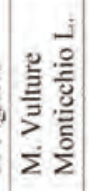 & 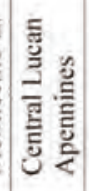 & 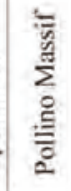 & 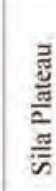 & & \\
\hline Lymexylon navale (Linnaeus, 1758) & $\mathrm{X}$ & & & & & & & & \\
\hline
\end{tabular}

CLEROIDEA Latreille, 1802

sensu Robertson et al., 2015; Gimmel et al., 2019

BYTURIDAE Jacquelin du Val, 1858

Löbl in Cat. Pal. Col., vol. 4, 2007; Robertson et al., 2015

Byturus ochraceus (L.G. Seriba, 1790) Byturus tomentosus (DeGeer, 1774)

\begin{tabular}{|l|l|l|l|l|l|l|l|l|}
\hline $\mathrm{X}$ & & & & $\mathrm{N}$ & & & \\
\hline
\end{tabular}

BIPHYLLIDAE LeConte, 1861

Jelinek in Cat. Pal. Col., vol. 4, 2007; Robertson et al., 2015

Biphyllus frater (Aubé, 1850)

Biphyllus lunatus (Fabricius, 1787)

Diplocoelus fagi (Chevrolat, 1837)

\begin{tabular}{|l|l|l|l|l|l|l|l|l|} 
& $\mathrm{N}$ & & & $\mathrm{N}$ & $\mathrm{N}$ & $\mathrm{X}$ & $\mathrm{N}$ & LC \\
\hline $\mathrm{X}$ & $\mathrm{X}$ & & & & $\mathrm{N}$ & & & LC \\
\hline $\mathrm{H}$ & $\mathrm{X}$ & & $\mathrm{N}$ & $\mathrm{N}$ & $\mathrm{X}$ & $\mathrm{X}$ & $\mathrm{N}$ & LC \\
\hline
\end{tabular}

PELTIDAE Kirby, 1837

Peltini Kirby, 1837

Kolibáč in Cat. Pal. Col., vol. 4, 2007; Gimmel et al., 2019

Peltis ferruginea (Linnaeus, 1758)

Peltis grossa (Linnaeus, 1758)

LOPHOCATERIDAE Crowson, 1964

Kolibáč in Cat. Pal. Col., vol. 4, 2007; Gimmel et al., 2019

Grynocharis oblonga (Linnaeus, 1758)

TROGOSSITIDAE Latreille, 1802

Trogossitinae Latreille, 1802

Trogossitini Latreille, 1802

Kolibáč in Cat. Pal. Col., vol. 4, 2007; Gimmel et al., 2019;

Nemozoma elongatum (Linnaeus, 1761)

Temnoscheila caerulea (A.G. Olivier, 1790)

Tenebroides mauritanicus (Linnaeus, 1758)

\begin{tabular}{|c|c|c|c|c|c|c|c|c|}
\hline $\mathrm{N}$ & & & & $\mathrm{N}$ & $\mathrm{X}$ & & $\mathrm{N}$ & $\mathrm{LC}$ \\
$\mathrm{X}$ & $\mathrm{X}$ & & $\mathrm{N}$ & $\mathrm{N}$ & $\mathrm{X}$ & $\mathrm{X}$ & $\mathrm{N}$ & LC \\
\hline $\mathrm{X}$ & $\mathrm{X}$ & & $\mathrm{N}$ & & & $\mathrm{B}$ & $\mathrm{B}$ & LC \\
\hline
\end{tabular}

THYMALIDAE Léveillé, 1888

Thymalinae Léveillé, 1888

Kolibáč in Cat. Pal. Col., vol. 4, 2007; Gimmel et al., 2019

Thymalus limbatus (Fabricius, 1787)

\section{RHADALIDAE LeConte, 1862 \\ Rhadalinae LeConte, 1862 \\ Aplocnemini Majer, 1987}

Mayor in Cat. Pal. Col., vol. 4, 2007; Bocakova et al., 2011; Gimmel et al., 2019

Aplocnemus angelinii Liberti, 1994

Aplocnemus chalconatus (Germar, 1817)

Aplocnemus corcyricus (L. Miller, 1866)

Aplocnemus etruscus Liberti \& Zinetti, 2009

Aplocnemus integer (Baudi di Selve, 1873)

Aplocnemus jejumus (Kiesenwetter, 1863)

Aplocnemus marginatus (Rottenberg, 1871)

Aplocnemus nigricornis garganicus Liberti, 1994 [E]

Aplocnemus nigricornis nigricornis (Fabricius, 1792)

Trichoceble floralis floralis (A.G. Olivier, 1790)

Trichoceble memnonia (Kiesenwetter, 1861)

\begin{tabular}{|c|c|c|c|c|c|c|c|c|}
\hline & B & & & & & & LC \\
\hline & B & & & B & & & \\
\hline & B & & B & & & B & LC \\
\hline & & & B & & & & NT \\
\hline & & & & B & & X? & B & LC \\
\hline & & & & & & & B & LC \\
\hline & & & & & & & & NT \\
\hline & & & B & B & X & X & B & LC \\
\hline & & & & & B & & & \\
\hline
\end{tabular}


Species

MELYRIDAE Leach, 1815

Malachiinae Fleming, 1821

Malachiini Fleming, 1821

Apalochrina Mulsant \& Rey, 1867

Mayor in Cat. Pal. Col., vol. 4, 2007; Gimmel et al., 2019

Protapalochrus flavolimbatus Mulsant \& Rey, 1853

Attalina Abeille de Perrin, 1890

Attalus amictus (Erichson, 1840)

Attalus analis (Panzer, 1798)

Attalus cyaneus (Fabricius, 1787)

Attalus minimus (P. Rossi, 1790)

Attalus nourricheli nourricheli (Laporte, 1838)

Attalus sericans (Erichson, 1840)

Attalus varitarsis (Kraatz, 1862)

Sphinginus coarctatus (Erichson, 1840)

Sphinginus lobatus lobatus (A.G. Olivier, 1790)

Colotina Abeille de Perrin, 1890

\begin{tabular}{|c|c|c|c|c|c|c|c|c|}
\hline B? & & & & B? & X? & & & \\
\hline & & & & N & X & X & & LC \\
\hline & & & N & & N & & N & \\
\hline X & X & X & N & B & X & X & B & \\
\hline & B & & & B & N & & & \\
\hline & B & & & & B & & & \\
\hline H & X & & B & B & & & B & LC \\
\hline
\end{tabular}

Colotes maculatus (Laporte, 1838)

Colotes punctatus (Erichson, 1840)

\begin{tabular}{l|l|}
$\mathrm{X}$ & $\mathrm{X}$ \\
\hline
\end{tabular}

Ebaeina Portevin, 1931

Ebaets ater Kiesenwetter, 1863

Ebaens battonii Pardo Alcaide, 1962

Ebaeus gibbus (Drapiez, 1819)

Hypebaeus flavicollis (Erichson, 1840)

Hypebaeus flavipes (Fabricius, 1787)

Malachiina Fleming, 1821

Anthocomus fasciatus (Linnaeus, 1758)

Anthocomus rufus rufus (Herbst, 1784)

Anthomalachius spinosus (Erichson, 1840)

Axinotarsus insularis Abeille de Perrin, 1881

Axinotarsus italicus Escalera, 1926 [E]

Axinotarsus longicornis (Kiesenwetter, 1859)

Axinotarsus marginalis (Laporte, 1840)

Axinotarsus pulicarius (Fabricius, 1777)

Axinotarsus ruficollis (A.G. Olivier, 1790)

Charopus apicalis Kiesenwetter, 1859

Charopus concolor (Fabricius, 1801)

Charopus docilis Kiesenwetter, 1852

Charopus pallipes (A.G. Olivier, 1790)

Charopus philoctetes Abeille de Perrin, 1885

Charopus rotundatus Erichson, 1840

Clanoptilus affinis subconcolor Pic, 1911

Clanoptilus bellieri (Peyron, 1877)

Clanoptilus calabrus (Baudi di Selve, 1873) [E]

Clanoptilus emarginatus (Krauss, 1902)

Clanoptilus geniculatus (Germar, 1824)

Clanoptilus italicus (Pardo Alcaide, 1967) [E]

Clanoptilus spinipennis brevispina (Kiesenwetter, 1871)

Cordylepherus viridis (Fabricius, 1787)

Cyrtosus cyanipennis (Erichson, 1840)

Cyrtosus ovalis (Laporte, 1838)

Malachius aeneus (Linnaeus, 1758)

\begin{tabular}{|c|c|c|c|c|c|c|c|c|}
\hline $\mathrm{H}$ & $X$ & & & & $\mathrm{~N}$ & & & \\
\hline \multirow[t]{7}{*}{ X } & $\mathrm{X}$ & B & & & $\mathrm{X}$ & $\mathrm{X}$ & & \\
\hline & $\mathrm{X}$ & B & B & & & B & & \\
\hline & $X$ & & & B & & & $\mathrm{N}$ & \\
\hline & & & & B & $\mathrm{X}$ & & B & \\
\hline & & & B? & & & & & \\
\hline & $\mathrm{X}$ & & & & & & & \\
\hline & & & & $\mathrm{N}$ & B & X & B & \\
\hline $\mathrm{X}$ & B & & B & B & $\mathrm{X}$ & $\mathrm{X}$ & B & \\
\hline $\mathrm{H}$ & X & & $\mathrm{N}$ & B & $\mathrm{N}$ & & B & \\
\hline \multirow[t]{2}{*}{$\mathrm{H}$} & & & & & B & B & $\mathrm{N}$ & \\
\hline & & & & & B & B & B & \\
\hline \multirow[t]{2}{*}{$\mathrm{H}$} & B & & B & B & X & $\mathrm{X}$ & B & \\
\hline & & & & & & B & & \\
\hline \multirow[t]{4}{*}{$\mathrm{X}$ ? } & & & & & & $\mathrm{X}$ ? & & \\
\hline & & & & B & & & & \\
\hline & B & & & & B & & & \\
\hline & & & & & & $X$ & B & LC \\
\hline $\mathrm{H}$ & & $\mathrm{X}$ & B & B & $X$ & $\mathrm{X}$ & B & \\
\hline $\mathrm{H}$ & $X$ & $\mathrm{X}$ & B & B & $\mathrm{N}$ & B & & \\
\hline $\mathrm{H}$ & & $\mathrm{X}$ & B & B & $\mathrm{X}$ & $\mathrm{X}$ & & LC \\
\hline $\mathrm{H}$ & $\mathrm{X}$ & B & B & $\mathrm{N}$ & X & $\mathrm{X}$ & B & \\
\hline \multirow[t]{2}{*}{$\mathrm{X}$} & & B & B & B & $\mathrm{X}$ & $\mathrm{X}$ & B & \\
\hline & & & & & & & B & \\
\hline $\mathrm{H}$ & & & B & B & $\mathrm{X}$ & $\mathrm{X}$ & B & \\
\hline $\mathrm{H}$ & B & X & $\mathrm{N}$ & B & $\mathrm{X}$ & X & $\mathbf{N}$ & \\
\hline
\end{tabular}


Species

Malachius australis Mulsant \& Rey, 1867

Malachius bipustulatus (Linnaeus, 1758)

Troglopina Mulsant \& Rey, 1867

Troglops albicans (Linnaeus, 1767)

Troglops italicus Wittmer, 1984

\begin{tabular}{|c|c|c|c|c|c|c|c|c|}
\hline 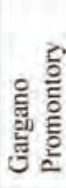 & $\begin{array}{l}\bar{b} \\
\vdots \\
3 \\
0 \\
\frac{3}{3} \\
\frac{.3}{0} \\
2\end{array}$ & 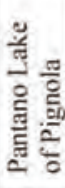 & 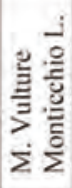 & 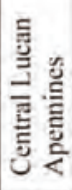 & 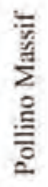 & 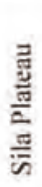 & 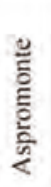 & 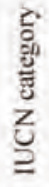 \\
\hline $\mathrm{H}$ & $\mathrm{N}$ & $\mathrm{X}$ & B & B & $\mathrm{X}$ & $\mathrm{X}$ & B & \\
\hline & & & B & B & $X$ & & B & \\
\hline
\end{tabular}

\begin{tabular}{|l|l|l|l|l|l|l|l|}
\hline & & & B & & & B & LC \\
\hline
\end{tabular}

Dasytinae Laporte, 1840

Danaceini Kiesenwetter, 1863

\begin{tabular}{|c|c|c|c|c|c|c|c|c|}
\hline Danacea ambigua Mulsant \& Rey, 1868 & $\mathrm{X}$ & $\mathrm{X}$ & & $\mathrm{N}$ & $\mathrm{N}$ & $\mathrm{X}$ & $\mathrm{X}$ & \\
\hline Danacea apula Liberti, 2007 [E] & B & & & & & & & \\
\hline Danacea aurichalcea (Küster, 1850) & $\mathrm{H}$ & $\mathrm{X}$ & $\mathrm{X}$ & $\mathrm{N}$ & $\mathrm{N}$ & $\mathrm{X}$ & $\mathrm{X}$ & $\mathrm{N}$ \\
\hline Danacea concii Liberti, 1984 [E] & & & $\mathrm{X}$ & & B & $\mathrm{X}$ & & \\
\hline Danacea cusanensis (A. Costa, 1847) [E] & $\mathrm{X}$ & $\mathrm{X}$ & $\mathrm{X}$ & & B & $\mathrm{X}$ & $\mathrm{X}$ & B \\
\hline Danacea jonica Liberti, 1985 [E] & & & & & & & & B \\
\hline Danacea lucana Liberti, 1989 [E] & & & & & & B & $\mathrm{X}$ & \\
\hline Danacea marginata (Küster, 1851) & & & & & & & & B \\
\hline Danacea nigritarsis brumneipes Pic, 1927 [E] & $\mathrm{B}$ & & & $\mathrm{N}$ & & $\mathrm{X}$ & $\mathrm{X}$ & \\
\hline Danacea scyllea Liberti, 1981 [E] & & & & & & $\mathrm{N}$ & $\mathrm{X}$ & B \\
\hline
\end{tabular}

Danacea scyllea Liberti, 1981 [E]

Dasytini Laporte, 1840

\begin{tabular}{|c|c|c|c|c|c|c|c|c|c|}
\hline Dasytes aeneiventris Küster, 1850 & $\mathrm{H}$ & $\mathrm{X}$ & $\mathrm{X}$ & B & B & $\mathrm{X}$ & $\mathrm{X}$ & B & LC \\
\hline Dasytes aeratus Stephens, 1830 & $\mathrm{~B}$ & & $\mathrm{X}$ & $\mathrm{N}$ & B & $\mathrm{X}$ & $\mathrm{X}$ & B & LC \\
\hline Dasytes caeruleus (DeGeer, 1774) & & & & B & B & $\mathrm{X}$ & & B & LC \\
\hline Dasytes flavescens Gené, 1839 & & $\mathrm{X}$ & & & & $\mathrm{B}$ & & B & \\
\hline Dasytes nigroaeneus Küster, 1850 & & & & & & & & B & LC \\
\hline Dasytes pauperculus Laporte, 1840 & B & & & & & & & & LC \\
\hline Dasytes plumbeus (O.F. Müller, 1776) & $\mathrm{H}$ & $\mathrm{X}$ & B & B & B & $\mathrm{X}$ & $\mathrm{X}$ & B & LC \\
\hline Dasytes productus Schilsky, 1894 & & & & & & & & B & \\
\hline Dasytes striatulus Brullé, 1832 & $\mathrm{H}$ & $\mathrm{X}$ & $\mathrm{X}$ & & B & B & & & \\
\hline Dasytes subaeneus Schönherr, 1817 & B & & & & & B & & & \\
\hline Dasytes tardus L.W. Schaufuss, 1876 & & & & & B & & $\mathrm{X}$ & & \\
\hline Dasytes thoracicus lucamus Wittmer, 1935 & & & & & & $\mathrm{X}$ & & & $\mathrm{VU}$ \\
\hline Dasytes tristiculus Mulsant \& Rey, 1868 & $\mathrm{H}$ & $\mathrm{B}$ & & & & B & B & B & \\
\hline Divales bipustulatus (Fabricius, 1781) & $\mathrm{X}$ & & & B & $\mathrm{N}$ & $\mathrm{X}$ & $\mathrm{X}$ & $\mathrm{N}$ & \\
\hline Divales cinctus (Gené, I839) & & $\mathrm{X}$ & & & $\mathrm{N}$ & $\mathrm{X}$ & & $\mathrm{N}$ & \\
\hline Dolichosoma simile (Brullé, 1832) & $\mathrm{H}$ & $\mathrm{X}$ & & & & & & & \\
\hline Enicopus ater (Fabricius, 1787) & $!$ & & & & & & & & \\
\hline Enicopus pilosus (Scopoli, 1763) & $\mathrm{X}$ & $\mathrm{X}$ & $\mathrm{X}$ & B & B & $\mathrm{X}$ & $\mathrm{X}$ & B & \\
\hline Psilothrix melanostoma (Brullé, 1832) & $\mathrm{H}$ & & $\mathrm{X}$ & $\mathrm{N}$ & $\mathrm{N}$ & $\mathrm{X}$ & $\mathrm{X}$ & $\mathrm{N}$ & \\
\hline Psilothrix viridicoernilea (Geoffroy, 1785) & $\mathrm{H}$ & $\mathrm{X}$ & $X$ & $\mathrm{~N}$ & $\mathrm{~N}$ & $X$ & $\mathrm{X}$ & $\mathrm{N}$ & \\
\hline
\end{tabular}

CLERIDAE Latreille, 1802

Tillinae Leach, 1815

Löbl, Rolčik, Kolibáč \& Gerstmeier in Cat. Pal. CoL, vol 4, 2007; Gimmel et al., 2919

\begin{tabular}{|l|c|c|c|c|c|c|c|c|c|}
\hline Denops albofasciatus (Charpentier, I825) & $\mathrm{X}$ & $\mathrm{X}$ & & & ! & $\mathrm{N}$ & & & NT \\
\hline Tilloidea transversalis (Charpentier, 1825) & $\mathrm{X}$ & $\mathrm{X}$ & $\mathrm{X}$ & $\mathrm{N}$ & ! & $\mathrm{X}$ & $\mathrm{X}$ & $\mathrm{B}$ & \\
\hline Tilloidea unifasciata (Fabricius, 1787) & & & & & $\mathrm{N}$ & & & $\mathrm{N}$ & NT \\
\hline Tillus elongatus (Linnaeus, 1758) & & & & & & $\mathrm{X}$ & $\mathrm{X}$ ? & & NT \\
\hline
\end{tabular}

Tillus elongatus (Linnaeus, 1758)

Korynetinae Laporte, 1836

\begin{tabular}{|c|c|c|c|c|c|c|c|c|c|}
\hline Enoplium doderoi Luigioni, 1926 & & $\mathrm{X}$ & & & & & & & EN \\
\hline Enoplium serraticorne (A.G. Olivier, 1790) & & & & & & B & & B & NT \\
\hline Korynetes pusillus (Klug, 1842) & $!$ & $\mathrm{X}$ & $\mathrm{X}$ & & $\mathrm{N}$ & $\mathrm{N}$ & $\mathrm{X}$ & 1 & NT \\
\hline Necrobia ruficollis (Fabricius, 1775) & $\mathrm{H}$ & & & & & & & & \\
\hline Necrobia rufipes (DeGeer, 1775) & $\mathrm{X}$ & $\mathrm{X}$ & & & $!$ & $\mathrm{X}$ & & $\mathrm{N}$ & \\
\hline Necrobia violacea (Linnaeus, 1758) & $!$ & $\mathrm{X}$ & $\mathrm{N}$ & $\mathrm{N}$ & ! & $\mathrm{N}$ & $\mathrm{X}$ & 1 & \\
\hline
\end{tabular}


Species

Tarsostenus univittatus (P. Rossi, 1792)

Clerinae Latreille, 1802

Clerus mutillarius mutillarius Fabricius, 1775

Opilio domesticus (Sturm, 1837)

Opilio mollis (Linnaeus, 1758)

Opilio pallidus (A.G. Olivier, 1795)

Opilio taeniatus (Klug, 1842)

Thanasimus formicarius formicarius (Linnaeus, 1758)

Trichodes alvearius (Fabricius, 1792)

Trichodes apiarius (Linnaeus, 1758)

Trichodes lencopsideus (A.G. Olivier, 1795)

\begin{tabular}{|c|c|c|c|c|c|c|c|c|}
\hline 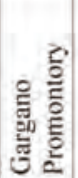 & $\begin{array}{l}\text { Do } \\
0 \\
\frac{0}{0} \\
\frac{3}{0} \\
\frac{3}{0} \\
2\end{array}$ & 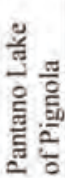 & 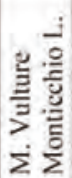 & 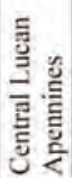 & 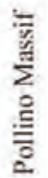 & 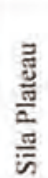 & $\begin{array}{l}\frac{\dddot{g}}{\overline{0}} \\
\frac{\mathrm{E}}{\mathrm{E}} \\
\frac{\mathrm{c}}{\mathrm{c}}\end{array}$ & 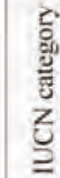 \\
\hline$!$ & & & & ! & & & & \\
\hline
\end{tabular}

CUCUJOIDEA Latreille, 1802

sensu Robertson et al., 2015

SPHINDIDAE Jacquelin du Val, 1860

Sphindinae Jacquelin du Val, 1860

Jelinek in Cat. Pal. Col., vol. 4, 2007

Sphindus dubius (Gyllenhal, 1808)

\begin{tabular}{|c|c|c|c|c|c|c|}
$\mathrm{H} \mid \mathrm{X}$ & & & $\mathrm{X}$ & & $\mathrm{N}$ & $\mathrm{NT}$ \\
\hline
\end{tabular}

Aspidiphorinae Kiesenwetter, 1877

Aspidiphoms lareyniei Jacquelin du Val, 1859

Aspidiphorus orbiculatus (Gyllenhal, 1808)

\begin{tabular}{|l|l|l|l|l|l|l|l|l|}
\hline & $\mathrm{X}$ & & & & & & & $\mathrm{NT}$ \\
\hline $\mathrm{X}$ & $\mathrm{X}$ & & $\mathrm{N}$ & $\mathrm{N}$ & $\mathrm{X}$ & $\mathrm{X}$ & $\mathrm{N}$ & $\mathrm{LC}$ \\
\hline
\end{tabular}

EROTYLIDAE Latreille, 1802

Cryptophilinae Casey, 1900

Cryptophilini Casey, 1900

Wegrzynowic in Cat. Pal. Col., vol. 4, 2007

Cryptophilus angustus (Rosenhauer, 1856) \begin{tabular}{|l|l|l|l|l|l|l|l|l|}
$\mathrm{X}$ & $\mathrm{N}$ & & $\mathrm{N}$ & $\mathrm{N}$ & $\mathrm{N}$ & $\mathrm{X}$ & $\mathrm{N}$ & $\mathrm{LC}$ \\
\hline
\end{tabular}

Xenoscelinae Ganglbauer, 1899

Xenoscelini Ganglbauer, 1899

Leucohimatium arundinaceum (Forskâl, 1775)

Setariola sericea (Mulsant \& Rey, 1863)

Xenoscelis costipennis (Fairmaire, 1852)

Erotylinae Latreille, 1802

\begin{tabular}{|c|c|c|c|c|c|c|c|c|}
$\mathrm{H}$ & $\mathrm{X}$ & & & $\mathrm{N}$ & $\mathrm{N}$ & & & \\
$\mathrm{X}$ & $\mathrm{X}$ & & & & & & $\mathrm{N}$ & LC \\
\hline
\end{tabular}

Dacnini Crotch, 1876

Dacne bipustulata (Thunberg, 1781)

Dacne pontica (Bedel, 1868)

Dacne rufifions (Fabricius, 1775)

\begin{tabular}{|c|c|c|c|c|c|c|c|}
\hline $\mathrm{X}$ & & & & & & & LC \\
& & $\mathrm{N}$ & & $\mathrm{X}$ & $\mathrm{X}$ & & NT \\
& & & & $\mathrm{X}$ & $\mathrm{X}$ & $\mathrm{NT}$ \\
\hline
\end{tabular}

Triplax aenea (Schaller, 1783)

Triplax collaris (Schaller, 1783)

Triplax lacordairii Crotch, 1870

Triplax lepida (Faldermann, 1837)

Triplax marseuli Bedel, 1868

Triplax russica (Linnaeus, 1758)

Triplax scutellaris Charpentier, 1825

Tritoma bipustulata Fabricius, 1775

MONOTOMIDAE Laporte, 1840

Tritomini Curtis, 1834

Rhizophaginae L. Redtenbacher, 1845

Jelinek in Cat. Pal. Col., vol. 4, 2007; Otero, 2011

Rhizophaguis bipustulatus (Fabricius, 1792)

Rhizophagus depressus (Fabricius, 1792)

Rhizophaguis dispar (Paykull, 1800)

\begin{tabular}{|l|l|l|l|l|l|l|l|l|}
\hline $\mathrm{H}$ & $\mathrm{X}$ & $\mathrm{N}$ & $\mathrm{N}$ & $\mathrm{N}$ & $\mathrm{X}$ & $\mathrm{X}$ & $\mathrm{N}$ & LC \\
\hline $\mathrm{X}$ & & & & & & & & DD \\
\hline $\mathrm{X}$ & $\mathrm{X}$ & & $\mathrm{N}$ & $\mathrm{N}$ & $\mathrm{X}$ & $\mathrm{X}$ & $\mathrm{N}$ & LC \\
\hline
\end{tabular}


Species

Rhizophagus ferrugineus (Paykull, 1800)

Rhizophagus nitidulus (Fabricius, 1798)

Rhizophagus unicolor P.H. Lucas, 1849

\section{Monotominae Laporte, 1840}

\begin{tabular}{|c|c|c|c|c|c|c|c|c|}
\hline 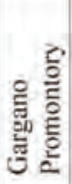 & $\begin{array}{l}7 \\
0 \\
\frac{0}{0} \\
0 \\
\frac{0}{0} \\
\frac{.0}{0} \\
2\end{array}$ & 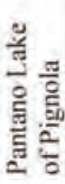 & 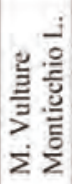 & 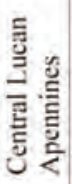 & 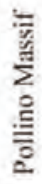 & & 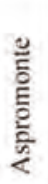 & 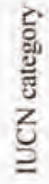 \\
\hline & & & & & & $\mathrm{X}$ & & LC \\
\hline X & & & & & X & & & NT \\
\hline $\mathrm{H}$ & & & & & & & & LC \\
\hline
\end{tabular}

Monotoma bicolor A. Villa \& G.B. Villa, 1835

Monotoma brevicollis brevicollis Aubé, 1838

Monotoma diecki Reitter, 1877

Monotoma johnsoni Bousquet \& Laplante, 1999

Monotoma longicollis (Gyllenhal, 1827)

Monotoma picipes Herbst, 1793

Monotoma spinicollis Aubé, 1837

\section{KATERETIDAE Erichson, 1846}

\begin{tabular}{|c|c|c|c|c|c|c|c|c|} 
& $\mathrm{N}$ & & & $\mathrm{N}$ & $\mathrm{N}$ & $\mathrm{N}$ & & LC \\
\hline $\mathrm{X}$ & $\mathrm{N}$ & $\mathrm{X}$ & $\mathrm{N}$ & $\mathrm{N}$ & $\mathrm{N}$ & & $\mathrm{N}$ & LC \\
\hline & $\mathrm{N}$ & & & & & & & LC \\
\hline & & $\mathrm{N}$ & & $\mathrm{N}$ & & & & LC \\
\hline $\mathrm{X}$ & $\mathrm{X}$ & $\mathrm{X}$ & & & $\mathrm{X}$ & & & LC \\
\hline & $\mathrm{X}$ & $\mathrm{X}$ & $\mathrm{N}$ & & & & $\mathrm{N}$ & LC \\
\hline $\mathrm{X}$ & $\mathrm{X}$ & & $\mathrm{N}$ & & & & $\mathrm{N}$ & LC \\
\hline
\end{tabular}

Jelinek in Cat. Pal. Col., vol. 4, 2007

Brachypterolus antirrhini (Murray, 1864)

Brachypterolus linariae (Stephens, 1830)

Brachypterolus pulicarius (Linnaeus, 1758)

Brachypterus curtulus Wollaston, 1864

Brachypterus glaber (Newman, 1834)

Brachypterus urticae (Fabricius, 1792)

Kateretes dalmatimus (Sturm, 1844)

Kateretes pusillus (Thunberg, 1794)

Kateretes rufilabris (Latreille, 1807)

\section{NITIDULIDAE Latreille, 1802}

\begin{tabular}{|c|c|c|c|c|c|c|c|c|}
\hline H & & & & & & X & & \\
\hline B & & & N & N & X & & B & \\
\hline H & & & & & & & & \\
\hline & B & & & & & & & \\
\hline X & X & & & & & X & N & \\
\hline & X & & N & & X & X & N & \\
\hline & & & & B & & & & \\
\hline X & X & & & N & N & & \\
\hline
\end{tabular}

Epuraeinae Kirejtshuk, 1986

Jelinek \& Audisio in Cat. Pal. Col., vol. 4, 2007; Cline et al., 2014

\begin{tabular}{|c|c|c|c|c|c|c|c|c|c|}
\hline Epuraea unicolor (A.G. Olivier, 1790) & $\mathrm{H}$ & $\mathrm{X}$ & $\mathrm{X}$ & $\mathrm{N}$ & $\mathrm{N}$ & $\mathrm{X}$ & $\mathrm{X}$ & $\mathrm{N}$ & LC \\
\hline Epuraea thoracica Tournier, 1872 & & & & & & B & & & VU \\
\hline Epuraea terminalis (Mannerheim, 1843) & & & & & & $\mathrm{CK}$ & & & LC \\
\hline Epuraea silacea (Herbst, 1783) & & & & & CK & $\mathrm{X}$ & & & VU \\
\hline Epuraea rufomarginata (Stephens, 1830) & & & & & & B & & & VU \\
\hline Epuraea pallescens (Stephens, 1832) & $\mathrm{H}$ & & & $\mathrm{N}$ & & $\mathrm{X}$ & $\mathrm{X}$ & & LC \\
\hline Epuraea ocularis Fairmaire, 1849 & & & & & & & & B & \\
\hline Epuraea melina Erichson, 1843 & CK & & & & CK & $\mathrm{X}$ & $\mathrm{X}$ & CK & \\
\hline Epuraea melanocephala (Marsham, 1802) & $\mathrm{H}$ & & CK & & $\mathrm{CK}$ & CK & B & & LC \\
\hline Epuraea marseuli Reitter, 1873 & & & & & & & $\mathrm{X}$ & B & LC \\
\hline Epuraea luteola Erichson, 1843 & & & & $\mathrm{~N}$ & $\mathrm{~N}$ & $\mathrm{~N}$ & & B & \\
\hline Epuraea longula Erichson, 1845 & & & $\mathrm{X}$ & & & $\mathrm{X}$ & & $\mathrm{CK}$ & LC \\
\hline Epuraea imperialis (Reitter, 1877) & & & & & & & & B & \\
\hline Epuraea guttata (A.G. Olivier, 1811) & & $\mathrm{X}$ & & & CK & $\mathrm{X}$ & $\mathrm{B}$ & & LC \\
\hline Epuraea fuscicollis (Stephens, 1835) & & $\mathrm{N}$ & $\mathrm{X}$ & $\mathrm{N}$ & CK & $\mathrm{X}$ & $\mathrm{X}$ & CK & LC \\
\hline Epuraea fageticola Audisio, 1991 & $\mathrm{X}$ & $\mathrm{X}$ & & & B & B & B & & VU \\
\hline Epuraea biguttata (Thunberg, 1784) & & & & & & B & & & LC \\
\hline Epuraea angustula Sturm, 1844 & & $\mathrm{X}$ & & & $\mathrm{N}$ & B & & & $\mathrm{VU}$ \\
\hline Epuraea aestiva (Linnaeus, 1758) & $\mathrm{X}$ & & & $\mathrm{N}$ & CK & $\mathrm{X}$ & $\mathrm{X}$ & CK & \\
\hline \multicolumn{10}{|c|}{ Carpophilinae Erichson, 1842} \\
\hline Carpophilus bifenestratus Murray, 1864 & & $\mathrm{X}$ & & $\mathrm{N}$ & $\mathrm{N}$ & $\mathrm{N}$ & & $\mathrm{N}$ & \\
\hline Carpophilus bipustulatus (Heer, 1841) & & $\mathrm{X}$ & CK & & & B & $\mathrm{B}$ & & LC \\
\hline Carpophilus hemipterus (Linnaeus, 1758) & $\mathrm{X}$ & $\mathrm{X}$ & $\mathrm{X}$ & $\mathrm{N}$ & $\mathrm{N}$ & CK & & & \\
\hline Carpophilus marginellus Motschulsky, 1858 & & CK & $\mathrm{X}$ & & & CK & & $\mathrm{N}$ & \\
\hline Carpophilus mutilatus Erichson, 1843 & $\mathrm{X}$ & $\mathrm{X}$ & & $\mathrm{N}$ & $\mathrm{N}$ & CK & & $\mathrm{N}$ & \\
\hline Carpophilus nepos Murray, 1864 & $\mathrm{X}$ & $\mathrm{X}$ & $\mathrm{X}$ & & $\mathrm{N}$ & CK & & B & \\
\hline Carpophilus obsoletus Erichson, 1843 & B & $\mathrm{X}$ & & & & $\mathrm{CK}$ & & & \\
\hline
\end{tabular}


Species

Carpophilus quadrisignatus Erichson, 1843

Carpophilus sexpustulatus (Fabricius, 1792)

Urophorns humeralis (Fabricius, 1798)

Urophorus rubripennis (Heer, 1841)

Cryptarchinae C.G. Thomson, 1859

Cryptarcha strigata (Fabricius, 1787)

Cryptarcha undata (A.G. Olivier, 1790)

Glischrochilus quadriguttatus (Fabricius, 1777)

Glischrochilus quadripunctatus (Linnaeus, 1758)

Pityophagus ferrugineus (Linnaeus, 1761)

Nitidulinae Latreille, 1802

Aethina tumida Murray, 1867

Amphotis marginata (Fabricius, 1781)

Cycramus luteus (Fabricius, 1787)

Ipidia binotata Reitter, 1875

Nitidula carnaria (Schaller, 1783)

Nitidula flavomaculata P. Rossi, 1790

Nitidula rufipes (Linnaeus, 1767)

Omosita colon (Linnaeus, 1758)

Omosita discoidea (Fabricius, 1775)

Pocadius ferrugineus (Fabricius, 1775)

Soronia grisea (Linnaeus, 1758)

Soronia oblonga C.N.F. Brisout de Barneville, 1863

Stelidota geminata (Say, 1825)

Thalycra fervida (A.G. Olivier, 1790)

Xenostrongylus arcuatus Kiesenwetter, 1859

Xenostrongylus lateralis Chevrolat, 1861

\begin{tabular}{|c|c|c|c|c|c|}
\hline $\mathrm{X}$ & $\mathrm{X}$ & CK & $\mathrm{X}$ & $\mathrm{X}$ & LC \\
\hline & & CK & X & & NT \\
\hline $\mathrm{H}$ & & & & & $\mathrm{VU}$ \\
\hline & & & B & $X$ & NT \\
\hline & & & B & $\mathrm{X}$ & LC \\
\hline
\end{tabular}

\section{Nitidulinae Latreille, 1802}

\begin{tabular}{|c|c|c|c|c|c|c|c|c|}
\hline & & & & & & & B & \\
\hline \multirow{3}{*}{$X$} & & & & B & CK & $X$ & & LC \\
\hline & CK & & & & CK & & & LC \\
\hline & & & & & $X$ & B & B & $\mathrm{VU}$ \\
\hline $\mathrm{X}$ & $\mathrm{X}$ & $\mathrm{X}$ & $\mathrm{N}$ & & $X$ & $\mathrm{X}$ & & \\
\hline $\mathrm{X}$ & $\mathrm{X}$ & & & & CK & & & \\
\hline \multirow[t]{2}{*}{ CK } & $X$ & CK & CK & & CK & CK & $\mathrm{N}$ & \\
\hline & & & & & & B & B & \\
\hline CK & $X$ & CK & & CK & CK & CK & CK & \\
\hline \multirow{2}{*}{$\mathrm{X}$} & & & $\mathrm{N}$ & $\mathrm{N}$ & $X$ & $X$ & & \\
\hline & $X$ & $\mathrm{X}$ & & $\mathrm{N}$ & & $X$ & $\mathrm{~N}$ & LC \\
\hline \multirow[t]{3}{*}{$\mathrm{X}$} & $X$ & & CK & CK & $X$ & $X$ & CK & LC \\
\hline & & & & & & & B & \\
\hline & & & & CK & $\mathrm{CK}$ & $X$ & CK & \\
\hline \multirow[t]{2}{*}{$\mathrm{X}$} & $X$ & $x$ & & & $\mathrm{CK}$ & $X$ & CK & \\
\hline & & & & & & & B & \\
\hline
\end{tabular}

Cillaeinae Kirejtshuk \& Audisio, 1986

Colopterus abdominalis (Erichson, 1843)

\section{Meligethinae C.G. Thomson, 1859}

Audisio et al., 2009 for ex genus Meligethes

Acanthogethes brevis (Sturm, 1845)

Acanthogethes fuscus (A.G. Olivier, 1790)

Acanthogethes reyi (Guillebeau, 1885)

Acanthogethes solidus (Kugelann, 1794)

Afrogethes buduensis (Ganglbauer, 1899)

Afrogethes planiusculus (Heer, 1841)

Astylogethes substrigosus (Erichson, 1845)

Brassicogethes aeneus (Fabricius, 1775)

Brassicogethes anthracinus (C.N.F. Brisout de Barneville, 1863)

Brassicogethes arankae (Audisio \& De Biase, 2005)

Brassicogethes coracinus (Sturm, 1845)

Brassicogethes erysimicola (Audisio \& De Biase, 2001)

Brassicogethes lunariae (Audisio \& De Biase, 1999)

Brassicogethes matronalis (Audisio \& Spornraft, 1990)

Brassicogethes spornrafti (Audisio, 1977)

Brassicogethes viridescens (Fabricius, 1787)

Clypeogethes lepidii (L. Miller, 1851)

Clypeogethes tener (Reitter, 1873)

Fabogethes brachialis Erichson, 1845

Fabogethes nigrescens (Stephens, 1830)

Genistogethes bidentatus (C.N.F. Brisout de Barneville, 1863)

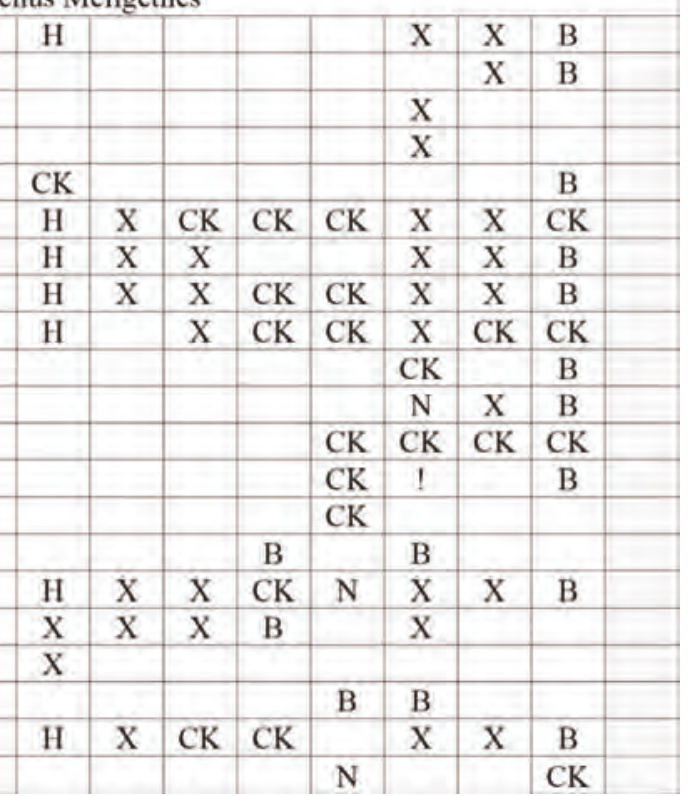


Species

Genistogethes carimulatus (Forster, 1849)

Genistogethes erichsoni (C.N.F. Brisout de Barneville, 1863)

Genistogethes immundus (Kraatz, 1858)

Genistogethes punctatus (C.N.F. Brisout de Barneville, 1863)

Lamiogethes angustatus (Küster, 1848)

Lamiogethes bidens (C.N.F. Brisout de Barneville, 1863)

Lamiogethes brunnicornis (Sturm, 1845)

Lamiogethes bucciarellii (Audisio, 1976)

Lamiogethes difficilis (Heer, 1841)

Lamiogethes jelineki (Audisio, 1976)

Lamiogethes kunzei (Erichson, 1845)

Lamiogethes morosus (Erichson, 1845)

Lamiogethes paschalis (Spornraft, 1975) [E]

Lamiogethes pedicularius (Gyllenhal, 1808)

Lamiogethes persicus (Faldermann, 1835)

Meligethes atratus (A.G. Olivier, 1790)

Meligethes denticulatus (Heer, 1841)

Meligethes flavimamus Stephens, 1830

Pria dulcamarae (Scopoli, 1763)

Sagittogethes distinctus Sturm, 1845

Sagittogethes hoffmanni (Reitter, 1871)

Sagitlogethes lindbergi (Rebmann, 1940)

Sagittogethes maurus (Sturm, 1845)

Sagittogethes minutus (C.N.F. Brisout de Barneville, 1863)

Sagittogethes obscurus (Erichson, 1845)

Sagittogethes ovatus (Sturm, 1845)

Sagittogethes umbrosus (Sturm, 1845)

Stachygethes namus (Erichson, 1845)

Stachygethes ruficornis (Marsham, 1802)

Stachygethes scholzi (Easton, 1960)

Stachygethes villosus (C.N.F. Brisout de Barneville, 1863)

Thymogethes acicularis (C.N.F. Brisout de Barneville, 1863)

Thymogethes egemus (Erichson, 1845)

Thymogethes exilis Sturm, 1845

Thymogethes fumatus (Erichson, 1845)

Thymogethes funereus (Jelinek, 1967)

Thymogethes gagathimus (Erichson, 1845)

Thymogethes lugubris (Sturm, 1845)

Thymogethes submetallicus (Sainte-Claire Deville, 1908)

Xerogethes rotundicollis (C.N.F. Brisout de Barneville, 1863)

\begin{tabular}{|c|c|c|c|c|c|c|c|c|}
\hline 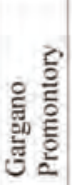 & $\begin{array}{l}70 \\
0 \\
3 \\
0 \\
0 \\
\frac{3}{0} \\
2\end{array}$ & 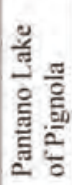 & 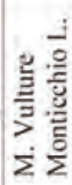 & 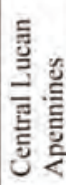 & 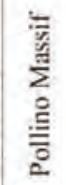 & 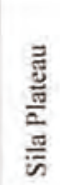 & 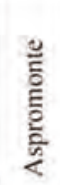 & 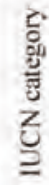 \\
\hline $\mathrm{H}$ & $\mathrm{X}$ & $X$ & $\mathrm{~N}$ & $\mathrm{~N}$ & CK & $\mathrm{X}$ & CK & \\
\hline \multirow[t]{2}{*}{$\mathrm{H}$} & & & & & $\mathrm{X}$ & $\mathrm{X}$ & CK & \\
\hline & & & & & & CK & B & \\
\hline $\mathrm{X}$ & $\mathrm{X}$ & $\mathrm{X}$ & $\mathrm{N}$ & CK & $X$ & $X$ & B & \\
\hline$X$ & & & & CK & $\mathrm{X}$ & & $\mathrm{CK}$ & \\
\hline $\mathrm{H}$ & & & & & CK & $X$ & CK & \\
\hline $\mathrm{H}$ & B & & & & CK & B & & \\
\hline$X$ & & & & B & B & & & \\
\hline \multirow[t]{3}{*}{$\mathrm{X}$} & & & & & CK & $X$ & B & \\
\hline & & & & B & B & B & & \\
\hline & & & & & B & & B & \\
\hline \multirow[t]{4}{*}{$\mathrm{H}$} & & & & & CK & & B & \\
\hline & & & & B & $\mathrm{X}$ & & B & \\
\hline & & & & & B & & & \\
\hline & & & & & B & & & \\
\hline \multirow[t]{3}{*}{ CK } & $\mathrm{X}$ & & $\mathrm{N}$ & CK & $\mathrm{X}$ & $\mathrm{X}$ & CK & \\
\hline & & & & & & B & & \\
\hline & & $X$ & $\mathrm{~N}$ & CK & $\mathrm{X}$ & $X$ & & \\
\hline \multirow[t]{3}{*}{$\mathrm{H}$} & & CK & CK & & & CK & CK & \\
\hline & & & & & B & & B & \\
\hline & CK & $\mathrm{X}$ & & & & & B & \\
\hline B & & & & B & & & B & \\
\hline \multirow[t]{4}{*}{ CK } & & & & & & & & \\
\hline & & & & & & & B & \\
\hline & & & & & B & & B & \\
\hline & & & B & & & & & \\
\hline $\mathrm{H}$ & $\mathrm{X}$ & $\mathrm{X}$ & $\mathrm{N}$ & CK & $\mathrm{X}$ & $\mathrm{X}$ & B & \\
\hline $\mathrm{X}$ & $\mathrm{X}$ & & & & CK & & B & \\
\hline $\mathrm{H}$ & $\mathrm{X}$ & CK & & & CK & & B & \\
\hline CK & & & & & B & & B & \\
\hline $\mathrm{H}$ & $\mathrm{X}$ & & & & CK & & CK & \\
\hline \multirow[t]{2}{*}{ CK } & & & & & CK & $X$ & B & \\
\hline & $\mathrm{N}$ & $X$ & B & $\mathrm{N}$ & & CK & B & \\
\hline $\mathrm{H}$ & & & & & CK & & & \\
\hline $\mathrm{H}$ & $\mathrm{X}$ & & & & CK & & & \\
\hline \multirow[t]{3}{*}{$\mathrm{X}$} & & $\mathrm{X}$ & & & CK & & CK & \\
\hline & CK & $\mathrm{X}$ & CK & & CK & & B & \\
\hline & & & & & & B & & \\
\hline $\mathrm{H}$ & $\mathrm{X}$ & $\mathrm{X}$ & & B & B & & B & \\
\hline $\mathrm{H}$ & $\mathrm{X}$ & $\mathrm{X}$ & $\mathrm{N}$ & & CK & CK & CK & \\
\hline
\end{tabular}

\section{CRYPTOPHAGIDAE Kirby, 1837}

\section{Cryptophaginae Kirby, 1837}

Caenoscelini Casey, 1900

Johnson, Otero \& Leschen in Cat. Pal. Col., vol. 4, 2007; Otero, 2011, 2013

Caenoscelis angelinii C. Johnson \& S. Bowestead, 2003 [E]

Caenoscelis ferruginea (C.R. Sahlberg, 1820)

Sternodea baudii Reitter, 1875

\section{Cryptophagini Kirby, 1837}

Anterophagus pallens (Linnaeus, 1758)

Anterophaguis silaceus (Herbst, 1792)

Cryptophagus badius Sturm, 1845

Cryptophagus cylindrellus C. Johnson, 2007

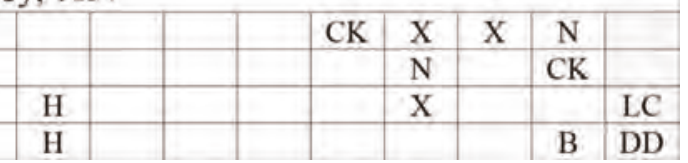


Species

Cryptophagus dentatus (Herbst, 1793)

Cryptophagus denticulatus Heer, 1841

Cryptophagus distinguendus Sturm, 1845

Cryplophagus dorsalis C.R. Sahlberg, 1819

Cryptophagus falcozi Roubal, 1927

Cryptophagus fasciatus Kraatz, 1852

Cryplophagus jakowlewi Reitter, 1888

Cryptophagus labilis Erichson, 1846

Cryptophagus laticollis P.H. Lucas, 1846

Cryptophagus lycoperdi (Scopoli, 1763)

Cryptophagus micaceus Rey, 1889

Cryptophagus pallidus Sturm, 1845

Cryptophagus parallelus C.N.F. Brisout de Barneville, 1863

Cryptophagus populi Paykull, 1800

Cryptophagus pubescens Sturm, 1845

Cryptophagus puncticollis P.H. Lucas, 1846

Cryptophagus ruficormis Stephens, 1830

Cryptophagus saginatus Sturm, 1845

Cryptophagus scanicus (Linnaeus, 1758)

Cryptophagus schroetteri Reitter, 1912

Cryptophagus setulosus Sturm, 1845

Cryptophagus sporadum Bruce, 1934

Cryptophagus uncinatus Stephens, 1830

Henoticus serratus (Gyllenhal, 1808)

Micrambe abietis (Paykull, 1798)

Micrambe ulicis (Stephens, 1830)

Micrambe umbripennis (Reitter, 1888)

Micrambe woodroffei C. Johnson, 2007

Paramecosoma melanocephalum (Herbst, 1793)

Pteryngium cremulatum (Erichson, 1846)

\begin{tabular}{|c|c|c|c|c|c|c|c|c|}
\hline 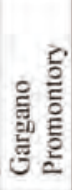 & $\begin{array}{l}7 \\
8 \\
3 \\
0 \\
0 \\
\frac{3}{0} \\
2\end{array}$ & 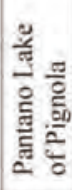 & 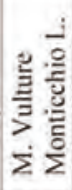 & 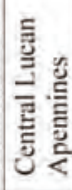 & 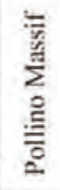 & 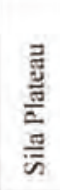 & 总 & 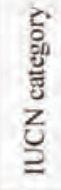 \\
\hline $\mathrm{H}$ & $X$ & & $\mathrm{~N}$ & & $\mathrm{X}$ & & B & LC \\
\hline $\mathrm{H}$ & $\mathrm{X}$ & $X$ & $\mathrm{~N}$ & B & $\mathrm{X}$ & $\mathrm{X}$ & CK & LC \\
\hline \multirow[t]{3}{*}{$\mathrm{N}$} & & & & $\mathrm{N}$ & & & $\mathrm{N}$ & \\
\hline & & & & & & & $\mathrm{N}$ & DD \\
\hline & & & & CK & CK & & & DD \\
\hline \multirow[t]{3}{*}{$\mathrm{N}$} & $\mathrm{X}$ & & & & & & CK & LC \\
\hline & $X$ & & & & & & & LC \\
\hline & & & & & CK & $\mathrm{N}$ & & VU \\
\hline $\mathrm{H}$ & $\mathrm{X}$ & & & $\mathrm{N}$ & $\mathrm{N}$ & CK & $\mathrm{N}$ & LC \\
\hline \multirow[t]{2}{*}{$\mathrm{X}$} & CK & & CK & $\mathrm{N}$ & & & CK & \\
\hline & & $\mathrm{N}$ & & $\mathrm{N}$ & & & & \\
\hline \multirow[t]{2}{*}{$\mathrm{X}$} & $\mathrm{X}$ & $\mathrm{X}$ & B & CK & $\mathrm{X}$ & $\mathrm{X}$ & B & LC \\
\hline & & & & $\mathrm{N}$ & & & & DD \\
\hline CK & CK & & & & & & & DD \\
\hline $\mathrm{H}$ & & & & B & & & CK & \\
\hline X & $\mathrm{X}$ & & & B & CK & & & DD \\
\hline \multirow[t]{2}{*}{$\mathrm{H}$} & & & & $\mathrm{N}$ & & & & LC \\
\hline & CK & & & $\mathrm{N}$ & $X$ & $X$ & CK & \\
\hline \multirow[t]{3}{*}{$\mathrm{H}$} & CK & $\mathrm{X}$ & CK & B & $X$ & $\mathrm{X}$ & CK & LC \\
\hline & & & & & & & B & DD \\
\hline & & & & CK & & & & DD \\
\hline $\mathrm{X}$ & & & & & & & & DD \\
\hline $\mathrm{H}$ & CK & $X$ & CK & CK & $\mathrm{N}$ & & CK & LC \\
\hline \multirow[t]{2}{*}{$\mathrm{H}$} & $X$ & & & & & $\mathrm{X}$ & & LC \\
\hline & & & & & $\mathrm{N}$ & $X$ & B & DD \\
\hline \multirow[t]{2}{*}{$\mathrm{H}$} & $\mathrm{X}$ & & & & $\mathrm{N}$ & & CK & \\
\hline & & & & & & & B & DD \\
\hline \multirow[t]{3}{*}{ CK } & $X$ & & $\mathrm{~N}$ & & $\mathrm{~N}$ & CK & CK & \\
\hline & & & & & CK & & $\mathrm{N}$ & LC \\
\hline & & & & & CK & & & LC \\
\hline
\end{tabular}

Telmatophilinae Jacquelin du Val, 1858

Telmatophilus brevicollis Aubé, 1862

Telmatophilus caricis (A.G. Olivier, 1790)

Telmatophilus typhae (Fallén, 1802)

\begin{tabular}{|c|c|c|c|c|c|c|c|}
\hline B & $\mathrm{X}$ & & & & & & \\
\hline $\mathrm{X}$ & $\mathrm{X}$ & & & & & $\mathrm{CK}$ & \\
\hline $\mathrm{B}$ & $\mathrm{X}$ & & & & & $\mathrm{N}$ & \\
\hline
\end{tabular}

Atomariinae LeConte, 1861

Atomariini LeConte, 1861

\begin{tabular}{|c|c|c|c|c|c|c|c|c|c|}
\hline Atomaria affinis (R.F. Sahlberg, 1834) & CK & & & & & CK & & & \\
\hline Atomaria analis Erichson, 1846 & $\mathrm{H}$ & & $\mathrm{N}$ & CK & CK & $\mathrm{N}$ & & $\mathrm{N}$ & \\
\hline Atomaria apicalis Erichson, 1846 & $\mathrm{H}$ & $\mathrm{X}$ & $\mathrm{X}$ & & B & & $\mathrm{X}$ & & \\
\hline Atomaria atricapilla Stephens, 1830 & $\mathrm{H}$ & $\mathrm{N}$ & CK & CK & $\mathrm{N}$ & CK & CK & $\mathrm{CK}$ & \\
\hline Alomaria gibbula Erichson, 1846 & & & & & CK & & & & \\
\hline Atomaria gravidula Erichson, 1846 & & & $\mathrm{~N}$ & $\mathrm{~N}$ & & $\mathrm{~N}$ & & & \\
\hline Atomaria gutta Newman, 1834 & & $\mathrm{X}$ & & & & & & & \\
\hline Atomaria impressa Erichson, 1846 & & & & & & & & $\mathrm{CK}$ & \\
\hline Atomaria linearis Stephens, 1830 & & & $\mathrm{X}$ & & & & & & DD \\
\hline Atomaria nigripennis (Kugelann, 1794) & & & & & $\mathrm{N}$ & & & & \\
\hline Atomaria nigrirostris Stephens, 1830 & $\mathrm{H}$ & $\mathrm{N}$ & $\mathrm{X}$ & $\mathrm{N}$ & $\mathrm{N}$ & CK & CK & CK & LC \\
\hline Atomaria nigriventris Stephens, 1830 & CK & & & & CK & & $\mathrm{CK}$ & & \\
\hline Atomaria ornata Heer, 1841 & & & & & & $\mathrm{~N}$ & & CK & \\
\hline Atomaria pallidipennis Holdhaus, 1903 & & $\mathrm{~N}$ & & & $\mathrm{~N}$ & & & & \\
\hline Atomaria parvula Reitter, 1875 [E] & & & & & CK & CK & & & \\
\hline Atomaria pulchra Erichson, 1846 & & & $\mathrm{X}$ & & & & & & \\
\hline Atomaria puncticollis C.G. Thomson, 1868 & & & & & CK & & & & \\
\hline
\end{tabular}


Species

Atomaria pusilla (Paykull, 1798)

Atomaria rubida Reitter, 1875

Atomaria scutellaris Motschulsky, 1849

Atomaria slavonica C. Johnson, 1971

Atomaria testacea Stephens, 1830

Curelius exiguus (Erichson, 1846)

Curelius japonicus (Reitter, 1878)

Ephistemus globulus (Paykull, 1798)

Ephistemus reitteri Casey, 1900

Ootypus globosus Waltl, 1838

Hypocoprus lathridioides Motschulsky, 1839

Hypocoprinae Reitter, 1879

\begin{tabular}{|c|c|c|c|c|c|c|c|c|}
\hline 㝴 & $\begin{array}{l}3 \\
8 \\
3 \\
0 \\
\frac{0}{0} \\
\frac{0}{0} \\
0\end{array}$ & 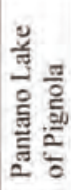 & 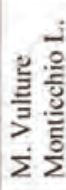 & 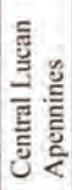 & 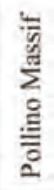 & 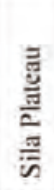 & 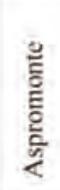 & 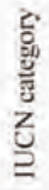 \\
\hline $\mathrm{H}$ & & $\mathrm{X}$ & $\mathrm{N}$ & CK & $\mathrm{X}$ & $\mathrm{X}$ & CK & \\
\hline $\mathrm{X}$ & $X$ & & & & & CK & & \\
\hline $\mathrm{H}$ & $\mathrm{X}$ & CK & CK & CK & $\mathrm{N}$ & $X$ & $\mathrm{~N}$ & \\
\hline & $\mathrm{X}$ & & & CK & X & & CK & \\
\hline $\mathrm{H}$ & $\mathrm{X}$ & $\mathrm{X}$ & $\mathrm{N}$ & B & $\mathrm{X}$ & $\mathrm{X}$ & CK & \\
\hline CK & $X$ & $\mathrm{X}$ & & $\mathrm{N}$ & & & CK & LC \\
\hline & $\mathrm{N}$ & & & $\mathrm{N}$ & & & CK & \\
\hline $\mathrm{X}$ & $\mathrm{X}$ & $\mathrm{X}$ & & CK & CK & $\mathrm{X}$ & CK & \\
\hline & & $\mathrm{N}$ & & $\mathrm{N}$ & & & & \\
\hline & & X & & & & & $\mathrm{N}$ & \\
\hline
\end{tabular}

SILVANIDAE Kirby, 1837

Brontinae Erichson, 1845

Brontini Erichson, 1845

Halstead, Löbl \& Jelinek in Cat. Pal. Col., vol. 4, 2007

Uleiota planatus (Linnaeus, 1761)

Telephanini LeConte, 1861

\begin{tabular}{|c|c|c|c|c|c|c|c|c|c|}
\hline Psammoecus bipumctanus (Fabricius, 1792) & $\mathrm{X}$ & $\mathrm{X}$ & $\mathrm{X}$ & & & $\mathrm{X}$ & & $\mathrm{N}$ & \\
\hline \multicolumn{10}{|c|}{ Silvaninae Kirby, 1837} \\
\hline Ahasverus advena (Waltl, 1834) & $\mathrm{X}$ & $\mathrm{X}$ & $\mathrm{X}$ & $\mathrm{N}$ & $\mathrm{N}$ & $\mathrm{X}$ & & $\mathrm{N}$ & \\
\hline Airaphilus calabricus Obenberger, 1914 [E] & & & & & & & & B & \\
\hline Airaphilus elongatus (Gyllenhal, 1813) & $\mathrm{X}$ & $\mathrm{X}$ & $\mathrm{X}$ & $\mathrm{N}$ & $\mathrm{N}$ & & & $\mathrm{N}$ & \\
\hline Airaphilus talpa (Kraatz, 1862) [E] & B & B & & & & & & & \\
\hline Oryzaephilus surinamensis (Linnaeus, 1758) & $\mathrm{X}$ & $\mathrm{X}$ & $\mathrm{X}$ & $\mathrm{N}$ & $\mathrm{N}$ & $\mathrm{X}$ & & B & \\
\hline Silvamus unidentatus (A.G. Olivier, 1790) & $\mathrm{H}$ & $\mathrm{X}$ & $\mathrm{X}$ & $\mathrm{N}$ & B & $\mathrm{X}$ & $\mathrm{X}$ & $\mathrm{N}$ & LC \\
\hline
\end{tabular}

CUCUJIDAE Latreille, 1802

Wegrzynowic in Cat. Pal. Col., vol. 4, 2007

Cucujus cinnaberimus (Scopoli, 1763)

Cucujus haematodes (Erichson, 1845)

Cucujus tulliae Bonacci, Mazzei, Horák \& Brandmayr, 2012 [E]

Pediacus dermestoides (Fabricius, 1792)

PHALACRIDAE Leach, 1815

Phalacrinae Leach, 1815

Olibrus aenescens Küster, 1852

Olibrus aeneus (Fabricius, 1792)

Olibrus affinis (Sturm, 1807)

Olibrus bicolor (Fabricius, 1792)

Olibrus bimaculatus Küster, 1848

Olibrus bisignatus (Ménétriés, 1849)

Olibrus castaneus Baudi di Selve, 1870

Olibrus corticalis (Panzer, 1797)

Olibrus demarzoi Švee \& Angelini, 1996

Olibrus fallax Flach, 1888

Olibrus flavicornis (Sturm, 1807)

Olibrus liquidus Erichson, 1845

Olibrus millefolii (Paykull, 1800)

Olibrus pygmaeus (Sturm, 1807)

Olibrus reitleri Flach, 1888

Olibruis stierlini Flach, 1888

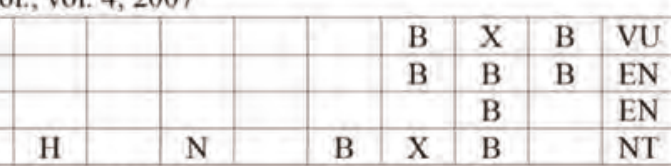

\section{Svec in Cat. Pal. Col., vol. 4, 2007}




\begin{tabular}{|c|c|c|c|c|c|c|c|c|c|}
\hline Species & 总 & $\begin{array}{l}\bar{\zeta} \\
0 \\
3 \\
0 \\
0 \\
\frac{0}{0} \\
2\end{array}$ & 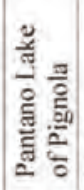 & 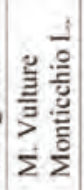 & 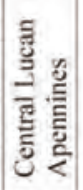 & 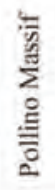 & 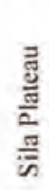 & $\frac{\mathscr{\Xi}}{\mathrm{E}}$ & 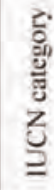 \\
\hline Phalacrus corruscus (Panzer, 1797) & & $\mathrm{B}$ & $\mathrm{X}$ & B & B & $\mathrm{B}$ & B & B & \\
\hline Phalacrus fimetarius (Fabricius, 1775) & $\mathrm{H}$ & B & $\mathrm{X}$ & B & B & $\mathrm{N}$ & & $\mathrm{N}$ & \\
\hline Stilbus atomarius (Linnaeus, 1767) & $\mathrm{H}$ & & & & & & & & \\
\hline Stilbus oblongus (Erichson, 1845) & & & $\mathrm{N}$ & & & $\mathrm{B}$ & & & \\
\hline Stilbus testaceus (Panzer, 1797) & $\mathrm{H}$ & B & $\mathrm{X}$ & B & $\mathrm{N}$ & $\mathrm{B}$ & & $\mathrm{N}$ & \\
\hline Tolyphus granulatus (Guérin-Méneville, 1834) & & & $\mathrm{X}$ & & B & $\mathrm{B}$ & & $\mathrm{N}$ & \\
\hline Tolyphus punctulatus Rosenhauer, 1856 & $\mathrm{X}$ & $\mathrm{X}$ & & & B & $\mathrm{X}$ & & & \\
\hline \multicolumn{10}{|c|}{$\begin{array}{c}\text { LAEMOPHLOEIDAE Ganglbauer, } 1899 \\
\text { Laemophloeinae Ganglbauer, 1899 } \\
\text { Wegrzynowic in Cat. Pal. Col., vol. 4, 2007 }\end{array}$} \\
\hline Cryptolestes capensis (Waltt, 1834) & $\mathrm{X}$ & $\mathrm{X}$ & & & & & & & \\
\hline Cryptolestes duplicatus (Waltl, 1839) & $\mathrm{H}$ & $\mathrm{X}$ & & & B & $\mathrm{N}$ & & $\mathrm{N}$ & NT \\
\hline Cryptolestes ferrugineus (Stephens, 1831) & $\mathrm{H}$ & $\mathrm{X}$ & & & CK & $\mathrm{X}$ & $\mathrm{X}$ & $\mathrm{N}$ & LC \\
\hline Cryptolestes fractipennis (Motschulsky, 1845) & $\mathrm{X}$ & B & & $\mathrm{N}$ & $\mathrm{B}$ & $\mathrm{B}$ & $\mathrm{X}$ & & LC \\
\hline Cryptolestes spartii (Curtis, 1834) & & $\mathrm{X}$ & & & & $\mathrm{X}$ & & $\mathrm{N}$ & LC \\
\hline Laemophloeus monilis (Fabricius, 1787) & $\mathrm{X}$ & $\mathrm{X}$ & & & $\mathrm{N}$ & $\mathrm{X}$ & $\mathrm{X}$ & $\mathrm{N}$ & LC \\
\hline Laemophloens nigricollis P.H. Lucas, 1849 & & $\mathrm{~B}$ & $\mathrm{X}$ & & & $\mathrm{B}$ & & & NT \\
\hline Leptophloeus jumiperi (Grouvelle, 1874) & B & $\mathrm{X}$ & & & & & & & LC \\
\hline Placonotus testaceus (Fabricius, 1787) & $\mathrm{H}$ & $\mathrm{X}$ & $\mathrm{X}$ & $\mathrm{N}$ & $\mathrm{N}$ & $\mathrm{X}$ & $\mathrm{X}$ & B & \\
\hline
\end{tabular}

CYBOCEPHALIDAE Jacquelin du Val, 1858

Jelinek \& Audisio in Cat. Pal. Col., vol. 4, 2007; Cline et al., 2014

Cybocephalus rufifrons rufifrons Reitter, 1874 B $\mathrm{N}$ $\mathrm{N}$

COCCINELLOIDEA Latreille, 1807

Robertson et al., 2015

BOTHRIDERIDAE Erichson, 1845

Bothriderinae Erichson, 1845

Ślipiński in Cat. Pal. Col, vol. 4, 2007; Robertson et al., 2015

Bothrideres bipumctatus (Gmelin, 1790)

Ogmoderes angusticollis (C.N.F. Brisout de Bameville, 1862)

\begin{tabular}{|l|l|l|l|l|l|l|}
\hline $\mathrm{X}$ & & B & X & X & B & NT \\
\hline
\end{tabular}

TEREDIDAE Seidlitz, 1888

Anommatinae Ganglbauer, 1899

Ślipiński in Cat. Pal. Col., vol. 4, 2007; Robertson et al., 2015

\begin{tabular}{|l|l|l|}
\hline Anommatus duodecimstriatus (P.W.J. Müller, 1821) & H & X
\end{tabular}

Teredinae Seidlitz, 1888

Oxylaemus cylindricus (Creutzer, 1796)

Teredus cylindricus (A.G. Olivier, 1790)

\begin{tabular}{|l|l|l|l|l|l|l|l|l|}
$\mathrm{X}$ & & & $\mathrm{N}$ & & $\mathrm{X}$ & & $\mathrm{N}$ & $\mathrm{NT}$ \\
$\mathrm{X}$ & $\mathrm{X}$ & & & $\mathrm{N}$ & $\mathrm{X}$ & & $\mathrm{N}$ & LC \\
\hline
\end{tabular}

CERYLONIDAE Billberg, 1820

Ceryloninae Billberg, 1820

Ślipinski in Cat. Pal. Col., vol. 4, 2007; Robertson et al., 2015

Cerylon fagi C.N.F. Brisout de Barneville, 1867

Cerylon ferrugineum Stephens, 1830

Cerylon histeroides (Fabricius, 1792)

Cerylon impressum Erichson, 1845

Philothermus evanescens (Reitter, 1876)

Philothermus semistriatus (Perris, 1865)

\begin{tabular}{|c|c|c|c|c|c|c|c|c|}
\hline X & X & & N & N & X & X & N & LC \\
\hline H & & & N & N & X & X & N & LC \\
\hline H & X & & N & N & X & X & N & LC \\
\hline & & & & N & & & & NT \\
\hline H & N & & & N & X & X & & NT \\
\hline H & X & N & & N & X & & N & LC \\
\hline
\end{tabular}

LATRIDIIDAE Erichson, 1842

Latridiinae Erichson, 1842

Latridiini Erichson, 1842

Johnson in Cat. Pal. Col., vol. 4, 2007; Robertson et al., 2015; Otero \& Lopez, 2016; Rücker, 2018

Cartodere constricta (Gyllenhal, 1827) 
Species

Cartodere nodifer (Westwood, 1839)

Dienerella anatolica (Mannerheim, 1844)

Dienerella angelinii Rücker, I998 [E]

Dienerella clathrata (Mannerheim, 1844)

Dienerella elegans (Aubé, 1850)

Dienerella elongata (Curtis, 1830)

Dienerella parilis (Rey, 1889)

Dienerella polyhymnia Rücker \& Poggi, 2013

Dienerella ruficollis (Marsham, 1802)

Dienerella siciliana Vincent, 1990 [E]

Enicmus atriceps V. Hansen, 1962

Enicmus brevicornis (Mannerheim, 1844)

Enicmus fungicola C.G. Thomson, 1868

Enicmus histrio Joy \& Tomlin, 1910

Enicmus rugosus (Herbst, 1793)

Enicmus testaceus (Stephens, 1830)

Enicmus transversus (A.G. Olivier, 1790)

Latridius assimilis (Mannerheim, 1844)

Latridius brevicollis (C.G. Thomson, 1868)

Latridius consimilis (Mannerheim, 1844)

Latridius mimutus (Linnaeus, 1767)

Latridius porcatus Herbst, 1793

Metophthalmus solarii Binaghi, 1946

Revelieria genei (Aubé, 1850)

Stephostethus angusticollis (Gyllenhal, 1827)

Stephostethus productus (Rosenhauer, 1856)

\section{Corticariinae Curtis, 1829}

Corticaria cremulata (Gyllenhal, 1827)

Corticaria cucujiformis Reitter, 1880

Corticaria elongata (Gyllenhal, 1827)

Corticaria fagi Wollaston, 1854

Corticaria fulva (Comolli, 1837)

Corticaria laertes Rücker, 2006

Corticaria obscura C.N.F. Brisout de Barneville, 1863

Corticaria olympiaca Reitter, 1875

Corticaria pineti Lohse, 1960

Corticaria pubescens (Gyllenhal, 1827)

Corticaria saginata Mannerheim, 1844

Corticaria serrata (Paykull, 1798)

Corticarina curta (Wollaston, 1854)

Corticarina similata (Gyllenhal, 1827)

Cortinicara gibbosa (Herbst, 1793)

Melanophthalma distinguenda (Comolli, 1837)

Melanophthalma extensa Rey, 1889

Melanophthalma fuscipennis (Mannerheim, 1844)

Melanophthalma maura Motschulsky, 1866

Melanophthalma parvicollis (Mannerheim, 1844)

Melanophthalma rispini Rücker \& C. Johnson, 2007

Melanophthalma sericea (Mannerheim, 1844)

Melanophthalma suturalis (Mannerheim, 1844)

Melanophthalma taurica (Mannerheim, 1844)

Melanophthalma transversalis (Gyllenhal, 1827)

\begin{tabular}{|c|c|c|c|c|c|c|c|c|}
\hline 总 & $\begin{array}{l}\bar{b} \\
\vdots \\
3 \\
0 \\
\frac{0}{5} \\
\frac{3}{0} \\
2\end{array}$ & 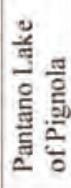 & 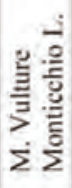 & 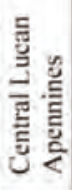 & 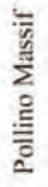 & $\frac{\frac{\vec{g}}{a}}{\frac{\vec{g}}{a}}$ & 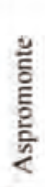 & 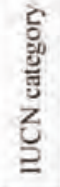 \\
\hline $\mathrm{H}$ & $\mathrm{X}$ & & B & B & $\mathrm{X}$ & $X$ & B & LC \\
\hline \multirow[t]{4}{*}{$\mathrm{N}$} & B & $\mathrm{N}$ & & B & $\mathrm{N}$ & & B & LC \\
\hline & & & $\mathrm{N}$ & B & B & B & B & \\
\hline & & & & & & & B & LC \\
\hline & & & & & & & B & LC \\
\hline $\mathrm{H}$ & $\mathrm{X}$ & & B & B & $\mathrm{X}$ & $\mathrm{X}$ & B & LC \\
\hline$X$ & & B & B & B & $X$ & $X$ & B & LC \\
\hline \multirow[t]{4}{*}{ B } & & B & B & B & B & B & B & LC \\
\hline & & & & & & & $\mathrm{N}$ & LC \\
\hline & & & & & & $X$ & & DD \\
\hline & & & & & & & B & DD \\
\hline $\mathrm{X}$ & $\mathrm{X}$ & & & & $X$ & $X$ & B & LC \\
\hline \multirow[t]{2}{*}{$\mathrm{X}$} & $\mathrm{X}$ & & & & & & B & LC \\
\hline & $\mathrm{X}$ & $X$ & & B & $X$ & & B & LC \\
\hline $\mathrm{H}$ & $\mathrm{X}$ & & B & B & $X$ & $X$ & B & LC \\
\hline $\mathrm{X}$ & & & & B & B & $\mathrm{X}$ & B & LC \\
\hline \multirow[t]{2}{*}{$\mathrm{H}$} & $X$ & X & B & B & $X$ & X & B & LC \\
\hline & & & & & & & B & DD \\
\hline \multirow[t]{2}{*}{$\mathrm{X}$} & $\mathrm{X}$ & & & B & $\mathrm{X}$ & $\mathrm{X}$ & B & DD \\
\hline & & & & B & $X$ & & $\mathrm{~N}$ & LC \\
\hline $\mathrm{X}$ & $\mathrm{X}$ & $\mathrm{X}$ & $\mathrm{N}$ & B & $X$ & X & B & LC \\
\hline \multirow[t]{5}{*}{ B } & & $\mathrm{X}$ & $\mathrm{N}$ & B & $\mathrm{X}$ & & & \\
\hline & & & & B & & & B & DD \\
\hline & & & & & & & B & LC \\
\hline & B & & & B & & & B & LC \\
\hline & $\mathrm{X}$ & & & & & & & DD \\
\hline
\end{tabular}

Migneauxia crassiuscula (Aubé, 1850)

\begin{tabular}{|c|c|c|c|c|c|c|c|c|}
\hline & & & & $\mathrm{N}$ & & & & LC \\
\hline & $\mathrm{X}$ & B & & & & & & DD \\
\hline & & $\mathrm{X}$ & & B & & & $\mathrm{N}$ & LC \\
\hline & & $\mathrm{X}$ & & & & & & \\
\hline $\mathrm{X}$ & & & & & $\mathrm{X}$ & & & LC \\
\hline & B & B & & & $\mathrm{N}$ & & & \\
\hline & & $X$ & B & B & $\mathrm{X}$ & $X$ & B & \\
\hline $\mathrm{H}$ & & & & & & & & \\
\hline $\mathrm{X}$ & $\mathrm{X}$ & & & & & & & LC \\
\hline $\mathrm{H}$ & $\mathrm{X}$ & & & & $\mathrm{N}$ & & B & LC \\
\hline & & B & & & & & & LC \\
\hline & $\mathrm{X}$ & & & $\mathrm{N}$ & $X$ & $X$ & B & LC \\
\hline $\mathrm{N}$ & $\mathrm{N}$ & $\mathrm{N}$ & & $\mathrm{N}$ & $\mathrm{N}$ & & $\mathrm{N}$ & \\
\hline$X$ & $\mathrm{X}$ & X & & & & & B & LC \\
\hline $\mathrm{H}$ & $\mathrm{X}$ & $\mathrm{X}$ & $\mathrm{N}$ & $\mathrm{N}$ & $\mathrm{X}$ & $\mathrm{X}$ & B & LC \\
\hline $\mathrm{H}$ & B & $\mathrm{X}$ & $\mathrm{N}$ & B & B & $\mathrm{X}$ & B & LC \\
\hline & B & & & B & & & & \\
\hline $\mathrm{H}$ & X & X & $\mathrm{N}$ & B & $\mathrm{X}$ & & B & LC \\
\hline & & B & & B & & & B & LC \\
\hline & B & & & & B & & & \\
\hline & B & & & & & & & \\
\hline & $\mathrm{X}$ & $\mathrm{X}$ & & & & & & LC \\
\hline & B & $\mathrm{X}$ & $\mathrm{N}$ & B & & $\mathrm{X}$ & B & LC \\
\hline $\mathrm{X}$ & $\mathrm{X}$ & $\mathrm{X}$ & & B & $\mathrm{X}$ & $\mathrm{X}$ & $\mathrm{N}$ & LC \\
\hline $\mathrm{H}$ & $\mathrm{X}$ & & & & & & & \\
\hline & $\mathrm{X}$ & $\mathrm{X}$ & $\mathrm{N}$ & B & $\mathrm{N}$ & & B & LC \\
\hline
\end{tabular}


Species

Migneanxia lederi Reitter, 1875

Migneauxia ottoi C. Johnson, 2007

\begin{tabular}{|c|c|c|c|c|c|c|c|c|}
\hline 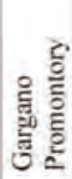 & $\begin{array}{l}7 \\
8 \\
3 \\
0 \\
0 \\
\frac{3}{0} \\
2\end{array}$ & 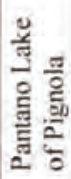 & 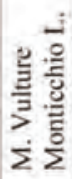 & 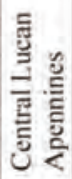 & 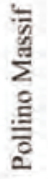 & $\begin{array}{l}\frac{\overline{\mathrm{g}}}{\mathrm{g}} \\
\frac{\mathrm{w}}{\mathrm{a}} \\
\frac{\mathrm{m}}{\bar{b}}\end{array}$ & है & 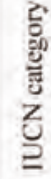 \\
\hline & & & & & & & B & \\
\hline & & & & $\mathrm{N}$ & & & $\mathrm{N}$ & \\
\hline
\end{tabular}

ALEXIIDAE Imhoff, 1856

Tomaszewska in Cat. Pal. Col., vol. 4, 2007; Robertson et al., 2015

Sphaerosoma globosum (Sturm, 1807)

Sphaerosoma reitteri (Ormay, 1888)

Sphaerosoma solarii Reitter, 1904 [E]

Sphaerosoma sparsum Reitter, 1909 [E]

Sphaerosoma vallombrosae (Reitter, 1885) [E]

\begin{tabular}{|l|l|l|l|l|l|l|l|}
\hline & & & & & N & LC \\
\hline N & & N & N & N & & B & LC \\
\hline N & B & N & N & X & N & LC \\
\hline N & N & N & N & N & B & LC \\
\hline
\end{tabular}

CORYLOPHIDAE LeConte, 1852

Corylophinae LeConte, 1852

Corylophini LeConte, 1852

Bowestead in Cat. Pal. Col., vol. 4, 2007; Robertson et al., 2015

Corylophus cassidoides (Marsham, 1802)

Corylophus sublaevipennis Jacquelin du Val, 1859

Parmulini Poey, 1854

Arthrolips convexiuscula (Motschulsky, 1849)

Arthrolips hetschkoi (Reitter, 1913)

Arthrolips nana (Mulsant \& Rey, 1861)

Arthrolips obscura (C.R. Sahlberg, 1833)

Arthrolips picea (Comolli, 1837)

Clypastraea reitteri Bowestead, 1999

Sericoderini Matthews, 1888

\begin{tabular}{|c|c|c|c|c|c|c|c|c|}
\hline & B & X & N & N & N & & N & \\
\hline & & & & & B & & & \\
\hline X & N & & & & X & X & & DD \\
\hline H & B & X & N & N & B & X & B & DD \\
\hline & & & & B & B & N & B & DD \\
\hline
\end{tabular}

Aposericoderus revelierei Reitter, 1878

Sericoderus lateralis (Gyllenhal, 1827)

\begin{tabular}{l|l|l|}
\hline & $\mathrm{B}$ & \\
$\mathrm{H}$ & $\mathrm{X}$ & $\mathrm{X}$ \\
\hline
\end{tabular}

Orthoperinae Jacquelin du Val, 1857

Orthoperus aequalis Sharp, 1885

Orthoperus anxius Mulsant \& Rey, 1861

Orthoperus atomus (Gyllenhal, 1808)

Orthoperus brumnipes (Gyllenhal, 1808)

Orthoperus corticalis (L. Redtenbacher, 1845)

Orthoperus nigrescens Stephens, 1829

du Val, 1857
\begin{tabular}{|c|c|c|c|c|c|c|c|c|}
\hline & B & & & & & & N & DD \\
\hline B & B & X & & & & & & \\
\hline & & & & & N & & & \\
\hline & & & & B & N & & B & DD \\
\hline H & & & & & & & & \\
\hline
\end{tabular}

Rypobiinae Paulian, 1950

Gloeosomatini Bowestead, 1999

Gloeosoma velox Wollaston, 1854

Rypobiini Paulian, 1950

B

Rypobius fioriamus Matthews, 1886

ANAMORPHIDAE Strohecker, 1953

Tomaszewska in Cat. Pal. Col., vol. 4, 2007; Robertson et al., 2015

Aclemmysa solarii Reitter, 1904 [E]

Mychothenus mimutus (Frivaldszky, 1877)

Symbiotes gibberosus (P.H. Lucas, 1846)

\begin{tabular}{|l|l|l|l|l|l|l|l|l|}
\hline & & X & B & B & N & & N & NT \\
\hline & & & & & N & & & NT \\
\hline & X & & N & & N & & N & LC \\
\hline
\end{tabular}

ENDOMYCHIDAE Leach, 1815

Endomychinae Leach, 1815

Tomaszewska in Cat. Pal. Col., vol. 4, 2007; Robertson et al., 2015

Endomychus coccineus (Linnaeus, 1758)

Leiestinae C.G. Thomson, 1863

Leiestes seminiger (Gyllenhal, 1808)
Lycoperdininae L. Redtenbacher, 1844 \begin{tabular}{|l|l|l|l|l|l|}
$\mathrm{N}$ & $\mathrm{N}$ & $\mathrm{X}$ & $\mathrm{X}$ & $\mathrm{B}$ & $\mathrm{LC}$ \\
\hline
\end{tabular}

Ancylopus melanocephalus (A.G. Olivier, 1808)

\begin{tabular}{l|l|l}
$\mathrm{X}$ & $\mathrm{X}$
\end{tabular}

\begin{tabular}{|l|l|l|l|}
\hline N & B & VU \\
\hline
\end{tabular}


Dapsa trimaculata Motschulsky, 1835

Lycoperdina bovistae (Fabricius, 1792)

Lycoperdina validicornis Gerstaecker, 1858

Mycetina cruciata (Schaller, 1783)

\section{Merophysiinae Seidlitz, 1872}

\begin{tabular}{|c|c|c|c|c|c|c|c|c|}
\hline 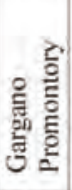 & $\begin{array}{l}0 \\
0 \\
3 \\
0 \\
0 \\
\frac{0}{0} \\
\frac{0}{0} \\
0\end{array}$ & 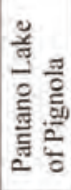 & 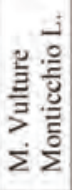 & 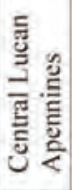 & 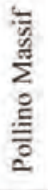 & 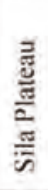 & 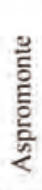 & 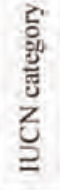 \\
\hline $\mathrm{H}$ & $X$ & $\mathrm{~N}$ & B & B & B & B & B & \\
\hline & $X$ & & & & & & $\mathrm{~N}$ & \\
\hline $\mathrm{H}$ & $X$ & & $\mathrm{~N}$ & $\mathrm{~N}$ & $X$ & $X$ & $\mathrm{~N}$ & LC \\
\hline $\mathrm{X}$ & $X$ & & & & $\mathrm{X}$ & $\mathrm{X}$ & $\mathrm{N}$ & $\mathrm{VU}$ \\
\hline $\mathrm{X}$ & $X$ & & $\mathrm{~N}$ & $\mathrm{~N}$ & $\mathrm{X}$ & $\mathrm{X}$ & $\mathrm{N}$ & LC \\
\hline
\end{tabular}

Merophysiini Seidlitz, 1872

Rücker \& Löbl in Cat. Pal. Col., vol. 4, 2007; Robertson et al., 2015; Rücker, 2018

Cholovocera attae (Kraatz, 1858)

Cholovocera fleischeri Reitter, 1902

Cholovocera formicaria Motschulsky, 1838

Cholovocera punctata Märkel, 1844

Merophysia formicaria P.H. Lucas, 1852

Merophysia oblonga Kiesenwetter, 1872

Holoparamecini Seidlitz, 1888

\begin{tabular}{|c|c|c|c|c|c|c|c|c|}
\hline & B & & & N & & N & \\
\hline H & & & & & & & N & \\
\hline & & & & B & N & & N & \\
\hline & & & & & N & N & \\
\hline H & B & & & B & & & B & \\
\hline
\end{tabular}

\begin{tabular}{l}
\hline Holoparamecus caularum (Aubé, 1843 \\
\hline Holoparamecus depressus Curtis, 1833 \\
\hline Holoparamecus niger (Aubé, 1843) \\
\hline Holoparamecus ragusae Reitter, 1875 \\
\hline Holoparamecus singularis (Beck, 1817
\end{tabular}

\begin{tabular}{|l|l|l|l|l|l|l|l|l|}
$\mathrm{X}$ & $\mathrm{X}$ & & & & $\mathrm{X}$ & & & \\
\hline & $\mathrm{B}$ & & & & & & & \\
$\mathrm{X}$ & $\mathrm{X}$ & & & $\mathrm{N}$ & $\mathrm{B}$ & & $\mathrm{B}$ & \\
\hline $\mathrm{B}$ & & & & & & & \\
\hline & $\mathrm{X}$ & & & & & & \\
\hline
\end{tabular}

MYCETAEIDAE Jacquelin du Val, 1857

Tomaszewska in Cat. Pal. Col., vol. 4, 2007; Robertson et al., 2015

Mycetaea subterranea (Fabricius, 1801) $\mathrm{N}$ \begin{tabular}{|l|l|l|l|l|}
$\mathrm{N}$ & $\mathrm{X}$ & $\mathrm{X}$ & $\mathrm{N}$ & $\mathrm{LC}$ \\
\hline
\end{tabular}

\section{COCCINELLIDAE Latreille, 1807 \\ Coccinellinae Latreille, 1807 \\ Chilocorini Mulsant, 1846}

Kováŕ in Cat. Pal. Col., vol. 4, 2007; Seago et al., 2011; Robertson et al., 2015

Chilocorus bipustulatus (Linnaeus, 1758)

Exochomus quadripustulatus (Linnaeus, 1758)

Parexochomus nigromaculatus (Goeze, 1777)

\begin{tabular}{|l|l|l|l|l|l|l|l|l|}
$\mathrm{H}$ & $\mathrm{X}$ & $\mathrm{X}$ & $\mathrm{N}$ & & $\mathrm{X}$ & $\mathrm{X}$ & $\mathrm{N}$ & \\
\hline $\mathrm{X}$ & $\mathrm{N}$ & $\mathrm{X}$ & $\mathrm{N}$ & $\mathrm{N}$ & $\mathrm{X}$ & $\mathrm{X}$ & $\mathrm{N}$ & \\
\hline & $\mathrm{X}$ & $\mathrm{N}$ & & & $\mathrm{N}$ & & & \\
\hline
\end{tabular}

Coccidulini Mulsant, 1846

Clitostethus arcuatus (P. Rossi, 1794)

Coccidula rufa (Herbst, 1783)

Coccidula scutellata (Herbst, 1783)

Nephus bipunctanus (Kugelann, 1794)

Nephus bisignatus bisignatus (Boheman, 1850)

Nephus kiesenwetteri (Mulsant, 1850)

Nephus nigricans nigricans (J. Weise, 1879)

Nephus quadrimaculatus (Herbst, 1783)

Nephus schatzmayri Canepari \& Tedeschi, 1977

Rhyzobius litura (Fabricius, 1787)

Rhyzobius lophantae (Blaisdéll, 1892)

Scymniscus amomus (Mulsant \& Rey, 1852)

Scymmiscus helgae (Fürsch, 1965)

Scymniscus tristiculus (J. Weise, 1929)

Scymmus apetzi Mulsant, 1846

Scymmis ater Kugelann, 1794

Scymmus auritus Thunberg, 1795

Scymmus bivulnerus Baudi di Selve, 1894

Scymmus femoralis (Gyllenhal, 1827)

Scymmus ferrugatus (Moll, 1785)

Scymmus flagellisiphonatus (Fürsch, 1970)

\begin{tabular}{|c|c|c|c|c|c|c|c|c|}
\hline H & & & & & & X & & \\
\hline X & N & X & & & & & N & \\
\hline H & & X & N & & N & & & \\
\hline & & X & & & X & & & \\
\hline X & & & N & & X & X & N & \\
\hline H & X & & & & X & X & & \\
\hline H & X & X & B & N & X & X & N & \\
\hline N & X & B & & B & & & & \\
\hline & & & & & & & B & \\
\hline B & X & X & N & N & X & X & N & \\
\hline H & N & & & & & & & \\
\hline H & N & X & N & N & X & X & N & \\
\hline & X & X & & & & & B & \\
\hline H & & X & & & & & N & \\
\hline & X & X & & & & & N & \\
\hline
\end{tabular}


Species

Scymnus fraxini Mulsant, 1850

Scymmus frontalis (Fabricius, 1787)

Scymmus haemorrhoidalis Herbst, 1797

Scymmus impexus Mulsant, 1850

Scymmus inderihensis Mulsant, 1850

Scymmus intermuptus (Goeze, 1777)

Scymmus limbatus Stephens, 1832

Scymmus marginalis (P. Rossi, 1794)

Scymmus marimus (Mulsant, 1850)

Scymmus pharaonis Motschulsky, 1851

Scymnus rubromaculatus (Goeze, 1777)

Scymnus rufipes (Fabricius, 1798)

Scymmus sacium (Roubal, 1927)

Scymmus subvillosus (Goeze, 1777)

Scymmus suffrianioides apetzoides Capra \& Fürsch, 1967

Scymmus suturalis Thunberg, 1795

Stethorus gilvifrons (Mulsant, 1850)

Stethorus pusillus (Herbst, 1797)

\begin{tabular}{|c|c|c|c|c|c|c|c|c|}
\hline 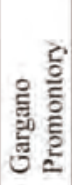 & $\begin{array}{l}\overline{0} \\
\vdots \\
\vdots \\
0 \\
\frac{0}{0} \\
\frac{.0}{0}\end{array}$ & 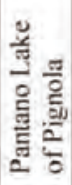 & 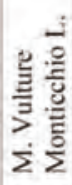 & 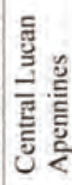 & 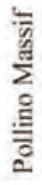 & 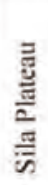 & $\begin{array}{l}\text { है } \\
\text { हू } \\
\text { है }\end{array}$ & 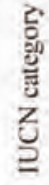 \\
\hline & & $X$ & & $\mathrm{~N}$ & & & $\mathrm{~N}$ & \\
\hline \multirow{4}{*}{$\mathrm{H}$} & $X$ & X & $\mathrm{N}$ & $\mathrm{N}$ & X & X & $\mathrm{N}$ & \\
\hline & & $X$ & & & & & & \\
\hline & & & & $\mathrm{N}$ & & & & \\
\hline & $\mathrm{X}$ & & & & & & & \\
\hline \multirow[t]{2}{*}{$\mathrm{H}$} & $\mathrm{X}$ & $\mathrm{X}$ & & $\mathrm{N}$ & $\mathrm{N}$ & $\mathrm{X}$ & $\mathrm{N}$ & \\
\hline & & X & & & & & & \\
\hline $\mathrm{X}$ & $\mathrm{X}$ & & & $\mathrm{N}$ & $\mathrm{X}$ & $\mathrm{X}$ & B & \\
\hline \multirow[t]{2}{*}{$\mathrm{X}$} & $X$ & $X$ & $\mathrm{~N}$ & $\mathrm{~N}$ & $\mathrm{X}$ & $X$ & $\mathrm{~N}$ & \\
\hline & & $X$ & & & & & $\mathrm{~N}$ & \\
\hline \multirow[t]{3}{*}{$\mathrm{H}$} & $\mathrm{X}$ & $\mathrm{X}$ & $\mathrm{N}$ & $\mathrm{N}$ & $\mathrm{X}$ & $\mathrm{X}$ & $\mathrm{N}$ & \\
\hline & $\mathrm{X}$ & $\mathrm{X}$ & & & & & $\mathrm{N}$ & \\
\hline & & & & $\mathrm{N}$ & & & & \\
\hline $\mathrm{H}$ & $\mathrm{X}$ & $\mathrm{X}$ & $\mathrm{N}$ & $\mathrm{N}$ & $\mathrm{X}$ & $\mathrm{X}$ & $\mathrm{N}$ & \\
\hline \multirow[t]{2}{*}{$\mathrm{N}$} & & & & & & $X$ & & \\
\hline & & & & $\mathrm{N}$ & $\mathrm{X}$ & $\mathrm{X}$ & & \\
\hline $\mathrm{H}$ & $\mathrm{X}$ & $\mathrm{X}$ & & $\mathrm{N}$ & $\mathrm{N}$ & & & \\
\hline$X$ & $X$ & $\mathrm{X}$ & $\mathrm{N}$ & $\mathrm{N}$ & $\mathrm{X}$ & & & \\
\hline
\end{tabular}

Adalia bipunctata (Linnaeus, 1758)

Coccinellini Latreille, 1807

Adalia decempunctata (Linnaeus, 1758)

Anatis ocellata (Linnaeus, 1758)

Anisosticta novemdecimpunctata (Linnaeus, 1758)

Aphidecta obliterata (Linnaeus, 1758)

Calvia quatuordecimguttata (Linnaeus, 1758)

Ceratomegilla undecimnotata (D.H. Schneider, 1792)

Coccinella hieroglyphica hieroglyphica Linnaeus, 1758

Coccinella septempumctata Linnaeus, 1758

Coccinella undecimpunctata undecimpunctata Linnaeus, 1758

Coccimula quatuordecimpustulata (Linnaeus, 1758)

Halyzia sedecimguttata (Linnaeus, 1758)

Harmonia quadripunctata (Pontoppidan, 1763)

Hippodamia variegata (Goeze, 1777)

Myrrha octodecimguttata (Linnaeus, 1758)

Myzia oblongoguttata oblongoguttata (Linnaeus, 1758)

Oenopia conglobata conglobata (Linnaeus, 1758)

Oenopia doublieri (Mulsant, 1846)

Oenopia byncea agnata (Rosenhauer, 1847)

Oenopia lyncea lyncea (A.G. Olivier, 1808)

Psyllobora vigintiduopumctata (Linnaeus, 1758)

Sospita vigintiguttata (Linnaeus, 1758)

Tytthaspis sedecimpunctata (Linnaeus, 1758)

Vibidia duodecimguttata (Poda von Neuhaus, 1761)

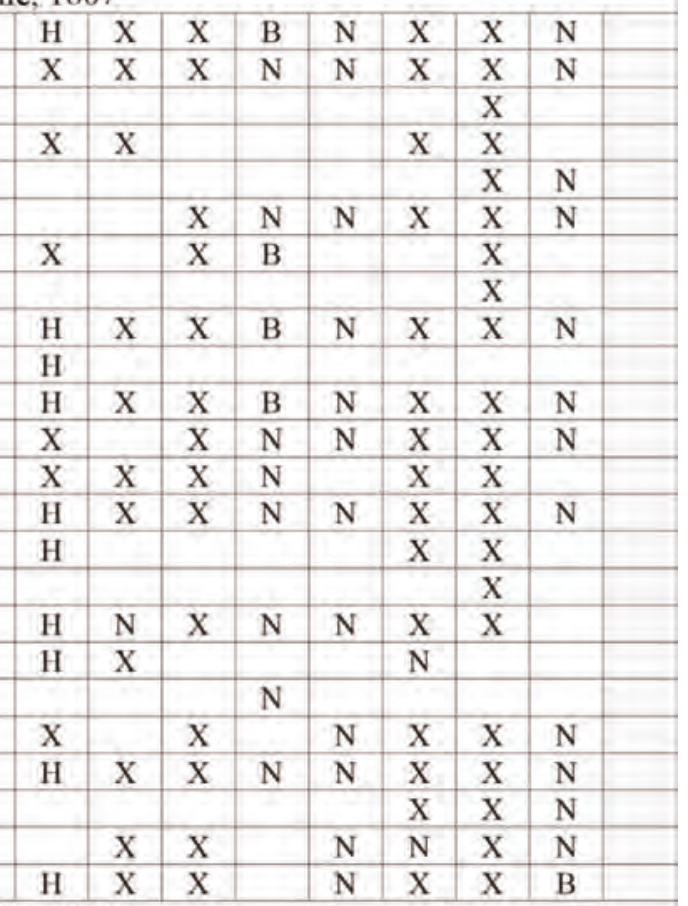

Diomini R.D. Gordon, 2000

Diomus rubidus (Motschulsky, 1837)

$\mathrm{X} \mid \mathrm{X}$

Epilachnini Mulsant, 1850

Chnootriba elaterii elaterii (P. Rossi, 1794)

Henosepilachna vigintioctomaculata Motschulsky, 1858

Subcoccinella vigintiquatuorpunctata (Linnaeus, 1758)

\begin{tabular}{|l|l|l|l|l|l|l|l|l|} 
& X & & & & & & & \\
\hline H & & & & & & & B & \\
\hline H & X & X & B & N & X & X & N & \\
\hline
\end{tabular}

Hyperaspis campestris (Herbst, 1783)

Hyperaspis concolor (Suffrian, 1843)

Hyperaspis duvergeri Fürsch, 1985

Hyperaspidini Mulsant, 1846

\begin{tabular}{|c|c|c|c|c|c|c|c|c|}
\hline $\mathrm{X}$ & & & & $\mathrm{N}$ & & & & \\
\hline & & $\mathrm{X}$ & $\mathrm{N}$ & & $\mathrm{B}$ & $\mathrm{X}$ & $\mathrm{B}$ & \\
\hline $\mathrm{X}$ & $\mathrm{X}$ & $\mathrm{X}$ & & & & $\mathrm{X}$ & $\mathrm{B}$ & \\
\hline
\end{tabular}




\begin{tabular}{|c|c|c|c|c|c|c|c|c|c|}
\hline Species & 总 & 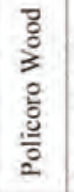 & 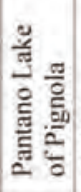 & 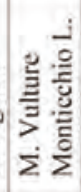 & 总 & 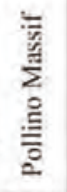 & 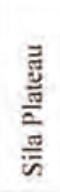 & 总 & 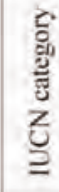 \\
\hline Hyperaspis peezi Fürsch, 1976 & & & & & & B & B & B & \\
\hline Hyperaspis stigma (A.G. Olivier, 1808) & & $\mathrm{N}$ & $\mathrm{X}$ & & & & & & \\
\hline \multicolumn{10}{|c|}{ Noviini Mulsant, 1850} \\
\hline Rodolia cardinalis (Mulsant, 1850) & & $\mathrm{X}$ & & & & & & & \\
\hline \multicolumn{10}{|c|}{ Platynaspidini Mulsant, 1846} \\
\hline Platynaspis luteorubra Goeze, 1777 & & $\mathrm{X}$ & $\mathrm{X}$ & $\mathrm{N}$ & $\mathrm{N}$ & $\mathrm{x}$ & $\mathrm{x}$ & & \\
\hline \multicolumn{10}{|c|}{ TENEBRIONOIDEA Latreille, 1802} \\
\hline \multicolumn{10}{|c|}{$\begin{array}{c}\text { MYCETOPHAGIDAE Leach, } 1815 \\
\text { Mycetophaginae Leach, } 1815 \\
\text { Mycetophagini Leach, } 1815 \\
\text { Nikitsky in Cat. Pal. Col., vol. } 5,2008\end{array}$} \\
\hline Litargus balteatus Leeonte, 1856 & $\mathrm{~N}$ & & & & $\mathrm{~N}$ & $\mathrm{~N}$ & $\mathrm{~N}$ & $\mathrm{~N}$ & \\
\hline Litargus coloratus Rosenhauer, 1856 & $\mathrm{X}$ & $\mathrm{X}$ & & & & & & & NT \\
\hline Litargus connexus (Geoffroy, 1785) & $\mathrm{X}$ & $\mathrm{X}$ & $\mathrm{X}$ & $\mathrm{N}$ & $\mathrm{N}$ & $\mathrm{X}$ & $\mathrm{X}$ & $\mathrm{N}$ & LC \\
\hline Mycetophagus atomarius (Fabricius, 1787) & $\mathrm{H}$ & & & $\mathrm{N}$ & $\mathrm{N}$ & $\mathrm{x}$ & $\mathrm{x}$ & $\mathrm{N}$ & LC \\
\hline Mycetophagus fulvicollis Fabricius, 1792 & & $\mathrm{~N}$ & & & & & & & NT \\
\hline Mycetophagus quadriguttatus P.W.J. Müller, 1821 & & & & $\mathrm{~N}$ & & & & & LC \\
\hline Mycetophagus quadripustulatus (Linnaeus, 1760) & $\mathrm{X}$ & $\mathrm{X}$ & & & $\mathrm{N}$ & $\mathrm{x}$ & $\mathrm{X}$ & $\mathrm{N}$ & LC \\
\hline Psendotriphyllus suturalis (Fabricius, 1801) & & $\mathrm{X}$ & & & & & $\mathrm{X}$ & & NT \\
\hline Triphyllus bicolor (Fabricius, 1777) & $\mathrm{X}$ & & & & $\mathrm{N}$ & $\mathrm{X}$ & $\mathrm{x}$ & $\mathrm{N}$ & LC \\
\hline Typhaea stercorea (Linnaeus, 1758) & $\mathrm{H}$ & $\mathrm{X}$ & $\mathrm{x}$ & $\mathrm{N}$ & $\mathrm{N}$ & $\mathrm{x}$ & $\mathrm{x}$ & $\mathrm{N}$ & LC \\
\hline Typhaeola umbrata (Baudi di Selve, 1870) & & $\mathrm{N}$ & & $\mathrm{N}$ & $\mathrm{N}$ & B & & B & LC \\
\hline \multicolumn{10}{|c|}{ Bergininae Lend, 1920} \\
\hline Berginus tamarisci Wollaston, 1854 & $\mathrm{X}$ & $\mathrm{X}$ & $\mathrm{X}$ & $\mathrm{N}$ & & $\mathrm{N}$ & $\mathrm{X}$ & $\mathrm{N}$ & LC \\
\hline \multicolumn{10}{|c|}{ Esarcinae Reitter, 1882} \\
\hline Esarcus fiorii Reitter, 1887 [E] & & $\mathrm{N}$ & & & & & & B & VU \\
\hline \multicolumn{10}{|c|}{$\begin{array}{c}\text { CIIDAE Leach, } 1819 \\
\text { Ciinae Leach, } 1819 \\
\text { Ciini Leach, 1819 } \\
\text { Jelinek in Cat. Pal. Col., vol. 5, 2008; Reibnitz et al., } 2013\end{array}$} \\
\hline Cis bidentatus (A.G. Olivier, 1790) & & & & & & & & $\mathrm{N}$ & LC \\
\hline Cis boleti (Scopoli, 1763) & $\mathrm{H}$ & $\mathrm{X}$ & & $\mathrm{N}$ & $\mathrm{N}$ & $\mathrm{X}$ & $\mathrm{X}$ & $\mathrm{N}$ & LC \\
\hline Cis castaneus (Herbst, 1793) & $\mathrm{X}$ & $\mathrm{N}$ & $\mathrm{N}$ & $\mathrm{N}$ & $\mathrm{N}$ & $\mathrm{x}$ & $\mathrm{x}$ & $\mathrm{N}$ & LC \\
\hline Cis comptus Gyllenhal, 1827 & $\mathrm{H}$ & $\mathrm{X}$ & & $\mathrm{N}$ & $\mathrm{N}$ & $\mathrm{X}$ & & & LC \\
\hline Cis dentatus Mellié, 1849 & & & & & & $\mathrm{X}$ & & & LC \\
\hline Cis fissicollis Mellié, 1849 & & & $\mathrm{X}$ & & $\mathrm{N}$ & $\mathrm{N}$ & & & LC \\
\hline Cis glabratus Mellié, 1849 & & & & & & $\mathrm{~N}$ & & & LC \\
\hline Cis hanseni A. Strand, 1965 & $\mathrm{~N}$ & & & & $\mathrm{~N}$ & $\mathrm{~N}$ & & & $\mathrm{VU}$ \\
\hline Cis jacquemartii Mellié, 1849 & & & & & & $\mathrm{~N}$ & & & LC \\
\hline Cis lineatocribratus Mellié, 1849 & $\mathrm{H}$ & $\mathrm{X}$ & & $\mathrm{N}$ & $\mathrm{N}$ & $\mathrm{x}$ & $\mathrm{X}$ & $\mathrm{N}$ & LC \\
\hline Cis micans (Fabricius, 1792) & $\mathrm{H}$ & $\mathrm{X}$ & & $\mathrm{N}$ & $\mathrm{N}$ & $\mathrm{x}$ & $\mathrm{x}$ & $\mathrm{N}$ & LC \\
\hline Cis pygmaeus pygmaeus (Marsham, 1802) & $\mathrm{x}$ & & & & & & & & VU \\
\hline Cis quadridentulus Perris, 1874 & & $\mathrm{~N}$ & & & & & & & LC \\
\hline Cis rugulosus Mellié, 1849 & & $\mathrm{X}$ & & & $\mathrm{N}$ & $\mathrm{N}$ & & & LC \\
\hline Cis vestitus Mellié, 1849 & $\mathrm{H}$ & $\mathrm{x}$ & & & & $\mathrm{X}$ & $\mathrm{X}$ & $\mathrm{N}$ & LC \\
\hline Ennearthron cornutum (Gyllenhal, 1827) & & & & & & $\mathrm{x}$ & & & LC \\
\hline Ennearthron palmi Lohse, 1966 & & & & & & $\mathrm{~N}$ & & & \\
\hline Ennearthron pruinosulum (Perris, 1864) & & & & & & $\mathrm{N}$ & & & VU \\
\hline Orthocis alni (Gyllenhal, 1813) & & & & & & $\mathrm{N}$ & & & \\
\hline Orthocis reflexicollis (Abeille de Perrin, 1874) & & $\mathrm{N}$ & & & & & & & VU \\
\hline Strigocis bicornis (Mellié, 1849) & $\mathrm{H}$ & $\mathrm{X}$ & & $\mathrm{N}$ & & $\mathrm{X}$ & $\mathrm{X}$ & $\mathrm{N}$ & LC \\
\hline
\end{tabular}


Species

Sulcacis bidentulus (Rosenhauer, 1847)

Sulcacis fronticornis (Panzer, 1805)

Sulcacis nitidus (Fabricius, 1792)

Orophiini C.G. Thomson, 1863

\begin{tabular}{|c|c|c|c|c|c|c|c|c|}
\hline 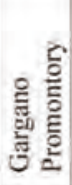 & $\begin{array}{l}7 \\
\delta \\
3 \\
0 \\
\frac{0}{0} \\
\frac{.0}{0} \\
2\end{array}$ & 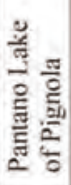 & 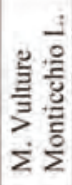 & 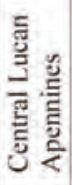 & 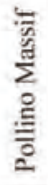 & 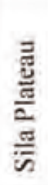 & 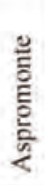 & 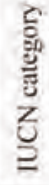 \\
\hline & & & & & $\mathrm{N}$ & & & LC \\
\hline & $X$ & & & & $\mathrm{~N}$ & & & LC \\
\hline & $\mathrm{X}$ & & $\mathrm{N}$ & & $\mathrm{X}$ & $X$ & $\mathrm{~N}$ & LC \\
\hline
\end{tabular}

Octotemmus glabriculus (Gyllenhal, 1827)

Octotemnus mandibularis (Gyllenhal, 1813)

Ropalodontus baudueri Abeille de Perrin, 1874

Ropalodontus novorossicus Reitter, 1902

Ropalodontus perforatus (Gyllenhal, 1813)

Xylographus bostrichoides (Dufour, 1843)

\begin{tabular}{|c|c|c|c|c|c|c|c|c|}
\hline & $\mathrm{X}$ & $\mathrm{X}$ & $\mathrm{N}$ & $\mathrm{N}$ & $\mathrm{X}$ & $\mathrm{X}$ & $\mathrm{N}$ & $\mathrm{LC}$ \\
\hline $\mathrm{X}$ & & & & & & & & $\mathrm{VU}$ \\
\hline & & & & & & & $\mathrm{N}$ & $\mathrm{EN}$ \\
\hline & $\mathrm{X}$ & & & & & & & $\mathrm{EN}$ \\
\hline $\mathrm{X}$ & $\mathrm{X}$ & $\mathrm{X}$ & $\mathrm{N}$ & $\mathrm{N}$ & $\mathrm{X}$ & $\mathrm{X}$ & $\mathrm{N}$ & $\mathrm{LC}$ \\
\hline $\mathrm{X}$ & $\mathrm{X}$ & & & $\mathrm{N}$ & $\mathrm{X}$ & & & \\
\hline
\end{tabular}

TETRATOMIDAE Billberg, 1820

Eustrophinae Gistel, 1856

Eustrophini Gistel, 1856

Nikitsky in Cat. Pal. Col., vol. 5, 2008

Eustrophus dermestoides (Fabricius, 1792)

Hallomeninae Mulsant, 1856

Hallomenus binotatus (Quensel, 1790)

MELANDRYIDAE Leach, 1815

Melandryinae Leach, 1815

Dircaeini Mulsant, 1856

Nikitsky \& Pollock in Cat. Pal. Col., vol. 5, 2008

Abdera quadrifasciata (Curtis, 1829

Phloiotrya tenuis (Hampe, 1850)

\begin{tabular}{|l|l|}
$\mathrm{X}$ & $\mathrm{X}$ \\
$\mathrm{X}$ & $\mathrm{X}$ \\
\hline
\end{tabular}

Melandryini Leach, 1815

Melandrya barbata (Fabricius, 1787)

Melandrya caraboides (Linnaeus, 1760)

Orchesiini Mulsant, 1856

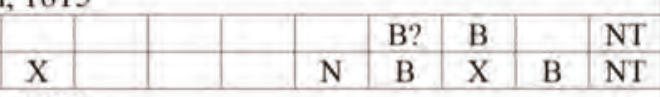

Orchesia micans (Panzer, 1793)

Orchesia undulata Kraatz, 1853

Serropalpini Latreille, 1829

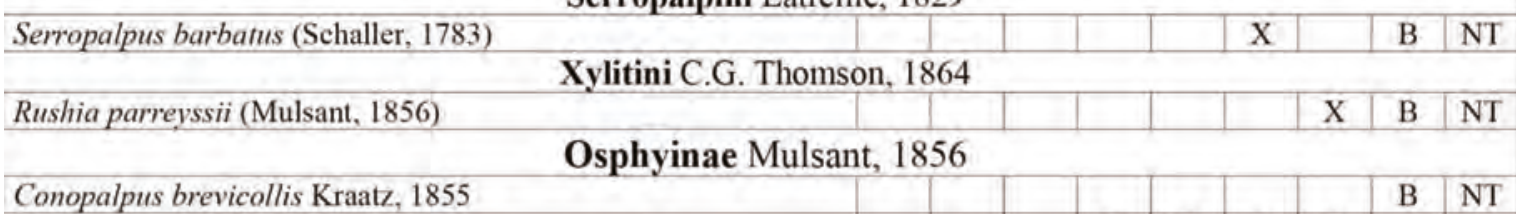

Osphya bipunctata (Fabricius, 1775)

MORDELLIDAE Latreille, 1802

Mordellinae Latreille, 1802

Mordellini Latreille, 1802

Horák in Cat. Pal. Col., vol. 5, 2008

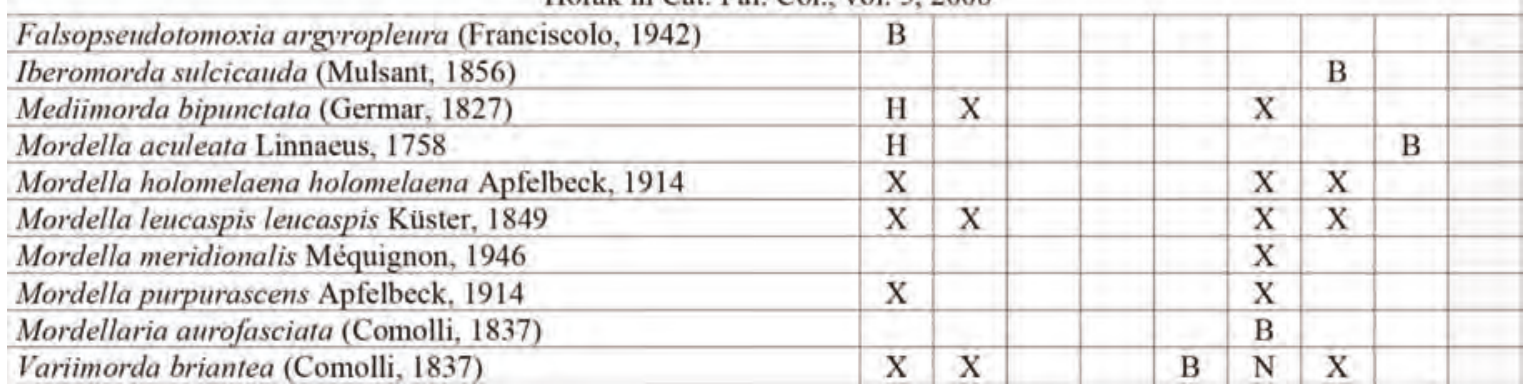


Variimorda ragusai (Emery, 1876)

Variimorda villosa (Schrank, 1781)

Mordellistenini Ermisch, 1941

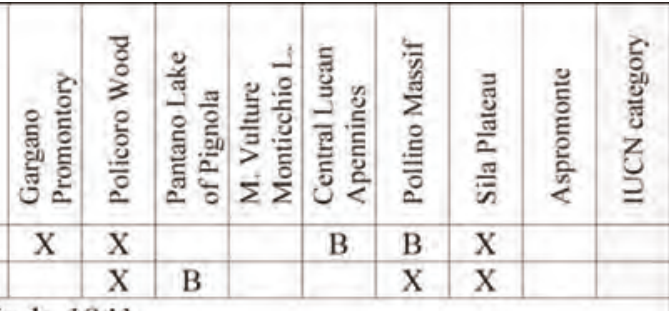

Mordellistena austriaca Schilsky, 1899

Mordellistena brevicauda (Boheman, 1849)

Mordellistena emeryi Schilsky, 1895

Mordellistena episternalis Mulsant, 1856

Mordellistena falsoparvula Ermisch, 1956

Mordellistena humeralis Fabricius, 1758

Mordellistena micans (Germar, 1817)

Mordellistena minima A. Costa, 1854

Mordellistena netrwaldeggiana (Panzer, 1796)

Mordellistena paraepisternalis Ermisch, 1965

Mordellistena paraintersecta Ermisch, 1956

Mordellistena parvula (Gyllenhal, 1827)

Mordellistena pseudohirtipes pseudohirtipes Ermisch, 1965

Mordellistena pumila (Gyllenhal, 1810) gr,

Mordellistena stenidea Mulsant, 1856

Mordellistena subepistermalis Ermisch, 1965

Mordellistena tarsata Mulsant, 1856

Mordellistenula perrisi (Mulsant, 1857)

Mordellistenula planifrons Stchegoleva-Barovskaya, 1930

Mordellochroa abdominalis (Fabricius, 1775)

Mordellochroa pulchella (Mulsant \& Rey, 1859)

Stenaliini Franciscolo, 1955

\begin{tabular}{|c|c|c|c|c|c|c|c|}
\hline & $X$ & & & & $\mathrm{X}$ & $X$ & \\
\hline \multirow[t]{2}{*}{$\mathrm{H}$} & & & & & $X$ & & \\
\hline & X & & & & X & X & \\
\hline \multirow[t]{2}{*}{$\mathrm{H}$} & & & & & $\mathrm{X}$ & & \\
\hline & & & & B & B & & \\
\hline $\mathrm{H}$ & & $\mathrm{N}$ & & & & & \\
\hline \multirow[t]{3}{*}{$\mathrm{H}$} & & & & & $X$ & & \\
\hline & $\mathrm{X}$ & & & & $X$ & X & \\
\hline & X & & & & & & \\
\hline $\mathrm{N}$ & $\mathrm{X}$ & & & & & & \\
\hline $\mathrm{X}$ & & & & B & & & \\
\hline$X$ & & & & & $X$ & & \\
\hline $\mathrm{X}$ & $X$ & & & & $X$ & $X$ & \\
\hline \multirow[t]{2}{*}{$\mathrm{H}$} & X & & B & & $\mathrm{X}$ & $\mathrm{X}$ & \\
\hline & & & & & X & & \\
\hline $\mathrm{X}$ & X & & & & & & \\
\hline \multirow[t]{4}{*}{$\mathrm{X}$} & & & & & X & & \\
\hline & & & & & $\mathrm{X}$ & $\mathrm{X}$ & \\
\hline & & & & & & X & \\
\hline & $\mathrm{X}$ & & & & & & LC \\
\hline B & & & & & & & \\
\hline
\end{tabular}

Stenalia bisecta Baudi di Selve, 1883 [E]

Stenalia testacea testacea (Fabricius, 1787)

\begin{tabular}{|l|l|l|l|l|l|l|l|l|}
\hline & B & & & & & & & \\
\hline X & X & & & & N & B & B & \\
\hline
\end{tabular}

RIPIPHORIDAE Gemminger \& Harold, 1870

Ptilophorinae Gerstaecker, 1855

Batelka in Cat. Pal. Col., vol. 5, 2008

Ptilophorus dufourii (Latreille, 1817)

Ripiphorinae Gemminger \& Harold, 1870

Ripiphorini Reitter, 1911

Rhipiphorus subdipterus Bosc, 1792

ZOPHERIDAE Solier, 1834

Zopherinae Solier, 1834

Pycnomerini Erichson, 1845

Ślipinsky \& Schuh in Cat. Pal. Col., vol. 5, 2008

Pycnomerus italicus (Ganglbauer, 1899)

Pycnomerus terebrans (A.G. Olivier, 1790)

\section{Colydiinae Erichson, 1842}

Colydiini Erichson, 1842

Aulonium muficorne (A.G. Olivier, 1790)

Colydium elongatum (Fabricius, 1787)

Colydium filiforme Fabricius, 1792

\begin{tabular}{|c|c|c|c|c|c|c|c|c|}
\hline $\mathrm{X}$ & $\mathrm{X}$ & & & & & & & LC \\
\hline & & & & $\mathrm{N}$ & $\mathrm{N}$ & $\mathrm{B}$ & & LC \\
\hline $\mathrm{X}$ & & & & & & $\mathrm{X}$ & $\mathrm{N}$ & $\mathrm{NT}$ \\
\hline
\end{tabular}

Synchitini Erichson, 1845

Bitoma crenata (Fabricius, 1775)

Colobicus hirtus (P. Rossi, 1790)

Corticus celtis (Germar, 1824)

Coxelus pictus (J. Sturm, 1807)

Diodesma denticincta Abeille de Perrin, 1899

\begin{tabular}{|c|c|c|c|c|c|c|c|c|}
\hline $\mathrm{H}$ & $\mathrm{X}$ & $\mathrm{X}$ & $\mathrm{N}$ & $\mathrm{N}$ & $\mathrm{X}$ & $\mathrm{X}$ & $\mathrm{N}$ & $\mathrm{LC}$ \\
\hline $\mathrm{X}$ & $\mathrm{X}$ & & & & $\mathrm{X}$ & & $\mathrm{N}$ & $\mathrm{NT}$ \\
\hline $\mathrm{H}$ & $\mathrm{X}$ & $\mathrm{X}$ & $\mathrm{N}$ & $\mathrm{N}$ & $\mathrm{X}$ & $\mathrm{X}$ & $\mathrm{B}$ & LC \\
\hline & & & $\mathrm{N}$ & $\mathrm{N}$ & $\mathrm{X}$ & $\mathrm{X}$ & $\mathrm{N}$ & LC \\
\hline & & & $\mathrm{N}$ & $\mathrm{B}$ & $\mathrm{N}$ & $\mathrm{N}$ & $\mathrm{N}$ & $\mathrm{NT}$
\end{tabular}


Species

Endophloeus markovichianus (Piller \& Mitterpacher, 1783)

Langelandia anophthalma Aubé, 1842

Langelandia ausonica Obenberger, 1914 [E]

Langelandia reitteri Belon, 1882

Nosodomodes tuberculatus (Germar, 1832)

Synchita mediolanensis A. Villa \& G.B. Villa, 1833

Synchita separanda (Reitter, 1882)

Synchita undata Guérin-Méneville, 1844

Synchita variegata Hellwig, 1792

Tarphius gibbulus Erichson, 1845 [E]

TENEBRIONIDAE Latreille, 1802

Lagriinae Latreille, 1825

Belopini Reitter, 1917

Löbl, Merkl, Ando, Bouchard, Lillig, Masomutu \& Schawaller in Cat. Pal. Col., vol. 5, 2008

Centorus elongatus ecalcaratus (Seidlitz, 1896)

Centorus crassipes crassipes (Fischer von Waldheim, 1844)

\begin{tabular}{|l|l|l|l|l|l|l|l|l|}
\hline $\mathrm{X}$ & & & & & & & & \\
\hline
\end{tabular}

Cossyphini Latreille, 1802

Cossyphus moniliferus moniliferus Chevrolat, 1829

Cossyphus tauricus Steven, 1832

Lagriini Latreille, 1825

Lagriina Latreille, 1825

Merkl in Cat. Pal. Col., vol. 5, 2008

Lagria atripes Mulsant \& Guillebeau, 1855

Lagria hirta (Linnaeus, 1758)

Lagria rugosula Rosenhauer, 1856

\begin{tabular}{|l|l|l|l|l|l|}
\hline $\mathrm{X}$ & & & & & \\
\hline
\end{tabular}

Pimeliinae Latreille, 1802

Akidini Billberg, 1820

Akis trilineata Herbst, 1799

Akis subterranea Solier, 1836

Asidini Fleming, 1821

Soldati in Cat. Pal. Col., vol. 5, 2008

Alphasida grossa grossa (Solier, 1836) [E]

Asida bayardi bayardi Solier, 1836 [E]

Asida calabra Leoni, 1909 [E]

Asida fascicularis fiorii Leoni, 1909 [E]

Asida goryi Solier, 1836 [E]

Asida novasiriensis Grimm, 1985 [E]

Asida piligera Leoni, 1909 [E]

Boromorphus italicus Gardini, 2010 [E]
Cnemeplatiini Jacquelin du Val, 1861

Boromorphini Skopin, 1978

\begin{tabular}{|c|c|c|c|c|c|c|c|c|}
\hline B & & & B & B & X & & & \\
\hline H & & & & & & & & \\
\hline & & & & & & & B & \\
\hline & N & X & & & & & & \\
\hline B & & & & & & & & \\
\hline
\end{tabular}

\begin{tabular}{|l|l|l|l|l|}
\hline B & & & & \\
\hline
\end{tabular}

Cnemeplatia atropos atropos A. Costa, 1847

Elenophorini Solier, 1837

Elenophorus italicus (Ferrer, 2015)

Erodiini Billberg, 1820

Erodius siculus dalmatimus Kraatz, 1865

Erodius siculus neapolitamus Solier, 1834 [E]

Pimeliini Latreille, 1802

Pimelia rugulosa apula Gridelli, 1950 [E]

Pimelia rugulosa rugulosa Germar, 1824 [E]

Stenosini Lacordaire, 1859

\begin{tabular}{|l|l|l|l|l|l|l|l|}
\hline $\mathrm{X}$ & $\mathrm{X}$ & & & & & & \\
\hline
\end{tabular}

\begin{tabular}{|c|c|c|c|c|c|c|c|c|}
\hline $\mathrm{X}$ & $\mathrm{X}$ & & & & & & & \\
\hline
\end{tabular}




\begin{tabular}{|c|c|c|c|c|c|c|c|c|c|}
\hline Species & 递 & $\begin{array}{l}70 \\
0 \\
3 \\
0 \\
0 \\
\frac{3}{0} \\
0\end{array}$ & 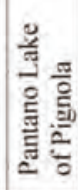 & 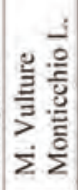 & 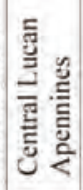 & 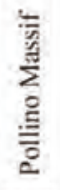 & 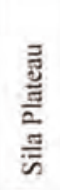 & 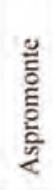 & 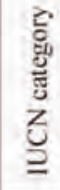 \\
\hline Dichillus corsicus (Solier, 1838) & & & & & & B & $\mathrm{B}$ & B & \\
\hline Dichillus minutus (Solier, 1838) & & & & & B & & & & \\
\hline Dichillus pertusus (Kiesenwetter, 1861) & & & & & & & & B & \\
\hline Eutagenia elvirae Marcuzzi \& Turchetto, 1982 [E] & & $\mathrm{N}$ & & & & & & & \\
\hline Stenosis brenthoides brenthoides (P. Rossi, 1790) & $\mathrm{X}$ & $\mathrm{X}$ & & $\mathrm{N}$ & & $\mathrm{X}$ & $\mathrm{X}$ & $\mathrm{B}$ & \\
\hline Stenosis intermedia intermedia (Solier, 1838) & B & B & & B & $\mathrm{N}$ & $\mathrm{N}$ & $\mathrm{B}$ & $\mathrm{B}$ & \\
\hline
\end{tabular}

Stenosis intermedia intermedia (Solier, 1838)

Tentyriini Eschscholtz, 1831

Pachychila dejeani dejeani (Besser, 1832)

Pachychila italica Ferrer, 2018

Tentyria grossa grossa Besser, 1832

Tentyria italica Solier, 1835

\begin{tabular}{|c|c|c|c|c|c|c|c|}
\hline & N & & & & & B \\
\hline X & X & & & & & & B \\
\hline & X & & & & B \\
\hline H & X & B & & B & B & \\
\hline
\end{tabular}

Tenebrioninae Latreille, 1802

Alphitobiini Reitter, 1917

Alphitobius diaperimus (Panzer, 1796)

Diaclina fagi (Panzer, 1799)

Diaclina testudinea (Piller \& Mitterpacher, 1783)

\begin{tabular}{|c|c|c|c|c|c|c|c|c|}
\hline $\mathrm{X}$ & & & $\mathrm{N}$ & $\mathrm{N}$ & $\mathrm{X}$ & $\mathrm{X}$ & $\mathrm{N}$ & \\
\hline & $\mathrm{X}$ & & & $\mathrm{N}$ & & & & DD \\
\hline
\end{tabular}

Blaptini Leach, 1815

Blaptina Leach, 1815

Löbl, Nabozhenko \& Merkl in Cat. Pal. Col., vol. 5, 2008

Blaps gibba Laporte, 1840

Blaps gigas (Linnaeus, 1767)

Blaps lethifera lethifera Marsham, 1802

Blaps mucronata Latreille, 1804

\begin{tabular}{|c|c|c|c|c|c|c|c|c|}
\hline H & X & X & B & N & X & X & B & \\
\hline B & X & & & & & & B & \\
\hline & & & & & X & & & \\
\hline X & X & & & & & & B & \\
\hline
\end{tabular}

Bolitophagini Kirby, 1837

Bolitophagina Kirby, 1837

Bolitophagus interruptus Illiger, 1800

Bolitophagus reticulatus (Linnaeus, 1767)

Eledona agricola (Herbst, 1783)

Eledonoprius armatus (Panzer, 1799)

Eledonoprius serrifrons (Reitter, 1890)

\begin{tabular}{|c|c|c|c|c|c|c|c|c|}
\hline $\mathrm{X}$ & $\mathrm{X}$ & & & & $\mathrm{X}$ & $\mathrm{X}$ & & $\mathrm{EN}$ \\
\hline $\mathrm{X}$ & $\mathrm{X}$ & & $\mathrm{N}$ & $\mathrm{N}$ & $\mathrm{X}$ & $\mathrm{X}$ & $\mathrm{N}$ & $\mathrm{VU}$ \\
\hline & & & & $\mathrm{N}$ & & $\mathrm{X}$ & & $\mathrm{NT}$ \\
\hline $\mathrm{B}$ & & & & & & & & $\mathrm{CR}$ \\
\hline $\mathrm{B}$ & & & & & $\mathrm{B}$ & & & $\mathrm{CR}$ \\
\hline
\end{tabular}

Helopini Latreille, 1802

Cylindrinotina Espanol, 1956

Nabozhenko \& Löbl in Cat. Pal. Col., vol. 5, 2008

Nalassus dermestoides (Illiger, 1798)

Nalassus dryadophilus (Mulsant, 1854)

Nalassus planipennis (Küster, 1850) [E]

Nalassus plebejus (Küster, 1850)

Odocnemis exaratus (Germar, 1817)

Stenomax aeneus (Scopoli, 1763)

Xanthomus pallidus (Curtis, 1830)

Xanthomus pellucidus (Mulsant \& Rey, 1856)

Helopina Latreille, 1802

\begin{tabular}{|l|c|c|c|c|c|c|c|c|c|}
\hline Accanthopus velikensis (Piller \& Mitterpacher, 1783) & H & X & X & B & N & X & X & B & LC \\
\hline Catomus rotundicollls (Guérin-Méneville, 1825) & X & X & & & & & & B & \\
\hline Helops caeruleus caeruleus (Linnaeus, 1758) & X & X & X & N & N & X & X & B & LC \\
\hline Helops rossii (Germar, 1817) & H & X & & & & & & B & LC \\
\hline Italohelops subchalybaeus (Reitter, 1907) [E] & & X & & & & X & & NT \\
\hline Probaticus anthrax (Seidlitz, 1896) [E] & & & & & & & & B & DD \\
\hline Probaticus sphaericollis (Küster, 1850 [E]) & & N & X & & & & B & NT \\
\hline Raiboscelis azureus azureus (Brullé, 1832) & B & & & & & & & DD \\
\hline
\end{tabular}

Melanimini Seidlitz, 1894

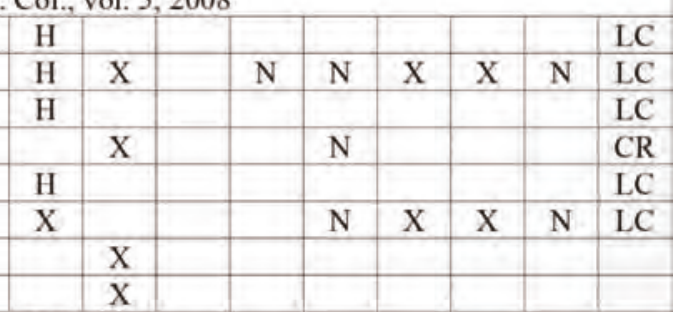


Species

Melanimon tibialis tibialis (Fabricius, 1781)

Opatrini Brullé, 1832

Opatrina Brullé, 1832

Iwan \& Löbl in Cat. PaL. Col., vol. 5, 2008

Ammobius rufus (P.H. Lucas, 1846)

Gonocephalum granulatum pusillum (Fabricius, 1792)

Gonocephalum obscurum obscurum (Küster, 1849)

Gonocephalum prolixum (Erichson, 1843)

Gonocephalum rusticum (A.G. Olivier, 1811)

Opatroides punctulatus punctulatus Brullé, 1832

Opatrum obesum A.G. Olivier, 1811

Opatrum sabulosum sculptum Rey, 1892

Opatrum verrucosum Germar, 1817

Palorini Matthews, 2003

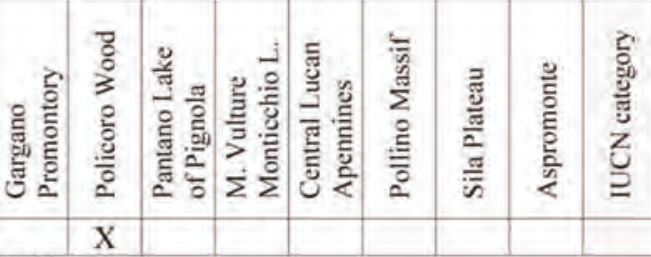

\begin{tabular}{|c|c|c|c|c|c|c|c|}
\hline X & X & & & & & B \\
\hline H & X & & & X & X & B & \\
\hline & & & & & & & B \\
\hline H & & & & & & & B \\
\hline B & N & & N & N & X & B & \\
\hline & & & & & & & B \\
\hline & X & & & & & B \\
\hline B & X & & N & X & X & B \\
\hline B & & & B & X & & B \\
\hline
\end{tabular}

Palorus depressus (Fabricius, 1790)

Palorus ratzeburgii (Wissmann, 1848)

Palorus subdepressus (Wollaston, 1864)

Pedinini Eschscholtz, 1829

\begin{tabular}{|c|c|c|c|c|c|c|c|c|}
\hline $\mathrm{X}$ & $\mathrm{X}$ & & $\mathrm{N}$ & $\mathrm{N}$ & $\mathrm{X}$ & $\mathrm{X}$ & $\mathrm{N}$ & $\mathrm{LC}$ \\
\hline & $\mathrm{X}$ & & & & & & & \\
\hline
\end{tabular}

Dendarina Seidlitz, 1889

Iwan \& Löbl in Cat. Pal. Col., vol. 5, 2008

Dendarus dalmatinus (Germar, 1824)

Dendarus lugens (Mulsant \& Rey, 1854)

Dendarus peslieri Soldati, 2012

\begin{tabular}{|c|c|c|c|c|c|c|c|c|}
\hline $\mathrm{H}$ & $\mathrm{X}$ & & & & & & & \\
\hline & & & $\mathrm{B}$ & & $\mathrm{X}$ & $\mathrm{X}$ & $\mathrm{B}$ & \\
\hline $\mathrm{X}$ & & & & & & & & \\
\hline
\end{tabular}

Leichenina Mulsant, 1854

Leichenum pictum (Fabricius, 1801)

\begin{tabular}{|c|c|}
$\mathrm{X}$ & $\mathrm{X}$ \\
\hline
\end{tabular}

Allophylax picipes (A.G. Olivier, 1811) Melambiina Mulsant \& Rey, 1854

\begin{tabular}{|l|l|l|l|l|}
\hline $\mathrm{X}$ & & & & \\
\hline
\end{tabular}

Pedinina Eschscholtz, 1829

Colpotus strigosus ganglbaueri D'Amore Fracassi, 1907

Colpotus strigosus ragusae D'Amore Fracassi, 1907

Colpotus strigostus strigosus (A. Costa, 1847)

Pedinus helopioides Germar, 1814

Pedinus meridiamus Mulsant \& Rey, 1853

Pedinus ragusae Baudi di Selve, 1876

\begin{tabular}{|c|c|c|c|c|c|c|c|c|}
\hline & X & & & & & & B & \\
\hline & & & & & & X & & \\
\hline H & N & X & B & N & X & X & N & \\
\hline & & & & & & & B & \\
\hline
\end{tabular}

Scaurus striatus Fabricius, 1792

Scaurus tristis A.G. Olivier, 1795

Scaurini Billberg, 1820

\begin{tabular}{|c|c|c|c|c|c|c|c|c|}
\hline $\mathrm{X}$ & $\mathrm{X}$ & & & & & $\mathrm{B}$ & $\mathrm{B}$ & \\
\hline $\mathrm{B}$ & & & & & & & $\mathrm{B}$ & \\
\hline
\end{tabular}

Tenebrionini Latreille, 1802

Neatus noctivagus (Mulsant \& Rey, 1853)

Tenebrio molitor Linnaeus, 1758

Tenebrio obscurus Fabricius, 1792

Lyphia tetraphylla (Fairmaire, 1856)

Triboliini Gistel, 1848

\begin{tabular}{|c|c|c|c|c|c|c|c|c|}
\hline $\mathrm{X}$ & $\mathrm{X}$ & & & & $\mathrm{X}$ & $\mathrm{X}$ & & $\mathrm{VU}$ \\
\hline $\mathrm{X}$ & $\mathrm{X}$ & & & $\mathrm{X}$ & & $\mathrm{B}$ & \\
\hline & $\mathrm{X}$ & & $\mathrm{N}$ & & $\mathrm{X}$ & & $\mathrm{B}$ & $\mathrm{LC}$ \\
\hline
\end{tabular}

Uloma culinaris (Linnaeus, 1758)

Uloma rufa (Piller \& Mitterpacher, 1783)

Ulomini Blanchard, 1845

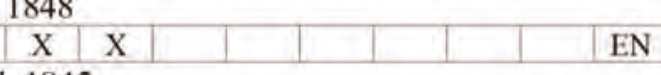

\begin{tabular}{|l|l|l|l|l|l|l|l|l|l|}
\hline $\mathrm{X}$ & $\mathrm{X}$ & & $\mathrm{N}$ & $\mathrm{N}$ & $\mathrm{X}$ & $\mathrm{X}$ & $\mathrm{N}$ & $\mathrm{LC}$ \\
\hline
\end{tabular}

Diaperinae Latreille, 1802

Crypticini Brullé, 1832

Oochrotus unicolor ardoini Canzoneri, 1961 [E]

Pseudoseriscius helvolus adriaticus (Espanol, 1949)

Pseudoseriscius helvolus helvolus (Küster, 1852)

\begin{tabular}{|c|c|c|c|c|c|c|c|c|}
\hline $\mathrm{H}$ & $\mathrm{X}$ & $\mathrm{X}$ & & & & & $\mathrm{N}$ & \\
\hline $\mathrm{X}$ & & & & & & & & \\
\hline
\end{tabular}

Diaperini Latreille, 1802 
Species

Alphitophagus bifasciatus (Say, 1824)

Diaperis boleti boleti (Linnaeus, 1758)

Gnathocerus cormutus (Fabricius, 1798)

Neomida haemorrhoidalis (Fabricius, 1787)

Pentaphyllus chrysomeloides (P. Rossi, 1792)

Pentaphyllus testaceus (Hellwig, 1792)

Platydema europaeum Laporte \& Brullé, 1831

Platydema violaceum (Fabricius, 1790)

Hypophlaeini Billberg, 1820

\begin{tabular}{|c|c|c|c|c|c|c|c|c|}
\hline 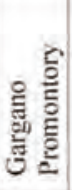 & $\begin{array}{l}30 \\
\vdots \\
3 \\
0 \\
0 \\
\frac{0}{0} \\
2 \\
2\end{array}$ & 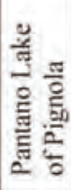 & 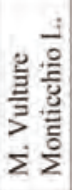 & 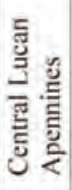 & 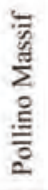 & $\frac{\vec{z}}{\frac{\vec{g}}{\tilde{g}}}$ & 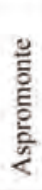 & 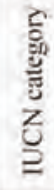 \\
\hline & $X$ & $\mathrm{~N}$ & & $\mathrm{~N}$ & $\mathrm{~N}$ & X & & \\
\hline $\mathrm{X}$ & $\mathrm{X}$ & & $\mathrm{N}$ & $\mathrm{N}$ & $\mathrm{X}$ & $\mathrm{X}$ & $\mathrm{N}$ & LC \\
\hline$X$ & $X$ & & & & $\mathrm{X}$ & $\mathrm{X}$ & & \\
\hline$X$ & $\mathrm{X}$ & & & $\mathrm{N}$ & $\mathrm{X}$ & $\mathrm{X}$ & $\mathrm{N}$ & EN \\
\hline X & $\mathrm{X}$ & $\mathrm{X}$ & & $\mathrm{N}$ & $\mathrm{N}$ & $\mathrm{X}$ & $\mathrm{N}$ & EN \\
\hline & $\mathrm{N}$ & & & $\mathrm{N}$ & & & & EN \\
\hline & & & & & & $\mathrm{X}$ & & CR \\
\hline X & $X$ & & & & & & & NT \\
\hline
\end{tabular}

Corticeus bicolor (A.G. Olivier, 1790)

Corticeus fasciatus Fabricius, 1790

Corficeus fraxini (Kugelann, 1794)

Corticeus pini (Panzer, 1799)

Corticeus unicolor Piller \& Mitterpacher, 1783

Myrmechixenini Jacquelin du Val, 1858

Myrmechixemus vaporariortum Guérin-Méneville, 1843

Phaleriini Blanchard, 1845

Phaleria acuminata acuminata Küster, 1852

Phaleria bimaculata bimaculata (Linnaeus, 1767)

Phtora crenata Germar, 1836

Scaphidemini Reitter, 1922

Scaphidema metallicum metallicum (Fabricius, 1792)

Trachyscelini Blanchard, 1845

Trachyscelis aphodioides aphodioides Latreille, 1809

\begin{tabular}{|r|r|l|l|l|l|l|l|l|}
$\mathrm{X}$ & $\mathrm{X}$ & & & & & & $\mathrm{N}$ & \\
\hline $\mathrm{N}$ & & & & & & $\mathrm{B}$ & \\
\hline & $\mathrm{X}$ & & & & & & & \\
\hline
\end{tabular}

\begin{tabular}{|l|l|l|l|l|l|l|l|l|}
\hline $\mathrm{X}$ & $\mathrm{X}$ & & $\mathrm{N}$ & $\mathrm{N}$ & $\mathrm{X}$ & $\mathrm{X}$ & $\mathrm{N}$ & $\mathrm{LC}$ \\
\hline
\end{tabular}

Alleculinae Laporte, 1840

Alleculini Laporte, 1840

Alleculina Laporte, 1840

Novák \& Pettersson in Cat. Pal. Col., vol. 5, 2008

Allecula morio (Fabricius, 1787)

Hymenalia rufipes (Fabricius, 1792)

Prionychus ater (Fabricius, 1775)

Gonodera luperus luperus (Herbst, 1783)

Gonodera metallica (Küster, 1850)

Isomira genistae (Rottenberg, 1871) [E]

Isomira icteropa (Küster, 1852)

Isomira marcida Kiesenwetter, 1863

Isomira melanophthalma (P.H. Lucas, 1846)

Isomira murina murina (Linnaeus, 1758)

Isomira ochropus (Küster, 1850)

Isomira testacea Seidlitz, 1896

Isomira umbellatarum (Kiesenwetter, 1863)

Pseudocistela ceramboides ceramboides (Linnaeus, 1761)

Gonoderina Seidlitz, 1896

\begin{tabular}{|c|c|c|c|c|c|c|c|c|}
\hline & & & N & & & N & LC \\
\hline & N & & & & & & LC \\
\hline
\end{tabular}

Mycetochara axillaris axillaris (Paykull, 1799)

Mycetochara maura (Fabricius, 1792)

Mycetochara quadrimaculata (Latreille, 1804)

Cteniopodini Solier, 1835

Cteniopus neapolitamus Baudi di Selve, 1877 [E]

Cteniopus sulphureus (Linnaeus, 1758)

Cteniopus sulphuripes (Germar, 1824)

Megischia curvipes curvipes (Brullé, 1832)

\begin{tabular}{|c|c|c|c|c|c|c|c|c|}
\hline H & & X & N & N & X & X & B & \\
\hline H & & X & N & N & X & X & & \\
\hline & & & & N & N & & & \\
\hline X & & & N & & X & X & N & \\
\hline X & X & X & & & X & X & & \\
\hline H & X & & & N & & & N & \\
\hline & & & B & & X & X & B & \\
\hline H & N & & & & & & N & \\
\hline & & X & N & N & X & X & B & \\
\hline
\end{tabular}

Mycetocharina Gistel, 1848 
Species

Megischina armillata (Brullé, 1832)

Omophlus dispar A. Costa, 1847 [E

Omophlus flavipennis Küster, 1849

Omophlus lepturoides (Fabricius, 1787)

Omophlus longicornis Bertolini, 1868

Omophlus picipes (Fabricius, 1792)

Omophlus pubescens (Linnaeus, 1758)

Omophlus rugosicollis (Brullé, 1832)

Podonta italica Baudi di Selve, 1877 [E]

\section{Stenochiinae Kirby, 1837}

Cnodalonini Gistel, 1856

Iphthiminus italicus italicus (Truqui, 1857)

Menephilus cylindricus cylindricus (Herbst, 1784)

\begin{tabular}{|l|l|l|l|l|l|l|}
\hline $\mathrm{X}$ & & $\mathrm{B}$ & $\mathrm{X}$ & $\mathrm{X}$ & $\mathrm{B}$ & $\mathrm{VU}$ \\
\hline & & $\mathrm{N}$ & $\mathrm{X}$ & $\mathrm{X}$ & $\mathrm{N}$ & $\mathrm{NT}$ \\
\hline
\end{tabular}

PROSTOMIDAE C.G. Thomson, 1859

Schawaller in Cat. Pal. Col. Vol. 5, 2008

\begin{tabular}{|l|l|l|l|l|l|l|l|}
\hline Prostomis mandibularis (Fabricius, 1801) & & & & N & & LC
\end{tabular}

OEDEMERIDAE Latreille, 1810

Calopodinae A. Costa, 1852

Švihla in Cat. Pal. Col., vol. 5, 2008

Sparedrus orsinii A. Costa, 1852 [E]

Oedemerinae Latreille, 1810

\begin{tabular}{|l|l|l|l|l|l|l|l|l|}
$\mathrm{B}$ & $\mathrm{X}$ & $\mathrm{X}$ & & $\mathrm{N}$ & & & $\mathrm{N}$ & LC \\
\hline
\end{tabular}

Asclerini Semenov, 1894

Ischnomera caerulea (Linnaeus, 1758)

Ischnomera cinerascens cinerascens (Pandellé, 1867)

Ischnomera cyanea (Fabricius, 1792)

Ischnomera sanguinicollis (Fabricius, 1787)

Ditylini Mulsant, 1858

\begin{tabular}{|l|l|l|l|l|l|l|l|l|l|}
\hline Chrysanthia viridissima (Linnaeus, 1758) & & & N & N & X & X & B & LC
\end{tabular}

Nacerdini Mulsant, 1858

Anogcodes ruficollis (Fabricius, 1781

Anogcodes rufiventris (Scopoli, 1763)

Nacerdes melamura Linnaeus, 1758

\begin{tabular}{|c|c|c|c|c|c|c|c|c|}
\hline & X & & N & N & X & & N & LC \\
\hline & X & & & & & X & & LC \\
\hline I & N & & & & & & LC \\
\hline
\end{tabular}

Oedemerini Latreille, 1810

Oedemera atrata W.L.E. Schmidt, 1846

Oedemera barbara (Fabricius, 1792)

Oedemera crassipes Ganglbauer, $188 \mathrm{I}$

Oedemera croceicollis Gyllenhal, 1827

Oedemera femoralis femoralis A.G. Olivier, 1803

Oedemera femorata (Scopoli, 1763)

Oedemera flavipes (Fabricius, 1792)

Oedemera lurida (Marsham, 1802)

Oedemera melanopyga W.L.E. Schmidt, 1846 [E]

Oedemera nobilis (Scopoli, 1763)

Oedemera podagrariae podagrariae (Linnaeus, 1767)

Oedemera pthysica (Scopoli, 1763)

Oedemera simplex (Linnaeus, 1767)

Stenostomini Mulsant, 1858

\begin{tabular}{|c|c|c|c|c|c|c|c|c|}
\hline X & X & & B & N & X & X & B & \\
\hline H & X & & & N & N & X & B & \\
\hline H & X & & B & N & X & X & N & \\
\hline & X & & & & & & & \\
\hline & & & & N & X & & N & LC \\
\hline B & X & N & B & B & X & X & B & \\
\hline X & X & X & B & B & X & X & B & \\
\hline H & & X & B & B & X & X & B & \\
\hline H & X & X & B & B & X & X & B & \\
\hline H & X & X & N & N & X & X & B & \\
\hline & X & X & B & N & X & X & B & \\
\hline H & X & X & N & B & X & X & B & \\
\hline
\end{tabular}

Stenostoma rostratum septentrionale Śvihla, 2005

\begin{tabular}{l|l}
$\mathrm{X}$ & $\mathrm{X}$
\end{tabular} 
Species

MELOIDAE Gyllenhal, 1810

Meloinae Gyllenhal, 1810

Cerocomini Leach, 1815

Bologna in Cat. Pal. Col., vol. 5, 2008; Bologna et al., 2008

\begin{tabular}{|l|l|l|l|l|l|l|l|l|} 
Cerocoma schaefferi (Linnaeus, 1758) & $\mathrm{B}$ & $\mathrm{X}$ & $\mathrm{B}$ & $\mathrm{B}$ & $\mathrm{X}$ & $\mathrm{X}$ & $\mathrm{B}$ \\
\hline
\end{tabular}

Epicautini Denier, 1935

Epicauta rufidorsum (Goeze, 1777)

Lyttini Solier, 1851

Lydus trimaculatus italicus Kaszab, 1952 [E]

Lytta vesicatoria vesicatoria (Linnaeus, 1758)

Mylabrini Laporte, 1840

Mylabris fabricii Sumakov, 1924

Mylabris flexuosa A.G. Olivier, 1811

Mylabris absoleta Nowicki, 1872 [E]

Mylabris quadripumctata quadripunctata (Linnaeus, 1767)

Mylabris variabilis (Pallas, 1781)

Meloini Gyllenhal, 1810

\begin{tabular}{|c|c|c|c|c|c|c|c|c|}
\hline Meloe apenninicus Bologna, 1988 [E] & & & B & & & B & B & B \\
\hline Meloe autumnalis autumnalis A.G. Olivier, 1797 & B & $\mathrm{X}$ & & & $\mathrm{N}$ & & B & B \\
\hline Meloe baudii Leoni, 1907 & B & & & & & & B & \\
\hline Meloe brevicollis brevicollis Panzer, 1793 & $\mathrm{X}$ & & & B & & $\mathrm{X}$ & $\mathrm{X}$ & B \\
\hline Meloe cavensis L. Petagna, 1819 & & & & & & & & B \\
\hline Meloe cicatricosus Leach, 1815 & & & & B & B & B & & B \\
\hline Meloe erythrocnemus Pallas, 1782 & $\mathrm{~B}$ & & & & & & $\mathrm{~B}$ & B \\
\hline Meloe ganglbaueri Apfelbeck, 1905 & B & & & & & & & B \\
\hline Meloe mediterranens G. Müller, 1925 & $\mathrm{X}$ & $\mathrm{N}$ & & & & & & B \\
\hline Meloe proscarabaeus proscarabaeus Linnaeus, 1758 & B & $\mathrm{X}$ & $\mathrm{X}$ & & & B & B & $\mathrm{B}$ \\
\hline Meloe rugosus Marsham, 1802 & & & & & & & $\mathrm{X}$ & \\
\hline Meloe tuccius corrosus Brandt \& Erichson, 1832 [E] & & $\mathrm{X}$ & & & & & $\mathrm{B}$ & B \\
\hline Meloe violaceus Marsham, 1802 & $\mathrm{H}$ & & & & B & & B & \\
\hline
\end{tabular}

Meloe violaceus Marsham, 1802

Nemognathinae Laporte, 1840

Nemognathini Laporte, 1840

Sitaris muralis (Forster, 1771)

Stenoria apicalis apicalis (Latreille, 1804)

Zonitis flava Fabricius, 1775

Zonitis immaculata (A.G. Olivier, 1789)

Zomitis nana Ragusa, 1881

\begin{tabular}{|c|c|c|c|c|c|c|c|c|}
\hline B & & & & & & & B & \\
\hline B & & & & & & & & \\
\hline H & X & X & & B & X & X & N & \\
\hline H & X & & N & B & X & X & B & \\
\hline & & & & & X & & & \\
\hline
\end{tabular}

MYCTERIDAE Blanchand, 1845

Mycterinae Blanchard, 1845

Löbl in Cat. Pal. Col., vol. 5, 2008

Mycterus curculionoides (Fabricius, 1781)

Mycterus tibialis Küster, 1850

Mycterus umbellatarum (Fabricius, 1787)

\begin{tabular}{|l|l|l|l|l|l|l|l|l|}
\hline B & X & & & & N & X & N & \\
\hline X & X & X & B & N & X & X & N & \\
\hline X & & & & & & & & \\
\hline
\end{tabular}

PYROCHROIDAE Latreille, 1807

Pyrochroinae Latreille, 1807

Pollock \& Young in Cat. Pal. Col., vol. 5, 2008

Pyrochroa coccinea (Linnaeus, 1760)

Pyrochroa serraticornis kiesenwetteri Fairmaire, 1849

Pyrochroa serraticormis serraticornis Scopoli, 1763)

\begin{tabular}{|c|c|c|c|c|c|c|c|c|} 
& $\mathrm{X}$ & $\mathrm{X}$ & $\mathrm{N}$ & $\mathrm{N}$ & $\mathrm{X}$ & $\mathrm{X}$ & $\mathrm{N}$ & $\mathrm{LC}$ \\
\hline & & & & & & $\mathrm{X}$ & & $\mathrm{NT}$ \\
\hline $\mathrm{X}$ & & & & $\mathrm{N}$ & $\mathrm{X}$ & & $\mathrm{N}$ & $\mathrm{LC}$ \\
\hline
\end{tabular}


Species

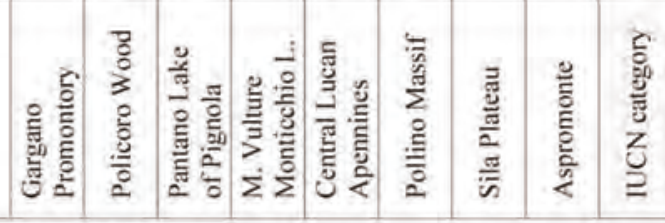

SALPINGIDAE Leach, 1815

Agleninae Horn, 1878

Pollock \& Löbl in Cat. Pal. Col., vol. 5, 2008

Aglemus brunneus (Gyllenhal, 1813)

Salpinginae Leach, 1815

Lissodema denticolle (Gyllenhal, 1813)

Lissodema lituratum (A. Costa, 1847)

Rabocerus foveolatus (Ljungh, 1823)

Salpingus planirostris (Fabricius, 1787)

Salpingus ruficollis (Linnaeus, 1760)

Vincenzellus ruficollis (Panzer, 1794)

ANTHICIDAE Latreille, 1819

Anthicinae Latreille, 1819

Chandler, Uhmann, Nardi \& Telnov in Cat. Pal. Col., vol. 5, 2008

Amblyderus scabricollis (LaFerté-Sénectère, 1847)

Anthelephila pedestris (P. Rossi, 1790)

Anthicus antherinus antherinus (Linnaeus, 1760)

Anthicus biguttatus LaFerté-Sénectère, 1849

Anthicus fenestratus. W.L.E. Schmidt, 1842

Anthicus invreai Koch, 1933

Anthicus laeviceps Baudi di Selve, 1877

Anthicus luteicornis W.L.E. Schmidt, 1842

Anthicus niger (A.G. Olivier, 1811)

Anthicus quadrioculatus LaFerté-Sénectère, 1849

Anthicus tristis tristis W.L.E. Schmidt, 1842

Clavicomus paganettii (Pic, 1909) [E]

Cordicomus gracilior (Abeille de Perrin, 1885)

Cordicomus instabilis instabilis (W.L.E. Schmidt, 1842)

Cyclodinus blandulus blandulus (Baudi di Selve, 1877)

Cyclodinus bremei bremei (LaFerté-Sénectẻre, 1842)

Cyclodinus coniceps (Marseul, 1879)

Cyclodimus constrictus constrictus (Curtis, 1838)

Cyclodinus humilis (Germar, 1824)

Cyclodinus italicus (Pic, 1901) [E]

Cyclodinus larvipennis (Marseul, 1879)

Cyclodinus mimutus minutus (LaFerté-Sénectère, 1842)

Endomia occipitalis (Dufour, 1843)

Endomia tenuicollis temuicollis (P. Rossi, 1792)

Endomia unifasciata unifasciata (Bonelli, 1812)

Hirticomus hispidus (P. Rossi, 1792)

Hirticomus quadriguttatus (P. Rossi, 1792)

Leptaleus rodriguesi (Latreille, 1804)

Microhoria fasciata fasciata (Chevrolat, 1834)

Microhoria helenae (Koch, 1933) [E]

Microhoria leonhardi (Krekich-Strassoldo, 1913) [E]

Microhoria raveli (Pic, 1899)

Microhoria terminata (W.L.E. Schmidt, 1842)

Omonadus bifasciatus (P. Rossi, 1792)

Omonadus floralis (Linnaeus, 1758)

Omonadus formicarius formicarius (Goeze, 1777)

Stricticomus longicollis (W.L.E. Schmidt, 1842)

Stricticomus tobias (Marseul, 1879)

\begin{tabular}{|c|c|c|c|c|c|c|c|c|}
\hline \\
\hline & & & & & & $\mathrm{N}$ & & \\
\hline $\mathrm{H}$ & $\mathrm{X}$ & $\mathrm{X}$ & $\mathrm{N}$ & $\mathrm{N}$ & $X$ & $\mathrm{X}$ & B & \\
\hline \multirow[t]{2}{*}{$\mathrm{H}$} & & X & & & $X$ & $X$ & $\mathrm{~N}$ & \\
\hline & & & & & & & $\mathrm{N}$ & \\
\hline $\mathrm{X}$ & $\mathrm{X}$ & & & & & & $\mathrm{N}$ & \\
\hline $\mathrm{X}$ & $\mathrm{X}$ & $\mathrm{X}$ & & & & & & \\
\hline \multirow[t]{2}{*}{$\mathrm{X}$} & $X$ & $\mathrm{X}$ & $\mathrm{N}$ & $\mathrm{N}$ & $X$ & $\mathrm{X}$ & $\mathrm{N}$ & \\
\hline & & & & $\mathrm{N}$ & & & & \\
\hline \multirow[t]{5}{*}{$\mathrm{H}$} & $\mathrm{X}$ & $\mathrm{X}$ & $\mathrm{N}$ & $\mathrm{N}$ & $\mathrm{X}$ & $\mathrm{N}$ & B & \\
\hline & $\mathrm{N}$ & & & B & & & & \\
\hline & $\mathrm{X}$ & & & & & & $\mathrm{N}$ & \\
\hline & $\mathrm{X}$ & & & & & & & \\
\hline & $\mathrm{X}$ & & & & & & & \\
\hline \multirow[t]{3}{*}{$\mathrm{H}$} & $\mathrm{X}$ & $\mathrm{X}$ & & $\mathrm{N}$ & $\mathrm{N}$ & & B & \\
\hline & & & & & & & B & \\
\hline & $\mathrm{X}$ & & & & & & & \\
\hline $\mathrm{X}$ & $\mathrm{X}$ & & & & & & $\mathrm{N}$ & \\
\hline \multirow[t]{2}{*}{$X$} & & & & & $\mathrm{~N}$ & & B & \\
\hline & $\mathrm{N}$ & $\mathrm{X}$ ? & & & & & B & \\
\hline \multirow[t]{2}{*}{$\mathrm{X}$} & $\mathrm{X}$ & & & & & & & \\
\hline & $\mathrm{X}$ & & & & & & & \\
\hline \multirow[t]{2}{*}{$\mathrm{N}$} & & & & & & & & \\
\hline & & & & $\mathrm{N}$ & & $\mathrm{X}$ & B & \\
\hline \multirow[t]{2}{*}{$\mathrm{H}$} & $\mathrm{X}$ & $\mathrm{X}$ & $\mathrm{N}$ & & $\mathrm{X}$ & $\mathrm{N}$ & $\mathrm{N}$ & \\
\hline & $\mathrm{X}$ & $\mathrm{X}$ & & $\mathrm{N}$ & & & & \\
\hline $\mathrm{H}$ & $\mathrm{X}$ & $\mathrm{X}$ & $\mathrm{N}$ & $\mathrm{N}$ & $\mathrm{X}$ & X & $\mathrm{N}$ & \\
\hline$X$ & $\mathrm{X}$ & $\mathrm{X}$ & $\mathrm{N}$ & & $\mathrm{X}$ & & B & \\
\hline $\mathrm{N}$ & & $\mathrm{X}$ & & B & B & B & $\mathrm{N}$ & \\
\hline $\mathrm{H}$ & $\mathrm{X}$ & & & & $\mathrm{X}$ & $\mathrm{X}$ & $\mathrm{N}$ & \\
\hline \multicolumn{9}{|l|}{$\mathrm{X}$} \\
\hline \multicolumn{8}{|l|}{$\mathrm{H}$} & \\
\hline$X$ & $\mathrm{X}$ & & & & $\mathrm{X}$ & & & \\
\hline $\mathrm{H}$ & $\mathrm{X}$ & $\mathrm{N}$ & $\mathrm{N}$ & & $X$ & $\mathrm{X}$ & B & \\
\hline $\mathrm{X}$ & $\mathrm{X}$ & $X$ & & & $\mathrm{X}$ & $\mathrm{X}$ & B & \\
\hline \multirow[t]{4}{*}{$\mathrm{H}$} & $\mathrm{X}$ & $\mathrm{X}$ & $\mathrm{N}$ & & $\mathrm{X}$ & $\mathrm{X}$ & $\mathrm{N}$ & \\
\hline & & $\mathrm{X}$ & & & & & $\mathrm{N}$ & \\
\hline & & & & B & B & & B & \\
\hline & $\mathrm{X}$ & & & & $\mathrm{X}$ & & & \\
\hline
\end{tabular}


Species

Stricticomus transversalis transversalis (A. Villa \& G.B. Villa, 1833)

Temuicomus velox velox (LaFerté-Sénectère, 1849)

Notoxinae Stephens, 1829

Mecynotarsus serricornis (Panzer, 1796)

Notoxus appendicimus Desbrochers des Loges, 1874

Notoxus brachycerus (Faldermann, 1837)

Notoxus lonai Bucciarelli, 1973 [E]

Notoxus miles italicus Bucciarelli, 1978 [E]

Notoxus trifasciatus P. Rossi, 1792

Tomoderinae Bonadona, 1961

\begin{tabular}{|c|c|c|c|c|c|c|c|c|}
\hline 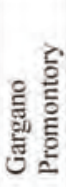 & $\begin{array}{l}3 \\
0 \\
3 \\
0 \\
\frac{0}{0} \\
\frac{0}{0}\end{array}$ & 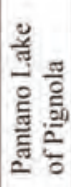 & 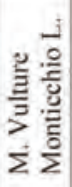 & 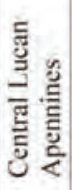 & 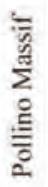 & 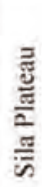 & $\begin{array}{l}\frac{a}{0} \\
\vdots \\
\frac{2}{2} \\
\frac{2}{n}\end{array}$ & 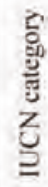 \\
\hline $\mathrm{X}$ & $X$ & & & $\mathrm{~N}$ & $\mathrm{~N}$ & & B & \\
\hline & & & & & & & B & \\
\hline
\end{tabular}

\begin{tabular}{|c|c|c|c|c|c|c|c|c|}
\hline $\mathrm{X}$ & $\mathrm{X}$ & & & & & & & \\
\hline $\mathrm{X}$ & $\mathrm{X}$ & & & & & & $\mathrm{N}$ & \\
\hline & $\mathrm{X}$ & & & & & $\mathrm{B}$ & \\
\hline & $\mathrm{X}$ & & & & & $\mathrm{X}$ & & \\
\hline $\mathrm{X}$ & & & $\mathrm{N}$ & $\mathrm{X}$ & $\mathrm{X}$ & $\mathrm{N}$ & \\
\hline $\mathrm{X}$ & & $\mathrm{N}$ & & $\mathrm{X}$ & $\mathrm{X}$ & $\mathrm{B}$ & \\
\hline
\end{tabular}

Psendotomoderus compressicollis compressicollis (Motschulsky, 1839)

Tomoderus italicus Marseul, 1879 [E]

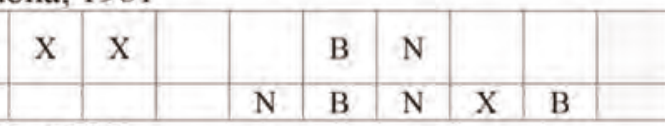

ADERIDAE Csiki, 1909

Nardi in Cat. Pal. Col., vol. 5, 2008

Aderus populneus (Creutzer, 1796

Anidornis nigrinus (Germar, 1842)

Cobosia pruinosa pruinosa (Kiesenwetter, 1861)

Gompelia ruficollis (P. Rossi, 1794)

Phytobaenus amabilis amabilis R.F. Sahlberg, 1834

\begin{tabular}{|c|c|c|c|c|c|c|c|c|}
\hline & & X & & & & & B & LC \\
\hline H & & & & N & & & & \\
\hline X & & & & & & X & B & NT \\
\hline X & N & X & & & X & X & B & NT \\
\hline
\end{tabular}

SCRAPTIIDAE Gistel, 1848

Scraptiinae Gistel, 1848

Scraptiini Gistel, 1848

Leblanc, Levey \& Horák in Cat. Pal. Col., vol. 5, 2008

Scraptia bifoveolata Küster, 1853

Scraptia dubia (A.G. Olivier, 1790)

Scraptia ferruginea Kiesenwetter, 1861

Scraptia ophthalmica Mulsant, 1856

Trotomma pubescens Kiesenwetter, 1851

Anaspidinae Mulsant, 1856

\begin{tabular}{|c|c|c|c|c|c|c|c|c|}
\hline X & X & X & & & X & X & & \\
\hline H & X & & N & & X & X & & \\
\hline H & X & & & & & & & \\
\hline & X & & & & & & & VU \\
\hline H & N & & N & N & N & & N & \\
\hline
\end{tabular}

Anaspidini Mulsant, 1856

\begin{tabular}{|c|c|c|c|c|c|c|c|c|c|}
\hline Anaspis brumnipes (Mulsant, 1856) & $\mathrm{H}$ & & & & & & & & \\
\hline Anaspis costai Emery, 1876 & $\mathrm{X}$ & $\mathrm{X}$ & & & & $\mathrm{X}$ & & & VU \\
\hline Anaspis fasciata (Forster, 1771) & $\mathrm{X}$ & $\mathrm{X}$ & & & & & & & \\
\hline Anaspis flava (Linnaeus, 1758) & $\mathrm{H}$ & & & $\mathrm{N}$ & & $\mathrm{X}$ & $\mathrm{X}$ & $\mathrm{N}$ & LC \\
\hline Anaspis frontalis (Linnaeus, 1758) & $\mathrm{H}$ & $\mathrm{X}$ & $\mathrm{N}$ & $\mathrm{N}$ & & $\mathrm{X}$ & $\mathrm{X}$ & & LC \\
\hline Anaspis labiata A. Costa, 1854 & & $\mathrm{X}$ & $\mathrm{N}$ & & & & & & \\
\hline Anaspis lurida Stephens, 1832 & $\mathrm{H}$ & $\mathrm{X}$ & & & & $\mathrm{X}$ & $\mathrm{X}$ & $\mathrm{N}$ & LC \\
\hline Anaspis maculata (Geoffroy, 1785) & $\mathrm{H}$ & $\mathrm{X}$ & & $\mathrm{N}$ & $\mathrm{N}$ & $\mathrm{X}$ & $\mathrm{X}$ & $\mathrm{N}$ & \\
\hline Anaspis melanostoma A. Costa, 1854 & & & & & & $\mathrm{X}$ & & & \\
\hline Anaspis nigripes C.N.F. Brisout de Barneville, 1866 & $\mathrm{H}$ & $\mathrm{X}$ & & & $\mathrm{N}$ & $\mathrm{X}$ & & $\mathrm{N}$ & \\
\hline Anaspis palpalis (Gerhardt, 1876 ) & $\mathrm{H}$ & $\mathrm{X}$ & & & $\mathrm{N}$ & & $\mathrm{X}$ & $\mathrm{N}$ & \\
\hline Anaspis pulicaria A. Costa, 1854 & $\mathrm{H}$ & $\mathrm{X}$ & & $\mathrm{N}$ & $\mathrm{N}$ & $\mathrm{X}$ & $\mathrm{X}$ & $\mathrm{N}$ & LC \\
\hline Anaspis quadrimaculata Gyllenhal, 1817 & $\mathrm{H}$ & & & & & & & & \\
\hline Anaspis regimbarti Schilsky, 1895 & & $\mathrm{~N}$ & & & & & & $\mathrm{~N}$ & \\
\hline Anaspis ruficollis (Fabricius, 1792) & $\mathrm{H}$ & $\mathrm{X}$ ? & & & & $\mathrm{X}$ & & & EN \\
\hline Anaspis rufilabris (Gyllenhal, 1827) & $\mathrm{X}$ & & & & & & $\mathrm{X}$ & & EN \\
\hline Anaspis serbica Apfelbeck, 1931 & & & & & & $\mathrm{X}$ & $\mathrm{X}$ & & \\
\hline Anaspis thoracica (Linnaeus, 1758) & & & & & & & $\mathrm{X}$ & & \\
\hline Anaspis varians varians (Mulsant, 1856 ) & $\mathrm{H}$ & $\mathrm{X}$ & & & & $\mathrm{X}$ & $\mathrm{X}$ & & \\
\hline Anaspis viennensis Schilsky, 1895 & & & & & & $\mathrm{X}$ & & & \\
\hline
\end{tabular}


Species

CHRYSOMELOIDEA Latreille, 1802

VESPERIDAE Mulsant, 1839

Vesperinae Mulsant, 1839

Vesperini Mulsant, 1839

Sama \& Löbl in Cat. Pal. Col., vol. 6, 2010; Bouchard et al., 2011

Vesperus luridus (P. Rossi, 1794)

$$
\begin{array}{l|l|l|}
\mathrm{X} & \mathrm{B} & \mathrm{X} \\
\hline
\end{array}
$$

CERAMBYCIDAE Latreille, 1802

Sama \& Löbl in Cat. Pal. Col., vol. 6, 2010 (except Prioninae and Dorcadionini)

Prioninae Latreille, 1802

Aegosomatini J. Thomson, 1861

Drumont \& Komiya in Cat. Pal. Col., vol. 6, 2010

Aegosoma scabricorne (Scopoli, 1763)

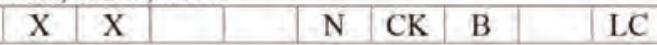

Ergatini Fairmaire, 1864

Ergates faber faber (Linnaeus, 1751)

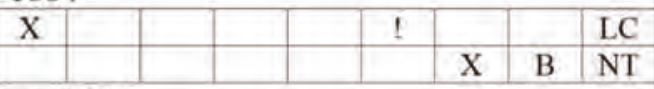

Ergates faber opifex Mulsant, 1851

Macrotomini J. Thomson, 1861

Prinobius myardi slamorum Danilevsky, 2012

Prionini Latreille, 1802

Prionus coriarius (Linnaeus, 1758) B $\mathrm{X}$

\begin{tabular}{|l|l|l|l|l|l|l|l|}
\hline $\mathrm{X}$ & & $\mathrm{B}$ & $\mathrm{N}$ & $\mathrm{X}$ & $\mathrm{X}$ & $\mathrm{B}$ & $\mathrm{NT}$ \\
\hline
\end{tabular}

Lepturinae Latreille, 1802

Lepturini Latreille, 1802

\begin{tabular}{|c|c|c|c|c|c|c|c|c|c|}
\hline Alosterna tabacicolor tabacicolor (DeGeer, 1775 ) & $\mathrm{X}$ & & & & CK & $\mathrm{CK}$ & B & $\mathrm{N}$ & LC \\
\hline Anastrangalia dubia dubia (Scopoli, 1763) & & & & $\mathrm{N}$ & & $\mathrm{X}$ & B & B & LC \\
\hline Anastrangalia sanguinolenta (Linnaeus, 1760) & & & & $\mathrm{N}$ & $\mathrm{N}$ & $\mathrm{X}$ & $\mathrm{X}$ & CK & LC \\
\hline Anoplodera rufipes izzilloi Sama, 1999 [E] & & & & B & B & $\mathrm{CK}$ & & & NT \\
\hline Anoplodera sexguttata (Fabricius, 1775) & & & & & B & & & & LC \\
\hline Grammoptera ruficornis ruficornis (Fabricius, 1781) & B & & & CK & $\mathrm{CK}$ & $\mathrm{X}$ & $\mathrm{X}$ & $\mathrm{N}$ & LC \\
\hline Grammoptera ustulata ustulata (Schaller, 1783) & CK & & & B & & $\mathrm{X}$ & $\mathrm{X}$ & $\mathrm{N}$ & NT \\
\hline Leptura aurulenta Fabricius, 1793 & & & & & & $\mathrm{X}$ & $\mathrm{X}$ & & LC \\
\hline Pachytodes erraticus (Dalman, 1817) & $\mathrm{X}$ & & & B & CK & $\mathrm{X}$ & $\mathrm{X}$ & & LC \\
\hline Pedostrangalia revestita (Linnaeus, 1767) & B & B & & & CK & B & & & NT \\
\hline Pseudovadonia livida pecta K. Daniel \& J. Daniel, 189I & $\mathrm{H}$ & $\mathrm{X}$ & & $\mathrm{N}$ & B & $\mathrm{X}$ & $\mathrm{X}$ & CK & LC \\
\hline Rutpela maculata maculata (Poda von Neuhaus, 1761) & $\mathrm{X}$ & $\mathrm{X}$ & $\mathrm{X}$ & $\mathrm{N}$ & CK & $\mathrm{X}$ & $\mathrm{X}$ & $\mathrm{N}$ & LC \\
\hline Stenurella bifasciata bifasciata (O.F. Müller, 1776) & $\mathrm{H}$ & $\mathrm{X}$ & $\mathrm{X}$ & B & $\mathrm{B}$ & $\mathrm{X}$ & $\mathrm{X}$ & $\mathrm{N}$ & LC \\
\hline Stenurella melanura melanura (Linnaeus, 1758 ) & $\mathrm{X}$ & $\mathrm{X}$ & & & $\mathrm{N}$ & $\mathrm{X}$ & $\mathrm{X}$ & $\mathrm{N}$ & LC \\
\hline Stentrella nigra nigra (Linnaeus, 1758) & $\mathrm{X}$ & $\mathrm{X}$ & & B & B & $\mathrm{X}$ & $\mathrm{X}$ & B & LC \\
\hline Stictoleptura cordigera cordigera (Fuessly, 1775) & $\mathrm{H}$ & $\mathrm{X}$ & $\mathrm{X}$ & B & B & $\mathrm{X}$ & $\mathrm{X}$ & B & LC \\
\hline Stictoleptura fulva (DeGeer, 1775) & CK & & & B & B & $\mathrm{X}$ & $\mathrm{X}$ & $\mathrm{N}$ & \\
\hline Stictoleptura rubra rubra (Linnaeus, 1758) & & & & & & $\mathrm{X}$ & & & LC \\
\hline Stictoleptura rufa rufa (Brullé, 1832) & B & $\mathrm{X}$ & & 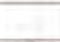 & B & CK & $\mathrm{B}$ & B & NT \\
\hline Stictoleptura scutellata scutellata (Fabricius, 1781) & $\mathrm{X}$ & $\mathrm{X}$ & & $\mathrm{B}$ & CK & $\mathrm{X}$ & $\mathrm{X}$ & B & NT \\
\hline
\end{tabular}

Rhagiini Kirby, 1837

Acmaeops marginatus (Fabricius, 1781)

Cortodera humeralis aspromontana G. Müller, 1948

Dinoptera collaris (Linnaeus, 1758)

Evodinellus clathratus (Fabricius, 1792)

Pidonia lurida (Fabricius, 1793)

Rhagium bifasciatum Fabricius, 1775

Rhagium inquisitor inquisitor (Linnaeus, 1758)

Rhagium mordax (DeGeer, 1775)

Rhagium sycophanta (Schrank, 1781)

\begin{tabular}{|c|c|c|c|c|c|c|c|}
\hline & & & & B & & & NT \\
\hline B & & B & B & $X$ & & B & NT \\
\hline $\mathrm{H}$ & $\mathrm{N}$ & $\mathrm{N}$ & B & $\mathrm{X}$ & $X$ & $\mathrm{~N}$ & LC \\
\hline $\mathrm{X}$ & & & & & & & NT \\
\hline $\mathrm{X}$ & & & & $\mathrm{X}$ & & & NT \\
\hline & & & $\mathrm{N}$ & CK & $X$ & & LC \\
\hline & & $\mathrm{N}$ & & $\mathrm{X}$ & $\mathrm{X}$ & CK & LC \\
\hline & & & $\mathrm{N}$ & $\mathrm{X}$ & $X$ & B & LC \\
\hline & & B & $\mathrm{N}$ & $\mathrm{X}$ & & & NT \\
\hline
\end{tabular}


Species

Stenocortus meridianus (Linnaeus, 1758)

Stenocorus quercus quercus (Götz, 1783)

Rhamnusiini Danilevsky in Althoff \& Danilevsky, 1997

Rhammusium bicolor italicum G. Müller, 1966 [E]

Necydalinae Latreille, 1825

\begin{tabular}{|l|l|}
\hline Necydalis ulmi (Chevrolat, 1838) & CK
\end{tabular}

\begin{tabular}{|c|c|c|c|c|c|c|c|}
\hline 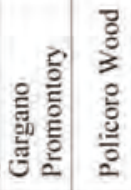 & 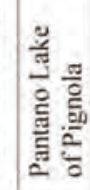 & 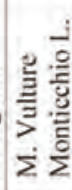 & 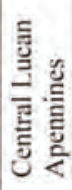 & 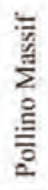 & 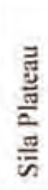 & 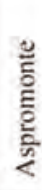 & \\
\hline & & & CK & $X$ & $X$ & B & $\mathrm{N}$ \\
\hline & & E & & & & & \\
\hline
\end{tabular}

pondylidinae Audinet-Serville, 1832

Asemini J. Thomson, 1861

Arhopalus ferus Mulsant, 1839

Arhopalus rusticus rusticus (Linnaeus, 1758)

Arhopalus syriacus (Reitter, 1895)

Asemum striatum striatum (Linnaeus, 1758)

Nothorhina punctata (Fabricius, 1798)

\begin{tabular}{|c|c|c|c|c|c|c|c|c|c|}
\hline & $\mathrm{X}$ & $\mathrm{B}$ & & & & $\mathrm{X}$ & $\mathrm{X}$ & $\mathrm{B}$ & $\mathrm{LC}$ \\
\hline & & & & $\mathrm{N}$ & & $\mathrm{X}$ & $\mathrm{X}$ & $\mathrm{B}$ & LC \\
\hline & $\mathrm{CK}$ & & & & & & $\mathrm{B}$ & $\mathrm{CK}$ & LC \\
\hline & & & & & & $\mathrm{X}$ & $\mathrm{X}$ & & LC \\
\hline
\end{tabular}

Spondylis buprestoides (Linnaeus, 1758)

Spondylidini Audinet-Serville, 1832

\begin{tabular}{|c|c|c|c|c|c|}
\hline B & B & X & & & CR \\
\hline
\end{tabular}

Saphanini Gistel, 1848

Cerambycinae Latreille, 1802

Achrysonini Lacordaire, 1868

Icosium tomentoszm atticum Ganglbauer, 1881 CK $\quad$ B

Anaglyptini Lacordaire, 1868

Anaglyptus gibbosus (Fabricius, 1787)

Anaglyptus mysticus (Linnaeus, 1758)

Anaglyptus zappii Rapuzzi \& Sama, 2014 [E]

\section{Brachypteromatini Sama, 2008}

Dolocerus reichii Mulsant, 1862

Callichromatini Swainson \& Shuckard, 1840

Aromia moschata moschata (Linnaeus, 1758)

Callidium aeneum aeneum DeGeer, 1775

Leioderes kollari kollari L. Redtenbacher, 1849

Phymatodes alni alni (Linnaeus, 1767)

Phymatodes fasciatus (Villers, 1789)

Phymatodes glabratus (Charpentier, 1825)

Phymatodes lividus (P. Rossi, 1794)

Phymatodes pusillus pusillus (Fabricius, 1787)

Phymatodes rufipes rufipes (Fabricius, 1777)

Phymatodes testacens (Linnaeus, 1758)

Pyrrhidium sanguineum (Linnaeus, 1758)

Ropalopus clavipes (Fabricius, 1775)

Ropalopus ungaricus (Herbst, 1784)

Ropalopus varini (Bedel, 1870)

Semanotus russicus russicus (Fabricius, 1777)

Cerambycini Latreille, 1802

Callidiini Kirby, 1837

Cerambyx cerdo cerdo Linnaeus, 1758

Cerambyx miles Bonelli, 1812

Cerambyx scopolii scopolii Fuessly, 1775

Cerambyx welensii welensii (Küster, 1845)

Certallum ebulinum (Linnaeus, 1767)

Certallini Fairmaire, 1864

\begin{tabular}{|c|c|c|c|c|c|c|c|c|}
\hline \\
\hline & & & & & $\mathrm{X}$ & $\mathrm{X}$ & CK & LC \\
\hline B & & & & & & & & NI \\
\hline $\mathrm{X}$ & $\mathrm{N}$ & & B & $\mathrm{N}$ & $\mathrm{X}$ & $\mathrm{X}$ & $\mathrm{N}$ & LC \\
\hline CK & & & & & & & & LC \\
\hline & B & & & & & & & NT \\
\hline $\mathrm{X}$ & & & & & & & & LC \\
\hline CK & & & & $\mathrm{N}$ & & & & NT \\
\hline & & & B & B & & X & & NT \\
\hline $\mathrm{H}$ & $\mathrm{X}$ & $X$ & $\mathrm{~N}$ & B & $\mathrm{X}$ & $\mathrm{X}$ & $\mathrm{CK}$ & LC \\
\hline & & & & B & $\mathrm{X}$ & & & LC \\
\hline $\mathrm{X}$ & $X$ & & & $\mathrm{~N}$ & $\mathrm{X}$ & B & & LC \\
\hline B & & & & B & & & & NT \\
\hline & & & & B & & & & NT \\
\hline B & & & & ! & & & & NT \\
\hline
\end{tabular}

X X

\begin{tabular}{rr} 
CKytini Mulsant, 1839 \\
\hline
\end{tabular}

\begin{tabular}{|c|c|c|c|c|c|c|c|}
\hline$X$ & $\mathrm{X}$ & & B & X & B & B & LC \\
\hline & & & B & B & & & LC \\
\hline $\mathrm{H}$ & $\mathrm{X}$ & $\mathrm{N}$ & B & $\mathrm{X}$ & X & B & LC \\
\hline $\mathrm{X}$ & $\mathrm{X}$ & & B & $\mathrm{X}$ & B & B & LC \\
\hline
\end{tabular}
CK

B


Stenopterini Gistel, 1848

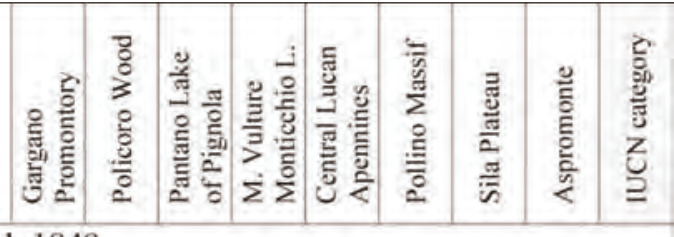

Species

\begin{tabular}{|c|c|c|c|c|c|c|c|c|c|}
\hline \multicolumn{10}{|c|}{ Stenopterini Gistel, 1848} \\
\hline Callimus abdominalis (A.G. Olivier, 1795) & $X$ & $\mathrm{~N}$ & & & CK & $\mathrm{N}$ & & & LC \\
\hline Callimus angulatus angulatus (Schrank, 1789) & & & & B & $\mathrm{N}$ & $\mathrm{N}$ & $\mathrm{X}$ & & LC \\
\hline Stenopterus ater (Linnaeus, 1767) & $\mathrm{H}$ & $\mathrm{X}$ & $\mathrm{X}$ & B & B & $\mathrm{X}$ & $\mathrm{X}$ & CK & LC \\
\hline Stenopterus flavicornis Küster, 1846 & & & & & & $\mathrm{X}$ & $\mathrm{X}$ & & NT \\
\hline Stenopterus rufius rufus (Linnaeus, 1767) & $\mathrm{H}$ & $\mathrm{X}$ & $\mathrm{X}$ & $\mathrm{N}$ & B & $\mathrm{X}$ & $\mathrm{X}$ & CK & LC \\
\hline
\end{tabular}

Lamiinae Latreille, 1825

Acanthocinini Blanchard, 1845

Acanthocinus aedilis (Linnaeus, 1758)

Acanthocimus griseus (Fabricius, 1793)

Acanthocinus reticulatus (Razoumov, 1789)

Acanthocinus xanthoneurus (Mulsant \& Rey, 1852) [E]

Leiopus femoratus Fairmaire, 1859

Leiopus nebulosus nebulosus (Linnaeus, 1758)

Acanthoderini J. Thomson, 1860

\begin{tabular}{|c|c|c|c|c|c|c|c|c|}
\hline & & & & & $\mathrm{X}$ & $\mathrm{X}$ & $\mathrm{N}$ & LC \\
\hline B & & & & & & $\mathrm{X}$ & $\mathrm{N}$ & LC \\
\hline & & & & & $\mathrm{X}$ & & & LC \\
\hline & & & & ! & B & $\mathrm{X}$ & B & NT \\
\hline & & & & & & & B & NT \\
\hline $\mathrm{X}$ & $\mathrm{N}$ & & $\mathrm{N}$ & $\mathrm{N}$ & $\mathrm{X}$ & $\mathrm{X}$ & B & LC \\
\hline
\end{tabular}

Aegomorphus clavipes (Schrank, 1781)

\begin{tabular}{l|l|l}
$\mathrm{X}$ & $\mathrm{X}$ \\
$\mathrm{n}$
\end{tabular}

Agapanthiini Mulsant, 1839

\begin{tabular}{|l|c|c|c|c|c|c|c|c|c|}
\hline Agapanthia asphodeli (Latreille, 1804) & $\mathrm{B}$ & & $\mathrm{B}$ & & $\mathrm{CK}$ & & & \\
\hline Agapanthia cardui (Linnaeus, 1767) & $\mathrm{H}$ & $\mathrm{X}$ & $\mathrm{X}$ & $\mathrm{B}$ & $\mathrm{B}$ & $\mathrm{X}$ & $\mathrm{X}$ & $\mathrm{B}$ & \\
\hline Agapanthia cynarae cynarae (Gyllenhal, 1817) & $\mathrm{X}$ & $\mathrm{X}$ & & $\mathrm{N}$ & $\mathrm{B}$ & $\mathrm{X}$ & $\mathrm{X}$ & & \\
\hline Agapanthia irrorata (Fabricius, 1787) & $\mathrm{CK}$ & & & & ! & & $\mathrm{X}$ & \\
\hline Agapanthia maculicornis davidi Släma, 1986 [E] & & $\mathrm{B}$ & & $\mathrm{CK}$ & $\mathrm{CK}$ & $\mathrm{B}$ & & \\
\hline Agapanthia sicula malmerendii Sama, 1981 & $\mathrm{H}$ & $\mathrm{X}$ & $\mathrm{CK}$ & $\mathrm{B}$ & $\mathrm{B}$ & $\mathrm{CK}$ & & $\mathrm{CK}$ & \\
\hline Agapanthia suturalis (Fabricius, 1787) & & & & $\mathrm{B}$ & & & & $\mathrm{B}$ & \\
\hline Agapanthia villosoviridescens (DeGeer, 1775) & & $\mathrm{X}$ & & $\mathrm{N}$ & $\mathrm{B}$ & $\mathrm{X}$ & $\mathrm{X}$ & & \\
\hline Agapanthia violacea (Fabricius, 1775) & $\mathrm{X}$ & & & $\mathrm{B}$ & $\mathrm{B}$ & $\mathrm{X}$ & $\mathrm{X}$ & $\mathrm{N}$ & \\
\hline Calamobius filum (P. Rossi, 1790) & $\mathrm{H}$ & $\mathrm{X}$ & $\mathrm{X}$ & $\mathrm{B}$ & $\mathrm{N}$ & $\mathrm{X}$ & $\mathrm{X}$ & $\mathrm{N}$ & \\
\hline
\end{tabular}

Apodasyini Lacordaire, 1872

Anaesthetis testacea testacea (Fabricius, 1781)

Deroplia genei genei (Aragona, 1830)

Deroplia troberti troberti (Mulsant, 1843)

\begin{tabular}{|c|c|c|c|c|c|c|}
\hline X & $X$ & CK & $X$ & $\mathrm{X}$ & B & LC \\
\hline & $X$ & & & & & NT \\
\hline B & & & & & & NT \\
\hline
\end{tabular}

Dorcadionini Swainson \& Shuckard, 1840

Danilevsky in Cat. Pal. Col., vol. 6, 2010

Dorcadion arenarium marsicamum D'Amore Fracassi, 1905

Dorcadion etruscum etruscum (P. Rossi, 1790) [E]

Neodorcadion calabricum (Reitter, 1889) [E]

Lamiini Latreille, 1825

Herophila tristis martinascoi (Contarini \& Gargagni, 1983)

Herophila tristis tristis (Linnaeus, 1767)

Lamia textor (Linnaeus, 1758)

Morimus asper asper (Sulzer, 1776)

\begin{tabular}{|c|c|c|c|c|c|c|c|}
\hline $\mathrm{H}$ & $\mathrm{B}$ & & & & & & \\
\hline $\mathrm{H}$ & $\mathrm{X}$ & $\mathrm{X}$ & $\mathrm{B}$ & $\mathrm{B}$ & $\mathrm{X}$ & $\mathrm{X}$ & $\mathrm{CK}$ \\
\hline
\end{tabular}

B

Mesosa curculionoides (Linnaeus, 1760)

Mesosa nebulosa nebulosa (Fabricius, 1781)

Mesosini Mulsant, 1839

Monochamini Gistel, 1848

Monochamus galloprovincialis galloprovincialis (A.G. Olivier,

1795)

Parmenini Mulsant, 1839

\begin{tabular}{|c|c|c|c|c|c|c|c|c|}
\hline & X & & & & & & CK & NT \\
\hline H & & & CK & B & X & X & & LC \\
\hline & X & & & $!$ & & & B & NT \\
\hline H & X & & N & CK & X & X & B & LC \\
\hline
\end{tabular}

\begin{tabular}{|c|c|c|c|c|c|c|c|c|}
\hline $\mathrm{X}$ & $\mathrm{X}$ & $\mathrm{X}$ & & $\mathrm{B}$ & $\mathrm{B}$ & & $\mathrm{B}$ & $\mathrm{LC}$ \\
\hline $\mathrm{B}$ & $\mathrm{X}$ & & $\mathrm{B}$ & $\mathrm{CK}$ & & $\mathrm{B}$ & & LC \\
\hline
\end{tabular}

Parmena pubescens (Dalman, 1817)

Parmena subpubescens Hellrigl, 1971 [E]

Parmena unifasciata (P. Rossi, 1790)

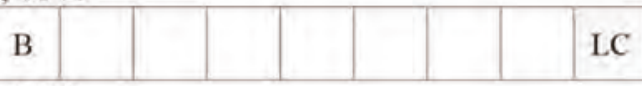

Parme

Phytoeciini Mulsant, 1839 
Species

Oberea linearis (Linnaeus, 1760)

Oberea oculata (Linnaeus, 1758)

Phytoecia affinis nigrohirta (G. Müller, 1948) [E]

Phytoecia caerulea caerulea (Scopoli, 1772)

Phytoecia coerulescens coerulescens (Scopoli, 1763)

Phytoecia cylindrica (Linnaeus, 1758)

Phytoecia nigricornis (Fabricius, 1782)

Phytoecia pustulata pustulata (Schrank, 1776)

Phytoecia mufipes mufipes (A.G. Olivier, 1795)

Phytoecia virgula virgula (Charpentier, 1825 )

Phytoecia vulneris eremita Sama, 1999 [E]

Phytoecia vulneris vulneris Aurivillius, 1923

Pogonocherini Mulsant, 1839

\begin{tabular}{|c|c|c|c|c|c|c|c|c|}
\hline 总 & 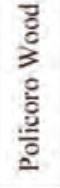 & 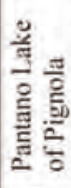 & 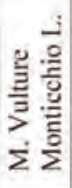 & 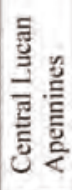 & 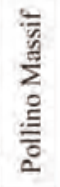 & 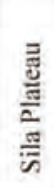 & 䒓 & 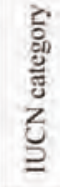 \\
\hline & B & & & B & & $\mathrm{X}$ & & LC \\
\hline & B & & & & $\mathrm{X}$ & & & LC \\
\hline & & & B & & & & & \\
\hline $\mathrm{H}$ & $\mathrm{X}$ & $X$ & B & & $\mathrm{X}$ & $\mathrm{X}$ & B & \\
\hline $\mathrm{H}$ & CK & & B & $\mathrm{N}$ & $\mathrm{X}$ & $\mathrm{X}$ & $\mathrm{N}$ & \\
\hline $\mathrm{X}$ & B & $\mathrm{X}$ & B & B & B & B & CK & \\
\hline & & & & $\mathrm{N}$ & & $\mathrm{X}$ & & \\
\hline$X$ & & $X$ & CK & $\mathrm{N}$ & B & $\mathrm{X}$ & $\mathrm{N}$ & \\
\hline $\mathrm{H}$ & & $\mathrm{X}$ & & & CK & CK & & \\
\hline $\mathrm{H}$ & $\mathrm{X}$ & $\mathrm{X}$ & $\mathrm{N}$ & B & B & $\mathrm{X}$ & & \\
\hline & & & & & CK & & & \\
\hline $\mathrm{H}$ & $\mathrm{X}$ & & B & B & & CK & $\mathrm{N}$ & \\
\hline
\end{tabular}

Exocentrus adspersus Mulsant, 1846

Exocentrus lusitanicus (Linnaeus, 1767)

Exocentrus punctipennis punctipennis Mulsant \& Guillebeau,

1856

Pogonocherus decoratus Fairmaire, 1855

Pogonocherus eugeniae engeniae Ganglbauer, 1891

Pogonocherus fasciculatus fasciculatus (DeGeer, 1775)

Pogonocherus hispidulus (Piller \& Mitterpacher, 1783)

Pogonocherus hispidus (Linnaeus, 1758)

Pogonocherus neuhausi (G. Müller, 1916)

Pogonocherus ovatoides Rapuzzi \& Sama, 2014

Pogonocherus perroudi perroudi Mulsant, 1839

Pteropliini J. Thomson, 1860

\begin{tabular}{|c|c|c|c|c|c|c|c|c|}
\hline B & & X & & CK & & B & & LC \\
\hline & & & & B & & & & NT \\
\hline B & X & & & CK & & & & LC \\
& & & & & CK & X & & NT \\
\hline & & & & & X & & B & NT \\
\hline & & & & N & B & & & LC \\
\hline & B & & & & & & CK & LC \\
\hline X & B & X & B & & B & X & & LC \\
\hline & & & & & & & & NT \\
\hline X & & & & & & X & & LC \\
\hline
\end{tabular}

Niphona picticornis Mulsant, 1839

H X

Saperdini Mulsant, 1839

Saperda carcharias (Linnaeus, 1758)

Saperda octopunctata (Scopoli, 1772)

Saperda populnea populnea (Linnaeus, 1758)

Saperda punctata (Linnaeus, 1767)

Saperda scalaris scalaris (Linnaeus, 1758)

Saperda similis Laicharting, 1784

Stenostola ferrea ferrea (Schrank, 1776)

Tetrops praeustus praeustus (Linnaeus, 1758

Tetrops starkii starkii Chevrolat, 1859

Tetropini Portevin, 1927

\begin{tabular}{|c|c|c|c|c|c|c|c|c|}
\hline & B & $\mathrm{X}$ & & & & & B & NT \\
\hline & & & & & & $X$ & & NT \\
\hline \multirow[t]{5}{*}{ 88) } & $\mathrm{X}$ & & & & B & $\mathrm{X}$ & & \\
\hline & $\mathrm{X}$ & & B & B & $\mathrm{N}$ & & $\mathrm{N}$ & LC \\
\hline & & & & $!$ & B & $\mathrm{X}$ & & LC \\
\hline & & & & & & $\mathrm{X}$ & & NT \\
\hline & & & & CK & & $\mathrm{X}$ & & NT \\
\hline
\end{tabular}

\author{
(1)
}

MEGALOPODIDAE Latreille, 1802

Zeugophorinae Böving \& Craighead, 1931

Silfverberg in Cat. Pal. Col., vol. 6, 2010

Zetugophora subspinosa (Fabricius, 1781)

ORSODACNIDAE C.G. Thomson, 1859

Silfverberg in Cat. Pal. Col., vol. 6, 2010

Orsodacne humeralis humeralis Latreille, 1804

\begin{tabular}{|l|l|l|l|l|l|l|}
\hline $\mathrm{N}$ & $\mathrm{N}$ & $\mathrm{B}$ & $\mathrm{N}$ & $\mathrm{N}$ & $\mathrm{N}$ & $\mathrm{N}$ \\
\hline
\end{tabular}

CHRYSOMELIDAE Latreille, 1802

Bruchinae Latreille, 1802

Amblycerini Bridwell, 1932

Spermophagina Borowiec, 1987

Anton in Cat. Pal. Col., vol. 6, 2010

Spermophagus calystegiae (Lukjanovitch \& Ter-Minassian, 1957) 


\section{Species}

Spermophagus kuesteri Schilsky, 1905

Spermophagus sericeus (Geoffroy, 1785)

Bruchini Latreille, 1802

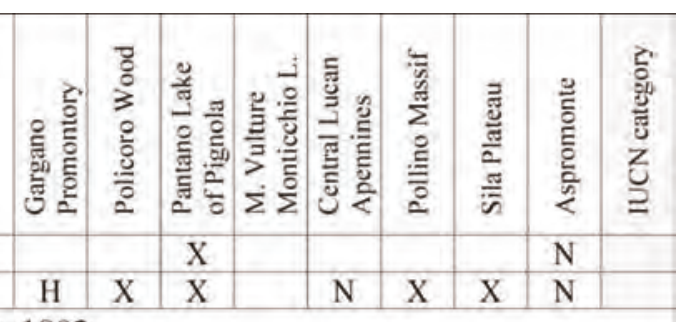

Acanthoscelidina Bridwell, 1946

\begin{tabular}{|c|c|c|c|c|c|c|c|c|c|}
\hline Acanthoscelides obtectus (Say, 1831) & $\mathrm{X}$ & $\mathrm{X}$ & & & $\mathrm{B}$ & & & & \\
\hline Acanthoscelides pallidipennis (Motschulsky, 1874) & & & $\mathrm{X}$ & & $\mathrm{N}$ & & & & \\
\hline Bruchidius biguttatus (A.G. Olivier, 1795) & B & & & & & & $\mathrm{X}$ & $\mathrm{N}$ & \\
\hline Bruchidius bimaculatus (A.G. Olivier, 1795) & $\mathrm{H}$ & $\mathrm{X}$ & $\mathrm{X}$ & & $\mathrm{N}$ & $\mathrm{N}$ & & $\mathrm{N}$ & \\
\hline Bruchidius calabrensis (Blanchard, 1844) & & $\mathrm{X}$ & & & $\mathrm{N}$ & & & $\mathrm{N}$ & \\
\hline Bruchidius canimus (Kraatz, 1869) & $\mathrm{B}$ & & $\mathrm{X}$ & & B & $\mathrm{X}$ & $\mathrm{X}$ & & \\
\hline Bruchidins cinerascens (Gyllenhal, 1833) & & $\mathrm{N}$ & $\mathrm{X}$ & & $\mathrm{N}$ & $\mathrm{N}$ & & & \\
\hline Bruchidius cisti (Fabricius, 1775) & $\mathrm{H}$ & & $\mathrm{X}$ & & $\mathrm{B}$ & $\mathrm{X}$ & $\mathrm{X}$ & $\mathrm{N}$ & \\
\hline Bruchidius corsicus (Baudi di Selve, 1890) & $\mathrm{H}$ & & $\mathrm{X}$ & & & & $\mathrm{X}$ & & \\
\hline Bruchidius dispar (Gyllenhal, 1833) & $\mathrm{H}$ & & $\mathrm{X}$ & & $\mathrm{N}$ & $\mathrm{N}$ & $\mathrm{X}$ & $\mathrm{N}$ & \\
\hline Bruchidius foveolatus (Gyllenhal, 1833) & $\mathrm{H}$ & $\mathrm{X}$ & $\mathrm{X}$ & $\mathrm{N}$ & $\mathrm{N}$ & $\mathrm{X}$ & $\mathrm{X}$ & $\mathrm{N}$ & \\
\hline Bruchidius jocosus (Gyllenhal, 1833) & & & & & & & $\mathrm{X}$ & & \\
\hline Bruchidius lineatus (Allard, 1868) & & & & & B & $\mathrm{N}$ & B & B & \\
\hline Bruchidius lividimamus (Gyllenhal, 1833) & $\mathrm{H}$ & $\mathrm{X}$ & $\mathrm{X}$ & & $\mathrm{N}$ & $\mathrm{N}$ & $\mathrm{X}$ & $\mathrm{N}$ & \\
\hline Bruchidius lutescens (Blanchard, 1844) & B & & & & & B & & & \\
\hline Bruchidius marginalis (Fabricius, 1776) & $\mathrm{H}$ & & $X$ & $\mathrm{~N}$ & & $\mathrm{X}$ & & $\mathrm{N}$ & \\
\hline Bruchidiuls martinezi (Allard, 1868) & & & $\mathrm{X}$ & & & & & & \\
\hline Bruchidius meleagrinus (Gené, 1839) & $\mathrm{H}$ & $\mathrm{X}$ & $\mathrm{X}$ & $\mathrm{N}$ & $\mathrm{N}$ & $\mathrm{X}$ & $\mathrm{X}$ & $\mathrm{N}$ & \\
\hline Bruchidius mulsanti (C.N.F. Brisout de Barneville, I863) & $\mathrm{H}$ & $\mathrm{X}$ & $\mathrm{X}$ & & $\mathrm{N}$ & & & & \\
\hline Bruchidius murinus (Boheman, 1829) & $\mathrm{H}$ & $\mathrm{X}$ & $\mathrm{X}$ & $\mathrm{N}$ & B & $\mathrm{X}$ & $\mathrm{X}$ & $\mathrm{N}$ & \\
\hline Bruchidius nanus (Germar, 1824) & $\mathrm{H}$ & $\mathrm{X}$ & $\mathrm{X}$ & B & $\mathrm{N}$ & $\mathrm{X}$ & $\mathrm{X}$ & $\mathrm{N}$ & \\
\hline Bruchidius mudus (Allard, 1868) & $\mathrm{H}$ & & $\mathrm{X}$ & & $\mathrm{N}$ & & $\mathrm{X}$ & $\mathrm{N}$ & \\
\hline Bruchidius obscuripes (Gyllenhal, 1839) & $\mathrm{N}$ & & & & & & & & \\
\hline Bruchidius olivaceus (Germar, 1824) & & $\mathrm{X}$ & & & $\mathrm{B}$ & B & & $\mathrm{N}$ & \\
\hline Bruchidius picipes (Germar, 1824) & B & & $\mathrm{N}$ & & $\mathrm{N}$ & $\mathrm{X}$ & $\mathrm{X}$ & $\mathrm{N}$ & \\
\hline Bruchidius poecilus (Germar, 1824) & $\mathrm{H}$ & & $\mathrm{X}$ & & B & $\mathrm{B}$ & & & \\
\hline Bruchidius poupillieri (Allard, 1868) & & $\mathrm{N}$ & $\mathrm{X}$ & & & B & & & \\
\hline Bruchidius pygmaeus (Boheman, 1833) & $\mathrm{H}$ & & $\mathrm{X}$ & $\mathrm{N}$ & $\mathrm{N}$ & $\mathrm{X}$ & $\mathrm{X}$ & $\mathrm{N}$ & \\
\hline Bruchidius seminarius (Linnaeus, 1767) & $\mathrm{H}$ & $\mathrm{X}$ & $\mathrm{X}$ & $\mathrm{N}$ & $\mathrm{N}$ & $\mathrm{X}$ & $\mathrm{X}$ & $\mathrm{N}$ & \\
\hline Bruchidius sericatus (Germar, 1824) & $\mathrm{H}$ & $\mathrm{X}$ & $\mathrm{X}$ & $\mathrm{N}$ & $\mathrm{N}$ & $\mathrm{X}$ & $\mathrm{X}$ & $\mathrm{N}$ & \\
\hline Bruchidius tibialis (Boheman, 1829) & $\mathrm{H}$ & $\mathrm{N}$ & $\mathrm{X}$ & & $\mathrm{N}$ & $\mathrm{N}$ & & & \\
\hline Bruchidius tuberculatus (Hochhuth, 1847) & $\mathrm{H}$ & & & & & & & & \\
\hline Bruchidius unicolor (A.G. Olivier, 1795) & & & & & & $\mathrm{N}$ & & & \\
\hline Bruchidius varipictus (Motschulsky, 1874) & & & $\mathrm{X}$ & & $\mathrm{N}$ & & & & \\
\hline Bruchidius varius (A.G. Olivier, 1795) & $\mathrm{H}$ & & $\mathrm{X}$ & & $\mathrm{N}$ & $\mathrm{N}$ & $\mathrm{X}$ & $\mathrm{N}$ & \\
\hline Bruchidius villosus (Fabricius, 1792) & $\mathrm{X}$ & & & & $\mathrm{N}$ & B & $\mathrm{X}$ & $\mathrm{B}$ & \\
\hline Callosobruchus maculatus (Fabricius, 1775) & $\mathrm{B}$ & $\mathrm{X}$ & & & & & & & \\
\hline Paleoacanthoscelides gilvus (Gyllenhal, 1839) & $\mathrm{X}$ & $\mathrm{X}$ & $\mathrm{X}$ & $\mathrm{N}$ & $\mathrm{N}$ & $\mathrm{X}$ & $\mathrm{X}$ & $\mathrm{N}$ & \\
\hline
\end{tabular}

Paleoacanthoscelides gilvus (Gyllenhal, 1839)

Bruchina Latreille, 1802

\begin{tabular}{|c|c|c|c|c|c|c|c|c|}
\hline Bruchus affinis J.A. Frölich, 1799 & & $\mathrm{X}$ & & & & & & \\
\hline Bruchus atomarius (Linnaeus, 1760) & & & & $\mathrm{N}$ & $\mathrm{N}$ & B & $\mathrm{X}$ & \\
\hline Bruchus brachialis Fähraeus, 1839 & $\mathrm{H}$ & $\mathrm{X}$ & & $\mathrm{N}$ & $\mathrm{N}$ & $\mathrm{X}$ & $\mathrm{X}$ & $\mathrm{N}$ \\
\hline Bruchus emarginatus Allard, 1868 & & & $\mathrm{X}$ & & & & & \\
\hline Bruchus ervi J.A. Frölich, 1799 & & & & & & & & $\mathrm{~N}$ \\
\hline Bruchus griseomaculatus Gyllenhal, 1833 & & & $\mathrm{X}$ & & $\mathrm{N}$ & & & \\
\hline Bruchus laticollis Boheman, 1833 & $\mathrm{H}$ & $\mathrm{X}$ & $\mathrm{X}$ & & $\mathrm{N}$ & B & & $\mathrm{N}$ \\
\hline Bruchus lentis J,A. Frölich, 1799 & & & & & & B & & \\
\hline Bruchus loti Paykull, 1800 & $\mathrm{H}$ & & $\mathrm{X}$ & & $\mathrm{N}$ & $\mathrm{X}$ & $\mathrm{X}$ & $\mathrm{N}$ \\
\hline Bruchus luteicornis Illiger, 1794 & $\mathrm{H}$ & $\mathrm{X}$ & $\mathrm{X}$ & & $\mathrm{N}$ & & $\mathrm{N}$ & $\mathrm{N}$ \\
\hline
\end{tabular}




\title{
Species
}

Bruchus pisorum (Linnaeus, 1758)

Bruchus rufimanus Boheman, 1833

Bruchus rufipes Herbst, 1783

Bruchus signaticornis Gyllenhal, 1833

Bruchus tristiculus Fãhraeus, 1839

Bruchus ulicis Mulsant \& Rey, 1858

Bruchus viciae A.G. Olivier, 1795

\begin{tabular}{|c|c|c|c|c|c|c|c|c|}
\hline 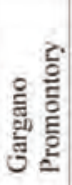 & $\begin{array}{l}\vec{z} \\
0 \\
3 \\
0 \\
\frac{0}{0} \\
\frac{.0}{0} \\
0\end{array}$ & 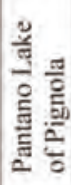 & 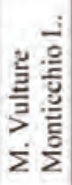 & 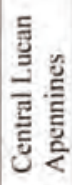 & $\begin{array}{l}\bar{a} \\
\frac{a}{a} \\
0 \\
\frac{g}{\bar{a}} \\
0\end{array}$ & 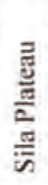 & 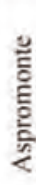 & 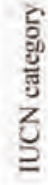 \\
\hline $\mathrm{H}$ & $X$ & & & $\mathrm{~N}$ & B & & & \\
\hline $\mathrm{H}$ & $X$ & $X$ & & B & $X$ & & & \\
\hline $\mathrm{H}$ & $X$ & $X$ & $\mathrm{~N}$ & $\mathrm{~N}$ & $X$ & $\mathrm{X}$ & $\mathrm{N}$ & \\
\hline $\mathrm{H}$ & $\mathrm{X}$ & & & & & $\mathrm{X}$ & & \\
\hline $\mathrm{H}$ & $X$ & $\mathrm{X}$ & & $\mathrm{N}$ & & & & \\
\hline & & & & $\mathrm{N}$ & & & & \\
\hline $\mathrm{H}$ & & $X$ & & $\mathrm{~N}$ & $\mathrm{~N}$ & $\mathrm{X}$ & $\mathrm{N}$ & \\
\hline
\end{tabular}

Donaciinae Kirby, 1837

Donaciini Kirby, 1837

Silfverberg in Cat. Pal. Col., vol. 6, 2010

Donacia bicolora meridionalis Weise, 1886 [E]

Donacia cinerea Herbst, 1784

Donacia impressa Paykull, 1799

Donacia reticulata Gyllenhal, 1817

Donacia simplex Fabricius, 1775

Donacia versicolorea (Brahm, 1790)

Donacia vulgaris vulgaris Zschach, 1788

Plateumarini Baving, 1922

Plateumaris sericea sericea (Linnaeus, 1760)

\begin{tabular}{|c|c|c|c|c|c|c|c|c|}
\hline & N & & & & & & & \\
\hline $\mathrm{N}$ & $\mathrm{X}$ & & & & $\mathrm{N}$ & & & \\
\hline & $\mathrm{X}$ & & & & & & & \\
\hline & $\mathrm{X}$ & & $\mathrm{B}$ & & & $\mathrm{X}$ & & \\
\hline $\mathrm{X}$ & $\mathrm{X}$ & $\mathrm{X}$ & & & $\mathrm{X}$ & & \\
\hline & & & $\mathrm{B}$ & & & & \\
\hline & & & $\mathrm{N}$ & & & & & \\
\hline
\end{tabular}

\author{
, 1922
}

Criocerinae Latreille, 1804

Schmitt in Cat. Pal. Col., vol. 6, 2010

Crioceris duodecimpunctata (Linnaeus, 1758)

Crioceris macilenta Weise, 1881

Crioceris paracenthesis (Linnaeus, 1767)

Lema cyanella (Linnaeus, 1758)

Lilioceris lilii (Scopoli, 1763)

Lilioceris merdigera (Linnaeus, 1758)

Lilioceris stercoraria (Limnaeus, 1767)

Oulema erichsonii Suffrian, 1841

Oulema magistrettiortum Ruffo, 1964 [E]

Oulema melanopus (Linnaeus, I758)

Oulema rufocyanea (Suffrian, 1847)

\begin{tabular}{|c|c|c|c|c|c|c|c|}
\hline $\mathrm{H}$ & X & & & & $\mathrm{X}$ & $\mathrm{X}$ & B \\
\hline X & X & & & & X & & \\
\hline \multirow[t]{7}{*}{$\mathrm{H}$} & $\mathrm{X}$ & & $\mathrm{N}$ & & $\mathrm{X}$ & $X$ & B \\
\hline & $\mathrm{X}$ & $\mathrm{N}$ & & & & & \\
\hline & & & $\mathrm{N}$ & & $\mathrm{X}$ & & \\
\hline & & & B & & & & B \\
\hline & & & & & & & B \\
\hline & & & B & & & & \\
\hline & & & & $\mathrm{N}$ & & & \\
\hline $\mathrm{H}$ & $\mathrm{X}$ & X & $\mathrm{N}$ & $\mathrm{N}$ & X & $\mathrm{X}$ & B \\
\hline & & & B & & & & \\
\hline
\end{tabular}

Cassidinae Gyllenhal, 1813

Cassidini Gyllenhal, 1813

Borowiec \& Sekerka in Cat. Pal. Col., vol. 6, 2010

Cassida algitica P.H. Lucas, 1849

Cassida deflorata Suffrian, 1844

Cassida denticollis Suffrian, 1844

Cassida ferruginea Goeze, 1777

Cassida flaveola Thurnberg, 1794

Cassida hemisphaerica Herbst, 1799

Cassida hexastigma Suffrian, 1844

Cassida inopinata Sassi \& Borowiec, 2006

Cassida inquinata Brullé, 1832

Cassida margaritacea Schaller, 1783

Cassida nebulosa Linnaeus, 1758

Cassida nobilis Linnaeus, 1758

Cassida prasina Illiger, 1798

Cassida pusilla Waltl, 1835

Cassida rubiginosa rubiginosa O.F. Müller, 1776

Cassida sanguinolenta O.F. Müller, 1776

Cassida seladonia Gyllenhal, 1827

\begin{tabular}{|c|c|c|c|c|c|c|c|c|}
\hline X & X & & & ! & B & & B & \\
\hline B & & & B & ! & B & & & \\
\hline & & X & & ! & B & X & N & \\
\hline & & ! & & & & B & & \\
\hline B & & & & & & B & & \\
\hline B & & & & & B & & & \\
\hline B & B & B & & & B & & & \\
\hline B & X & X & & & N & & & \\
\hline H & X & ! & B & N & N & X & B & \\
\hline & & & & N & & & & \\
\hline & N & & & & & & & \\
\hline B & X & & & & X & X & & \\
\hline & X & & & ! & & & & \\
\hline H & X & X & N & B & B & X & B & \\
\hline & & & ! & & B & X & & \\
\hline & & & & & B & & & \\
\hline
\end{tabular}




\begin{tabular}{|c|c|c|c|c|c|c|c|c|c|}
\hline Species & 焉 & $\begin{array}{l}3 \\
0 \\
3 \\
0 \\
0 \\
\frac{0}{0} \\
:\end{array}$ & 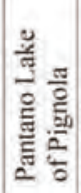 & 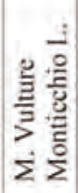 & 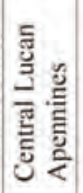 & 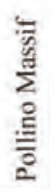 & 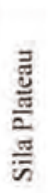 & $\frac{\stackrel{y}{5}}{\grave{E}}$ & 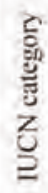 \\
\hline Cassida vibex Linnaeus, 1767 & & $\mathrm{X}$ & $\mathrm{X}$ & & B & $\mathrm{X}$ & & & \\
\hline Cassida viridis Linnaeus, 1758 & $\mathrm{H}$ & $\mathrm{X}$ & $\mathrm{X}$ & B & $\mathrm{N}$ & $\mathrm{B}$ & $\mathrm{X}$ & B & \\
\hline Cassida vittata Villers, 1789 & B & $\mathrm{X}$ & & & $\mathrm{N}$ & & & & \\
\hline Hypocassida subferruginea (Schrank, 1776) & $\mathrm{H}$ & $\mathrm{X}$ & $\mathrm{X}$ & $\mathrm{N}$ & & $\mathrm{X}$ & & $\mathrm{N}$ & \\
\hline \multicolumn{10}{|c|}{ Hispini Gyllenhal, 1813} \\
\hline Dicladispa testacea (Linnaeus, 1767) & $\mathrm{X}$ & $\mathrm{X}$ & & & $\mathrm{N}$ & $\mathrm{N}$ & $\mathrm{X}$ & $\mathrm{N}$ & \\
\hline Hispa atra Linnaeus, 1767 & $\mathrm{H}$ & $\mathrm{X}$ & $\mathrm{X}$ & & $\mathrm{N}$ & $\mathrm{N}$ & $\mathrm{X}$ & $\mathrm{N}$ & \\
\hline \multicolumn{10}{|c|}{ Leptispini Fairmaire, 1864} \\
\hline Lepthispa filiformis (Germar, 1842) & $\mathrm{X}$ & & & & & & & & \\
\hline \multicolumn{10}{|c|}{$\begin{array}{c}\text { Chrysomelinae Latreille, } 1802 \\
\text { Chrysomelini Latreille, } 1802 \\
\text { Chrysomelina Latreille, } 1802 \\
\text { Kippenberg in Cat. Pal. Col, vol. } 6,2010\end{array}$} \\
\hline
\end{tabular}

Chrysomela populi Linnaeus, 1758

Plagiodera versicolora (Laicharting, 1781)

Plagiosterna aenea aenea (Linnaeus, 1758)

\begin{tabular}{|l|l|l|l|l|l|l|l|l|}
$\mathrm{X}$ & $\mathrm{X}$ & $\mathrm{X}$ & $\mathrm{N}$ & $\mathrm{N}$ & $\mathrm{X}$ & $\mathrm{X}$ & $\mathrm{N}$ & \\
$\mathrm{X}$ & $\mathrm{X}$ & $\mathrm{X}$ & $\mathrm{N}$ & $\mathrm{N}$ & $\mathrm{X}$ & $\mathrm{X}$ & $\mathrm{N}$ & \\
\hline & & & & $\mathrm{N}$ & $\mathrm{N}$ & $\mathrm{X}$ & $\mathrm{B}$ & \\
\hline
\end{tabular}

Gastrophysina Kippenberg, 2010

Gastrophysa polygoni polygoni (Linnaeus, 1758) $\mathrm{H}$ X

Phratorina Motschulsky, 1860

Phratora laticollis (Suffrian, 1851)

\begin{tabular}{|l|l|l|l|l|l|l|l|l|}
\hline $\mathrm{X}$ & $\mathrm{X}$ & $\mathrm{X}$ & $\mathrm{N}$ & $\mathrm{N}$ & $\mathrm{X}$ & $\mathrm{X}$ & & \\
$\mathrm{X}$ & & $\mathrm{X}$ & $\mathrm{N}$ & & $\mathrm{X}$ & & $\mathrm{B}$ & \\
\hline
\end{tabular}

Phratora vitellinae (Linnaeus, 1758)

\section{Prasocurina Reitter, 1913}

Phaedon armoraciae (Linnaeus, 1758)

Phaedon cochleariae cochleariae (Fabricius, 1792)

Phaedon laevigatus laevigatus (Duftschmid, 1825)

Prasocuris flavocincta (Brullé, 1832)

Prasocuris glabra (Herbst, 1783)

Prasocuris junci (Brahm, 1790)

Prasocuris vicina (P.H. Lucas, 1849)

\section{Doryphorini Motschulsky, 1860}

Chrysolinina S.-H. Chen, 1936

\begin{tabular}{|c|c|c|c|c|c|c|c|c|}
\hline Chrysolina affinis bruttiana Bourdonné, 1999 [E] & & & & & & & & $\mathrm{B}$ \\
\hline Chrysolina americana (Linnaeus, 1758) & $\mathrm{H}$ & $\mathrm{X}$ & & & $\mathrm{N}$ & & & B \\
\hline Chrysolina bankii (Fabricius, 1775) & $\mathrm{H}$ & $\mathrm{X}$ & & & $\mathrm{N}$ & & & $!$ \\
\hline Chrysolina cerealis mixta (Küster, 1844) & & & & & & $\mathrm{B}$ & & B \\
\hline Chrysolina chalcites (Germar, 1824) & $\mathrm{H}$ & & & & & & & \\
\hline Chrysolina corcyria (Suffrian, 1851) & $\mathrm{H}$ & & & & & & & $!$ \\
\hline Chrysolina cribrosa sirentensis (Meier, 1900) [E] & $\mathrm{X}$ & & & & B & $\mathrm{X}$ & & \\
\hline Chrysolina fastuosa fastuosa (Scopoli, 1763) & $\mathrm{X}$ & & & & $\mathrm{N}$ & $\mathrm{X}$ & $\mathrm{X}$ & $\mathrm{N}$ \\
\hline Chrysolina grossa grossa (Fabricius, 1792) & $\mathrm{H}$ & & & & & $\mathrm{X}$ & $\mathrm{X}$ & $\mathrm{N}$ \\
\hline Chrysolina haemoptera corvina (Weise, 1916) [E] & $\mathrm{X}$ & $\mathrm{X}$ & & & & B & $\mathrm{X}$ & B \\
\hline Chrysolina herbacea herbacea (Duftschmid, 1825) & $\mathrm{X}$ & $\mathrm{X}$ & $\mathrm{X}$ & B & $\mathrm{N}$ & $\mathrm{N}$ & $\mathrm{X}$ & ! \\
\hline Chrysolina hyperici hyperici (Forster, 1771) & $\mathrm{X}$ & $\mathrm{X}$ & $\mathrm{X}$ & $\mathrm{N}$ & & $\mathrm{X}$ & $\mathrm{X}$ & $!$ \\
\hline Chrysolina interstincta interstincta (Suffrian, 1851) [E] & $\mathrm{H}$ & & & & & $\mathrm{N}$ & $\mathrm{N}$ & B \\
\hline Chrysolina lutea (L. Petagna, 1819) & $\mathrm{H}$ & $\mathrm{X}$ & & & $\mathrm{N}$ & $\mathrm{X}$ & $\mathrm{X}$ & B \\
\hline Chrysolina oricalcia (O.F. Müller, 1776) & $\mathrm{X}$ & $\mathrm{X}$ & & $\mathrm{N}$ & & & & \\
\hline Chrysolina peregrina (Herrich-Schäffer, 1838) & & & & & & & & $\mathrm{B}$ \\
\hline Chrysolina polita polita (Linnaeus, 1758) & $\mathrm{X}$ & $\mathrm{X}$ & $\mathrm{X}$ & B & $\mathrm{N}$ & ! & $\mathrm{X}$ & B \\
\hline Chrysolina pseudolurida obscurefacta Bechyné, 1952 [E] & $\mathrm{X}$ & & & & & $\mathrm{X}$ & & \\
\hline Chrysolina rossia (Illiger, 1802) & $\mathrm{H}$ & $\mathrm{X}$ & & & $\mathrm{N}$ & $\mathrm{X}$ & $\mathrm{X}$ & $\mathrm{B}$ \\
\hline Chrysolina vernalis italica (Weise, 1882) & $\mathrm{H}$ & $\mathrm{X}$ & $\mathrm{N}$ & & $\mathrm{N}$ & $\mathrm{X}$ & $\mathrm{X}$ & B \\
\hline Chrysolina viridana viridana (Küster, 1844) & & $\mathrm{X}$ & & $\mathrm{B}$ & & & & $\mathrm{N}$ \\
\hline
\end{tabular}


Species

Oreina alpestris marsicana (Luigioni, 1933) [E]

Oreina cacaliae magistrettii (Schatzmayr, 1941) [E]

Doryphorina Motschulsky, 1860

\begin{tabular}{|c|c|c|c|c|c|c|c|c|}
\hline 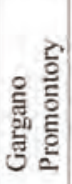 & 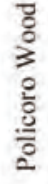 & 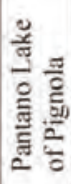 & 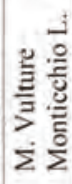 & 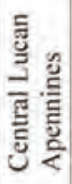 & 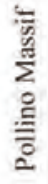 & 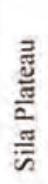 & 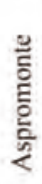 & 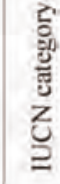 \\
\hline & & & & & $\mathrm{X}$ & & & \\
\hline & & & & & $\mathrm{X}$ & & & \\
\hline
\end{tabular}

Leptinotarsa decemlineata (Say, 1824)

\begin{tabular}{|l|l|l|l|l|l|l|}
\hline $\mathrm{X}$ & $\mathrm{X}$ & & & $\mathrm{N}$ & $\mathrm{X}$ & $\mathrm{N}$ \\
\hline
\end{tabular}

Sclerophaedon orbicularis (Suffrian, 1851)

Entomoscelini Reitter, 1913

Gonioctenini Motschulsky, 1860

Gonioctena quinquepunctata quinquepunctata (Fabricius, 1787)

Timarchini Motschulsky, 1860

Gomez-Zurita \& Kippenberg in Cat. Pal. Col., vol. 6, 2010

Timarcha metallica (Laicharting, 1781)

Timarcha nicaeensis A. Villa \& G.B. Villa, 1835

Timarcha pimelioides Herrich-Schäffer, $1838[\mathrm{E}]$

\begin{tabular}{|l|l|l|l|l|l|l|l} 
& & & & & & X & \\
\hline H & X & & & N & X & X & B \\
\hline & X & X & N & N & X & & N \\
\hline
\end{tabular}

Galerucinae Latreille, 1802

Galerucini Latreille, 1802

Beenen in Cat. Pal. Col., vol. 6, 2010

Diorhabda elongata (Brullé, 1836)

Galeruca interrupta (Illiger, 1802)

Galeruca littoralis (Fabricius, 1787)

Galeruca pomonae pomonae (Scopoli, 1763 )

Galeruca reichei (Joannis, 1865)

Galeruca rufa Germar, 1824

Galeruca tanaceti tanaceti (Linnaeus, 1758)

Galerucella calmariensis (Linnaeus, 1767)

Galerucella lineola lineola (Fabricius, 1781)

Galerucella lineola solarii Burlini, 1942 [E]

Lochmaea caprea (Linnaeus, 1758)

Lochmaea crataegi (Forster, 1771)

Xanthogaleruca luteola (O.F. Müller, 1766)

Agelastica alni alni (Linnaeus, 1758)

Sermylassa halensis (Linnaeus, 1767)

Hylaspini Chapuis, 1875

\begin{tabular}{|c|c|c|c|c|c|c|c|c|}
\hline H & X & & & & & & & \\
\hline X & & & & & & X & & \\
\hline X & & & & N & & & & \\
\hline H & & & & & & & & \\
\hline H & X & & & & & & & \\
\hline X & X & & N & N & X & X & N & \\
\hline & & X & & N & & & & \\
\hline X & & X & B & & & & & \\
\hline & & & & & X & X & N & \\
\hline & & & & & X & & & \\
\hline H & X & & N & N & N & X & N & \\
\hline X & & & N & X & & & \\
\hline
\end{tabular}

Luperini Gistel, 1848

Luperina Gistel, 1848

Calomicrus circumfusus (Marsham, 1802)

Calomicrus rottenbergi Ragusa, 1873 [E]

Exosoma lusitanicum (Linnaeus, 1767)

Luperus biraghii Ragusa, 1871 [E]

Luperus calabricus Laboissiére, 1912 [E]

Luperus leonardii Fogato, 1979 [E]

Luperus longicornis (Fabricius, 1781)

Luperus pygmaeus Joannis, 1865 [E]

Luperus ragusai Laboissiére, 1919 [E]

\begin{tabular}{|l|l|l|l|l|l|l|l|l|}
\hline X & & & & & & & & \\
\hline & & & & N & N & N & & \\
\hline H & X & X & N & N & X & X & N & \\
\hline H & & & N & N & X & X & N & \\
\hline H & & & & N & & & & \\
\hline X & & & B & N & X & X & N & \\
\hline & & & N & N & N & & & \\
\hline H & & X & & N & & B & & \\
\hline & & X & & & & N & N & \\
\hline
\end{tabular}

Alticinae Newman, 1835

Doberl in Cat. Pal. Col, vol. 6, 2010

Altica aenescens (Weise, 1888)

Altica ampelophaga (Guérin-Méneville, 1858)

Altica brevicollis Foudras, 1861

Altica carduorum Guérin-Méneville, 1858

Altica lythri Aubé, 1843

Altica oleracea (Linnaeus, 1758)

Altica quercetorum Foudras, 1861

\begin{tabular}{|c|c|c|c|c|c|c|c|c|}
\hline & & & & B & B & & & \\
\hline H & & N & & & & X & & \\
\hline X & X & & & & N & & & \\
\hline & & N & & N & & & & \\
\hline H & X & N & B & N & X & X & B & \\
\hline X & & & & & & & & \\
\hline
\end{tabular}


Species

Altica lamaricis Schrank, 1785

Aphthona carbonaria Rosenhauer, 1856

Aphthona euphorbiae (Schrank, 1781)

Aphthona flaviceps Allard, 1859

Aphthona herbigrada (Curtis, 1837)

Aphthona lutescens (Gyllenhal, 1813)

Aphthona nigriceps (W. Redtenbacher, 1842)

Aphthona nonstriata (Goeze, 1777)

Aphthona pygmaea (Kutschera, 1861)

Aphthona semicyanea Allard, 1859

Aphthona sicelidis Weise, 1888

Aphthona venustula Kutschera, 1861

Apteropeda orbiculata (Marsham, 1802)

Argopus ahrensii (Germar, 1817)

Arrhenocoela lineata (P. Rossi, 1790)

Batophila aerata (Marsham, 1802)

Chaetocnema arenacea (Allard, 1860)

Chaetocnema arida Foudras, 1860

Chaetocnema aridula (Gyllenhal, 1827)

Chaetocnema chlorophana (Duftschmid, 1825)

Chaetocnema concinna (Marsham, 1802)

Chaetocnema conducta (Motschulsky, 1838)

Chaetocnema depressa (Boieldieu, 1859)

Chaetocnema hortensis (Geoffroy, 1785)

Chaetocnema mannerheimii (Gyllenhal, 1827)

Chaetocnema montenegrina Heikertinger, 1912

Chaelocnema obesa (Boieldieu, 1859)

Chaetocnema picipes Stephens, 1831

Chaetocnema procerula (Rosenhauer, 1856)

Chaetocnema punctifrons Abeille de Perrin, 1907

Chaetocnema scheffleri (Kutschera, 1864)

Chaetocnema semicoerulea (Koch, 1803)

Chaetocnema subcoerulea (Kutschera, 1864)

Chaetocnema tibialis (Illiger, 1807)

Crepidodera aurata (Marsham, 1802)

Crepidodera aurea (Geoffroy, 1785)

Crepidodera nitidula (Linnaeus, 1758)

Crepidodera plutus (Latreille, 1804)

Derocrepis sodalis (Kutschera, 1860)

Dibolia chryptocephala (Koch, 1803)

Dibolia femoralis femoralis L. Redtenbacher, 1849

Dibolia occultans (Koch, 1803)

Dibolia timida (Illiger, 1807)

Epitrix atropae Foudras, 1861

Epitrix hirtipennis (Melsheimer, 1847)

Epitrix pubescens (Koch, 1803)

Hermaeophaga mercurialis (Fabricius, 1792)

Hermaeophaga ruficollis (P.H. Lucas, 1849)

Hippuriphila modeeri (Linnaeus, 1760)

Longitarsus aeneicollis (Faldermann, 1837)

Longitarsus aeneus Kutschera, 1862

Longitarsus aeruginosus (Foudras, 1860)

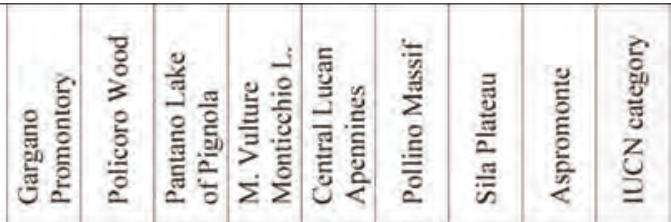


Species

Longitarsus albinens (Foudras, 1860)

Longitarsus anacardius (Allard, 1866)

Longitarsus anchusae (Paykull, 1799)

Longitarsus atricillus (Linnaeus, 1760)

Longitarsus ballotae (Marsham, 1802)

Longitarsus brisouti Heikertinger, 1912

Longitarsus brumneus (Duftschmid, 1825)

Longitarsus cerinthes (Schrank, 1798)

Longitarsus codinai Madar \& Madar, 1965

Longitarsus corynthius (Reiche, 1858)

Longitarsus exchii (Koch, 1803)

Longitarsus exsoletus (Linnaeus, 1758)

Longitarsus foudrasi Weise, 1893

Longitarsus gracilis Kutschera, 1864

Longitarsus helvolus Kutschera, 1864

Longitarsus holsaticus (Linnaeus, 1758)

Longitarsus juncicola (Foudras, 1860)

Longitarsus kutscherai (Rye, 1872)

Longitarsus lateripunctatus Rosenhauer, 1856

Longitarsus laureolae Biondi, 1988 [E]

Longitarsus linnaei (Duftschmid, 1825)

Longitarsus longipennis Kutschera, 1863

Longilarsus luridus (Scopoli, 1763)

Longitarsus lycopi (Foudras, 1860)

Longitarsus melanocephalus (DeGeer, 1775)

Longitarsus minusculus (Foudras, 1860)

Longitarsus monticola Kutschera, 1864

Longitarsus nasturtii (Fabricius, 1792)

Longitarsus niger (Koch, I803)

Longitarsus nigrocillus (Motschulsky, 1849)

Longitarsus nigrofasciatus (Goeze, 1777)

Longitarsus obliteratoides Gruev, 1973

Longitarsus obliteratus (Rosenhauer, 1847)

Longitarsus ochroleucus (Marsham, 1802)

Longitarsus ordinatus (Foudras, 1860)

Longitarsus parvulus (Paykull, 1799)

Longitarsus pellucidus (Foudras, 1860)

Longitarsus pratensis (Panzer, 1794)

Longitarsus rectelineatus (Foudras, 1860

Longitarsus rubiginosus (Foudras, 1860)

Longitarsus salviae Gruev, 1975

Longitarsus strigicollis Wollaston, 1864

Longitarsus substriatus Kutschera, 1864

Longitarsus succineus (Foudras, 1860)

Longitarsus suturellus (Duftschmid, 1825)

Longitarsus tabidus (Fabricius, 1775)

Longitarsus vilis Wollaston, 1864

Lythraria salicariae (Paykull, I800)

Mantura cylindrica Miller, 1881

Mantura lutea (Allard, 1859)

Mantura obtusata (Gyllenhal, 1813)

Mniophila bosnica Apfelbeck, 1914

Mniophila muscorum muscorum (Koch, 1803)

\begin{tabular}{|c|c|c|c|c|c|c|c|c|}
$\mathrm{N}$ & 1 & $\mathrm{~N}$ & & $\mathrm{~N}$ & $!$ & & & \\
\hline & & & & ! & & & & \\
& & $\mathrm{N}$ & $\mathrm{N}$ & $\mathrm{CK}$ & & & $\mathrm{B}$ & \\
\hline
\end{tabular}

\begin{tabular}{|c|c|c|c|c|c|}
\hline $\mathrm{H}$ & & & & $\mathrm{CK}$ & $\mathrm{CK}$ \\
\hline & $\mathrm{X}$ & & & & $\mathrm{B}$ \\
\hline
\end{tabular}

\begin{tabular}{|l|l}
\hline-1 \\
\hline
\end{tabular}

\begin{tabular}{|l|l|l|l|l|} 
& $\mathrm{X}$ & & & \\
$\mathrm{B}$ & $\mathrm{X}$ & & & \\
$\mathrm{H}$ & & & & \\
$\mathrm{H}$ & & $\mathrm{N}$ & & \\
$\mathrm{X}$ & & & & \\
\hline
\end{tabular}

$\mathrm{N} \quad \mathrm{X}$

B
CK

\begin{tabular}{|l|l|l|l|}
\hline & $\mathrm{N}$ & & $\mathrm{N}$ \\
\hline & & & $\mathrm{N}$ \\
\hline $\mathrm{N}$ & & $\mathrm{N}$ \\
\hline & $\mathrm{N}$ & \\
\hline
\end{tabular}

X $\mathrm{X}$

\begin{tabular}{|l|l|l|l|l|}
\hline & $\mathrm{N}$ & & $\mathrm{B}$ & \\
\hline $\mathrm{N}$ & & & & \\
\hline $\mathrm{N}$ & & & & \\
\hline
\end{tabular}

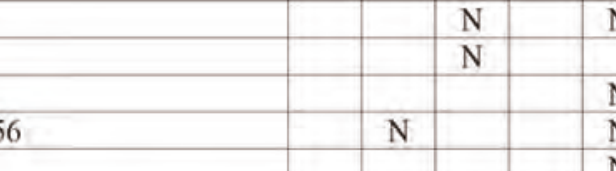

\begin{tabular}{|c|c|c|}
\hline & $\mathrm{X}$ & $\mathrm{CK}$ \\
\hline & & $\mathrm{B}$ \\
\hline
\end{tabular}

\begin{tabular}{l|l}
$\mathrm{N}$ & $\mathrm{X}$
\end{tabular}

\begin{tabular}{|l|l}
\hline & H \\
X & \\
\hline
\end{tabular}

\begin{tabular}{|l|l|l|l|l|l|l|l|l|}
\hline $\mathrm{H}$ & & & $\mathrm{N}$ & & $\mathrm{B}$ & $\mathrm{B}$ & \\
\hline $\mathrm{X}$ & & $\mathrm{N}$ & & & & $\mathrm{X}$ & & \\
\hline $\mathrm{H}$ & $\mathrm{X}$ & $\mathrm{N}$ & $\mathrm{N}$ & $\mathrm{N}$ & $\mathrm{X}$ & $\mathrm{X}$ & $\mathrm{B}$ & \\
\hline
\end{tabular}

$x$
$x$

\begin{tabular}{ll|l}
$\mathrm{X}$ & $\mathrm{X}$ \\
$\mathrm{H}$ & $\mathrm{X}$ \\
\hline
\end{tabular}

(2)

\begin{tabular}{|c|c|c|c|c|c|c|c|c|}
\hline H & X & N & & N & & & CK & \\
\hline H & X & & & & & & & \\
\hline & & ! & & N & & & & \\
\hline H & & & & & & B & & \\
\hline H & & & & & N & X & B & \\
\hline H & & N & N & N & N & & & \\
\hline B & & & & ! & & & CK & \\
\hline
\end{tabular}

\begin{tabular}{|c|c|}
\hline $\mathrm{N}$ & $\mathrm{N}$ \\
\hline$x$ & \\
\hline $\mathrm{N}$ & \\
\hline
\end{tabular}


Species

Neocrepidodera brevicollis (J. Daniel, 1904)

Neocrepidodera corpulenta (Kutschera, 1860)

Neocrepidodera ferruginea (Scopoli, 1763)

Neocrepidodera impressa impressa (Fabricius, 1801)

Neacrepidodera melanostoma (L. Redtenbacher, 1849)

Neocrepidodera transversa (Marsham, 1802)

Ochrosis ventralis (Illiger, 1807)

Orestia alpina (Germar, 1824)

Orestia apennina Weise, 1886 [E]

Orestia calabra calabra Heikertinger, 1909 [E]

Orestia kraatzi Allard, 1861

Phyllotreta astrachanica Lopatin, 1977

Phyllotreta atra (Fabricius, 1775)

Phyllotreta balcanica Heikertinger, 1909

Phyllotreta cruciferae (Goeze, 1777)

Phyllotreta lativittata Kutschera, 1860

Phyllotreta nemorum (Linnaeus, 1758)

Phyllotreta nigripes (Fabricius, 1775)

Phyllotreta nodicornis (Marsham, 1802)

Phyllotreta ochripes (Curtis, 1837)

Phyllotreta parallela (Boieldieu, I859)

Phyllotreta procera (L. Redtenbacher, 1849)

Phyllotreta punctulata (Marsham, 1802)

Phyllotreta rugifrons Küster, 1849

Phyllotreta striolata (Illiger, I803)

Phyllotreta undulata Kutschera, 1860

Phyllotreta variipennis (Boieldieu, 1859)

Phyllotreta vittula (L. Redtenbacher, 1849)

Podagrica fuscicornis (Linnaeus, 1767)

Podagrica malvae (Illiger, 1807)

Podagrica menetriesii (Faldermann, 1837)

Psylliodes affinis (Paykull, 1799)

Psylliodes algirica Allard, 1859

Psylliodes caneparii Leonardi, 2007 [E]

Psylliodes chalcomera (Illiger, 1807)

Psylliodes chrysocephala (Linnaeus, 1758)

Psylliodes circumdata (W. Redtenbacher, 1842)

Psylliodes cuprea Koch, 1803

Psylliodes dulcamarae Koch, 1803

Psylliodes feromiae Leonardi, 1978 [E]

Psylliodes fusiformis (Illiger, 1807)

Psylliodes gibbosa Allard, 1860

Psylliodes hyoscyami (Linnaeus, 1758)

Psylliodes instabilis Foudras, 1860

Psylliodes isatidis Heikertinger, 1913

Psylliodes kiesenwetteri Kutschera, 1864

Psylliodes laevifrons Kutschera, 1864

Psylliodes laticollis Kutschera, 1864

Psylliodes leonhardi Heikertinger, 1926 [E]

Psylliodes libertii Leonardi, 2013 [E]

Psylliodes Iuteola (O.F. Müller, 1776)

Psylliodes marcida (Illiger, 1807)

Psylliodes milleri Kutschera, 1864

\begin{tabular}{|l|l|l|l|l|l|l|l|} 
& & & & & X & X & \\
\hline
\end{tabular}

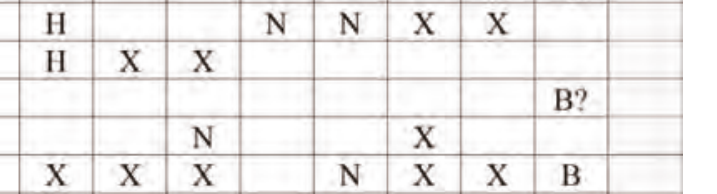


Species

Psylliodes napi (Fabricius, 1792)

Psylliodes obscuroaenea Rosenhauer, 1856

Psylliodes pallidipennis Rosenhauer, 1856

Psylliodes puncticollis Rosenhauer, 1856

Psylliodes pyritosa Kutschera, 1864

Psylliodes ruffoi Leonardi, 1975 [E]

Psylliodes thlaspis Foudras, 1860

Sphaeroderma rubidum (Graells, 1858)

Sphaeroderma testaceum (Fabricius, 1775)

Lamprosomatinae Lacordaire, 1848

Konstantinov in Cat. Pal. Col., vol. 6, 2010

Oomorphus concolor (Sturm, 1807)

Cryptocephalinae Gyllenhal, 1813

Clytrini Kirby, 1837

Clytrina Kirby, 1837

Regalin \& Medvedev in Cat. Pal. Col., vol. 6, 2010

Cheilotoma erythrostoma italica Leoni, 1906

Cheilotoma musciformis musciformis (Goeze, 1777)

Clytra laeviuscula Ratzeburg, 1837

Clytra quadripunctata quadripunctata (Linnaeus, 1758)

Coptocephala scopolina kuesteri Kraatz, 1872

Coptocephala unicolor (P.H. Lucas, 1845)

Coptocephala unifasciata unifasciata (Scopoli, 1763)

Labidostomis humeralis (D.H. Schneider, 1792)

Labidostomis longimana (Linnaeus, 1760)

Labidostomis pallidipennis (Gebler, 1830)

Labidostomis taxicornis taxicornis (Fabricius, 1792)

Labidostomis tridentata (Linnaeus, 1758)

Lachnaia italica italica Weise, 1881

Lachnaia sexpunctata (Scopoli, 1763)

Macrolenes dentipes (A.G. Olivier, 1808)

Smaragdina affinis affinis (Illiger, 1794)

Smaragdina aurita aurita (Linnaeus, 1767)

Smaragdina concolor concolor (Fabricius, 1792)

Smaragdina salicina (Scopoli, 1763)

Tituboea biguttata (A.G. Olivier, 1791)

\begin{tabular}{|c|c|c|c|c|c|c|c|c|}
\hline $\mathrm{X}$ & $\mathrm{X}$ & $X$ & & $\mathrm{~N}$ & $\mathrm{X}$ & $\mathrm{X}$ & & \\
\hline $\mathrm{H}$ & & X & & & & $\mathrm{X}$ & B & \\
\hline \multirow[t]{2}{*}{$\mathrm{X}$} & $\mathrm{X}$ & & $\mathrm{N}$ & & $X$ & $\mathrm{X}$ & $\mathrm{N}$ & \\
\hline & & $\mathrm{X}$ & & & X & & B & \\
\hline \multirow[t]{2}{*}{$\mathrm{H}$} & $\mathrm{X}$ & $\mathrm{X}$ & & $\mathrm{N}$ & $\mathrm{N}$ & $\mathrm{X}$ & $\mathrm{N}$ & \\
\hline & & $\mathrm{N}$ & & $\mathrm{N}$ & & & $\mathrm{N}$ & \\
\hline $\mathrm{X}$ & $\mathrm{X}$ & $\mathrm{X}$ & $\mathrm{N}$ & $\mathrm{N}$ & $\mathrm{X}$ & $\mathrm{X}$ & & \\
\hline \multicolumn{9}{|l|}{$\mathrm{X}$} \\
\hline $\mathrm{X}$ & & $\mathrm{X}$ & B & $\mathrm{N}$ & $\mathrm{X}$ & $\mathrm{X}$ & & \\
\hline $\mathrm{X}$ & & & & $\mathrm{N}$ & $\mathrm{X}$ & & & \\
\hline \multirow[t]{2}{*}{$\mathrm{H}$} & & & $\mathrm{N}$ & $\mathrm{N}$ & $\mathrm{X}$ & $\mathrm{X}$ & $\mathrm{N}$ & \\
\hline & & & & & & & B & \\
\hline \multirow[t]{2}{*}{$\mathrm{H}$} & $\mathrm{X}$ & & $\mathrm{N}$ & $\mathrm{N}$ & $\mathrm{X}$ & $\mathrm{X}$ & & \\
\hline & & & & $\mathrm{N}$ & & & & \\
\hline $\mathrm{H}$ & $\mathrm{X}$ & $\mathrm{N}$ & $\mathrm{N}$ & $\mathrm{N}$ & $\mathrm{X}$ & $\mathrm{X}$ & $\mathrm{N}$ & \\
\hline H & & $\mathrm{X}$ & B & $\mathrm{N}$ & $X$ & & & \\
\hline \multirow[t]{2}{*}{$\mathrm{H}$} & & & & & $X$ & $\mathrm{X}$ & $\mathrm{N}$ & \\
\hline & & & $\mathrm{N}$ & $\mathrm{N}$ & $\mathrm{X}$ & $\mathrm{X}$ & $\mathrm{N}$ & \\
\hline $\mathrm{H}$ & & & & $\mathrm{N}$ & $\mathrm{X}$ & & & \\
\hline $\mathrm{H}$ & & & $\mathrm{N}$ & & $\mathrm{X}$ & $\mathrm{X}$ & & \\
\hline
\end{tabular}

Cryptocephalini Gyllenhal, 1813

Cryptocephalina Gyllenhal, 1813

Lopatin, Smetana \& Schöller in Cat. Pal. Col., vol. 6, 2010

\begin{tabular}{|c|c|c|c|c|c|c|c|c|c|}
\hline Cryptocephalus anticus Suffrian, 1848 & $\mathrm{X}$ & $\mathrm{X}$ & $\mathrm{X}$ & & & & & & \\
\hline Cryptocephalus aureolus aureolus Suffrian, 1847 & & & & $\mathrm{~N}$ & & $\mathrm{X}$ & $\mathrm{X}$ & & \\
\hline Cryptocephalus bameuli Duhaldeborde, 1999 & & & & & & CK & & & \\
\hline Cryptocephalus bilineatus (Linnaeus, 1767) & & & & & & & CK & & \\
\hline Cryptocephalus bimaculatus Fabricius, 1781 & & & & & & $!$ & CK & & \\
\hline Cryptocephalus bipunctatus bipunctatus (Linnaeus, 1758) & $\mathrm{H}$ & $\mathrm{X}$ & $\mathrm{X}$ & & CK & B & $\mathrm{X}$ & B & \\
\hline Cryptocephalus connexus A.G. Olivier, 1807 & $\mathrm{X}$ & & $\mathrm{X}$ & $\mathrm{N}$ & CK & $\mathrm{X}$ & $\mathrm{X}$ & & \\
\hline Cryptocephahus cyanipes Suffrian, 1847 & & & & & & $\mathrm{~N}$ & $\mathrm{X}$ & & \\
\hline Cryptocephalus czwalinae Weise, 1882 [E] & $\mathrm{H}$ & & & & CK & $\mathrm{X}$ & $\mathrm{X}$ & B & \\
\hline Cryptocephahus daccordii Biondi, 1995 [E] & & $\mathrm{B}$ & & & & CK & & B & \\
\hline Cryptocephalus decemmaculatus (Linnaeus, 1758) & $\mathrm{H}$ & & & & & & & & \\
\hline Cryptocephalus elegantulus Gravenhorst, 1807 & $\mathrm{~N}$ & & & & & & & & \\
\hline Cryptocephalus etruscus Suffrian, 1847 & B & B & $\mathrm{N}$ & B & B & $\mathrm{X}$ & B & B & \\
\hline
\end{tabular}


Species

Cryptocephalus falzonii Burlini, 1956 [E]

Cryptocephalus flavipes Fabricius, 1781

Cryptocephalus frenatus Laicharting, 1781

Cryplocephalus frontalis Marsham, 1802

Cryptocephalus fulvus fulvus (Goeze, 1777)

Cryptocephalus imperialis Laicharting, 1781

Cryptocephalus informis Suffrian, 1847

Cryptocephalus labiatus (Linnaeus, 1760)

Cryptocephalus leonhardi Breit, 1918

Cryptocephalus lorevi Solier, 1837

Cryptocephalus macellus Suffrian, 1860

Cryptocephalus marginatus Fabricius, 1781

Cryptocephalus moraei (Linnaeus, 1758)

Cryptocephalus nitidus (Linnaeus, 1758)

Cryptocephalus ocellatus ocellatus Drapiez, 1819

Cryptocephalus ochroleucus ochroleucus Fairmaire, 1859

Cryptocephalus octomaculatus P. Rossi, 1790

Cryptocephalus parvulus O.F. Müller, 1776

Cryptocephalus pini pini (Linnaeus, 1758)

Cryptocephalus politus politus Suffrian, 1853

Cryptocephahus populi Suffrian, 1848

Cryptocephalus pygmaeus vittula Suffrian, 1848

Cryptocephalus renatae Sassi, 2001

Cryptocephalus rufipes (Goeze, 1777)

Cryptocephahus migicollis A.G. Olivier, 1791

Cryptocephalus sammiticus Leonardi \& Sassi, 2001 [E]

Cryptocephalus scapularis Suffrian, 1848

Cryptocephalus sexpumctatus sexpunctatus Linnaeus, 1758

Cryptocephalus signatifrons Suffrian, 1847

Cryptocephalus stragula P. Rossi, 1794

Cryptocephalus strigosus Germar, 1824

Cryptocephalus tricolor P. Rossi, 1792

Cryptocephalus trimaculatus P. Rossi, 1790

Cryptocephalus turcicus Suffrian, 1847

Cryptocephalus zambanellus Marsham, 1875

Pachybrachina Chapuis, 1874

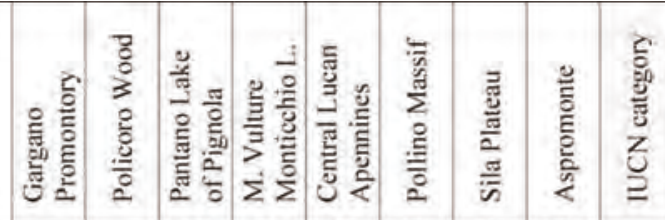

Schöller, Löbl \& Lopatin in Cat. Pal. Col., vol. 6, 2010

Pachybrachis exclusus (Rey, 1883)

Pachybrachis fimbriolatus (Suffrian, 1848)

Pachybrachis hieroglyphicus (Laicharting, 1781)

Pachybrachis hippophaes (Suffrian, 1848)

Pachybrachis karamani (Weise, 1893)

Pachybrachis ruffoi ruffoi Burlini, 1957 [E]

Pachybrachis salfii Burlini, 1957

Pachybrachis scriptus Herrich-Schäffer, 1838

Pachybrachis tessellatus (A.G. Olivier, 1791)

Stylosomina Chapuis, 1874

Stylosomus mimutissimus (Germar, 1824)

Stylosomus tamaricis (Herrich-Schäffer, 1836)

\begin{tabular}{|c|c|c|c|c|c|c|c|c|}
\hline $\mathrm{H}$ & $\mathrm{X}$ & & $\mathrm{N}$ & $\mathrm{N}$ & $\mathrm{X}$ & $\mathrm{X}$ & $\mathrm{B}$ & \\
\hline & & $\mathrm{X}$ & & & $\mathrm{X}$ & & & \\
\hline $\mathrm{CK}$ & $\mathrm{CK}$ & & & $\mathrm{N}$ & $\mathrm{N}$ & $\mathrm{N}$ & & \\
\hline & & & & & & $\mathrm{N}$ & & \\
\hline & $\mathrm{CK}$ & & & & & & & \\
\hline $\mathrm{CK}$ & & & & & $\mathrm{X}$ & & & \\
\hline $\mathrm{X}$ & & & & & & & & \\
\hline $\mathrm{X}$ & & & & & & & & \\
\hline
\end{tabular}

\section{Eumolpinae Hope, 1840}

Bromiini Chapuis, 1874

Moseyko \& Sprecher-Uebersax in Cat. Pal. Col., vol. 6, 2010

Bromius obscurus (Linnaeus, 1758) 


\begin{tabular}{|c|c|c|c|c|c|c|c|c|c|}
\hline Species & 总 & 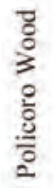 & 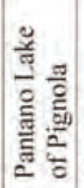 & 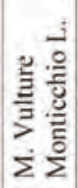 & 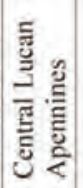 & 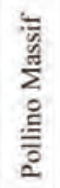 & $\frac{\text { 胥 }}{\frac{\underline{m}}{2}}$ & 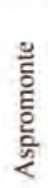 & 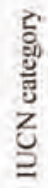 \\
\hline Colaspidea nitida P.H. Lucas, 1846 & $\mathrm{H}$ & $\mathrm{X}$ & & & & & & B & \\
\hline Pachnephortis bistriatus Mulsant \& Wachanru, 1852 & $\mathrm{X}$ & $\mathrm{X}$ & & & & & & & \\
\hline Pachnephorus cylindricus P.H. Lucas, 1849 & & $\mathrm{X}$ & & & & & & $\mathrm{N}$ & \\
\hline Pachnephorus tessellatus (Duftschmid, 1825) & & $\mathrm{N}$ & $\mathrm{N}$ & & $\mathrm{N}$ & & & & \\
\hline Pachmephorus villosus (Duftschmid, 1825) & $\mathrm{X}$ & $\mathrm{X}$ & & & & & & & \\
\hline
\end{tabular}

CURCULIONOIDEA Latreille, 1802

Alonso-Zarazaga et. al, 2017, Coop. Cat. Curculionoidea

NEMONYCHIDAE Bedel, 1882

Nemonyx lepturoides (Fabricius, 1801)

ANTHRIBIDAE Billberg, 1820

Anthribinae Billberg, 1820

Anthribini Billberg, 1820

\begin{tabular}{|c|c|c|c|c|c|c|c|c|}
\hline Anthribus fasciatus Forster, 1770 & B & $\mathrm{X}$ & & $\mathrm{N}$ & $\mathrm{X}$ & $\mathrm{X}$ & $\mathrm{N}$ & LC \\
\hline \multicolumn{9}{|c|}{ Discotenini Lacordaire, 1865} \\
\hline Enedreytes hilaris Făhraeus, 1839 & $\mathrm{X}$ & $\mathrm{X}$ & & & $\mathrm{N}$ & & B & LC \\
\hline Psendeuparius centromaculatus (Gyllenhal, 1833) & B & $\mathrm{X}$ & B & & & & $\mathrm{B}$ & LC \\
\hline
\end{tabular}

Piesocorynini Valentine, 1960

\begin{tabular}{|l|l|l|l|l|l|l|l|l|}
\hline Phaenotherion fasciculatum fasciculatum Reitter, 1891 [E] & X & X & N & B & X & X & B & LC
\end{tabular} Platyrhinini Imhoff, 1856

Platyrhinus resinosus (Scopoli, 1763)

Ulorhimus bilineatus (Germar, 1819)

Platystomini Pierce, 1916

\begin{tabular}{|l|l|l|l|l|l|l|l|l|}
\hline $\mathrm{X}$ & $\mathrm{X}$ & & $\mathrm{N}$ & $\mathrm{B}$ & $\mathrm{X}$ & $\mathrm{B}$ & $\mathrm{B}$ & $\mathrm{LC}$ \\
\hline $\mathrm{H}$ & $\mathrm{X}$ & & & $\mathrm{B}$ & $\mathrm{B}$ & & & LC \\
\hline
\end{tabular}

Platystomos albinus (Linnaeus, 1758) \begin{tabular}{l|l}
$\mathrm{H}$ & $\mathrm{X}$
\end{tabular}

Zygaenodini Lacordaire, 1865

Dissolencas niveirostris (Fabricius, 1798)

Noxius curtirostris (Mulsant \& Rey, 1861)

Rhaphitropis marchica (Herbst, 1797)

Rhaphitropis oxyacanthae (C.N.F. Brisout de Barneville, 1863)

\begin{tabular}{|c|c|c|c|c|c|c|c|c|}
\hline $\mathrm{X}$ & & $\mathrm{B}$ & & $\mathrm{B}$ & $\mathrm{X}$ & $\mathrm{X}$ & $\mathrm{B}$ & $\mathrm{LC}$ \\
\hline $\mathrm{B}$ & $\mathrm{B}$ & & & & & & & LC \\
\hline & & & & $\mathrm{B}$ & & & & LC \\
\hline
\end{tabular}

Choraginae Kirby, 1819

Choragini Kirby, 1819

Choragus sheppardi Kirby, 1819

\begin{tabular}{|l|l|l|l|l|l|l|l|}
\hline B & & & & & & & LC \\
\hline
\end{tabular}

Urodontinae C.G. Thomson, 1859

Bruchela flavescens (Küster, 1850)

Brichela rufipes rufipes (A.G. Olivier, 1790

\begin{tabular}{|c|c|c|c|c|c|c|c|c|}
\hline H & X & X & & & & & N & \\
\hline B & & & & & & & & \\
\hline
\end{tabular}

ATTELABIDAE Billberg, 1820

Attelabinae Billberg, 1820

Apoderini Jekel, 1860

Apoderina Jekel, 1860

Apoderus coryli (Linnaeus, 1758)

Attelabini Billberg, 1820

\begin{tabular}{|l|l|l|l|l|l|l|}
\hline CK & & CK & CK & $\mathrm{X}$ & $\mathrm{X}$ & $\mathrm{CK}$ \\
\hline
\end{tabular}

Attelabus nifens (Scopoli, 1763)

\begin{tabular}{|l|l|l|l|l|l|l|l|}
\hline$H$ & $X$ & $X$ & $C K$ & $C K$ & $X$ & $X$ & $B$
\end{tabular}

Rhynchitinae Gistel, 1848

Auletini Desbrochers des Loges, 1908

Auletina Desbrochers des Loges, 1908

Auletes tubicen Schönherr, 1826

Pseudomesauletina Legalov, 2003

Aletinus maculipennis (Jacquelin du Val, 1854)

Eomesauletes politus (Lepeletier \& Audinet-Serville, 1825)

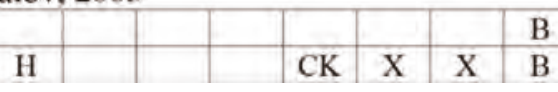




\begin{tabular}{|c|c|c|c|c|c|c|c|c|c|}
\hline Species & 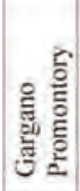 & $\begin{array}{l}3 \\
0 \\
3 \\
0 \\
\frac{0}{0} \\
\frac{0}{0} \\
2\end{array}$ & 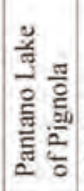 & 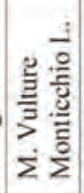 & 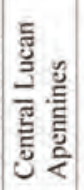 & 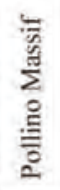 & 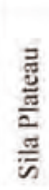 & 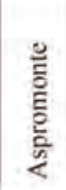 & 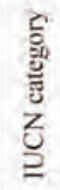 \\
\hline Mesauletobius pubescens (Kiesenwetter, 1852) & $\mathrm{CK}$ & & & CK & CK & & & & \\
\hline \multicolumn{10}{|c|}{$\begin{array}{l}\text { Byctiscini Voss, } 1923 \\
\text { Byctiscina Voss, } 1923\end{array}$} \\
\hline Byctiscus betulae (Linnaeus, 1758) & $\mathrm{H}$ & & & $\mathrm{N}$ & & $\mathrm{X}$ & $\mathrm{X}$ & B & \\
\hline Byctiscus populi (Linnaeus, 1758 ) & & & & CK & & $\mathrm{X}$ & & CK & \\
\hline \multicolumn{10}{|c|}{$\begin{array}{l}\text { Deporaini Voss, } 1929 \\
\text { Chonostropheina Morimoto, } 1962\end{array}$} \\
\hline Chonostropheus seminiger (Reitter, 1881) & & & & $\mathrm{N}$ & & $\mathrm{X}$ & $\mathrm{X}$ & B & \\
\hline \multicolumn{10}{|c|}{ Deporaina Voss, 1929} \\
\hline Caenorhinus megacephalus (Germar, 1823) & & & & B & & & & & \\
\hline Deporaus betulae (Linnaeus, 1758 ) & $\mathrm{H}$ & CK & & CK & & $\mathrm{N}$ & $\mathrm{X}$ & CK & \\
\hline \multicolumn{10}{|c|}{ Rhynchitini Gistel, 1848} \\
\hline Involvulus icosandriae icosandriae (Scopoli, 1763) & $\mathrm{H}$ & CK & $\mathrm{X}$ & & CK & $\mathrm{X}$ & $\mathrm{X}$ & B & \\
\hline Involvulus pubescens (Fabricius, 1775) & & & & $\mathrm{CK}$ & $\mathrm{CK}$ & & $\mathrm{X}$ & & \\
\hline Lasiorhynchites cavifrons (Gyllenhal, 1833) & $\mathrm{H}$ & $\mathrm{X}$ & & & CK & & & & \\
\hline Lasiorhynchites comatus (Gyllenhal, 1833) & CK & & & & CK & & & & \\
\hline Lasiorhynchites praeustus (Boheman, 1845) & $\mathrm{X}$ & $\mathrm{X}$ & & $\mathrm{N}$ & CK & $\mathrm{X}$ & $\mathrm{X}$ & $\mathrm{CK}$ & \\
\hline Lasiorhynchites sericeus (Herbst, 1797) & $\mathrm{H}$ & & & CK & CK & $\mathrm{X}$ & & B & \\
\hline Mecorhis aethiops (Bach, 1854) & & & & & B & & & & \\
\hline Neocoenorrhimus abeillei (Desbrochers des Loges, 1869) & $\mathrm{H}$ & & & & CK & CK & & & \\
\hline Neocoenorrhinus germanicus (Herbst, 1797) & $\mathrm{H}$ & & $\mathrm{X}$ & & $\mathrm{CK}$ & $\mathrm{X}$ & $\mathrm{X}$ & B & \\
\hline Neocoenorrhimus interpumctatus (Stephens, 1831) & & $\mathrm{X}$ & & & CK & $\mathrm{X}$ & & B & \\
\hline Neocoenorrhimus minutus (Herbst, 1797) & $\mathrm{H}$ & & & & CK & & & & \\
\hline Neocoenorrhimus pauxillus (Germar, 1823) & $\mathrm{H}$ & $\mathrm{X}$ & $\mathrm{X}$ & $\mathrm{N}$ & $\mathrm{CK}$ & $\mathrm{X}$ & $\mathrm{X}$ & CK & \\
\hline Rhodocyrtus cribripennis (Desbrochers des Loges, 1869) & $\mathrm{X}$ & & & & CK & & & & \\
\hline Rhynchites auratus (Scopoli, 1763) & $\mathrm{H}$ & & $\mathrm{X}$ & $\mathrm{CK}$ & CK & CK & $\mathrm{X}$ & CK & \\
\hline Rhynchites bacchus (Linnaeus, 1758) & $\mathrm{H}$ & $\mathrm{X}$ & & $\mathrm{N}$ & CK & $\mathrm{X}$ & $\mathrm{X}$ & CK & \\
\hline Rhynchites giganteus Schönherr, 1832 & $\mathrm{X}$ & $\mathrm{X}$ & & CK & CK & $\mathrm{X}$ & $\mathrm{X}$ & CK & \\
\hline Tatyanaerhynchites aequatus (Linnaeus, 1767) & $\mathrm{H}$ & $\mathrm{X}$ & $\mathrm{X}$ & $\mathrm{N}$ & $\mathrm{CK}$ & $\mathrm{X}$ & $\mathrm{X}$ & B & \\
\hline Temnocerus coeruleus (Fabricius, 1798) & $\mathrm{H}$ & $\mathrm{X}$ & CK & & CK & $\mathrm{X}$ & $\mathrm{X}$ & B & \\
\hline Temnocerus namus (Paykull, 1792) & CK & $\mathrm{X}$ & & $\mathrm{N}$ & CK & $\mathrm{X}$ & $\mathrm{X}$ & & \\
\hline
\end{tabular}

BRENTIDAE Billberg, 1820 (sensu lato)

Apioninae Schönherr, 1823

Apionini Schönherr, 1823

Apionina Schönherr, 1823

\begin{tabular}{|l|l|l|l|l|l|l|l|l|l|}
\hline Apion cruentatum Walton, 1844 & & & & & $\mathrm{X}$ & $\mathrm{X}$ & $\mathrm{B}$ & \\
\hline Apion frumentarium (Linnaeus, 1758) & $\mathrm{X}$ & $\mathrm{X}$ & $\mathrm{X}$ & $\mathrm{N}$ & & & $\mathrm{N}$ & $\mathrm{B}$ & \\
\hline Apion haematodes haematodes Kirby, 1808 & $\mathrm{X}$ & & & $\mathrm{N}$ & & $\mathrm{X}$ & $\mathrm{X}$ & $\mathrm{N}$ & \\
\hline Apion longithorax Desbrochers des Loges, 1889 & & & & & & & $\mathrm{X}$ & $\mathrm{B}$ & \\
\hline
\end{tabular}

Aplemonina Kissinger, 1968

Perapion affine (Kirby, 1808)

Perapion curtirostre (Germar, 1817)

Perapion hydrolapathi (Marsham, 1802)

Perapion ilvense (Wagner, 1905)

Perapion marchicum (Herbst, 1797)

Perapion violaceum violaceum (Kirby, 1808)

Phrissotrichum tubiferum (Gyllenhal, 1833)

Pseudoperapion brevirostre (Herbst, 1797)

Pseudostenapion simum (Germar, 1817)

\begin{tabular}{|c|c|c|c|c|c|c|c|c|}
\hline X & & & N & & X & X & N & \\
\hline H & X & X & N & N & X & X & & \\
\hline X & X & & & & X & X & B & \\
\hline & & & & & & X & B & \\
\hline H & X & X & N & N & X & X & B & \\
\hline X & & & & N & N & & B & \\
\hline H & & X & N & N & X & X & & \\
\hline & X & & & N & X & X & N & \\
\hline
\end{tabular}

Aspidapiina Alonso-Zarazaga, 1990

Aspidapion aeneum (Fabricius, 1775)

Aspidapion caprai Giusto, 1993 [E]

\begin{tabular}{|l|l|l|l|l|l|l|l|}
\hline $\mathrm{H}$ & $\mathrm{X}$ & $\mathrm{X}$ & $\mathrm{N}$ & $\mathrm{N}$ & $\mathrm{X}$ & $\mathrm{X}$ & $\mathrm{B}$ \\
\hline & & & B & & & & B \\
\hline
\end{tabular}


Species

Aspidapion radiolus (Marsham, 1802) Aspidapion soror (Rey, 1895)

\section{Catapiina Alonso-Zarazaga, 1990}

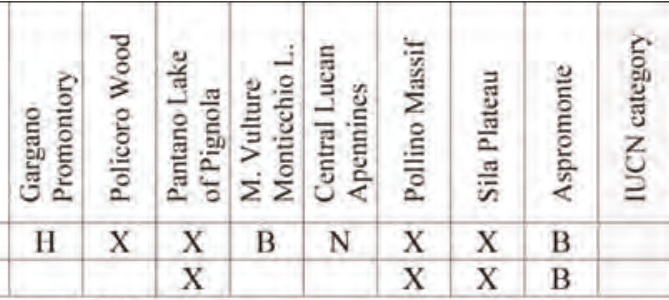

Catapion burdigalense (Wencker, 1859)

Catapion calabricum (A. Solari \& F. Solari, 1922) [E]

Catapion corsicum (Desbrochers des Loges, 1888)

Catapion curtisii (Stephens, 1831)

Catapion jaffense (Desbrochers des Loges, 1896)

Catapion meieri (Desbrochers des Loges, 1901)

Catapion pubescens (Kirby, 1811)

Catapion seniculus (Kirby, 1808)

Catapion seriatosetosulum (Wencker, 1864)

\begin{tabular}{|l|l|l|l|l|l|l|l|l|}
\hline H & & & & & & & & \\
\hline H & & & & & & & N & \\
\hline H & & & & & & & B & \\
\hline X & & X & & N & & X & & \\
\hline & B & & & & & & & \\
\hline H & X & X & & N & X & X & B & \\
\hline H & & X & N & N & X & X & N & \\
\hline H & & X & & & X & & B & \\
\hline
\end{tabular}

Ceratapiina Alonso-Zarazaga, 1990

Acentrotypus brumnipes (Boheman, 1839)

Ceratapion armatum (Gerstaecker, 1854)

Ceratapion basicorne (Illiger, 1807)

Ceratapion carduorum (Kirby, 1808)

Ceratapion cylindricolle (Gyllenhal, 1839)

Ceratapion damryi (Desbrochers des Loges, 1894)

Ceratapion gibbirostre (Gyllenhal, 1813)

Ceratapion onopordi onopordi (Kirby, 1808)

Ceratapion penetrans penetrans (Germar, 1817)

Ceratapion robusticorne (Desbrochers des Loges, 1866)

Ceratapion scalptum scalptum (Mulsant \& Rey, 1859)

Diplapion confluens (Kirby, 1808)

Diplapion detritum (Mulsant \& Rey, 1859)

Diplapion siolidum (Germar, 1817)

Omphalapion dispar (Germar, 1817)

Omphalapion hookerorum (Kirby, 1808)

Omphalapion laevigatum (Paykull, 1792)

Exapiina Alonso-Zarazaga, 1990

\begin{tabular}{|l|l|l|l|l|l|l|l|l|}
\hline B & & & & & & & & \\
\hline & & & & & & & B & \\
\hline H & X & X & & & & & & \\
\hline & & X & & & B & X & B & \\
\hline & & X & & & X & X? & & \\
\hline H & & X & N & & X & X & B & \\
\hline B & & X & & & X & & & \\
\hline & & & & & & X & & \\
\hline H & X & X & & & & & B & \\
\hline X & & X & & & & & & \\
\hline & X & & & & X & X & N & \\
\hline & & & & & & X & & \\
\hline
\end{tabular}

Exapion difficile (Herbst, 1797)

Exapion elongatulum (Desbrochers des Loges, 1891)

Exapion formaneki (Wagner, 1929)

Exapion fuscirostre fuscirostre (Fabricius, 1775)

Exapion genistae (Kirby, 1811)

Exapion subparallehum (Desbrochers des Loges, 1888)

\begin{tabular}{|l|l|l|l|l|l|l|l|l|}
\hline X & & & & & & X & & \\
\hline & & & & & X & & & \\
\hline H & & & N & N & X & & & \\
\hline & & & & N & X & & & \\
\hline & & & & & & & & \\
\hline
\end{tabular}

Ixapiina Alonso-Zarazaga, 1990

Trichopterapion holosericeum (Gyllenhal, I833)

\begin{tabular}{l|l|l|l|l}
$\mathrm{H}$ & $\mathrm{X}$ & & $\mathrm{N}$
\end{tabular}

Kalcapiina Alonso-Zarazaga, 1990

Kalcapion semivittatum semivittatum (Gyllenhal, 1833)

Melanapion minimum (Herbst, 1797)

Squamapion atomarium (Kirby, 1808)

Squamapion leucophaeatum (Wencker, 1864)

Squamapion mimutissimum (Rosenhauer, 1856)

Squamapion vicimum (Kirby, 1808)

Taeniapion rufescens (Gyllenhal, 1833)

Taeniapion urticarium urticarium (Herbst, 1784)

\begin{tabular}{|c|c|c|c|c|c|c|}
\hline $\mathrm{H}$ & & $\mathrm{N}$ & & $\mathrm{X}$ & $\mathrm{N}$ & B \\
\hline$X$ & $\mathrm{X}$ & $\mathrm{N}$ & $\mathrm{N}$ & $X$ & $\mathrm{X}$ & B \\
\hline & & & & $\mathrm{X}$ & & \\
\hline & $\mathrm{X}$ & & & & & $\mathrm{N}$ \\
\hline & & & & & B & \\
\hline & & & & & $\mathrm{N}$ & \\
\hline $\mathrm{H}$ & & & & & & $\mathrm{N}$ \\
\hline $\mathrm{H}$ & & B & $\mathrm{N}$ & $X$ & & B \\
\hline
\end{tabular}

Malvapiina Alonso-Zarazaga, 1990

Malvapion malvae (Fabricius, 1775)

Pseudapion fulvirosire (Gyllenhal, 1833)

Pseudapion rufirostre (Fabricius, 1775)

\begin{tabular}{|l|l|l|l|l|l|l|l|l}
$\mathrm{H}$ & $\mathrm{X}$ & & $\mathrm{N}$ & $\mathrm{N}$ & $\mathrm{X}$ & & $\mathrm{B}$ & \\
\hline $\mathrm{H}$ & $\mathrm{X}$ & $\mathrm{N}$ & & $\mathrm{N}$ & $\mathrm{N}$ & & & \\
\hline $\mathrm{H}$ & $\mathrm{X}$ & & $\mathrm{N}$ & $\mathrm{N}$ & $\mathrm{X}$ & $\mathrm{X}$ & & \\
\hline
\end{tabular}


Species

Rhopalapion longirostre (A.G. Olivier, 1807)

Oxystomatina Alonso-Zarazaga, 1990

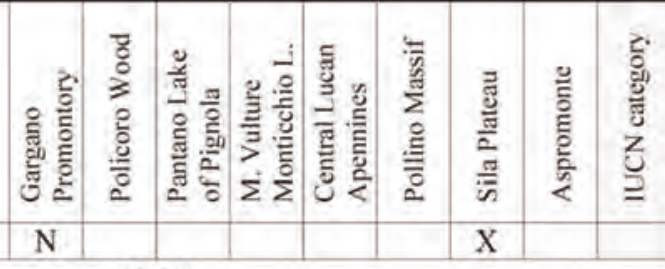

Cyanapion afer (Gyllenhal, 1833)

Cyanapion alcyoneum (Germar, 1817)

Cyanapion gyllenhali (Kirby, 1808)

Cyanapion platalea (Germar, 1817)

Cyanapion spencii (Kirby, 1808)

Eutrichapion ervi (Kirby, 1808)

Eutrichapion facetum (Gyllenhal, 1839)

Eutrichapion melancholicum (Wencker, 1864)

Eutrichapion punctiger (Paykull, 1792)

Eutrichapion viciae (Paykull, 1800)

Eutrichapion vorax (Herbst, 1797)

Hemitrichapion filicorne (Wencker, 1864)

Hemitrichapion juniperi (Boheman, 1839)

Hemitrichapion lethierryi (Desbrochers des Loges, 1870)

Hemitrichapion pavidum (Germar, 1817)

Hemitrichapion reflexum (Gyllenhal, 1833)

Holotrichapion aethiops (Herbst, 1797)

Holotrichapion antennale (Desbrochers des Loges, 1896)

Holotrichapion gracilicolle (Gyllenhal, 1839)

Holotrichapion ononis (Kirby, 1808)

Holotrichapion pisi (Fabricius, 1801)

Ischnopterapion cognatum (Hochhuth, 1851)

Ischnopterapion fallens (Marseul, 1888)

Ischnopterapion loti (Kirby, 1808)

Ischnopterapion modestum (Germar, 1817)

Ischnopterapion plumbeomicans plumbeomicans (Rosenhauer,

1856)

Ischnopterapion virens (Herbst, 1797)

Oryxolaemus croceifemoratus (Gyllenhal, 1839)

Oryxolaemus scabiosus (Weise, 1889)

Oxystoma cerdo (Gerstaecker, 1854)

Oxystoma craccae (Linnaeus, 1767)

Oxystoma dimidiatum (Desbrochers des Loges, 1897)

Oxystoma ochropus (Germar, 1818)

Oxystoma opeticum Bach, 1854

Oxystoma pomonae (Fabricius, 1798)

Oxystoma subulatum (Kirby, 1808)

Pirapion immume (Kirby, 1808)

Stenopterapion meliloti (Kirby, 1808)

Stenopterapion temue (Kirby, 1808)

Synapion falzonii (Schatzmayr, 1922)

\begin{tabular}{|c|c|c|c|c|c|c|c|c|}
\hline X & & X & & & & & & \\
\hline H & & & N & & X & X & B & \\
\hline H & & & & & & & & \\
\hline H & & & & & & X & & \\
\hline H & & & N & N & X & & B & \\
& & & N & N & X & X & N & \\
\hline & & & & & X & X & & \\
\hline H & & & N & N & N & & B & \\
\hline H & X & & N & N & X & X & B & \\
\hline H & X & X & N & N & X & X & B & \\
\hline
\end{tabular}

Protapion angusticolle (Gyllenhal, 1833)

Protapion apricans (Herbst, 1797)

Protapion assimile assimile (Kirby, 1808)

Protapion dentipes (Gerstaecker, 1854)

Protapion difforme (Germar, 1818)

Protapion dissimile (Germar, 1817)

Prolapion fulvipes fulvipes (Geoffroy, 1785)

Protapion interjectum interjectum (Desbrochers des Loges, 1895)

Protapion laevicolle (Kirby, 1811)

Piezotrachelina Voss, 1959

\begin{tabular}{|c|c|c|c|c|c|c|c|}
\hline & & & $\mathrm{N}$ & & X & & \\
\hline & & & & & $\mathrm{X}$ & & \\
\hline & & & & & X & & \\
\hline & & $\mathrm{X}$ & & & & & \\
\hline & & & $\mathrm{N}$ & & X & & $\mathrm{N}$ \\
\hline & & & & & $X$ & $\mathrm{X}$ & B \\
\hline & & & & $\mathrm{N}$ & X & & B \\
\hline $\mathrm{H}$ & & & & $\mathrm{N}$ & $\mathrm{X}$ & $\mathrm{X}$ & B \\
\hline $\mathrm{H}$ & & $\mathrm{X}$ & $\mathrm{N}$ & $\mathrm{N}$ & $\mathrm{X}$ & $\mathrm{N}$ & B \\
\hline $\mathrm{H}$ & $\mathrm{X}$ & $\mathrm{X}$ & $\mathrm{N}$ & $\mathrm{N}$ & $\mathrm{X}$ & $\mathrm{X}$ & B \\
\hline & & B & $\mathrm{N}$ & & $\mathrm{X}$ & & \\
\hline & & $\mathrm{X}$ & & $\mathrm{N}$ & $\mathrm{X}$ & & \\
\hline $\mathrm{H}$ & $\mathrm{X}$ & $\mathrm{X}$ & & & X & $X$ & B \\
\hline & $\mathrm{N}$ & & & & & & \\
\hline$X$ & $X$ & & & & & & \\
\hline $\mathrm{H}$ & $\mathrm{X}$ & $\mathrm{X}$ & B & $\mathrm{N}$ & $\mathrm{X}$ & $\mathrm{X}$ & B \\
\hline B & X & & & & X & X & B \\
\hline B & & & & $\mathrm{N}$ & B & $\mathrm{X}$ & B \\
\hline $\mathrm{H}$ & & & $\mathrm{N}$ & & $\mathrm{X}$ & $\mathrm{X}$ & B \\
\hline $\mathrm{H}$ & & & & & X & $\mathrm{X}$ & \\
\hline X & & X & $\mathrm{N}$ & $\mathrm{N}$ & X & & \\
\hline $\mathrm{H}$ & $\mathrm{X}$ & & & & $\mathrm{X}$ & $\mathrm{X}$ & $\mathrm{N}$ \\
\hline & & & & & $\mathrm{X}$ & & \\
\hline & $X$ & $\mathrm{X}$ & $\mathrm{N}$ & $\mathrm{N}$ & $\mathrm{X}$ & $\mathrm{X}$ & B \\
\hline & & $\mathrm{X}$ & B & $\mathrm{N}$ & & $\mathrm{X}$ & \\
\hline $\mathrm{N}$ & & & & & $\mathrm{X}$ & $\mathrm{X}$ & \\
\hline & $\mathrm{N}$ & & & & & & \\
\hline $\mathrm{H}$ & $\mathrm{X}$ & $\mathrm{X}$ & $\mathrm{N}$ & $\mathrm{N}$ & $\mathrm{X}$ & $\mathrm{N}$ & $\mathrm{N}$ \\
\hline & & & & & X & & \\
\hline
\end{tabular}

\begin{tabular}{|c|c|c|c|c|c|c|c|c|}
\hline & & $X$ & & & & & & \\
\hline $\mathrm{H}$ & X & $\mathrm{X}$ & $\mathrm{N}$ & $\mathrm{N}$ & X & $\mathrm{N}$ & B & \\
\hline $\mathrm{H}$ & & & $\mathrm{N}$ & $\mathrm{N}$ & X & X & B & \\
\hline $\mathrm{H}$ & $\mathrm{X}$ & $\mathrm{X}$ & $\mathrm{N}$ & $\mathrm{N}$ & X & & B & \\
\hline $\mathrm{N}$ & $X$ & & & & & & $\mathrm{~N}$ & \\
\hline $\mathrm{H}$ & & & & & $\mathrm{X}$ & & B & \\
\hline $\mathrm{N}$ & & $\mathrm{X}$ & & $\mathrm{N}$ & $\mathrm{X}$ & $\mathrm{N}$ & $\mathrm{N}$ & \\
\hline $\mathrm{H}$ & X & X & & $\mathrm{N}$ & X & & B & \\
\hline $\mathrm{H}$ & & $\mathrm{X}$ & $\mathrm{N}$ & $\mathrm{N}$ & $\mathrm{X}$ & $\mathrm{X}$ & B & \\
\hline
\end{tabular}


Species

Protapion nigricrus (Desbrochers des Loges, 1895)

Protapion nigritarse (Kirby, 1808)

Protapion ononidis (Gyllenhal, 1827)

Protapion schoenherri (Boheman, 1839)

Protapion trifolii (Linnaeus, 1768)

Protapion varipes (Germar, 1817)

Pseudoprotapion astragali (Paykull, 1800)

Psendoprotapion elegantulum (Germar, 1818)

Brentinae Billberg, 1820

Brentini Billberg, 1820

Eremoxenina Semenov, 1892

\begin{tabular}{|l|l|l|l|l|l|l|l|l|l|}
\hline Amorphocephala coronata $($ Germar, 1817) & X & B & X & & B & N & & B & LC \\
\hline
\end{tabular}

Nanophyinae Gistel, 1848

Corimaliini Alonso-Zarazaga, 1989

Allomalia quadrivirgata (A. Costa, 1863

Corimalia pallida (A.G. Olivier, 1807)

Corimalia postica (Gyllenhal, 1838)

Corimalia tamarisci (Gyllenhal, 1838)

Hypophyes pallidulus (Gravenhorst, 1807)

Nanophyini Gistel, 1848

\begin{tabular}{|c|c|c|c|c|c|c|}
\hline $\mathrm{N}$ & $\mathrm{X}$ & & & & $\mathrm{B}$ \\
\hline & $\mathrm{X}$ & & & & $\mathrm{B}$ \\
\hline & & & & & & $\mathrm{B}$ \\
\hline $\mathrm{X}$ & $\mathrm{X}$ & & & & $\mathrm{B}$ \\
\hline $\mathrm{N}$ & $\mathrm{N}$ & & & & $\mathrm{B}$ & \\
\hline
\end{tabular}

Dieckmanniellus chevrieri (Boheman, 1845)

Dieckmanniellus gracilis (L. Redtenbacher, 1847)

Dieckmanniellus nigritarsis (Aubé, 1862)

Dieckmanniellus nitidulus (Gyllenhal, 1838)

Nanomimus hemisphaericus (A.G. Olivier, 1807)

Nanophyes brevis brevis Boheman, 1845

Nanophyes brevis fallax Rey, 1893

Nanophyes marmoratus marmoratus (Goeze, 1777)

Nanophyes rubricus Rosenhauer, 1856

\begin{tabular}{|c|c|c|c|c|c|}
\hline & & $\mathrm{X}$ & & & \\
\hline & & & & & B \\
\hline & & & & & B \\
\hline $\mathrm{H}$ & X & & $\mathrm{X}$ & $\mathrm{X}$ & B \\
\hline$X$ & & $X$ & & & B \\
\hline$X$ & & X & & & \\
\hline & $\mathrm{X}$ & & & & \\
\hline & $X$ & $X$ & & & \\
\hline & & $\mathrm{N}$ & & & \\
\hline
\end{tabular}

CURCULIONIDAE Latreille, 1802 s.l.

Bagoinae C.G. Thomson, 1859

\begin{tabular}{|c|c|c|c|c|c|c|}
\hline Bagous argillaceus Gyllenhal, 1836 & & B & $\mathrm{X}$ & & & B \\
\hline Bagous biimpressus Fåhraeus, 1845 & & B & & & & \\
\hline Bagous costulatus Perris, 1869 & & & $\mathrm{X}$ & & $\mathrm{N}$ & $\mathrm{N}$ \\
\hline Bagous limosus (Gyllenhal, 1827) & CK & & $\mathrm{X}$ & $\mathrm{N}$ & & \\
\hline Bagous longirostris Vitale, 1904 & & & B & & & B \\
\hline Bagous lutulentus (Gyllenhal, 1813) & & $\mathrm{X}$ & $\mathrm{X}$ & & & CK \\
\hline Bagous robustus $\mathrm{H}$. Brisout de Barneville, 1863 & & CK & & & & \\
\hline Bagous rufimamis Péricart, 1989 & & & $\mathrm{X}$ & & & \\
\hline
\end{tabular}

Bagous rufimamis Péricart, 1989

\section{Brachycerinae Billberg, 1820 \\ Brachycerini Billberg, 1820 \\ Brachycerina Billberg, 1820}

Brachycerus albidentatus Gyllenhal, 1840

Brachycerus junix Liechtenstein, 1896

Brachycerus lutosus Gyllenhal, 1833

Brachycerus muricatus A.G. Olivier, 1790

Brachycerus undatus Fabricius, 1798

\begin{tabular}{|c|c|c|c|c|c|c|c|c|} 
& B & & & & & & B & \\
\hline X & N & & & & & & B & \\
\hline X & & & & & & & & \\
\hline H & & & & & & & B & \\
\hline H & X & & & N & N & & B & \\
\hline
\end{tabular}

Erirhinini Schönherr, 1825

Erirhinina Schönherr, 1825

Notaris acridulus (Linnaeus, 1758)

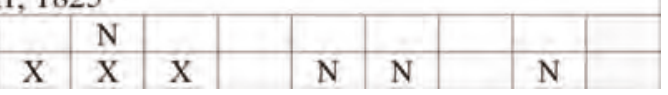

Notaris scirpi (Fabricius, 1792) 


\begin{tabular}{|c|c|c|c|c|c|c|c|c|c|}
\hline Species & 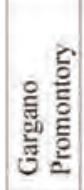 & $\begin{array}{l}\frac{0}{0} \\
3 \\
0 \\
0 \\
0 \\
\frac{0}{0} \\
2\end{array}$ & 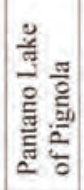 & 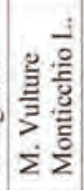 & 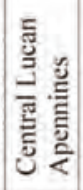 & $\begin{array}{l}\bar{y} \\
\sum_{0}^{0} \\
0 \\
\bar{\equiv} \\
0\end{array}$ & $\frac{\frac{\vec{g}}{\tilde{w}}}{\frac{\vec{w}}{\bar{n}}}$ & 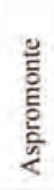 & 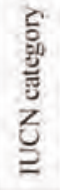 \\
\hline Thryogenes festucae (Herbst, 1795) & & $\mathrm{N}$ & & & & & & & \\
\hline \multicolumn{10}{|c|}{ Ocladiina Lacordaire, 1865} \\
\hline Jekelia notata Mulsant \& Godart, 1868 & $\mathrm{H}$ & & & & & & & & \\
\hline \multicolumn{10}{|c|}{ Raymondionymini Reitter, 1913} \\
\hline Alaocyba marcuzzii G. Osella, 1977 [E] & & & & & B & & & & \\
\hline Ferreria marqueti appenina (Dieck, 1869) [E] & $\mathrm{X}$ & & & B & B & & & & \\
\hline Raymondiellus pacei G. Osella, 1977 & $\mathrm{~N}$ & B & & & $\mathrm{N}$ & $\mathrm{B}$ & & & \\
\hline \multicolumn{10}{|c|}{ Tanysphyrini Gistel, 1848} \\
\hline Tanysphyrus lemnae (Paykull, 1792) & $\mathrm{H}$ & $\mathrm{N}$ & $\mathrm{X}$ & $\mathrm{N}$ & & & & & \\
\hline \multicolumn{10}{|c|}{$\begin{array}{c}\text { Conoderinae Schönherr, } 1833 \\
\text { Bariditae Schönherr, } 1836 \\
\text { Apostasimerini Schönherr, } 1844 \\
\text { Zygobaridina Pierce, } 1907\end{array}$} \\
\hline Limnobaris dolorosa (Goeze, 1777) & & & $\mathrm{X}$ & & & & & & \\
\hline Limnobaris t-album (Linnaeus, 1758) & & & $\mathrm{X}$ & & $\mathrm{N}$ & $\mathrm{X}$ & & & \\
\hline \multicolumn{10}{|c|}{$\begin{array}{l}\text { Baridini Schönherr, } 1836 \\
\text { Baridina Schönherr, } 1836\end{array}$} \\
\hline Aulacobaris angısta angusta (Brullé, 1832) & & $\mathrm{X}$ & & & & & $\mathrm{X}$ & & \\
\hline Aulacobaris caerulescens caerulescens (Scopoli, 1763) & & $\mathrm{X}$ & & & & & & & \\
\hline Aulacobaris chlorizans (Germar, 1823) & $\mathrm{H}$ & $\mathrm{N}$ & & & & & & & \\
\hline Aulacobaris cuprirostris cuprirostris (Fabricius, 1787) & $\mathrm{H}$ & & & & & & & & \\
\hline Aulacobaris fallax (Heyden, 1868) & $\mathrm{X}$ & $\mathrm{X}$ & & & & & & & \\
\hline Aulacobaris kaufimanni (Reitter, 1897) & B & & & & & $\mathrm{X}$ & $\mathrm{X}$ & & \\
\hline Aulacobaris pertusa (Kiesenwetter, 1864) & B & & $\mathrm{B}$ & & & & & & \\
\hline Aulacobaris picicornis picicornis (Marsham, 1802) & B & & $\mathrm{N}$ & & & & & & \\
\hline Aulacobaris prasina prasina (Boheman, 1836 ) & & & B & & B & & & & \\
\hline Baris analis analis (A.G. Olivier, 1791) & $\mathrm{X}$ & $\mathrm{X}$ & $\mathrm{N}$ & & & & & & \\
\hline Baris artemisiae (Panzer, 1794) & $\mathrm{X}$ & & $\mathrm{N}$ & & $\mathrm{N}$ & & & & \\
\hline Labiaticola atricolor (Boheman, 1844) & B & & & & & & & & \\
\hline Malvaevora timida timida (P. Rossi, 1792) & $\mathrm{X}$ & $\mathrm{N}$ & & $\mathrm{N}$ & & $\mathrm{X}$ & $\mathrm{X}$ & & \\
\hline Maurobaris spoliata (Boheman, 1836) & & $\mathrm{X}$ & & & & & & $\mathrm{N}$ & \\
\hline \multicolumn{10}{|c|}{$\begin{array}{c}\text { Madarini Jekel, } 1865 \\
\text { Leptoschoinina Lacordaire, } 1865\end{array}$} \\
\hline Cucubaris villae (Comolli, 1837) & $\mathrm{H}$ & & & & & & & & \\
\hline \multicolumn{10}{|c|}{ Neosharpiini A. Hoffmann, 1956} \\
\hline Amalus scortillum (Herbst, 1795) & & & & & & $\mathrm{X}$ & & & \\
\hline \multicolumn{10}{|c|}{ Ceutorhynchini Gistel, 1848} \\
\hline Calosirus terminatus (Herbst, 1795) & & B & $\mathrm{X}$ & B & & $\mathrm{X}$ & $\mathrm{X}$ & B & \\
\hline Centorhynchus aeneicollis Germar, 1823 & & $\mathrm{X}$ & & & & & & & \\
\hline Ceutorhynchus assimilis (Paykull, 1792) & $\mathrm{H}$ & $\mathrm{X}$ & $\mathrm{N}$ & B & $\mathrm{N}$ & $\mathrm{X}$ & $\mathrm{X}$ & $\mathrm{B}$ & \\
\hline Cetutorhynchus atomus Boheman, 1845 & $\mathrm{H}$ & & $\mathrm{X}$ & & $\mathrm{N}$ & $\mathrm{X}$ & $\mathrm{X}$ & $\mathrm{N}$ & \\
\hline Ceutorhynchus barbareae Suffrian, 1847 & & & & & & $\mathrm{X}$ & $\mathrm{X}$ & & \\
\hline Ceutorhynchus chalibaeus Germar, 1823 & $\mathrm{H}$ & $\mathrm{X}$ & & $\mathrm{N}$ & $\mathrm{N}$ & $\mathrm{X}$ & $\mathrm{X}$ & $\mathrm{B}$ & \\
\hline Ceutorhynchus cochleariae (Gyllenhal, 1813) & & & & & $\mathrm{N}$ & & & & \\
\hline Ceutorhynchus contractus (Marsham, 1802) & $\mathrm{H}$ & B & $\mathrm{X}$ & $\mathrm{N}$ & $\mathrm{N}$ & $\mathrm{X}$ & $\mathrm{X}$ & $\mathrm{B}$ & \\
\hline Ceutorhynchus declivis Colonnelli, 1988 [E] & & & & & $\mathrm{N}$ & $\mathrm{B}$ & & & \\
\hline Ceutorhynchus divali C.N.F. Brisout de Barneville, 1869 & $\mathrm{H}$ & $\mathrm{X}$ & $\mathrm{X}$ & $\mathrm{N}$ & $\mathrm{N}$ & $\mathrm{X}$ & $\mathrm{X}$ & $\mathrm{B}$ & \\
\hline Ceutorhynchus erysimi (Fabricius, 1787) & $\mathrm{H}$ & $\mathrm{X}$ & $\mathrm{X}$ & $\mathrm{N}$ & $\mathrm{N}$ & $\mathrm{X}$ & $\mathrm{X}$ & $\mathrm{B}$ & \\
\hline Ceutorhynchus fallax Boheman, 1845 & & $\mathrm{X}$ & $\mathrm{X}$ & & $\mathrm{N}$ & $\mathrm{X}$ & & & \\
\hline $\begin{array}{l}\text { Ceutorhynchus fulvitarsis Gougelet \& H. Brisout de Barneville, } \\
1860\end{array}$ & $\mathrm{H}$ & & & & $\mathrm{N}$ & & & & \\
\hline Ceutorhynchus gallorhenamus F. Solari, 1949 & & B & & & & & & & \\
\hline
\end{tabular}


Species

Ceutorhynchus grenieri Gougelet \& H. Brisout de Barneville, 1860

Ceutorhynchus griseus C.N.F. Brisout de Barneville, 1869

Ceutorhynchus hepaticus Gyllenhal, 1837

Ceutorhynchus hirtulus Germar, 1823

Ceutorhynchus inaffectatus Gyllenhal, 1837

Ceutorhynchus laetus Rosenhauer, 1856

Ceutorhynchus leonhardi F. Solari, 1932 [E]

Ceutorhynchus leprieuri C.N.F. Brisout de Barneville, 1881

Ceutorhynchus littoralis Schultze, 1898

Ceutorhynchus magnanoi Colonnelli, 2011 [E]

Ceutorhynchus matthiolae A. Hoffmann, 1955

Ceutorhynchus melitensis Schultze, 1900

Ceutorhynchus micans C.N.F. Brisout de Barneville, 1869

Ceutorhynchus namus Gyllenhal, 1837

Ceutorhynchus obstrictus (Marsham, 1802)

Ceutorhynchus pallidactylus (Marsham, 1802)

Ceutorhynchus pantellarianus A. Hoffmann, 1964

Ceutorhynchus picitarsis Gyllenhal, 1837

Ceutorhynchus posthumus Germar, 1823

Ceutorhynchus pumcticollis Boheman, 1845

Ceutorhynchus roberti Gyllenhal, 1837

Ceutorhynchus rusticus Gyllenhal, 1837

Ceutorhynchus scrobicollis Neresheimer \& Wagner, 1924

Ceutorhynchus striatellus Schultze, 1900

Ceutorhynchus sulcicollis (Paykull, 1800)

Ceutorhynchus typhae (Herbst, 1795)

Ceutorhynchus unguicularis C.G. Thomson, 1871

Ceutorhynchus viridipennis C.N.F. Brisout de Barneville, 1869

Coeliastes lamii (Fabricius, 1792)

Coeliodes angulipennis F. Solari, 1940 [E]

Coeliodes ilicis (Bedel, 1885)

Coeliodes rana (Fabricius, 1787)

Coeliodes ruber (Marsham, 1802)

Coeliodes transversealbofasciatus (Goeze, 1777)

Datonychus arquata (Herbst, 1795)

Datonychus melanostictus (Marsham, 1802)

Datonychus urticae (Boheman, 1845)

Drupenatus nasturtii (Germar, 1823)

Glocianus distinctus (C.N.F. Brisout de Barneville, 1870)

Glociamus maculaalba (Herbst, 1795)

Glocianus punctiger (C.R. Sahlberg, 1835)

Glociamus ragusae (C.N.F. Brisout de Barneville, 1884)

Hadroplontus trimaculatus (Fabricius, 1775)

Microplontus campestris (Gyllenhal, 1837)

Microplontus fairmairii (C.N.F. Brisout de Barneville, 1881)

Microplontus molitor (Gyllenhal, 1837)

Microplontus rugulosus (Herbst, 1795)

Mogulones amplipennis (Schultze, 1897)

Mogulones annibal (Desbrochers des Loges, 1896)

Mogulones asperifoliarum (Gyllenhal, 1813)

Mogulones aubei (Boheman, 1845)

Mogulones beckeri (Schultze, 1900)

Mogulones crucifer (Pallas, 1771)

\begin{tabular}{|c|c|c|c|c|c|c|c|c|}
\hline 窟 & $\begin{array}{l}\overline{0} \\
\vdots \\
0 \\
0 \\
\frac{0}{0} \\
0\end{array}$ & 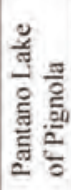 & 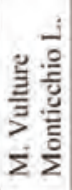 & 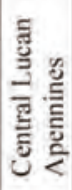 & 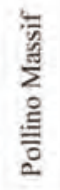 & 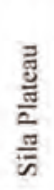 & 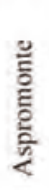 & 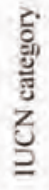 \\
\hline & $\mathrm{X}$ & & & & & & & \\
\hline \multicolumn{9}{|l|}{$X$} \\
\hline & & $\mathrm{N}$ & & & & & & \\
\hline \multirow[t]{2}{*}{$\mathrm{H}$} & B & & & & $\mathrm{X}$ & & B & \\
\hline & $\mathrm{X}$ & & & & $\mathrm{X}$ & & & \\
\hline $\mathrm{H}$ & & & & & $X$ & & & \\
\hline \multicolumn{9}{|l|}{$\mathrm{H}$} \\
\hline $\mathrm{X}$ & $\mathrm{X}$ & $\mathrm{X}$ & & & & & $\mathrm{N}$ & \\
\hline \multicolumn{9}{|l|}{$\mathrm{X}$} \\
\hline & B & & & & & & & \\
\hline & B & & & & & & & \\
\hline \multirow[t]{2}{*}{$\mathrm{X}$} & B & & & & & & & \\
\hline & B & & & & & & & \\
\hline \multicolumn{9}{|l|}{$\mathrm{H}$} \\
\hline & & $\mathrm{X}$ & & & & & & \\
\hline \multirow[t]{2}{*}{$\mathrm{H}$} & $\mathrm{X}$ & $\mathrm{X}$ & & $\mathrm{N}$ & $\mathrm{N}$ & & B & \\
\hline & B & & & & & & & \\
\hline $\mathrm{H}$ & $\mathrm{X}$ & $X$ & $\mathrm{~N}$ & $\mathrm{~N}$ & $\mathrm{X}$ & $X$ & B & \\
\hline \multicolumn{9}{|l|}{$\mathrm{H}$} \\
\hline \multicolumn{9}{|l|}{ B } \\
\hline & & & & $\mathrm{N}$ & & & & \\
\hline & & & $\mathrm{N}$ & & & & & \\
\hline & & & & B & & & & \\
\hline & & $\mathrm{X}$ & & & $\mathrm{X}$ & & & \\
\hline $\mathrm{H}$ & & $\mathrm{X}$ & $\mathrm{N}$ & $\mathrm{N}$ & $X$ & $\mathrm{X}$ & $\mathrm{N}$ & \\
\hline $\mathrm{H}$ & & & & & $\mathrm{X}$ & $X$ & & \\
\hline $\mathrm{H}$ & & $\mathrm{X}$ & & & $\mathrm{X}$ & & & \\
\hline \multirow[t]{2}{*}{$\mathrm{H}$} & $\mathrm{X}$ & & $\mathrm{N}$ & & & $\mathrm{X}$ & B & \\
\hline & & & & $\mathrm{N}$ & & & B & \\
\hline \multirow[t]{2}{*}{$\mathrm{X}$} & & & & & & $X$ & & \\
\hline & $\mathrm{X}$ & $\mathrm{X}$ & & & & & & \\
\hline $\mathrm{H}$ & & & & & & $\mathrm{N}$ & & \\
\hline $\mathrm{H}$ & & $\mathrm{X}$ & & $\mathrm{N}$ & & & $\mathrm{N}$ & \\
\hline \multirow[t]{3}{*}{$\mathrm{N}$} & & & & & & & $\mathrm{N}$ & \\
\hline & & & & & & & B & \\
\hline & $\mathrm{X}$ & $\mathrm{X}$ & & $\mathrm{N}$ & $\mathrm{N}$ & $\mathrm{X}$ & $\mathrm{N}$ & \\
\hline $\mathrm{H}$ & & & & $\mathrm{N}$ & $\mathrm{X}$ & & B & \\
\hline $\mathrm{X}$ & B & $\mathrm{X}$ & & & & & & \\
\hline \multirow[t]{3}{*}{$\mathrm{H}$} & $\mathrm{N}$ & $\mathrm{X}$ & $\mathrm{N}$ & & $\mathrm{X}$ & & B & \\
\hline & $\mathrm{X}$ & & & & $\mathrm{X}$ & & & \\
\hline & $\mathrm{X}$ & & & & $\mathrm{X}$ & $\mathrm{X}$ & & \\
\hline $\mathrm{H}$ & $X$ & & & & & & B & \\
\hline $\mathrm{H}$ & $\mathrm{X}$ & & $\mathrm{N}$ & $\mathrm{N}$ & $\mathrm{X}$ & $\mathrm{X}$ & B & \\
\hline \multirow[t]{2}{*}{$\mathrm{H}$} & & & & & & & & \\
\hline & & & $\mathrm{N}$ & & $\mathrm{X}$ & & & \\
\hline \multicolumn{9}{|l|}{$\mathrm{H}$} \\
\hline \multirow[t]{3}{*}{$\mathrm{H}$} & $\mathrm{X}$ & & & $\mathrm{N}$ & $\mathrm{N}$ & & B & \\
\hline & & $\mathrm{X}$ & & $\mathrm{N}$ & & & & \\
\hline & $\mathrm{X}$ & & & & & & & \\
\hline \multirow[t]{2}{*}{$\mathrm{H}$} & & $\mathrm{N}$ & & $\mathrm{N}$ & $\mathrm{N}$ & $\mathrm{X}$ & B & \\
\hline & & & & & & & B & \\
\hline \multirow[t]{2}{*}{$\mathrm{H}$} & & $\mathrm{N}$ & & & & & & \\
\hline & & & & $\mathrm{N}$ & $\mathrm{X}$ & $\mathrm{X}$ & B & \\
\hline
\end{tabular}


Species

Mogulones geographicus (Goeze, 1777)

Mogulones javetii (Gerhardt, 1867)

Mogulones lucanus (Colonnelli, 1983) [E]

Mogulones pallidicornis (Gougelet \& H. Brisout de Barneville,

1860)

Mogulones t-album (Gyllenhal, 1837)

Nedyus quadrimaculatus (Linnaeus, 1758)

Neoprohimus cinnamomeus (Schultze, 1897)

Oprohinus consputus (Germar, 1823)

Parethelcus pollinarius (Forster, 1771)

Phoeniconyx gobbii (Colonnelli, 1978) [E]

Phrydiuchus topiarius (Germar, 1823)

Pseudocoeliodes rubricus (Gyllenhal, 1837)

Sirocalodes depressicollis (Gyllenhal, 1813)

Sirocalodes mixtus (Mulsant \& Rey, 1859)

Stenocarus cardui (Herbst, 1784)

Stenocarns ruficornis (Stephens, 1831)

Thamiocolus pubicollis (Gyllenhal, 1837)

Thamiocolus sinapis (Desbrochers des Loges, 1893)

Thamiocolus uniformis (Gyllenhal, 1837)

Trichosirocalus horridus (Panzer, 1801)

Trichosirocalus rufulus (Dufour, 1851)

Trichosirocalus troglodytes (Fabricius, 1787)

Trichosirocalus urens (Gyllenhal, 1837)

Zacladus exiguus (A.G. Olivier, 1807)

Zacladus geranii (Paykull, 1800)

Mononychini LeConte, 1876

\begin{tabular}{|c|c|c|c|c|c|c|c|c|}
\hline 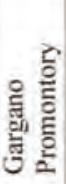 & $\begin{array}{l}7 \\
0 \\
3 \\
\frac{0}{0} \\
\frac{0}{0} \\
2\end{array}$ & 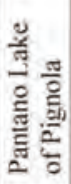 & 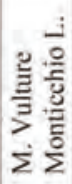 & 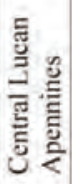 & 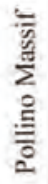 & 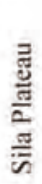 & 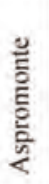 & 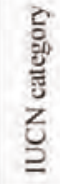 \\
\hline $\mathrm{H}$ & $X$ & & $\mathrm{~N}$ & & $\mathrm{X}$ & $\mathrm{X}$ & & \\
\hline \multirow[t]{3}{*}{$\mathrm{H}$} & $\mathrm{X}$ & & & & & & & \\
\hline & $\mathrm{X}$ & & & $\mathrm{N}$ & $\mathrm{X}$ & & & \\
\hline & & & & & $X$ & & & \\
\hline $\mathrm{X}$ & & & & & $\mathrm{X}$ & & & \\
\hline $\mathrm{H}$ & $\mathrm{X}$ & & $\mathrm{N}$ & $\mathrm{N}$ & $X$ & $\mathrm{X}$ & B & \\
\hline $\mathrm{H}$ & $X$ & & & & & $X$ & B & \\
\hline $\mathrm{H}$ & $X$ & $X$ & $\mathrm{~N}$ & & $X$ & & $\mathrm{~N}$ & \\
\hline \multirow[t]{2}{*}{$\mathrm{H}$} & & & & & & & & \\
\hline & $\mathrm{X}$ & & & & & & & \\
\hline \multicolumn{8}{|l|}{$\mathrm{H}$} & \\
\hline \multirow[t]{2}{*}{$\mathrm{H}$} & & & & & $\mathrm{X}$ & & & \\
\hline & B & & & & & & & \\
\hline \multirow[t]{3}{*}{$\mathrm{H}$} & & & $\mathrm{N}$ & & & & & \\
\hline & & $X$ & & C & & & & \\
\hline & $\mathrm{X}$ & & $\mathrm{N}$ & & $\mathrm{X}$ & & & \\
\hline$X$ & & & & & & $X$ & & \\
\hline \multicolumn{8}{|l|}{$\mathrm{H}$} & \\
\hline \multicolumn{8}{|l|}{$\mathrm{H}$} & \\
\hline $\mathrm{H}$ & & & $\mathrm{N}$ & & $X$ & $X$ & & \\
\hline \multirow[t]{3}{*}{$\mathrm{X}$} & $X$ & $\mathrm{X}$ & & & $X$ & $X$ & B & \\
\hline & $\mathrm{X}$ & $\mathrm{X}$ & $\mathrm{N}$ & $\mathrm{N}$ & $\mathrm{X}$ & $\mathrm{X}$ & B & \\
\hline & & & & & $X$ & & B & \\
\hline $\mathrm{H}$ & $\mathrm{X}$ & $\mathrm{X}$ & & $\mathrm{N}$ & $\mathrm{X}$ & & & \\
\hline $\mathrm{H}$ & $\mathrm{X}$ & & $\mathrm{N}$ & & $\mathrm{X}$ & & $\mathrm{N}$ & \\
\hline
\end{tabular}

Mononychus punctumalbum (Herbst, 1784)

Phytobiini Gistel, 1848

Neophytobius granatus (Gyllenhal, 1835)

Pelenomus quadrituberculatus (Fabricius, 1787)

Phytobius leucogaster (Marsham, 1802)

Rhinoncus bruchoides (Herbst, 1784)

Rhinoncus pericarpius (Linnaeus, 1758)

Rhinoncus perpendicularis (Reich, 1797)

Scleropterini Schultze, 1902

\begin{tabular}{|l|l|l|l|l|l|l|l|l|}
\hline & & & & N & & & & \\
\hline & N & X & & & X & & & \\
\hline & & & N & & & & \\
\hline H & X & N & N & N & X & X & & \\
\hline
\end{tabular}

Scleropteridius fallax Otto, 1897

Conoderitae Schönherr, 1833

Orobitidini C.G. Thomson, 1859

Orobitis nigrina Reitter, 1885

Choerorhinini Folwaczny, 1973

Choerorhimus squalidus Fairmaire, 1858

Cossonini Schönherr, 1825

Mesites cumipes Boheman, 1838

Mesites pallidipennis Boheman, 1838

Dryotribini LeConte, 1876

\begin{tabular}{|l|l|l|l|l|l|l|l|l|} 
& $\mathrm{X}$ & & $\mathrm{N}$ & $\mathrm{N}$ & $\mathrm{X}$ & & & LC \\
\hline $\mathrm{CK}$ & $\mathrm{X}$ & & & $\mathrm{N}$ & & & $\mathrm{CK}$ & LC \\
\hline
\end{tabular}

Amaurorhimus mediterraneus Folwaczny, 1973

Cotaster kostali Diotti, Pesarini \& Caldara, 2015 [E]

Cotaster uncipes (Boheman, I838)

Onycholipini Wollaston, 1873

Brachylemmus porcatus (Germar, 1823)

Hexarthrum exiguum (Boheman, 1838)

\begin{tabular}{|c|c|}
\hline & \\
\hline & \\
\hline & \\
\hline CK & B \\
\hline B & B \\
\hline
\end{tabular}

\begin{tabular}{|c|c|c|c|c|c|}
\hline & $\mathrm{CK}$ & & & & $\mathrm{DD}$ \\
\hline & & $\mathrm{B}$ & $\mathrm{B}$ & $\mathrm{B}$ & $\mathrm{LC}$ \\
\hline $\mathrm{N}$ & $\mathrm{B}$ & $\mathrm{X}$ & $\mathrm{X}$ & $\mathrm{B}$ & $\mathrm{LC}$ \\
\hline
\end{tabular}

\begin{tabular}{|c|c|c|c|c|c|}
\hline $\mathrm{N}$ & $\mathrm{N}$ & $\mathrm{X}$ & $\mathrm{X}$ & $\mathrm{CK}$ & $\mathrm{LC}$ \\
\hline & & & & $\mathrm{N}$ & $\mathrm{DD}$
\end{tabular}


Species

Stenoscelis submuricata (Schönherr, 1832)

Stereocorynes truncorum (Germar, 1823)

Rhyncolini Gistel, 1848

Phloeophagina Voss, 1955

Melicius cylindrus (Boheman, 1838)

Phloeophagus lignarius (Marsham, 1802)

\begin{tabular}{|c|c|c|c|c|c|c|c|c|}
\hline $\mathrm{CK}$ & $\mathrm{X}$ & & & $\mathrm{N}$ & $\mathrm{N}$ & ! & & LC \\
\hline $\mathrm{X}$ & $\mathrm{B}$ & & & $\mathrm{N}$ & $\mathrm{X}$ & $\mathrm{X}$ & & LC \\
\hline
\end{tabular}

Rhyncolina Gistel, 1848

Aphanommata filum (Mulsant \& Rey, 1859)

Rhyncolus ater ater (Linnaeus, 1758)

Rhyncolus elongatus (Gyllenhal, 1827)

Rhyncolus punctatulus Boheman, 1838

Rhyncolus reflexus Boheman, 1838

Rhyncolus sculpturatus Walt1, 1839

Curculioninae Latreille, 1802

Acalyptini C.G. Thomson, 1859

Acalyptus carpini (Fabricius, 1792)

Anoplini Bedel, 1884

Anoplus roboris Suffrian, 1840

Anoplus setulosus setulosus Kirsch, 1870

Anthonomini C.G. Thomson, 1859

Anthonomus bituberculatus C.G. Thomson, 1868

Anthonomus chevrolati chevrolati Desbrochers des Loges, 1868

Anthonomus kirschi Desbrochers des Loges, 1868

Anthonomus multifasciatus Pic, 1926

Anthonomus pedicularius (Linnaeus, 1758)

Anthonomus pomorum (Linnaeus, 1758)

Anthonomus pyri Gyllenhal, 1835

Anthonomus rubi (Herbst, 1795)

Anthonomus rufus Gyllenhal, 1835

Anthonomus stierlini Desbrochers des Loges, 1869

Anthonomus ulmi DeGeer, 1775

Bradybatus creutzeri Germar, 1823

Bradybatus elongatulus (Boheman, 1843)

Bradybatus kellneri Bach, 1845

Cionellus gibbifrons (Kiesenwetter, 1852)

Ciomus alauda (Herbst, 1784)

Cionus balianii F. Solari, 1932

Cionus colonnellii Koštál \& Caldara, 2019

Cionus hortulamus (Geoffroy, 1785)

Cionus montanus Wingelmüller, 1914

Cionus olens (Fabricius, 1792)

Cionus olivieri Rosenschöld, 1838

Cionus schoenherri C.N.F. Brisout de Barneville, 1863

Cionus scrophulariae (Linnaeus, 1758)

Cionus thapsus (Fabricius, 1792)

Cionus tuberculosus (Scopoli, 1793)

Cleopus solani (Fabricius, 1792)

Stereonychus fraxini (DeGeer, 1775)

Cionini Schönherr, 1825

\begin{tabular}{|c|c|c|c|c|c|c|c|c|}
\hline X & X & & & & & & & \\
\hline H & X & & & & X & & & \\
\hline & & & & & X & & N & \\
\hline X & X & & & & & & & \\
\hline H & X & X & & N & X & X & N & \\
\hline & X & & & & N & X & N & \\
\hline H & X & X & B & N & X & X & B & \\
\hline H & N & & & & & & & \\
\hline B & & & & & & & & \\
\hline H & X & & & & & & & \\
\hline H & X & & N & N & X & & & \\
\hline H & & & & N & & & & \\
\hline H & & N & & N & & & & \\
\hline H & & & & & & & & \\
\hline
\end{tabular}

\begin{tabular}{|c|c|c|c|c|c|c|c|}
\hline B & & & & & & NT \\
\hline & & & & CK & & & LC \\
\hline B & & N & N & X & X & B & LC \\
\hline B & & & & & & & LC \\
\hline CK & & & & & & & LC \\
\hline
\end{tabular}

$\mathrm{N}$

$\mathrm{N}$

\begin{tabular}{|c|c|c|c|c|c|c|c|}
\hline & & & & & & & B \\
\hline & & & & & $\mathrm{X}$ & & \\
\hline \multicolumn{8}{|l|}{ B } \\
\hline $\mathrm{H}$ & & $\mathrm{N}$ & $\mathrm{N}$ & $\mathrm{N}$ & $\mathrm{X}$ & $\mathrm{X}$ & B \\
\hline $\mathrm{X}$ & $\mathrm{N}$ & & & & $X$ & X & B \\
\hline$X$ & & $X$ & & & $X$ & & B \\
\hline \multirow[t]{3}{*}{$\mathrm{H}$} & $X$ & $\mathrm{X}$ & & & & & B \\
\hline & & $\mathrm{X}$ & & & & & \\
\hline & & $\mathrm{X}$ & & & & & B \\
\hline \multirow[t]{3}{*}{$\mathrm{H}$} & $\mathrm{X}$ & & $\mathrm{N}$ & & $\mathrm{X}$ & $\mathrm{X}$ & B \\
\hline & $\mathrm{X}$ & & $\mathrm{N}$ & & $\mathrm{X}$ & $\mathrm{X}$ & B \\
\hline & & & $\mathrm{N}$ & & $\mathrm{X}$ & & $\mathrm{N}$ \\
\hline $\mathrm{H}$ & B & & & & & & \\
\hline
\end{tabular}

Curculionini Latreille, 1802

Archariina Pelsue \& O'Brien, 2011

Archarius crux (Fabricius, 1777) 
Species

Archarius pyrrhoceras (Marsham, 1802) Archarius salicivorus (Paykull, 1792)

\begin{tabular}{|c|c|c|c|c|c|c|c|c|}
\hline 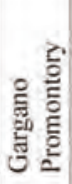 & $\begin{array}{l}7 \\
0 \\
3 \\
0 \\
0 \\
\frac{0}{0} \\
2\end{array}$ & 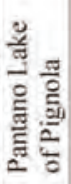 & 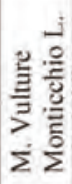 & 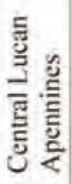 & $\begin{array}{l}\bar{y} \\
\sum_{0} \\
0 \\
\vdots \\
0\end{array}$ & 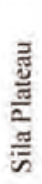 & 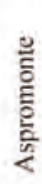 & 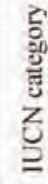 \\
\hline $\mathrm{H}$ & $\mathrm{X}$ & & $\mathrm{N}$ & $\mathrm{N}$ & $\mathrm{X}$ & $\mathrm{X}$ & & \\
\hline & $X$ & X & & $\mathrm{N}$ & X & $\mathrm{X}$ & $\mathrm{N}$ & \\
\hline
\end{tabular}

Curculionina Latreille, 1802

Curculio elephas (Gyllenhal, 1835)

Curculio glandium Marsham, 1802

Curculio nucum Linnaeus, 1758

Curculio pellitus (Boheman, 1843)

Curculio propinquus (Desbrochers des Loges, 1868)

Curculio villosus Fabricius, 1781

\begin{tabular}{|c|c|c|c|c|c|c|c|c|}
\hline X & & & & & & & & \\
\hline H & X & X & N & N & X & X & B & \\
\hline X & & & N & & X & & & \\
\hline H & & & N & & X & X & & \\
\hline X & & X & & & & & & \\
\hline H & & & N & & X & & & \\
\hline
\end{tabular}

Derelomini Lacordaire, 1865

Derelomina Lacordaire, 1865

Derelomus chamaeropis (Fabricius, 1798)

Ellescini C.G. Thomson, 1859

Dorytomina Bedel, 1886

Dorytomus dejeani Faust, 1883

Dorytomus, filirostris (Gyllenhal, 1835)

Dorytomus hirtipennis Bedel, 1884

Dorytomus ictor (Herbst, 1795)

Dorytomus longimamus (Forster, 1771)

Dorytomus melanophthalmus (Paykull, 1792)

Dorytomus mimutus (Gyllenhal, 1835)

Dorytomus nebulosus (Gyllenhal, 1835)

Dorytomus rubrirostris (Gravenhorst, 1807)

Dorytomus schoenherri schoenherri Faust, 1883

Dorytomus suratus (Gyllenhal, 1835)

Dorytomus taeniatus (Fabricius, 1781)

Dorytomus tremulae (Fabricius, 1787)

Dorytomus villosulus (Gyllenhal, 1835)

Ellescina C.G. Thomson, 1859

\begin{tabular}{|l|l|l|l|l|l|l|l|l|}
\hline $\mathrm{X}$ & $\mathrm{X}$ & & & & $\mathrm{N}$ & $\mathrm{N}$ & & \\
\hline $\mathrm{X}$ & $\mathrm{X}$ & $\mathrm{X}$ & & & & & & \\
\hline $\mathrm{X}$ & $\mathrm{X}$ & $\mathrm{X}$ & & $\mathrm{N}$ & $\mathrm{N}$ & & $\mathrm{N}$ & \\
\hline $\mathrm{N}$ & $\mathrm{X}$ & & & $\mathrm{N}$ & & & & \\
\hline & & $\mathrm{N}$ & $\mathrm{N}$ & & $\mathrm{X}$ & & & \\
\hline & $\mathrm{X}$ & & & & & & & \\
\hline $\mathrm{N}$ & $\mathrm{X}$ & & & & & & & \\
\hline & $\mathrm{N}$ & & & & & & & \\
\hline & $\mathrm{N}$ & & $\mathrm{N}$ & & & & & \\
\hline $\mathrm{N}$ & $\mathrm{X}$ & & $\mathrm{N}$ & $\mathrm{N}$ & $\mathrm{X}$ & $\mathrm{X}$ & $\mathrm{N}$ & \\
\hline & $\mathrm{X}$ & & & & & & & \\
\hline & $\mathrm{X}$ & & & & & & & \\
\hline
\end{tabular}

Ellescus scanicus (Paykull, 1792)

Mecinini Gistel, 1848

Cleopomiarus distinctus (Boheman, 1845)

Cleopomiarus graminis (Gyllenhal, 1813)

Cleopomiarus plantarum (Germar, 1823)

Gymnetron aper Desbrochers des Loges, 1892

Gymnetron niloticum Kirsch, 1881

Gymnetron rostellum (Herbst, 1795)

Gymnetron stimulosum (Germar, 1821)

Gymnetron tibiellum Desbrochers des Loges, 1900

Gymnetron veronicae (Germar, 1821)

Gymnetron villosulum Gyllenhal, 1838

Mecinus circulatus (Marsham, 1802)

Mecinus comosus Boheman, 1845

Mecinus haemorrhoidalis $(\mathrm{H}$. Brisout de Barneville, 1862)

Mecimus heydenii Wencker, 1866

Mecinus ictericus (Gyllenhal, 1838)

Mecinus janthimus (Germar, 1821)

Mecinus kaemmereri Wagner, 1927 [E]

Mecinus labilis (Herbst, 1795)

Mecinus latiusculus (Jacquelin du Val, 1855)

Mecinus pascuorum (Gyllenhal, 1813)

Mecinus pyraster (Herbst, 1795)

\begin{tabular}{|c|c|c|c|c|c|c|c|c|}
\hline & & & B & & $\mathrm{X}$ & & & \\
\hline & & & $\mathrm{N}$ & $\mathrm{N}$ & $\mathrm{X}$ & & & \\
\hline $\mathrm{H}$ & $X$ & $\mathrm{X}$ & & $\mathrm{N}$ & $X$ & $\mathrm{X}$ & & \\
\hline \multirow[t]{2}{*}{ B } & & & B & & & & & \\
\hline & & & & & & & B & \\
\hline \multirow[t]{7}{*}{$\mathrm{H}$} & & $\mathrm{X}$ & $\mathrm{N}$ & B & $\mathrm{X}$ & $\mathrm{X}$ & & \\
\hline & & & & B & & & & \\
\hline & & B & & & & & & \\
\hline & & $\mathrm{X}$ & B & B & $\mathrm{X}$ & $\mathrm{X}$ & B & \\
\hline & & $\mathrm{X}$ & & & & & B & \\
\hline & $\mathrm{X}$ & & & & & & & \\
\hline & & & & B & & & B & \\
\hline \multirow[t]{2}{*}{ B } & & & & B & B & & B & \\
\hline & & & & & & & B & \\
\hline $\mathrm{X}$ & $\mathrm{X}$ & & & $\mathrm{N}$ & & B & & \\
\hline \multirow[t]{2}{*}{$\mathrm{H}$} & & $\mathrm{X}$ & & $\mathrm{N}$ & $\mathrm{X}$ & $\mathrm{X}$ & & \\
\hline & & & & & & & B & \\
\hline \multirow[t]{2}{*}{$\mathrm{X}$} & & $\mathrm{X}$ & & $\mathrm{N}$ & & & & \\
\hline & & $\mathrm{N}$ & & & & & & \\
\hline $\mathrm{H}$ & B & $\mathrm{X}$ & & $\mathrm{N}$ & $\mathrm{X}$ & $\mathrm{X}$ & $\mathrm{N}$ & \\
\hline $\mathrm{H}$ & $\mathrm{X}$ & $\mathrm{X}$ & $\mathrm{N}$ & $\mathrm{N}$ & $\mathrm{X}$ & $\mathrm{X}$ & $\mathrm{N}$ & \\
\hline
\end{tabular}


Species

Mecinus raphaelis Baviera \& Caldara, 2014 [E]

Mecimus simus (Mulsant \& Rey, 1859)

Mecinus variabilis (Rosenhauer, 1856)

Miarus campanulae (Linnaeus, 1767)

Rhinusa anthirrhini (Paykull, 1800)

Rhimusa asellus (Gravenhorst, 1807)

Rhimusa bipustulata (P. Rossi, 1792)

Rhinusa comosa (Rosenschöld, 1838)

Rhinusa herbarum (H. Brisout de Barneville, 1862)

Rhinusa incana (Kirsch, 1881)

Rhimusa neta (Germar, 1821)

Rhimusa tetra (Fabricius, 1792)

Rhamphini Rafinesque, 1815

\begin{tabular}{|c|c|c|c|c|c|c|c|c|}
\hline 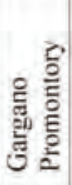 & $\begin{array}{l}70 \\
\vdots \\
3 \\
0 \\
0 \\
\frac{0}{0} \\
2 \\
2\end{array}$ & 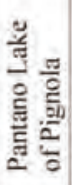 & 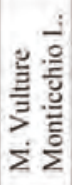 & 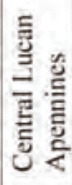 & 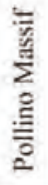 & 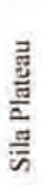 & & 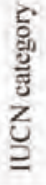 \\
\hline & & & & & & & B & \\
\hline & & & & & B & & & \\
\hline $\mathrm{H}$ & $X$ & & & & $\mathrm{~N}$ & $X$ & B & \\
\hline & & & & $\mathrm{N}$ & & $\mathrm{X}$ & . & \\
\hline $\mathrm{H}$ & $X$ & & & $\mathrm{~N}$ & $X$ & & B & \\
\hline$X$ & & & & & $\mathrm{X}$ & & B & \\
\hline $\mathrm{H}$ & $\mathrm{X}$ & $\mathrm{N}$ & $\mathrm{N}$ & $\mathrm{N}$ & $\mathrm{X}$ & $X$ & B & \\
\hline B & & & & & B & B & B & \\
\hline $\mathrm{H}$ & & & & & $\mathrm{X}$ & & B & \\
\hline & & & & & & & B & \\
\hline H & & X & & & & & B & \\
\hline$X$ & $X$ & $X$ & & $\mathrm{~N}$ & $X$ & $\mathrm{X}$ & B & \\
\hline
\end{tabular}

Rhamphina Rafinesque, 1815

Orchestes alni (Linnaeus, 1758)

Orchestes betuleti (Panzer, 1795)

Orchestes fagi fagi (Linnaeus, 1758)

Orchestes jota (Fabricius, 1787)

Orchestes pilosus (Fabricius, 1781)

Orchestes quedenfeldti Gerhardt, 1865

Orchestes quercus (Linnaeus, 1758)

Orchestes subfasciatus (Gyllenhal, 1835)

Pseudorchestes flavidus (H. Brisout de Barneville, 1865)

Pseudorchestes pratensis (Germar, 1821)

Pseudorchestes smrecynskii (Dieckmann, 1958)

Rhamphus oxyacanthae (Marsham, 1802)

Rhamphus pulicarius (Herbst, 1795)

Rhamphus subaeneus Illiger, 1807

Tachyerges decoratus (Germar, 1821)

Tachyerges rufitarsis (Germar, 1821)

Tachyerges salicis (Linnaeus, 1758)

Tachyerges stigma (Germar, 1821)

\begin{tabular}{|c|c|c|c|c|c|c|c|c|}
\hline & $X$ & & & & & & & \\
\hline & $X$ & & & & & & & \\
\hline $\mathrm{H}$ & $X$ & $X$ & $\mathrm{~N}$ & $\mathrm{~N}$ & $\mathrm{X}$ & $X$ & B & \\
\hline & & $\mathrm{X}$ & & & & & & \\
\hline & $X$ & & $\mathrm{~N}$ & $\mathrm{~N}$ & $\mathrm{X}$ & $X$ & $\mathrm{~N}$ & \\
\hline H & B & & & & & & & \\
\hline & & $X$ & & & $\mathrm{~N}$ & & & \\
\hline & & & & $\mathrm{N}$ & & & & \\
\hline $\mathrm{H}$ & & & & & & & & \\
\hline & & $X$ & & & & & & \\
\hline B & & & & & & & & \\
\hline & $X$ & $\mathrm{X}$ & $\mathrm{N}$ & $\mathrm{N}$ & $\mathrm{X}$ & & & \\
\hline $\mathrm{H}$ & $\mathrm{N}$ & $X$ & & $\mathrm{~N}$ & $\mathrm{~N}$ & $X$ & & \\
\hline$X$ & & & & & & & & \\
\hline & & & & & $\mathrm{X}$ & & $\mathrm{N}$ & \\
\hline & & & & B & & & & \\
\hline & & $X$ & & $\mathrm{~N}$ & & & & \\
\hline & X & $X$ & & & $\mathrm{X}$ & & & \\
\hline
\end{tabular}

Aubeonymus carinicollis (P.H. Lucas, 1846)

Pachytychius discithorax (Desbrochers des Loges, 1873)

Pachytychius haematocephalus (Gyllenhal, 1835)

Pachytychius hordei grandicollis (Waltl, 1835)

Pachytychius sparsutus (A.G. Olivier, 1807)

Smicronyx albosquamosus Wollaston, 1854

Smicronyx coecus (Reich, 1797)

Smicronyx cyaneus (Gyllenhal, 1835)

Smicronyx italicus Voss, 1953 [E]

Smicronyx jungermanniae (Reiche, 1797)

Smicronyx menozzii F. Solari, 1952

Smicronyx nebulosus Tournier, 1874

Smicronyx reichi (Gyllenhal, 1835)

Smicronyx syriacus Faust, 1887

Smicronychini Seidlitz, 1891

Orthochaetes insignis (Aubé, 1863)

Pseudostyphlus pillumus (Gyllenhal, 1835)

Styphlidius italicus G. Osella, 1981 [E]

\begin{tabular}{|c|c|c|c|c|c|c|c|c|}
\hline H & X & & & & & & & \\
\hline H & X & X & & & & & B & \\
\hline H & & & & B & & & B & \\
\hline X & & & & & & X & B & \\
\hline & X & & & & & & & \\
\hline & & & & & X & & & H \\
\hline & X & & & & & X & B & \\
\hline B & & & & N & & & N & \\
\hline & & & & & & & N & \\
\hline & X & & & & X & X & & \\
\hline X & X & & & & X & & & \\
\hline & & & & & X & & & \\
\hline
\end{tabular}

Styphlini Jekel, 1861

\begin{tabular}{|c|c|c|c|c|c|c|c|c|}
\hline H & & & & & & & \\
\hline B & & & & & & & & \\
\hline
\end{tabular}

Tychiini C.G. Thomson, 1859

Tychiina C.G. Thomson, 1859 


\section{Species}

Sibinia arenariae Stephens, 1831

Sibinia attalica Gyllenhal, 1835

Sibinia femoralis Germar, 1823

Sibinia pellucens (Scopoli, 1772)

Sibinia viscariae (Linnaeus, 1760)

Tychius amplicollis Aubé, 1850

Tychius argentatus Chevrolat, 1859

Tychius aureolus Kiesenwetter, 1852

Tychius bicolor C.N.F. Brisout de Barneville, 1863

Tychius breviusculus Desbrochers des Loges, 1873

Tychius caldarai Dieckmann, 1986

Tychius capucinus Boheman, 1843

Tychius consputus Kiesenwetter, 1864

Tychius cuprifer (Panzer, 1799)

Tychius cupriferoides Ragusa, 1922

Tychius curtirostris Desbrochers des Loges, 1873

Tychius decretus Tournier, 1874

Tychins depressus Desbrochers des Loges, 1873

Tychius flavus Becker, 1864

Tychius focarilei F. Solari, 1950 [E]

Tychius grandicollis Desbrochers des Loges, 1873

Tychius grenieri C.N.F. Brisout de Barneville, 1862

Tychius junceus (Reich, 1797)

Tychius kulzeri Penecke, 1934

Tychius lineatulus Stephens, 1831

Tychius longicollis C.N.F. Brisout de Barneville, 1863

Tychius medicaginis C.N.F. Brisout de Barneville, 1863

Tychius meliloti Stephens, 1831

Tychius naxiae Faust, 1889

Tychius picirostris (Fabricius, 1787)

Tychius polylineatus (Germar, 1823)

Tychius pusillus Germar, 1842

Tychius quinquepumctatus (Linnaeus, 1758)

Tychius rufipennis C.N.F. Brisout de Barneville, 1863

Tychius schmeideri (Herbst, 1795)

Tychius squamulatus Gyllenhal, 1835

Tychius stephensi Schönherr, 1835

Tychius striatulus Gyllenhal, 1835

Tychius thoracicus Boheman, 1843

Tychius tibialis Boheman, 1843

\begin{tabular}{|c|c|c|c|c|c|c|c|c|}
\hline 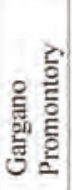 & $\begin{array}{l}70 \\
\frac{0}{0} \\
3 \\
0 \\
0 \\
\frac{0}{0} \\
0\end{array}$ & 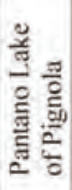 & 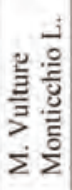 & 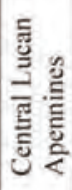 & 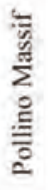 & 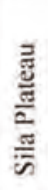 & 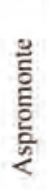 & 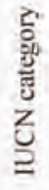 \\
\hline & $\mathrm{X}$ & & & & & & & \\
\hline $\mathrm{H}$ & $X$ & & B & & $\mathrm{X}$ & & B & \\
\hline X & $X$ & & $\mathrm{~N}$ & & $X$ & $X$ & B & \\
\hline $\mathrm{H}$ & & & B & $\mathrm{N}$ & $\mathrm{X}$ & $\mathrm{X}$ & B & \\
\hline \multirow[t]{2}{*}{$\mathrm{H}$} & & & & & & $X$ & B & \\
\hline & & & & & & & B & \\
\hline \multirow[t]{2}{*}{$\mathrm{X}$} & $\mathrm{X}$ & & & $\mathrm{N}$ & $\mathrm{N}$ & & B & \\
\hline & $\mathrm{X}$ & & & & & & & \\
\hline \multirow[t]{3}{*}{$\mathrm{X}$} & $\mathrm{X}$ & & $\mathrm{N}$ & $\mathrm{N}$ & $\mathrm{N}$ & $\mathrm{X}$ & & \\
\hline & & $\mathrm{X}$ & & & & & $\mathrm{N}$ & \\
\hline & & $\mathrm{X}$ & B & B & $\mathrm{X}$ & & $\mathrm{N}$ & \\
\hline $\mathrm{X}$ & $X$ & & & & & & B & \\
\hline $\mathrm{H}$ & & & B & B & & & & \\
\hline \multirow[t]{2}{*}{$\mathrm{X}$} & $X$ & $\mathrm{X}$ & $\mathrm{N}$ & $\mathrm{N}$ & $\mathrm{X}$ & $\mathrm{X}$ & B & \\
\hline & $\mathrm{N}$ & $\mathrm{N}$ & & & & B & & \\
\hline \multirow[t]{2}{*}{$\mathrm{X}$} & $X$ & $\mathrm{X}$ & $\mathrm{N}$ & $\mathrm{N}$ & $\mathrm{X}$ & $\mathrm{X}$ & B & \\
\hline & $\mathrm{N}$ & & & & & & B & \\
\hline \multirow[t]{3}{*}{$\mathrm{X}$} & & & & & & & & \\
\hline & & $\mathrm{X}$ & & & & & & \\
\hline & & & & & $\mathrm{X}$ & & & \\
\hline$X$ & $X$ & $X$ & & B & $\mathrm{X}$ & & $\mathrm{N}$ & \\
\hline \multicolumn{9}{|l|}{ B } \\
\hline \multirow[t]{2}{*}{$X$} & & X & & & $\mathrm{X}$ & & & \\
\hline & & $\mathrm{X}$ & $\mathrm{N}$ & $\mathrm{N}$ & $\mathrm{X}$ & & & \\
\hline \multirow[t]{3}{*}{ B } & & & & & & & B & \\
\hline & $\mathrm{X}$ & $\mathrm{X}$ & & $\mathrm{N}$ & $\mathrm{N}$ & $\mathrm{X}$ & $\mathrm{N}$ & \\
\hline & & $\mathrm{X}$ & & & & & & \\
\hline \multirow[t]{2}{*}{$\mathrm{X}$} & $\mathrm{X}$ & $\mathrm{N}$ & & & $\mathrm{N}$ & $\mathrm{X}$ & B & \\
\hline & B & & & & b & & & \\
\hline \multirow[t]{2}{*}{$\mathrm{X}$} & & $\mathrm{X}$ & $\mathrm{N}$ & $\mathrm{N}$ & $\mathrm{X}$ & $\mathrm{X}$ & $\mathrm{N}$ & \\
\hline & & $\mathrm{X}$ & & $\mathrm{N}$ & & $\mathrm{X}$ & B & \\
\hline $\mathrm{X}$ & $X$ & $\mathrm{X}$ & $\mathrm{N}$ & $\mathrm{N}$ & $\mathrm{X}$ & & B & \\
\hline $\mathrm{H}$ & $X$ & & & $\mathrm{~N}$ & $X$ & $\mathrm{X}$ & & \\
\hline $\mathrm{X}$ & & & $\mathrm{N}$ & & & B & & \\
\hline $\mathrm{X}$ & $\mathrm{X}$ & & & $\mathrm{N}$ & $\mathrm{X}$ & X & & \\
\hline \multirow[t]{3}{*}{$\mathrm{X}$} & $X$ & $\mathrm{X}$ & & $\mathrm{N}$ & & $\mathrm{X}$ & & \\
\hline & $\mathrm{X}$ & & $\mathrm{N}$ & $\mathrm{N}$ & $\mathrm{X}$ & & B & \\
\hline & & $\mathrm{X}$ & & & & & & \\
\hline$X$ & & $\mathrm{X}$ & & B & $\mathrm{X}$ & & B & \\
\hline$X$ & $\mathrm{X}$ & $\mathrm{X}$ & $\mathrm{N}$ & $\mathrm{N}$ & $\mathrm{X}$ & $X$ & B & \\
\hline
\end{tabular}

Cyclominae Schönherr, 1826

Dichotrachelini A. Hoffmann, 1957

Dichotrachelus bensae A. Solari \& F. Solari, 1904 [E]

Hipporhinini Lacordaire, 1863

Entomoderus deformis (Reiche \& Saulcy, 1858)

Entomoderus impressicollis peninsularis (F. Solari, 1940) [E]

Gronops lunatus (Fabricius, 1775)

Dryophthorinae Schönherr, 1825

Dryophthorini Schönherr, 1825

Diyophthorns corticalis (Paykull, 1792)

Rhynchophorini Schönherr, 1833

Litosomina Lacordaire, 1865 
Species

Sitophilus granarius (Linnaeus, 1758)

Sitophilus oryzae (Linnaeus, 1763)

Sitophìlus zeamais Motschulsky, 1855

Rhynchophorina Schönherr, 1833

\begin{tabular}{|c|c|c|c|c|c|c|c|}
\hline 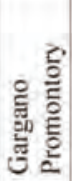 & $\begin{array}{l}\overline{0} \\
0 \\
3 \\
0 \\
\frac{3}{0} \\
\frac{3}{0} \\
2\end{array}$ & 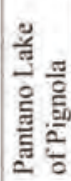 & 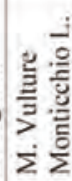 & 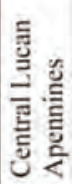 & 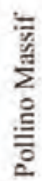 & 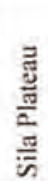 & 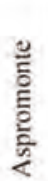 \\
\hline & & & & $\mathrm{N}$ & & & \\
\hline$X$ & $\mathrm{X}$ & & & $\mathrm{N}$ & $\mathrm{N}$ & $X$ & $\mathrm{~N}$ \\
\hline & & & & & $\mathrm{N}$ & & $\mathrm{N}$ \\
\hline
\end{tabular}

Rhynchophorus ferrugineus (A.G. Olivier, 1791)

Sphenophorina Lacordaire, 1865

Sphenophorus abbreviatus (Fabricius, 1787)

Sphenophorus meridionalis Gyllenhal, 1838

Sphenophorus partumpunctatus Gyllenhal, 1838

Sphenophorus piceus (Pallas, 1771)

Sphenophorus striatopunctatus (Goeze, 1777)

\begin{tabular}{|c|c|c|c|c|c|c|c|c|}
\hline X & B & & & & & & & \\
\hline & B & & & N & & & B & \\
\hline & & & & N & & & B & \\
\hline H & X & & & & & & & \\
\hline X & & & & & & & & \\
\hline
\end{tabular}

Entiminae Schönherr, 1823

Brachyderini Schönherr, 1826

Brachyderes incamus (Linnaeus, 1758)

Strophomorphis porcellus (Schönherr, 1832)

Strophosoma erinaceus (Chevrolat, 1865)

Strophosoma melanogrammus melanogrammus (Forster, 1771)

\begin{tabular}{|l|l|l|l|l|l|l|l|}
\hline $\mathrm{X}$ & $\mathrm{X}$ & & & $\mathrm{N}$ & $\mathrm{N}$ & $\mathrm{X}$ & $\mathrm{B}$ \\
\hline & & & & & & B \\
\hline & & $\mathrm{N}$ & & $\mathrm{X}$ & $\mathrm{X}$ & $\mathrm{B}$ \\
\hline
\end{tabular}

Byrsopagini Lacordaire, 1863

Graptus nictitans singularis (Jacquelin du Val, 1854)

Rhytideres plicatus (A.G. Olivier, 1790)

Tropiphorus imperator Pesarini, 1995 [E]

Tropiphorus obtusus (Bonsdorff, 1785)

Geonemini Gistel, 1848

Barynotus obscurus (Fabricius, 1775)

Barynotus solariortum Mainardi, 1907 [E]

Myorhinini Marseul, 1863

\begin{tabular}{|c|c|c|c|c|c|c|c|c|}
\hline H & X & & & N & & X & & \\
\hline & B & & & & & & & \\
\hline & & & & B & B & & & \\
\hline & & & & & X & & & \\
\hline
\end{tabular}

Haptomerus lepidus (Brullé, 1832)

Omiini Shuckard, 1840

Elytrodon bidentatus Boheman, 1834

Elytrodon italicus Pesarini, 1980 [E]

Euplatimus graecus (Stierlin, 1884)

Omias brancsiki (Reitter, 1906)

Rhinomias forticornis (Boheman, 1842)

\section{Otiorhynchini Schönherr, 1826}

Cirrorhynchus pilipes (Leoni, 1907) [E]

Cirrorhynchus sclanoi Abazzi \& Magnano, 2003 [E]

Dodecastichus brevipes tarentinus (A. Solari \& F. Solari, 1915)

[E]

Dodecastichus consentaneus (Boheman, 1842)

Dodecastichus dalmatinus (Gyllenhal, 1834)

Dodecastichus mastix (A.G. Olivier, 1807)

Dodecastichus ovoideus (Reitter, 1913) [E]

Limatogaster colonnellii (Magnano, 1986) [E]

Otiorhynchus abruzzensis Stierlin, 1893 [E]

Otiorhynchus affaber Boheman, 1842

Otiorhynchus alpicola Boheman, 1842

Otiorhynchus alutaceus alutaceus (Germar, 1817)

Otiorhynchus alutaceus coarctaticornis A. Solari \& F. Solari,

1907 [E]

Otiorhynchus anthracinus (Scopoli, 1763)

Otiorhynchus apulus A. Solari \& F. Solari, 1913 [E]

Otiorhynchus armatus armatus Boheman, 1842

\begin{tabular}{|c|c|c|c|c|c|c|c|c|}
\hline H & X & & & B & X & & & \\
\hline & & & & B & B & & & \\
\hline & & & & & X & & & \\
\hline & & & & CK & X & & & \\
\hline H & & & & CK & X & & & \\
\hline & & & & & & & & \\
\hline B & N & & B & & & & B & \\
\hline & & & I & CH & X & & & \\
\hline & & & & & X & X & & \\
\hline H & X & B & & & B & & & \\
\hline X & X & & N & CK & X & X & B & \\
\hline X & & & & & & & B & \\
\hline & X & & B & B & X & X & B & \\
\hline
\end{tabular}


Species

Otiorhynchus aurifer Boheman, 1842 [E]

Otiorhynchus calabrensis Stierlin, 1891 [E]

Otiorhynchus calabrolucamus Lona, 1958

Otiorhynchus corruptor corruptor (Host, 1790)

Otiorhynchus crataegi Germar, 1823

Otiorhynchus cribricollis Gyllenhal, 1834

Otiorhynchus desertus Rosenhauer, 1847

Otiorhynchus echidna K. Daniel \& J. Daniel, 1898 [E]

Otiorhynchus emanuelae Magrini, Meoli \& Abbazzi, 2005 [E

Otiorhynchus fernandoi Magnano, 2001 [E]

Otiorhynchus ferrarii Miller, I863

Otiorhynchus frescati Boheman, 1842

Otiorhynchus garibaldinus A. Solari \& F. Solari, 1909 [E]

Otiorhynchus holdhausi A. Solari \& F. Solari, 1913 [E]

Otiorhynchus indefinitus Reitter, 1912

Otiorhynchus juvencus juvencus Gyllenhal, 1834

Otiorhynchus ligneoides Stierlin, 1902 [E]

Otiorhynchus lubricus ausonius Pesarini, 1975 [E]

Otiorhynchus lugens Germar, 1817

Otiorhynchus lutosus Stierlin, 1858

Otiorhynchus matutimus Reitter, 1912 [E]

Otiorhynchus meridionalis Gyllenhal, 1834

Otiorhynchus minutesquamosus A. Solari \& F. Solari, 1909 [E]

Otiorhynchus perdix (A.G. Olivier, 1807)

Otiorhynchus pesarinit Diotti, 2008 [E]

Otiorhynchus porcellus K. Daniel \& J. Daniel, 1898 [E]

Otiorhynchus rugosostriatus (Goeze, 1777)

Otiorhynchus scopularis Hochhuth, 1847

Otiorhynchus setosulus Stierlin, 1861

Otiorhynchus transadriaticus Reitter, 1913 [E]

Otiorhynchus villosus Stierlin, 1872

Otiorhynchus volturinensis Magnano, 1999 [E]

Otiorhynchus vulturensis Lona, 1931 [E]

Stomodes puncticollis amorei Desbrochers des Loges, 1904 [E]

\begin{tabular}{|c|c|c|c|c|c|c|c|c|}
\hline 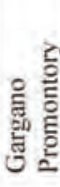 & $\begin{array}{l}3 \\
0 \\
3 \\
0 \\
0 \\
0 \\
\frac{0}{0} \\
2\end{array}$ & 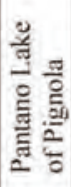 & 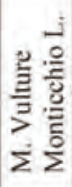 & 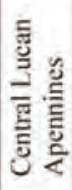 & $\begin{array}{l}\bar{y} \\
\frac{a}{2} \\
0 \\
\frac{g}{0} \\
0\end{array}$ & 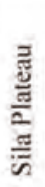 & 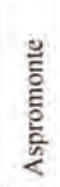 & 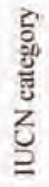 \\
\hline $\mathrm{H}$ & $\mathrm{X}$ & $\mathrm{X}$ & $\mathrm{N}$ & $\mathrm{N}$ & $\mathrm{N}$ & $\mathrm{X}$ & ! & \\
\hline \multirow[t]{4}{*}{$\mathrm{X}$ ? } & & & & & & $\mathrm{X}$ & B & \\
\hline & & & & & $X$ & & & \\
\hline & & & & & $X$ & $\mathrm{X}$ & $\mathrm{N}$ & \\
\hline & B & & & & & & & \\
\hline \multirow[t]{2}{*}{$\mathrm{X}$} & $\mathrm{X}$ & & $\mathrm{N}$ & & $\mathrm{X}$ & & $!$ & \\
\hline & & & & & $\mathrm{X}$ & & & \\
\hline \multirow[t]{3}{*}{$\mathrm{H}$} & & & & & & & & \\
\hline & & & & B & & & & \\
\hline & B & & & & & & & \\
\hline \multirow[t]{3}{*}{$X$} & $\mathrm{~N}$ & & & & & & & \\
\hline & & $X$ & & & & & & \\
\hline & & & & & & & B & \\
\hline $\mathrm{H}$ & & & & & B & & & \\
\hline $\mathrm{H}$ & & & B & B & $\mathrm{X}$ & B & B & \\
\hline \multirow[t]{3}{*}{$\mathrm{X}$} & $\mathrm{X}$ & & & & & & & \\
\hline & & & & & $\mathrm{X}$ & & & \\
\hline & & & & & & & B & \\
\hline $\mathrm{X}$ & $\mathrm{X}$ & $\mathrm{X}$ & $\mathrm{N}$ & $\mathrm{N}$ & $\mathrm{X}$ & $\mathrm{X}$ & B & \\
\hline \multicolumn{9}{|l|}{ B } \\
\hline \multirow[t]{3}{*}{$\mathrm{X}$} & $\mathrm{X}$ & & & & & & & \\
\hline & CK & & & CK & & & & \\
\hline & & & & & $X$ & & & \\
\hline \multirow[t]{3}{*}{$\mathrm{H}$} & & & $\mathrm{N}$ & B & $X$ & & & \\
\hline & & & & B & & & & \\
\hline & & & & & $\mathrm{X}$ & & & \\
\hline \multirow[t]{2}{*}{$\mathrm{H}$} & B & & $\mathrm{N}$ & $\mathrm{N}$ & $X$ & & CK & \\
\hline & & & B & ! & & $\mathrm{X}$ & & \\
\hline$X$ & & & & & $X$ & $X$ & & \\
\hline \multicolumn{9}{|l|}{$\mathrm{H}$} \\
\hline \multicolumn{9}{|l|}{ B } \\
\hline \multirow[t]{2}{*}{ B } & & & & B & & & & \\
\hline & & & B & & & & & \\
\hline $\mathrm{H}$ & & & & & & & & \\
\hline
\end{tabular}

Peritelini Lacordaire, 1863

Heteromeira margheritae Bellò \& Pierotti, 1992 [E]

Heteromeira marianii (Pesarini, 1972) [E]

Heteromeira neapolitana (Faust, 1890) [E]

Meira baudii Stierlin, 1892 [E]

Meira oenotria Pierotti, 2013 [E]

Meirella florentina (Stierlin, 1885)

Pseudomeira angelinii Pierotti, 2011 [E]

Pseudomeira binaghii F. Solari, 1955 [E]

Pseudomeira lucana Bellò \& Pierotti, 1992 [E]

Pseudomeira obscura (A. Solari \& F. Solari, 1907)

Pseudomeira paganettii (A. Solari \& F. Solari, 1907) [E]

Psendomeira parvula (Seidlitz, 1865) [E]

Pseudomeira pseudobscura Bellò \& Pierotti, 1992 [E]

Pseudomeira silvestrii (A. Solari \& F. Solari, 1907) [E]

Simo variegatus (Boheman, 1843) [E]

Solariola angelinii Bellò, G. Osella \& Baviera, 2019 [E]

Solariola diottii Bellò, G. Osella \& Baviera, 2019 [E]

Solariola fancelloi Bellò, G. Osella \& Baviera, 2019 [E]

\begin{tabular}{|l|l|l|l|l|l|l|l|l|l|l|l|}
\hline & & X & & & & B & & B & \\
\hline & H & & & B & B & & & & \\
\hline & & & & & & B & B & & \\
\hline & CK & & & & & & & & \\
\hline & & & & & & & & B & \\
\hline & & & & & & X & & N & \\
\hline & B & B & & & B & X & X & B & \\
\hline & H & & & & & & & & \\
\hline & & & & & B & B & B & & \\
\hline E] & & & & & & & & B & \\
\hline & & & & & & & & B & \\
\hline
\end{tabular}


Species

Solariola fraterna Baviera, 2015 [E]

Solariola hirtula A. Solari \& F. Solari, 1923 [E]

Solariola margaritae Bellò, G. Osella \& Baviera, 2019 [E]

Solariola pacei Bellò, G. Osella \& Baviera, 2019 [E]

Solariola paganettii (Flach, 1905) [E]

Solariola pentaphyllica Bellò, G. Osella \& Baviera, 2919 [E]

Solariola poggii Bellò, G. Osella \& Baviera, 2019 [E]

Solariola tedeschii Bellò, G. Osella \& Baviera, 2019 [E]

Phyllobiini Schönherr, 1826

Argoptochus schwarzi schwarzi (Reitter, 1888)

Metacinops rhinomacer Kraatz, 1862

Parascythopus baudii Stierlin, 1892 [E]

Phyllobius argentatus argentatus (Linnaeus, 1758)

Phyllobius chloropus (Linnaeus, 1758)

Phyllobius emeryi Desbrochers des Loges, 1873 [E]

Phyllobius etruscus Desbrochers des Loges, 1872

Phyllobius italicus A. Solari \& F. Solari, 1904 [E]

Phyllobius longipilis Boheman, 1843 [E]

Phyllobius maculicornis Germar, 1823

Phyllobius oblongus (Linnaeus, 1758)

Phyllobius pyri (Linnaeus, 1758)

Phyllobius raverae A. Solari \& F. Solari, 1904 [E]

Phyllobius reicheidius Desbrochers des Loges, 1873 [E]

Phyllobius romanus Faust, 1890 [E]

Phyllobius subdentatus Boheman, 1842 [E]

Phyllobius virideaeris pedestris Schilsky, 1911

Pseudomyllocerus neapolitanus (Pic, 1902)

Pseudomyllocerus simuatus (Fabricius, 1801)

\begin{tabular}{|c|c|c|c|c|c|c|c|}
\hline 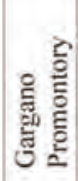 & $\begin{array}{l}\overline{0} \\
0 \\
3 \\
0 \\
\frac{0}{0} \\
\frac{.0}{0} \\
2\end{array}$ & 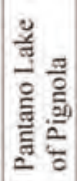 & 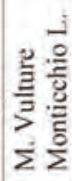 & 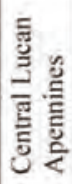 & $\begin{array}{l}\bar{n} \\
\sum_{0} \\
0 \\
\frac{E}{0}\end{array}$ & 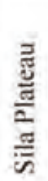 & 总 \\
\hline & & & & & & & B \\
\hline & & & & & & & B \\
\hline & & & & & & & B \\
\hline & & & & & & & B \\
\hline & & & & & & & B \\
\hline & & & & & & & B \\
\hline & & & & & & & B \\
\hline & & & & & & B & B \\
\hline
\end{tabular}

\begin{tabular}{|l|l|l|l|l|l|l|l|l|l|l|}
\hline & H & & & & N & X & X & B & \\
\hline & & & & N & B & X & X & B & \\
\hline & & & & N & N & X & X & B & \\
\hline [E] & & B & & & & & & & \\
\hline E] & X & & X & N & N & & X & B & \\
\hline & & & N & N & N & X & N & B & \\
\hline & H & & & N & N & X & X & B & \\
\hline & H & X & X & N & N & X & X & B & \\
\hline & & & X & B & & & & & \\
\hline & & & & & N & X & X & & \\
\hline & & & & & & & X & N & \\
\hline & & & & N & N & X & X & & \\
\hline & & & & & & & X & B & \\
\hline & H & N & & & N & & N & & \\
\hline
\end{tabular}

Polydrusini Schönherr, 1823

Auchmerestes kiesenwetteri Kraatz, 1862

Liophloeus tessulatus (O.F. Müller, 1776)

Polydrusus aeratus aeratus (Gravenhorst, 1807)

Polydrusus amplicollis (Desbrochers des Loges, 1902) [E]

Polvdrusus armipes Brullé, 1832

Polydrusus brevicollis Desbrochers des Loges, 1872

Polydrusus calabricus (Faust, 1890)

Polydrusus cervinus (Linnaeus, 1758)

Polydrusus formosus (Mayer, 1779)

Polydrusus frater Rottenberg, 1872

Polydrusus impar Gozis, 1882

Polydrusus kahri Kirsch, 1865

Polydrusus lateralis Gyllenhal, 1834

Polydrusus marginatus Stephens, 1831

Polydrusus modestus (Stierlin, 1864)

Polydrusus neapolitamus Desbrochers des Loges, 1872

Polydrusus picus picus (Fabricius, 1792)

Polydrusus pilosus italicus Schilsky, 1910 [E]

Polydrusus pirazzolii Stierlin, 1857

Polydrusus pterygomalis Boheman, 1840

Polydrusus raverae A. Solari \& F. Solari, 1904

Polydrusus scutellaris (Chevrolat, 1860)

Polydrusus siculus Desbrochers des Loges, 1872

Polydrusus sparsus Gyllenhal, 1834

\begin{tabular}{|l|l|l|l|l|l|l|l|l|l|}
\hline & & & & N & X & X & N & \\
\hline & & & & N & N & & N & \\
\hline & & & & N & N & & & \\
\hline H & X & X & N & N & X & X & & \\
\hline X & X & & & & N & & B & \\
\hline H & X & X & N & N & X & X & B & \\
\hline H & X & X & N & N & X & X & B & \\
\hline X & & & & N & X & X & B & \\
\hline & X & & & & X & X & N & \\
\hline & & & & & & & N & \\
\hline & & N & N & N & X & X & B & \\
\hline H & & & & & & & B & \\
\hline X & X & & & N & X & X & B & \\
\hline H & N & & N & N & X & X & & \\
\hline & & & & & X & & & \\
\hline & & & B & N & X & X & B & \\
\hline X & & & N & N & X & X & & \\
\hline & & & & & X & & B & \\
\hline X & X & X & N & N & X & X & B & \\
\hline & & & & & \\
\hline
\end{tabular}


Species

Polydrusus splendens A. Solari \& F. Solari, 1909

Polydrusus subnotatus Schilsky, 1910

Polydrusus tibialis (Gyllenhal, 1834)

Polydrusus viridicinctus Gyllenhal, 1834

\begin{tabular}{|c|c|c|c|c|c|c|c|c|}
\hline 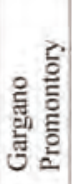 & 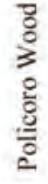 & 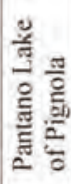 & 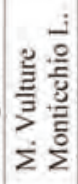 & 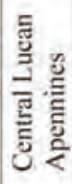 & 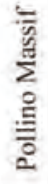 & 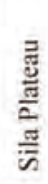 & $\begin{array}{l}\frac{U}{5} \\
\text { है } \\
\frac{0}{0} \\
\text { o }\end{array}$ & 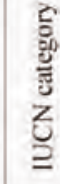 \\
\hline & & & & & $\mathrm{N}$ & & & \\
\hline & & & & & $X$ & X & B & \\
\hline X & X & $\mathrm{X}$ & & $\mathrm{N}$ & & X & & \\
\hline X & & & & & & & & \\
\hline
\end{tabular}

Psallidiini Lacordaire, 1863

Mesagroicus amicalis Magnano, G. Osella \& Abbazzi, 2008 [E]

Sciaphilini Sharp, 1891

Brachysomus hirtus (Boheman, 1845)

Pleurodirus doderoi (D'Amore Fracassi, 1907)

Sciaphobus abbreviatus (Desbrochers des Loges, 1871) [E]

\begin{tabular}{|l|l|l|l|l|l|l|l|l|}
\hline $\mathrm{H}$ & & & $\mathrm{N}$ & $\mathrm{N}$ & $\mathrm{N}$ & $\mathrm{X}$ & $\mathrm{N}$ & \\
\hline $\mathrm{H}$ & & & & & & & & \\
\hline $\mathrm{X}$ & & & & & & & & \\
\hline
\end{tabular}

Sitonini Gistel, 1848

Charagmus cachectus (Gyllenhal, 1834)

Charagmus gressorius (Fabricius, 1792)

Charagmus intermedius (Küster, 1847)

Charagmus variegatus (Fảhraeus, 1840)

Coelositona cambricus (Stephens, 1831)

Coelositona cinerascens (Fảhraeus, 1840)

Coelositona limosus (P. Rossi, 1792)

Sitona callosus Gyllenhal, 1834

Sitona cinnamomeus Allard, 1863

Sitona cylindricollis cylindricollis Fåhraeus, 1840

Sitona discoideus Gyllenhal, 1834

Sitona gemellatus Gyllenhal, 1834

Sitona goetzelmmi Reitter, 1909 [E]

Sitona hirsutus Desbrochers des Loges, 1884

Sitona hispidulus (Fabricius, 1777)

Sitona humeralis Stephens, 1831

Sitona lineatus (Linnaeus, 1758)

Sitona lividipes lividipes Fảhraeus, 1840

Sitona macularius macularius (Marsham, 1802)

Sitona obsoletus obsoletus (Gmelin, 1790)

Sitona ophthalmicus Desbrochers des Loges, 1869

Sitona puncticollis Stephens, 1831

Sitona sekerae Reitter, 1903 [E]

Sitona sulcifrons argutulus Gyllenhal, 1834

Sitona suturalis Stephens, 1831

Sitona temuis Rosenhauer, 1847

Sitona verecundus (P. Rossi, 1790)

Sitona waterhousei waterhousei Walton, 1846

\begin{tabular}{|c|c|c|c|c|c|c|c|c|}
\hline X & X & & & & & & & \\
\hline X & & & & & N & X & B & \\
\hline & & N & & & X & X & B & \\
\hline & & & & & & X & & \\
\hline H & X & & & & X & X & & \\
\hline X & & & & & X & X & B & \\
\hline X & & X & & N & & & N & \\
\hline H & X & & N & N & X & & N & \\
\hline & & & & & & & B & \\
\hline & & & & & & & B & \\
\hline H & X & X & N & N & X & X & B & \\
\hline H & X & X & B & N & X & X & B & \\
\hline & X & X & & & X & X & B & \\
\hline H & & X & N & N & & N & B & \\
\hline & & & & & & X & B & \\
\hline H & & N & & & & X & B & \\
\hline H & & X & B & & X & X & B & \\
\hline X & & & & & & X & & \\
\hline H & X & X & B & N & X & X & B & \\
\hline X & X & X & & N & X & X & B & \\
\hline & B & & & N & N & & N & \\
\hline H & & & & N & & & & \\
\hline & X & & & & & & & \\
\hline
\end{tabular}

Tanymecini Lacordaire, 1863

Tanymecina Lacordaire, 1863

Cycloderes canescens ( $\mathrm{P}+$ Rossi, 1792)

Cycloderes fritillum (Panzer, 1794)

Cycloderes pominii F. Solari, 1954 [E]

Tanymecus palliatus (Fabricius, 1787)

Tanymecus submaculatus Chevrolat, 1860

Trachyphloeini Gistel, 1848

\begin{tabular}{|c|c|c|c|c|c|c|c|c|}
\hline B & $\mathrm{B}$ & & & & & & & \\
\hline & $\mathrm{X}$ & & & & & & & \\
\hline & & & & & & & $\mathrm{B}$ & \\
\hline & $\mathrm{X}$ & & & & & $\mathrm{X}$ & $\mathrm{N}$ & \\
\hline & $\mathrm{X}$ & & & & & $\mathrm{B}$ & \\
\hline
\end{tabular}

Cathormiocerus aristatus (Gyllenhal, 1827)

Cathormiocerus discors Desbrochers des Loges, 1875

Cathormiocerus hircus Desbrochers des Loges, 1896

Cathormiocerns validiscapus Rouget, 1857

Pelletierellus calabricus (Borovec, 1986)

\begin{tabular}{|l|l|l|l|l|l|l|l|l|l|}
\hline & & & & & & & $!$ & \\
\hline & & & & $!$ & & $!$ & $!$ & \\
\hline & & & ! & & & & \\
\hline
\end{tabular}


Species

Romualdius canaliculatus (L.W. Schaufuss, 1867)

Romualdius peyerimhoffi (Formanek, 1910)

Romualdius scaber (Linnaeus, 1758)

Stuebenius amicalis (Borovec \& G. Osella, 1993)

Trachyphloeus alternans Gyllenhal, 1834

Trachyphloeus laticollis Boheman, 1842

Trachyphloeus nodipennis Chevrolat, 1860

Trachyphloeus spinimanus Germar, 1823

\section{Hyperinae Marseul, 1863 \\ Hyperini Marseul, 1863}

Brachypera crinita (Boheman, 1834)

Brachypera dauci (A.G. Olivier, 1807)

Brachypera zoilus (Scopoli, 1763)

Coniatus suavis Gyllenhal, 1834

Coniatus tamarisci (Fabricius, 1787)

Donus brucki (Capiomont, 1868) [E]

Donus cyrtus (Germar, 1821)

Donis füumanus (Petri, 1901)

Donus philanthus (A.G. Olivier, 1807)

Donus salviae (Schrank, 1789)

Hypera arator (Linnaeus, 1758)

Hypera conmaculata Herbst, 1795

Hypera contaminata (Herbst, 1795)

Hypera melancholica (Fabricius, 1792)

Hypera meles (Fabricius, 1792)

Hypera nigrirostris (Fabricius, 1775)

Hypera pastinacae (P. Rossi, 1790)

Hypera plantaginis (DeGeer, 1775)

Hypera postica (Gyllenhal, 1813)

Hypera rumicis (Linnaeus, 1758)

Hypera striata (Boheman, 1834)

Hypera venusta (Fabricius, 1781)

Hypera viciae (Gyllenhal, 1813)

Limobius borealis borealis (Paykull, 1792)

\begin{tabular}{|l|l|l|l|l|l|l|l|}
\hline H & X & & & N & X & & B \\
\hline H & X & & & N & X & X & B \\
\hline H & X & & & N & & & B \\
\hline X & & & & & & X & \\
\hline H & X & & & & N & & B \\
\hline H & & & & N & X & X & B \\
\hline & & & & & & & \\
\hline H & X & & & & & & \\
\hline H & X & & & N & N & X & N \\
\hline H & X & X & N & N & X & X & B \\
\hline H & & & & & & & \\
\hline & & N & & & & & B \\
\hline X & X & X & N & N & X & X & B \\
\hline X & & X & & & & & \\
\hline H & X & X & & & & X & \\
\hline X & & & & & & X & B \\
\hline H & X & X & N & N & X & & B \\
\hline H & & & & & \\
\hline
\end{tabular}

\section{Lixinae Schönherr, 1823}

Cleonini Schönherr, 1826

Asproparthenis albicans (Gyllenhal, 1834)

Asproparthenis maculicollis (Chevrolat, 1873)

Bothynoderes affinis (Schrank, 1781)

Cleonis pigra (Scopoli, 1763)

Coniocleonus excoriatus (Gyllenhal, 1834)

Coniocleonus megalographus (Făhraeus, 1842)

Coniocleonus nebulosus (Linnaeus, 1758)

Coniocleomus nigrosuturatus (Goeze, 1777)

Conorhynchus luigionii A. Solari \& F. Solari, 1905

Conorhynchus mendicus (Gyllenhal, 1834)

Cyphocleomus testatus (Gyllenhal, 1834)

Lelicophyes pedestris (Poda von Neuhaus, 1761)

Lixomorphus algirus (Linnaeus, 1758)

Mecaspis altermans (Hellwig, 1795)

Mecaspis emarginata (Fabricius, 1787)

Pachycerus madidus (A.G. Olivier, 1807)

Pachycerus segnis (Germar, 1823)

\begin{tabular}{|c|c|c|c|c|c|c|c|c|}
\hline \multirow{2}{*}{$\mathrm{X}$} & & & & & & & & \\
\hline & B & & & & & & & \\
\hline $\mathrm{X}$ & & & & & & & & \\
\hline $\mathrm{H}$ & $\mathrm{X}$ & $\mathrm{X}$ & & $\mathrm{N}$ & $\mathrm{X}$ & $X$ & $\mathrm{~N}$ & \\
\hline $\mathrm{X}$ & & & & & & & & \\
\hline $\mathrm{X}$ & & & & & & & & \\
\hline ! & & & & & & & B & \\
\hline $\mathrm{H}$ & & & & & & & & \\
\hline $\mathrm{X}$ & $\mathrm{X}$ & & & & & & & \\
\hline B & B & & & & & & & \\
\hline $\mathrm{H}$ & & & & & & & & \\
\hline $\mathrm{H}$ & & & & & & & & \\
\hline $\mathrm{H}$ & $\mathrm{X}$ & $\mathrm{X}$ & & & $\mathrm{X}$ & $\mathrm{X}$ & & \\
\hline $\mathrm{X}$ & $\mathrm{X}$ & & & & $\mathrm{X}$ & $\mathrm{X}$ & B & \\
\hline & & & B & $!$ & $\mathrm{X}$ & $!$ & B & \\
\hline & X & & & & & & & \\
\hline $\mathrm{H}$ & $\mathrm{X}$ & & & $!$ & $\mathrm{X}$ & $\mathrm{X}$ & & \\
\hline
\end{tabular}


Species

Pseudocleomus cinereus (Schrank, 1781)

Pseudocleomus grammicus (Panzer, 1789)

Pseudocleonus italicus F. Solari, 1950 [E]

Rhabdorhynchus echii (Brahm, 1790)

Temnorhimus mendicus (Gyllenhal, 1834)

Trachydemus rugosus (P.H. Lucas, 1846)

Lixini Schönherr, 1823

\begin{tabular}{|c|c|c|c|c|c|c|c|c|}
\hline 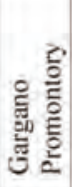 & $\begin{array}{l}7 \\
0 \\
3 \\
0 \\
\frac{0}{0} \\
\frac{3}{0} \\
2\end{array}$ & 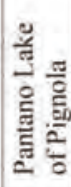 & 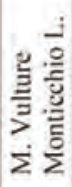 & 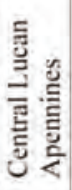 & 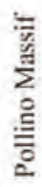 & 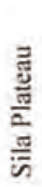 & 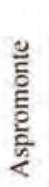 & 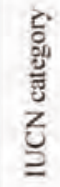 \\
\hline $\mathrm{H}$ & & & & & & & B & \\
\hline \multicolumn{9}{|l|}{$\mathrm{X}$} \\
\hline & & & & & $\mathrm{X}$ & & & \\
\hline $\mathrm{H}$ & $X$ & & & & 1 & B & & \\
\hline$X$ & & & & & ! & & & \\
\hline & B & & & & & & & \\
\hline
\end{tabular}

Bangastermus orientalis (Capiomont, 1873

Larinus carinirostris Gyllenhal, 1835

Larimus carlinae (A.G. Olivier, 1807)

Larimus cynarae (Fabricius, 1787)

Larimus iaceae (Fabricius, 1775)

Larinus latus (Herbst, 1783)

Larimus maurus (A.G. Olivier, 1807)

Larinus obtusus Gyllenhal, 1835

Larimus plamus (Fabricius, 1792)

Larinus pollinis (Laicharting, 1781)

Larimus scolymi (A.G. Olivier, 1807)

Larinus sturmus (Schaller, 1783)

Larinus turbinatus Gyllenhal, 1835

Larimus ursus (Fabricius, 1792)

Larimus vilpes (A.G. Olivier, 1807)

Lixus abdominalis Boheman, 1835

Lixus acicularis Germar, 1823

Lixus albomarginatus Boheman, 1842

Lixus anguinus (Linnaeus, 1767)

Lixus angustus (Herbst, 1795)

Lixus ascamii (Linnaeus, 1767)

Lixus bardanae (Fabricius, 1787)

Lixus cardui A.G. Olivier, 1807

Lixus cinerascens Schönherr, 1832

Lixus curvirostris Capiomont, 1875

Lixus cylindrus (Fabricius, 1781)

Lixus fasciculatus Boheman, 1835

Lixus filiformis (Fabricius, 1781)

Lixus iridis A.G. Olivier, 1807

Lixus juncii Boheman, 1835

Lixus lateralis (Panzer, 1789)

Lixus mucronatus (A.G. Olivier, 1791)

Lixus myagri A.G. Olivier, 1807

Lixus ochraceus Boheman, 1842

Lixus paraplecticus (Linnaeus, 1758)

Lixus pulverulentus (Scopoli, 1763)

Lixus scolopax Boheman, 1835

Lixus tibialis Boheman, 1842

Lixus umbellatarum (Fabricius, 1787)

Lixus vilis ( $\mathrm{P}$. Rossi, 1790)

Microlarinus lareynii (Jacquelin du Val, 1853)

Rhinocyllus conicus (Frölich, 1792)

Mesoptiliinae Lacordaire, 1863

\begin{tabular}{|c|c|c|c|c|c|c|c|c|}
\hline \multirow{3}{*}{$\begin{array}{l}\mathrm{H} \\
\mathrm{H}\end{array}$} & & & & & & & & \\
\hline & & & B & & $X$ & $X$ & B & \\
\hline & & & & & X & X & & \\
\hline \multirow{2}{*}{$\mathrm{H}$} & $X$ & & & $\mathrm{~N}$ & $\mathrm{~N}$ & & B & \\
\hline & $\mathrm{X}$ & $\mathrm{N}$ & B & $\mathrm{N}$ & $\mathrm{X}$ & $X$ & B & \\
\hline $\mathrm{H}$ & & & B & & & & & \\
\hline \multicolumn{8}{|l|}{$\mathrm{H}$} & \\
\hline $\mathrm{H}$ & & & & $\mathrm{N}$ & $\mathrm{X}$ & $\mathrm{X}$ & B & \\
\hline \multirow[t]{2}{*}{$\mathrm{H}$} & $X$ & $\mathrm{X}$ & $\mathrm{N}$ & & $\mathrm{X}$ & $\mathrm{X}$ & B & \\
\hline & B & & & & & $\mathrm{X}$ & B & \\
\hline $\mathrm{H}$ & $\mathrm{X}$ & & B & & $\mathrm{X}$ & & B & \\
\hline $\mathrm{X}$ & $\mathrm{X}$ & $\mathrm{X}$ & $\mathrm{N}$ & $\mathrm{N}$ & $\mathrm{X}$ & $\mathrm{X}$ & B & \\
\hline $\mathrm{X}$ & $X$ & & B & $\mathrm{N}$ & $\mathrm{X}$ & $\mathrm{X}$ & $\mathrm{N}$ & \\
\hline \multirow[t]{2}{*}{$\mathrm{H}$} & & & & $\mathrm{N}$ & & & & \\
\hline & & & & & $\mathrm{X}$ & & & \\
\hline $\mathrm{X}$ & $\mathrm{X}$ & & ! & & $X$ & $\mathrm{X}$ & B & \\
\hline $\mathrm{H}$ & & $\mathrm{X}$ & & & $\mathrm{X}$ & $\mathrm{X}$ & & \\
\hline \multirow[t]{3}{*}{$!$} & & & & & & $\mathrm{X}$ & & \\
\hline & $X$ & & & & & & B & \\
\hline & $X$ & $\mathrm{~N}$ & B & $\mathrm{N}$ & $X$ & $\mathrm{X}$ & B & \\
\hline $\mathrm{H}$ & & & B & & $\mathrm{X}$ & 1 & B & \\
\hline$!$ & $\mathrm{N}$ & $\mathrm{X}$ & & $!$ & & $\mathrm{X}$ & & \\
\hline $\mathrm{H}$ & $X$ & $\mathrm{X}$ & $\mathrm{N}$ & $\mathrm{N}$ & $\mathrm{X}$ & $\mathrm{X}$ & $\mathrm{N}$ & \\
\hline $\mathrm{X}$ & $\mathrm{N}$ & & & $\mathrm{N}$ & $\mathrm{N}$ & & & \\
\hline \multirow[t]{2}{*}{$\mathrm{X}$} & & & & & $\mathrm{X}$ & & & \\
\hline & $\mathrm{X}$ & & & & & & & \\
\hline $\mathrm{H}$ & $\mathrm{X}$ & & & & $X$ & $\mathrm{X}$ & B & \\
\hline $\mathrm{N}$ & $\mathrm{N}$ & $\mathrm{X}$ & $\mathrm{N}$ & $\mathrm{N}$ & & ! & $\mathrm{N}$ & \\
\hline $\mathrm{H}$ & $\mathrm{X}$ & $\mathrm{X}$ & $\mathrm{N}$ & ! & $\mathrm{X}$ & $\mathrm{X}$ & B & \\
\hline \multirow[t]{3}{*}{$\mathrm{X}$} & $\mathrm{X}$ & & B & $!$ & & 1 & B & \\
\hline & & & & & $\mathrm{X}$ & & & \\
\hline & & $\mathrm{X}$ & & & & & & \\
\hline \multirow[t]{3}{*}{$\mathrm{H}$} & & & & & $\mathrm{X}$ & & & \\
\hline & & & & & & $X$ & & \\
\hline & & & & & & & B & \\
\hline $\mathrm{X}$ & $\mathrm{X}$ & & & $!$ & & ! & B & \\
\hline \multirow[t]{2}{*}{$\mathrm{H}$} & $\mathrm{X}$ & & & & & & & \\
\hline & & & & & B & & & \\
\hline $\mathrm{H}$ & & & B & & & & & \\
\hline$X$ & $\mathrm{X}$ & & $\mathrm{N}$ & & & $\mathrm{X}$ & B & \\
\hline $\mathrm{X}$ & B & & & & & & & \\
\hline $\mathrm{X}$ & $X$ & $\mathrm{X}$ & $\mathrm{N}$ & $\mathrm{N}$ & $X$ & $\mathrm{X}$ & B & \\
\hline
\end{tabular}

Magdalidini Pascoe, 1870 
Species

Magdalis cerasi (Linnaeus, 1758)

Magdalis exarata C.N.F. Brisout de Barneville, 1862

Magdalis flavicornis (Gyllenhal, 1836)

Magdalis frontalis (Gyllenhal, 1827)

Magdalis memnonia (Gyllenhal, 1837)

Magdalis nitidipennis Boheman, 1843

Magdalis rufa Germar, 1823

Magdalis ruficornis (Linnaeus, 1758)

Magdalis scutellaris K. Daniel, 1903

Magdalis violacea (Linnaeus, 1758)

Molytinae Schönherr, 1823

Cryptorhynchini Schönherr, 1825

Cryptorhynchina Schönherr, 1825

Cryptorhynchus lapathi (Linnaeus, 1758)

Tylodina Lacordaire, 1865

Acalles camelis (Fabricius, 1792)

Acalles lemur cisalpimus Stüben, 2003 [E]

Acalles papei A. Solari \& F. Solari, 1905

Acalles parvulus Boheman, 1837

Acallocrates denticollis (Germar, 1823)

Acallocrates minutesquamosus (Reiche, 1861)

Acallorneuma reitteri Mainardi, 1906 [E]

Coloracalles humerosus (Fairmaire, 1862)

Dichromacalles diocletianus (Germar, 1817)

Dichromacalles dromedarius (Boheman, 1844)

Dichromacalles rolletii (Germar, 1839)

Echinodera aspromontensis Stüben, 2008 [E]

Echinodera bellieri (Reiche, 1861)

Echinodera brisouti brisouti Reitter, 1885

Echinodera hypocrita (Boheman, 1837)

Echinodera siciliensis Stüben, 2003 [E]

Echinodera variegata (Boheman, 1837)

Elliptacalles longus (Desbrochers des Loges, 1892)

Kyklioacalles aubei (Boheman, 1837)

Kyklioacalles fausti (P. Meyer, 1896)

Kyklioacalles navieresi (Boheman, 1837)

Kyklioacalles roboris (Curtis, 1834)

Kyklioacalles saccoi (Colonnelli, 1973) [E]

Kyklioacalles solarii (A. Fiori, 1903) [E]

Onyxacalles luigionii (A. Solari \& F. Solari, 1907)

\begin{tabular}{|l|l|l|l|l|l|l|}
\hline $\mathrm{B}$ & $\mathrm{N}$ & & & & LC \\
\hline
\end{tabular}

\begin{tabular}{|l|l|l|l|l|l|l|l|l|}
\hline & $\mathrm{X}$ & $\mathrm{X}$ & $\mathrm{N}$ & & & $\mathrm{N}$ & \\
\hline $\mathrm{X}$ & & & & & & & $\mathrm{B}$ & \\
\hline $\mathrm{X}$ & & & & & & & $\mathrm{N}$ & \\
\hline & & & & & & $\mathrm{X}$ & & \\
\hline
\end{tabular}

$\mathrm{X} \quad \mathrm{N}$

Camptorhinus simplex Seidlitz, 1867

Camptorhimus statua (P. Rossi, 1790)

Ithyporini Lacordaire, 1865

Lepyrini Kirby, 1837

\begin{tabular}{|l|l|l|l|l|l|l|l|}
\hline Lepyrus palustris palustris (Scopoli, 1763) & $\mathrm{X}$ & $\mathrm{X}$ & & $\mathrm{N}$ & & & \\
\hline
\end{tabular}

Molytini Schönherr, 1823

Hylobiina Kirby, 1837

Hylobius abietis (Linnaeus, 1758)

Hylobius transversovittatus (Goeze, 1777)

Molytina Schönherr, 1823

Anisorhynchus barbarus (Boheman, 1834)

Anisorhynchus monachus monachus (Germar, 1817)

H X

\begin{tabular}{|l|l|l|l|l|l|l|l|}
\hline & & & N & X & X & B & LC \\
\hline B & & & & & & & LC \\
\hline
\end{tabular}

\begin{tabular}{|c|c|c|c|c|c|c|}
\hline & & & & & $\mathrm{X}$ & $\mathrm{CK}$ \\
$\mathrm{X}$ & & & $\mathrm{CK}$ & & $\mathrm{CK}$ & $\mathrm{CK}$ \\
\hline
\end{tabular}




\begin{tabular}{|c|c|c|c|c|c|c|c|c|c|}
\hline Species & 을 & $\begin{array}{l}\bar{c} \\
0 \\
3 \\
\frac{2}{0} \\
\frac{0}{0} \\
0\end{array}$ & 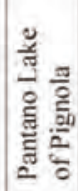 & 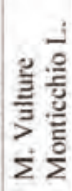 & 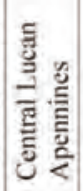 & $\begin{array}{l}\text { 氛 } \\
\frac{6}{2} \\
0 \\
\text { 言 } \\
0\end{array}$ & 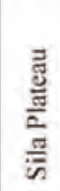 & 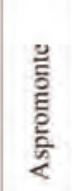 & 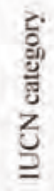 \\
\hline Leiosoma baudii Bedel, 1884 & $\mathrm{H}$ & & & & CK & $\mathrm{X}$ & CK & & \\
\hline Leiosoma colonnellii Pedroni, 2012 [E] & & & & & B & $\mathrm{B}$ & & & \\
\hline Leiosoma oblongulum Boheman, 1842 & & & & & & $\mathrm{X}$ & $\mathrm{X}$ & & \\
\hline Lipartus coronatus (Goeze, 1777) & & & & & & $\mathrm{X}$ & & & \\
\hline Liparus germanus (Linnaeus, 1758) & & & & & & & & B & \\
\hline Liparnis interruptus Magnano, 1955 [E] & & & & & & $\mathrm{B}$ & & & \\
\hline \multicolumn{10}{|c|}{ Plinthina Lacordaire, 1863} \\
\hline Hoplopteridius lutosus calaber G. Osella \& Lodos, 1979 [E] & & & & & & & & B & \\
\hline Minyops carinatus (Linnaeus, 1767) & & & & & & $\mathrm{B}$ & & CK & \\
\hline Minyops scrobiculatus Gyllenhal, 1834 [E] & & & & & & & & B & \\
\hline Mitoplinthus pubescens (Petri, 1896) & & & & & & & & B & \\
\hline Neoplinthus tigratus porculus (Fabricius, 1801) & $\mathrm{X}$ & & & & & & & & \\
\hline Neoplinthus tigratus tigratus (P. Rossi, 1792) [E] & B & & & & $\mathrm{N}$ & $\mathrm{X}$ & & CK & \\
\hline Plinthus illigeri colonnellii Meregalli, 1985 [E] & & & & & & $\mathrm{X}$ & & B & \\
\hline Plinthus megerlei (Panzer, 1802) & & & & & B & $\mathrm{X}$ & & & \\
\hline Plinthus squalidus gramulipennis Fairmaire, 1852 & $\mathrm{X}$ & & & B & CK & $\mathrm{X}$ & & B & \\
\hline
\end{tabular}

Pissodini Gistel, 1848

Pissodina Gistel, 1848

Pissodes castaneus (DeGeer, 1775)

Pissodes piceae (Illiger, 1807)

Pissodes validirostris (C.R. Sahlberg, 1834)

Torneumatini Bedel, 1884

\begin{tabular}{|l|l|l|l|l|l|l|l|}
\hline B & & N & & X & X & B & LC \\
\hline & & & N & X & X & B & LC \\
\hline & & & & X & X & & LC \\
\hline
\end{tabular}

Torneuma deplanatum deplanatum (Hampe, 1864)

Torneuma grouvellei grouvellei Desbrochers des Loges, 1890

Typoderini Voss, 1965

Aparopion chevrolati (Jacquelin du Val, 1855)

Aparopion suturidens Reitter, 1891

\begin{tabular}{|l|l|l|l|l|l|l|l|}
\hline $\mathrm{H}$ & $\mathrm{X}$ & & & $\mathrm{B}$ & $\mathrm{B}$ & $\mathrm{X}$ & $\mathrm{B}$ \\
\hline $\mathrm{X}$ & $\mathrm{X}$ & & $\mathrm{B}$ & $\mathrm{B}$ & $\mathrm{B}$ & $\mathrm{X}$ & $\mathrm{B}$ \\
\hline
\end{tabular}

Platypodinae Shuckard, 1840

Platypodini Shuckard, 1840

Platypus cylindrus (Fabricius, 1792)

Treptoplatypus oxyurus (Dufour, 1843)

Scolytinae Latreille, 1804

\begin{tabular}{|c|c|c|c|c|c|c|c|c|}
\hline H & X & & & & & X & N & LC \\
\hline
\end{tabular}

Corthylini LeConte, 1876

Pityophthorina Eichhoff, 1878

Pityophthonis glabratus Eichhoff, 1878

Pityophthorus lichrensteinii (Ratzeburg, 1837)

Pityophthorus pityographus pityographus (Ratzeburg, 1837)

Pityophthorus pubescens (Marsham, 1802)

Cryphalini Lindemann, 1877

Cryphalus asperatus (Gyllenhal, 1813

Ermoporicus fagi (Fabricius, 1798)

Crypturgus cinereus (Herbst, 1793)

Crypturgus numidicus Ferrari, 1867

Crypturgus pusillus (Gyllenhal, 1813)

Crypturgini LeConte, 1876

Coccotrypes dactyliperda (Fabricius, 1801)

Dactylotrypes longicollis (Wollaston, 1864)

Dryocoetes villosus villosus (Fabricius, 1792)

Taphrorhychus bicolor (Herbst, 1793)

Taphrorhychus villifrons (Dufour, 1843)

Thammurgus euphorbiae (Handschuch, 1845)

Dryocoetini Lindemann, 1877

\begin{tabular}{|l|l|l|l|l|l|l|l|l|}
\hline $\mathrm{X}$ & $\mathrm{X}$ & & & & & & & LC \\
\hline & & & & & $\mathrm{X}$ & & $\mathrm{N}$ & $\mathrm{LC}$ \\
\hline
\end{tabular}

\begin{tabular}{l|l|l|l|l|l|l|l|l|}
\hline \multicolumn{1}{|l|}{} & & & & & & B & \\
\hline & & & & & & & B & \\
\hline & & & & & N & & & LC \\
\hline X & X & & N & & X & X & N & LC \\
\hline H & & & & & & & & LC \\
\hline H & & & & & & & & LC \\
\hline
\end{tabular}


Species

Xylocleptes bispinus (Duftschmid, 1825)

Hylastini LeConte, 1876

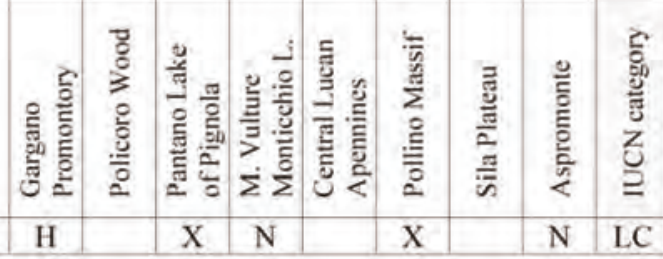

Hylastes angustatus (Herbst, 1763

Hylastes attenuatus Erichson, 1836

Hylastes pinicola (Bedel, 1888)

Hylurgops palliatus (Gyllenhal, 1813)

\begin{tabular}{|l|l|l|l|l|l|l|l|}
\hline & & N & & X & X & N & LC \\
\hline & & & & & X & & LC \\
\hline & & & & & X & & LC \\
\hline & & & & & X & N & LC \\
\hline
\end{tabular}

Hylesimus crenatus (Fabricius, 1787)

Hylesinus taranio (Danthoine, 1788)

Hylesinus varius (Fabricius, 1775)

Kissophagus novaki Reitter, I894

Kissophagus vicinus (Comolli, 1837)

Chaetoptelitis vestitus (Mulsant \& Rey, 1861)

Hylurgus ligniperda (Fabricius, 1787)

Hylurgus micklitzi Wachtl, 1881

Tomicus minor (Hartig, 1834)

Tomicus piniperda (Linnaeus, 1758)

Hylesinini Erichson, 1836

Hypoborus ficus Erichson, 1836

Hypoborini Nüsslin, 1911

Hylurgini Gistel, 1848

\begin{tabular}{|c|c|c|c|c|c|c|c|c|}
\hline & $\mathrm{X}$ & & & & & & & LC \\
\hline & $\mathrm{X}$ & & & & $\mathrm{N}$ & & \\
$\mathrm{X}$ & $\mathrm{X}$ & & & & $\mathrm{N}$ & & \\
\hline & $\mathrm{X}$ & $\mathrm{X}$ & & & & \\
\hline & $\mathrm{N}$ & & & & & & LC \\
\hline
\end{tabular}

\begin{tabular}{|c|c|c|c|c|c|c|c|}
\hline & $\mathrm{X}$ & & & & & & LC \\
\hline & & & & & $\mathrm{X}$ & & LC \\
\hline & & & & & & & LC \\
\hline & & & & & $\mathrm{X}$ & $\mathrm{B}$ & LC \\
\hline
\end{tabular}

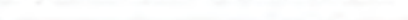

Ips acuminatus (Gyllenhal, 1827)

Ipini Bedel, 1888

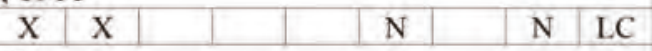

Ips sexdentatus (Boerner, 1776)

Ips typographus (Linnaeus, 1758)

Orthotomicus erosus (Wollaston, 1857)

Orthotomicus laricis (Fabricius, 1792)

Orthotomicus proximus (Eichhoff, 1868)

Orthotomicus suturalis (Gyllenhal, 1827)

Pityogenes bidentatus (Herbst, 1783)

Pityogenes bistridentatus (Eichhoff, 1878)

Pityogenes calcaratus (Eichhoff, 1878)

Pityogenes chalcographus (Linnaeus, 1760)

Pityogenes trepanatus (Nördlinger, 1848)

Pityokteines curvidens (Germar, 1824)

Phloeosinus thujae (Perris, 1855)

Phloeosinini Nüsslin, 1912

\begin{tabular}{|c|c|c|c|c|c|c|}
\hline & & & $\mathrm{X}$ & & & LC \\
\hline & & $\mathrm{N}$ & $\mathrm{X}$ & $\mathrm{X}$ & $\mathrm{N}$ & LC \\
\hline & & & & $\mathrm{X}$ & $\mathrm{N}$ & LC \\
\hline \multirow[t]{2}{*}{$X$} & $\mathrm{X}$ & & $\mathrm{N}$ & & & LC \\
\hline & $X$ & $\mathrm{~N}$ & $\mathrm{X}$ & $X$ & & LC \\
\hline \multirow[t]{3}{*}{ B } & & & & & & LC \\
\hline & & & $\mathrm{X}$ & & & LC \\
\hline & & & $\mathrm{X}$ & $\mathrm{X}$ & & LC \\
\hline B? & & & $\mathrm{X}$ & $X$ & $\mathrm{~N}$ & LC \\
\hline B & & & & $\mathrm{X}$ & & LC \\
\hline \multirow[t]{3}{*}{$X$} & & & & & & LC \\
\hline & & & $\mathrm{X}$ & & & LC \\
\hline & & & $X$ & & & LC \\
\hline
\end{tabular}

\section{Phloeotribini Chapuis, 1869}

Phloeotribus cristatus (Fauvel, 1889)

Phloeotribus rhododactylus (Marsham, 1802)

Phloeotribus scarabaeoides (Bernard, 1788)

Polygraphini Chapuis, 1869

Carphobortus pini Eichhoff, 188

Polygraphus grandiclava C.G. Thomson, 1886

Scolytini Latreille, 1804

Scolytus amygdali Guérin-Méneville, 1847

Scolytus ensifer Eichhoff, 1881

Scolytus intricatus (Ratzeburg, 1837)

Scolytus kirschii kirschii Skalitzky, 1876

Scolytus multistriatus (Marsham, 1802)

Scolytus pygmaeus (Fabricius, 1787)

Scolytus rugulosus (P.W.J. Müller, 1818)

Scolytus sulcifrons Rey, 1892

\begin{tabular}{|c|c|c|c|c|c|c|c|}
\hline $\mathrm{X}$ & & & & $\mathrm{N}$ & & $\mathrm{N}$ & LC \\
\hline & $\mathrm{X}$ & & & & & & VU \\
\hline & & & & & $X$ & & LC \\
\hline & $\mathrm{X}$ & & & & & & LC \\
\hline $\mathrm{X}$ & $\mathrm{X}$ & $\mathrm{N}$ & B & $\mathrm{N}$ & $\mathrm{X}$ & $\mathrm{N}$ & LC \\
\hline & $\mathrm{X}$ & & & & & & LC \\
\hline $\mathrm{H}$ & $\mathrm{X}$ & & & $\mathrm{X}$ & $\mathrm{X}$ & & LC \\
\hline & $\mathrm{N}$ & & B & & & & LC \\
\hline
\end{tabular}




\begin{tabular}{|c|c|c|c|c|c|c|c|c|c|}
\hline Species & 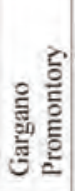 & 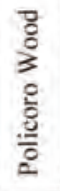 & 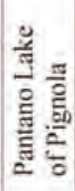 & 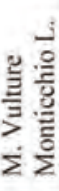 & 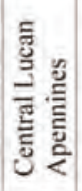 & 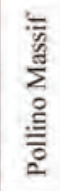 & 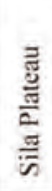 & $\begin{array}{l}\frac{3}{5} \\
\frac{5}{5} \\
\frac{5}{2} \\
\frac{5}{2}\end{array}$ & 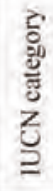 \\
\hline \multicolumn{10}{|c|}{ Xyleborini LeConte, 1876} \\
\hline Anisandrus dispar (Fabricius, 1792) & $\mathrm{X}$ & & & & & & & & LC \\
\hline Xyleborinus saxesenii (Ratzeburg, 1837) & $\mathrm{H}$ & $\mathrm{X}$ & $\mathrm{X}$ & $\mathrm{N}$ & & & & $\mathrm{N}$ & LC \\
\hline Xyleborus dryographis (Ratzeburg, 1837) & $\mathrm{H}$ & $\mathrm{N}$ & & & & & & & LC \\
\hline Xylebortus eurygraphus (Ratzeburg, 1837) & & & & & & & & B & LC \\
\hline Xylebortus monographus (Fabricius, 1792) & $\mathrm{H}$ & & $\mathrm{X}$ & & & $\mathrm{X}$ & $\mathrm{X}$ & & LC \\
\hline \multicolumn{10}{|c|}{ Xyloterini LeConte, 1876} \\
\hline Trypodendron domesticus (Linnaeus, 1758) & & $\mathrm{N}$ & & & & $\mathrm{X}$ & $\mathrm{X}$ & & LC \\
\hline Trypodendron lineatumsa (A.G. Olivier, 1800) & & & & & & $\mathrm{X}$ & & & LC \\
\hline Trypodendron signatus (Fabricius, 1792) & & & & & & $\mathrm{x}$ & & & LC \\
\hline \multicolumn{10}{|c|}{ Curculionidae taxa incertae sedis } \\
\hline Absoloniella servadeii (G. Osella, 1982) [E] & B & & & & & & & & \\
\hline
\end{tabular}

\section{CONCLUSIONS}

The latest author's research as well as the data available in literature have greatly increased the number of known species in the surveyed areas. The situation that emerges is summarized in following Table 2.

The presence of saproxylic species in the study sites is rather cospicuous; in fact, they amount to $56.1 \%$ of the overall saproxylic species known for Italy. However, this figure should not be misleading, since the last column of following Table 3 shows that the "Critically endangered" (CR) species make up just $25.9 \%$ of the total on Italy and the "Endangered" species (EN) and "Vulnerable" (VU) account for $27 \%$ and $40 \%$ respectively.

It can be deduced that the level of research, especially in some of the areas, is not yet sufficiently exhaustive and that the sites subject of the survey suffer from a considerable degree of anthropization or incorrect management of wooded areas, so that the entomofauna of special conservation interest proves to be, to some extent, impoverished. In many recent papers on saproxylic fauna the need to maintain a certain amount of dead wood in the forests is underlined, a good practice that unfortunately is often not applied.

According to the fifty-year experience of the author researching Coleoptera, it would be necessary to establish integral nature reserves in ancient woodland as well as regulating livestock grazing in grasslands that are increasingly depleted.
It is considered useful and necessary, mainly in the perspective of future research, to comment on the data presented here despite the fact that it is difficult to separate the mere judgment based on sterile numbers from personal sentiment, because these are places that the author investigated for more than four decades (starting from distant 1968) witnessing too many changes, often irreversible, with consequent progressive decline of the fauna.

Particularly, from an examination of the data summarized in Tables 2 and 4, it clearly emerges that only the Coleoptera fauna of Policoro Wood can be considered sufficiently studied. With its 2,353 species collected in an area of just 680 hectares, this site is perhaps one of those that show greater biodiversity throughout the Mediterranean basin. The reasons are many: it is an ancient floodplain forest with a quite varied and dense vegetation so that it preserves a certain microclimate and, although today reduced to a very small portion of those forests that once covered the entire Metapontino, for this same reason it represents a last exiguous shelter for all those forest dwelling species that have undergone the inexorable erosion of their habitat; it is also placed in a favorable position at the mouth of Sinni river which, especially before the construction of the Monte Cotugno dam, during the floods washed downstream huge quantities of debris, and with them species, from inland; last but not least, studies here have been conducted extensively by researchers of several disciplines. 


\begin{tabular}{|l|r|r|c|}
\hline Site & $\begin{array}{c}\text { Species in } \\
\text { Angelini's } \\
\text { catalogues } \\
(1986-1996)\end{array}$ & $\begin{array}{c}\text { New data } \\
\text { by Angelini }+ \\
\text { in literature }+ \\
\text { in CKmap }\end{array}$ & $\begin{array}{c}\text { Total species } \\
\text { known } \\
\text { to date }\end{array}$ \\
\hline Gargano Promontory & 2,291 & 301 & 2,592 \\
\hline Policoro Wood & 1,823 & 530 & 2,353 \\
\hline Pantano Lake of Pignola & 992 & 259 & 1,251 \\
\hline Mount Vulture-Monticchio Lakes & - & 1,427 & 1,427 \\
\hline Central Lucan Apennines & - & 2,315 & 2,315 \\
\hline Pollino Massif & 1,824 & 931 & 2,755 \\
\hline Sila Plateau & 1,741 & 350 & 2,091 \\
\hline Aspromonte & - & 2,715 & 2,715 \\
\hline
\end{tabular}

Table 2. The increase in number of known species, from the publication of the catalogues on Coleoptera in the 1980s-1990s to the present.

\begin{tabular}{|c|c|c|c|c|c|c|c|c|c|c|c|}
\hline Z & 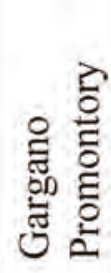 & 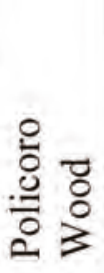 & 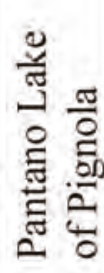 & 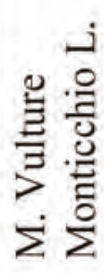 & 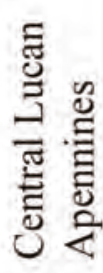 & 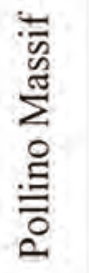 & 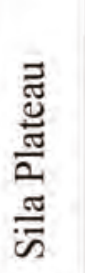 & 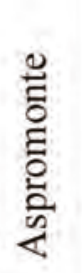 & 要 & $\begin{array}{l}\bar{\Xi} \\
\frac{\pi}{0} \\
\text { 焉 }\end{array}$ & $\therefore$ \\
\hline CR & 4 & 8 & & 5 & 8 & 9 & 3 & 6 & 21 & 81 & 25.9 \\
\hline $\mathrm{EN}$ & 10 & 15 & 2 & 3 & 11 & 14 & 15 & 8 & 33 & 122 & 27.0 \\
\hline VU & 31 & 31 & 1 & 7 & 31 & 55 & 36 & 38 & 86 & 215 & 40.0 \\
\hline NT & 80 & 64 & 12 & 20 & 69 & 92 & 77 & 90 & 191 & 348 & 54.9 \\
\hline $\mathrm{LC}$ & 395 & 393 & 122 & 243 & 368 & 484 & 372 & 415 & 708 & 977 & 72.5 \\
\hline DD & 25 & 26 & 11 & 8 & 26 & 23 & 14 & 36 & 74 & 240 & 30.8 \\
\hline NA & & 1 & & & & & & 1 & 2 & - & - \\
\hline Total & 545 & 538 & 148 & 286 & 513 & 677 & 517 & 594 & 1,115 & 1,986 & 56.1 \\
\hline
\end{tabular}

Table 3. Number of saproxylic species in each of the surveyed areas, compared to the total and percentage for Italy (CR: Critically Endangered; EN: Endangered; VU: Vulnerable; NT: Nearly Threatened; LC: Least Concern; DD: Data Deficient; NA: Not Applicable).

Even the Coleoptera fauna of Pantano Lake of Pignola can be considered sufficiently known taking into account the features and limited extent of this site, as evidenced by the high number of species typical of meadow areas and of lakes shores that it shows.

In the district of Mount Vulture-Monticchio
Lakes, despite the relatively small size, the number of species surveyed is probably less than the expected total considering the peculiar characteristics of the biotope.

Gargano Promontory, although explored at length by several researchers and given the variety of environments and the extension, records a num- 
ber of species $(2,591)$ decidedly lower than a realistic estimation. It can be assumed that especially the fauna of the coasts, where saved by the tourist encroachment and pressure, should still reveal many novelties.

Fauna of Central Lucan Apennines, known for over $2 / 3$ on the basis of published or unpublished personal data, could prove to be more abundant, as shown by particularly targeted research conducted by other entomologists in the Gallipoli-Cognato woods.

Faunal total of Pollino Massif, in the light of the many and often interdisciplinary researches it has been subjected to and the fact that most of the data comes from territories located over 1,000 meters above sea level, can be considered sufficiently investigated and close to the real one.

As far as Sila Plateau is concerned, the data possessed or deduced from bibliography are decidedly incomplete; it is to be regarded as the site whose fauna is less well known, especially that of the soil, as will be discussed later about Staphylinidae.

The Aspromonte, curiously, shows a species number almost similar to that of Gargano Promontory with which it shares, broadly, also the territorial extension, despite having been less investigated. And like Gargano Promontory, it is believed that the currently known fauna of Aspromonte, based for $1 / 3$ on mostly previously unpublished personal data, is partially incomplete and that more data may emerge from research aimed at the study mainly of floricolous species both of mountainous areas and of the shorelines least damaged by human impact.

A numerical analisys of the presence of the various families in each of the sites (Table 4) confirms and amplifies what has been remarked above. Taking into account the 12 families represented by over 100 species in this study, the following observations can be made:

- Ptinidae (103 species): in Pollino Massif, Policoro Wood, Aspromonte and Gargano Promontory the number of species varies between $47-54$ (equal to approximately $50 \%$ of the treated species); the 27 and 34 species recorded for Mount VultureMonticchio Lakes and Central Lucan Apennines are believed not to fully represent the actual presence of this family in these areas, while the low number of species known for Pantano Lake of Pignola is to be attributed to the particular features of the site.
- Nitidulidae (113 species): represented on average by $51-68$ species, the high and significant number of species known for Pollino Massif (87, that is $77 \%$ of total) stands out, while the 30 species known for Mount Vulture-Monticchio Lakes are to be considered heavily underestimated; regarding this family it should however be noted that 7,500 specimens collected by the author are still undetermined.

- Elateridae (117 species): represented on average with about 53-63 species, the positive peak of 77 species for Pollino Massif should be noted while the lesser total of 22 for the Pantano Lake of Pignola is largely imputable to the particular characteristics of the site; Sila Plateau and Aspromonte confirm that they have a similar trend (75 and 72 species respectively).

- Brentidae (sensu lato) (134 species): it is interesting to note how Gargano Promontory, Pollino Massif and Aspromonte are settled on 76-80 species while Policoro Wood has a strange smaller peak of 47 species, similar to that of Central Lucan Apennines. The number of species known for Pantano Lake of Pignola is significant, 52 species, reflecting the high biodiversity of this site regarding the floricolous fauna, a situation also found for other families not discussed here (Coccinellidae).

- Buprestidae (135 species): in this family a high number of known species is recorded for Pollino Massif (96, equal to $71.1 \%$ of the total) and Policoro Wood (95); the other sites have 70-77 species while the small number of Pantano Lake of Pignola stands out, 21 species collected only in meadows surrounding the lake, given the almost total absence of trees nearby.

- Tenebrionidae (147 species): family whose species have a remarkably diversified ecology, not by chance for Aspromonte, Gargano Promontory and Policoro Wood, therefore sites that include shorelines, 77-90 species are known (90 for the first site) while Central Lucan Apennines, Pollino Massif and Sila Plateau stand on 48-61 species; the lesser total of just 20 species of Pantano Lake of Pignola is due to its peculiar geomorphological characteristics.

- Scarabaeidae (169 species): the average number in most sites (five) varies between 87 and 99 species; the primacy of known species belongs to Aspromonte (120) and the negative one to Pantano Lake of Pignola (just 29). 


\begin{tabular}{|c|c|c|c|c|c|c|c|c|c|c|}
\hline Family & 总 & 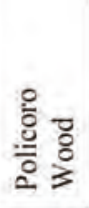 & 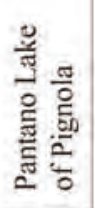 & 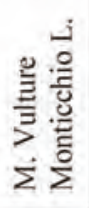 & 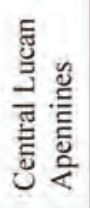 & 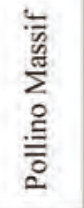 & 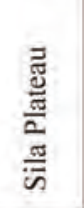 & 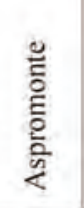 & 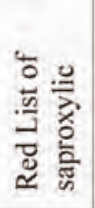 & $\frac{\sqrt{5}}{\circ}$ \\
\hline Sphaeriusidae & & 1 & & & & & & & & 1 \\
\hline Gyrinidae & 5 & 8 & 3 & 4 & 6 & 4 & 4 & 5 & & 9 \\
\hline Rhysodidae & 2 & 1 & & 2 & 3 & 2 & 1 & 2 & 3 & 3 \\
\hline Carabidae & 233 & 253 & 109 & 172 & 224 & 220 & 201 & 278 & & 475 \\
\hline Haliplidae & 8 & 9 & 3 & 4 & 6 & 5 & 5 & 5 & & 9 \\
\hline Noteridae & 1 & 2 & 1 & 1 & 1 & 1 & 2 & 1 & & 2 \\
\hline Hygrobiidae & 1 & 1 & & & 1 & 1 & & & & 1 \\
\hline Dytiscidae & 40 & 49 & 23 & 16 & 52 & 45 & 44 & 54 & & 89 \\
\hline Hydrophilidae & 24 & 40 & 26 & 18 & 19 & 30 & 30 & 34 & & 65 \\
\hline Helophoridae & 12 & 12 & 6 & 7 & 4 & 13 & 9 & 6 & & 18 \\
\hline Hydrochidae & 1 & 2 & 1 & 1 & 1 & 3 & 2 & 1 & & 3 \\
\hline Georissidae & & 2 & & & 1 & 2 & & 3 & & 3 \\
\hline Histeridae & 73 & 51 & 11 & 29 & 62 & 55 & 51 & 54 & 26 & 98 \\
\hline Hydraenidae & 15 & 11 & 9 & 6 & 21 & 20 & 18 & 27 & & 53 \\
\hline Ptiliidae & 19 & 18 & 11 & 12 & 20 & 17 & 11 & 27 & 28 & 45 \\
\hline Agyrtidae & & & & & & 1 & & 1 & & 1 \\
\hline Leiodidae & 40 & 30 & 17 & 31 & 47 & 50 & 32 & 44 & 24 & 75 \\
\hline Silphidae & 6 & 8 & 4 & 5 & 6 & 10 & 9 & 8 & & 13 \\
\hline Staphylinidae & 368 & 319 & 222 & 198 & 396 & 400 & 230 & 431 & 96 & 853 \\
\hline Geotrupidae & 7 & 5 & 3 & 5 & 5 & 6 & 6 & 7 & & 11 \\
\hline Trogidae & 1 & 2 & & & 1 & 1 & & 1 & 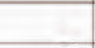 & 2 \\
\hline Lucanidae & 3 & 5 & I & 4 & 5 & 5 & 5 & 4 & 6 & 6 \\
\hline Ochodaeidae & & 2 & & & & & & & & 2 \\
\hline Hybosoridae & & 1 & 1 & & & & & 1 & & 1 \\
\hline Scarabaeidae & 97 & 87 & 29 & 68 & 88 & 99 & 90 & 120 & 14 & 169 \\
\hline Eucinetidae & & 1 & 1 & & & 1 & 2 & 2 & & 2 \\
\hline Clambidae & 5 & 7 & 4 & 3 & 3 & 7 & 3 & 5 & 12 & 12 \\
\hline Scirtidae & 4 & 8 & 5 & 6 & 9 & 8 & 7 & 8 & 1 & 18 \\
\hline Dascillidae & 1 & & & & 1 & 1 & 1 & 1 & & 1 \\
\hline Buprestidae & 70 & 95 & 21 & 39 & 77 & 96 & 74 & 72 & 96 & 135 \\
\hline Byrrhidae & 2 & 1 & 1 & & 2 & 5 & 3 & 2 & 1 & 9 \\
\hline Elmidae & & & 1 & 2 & 5 & 9 & 8 & 8 & & 12 \\
\hline Dryopidae & 2 & 7 & 3 & 3 & 3 & 4 & 4 & 5 & & 12 \\
\hline Limnichidae & 1 & 4 & 3 & 1 & 2 & 4 & & 3 & & 4 \\
\hline Heteroceridae & 5 & 6 & 2 & 3 & 5 & 6 & 2 & 4 & & 7 \\
\hline Artematopodidae & & & & & 1 & & & & & 1 \\
\hline Eucnemidae & 2 & 2 & & 1 & 1 & 4 & 5 & 5 & 8 & 8 \\
\hline Throscidae & 1 & 4 & 7 & 1 & 7 & 4 & 3 & 6 & 11 & 12 \\
\hline Lycidae & 3 & 2 & & 2 & 2 & 4 & 5 & 5 & 5 & 5 \\
\hline Lampyridae & 2 & 2 & 2 & & 2 & 3 & 3 & 2 & & 5 \\
\hline Cantharidae & 35 & 21 & 12 & 28 & 30 & 58 & 40 & 40 & & 77 \\
\hline Omalisidae & & 2 & & & 1 & & & & & 2 \\
\hline Elateridae & 62 & 53 & 22 & 39 & 63 & 77 & 75 & 72 & 36 & 117 \\
\hline Derodontidae & & 1 & & & 1 & 1 & & & 1 & 1 \\
\hline Nosodendridae & & & & & 1 & & & & 1 & 1 \\
\hline Dermestidae & 22 & 20 & 9 & 11 & 12 & 26 & 18 & 12 & 11 & 42 \\
\hline Bostrichidae & 9 & 11 & 5 & 4 & 8 & 7 & 6 & 12 & 17 & 18 \\
\hline
\end{tabular}

Table 4/1. Number of species, per family, in each of the sites, in the Italian Red List of saproxylic Coleoptera and total. 


\begin{tabular}{|c|c|c|c|c|c|c|c|c|c|c|}
\hline Family & 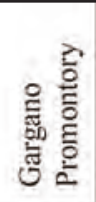 & $\begin{array}{l}\circ \\
\frac{0}{8} \\
\frac{.}{0} \\
\vdots\end{array}$ & 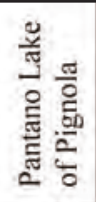 & 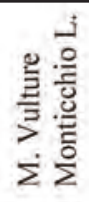 & 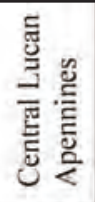 & 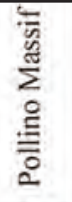 & 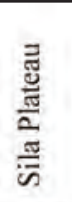 & 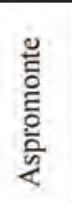 & 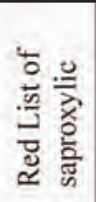 & 跑 \\
\hline Ptinidae & 47 & 53 & 10 & 27 & 34 & 54 & 30 & 50 & 65 & 103 \\
\hline Lymexylidae & 1 & & & & 1 & 1 & 1 & 2 & 2 & 2 \\
\hline Byturidae & 1 & & & & & 2 & 1 & & & 2 \\
\hline Biphyllidae & 2 & 3 & & 1 & 2 & 3 & 2 & 2 & 3 & 3 \\
\hline Peltidae & & & & & & 2 & 2 & 2 & 2 & 2 \\
\hline Lophocateridae & & & & & & 1 & & I & 1 & 1 \\
\hline Trogossitidae & 3 & 2 & & 2 & 2 & 2 & 2 & 3 & 3 & 3 \\
\hline Thymalidae & & & & & & 1 & 1 & 1 & 1 & 1 \\
\hline Rhadalidae & 2 & 3 & & 1 & 6 & 3 & 3 & 6 & 8 & 11 \\
\hline Melyridae & 41 & 32 & 22 & 30 & 41 & 52 & 37 & 45 & 17 & 77 \\
\hline Cleridae & 13 & 12 & 6 & 7 & 14 & 15 & 10 & 13 & 12 & 20 \\
\hline Sphindidae & 2 & 3 & & 1 & 1 & 2 & 1 & 2 & 3 & 3 \\
\hline Erotylidae & 8 & 8 & & 5 & 5 & 11 & 7 & 6 & 14 & 15 \\
\hline Monotomidae & 8 & 8 & 5 & 5 & 5 & 6 & 4 & 5 & 13 & 13 \\
\hline Kateretidae & 6 & 4 & & 2 & 2 & 4 & 5 & 3 & & 9 \\
\hline Nitidulidae & 56 & 45 & 32 & 30 & 45 & 87 & 51 & 68 & 26 & 113 \\
\hline Cryptophagidae & 31 & 31 & 22 & 15 & 36 & 32 & 20 & 39 & 30 & 68 \\
\hline Silvanidae & 7 & 7 & 6 & 4 & 4 & 5 & 2 & 7 & 2 & 8 \\
\hline Cucujidae & 1 & & 1 & & 1 & 3 & 4 & 2 & 4 & 4 \\
\hline Phalacridae & 11 & 11 & 11 & 9 & 14 & 17 & 10 & 12 & & 23 \\
\hline Laemophloeidae & 7 & 9 & 2 & 2 & 5 & 7 & 4 & 5 & 7 & 9 \\
\hline Cybocephalidae & & I & & & & 1 & & 1 & & 1 \\
\hline Bothrideridae & 1 & & & & 2 & 1 & 1 & 1 & 2 & 2 \\
\hline Teredidae & 3 & 2 & & 1 & 2 & 2 & & 3 & 2 & 3 \\
\hline Cerylonidae & 5 & 4 & 1 & 3 & 6 & 5 & 4 & 4 & 6 & 6 \\
\hline Latridiidae & 24 & 30 & 23 & 15 & 30 & 27 & 19 & 38 & 42 & 55 \\
\hline Alexiidae & 3 & & & 3 & 4 & 4 & 2 & 5 & 5 & 5 \\
\hline Corylophidae & 7 & 10 & 6 & 3 & 6 & 12 & 4 & 9 & 7 & 18 \\
\hline Anamorphidae & 1 & 1 & 1 & 2 & 1 & 3 & & 2 & 3 & 3 \\
\hline Endomychidae & 10 & 13 & 1 & 4 & 7 & 11 & 5 & 15 & 5 & 19 \\
\hline Mycetaeidae & 1 & & & & 1 & 1 & 1 & 1 & 1 & 1 \\
\hline Coccinellidae & 44 & 42 & 44 & 28 & 34 & 41 & 42 & 41 & & 77 \\
\hline Mycetophagidae & 8 & 9 & 3 & 6 & 7 & 8 & 8 & 9 & 12 & 13 \\
\hline Ciidae & 12 & 16 & 4 & 9 & 11 & 23 & 9 & 11 & 27 & 30 \\
\hline Tetratomidae & & & & & 1 & & 1 & 2 & 2 & 2 \\
\hline Melandryidae & 3 & 2 & & 1 & 2 & 7 & 7 & 8 & 10 & 10 \\
\hline Mordellidae & 21 & 16 & 2 & 1 & 4 & 23 & 14 & 2 & 1 & 35 \\
\hline Ripiphoridae & 1 & 1 & & & 1 & & & 2 & & 2 \\
\hline Zopheridae & 11 & 9 & 3 & 8 & 14 & 14 & 10 & 13 & 20 & 20 \\
\hline Tenebrionidae & 78 & 77 & 20 & 30 & 48 & 61 & 58 & 90 & 49 & 147 \\
\hline Prostomidae & & & & & & 1 & & & 1 & 1 \\
\hline Oedemeridae & 14 & 16 & 8 & 13 & 19 & 18 & 16 & 18 & 11 & 23 \\
\hline Meloidae & 20 & 11 & 6 & 9 & 13 & 15 & 19 & 20 & & 27 \\
\hline Mycteridae & 3 & 2 & 1 & 1 & 1 & 2 & 2 & & & 3 \\
\hline Pyrochroidae & 1 & I & 1 & 1 & 2 & 2 & 2 & 2 & 3 & 3 \\
\hline Salpingidae & 3 & 3 & 3 & 1 & 3 & 5 & 3 & 6 & 7 & 7 \\
\hline Anthicidae & 26 & 33 & 16 & 10 & 15 & 22 & 17 & 30 & & 48 \\
\hline
\end{tabular}

Table 4/2. Number of species, per family, in each of the sites, in the Italian Red List of saproxylic Coleoptera and total. 


\begin{tabular}{|c|c|c|c|c|c|c|c|c|c|c|}
\hline Family & 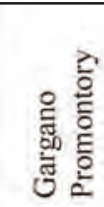 & 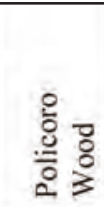 & 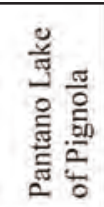 & 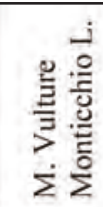 & 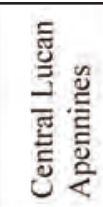 & 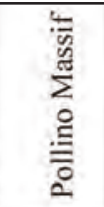 & 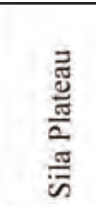 & 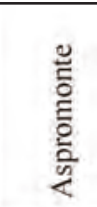 & 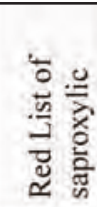 & 傿 \\
\hline Aderidae & 3 & 1 & 2 & & 2 & 1 & 3 & 3 & 4 & 5 \\
\hline Scraptiidae & 18 & 17 & 3 & 6 & 5 & 15 & 12 & 8 & 8 & 25 \\
\hline Vesperidae & 1 & 1 & 1 & & 1 & 1 & 1 & 1 & & 1 \\
\hline Cerambycidae & 107 & 84 & 25 & 73 & 105 & 120 & 101 & 89 & 150 & 181 \\
\hline Megalopodidae & & & & & & 1 & & & & 1 \\
\hline Orsodacnidae & I & & 1 & 1 & 1 & 1 & 1 & 1 & & 1 \\
\hline Chrysomelidae & 237 & 181 & 161 & 107 & 210 & 223 & 176 & 205 & & 427 \\
\hline Nemonychidae & & & & & & 1 & & & & 1 \\
\hline Anthribidae & 11 & 10 & 4 & 4 & 6 & 8 & 5 & 8 & 13 & 15 \\
\hline Attelabidae & 23 & 12 & 7 & 17 & 23 & 20 & 17 & 19 & & 30 \\
\hline Brentidae & 80 & 47 & 52 & 44 & 47 & 80 & 66 & 76 & 1 & 134 \\
\hline Curculionidae & 382 & 304 & 157 & 159 & 258 & 350 & 259 & 320 & 113 & 750 \\
\hline Totals & 2,592 & 2,353 & 1,251 & 1,427 & 2,315 & 2,755 & 2,091 & 2,715 & 1,115 & 5,130 \\
\hline
\end{tabular}

Table 4/3. Number of species, per family, in each of the sites, in the Italian Red List of saproxylic Coleoptera and total.

- Cerambycidae (181 species): the numerical dynamics of this family that accounts for the greatest quantity of saproxylic species is particularly important and significant; while Pollino Massif shows up for the greater number of species (120), Pantano Lake of Pignola is confirmed as having the least diverse fauna with just 25 species; indicative is the clear difference, generally not perceptible, between the fauna of Gargano Promontory and that of Aspromonte, 107 and 89 species respectively, while strangely Policoro Wood with 84 species places itself below Sila Plateau and Central Lucan Apennines which count, respectively, 101 and 105 .

- Chrysomelidae (427 species): Gargano Promontory with 237 species has the highest expression of biodiversity, while the discrepancy between Pollino Massif (223 species) and Aspromonte (205) validates what has already been said regarding the further research needed on the latter. Sila Plateau as well, with just 176 species (181 for Policoro Wood), confirms how little this area has been investigated. Mount Vulture-Monticchio Lakes with 107 species require further in-depth research, while Pantano Lake of Pignola with 161 species corroborate a high biodiversity for the species linked to the meadows.

- Carabidae (475 species): Aspromonte (278 species) is the significantly richest area, while four sites follow with a number of species ranging between 201 (Sila) and 233 (Gargano); Policoro Wood, despite its small size, with 253 known species far exceeds the remaining sites.

- Curculionidae (750 species): it is perhaps the only case in which Gargano Promontory shows the greatest richness with 382 species and reaffirms the contrast already noted with Aspromonte which counts only 320; Sila Plateau and Central Lucan Apennines stand respectively on 259 and 258 species, while Pantano Lake of Pignola and Mount Vulture-Monticchio Lakes have only 157 and 159 species; the Pollino Massif with 350 species sees its high biodiversity confirmed.

- Staphylinidae (853 species): particularly interesting and enlightening for future research and to understand the current state of the same is the analysis of the presence of this important family, mainly linked to the soil: Aspromonte stands out with 431 species that is 201 more than the nearby Sila Plateau (which would be the last, if not for the much smaller sites of Pantano Lake of Pignola and Mount Vulture-Monticchio Lakes); Gargano Promontory, Central Lucan Apennines and Pollino Massif vary between 368 and 400 species, while Policoro Wood, despite its small surface area, numbers 319 species; it should however be emphasized that the writer's contribution to the knowledge of this family was 
much lower than for other families, as 17,000 collected specimens are still unidentified.

On the basis of what has just been explained, it can be concluded that the research on the floricolous and saproxylic beetles of Aspromonte should be further increased, and that the fauna of the soil is still to be investigated more widely and in more detail, mainly in the Sila Plateau and Gargano Promontory. Policoro Wood and the Pollino Massif show a state of knowledge that can be considered acceptable, while Mount Vulture-Monticchio Lakes are further to be studied at a general level.

\section{ACKNOWLEDGEMENTS}

I would like to thank my colleagues and friends who, with effective collaboration and generosity, have spent time reviewing the families of their competence, sending papers and sharing valuable data, thus making it possible to accomplish this study and bring it up to date: Paolo Audisio (Università la Sapienza, Dipartimento di Biologia e Biotecnologie "Charles Darwin", Roma, Italy), Xavier Bellés (Institute of Evolutionary Biology (CSIC-UPF), Barcelona, Spain), Cesare Bellò (Castelfranco Veneto, Italy), Alessandro B. Biscaccianti (Laboratorio di Entomologia ed Ecologia Applicata (LEEA), Dipartimento PAU, Università Mediterranea di Reggio Calabria, Italy), Lech Borowiec (Department of Biodiversity and Evolutionary Taxonomy, University of Wrocław, Poland), Milan Boukal (Pardubice, Czech Republic), Roberto Caldara (Milano, Italy), Benjamin Calmont (Société d'Histoire Naturelle Alcide-d'Orbigny, Aubière, France), Enzo Colonnelli (Roma, Italy), Gianfranco Curletti (Museo Civico di Storia Naturale, Carmagnola, Italy), Mauro Daccordi (Verona, Italy), Luciano Diotti (Cinisello Balsamo, Italy), Michael Eifler (Pinneberg, Germany), Roberto Fabbri (Museo Civico, Sezione Naturalistica, Bagnacavallo, Italy), Gabriele Franzini (Milano, Italy), Carlo Giusto (Recco, Italy), Jiř́ Hájek (Department of Entomology, National Museum, Praha, Czech Republic), Jiř́i Háva (Private Entomological Laboratory and Collection, Prague-west, Czech Republic), Francesco Izzillo (Napoli, Italy), Jiř́ Kolibáč (Moravian Museum, Department of Entomology, Brno, Czech Republic), Ondřej Konvička (Institute of Entomology, Biology Centre AS CR, České Budějovice, Czech Republic), Robin Kundrata (Department of Zoology, Faculty of Science, Palacky University, Olomouc, Czech Republic), Leonardo Latella (Museo Civico di Storia Naturale, Verona, Italy), Piero Leo (Cagliari, Italy), Carlo Leonardi (Museo Civico di Storia Naturale, Milano, Italy), Gianfranco Liberti (Uboldo, Italy), Andrea Liberto (Roma, Italy), Gianluca Magnani (Cesena, Italy), Paolo Magrini (Firenze, Italy), Alessandro Mascagni (Firenze, Italy), Jan Matějíček (Hradec Králové, Czech republik), Antonio Mazzei (Università della Calabria, Museo di Storia Naturale della Calabria ed Orto Botanico, Rende, Italy), Josef Mertlik (Opatovice nad Labem, Czech Republic), Heinrich Meybohm (Großhansdorf, Germany), Vittorino Monzini (San Giuliano Milanese, Italy), Gianluca Nardi (Centro Nazionale Biodiversità Carabinieri "Bosco Fontana", Marmirolo, Italy), José Carlos Otero (Departamento de Zoología y Antropología Física, Universidad de Santiago de Compostela, Spain), Roberto A. Pantaleoni (Dipartimento di Protezione delle Piante, sezione di Entomologia agraria, Università degli Studi, Sassari, Italy), Guido Pedroni (Parco Regionale del Corno alle Scale, Marzabotto, Italy), Emanuele Piattella (Università la Sapienza, Dipartimento di Biologia e Biotecnologie "Charles Darwin", Roma, Italy), Giuseppe Platia (Gatteo, Italy), Pavel Průdek (Brno, Czech Republic), Pierpaolo Rapuzzi (Prepotto, Italy), Roberto Rattu (Cagliari, Italy), Johannes Reibnitz (Tamm, Germany), Antonio Rey (Genova, Italy), Saverio Rocchi (Firenze, Italy), Vito Antonio Romano (Potenza, Italy), Wolfgang Rücker (Neuwied, Germany), Rafal Ruta (Department of Biodiversity and Evolutionary Taxonomy, University of Wrocław, Poland), Jan Růžička (Department of Ecology, Faculty of Environmental Sciences, Czech University of Life Sciences Prague, Praha, Czech Republic), Enrico Ruzzier (Mirano, Italy), Davide Sassi (Castelmarte, Italy), Mikael Sörensson (Department of Biology, Lund University, Ecology Building, Lund, Sweden), Zdeněk Švec (Praha, Czech Republic), Dmitry Telnov (Natural History Museum, London, United Kingdom), Wioletta Tomaszewska (Museum and Institute of Zoology, Polish Academy of Sciences, Warszawa, Poland), Jiří Ch. Vávra (Ostrava Museum, Ostrava, Czech Republic), Pierpaolo Vienna (Lido di Venezia, Italy), Vincenzo Vomero (Roma, Italy), Piotr 
Węgrzynowicz (Institute of Forest Sciences, University of Łódź, Branch in Tomaszów, Tomaszów Mazowiecki, Poland), Rafael Yus Ramos (VélezMálaga, Málaga, Spain), Petr Zahradník (Forestry and Game Management Research Institute, Jíloviště, Czech Republic), Adriano Zanetti (Verona, Italy), Iuri Zappi (Casalecchio di Reno, Italy), Stefano Ziani (Imola, Italy).

My special gratitude for advice, support in bibliographical research and careful review of chapters during the manuscript realization goes to Luca Bartolozzi (Museo di Zoologia “La Specola” dell'Università di Firenze, Italy), Maura Bocci (Centro Nazionale Biodiversità Carabinieri "Bosco Fontana", Marmirolo, Italy), Roberto Poggi (Museo Civico di Storia Naturale, Genova, Italy) and Ignazio Sparacio (Palermo, Italy). I'd like to thank my friend Jonathan Cooter (Oxford University, Museum of Natural History, United Kingdom) for linguistic revision of the text.

\section{REFERENCES}

Abbazzi P., Bartolozzi L. \& Calamandrei S., 1999. Contributo alla conoscenza degli Anthribidae italiani (Insecta, Coleoptera, Curculionoidea). Annali del Museo civico di Storia naturale "G. Doria"di Genova, 93: 57-106.

Abbazzi P. \& Maggini L., 2009. Elenco sistematico-faunistico dei Curculionoidea italiani, Scolytidae e Platypodidae esclusi (Insecta, Coleoptera). Aldrovandia, 5: 29-216.

Abbazzi P. \& Magnano L., 2003. Una nuova specie di Cirrorhynchus Apfelbeck, 1898 dell'Italia meridionale (Coleoptera Curculionidae Entiminae). Atti Accademia Roveretana degli Agiati, ser.VIII, 3B: 7380 .

Abbazzi P. \& Zinetti F., 2013. Elenco sistematico-faunistico dei Curculionoidea italiani, Scolytidae e Platypodidae esclusi (Insecta, Coleoptera). 2. Addenda e corrigenda. Memorie della Società entomologica italiana, 90: 89-104.

Adorno A. \& Zanetti A., 2013. Descrizione di tre nuove specie del genere Sunius Curtis, 1829 con note sulle specie italiane e su alcune specie mediterranee occidentali (Coleoptera, Staphylinidae: Paederinae). Bollettino del Museo Civico di Storia Naturale di Verona, 27, Botanica Zoologia: 31-44.

Alekseev V.I., Bukejs A. \& Bellés X., 2019. Dignoptinus, a new genus for fossil Dignomus regiomontanus Alekseev from Eocene Baltic amber, and new status for Bruchoptinus Reitter and Pseudoptinus Reitter
(Coleoptera: Ptinidae). Fossil Record, 22: 65-72. https://doi.org/10.5194/fr-22-65-2019

Alekseev V.I., Bukejs A. \& Bellés X., 2019. Dignoptinus, a new genus for fossil Dignomus regiomontanus Alekseev from Eocene Baltic amber, and new status for Bruchoptinus Reitter and Pseudoptinus Reitter (Coleoptera: Ptinidae). Fossil Record, 22: 65-72.

Aliquò V. \& Massa B., 1976. Contributo allo studio di Anoxia scutellaris s.l. e descrizione di Anoxia scutellaris argentea $\mathrm{n}$. ssp. di Sicilia. Bollettino della Società entomologica italiana, 108: 151-157.

Alonso-Zarazaga M.A., 2010. Gompelia, a replacement name for Olotelus Mulsant \& Rey, 1866 (Coleoptera, Aderidae). Graellsia, 66: 299-301. https://doi:10. 3989/graellsia.2010.v66.026

Alonso-Zarazaga M.A., 2013. Case 3624. A proposal for the rejection of 38 names in Anthicidae (Coleoptera). Bulletin of Zoological Nomenclature, 70: 171-184.

Alonso-Zarazaga M.A., Barrios H., Borovec R., Bouchard P., Caldara R., Colonnelli E., Gültekin L., Hlavá P., Korotyaev B., Lyal C.H.C., Machado A., Meregalli M., Pierotti H., Ren L., Sánchez-Ruiz M., Sforzi A., Silfverberg H., Skuhrovec J., Trýzna M., Velázquez de Castro A.J. \& Yunakov N.N., 2017. Cooperative Catalogue of Palaearctic Coleptera Curculionoidea. Monografías electrónicas SEA 8, Sociedad Entomológica Aragonesa S.E.A., www.seaentomologia.org, November 2, 2017.

Angelini F., 1972. Hydroadephaga inediti per Puglia e Lucania. Bollettino della Società entomologica italiana, 104: 179-194.

Angelini F., 1973a. Hydroadephaga nuovi per Calabria e Sila. Bollettino della Società entomologica italiana, 105: 7-12.

Angelini F., 1973b. Hydrophilidae inediti per Puglia e Lucania. Bollettino della Società entomologica italiana, 105: 75-79.

Angelini F., 1973c. Descrizione di una nuova sottospecie dello Scarodytes halensis F. Memorie del Museo Civico di Storia Naturale di Verona, 20 (1972): 51-54.

Angelini F., 1975. Nuovi reperti di Hydroadephaga. Bollettino della Società entomologica italiana, 107: 5670 .

Angelini F., 1978. Haliplidae, Dytiscidae e Gyrinidae della Lucania. Entomologica, Bari, 14: 63-135.

Angelini F., 1984. Catalogo topografico dei Coleoptera Haliplidae, Hygrobiidae, Dytiscidae e Gyrinidae d'Italia. Memorie della Società entomologica italiana, 61/ A (1982): 45-126.

Angelini F., 1986. Coleotterofauna del Massiccio del Pollino (Basilicata-Calabria). Entomologica, 21: 37125.

Angelini F., 1987. Coleotterofauna del Promontorio del Gargano. Atti del Museo civico Storia naturale Grosseto, 11-12: 5-84. 
Angelini F., 1988. Gli Anisotomini del Museo Civico di Storia Naturale di Milano. Atti della Società italiana di Scienze naturali e del Museo civico di Storia naturale di Milano, 129: 305-366.

Angelini F., 1991. Coleotterofauna dell'Altipiano della Sila (Calabria, Italia) (Coleoptera). Memorie della Società entomologica italiana, 70: 171-254.

Angelini F., 1995. Revisione tassonomica delle specie paleartiche del genere Agathidium Panzer (Coleoptera, Leiodidae, Agathidiini). Monografie Museo regionale Scienze naturali di Torino, 18: 1484.

Angelini F., 1996. Coleotterofauna della Riserva Naturale WWF Lago di Pignola. Tipografia Alfagrafica Volonnino, Lavello, 135 pp.

Angelini F., 1998. Coleotterofauna reperita mediante trappola luminosa in due stazioni umide dalla Basilicata (Italia meridionale). Quaderni Stazione Ecologica Museo civico di Storia naturale di Ferrara, 11: 7-37.

Angelini F., 2005. Insecta Coleoptera Cryptophagidae. In: Ruffo S. \& Stoch F. (Eds.), Checklist e distribuzione della Fauna italiana. Memorie del Museo Civico di Storia Naturale di Verona, 2 serie, Sezione Scienze della Vita, 16: 215-216 + CD-ROM.

Angelini F. \& De Marzo L., 1985. Descrizione di una nuova specie e di una nuova sottospecie del genere Agathidium Panzer. Bollettino della Società entomologica italiana, 117: 172-177.

Angelini F. \& Ferro G., 1974. Hydrophilidae della Sila. Rivista di Idrobiologia, Istituto di Idrobiologia e Pescicultura G.B. Grassi Università Perugia, 13: 399-416.

Angelini F. \& Montemurro F., 1986. Coleotterofauna del bosco di Policoro (Matera). Biogeographia, Lavori della Società italiana di Biogeografia, 10 (1984): 545-604.

Angelini F. \& Rücker H.W., 1999. Contributo alla conoscenza dei Merophysiidae e Latridiidae dell'Italia meridionale e della Sicilia (Coleoptera). Memorie della Società entomologica italiana, 77 (1998): 213-240.

Angelini F. \& Sörensson M., 1997. Materiali per una coleotterofauna dell'Italia meridionale e della Sicilia. Ptiliidae. (Coleoptera). Annali del Museo civico di Storia naturale "G. Doria” di Genova, 91: 555-587.

Assing V., 1996. A revision of the European species of Calodera Mannerheim (Coleoptera, Staphylinidae, Aleocharinae). Beiträge zur Entomologie, 46: 3-24. https://doi:10.21248/contrib.entomol.69.1.001-032

Assing V., 2002. A taxonomic and phylogenetic revision of Amarochara Thomson. I. The species of the Holarctic region (Coleoptera: Staphylinidae, Aleocharinae, Oxypodini). Beiträge zur Entomologie, 52: 111-204.
Assing V., 2006. A revision of Western Palaearctic Medon: the species of the Atlantic Islands, the Western Mediterranean, and Europe, except for the southeast (Insecta: Coleoptera: Staphylinidae: Paederinae). Bonner zoologische Beiträge, 54 (2005): 25-95.

Assing V., 2007. A revision of the species of Pronomaea Erichson of the Western Palaearctic region, including Middle Asia (Coleoptera: Staphylinidae: Aleocharinae: Pronomaeini). Beiträge zur Entomologie, 57: 367-396.

Assing V., 2014. On the Bolitochara species of the West Palaearctic region (Coleoptera: Staphylinidae: Aleocharinae). Stuttgarter Beiträge zur Naturkunde A, Neue Serie 7: 33-63.

Assing V., 2019. Revision of the Baeoglena species of the West Palaearctic Region (Coleoptera: Staphylinidae: Aleocharinae). Contributions to Entomology, 69: 1-32.

Assing V. \& Wunderle P., 2008. On the Alevonota species of the Western Palaearctic region (Coleoptera: Staphylinidae: Aleocharinae: Athetini). Beiträge zur Entomologie, 58: 145-189.

Astrin J.J. \& Stüben P.E., 2008. Phylogeny in cryptic weevils: molecules, morphology and new genera of western Palaearctic Cryptorhynchinae (Coleoptera: Curculionidae). Invertebrate Systematics, 22: 503522.

Audisio P., 1973. Primo contributo alla conoscenza dei Silfidi d'Italia. Bollettino dell'Associazione romana di entomologia, 28: 55-58.

Audisio P., 1975. Note su alcune specie italiane del genere Meligethes Steph. Bollettino dell'Associazione romana di entomologia, 30: 2-16.

Audisio P., 1976. Note su alcune Epuraea italiane. Bollettino della Società entomologica italiana, 108: 9596.

Audisio P., 1986. I Kateretidae e i Nitidulidae dell'Italia Meridionale: distribuzione attuale e ipotesi sul popolamento. Biogeographia, Lavori Società italiana di Biogeografia, 10 (1984): 605-627.

Audisio P., 1993. Fauna d'Italia. XXXII. Coleoptera Nididulidae-Kateretidae. Ed. Calderini, Bologna, 971 pp.

Audisio P., Baviera C., Carpaneto G.M., Biscaccianti A.B., Battistoni A., Teofili C. \& Rondinini C., 2014. Lista Rossa IUCN dei Coleotteri saproxilici Italiani. Comitato Italiano IUCN e Ministero dell'Ambiente e della Tutela del Territorio e del Mare, Roma, 132 pp.

Audisio P., Brustel H., Carpaneto G.M., Coletti G., Mancini E., Piattella E., Trizzino M., Dutto M., Antonini G. \& De Biase A., 2007. Updating the taxonomy and distribution of the European Osmoderma, and strategies for their conservation (Coleoptera, Scarabaeidae, Cetoniinae). Fragmenta entomologica, 39: 273-290. 
Audisio P., Brustel H., Carpaneto G.M., Coletti G., Mancini E., Trizzino M., Antonini G. \& De Biase A., 2009. Data on molecular taxonomy and genetic diversification of the European Hermit beetles, a species-complex of endangered insects (Coleoptera: Scarabaeidae, Cetoniinae, Osmoderma). Journal of Zoological Systematics and Evolutionary Research, 47: 88-95. https://doi:10.1111/j.1439-0469.2008. 00475.x

Audisio P., Cline A.R., De Biase A., Antonini G., Mancini E., Trizzino M., Costantini L., Strika S., Lamanna F. \& Cerretti P., 2009. Preliminary reexamination of genus-level taxonomy of the Pollen Beetle subfamily Meligethinae (Coleoptera: Nitidulidae). Acta Entomologica Musei Nationalis Pragae, 49: 341-504.

Audisio P. \& De Biase A., 1995. Materiali per un'analisi biogeografica delle specie italiane dei generi $\mathrm{Hy}$ draena ed Haenydra (Coleoptera Hydraenidae). Fragmenta entomologica, 27: 163-189.

Audisio P. \& De Biase A., 1996. Taxonomic revision, phylogeny and biogeography of the beetle genus Dapsa Latreille (Coleoptera Endomychidae). Memorie della Società entomologica italiana, 74 (1995): 65-130.

Audisio P. \& De Biase A., 2005a. Insecta Coleoptera Hydraenidae. In: Ruffo S. \& Stoch F. (Eds.), Checklist e distribuzione della Fauna italiana. Memorie del Museo Civico di Storia Naturale di Verona, 2.serie, Sezione Scienze della Vita 16: 169-170 + CD-ROM.

Audisio P. \& De Biase A., 2005b. Insecta Coleoptera Nitidulidae. In: Ruffo S. \& Stoch F. (Eds.), Checklist e distribuzione della Fauna italiana. Memorie del Museo Civico di Storia Naturale di Verona, 2.serie, Sezione Scienze della Vita 16: 207-209 + CD-ROM.

Audisio P., De Biase A. \& Zampetti M.F., 1992. Le specie italiane del genere Dapsa Latreille. Bollettino della Società entomologica italiana, 124: 65-74.

Audisio P., Ferro G. \& De Biase A., 2000. Nuovi dati sulla distribuzione in Italia di alcune specie di Hydraenidae (Coleoptera). Bollettino della Società entomologica italiana, 132: 117-121.

Audisio P., Trizzino M., De Biase A., Rossetti G., Mancini E. \& Antonini G., 2010. Molecular and morphological evidence of a new sibling species of Calobius (Coleoptera: Hydraenidae) of the C. quadricollis complex from peninsular Italy. Italian Journal of Zoology, 77: 29-37. https://doi: 10.1080/11250000902 845738

Baiocchi D., 2015. Taxonomic notes on the genus $A n$ thaxia Eschscholtz, 1829 (Coleoptera: Buprestidae: Anthaxiini) 1. Zootaxa 3941 (3): 339-357. http://dx. doi.org/10.11646/zootaxa.3941.3.2

Baiocchi D. \& Magnani G., 2018. A revision of the $A n$ thaxia (Anthaxia) midas Kiesenwetter, 1857 species- group (Coleoptera: Buprestidae: Anthaxiini). Zootaxa 4370 (3): 201-254. https://doi.org/10.11646/zootaxa. 4370.3.1

Ballerio A., Krell F.-T., Bezděk A., Frolov A., Huchet J.B., Keith D., López-Colón J.I., Matthews E., Ocampo F., Rey A. \& Vaz-de-Mello F.Z., 2018. Case 3768 Hybosorus illigeri Reiche, 1853 (Insecta, Coleoptera): proposed conservation by giving it precedence over Hybosorus pinguis Westwood, 1845, Hybosorus roei Westwood, 1845 and Hybosorus carolinus LeConte, 1847. Bulletin of Zoological Nomenclature, 75: 152-164. http://dx.doi.org/10. 21805/bzn.v75.a032

Ballerio A., Rey A., Uliana A. \& Colla A., 2011. Coleoptera Scarabaeoidea nuovi o interessanti per la fauna italiana. Rivista del Museo Civico di Scienze Naturali "Enrico Caffi" di Bergamo, 25: 69-74.

Ballerio A., Rey A., Uliana A., Rastelli M., Rastelli S., Romano M. \& Colacurcio L., 2010. Piccole Faune. Coleotteri Scarabeoidei d'Italia. DVD. M. Serra Tarantola ed., Brescia: 1-13+ dvd.

Baraud J., 1965. Revision du genre Homaloplia Steph. (Col. Scarab.). Atti della Società italiana di Scienze naturali e del Museo civico di Storia naturale di Milano, Milano, 104: 393-448.

Baraud J., 1991. Revision des especes du genre Anisoplia Fischer, 1824 (Coleoptera Scarabaeoidea Rutelidae) (deuzieme partie). Beiträge zur Entomologie, 60: 353-384.

Bartolozzi L., 1989. Descrizione di una nuova sottospecie di Aesalus scarabaeoides (Panzer, 1794) di Basilicata. Bollettino della Società entomologica italiana, 121: 104-107.

Bartolozzi L. \& Maggini L., 2005. Insecta Coleoptera Lucanidae. In: Ruffo S. \& Stoch F. (Eds.), Checklist e distribuzione della Fauna italiana. Memorie del Museo Civico di Storia Naturale di Verona, 2 serie, Sezione Scienze della Vita, 16: 191-192 + CD-ROM.

Baudi di Selve F., Piccioli F. \& Cavanna G., 1882. Al Vulture ed al Pollino. Parte II. Catalogo degli animali raccolti al Vulture, al Pollino ed in altri luoghi dell'Italia mendionale e centrale. Coleoptera. Bollettino della Società entomologica italiana, 14: 63-81.

Baviera C. \& Audisio P., 2014. The Nitidulidae and Kateretidae (Coleoptera: Cucujoidea) of Sicily: recent records and updated checklist. Atti dell'Accademia peloritana dei Pericolanti, Classe di Scienze fisiche, matematiche e naturali, 92: A1-A32. http:// doi: 10.1478/AAPP.922A1

Baviera C. \& Biondi M., 2015. The Alticini (Coleoptera: Chrysomelidae, Galerucinae) of Sicily: recent records and updated checklist. Atti della Accademia Peloritana dei Pericolanti. Classe di Scienze Fisiche, Matematiche e Naturali, 93: 1-50. http://doi:10. 1478/AAPP.932A2 
Bellò C. \& Pierotti H., 1992. Nuovi Peritelini della fauna italiana (Coleoptera, Curculionidae). Fragmenta entomologica, 3: 369-387.

Bellò C., Osella G.B. \& Baviera C., 2019. A taxonomic monograph of the genus Solariola Flach, 1908 (Coleoptera: Curculionidae: Entiminae). Zootaxa 4676 (1): 1-261. http://dx.doi.org/10.11646/zootaxa. 4676.1

Bertolini S., 1876. Escursioni entomologiche nella Calabria. Bollettino della Società entomologica italiana, 8: 48-53.

Bertrand H., 1956. Ricerche Zoologiche sul Massiccio del Pollino. XX. Coleoptera. 10. Coléoptères acquatiques de Calabre. Annuario dell'Istituto e Museo di Zoologia della Università di Napoli, 8: 1-8.

Besuchet C., 1964. Psélaphides paléarctiques. Espèces nouvelles et notes synonymiques. II. Coleoptera. Revue Suisse de Zoologie, 71: 411-443.

Besuchet C., 1976. Contribution à l'etude des Ptiliides palaearctiques (Coleoptera). Mitteilungen der Schweizerischen Entomologischen Gesellschaft, 49: 51-71.

Besuchet C., 1980. Contribution a l'etude des Coleopteres Pselaphides d'Italie et du Tessin. Revue suisse de Zoologie, 87: 611-635.

Bezdek L. \& Baselga A., 2015. Revision of western Palaearctic species of the Oulema melanopus group, with description of two new species from Europe (Coleoptera: Chrysomelidae: Criocerinae). Acta Entomologica Musei Nationalis Pragae, 55: 273-304.

Binaghi G., 1934. Appunti sugli Elateridi italiani. I. Bollettino della Società entomologica italiana, 66: 150153.

Binaghi G., 1939. I Melanotini della fauna italiana. Spheniscosomus Schw. e Melanotus Eschs. Memorie della Società entomologica italiana, 17: 205-239.

Binaghi G., 1948, Note di caccia (III). Bollettino della Società entomologica italiana, 78: 77-79.

Binaghi G., 1955. Ricerche Zoologiche sul Massiccio del Pollino. XII. Coleoptera. 2. Elateridae, Melasidae, Throscidae e Dascillidae. Annuario dell'Istituto e Museo di Zoologia della Università di Napoli, 7: 1-19.

Binaghi G., 1958. Materiali per lo studio delle Hydraena italiane ( $1^{\circ}$ contributo). Bollettino della Società entomologica italiana, 88: 70-83.

Binaghi G., 1970. Revisione degli Scotonomus. Analisi di un genere paleotirrenico. Bollettino della Società entomologica italiana, 102: 118-137.

Binaghi G., 1974. Contributi alla geonemia della coleotterofauna italiana. II. Annali del Museo civico di Storia naturale "G. Doria" di Genova, 80: 119-129.

Binaghi G., 1978. Revisione degli Stenolophus del gruppo teutonus (Schrank) (Coleoptera Carabidae). Memorie della Società entomologica italiana, 56 (1977): 21-34.
Binaghi G., Dellacasa G. \& Poggi R., 1969. Nuovi caratteri diagnostici per la determinazione degli Onthophagus del gruppo ovatus (L.) e geonemia controllata delle specie italiane del gruppo. Memorie della Società entomologica italiana, 48 B: 29-46.

Biondi M., 1982. Le specie appenniniche del genere Crepidodera Chevrolat sensu Foudras. Bollettino del Museo Civico di Storia Naturale di Verona, 8: 45-66.

Biondi M., 1983. Contributo alla conoscenza delle Alticinae della fauna italiana. Fragmenta entomologica, 17: $151-158$.

Biondi M., 1989. Note faunistiche su alcune specie di Chrysomelidae Alticinae della fauna italiana. Bollettino della Società entomologica italiana, 121: 98103.

Biondi M., 1990. Elenco commentato dei Crisomelidi Alticini della fauna italiana. Fragmenta entomologica, 22: 109-183.

Biondi M., 1995. Descrizione di una nuova specie di Cryptocephalus del sottogenere Burlinius Lopatin dell'Italia Meridionale (Coleoptera Chrysomelidae). Bollettino della Società entomologica italiana, 127: 39-44.

Biondi M., 2005. Insecta Coleoptera Chrysomelidae Alticinae In: Ruffo S. \& Stoch F. (Eds.), Checklist e distribuzione della Fauna italiana. Memorie del Museo Civico di Storia Naturale di Verona, 2 serie, Sezione Scienze della Vita, 16: 227-229 + CD-ROM.

Biscaccianti A.B., 2002. Nuovi dati geonemici su alcuni Cerambicidi italiani (Coleoptera, Cerambycidae). Bollettino dell'Associazione romana di Entomologia, 57: 49-62.

Biscaccianti A.B., 2003. Dati faunistici e biologici inediti e rettifiche sulla geonemia di alcuni Longicorni della fauna italiana (Coleoptera, Cerambycidae). Bollettino dell'Associazione romana di entomologia, 58: 77-86.

Biscaccianti A.B., 2005. Osservazioni su alcuni longicorni della fauna italiana (Insecta, Coleoptera, Cerambycidae). Aldrovandia, 1: 71-80.

Biscaccianti A.B., Manti F., Castiglione E., Bonacci T., Siclari A., Pelle L. \& Bonsignore C.P., 2016. Coleotteri saproxilici e specie in Direttiva Habitat del Parco Nazionale dell'Aspromonte. $11^{\circ}$ Convegno Nazionale sulla Biodiversità, Matera 9 e 10 Giugno 2016, Sessione II, Biodiversità degli habitat naturali: 150 .

Bocek M., Fancello L., Motyka M., Bocakova M. \& Bocak L., 2017. The molecular phylogeny of Omalisidae (Coleoptera) defines the family limits and demonstrates low dispersal propensity and ancient vicariance patterns. Systematic Entomology, 42: 112. http://doi: 10.1111/syen.12271

Bologna M.A., 1975. Problemi sistematici e zoogeografici di alcune specie di Meloidae italiani. Bollettino dell'Associazione romana di entomologia, 30: 36-45. 
Bologna M.A., 1976. Reperti di Oedemeridae dell'Italia centromeridionale e insulare. Bollettino dell'Associazione romana di Entomologia, 31: 29-32.

Bologna M.A., 1988. Note su Eurymeloe e revisione delle specie euromediterranee del gruppo rugosus (Coleoptera, Meloidae). Fragmenta entomologica, 20: 233-301.

Bologna M.A., 1991. Fauna d'Italia. XXVIII. Coleoptera Meloidae. Ed. Calderini, Bologna, 541 pp.

Bologna M.A., 2005. Zonitis fernancastroi, a new Species for the Italian Fauna, and additional Records of Meloidae and Oedemeridae (Coleoptera). Bollettino della Società entomologica italiana, 137: 107114.

Bologna M.A., 2016. Range extension of Two Oedemeridae species in the italian peninsula and other faunistic new records. Bollettino dell'Associazione romana di entomologia, 71 (2015): 21-25.

Bocakova, M., Constantin, R. \& Bocak, L., 2011. Molecular phylogenetics of the melyrid lineage (Coleoptera: Cleroidea). Cladistics, 27: 1-13. https:// doi.org/10.1111/j.1096-0031.2011.00368.x

Bonacci T., Mazzei A., Horak J. \& Brandmayr P., 2012. Cucujus tulliae sp. n. an endemic Mediterranean saproxylic beetle from genus Cucujus Fabricius, 1775 (Coleoptera, Cucujidae), and keys for identification of adults and larvae native to Europe. ZooKeys 212: 63-79. https://doi: 10.3897/zookeys.212.3254

Bonavita P. \& Vigna Tagliati A., 1993. Note sulle specie di Ocydromus (Bembidionetolizkya) del gruppo fasciolatus (Coleoptera Carabidae). Fragmenta entomologica, 25: 67-90.

Bonavita P. \& Vigna Taglianti A., 2010. Ocydromus subg. Nepha Motschulsky, 1864: revisione tassonomica, filogenesi e biogeografia (Coleoptera Carabidae). Memorie della Società entomologica italiana, 89: 7-180.

Bongiovanni G.C., 1966. La geonemia italiana dei Cleoni della Bietola da zucchero. Bollettino dell'Istituto di Entomologia della Università di Bologna, 28: 1-12.

Bonsignore C.P., 2012. Apate monachus (Fabricius, 1775), a Bostrichid Pest of Pomegranate and Carob Trees in Nurseries. Short Communication. Plant Protection Science, 48: 94-97.

Bordoni A., 1972. Revisione degli Xantholinus della fauna italiana. Redia, 53: 151-237.

Bordoni A., 1973. Gli Astenus knizi Bernh. e le forme italiane del sottogenere Eurysunius Reitt. XXV Contributo alla conoscenza dei Coleoptera Staphylinidae. Bollettino della Società entomologica italiana, 105: 155-161.

Bordoni A., 1974. Studi sulla sistematica e la geonemia del genere Quedius Steph. III. Le specie italiane appartenenti al sottogenere Microsaurus (Steph.) sensu Gridelli, 1924. Redia, 55: 1-82.
Bordoni A., 1975. Neobisnius cerrutii Gridelli, 1943 è sinonimo di latrobioides (Baudi, 1848). Bollettino del Museo di Zoologia Università di Torino, 2: 81-84.

Bordoni A., 1976. Studi sulla sistematica e la geonemia del genere Quedius Steph. IV. I Quedius s. str. della fauna italiana. Redia, Firenze, 59: 85-107.

Bordoni A., 1982. Fauna d'Italia. XIX. Coleoptera Staphylinidae: Generalità-Xantholoninae. Ed. Calderini, Bologna, 434 pp.

Bordoni A., 1983. Una nuova Choleva della Calabria (Coleoptera, Catopidae). Fragmenta entomologica, $17: 101-104$

Bordoni A. \& Castellini G., 1973. Sulle specie paleartiche del genere Mastigus Latreille con osservazioni su due specie dell'Africa Australe (Coleoptera Scydmaenidae). Redia, 54: 295-323.

Borovec R. \& Osella G.B., 1993. Trachyphloeus amicalis n. sp. della regione appenninica (Coleoptera Curculionidae). Bollettino della Società entomologica italiana, 125: 179-185.

Bouchard P., Bousquet Y., Davies A.E., Alonso-Zarazaga M.A., Lawrence J.F., Lyal C.H.C., Newton A.F., Reid C.A.M., Schmitt M., Ślipiński S.A., Smith A.B.T., 2011. Family-group names in Coleoptera (Insecta). ZooKeys, 88: 1-972. https://doi:10.3897/zookeys. 88.807

Bousquet Y., 2016. Litteratura Coleopterologica (17581900): a guide to selected books related to the taxonomy of Coleoptera with publication dates and notes. ZooKeys, 583: 1-776. https://doi: 10.3897/zookeys. 583.7084

Bowestead S., 1999. A revision of the Corylophidae (Coleoptera) of the West Palaearctic Region. Muséum d'Histoire Naturelle, Genève, Instrumenta Biodiversitatis, 3: 1-203.

Brancucci M., 1979. Contribution a l'etude des Malthinini d'Italie. III. Malthodes cordiger Kiesw. et quelques especes voisines (Coleoptera Cantharidae). Bollettino della Società entomologica italiana, 111: 70-75.

Brandmayr P., Cagnin M., Mingozzi T. \& Pizzolotto R., 1996. Map of zoocoenoses and evaluation for the Menta River Dam in Aspromonte (Calabria, Italy), Zeitschrift zur Okologie und Naturhschutz, 5: 15-28.

Brandmayr P. \& Zetto Brandmayr T., 1986. Le comunità a Coleotteri geoadefagi di alcune faggete ed abetine appenniniche, dal Casentino al M. Pollino. Biogeographia, Lavori Società italiana di Biogeografia, Bologna, 10 (1984): 685-700.

Bruce N., 1936. Monographie der europäischen Arten der Gattung Cryptophagus Herbst. Acta Zoologica Fennica, 20: 1-167.

Bruno S., 1966. Sul Callicnemis latreillei Lap. in Italia (Col. Scarabaeidae). Bollettino dell'Associazione romana di entomologia, 21: 43-45. 
Bucciarelli I., 1959. Coleotteri Anthicidi dell'Aspromonte (Appennino Calabrese). Memorie del Museo Civico di Storia Naturale di Verona, 7: 183-189.

Bucciarelli I., 1961. Ricerche coleotterologiche sul litorale ionico della Puglia, Lucania e Calabria, Campagne 1956-1957-1958. VIII. Coleoptera Anthicidae. Bollettino della Società entomologica italiana, 91: 102-131.

Bucciarelli I., 1973. Note sui "Notoxus" mediterranei. Atti del Museo Civico di Storia naturale di Trieste, 28: 471-484.

Bucciarelli I., 1978. Anticidi nuovi, nuovi per l'ltalia e sinonimie. Bollettino della Società entomologica italiana, 110: 154-163.

Bucciarelli I., 1980. Coleoptera. Anthicidae. Fauna d'Italia, XVII. Ed. Calderini, Bologna, 240 pp.

Burlini M., 1939. Primo contributo alla maggiore conoscenza della distribuzione geografica dei Coleotteri in Italia. Bollettino della Società entomologica italiana, 71: 181-186.

Burlini M., 1942. Nuove forme di coleotteri italiani. Bollettino della Società entomologica italiana, 74: 126128.

Burlini M., 1955a. Revisione dei Cryptocephalus italiani e della maggior parte delle specie di Europa. Memorie della Società entomologica italiana, 34: 1-287.

Burlini M., 1955b. Ricerche Zoologiche sul Massiccio del Pollino. XIX. Coleoptera. 9. Due nuovi Pachybrachys italiani (Chrysomelidae). Annuario dell'Istituto e Museo di Zoologia della Università di Napoli, 8: $1-7$.

Burlini M., 1968. Revisione delle specie italiane e della maggior parte delle specie europee del genere Pachybrachis Chevr. (XXII contributo alla conoscenza dei Cryptocephalini). Memorie della Società entomologica italiana, 47: 11-116.

Caldara R., 1971. Note geonemiche su alcuni Curculionidi italiani. Bollettino della Società entomologica italiana, 103: 212-214.

Caldara R., 1975. Considerazioni su alcune specie del gruppo Tychius pusillus Germar (VII Contributo alla conoscenza dei Coleptera Curculionidae). Bollettino della Società entomologica italiana, 107: 31-40.

Caldara R., 1977. Revisione dei Pachytychius paleartici (Col. Curculionidae). Memorie della Società entomologica italiana, 56: 131-216.

Caldara R., 1978. I generi Apeltarius Desbrochers, Xenotychius Reitter e Pseudolignydes Pic. Bollettino della Società entomologica italiana, 110: 23-34.

Caldara R., 1985. Revisione delle Sibinia paleartiche (Col. Curculionidae). Memorie della Società entomologica italiana, 62/63 (1983/84): 24-105.

Caldara R., 1990. Revisione tassonomica delle specie paleartiche del genere Tychius Germar (Coleoptera Curculionidae). Memorie della Società italiana di
Scienze naturali e del Museo civico di Storia naturale di Milano, 25: 53-238.

Caldara R., 2008. Revisione delle specie paleartiche del genere Gymnetron (Insecta, Coleoptera, Curculionidae). Aldrovandia, 4: 27-103.

Caldara R., 2014. Rhinusa Stephens: a taxonomic revision of the species belonging to the $R$. tetra and $R$. bipustulata groups (Coleoptera Curculionidae). Journal of Insect Biodiversity, 2: 1-46. http://dx.doi.org/ 10.12976/jib/2014.2.19

Caldara R. \& Angelini F., 1997. Su alcuni Curculionoidea nuovi per l'Italia o per varie regioni italiane (Coleoptera). Bollettino della Società entomologica italiana, 129: 241-249.

Caldara R. \& Fogato V., 2013. Systematics of the weevil genus Mecinus Germar, 1821 (Coleoptera: Curculionidae). I. Taxonomic treatment of the species. Zootaxa 3654: 1-105.

Caldara R. \& O`Brien C.W., 1998. Systematics and evolution of Weevils of the genus Bagous. VI. Taxonomical treatment of the species of the Western Palearctic Region (Coleoptera Curculionidae). Memorie della Società entomologica italiana, 76 (1997): 131-347.

Canepari C., 1961. Ricerche coleotterologiche sul litorale ionico della Puglia, Lucania e Calabria, Campagne 1956-1957-1958. V11. Coleoptera Coccinellidae. Bollettino della Società entomologica italiana, 91: 154158.

Canepari C., 1983. Le specie italiane del gruppo dello Scymnus frontalis Fab. con descrizione di due nuove specie. Giornale Italiano di Entomologia, 1: 179-204.

Canepari C., 2000. The Nephus subgenus Sidis in Italy (Coleoptera Coccinellidae). Bollettino della Società entomologica italiana, 132: 49-62.

Canepari C., 2009. New data on some Coccinellidae (Coleoptera) from the Mediterranean Region. Zootaxa 2318: 394-399.

Canepari C., Fursch H. \& Kreissl E., 1985. Die Hyperaspis-Arten von Mittel-West- und Sudeuropa. Systematik und Verbreitung. Giornale Italiano di Entomologia, 2: 223-252.

Canzoneri S., 1968. Materiali per una monografia delle Phaleria del sottogenere Phaleria Latr. Memorie della Società entomologica italiana, Genova, 47: 117-167.

Canzoneri S., 1977. Contributo alla conoscenza dei Tenebrionidi Appenninici. Bollettino del Museo Civico di Storia Naturale di Verona, 4: 227-285.

Canzoneri S., 1981. Su alcuni interessanti coleotteri Tenebrionidi dell'Italia meridionale. Società Veneziana di Scienze Naturali. Lavori, Venezia, 6: 85-86.

Carpaneto G.M., 1974a. Endemismi italiani. 15. Aphodius (Acrossus) siculus Harold. Bollettino dell'Associazione romana di entomologia, 29: VI. 
Carpaneto G.M., 1974b. Note sulla distribuzione geografica ed ecologica dei Coleotteri Scarabaeoidea Laparosticti nell'Italia appenninica. Bollettino dell'Associazione romana di entomologia, 29: 32 54.

Carpaneto G.M., Baviera C., Biscaccianti A.B., Brandmayr P., Mazzei A., Mason F., Battistoni A., Teofili C., Rondinini C., Fattorini S. \& Audisio P. (Eds.), 2015. A Red List of Italian Saproxylic Beetles: taxonomic overview, ecological features and conservation issues (Coleoptera). Fragmenta entomologica, 47: 53-126.

Carpaneto G.M., Chiari S., Audisio P., Leo P., Liberto A., Jansson N. \& Zauli A., 2012. Biological and distributional overview of the genus Eledonoprius (Coleoptera: Tenebrionidae): Rare fungus-feeding beetles of European old-growth forests. European Journal of Entomology, 110: 173-176. http://www. eje.cz/pdfs/110/1/173

Carpaneto G.M., Piattella E. \& Valerio L., 2005. Insecta Coleoptera Scarabaeoidea. In: Ruffo S. \& Stoch F. (Eds.), Checklist e distribuzione della Fauna italiana. Memorie del Museo Civico di Storia Naturale di Verona, 2 serie, Sezione Scienze della Vita, 16: 193 $197+$ CD-ROM.

Casale A. \& Giachino P.M., 1985. Un nuovo Pterostichus dell'Appennino Lucano (Coleoptera, Carabidae). Bollettino del Museo Regionale di Scienze naturali di Torino, 3: 427-436.

Casale A., Sturani M. \& Vigna Taglianti A., 1982. Fauna d'Italia. XVIII. Coleoptera Carabidae. I. Introduzione, Paussinae, Carabinae. Ed. Calderini, Bologna, 499 pp.

Casale A., Vigna Taglianti A., Brandmayr P. \& Colombetta G., 2005. Insecta Coleoptera Carabidae (Carabini, Cychrini, Trechini, Abacetini, Stomini, Pterostichini. In: Ruffo S. \& Stoch F. (Eds.), Checklist e distribuzione della Fauna italiana. Memorie del Museo Civico di Storia Naturale di Verona, 2 serie, Sezione Scienze della Vita, 16: 159-163 + CD-ROM.

Cassola F., 1974. Studi sui Cicindelidi. XI. Validità specifica di Cicindela majalis Mandl. e problemi di conservazione degli ambienti golenali italiani (Coleoptera). Lavori della Società italiana di Biogeografia, 4 (1973): 57-75 + 3 tav.

Cassola F., 2012. Studi sui Cicindelidi. CLXXXVI. Le cicindele di Policoro (Coleoptera, Cicindelidae). Bollettino dell'Associazione romana di entomologia, 67: 37-42.

Castellini G., 1987. Scydmoraphes italiani nuovi o poco noti. Atti del Museo civico Storia naturale Grosseto, 11/12: 119-129.

Castellini G., 1994. Revisione del genere Leptomastax Pirazzoli, 1855. Atti del Museo civico Storia naturale Grosseto, Suppl. al n.15, 137 pp.
Castellini G., 2006. Pensieri sulla tassonomia e note su alcuni Scydmaenidae paleartici (Coleoptera). Annali del Museo civico di Storia naturale "G. Doria" di Genova, 98: 1-121.

Cavanna G., 1882. Al Vulture ed al Pollino. Parte I. Narrazione della escursione fatta al Vulture ed al Pollino nel luglio del 1880 da A. Biondi, C. Caroti e G. Cavanna. Bollettino della Società entomologica italiana, 14: 3-29.

Ceccolini F., Terzani F. \& Paggetti R., 2013. Contributo alla corologia dei Licidi italiani. (Coleoptera: Lycidae). Onychium, 10: 119-126.

Cecconi G., 1908, Contributo alla fauna delle Isole Tremiti. Bollettino del Museo di Zoologia, Anatomia comparata, Università di Torino, 23 (583): 1-53.

Chiesa A., 1959. Hydrophilidae Europae. Coleoptera Palpicornia. Tabelle di determinazione. Arnaldo Forni Editore, Bologna, 200 pp.

Ciceroni A., 1990. Una nuova specie di Atrechus dell'Italia meridionale (Coleoptera, Staphylinidae). Bollettino dell'Associazione romana di entomologia, 44 (1989): 55-59.

Ciceroni A., 1994. Revisione delle specie italiane del genere Leptacinus Erichson con note sinonimiche su alcuni Xantholinini europei e nordafricani (Coleoptera, Staphylinidae: Xantholininae). Bollettino del Museo Civico di Storia Naturale di Verona, 18 (1991): 97-119.

Cline A.R., Smith T.R., Miller K., Moulton M., Whiting M. \& Audisio P., 2014. Higher phylogenetics of Nitidulidae: assessment of subfamilial and tribal classifications, and formalization of the family Cybocephalidae (Coleoptera: Cucujoidea). Systematic Entomology, 39: 758-772. http://doi:10.1111/ syen. 12084

Colonnelli E., 1972. Una nuova specie di Acalles Schönherr del Massiccio del Pollino, Bollettino dell'Associazione romana di entomologia, 27: 25-30.

Colonnelli E., 1974a. Gli Attelabidi ed i Curculionidi del Massiccio del Pollino (Coleoptera). Fragmenta entomologica, Roma, 10 (2): 107-218.

Colonnelli E., 1974b. Osservazioni sulla distribuzione e sulla sistematica di alcune specie di Curculionidi. Bollettino dell'Associazione romana di entomologia, 29 (3-4): 33-63.

Colonnelli E., 1976. Note su alcuni Ceutorhynchinae italiani. Bollettino dell'Associazione romana di entomologia, 31: 51-54.

Colonnelli E., 1978. Una nuova specie di Paroxyonyx Hustache dell'Italia meridionale. Bollettino dell'Associazione romana di entomologia, 33: 80-87.

Colonnelli E., 1980. Revisione del Genere Acallocrates Reitter. Atti della Società italiana di Scienze naturali e del Museo civico di Storia naturale di Milano, 121: 3-16. 
Colonnelli E., 1991. Note sui Curculionidi della Riserva Naturale "Bosco Pantano" (Basilicata) e descrizione di un nuovo Bothynoderes (Coleoptera, Curculionidae). Bollettino dell'Associazione romana di entomologia, 45 (1990): 89-113.

Colonnelli E., 2003. A revised checklist of Italian Curculionoidea (Coleoptera). Zootaxa 337: 1-142. http:// dx.doi.org/10.11646/zootaxa.337.1

Colonnelli E., 2011. Two new italian Ceutorhynchus (Coleoptera, Curculionidae). Fragmenta entomologica, 43: 179-186.

Contarini E., Garagnani P. \& Zanetti A., 1985. La Coleotterofauna delle "Valli di Comacchio" (Ferrara) $\left(2^{\circ}\right.$ contributo: Staphylinidae). Bollettino del Museo Civico di Storia Naturale di Verona, 10: 121-140.

Costa A., 1863. Nuovi studi sulla Entomologia della Calabria ulteriore. Atti Reale Accademia Scienze fisica e matematica, Napoli, 1: 1-80.

Costa A., 1881. Relazione di un viaggio nelle Calabrie per ricerche zoologiche fatto nella state del 1876. Atti Reale Accademia Scienze fisica e matematica, Napoli, 9 (4): 1-62.

Costa O.G., 1839. Fauna di Aspromonte e sue adiacenze. Letta nella tornata de' 12 Febrajo 1828. Atti Reale Accademia Scienze Napoli, 4: 61-173.

Crovato P. \& Izzillo F., 1995. Nuovi reperti di Buprestidi dell'Italia centro-meridionale (Coleoptera, Buprestidae). Bollettino dell'Associazione Romana di Entomologia, 49 (3-4) (1994): 155-160.

Curletti G., 1990. Nuovi Buprestidi mediterranei. Bollettino della Società entomologica italiana, 122: 102106.

Curletti G., 1994. I Buprestidi d'Italia. Catalogo Tassonomico, Sinonimico, Biologico, Geonemico. Monografie Natura bresciana, 19: 1-318.

Curletti G., 2005. Insecta Coleoptera Buprestidae. In: Ruffo S. \& Stoch F. (Eds.), Checklist e distribuzione della Fauna italiana. Memorie del Museo Civico di Storia Naturale di Verona, 2 serie, Sezione Scienze della Vita, 16: 205-206 + CD-ROM.

Curletti G., 2013. Considerazioni su alcune specie di Agrilus Curtis, 1825 presenti in Italia e su alcuni sottogeneri proposti di recente (Coleoptera, Buprestidae). Fragmenta entomologica, 45: 71-82.

D'Alessandro P. \& Biondi M., 2007. Analisi zoogeografica della crisomelidofauna appenninica legata ai piani altitudinali montano, subalpino e alpino (Coleoptera, Chrysomelidae). Biogeographia, Lavori della Società italiana di Biogeografia, 28: 461-494.

Daccordi M., Lavarini N. \& Ruffo S., 1991. Considerazione faunistiche e biogeografiche sulle Gonioctena italiane (Coleoptera, Chrysomelidae). Atti XVI Congresso nazionale italiano di Entomologia, Bari-Martina Franca (TA): 93-101.
Daccordi M. \& Ruffo S., 1976. Le specie appenniniche del genere Oreina (Coleoptera, Chrysomelidae). Bollettino del Museo Civico di Storia Naturale di Verona, 3: $379-411$.

Daccordi M. \& Ruffo S., 1979. Le Chrysolina italiane del sottogere Threnosoma Motsch. (Coleoptera, Chrysomelidae). Bollettino del Museo Civico di Storia Naturale di Verona, 6: 305-332.

Daccordi M. \& Ruffo S., 1988. Considerazioni sistematiche e biogeografiche sulle Timarcha italiane. Atti XV Congresso nazionale italiano Entomologia, L'Aquila: 343-351.

Daccordi M. \& Ruffo S., 2005. Considerazioni biogeografiche sulle Chrysolina delle province appenninica e sicula con descrizione di Chrysolina (Stichoptera) bourdonnei n. sp. (Coleoptera, Chrysomelidae). Studi Trentini di Scienze Naturali, Acta Biologica, 81 (2004): 113-127.

Dahlgren G., 1964. Kafer aus Kalabrien. Atti del Museo Civico di Storia naturale di Trieste, 24: 133-137.

Danilevsky M.L., 2019. Catalogue of Palaearctic Cerambycoidea. On-line version Updated: 09.04.2019 (http://www.cerambycidae.net/catalog.pdf).

De Marzo L., 1995. Appunti sulla presenza di Cybocephalus rufifrons Reitt. in suditalia (Coleoptera: Cybocephalidae). Entomologica, 29: 135-147.

Degiovanni A., 1999. Coleotteri Anticidi del Museo civico di Scienze Naturali "E. Caffi" di Bergamo (Insecta Coleoptera Anthicidae). Quaderno di Studi e Notizie di Storia naturale della Romagna, 11, suppl.: 33-40.

Degiovanni A. \& Fancello L., 1987. Nuovi dati geonemici su alcuni Anthicidae italiani. Acta Coleopterologica, 3: 41-44.

Degiovanni A. \& Pezzi G., 2007. Anthicus catalanus Bonadonna, 1953 nuovo per l'Italia e reperti di altre specie (Insecta, Coleoptera Anthicidae). Quaderno di Studi e Notizie di Storia naturale della Romagna, 24: 69-77.

Dellacasa G., 1983. Sistematica e nomenclatura degli Aphodiini italiani. Museo Regionale Scienze naturali Torino, Monografie, I: 463 pp.

Dellacasa G. \& Dellacasa M., 2006. Fauna d'Italia XLI. Coleoptera Aphodiidae, Aphodiinae. Ed. Calderini, Bologna, I-XII + 484 pp.

Diotti L., 2008. Otiorhynchus (Nehrodistus) pesarinii, nuova specie del Massiccio del Monte Sirino, S Italia (Coleoptera Curculionidae). Atti della Società italiana di Scienze naturali e del Museo civico di Storia naturale di Milano, 149: 3-8.

Diotti L., Pesarini C. \& Caldara R., 2015. Revisione sistematica del genere Cotaster Motschulsky: nuove sinonimie e descrizione di due nuove specie (Coleoptera, Curculionidae). Giornale italiano di Entomologia, 14: 223-240. 
Dodero A., 1919a. Appunti Coleotterologici. IV. Bollettino della Società entomologica italiana, 54: 6776.

Dodero A., 1919b. Materiali per lo studio dei Coleotteri italiani con descrizioni di nuove specie. IV. Fam. Pselaphidae. Annali del Museo civico di Storia naturale "G. Doria" di Genova, ser. 3, 8 (48): 172-250.

Eberle J., Bazzato E., Fabrizi S., Rossini M., Colomba M., Cillo D., Uliana M., Sparacio I., Sabatinelli G., Warnock R.C.M., Carpaneto G. \& Ahrens D., 2018. Sex-Biased Dispersal Obscures Species Boundaries in Integrative Species Delimitation Approaches. Systematic Biology, 68: 441-459. https://doi.org/10. 1093/sysbio/syy072

Easton A.M., 1960. On the identity of Meligetes moestus Erichson and M. moestus Auctt. (Col. Nitidulidae). Memorie del Museo Civico di Storia Naturale di Verona, 8: 335-343.

Endrödy-Younga S., 1960. Monographie der Paläarktischen Arten der Gattung Clambus. Acta Zoologica Academiae Scientiarum Hungaricae, 6: 257-303.

Endrödy-Younga S., 1961. Revision der Gattung Calyptomerus Redtb. (Coleoptera: Clambidae). Acta Zoologica Academiae Scientiarum Hungaricae, 7: $401-412$

Esser J., 2016a. Revision der Gattung Typhaeola Ganglbauer, 1899 (Coleoptera: Mycetophagidae) - 1. Teil: Paläarktische Arten. Entomologische Zeitschrift, Schwanfeld, 126: 169-175.

Esser J., 2016b. Über die Identität von Cryptophilus integer (HEER, 1841) (Coleoptera: Erotylidae). Entomologische Nachrichten und Berichte, 60: 213-218.

Esser J., 2017. Revision der Gattung Pteryngium Reitter, 1888 (Coleoptera, Cryptophagidae). Entomologische Nachrichten und Berichte, 61: 103-105.

Evers M.A., 1965. Ricerche coleotterologiche sul litorale ionico della Puglia, Lucania e Calabria, Campagne 1956-1957-1958. XI. Coleoptera Malachidae. Bollettino della Società entomologica italiana, Genova, 95: 135-136.

Fabbri R. \& Allemand R., 1997. Nuovi dati corologici su Byrrhus (Byrrhus) numidicus Normand, 1935 (Insecta, Coleoptera, Byrrhidae). Quaderno di Studi e Notizie di Storia naturale della Romagna, 8: 49-54.

Faggioli D., 1955. Campagna di ricerche dell'Istituto di Entomologia dell'Università di Bologna nella "Foresta Umbra" (Gargano). II. Elenco delle specie raccolte (primo lotto). Bollettino dell'Istituto di Entomologia della Università di Bologna, 21: 167-177.

Fanti F., 2014. Catalogo critico delle Cantharidae d'Italia (Insecta, Coleoptera). Memorie della Società entomologica italiana, 91: 61-132.

Fanti F. \& Vitali F., 2013. Lygistopterus anorachilus Ragusa, 1883 (Coleoptera, Lycidae), morphological and faunistic remarks. Atti della Società italiana di
Scienze naturali e del Museo civico di Storia naturale di Milano, 154: 71-75.

Fattorini S., 2001. Nuovi dati regionali e altre note di distribuzione su Tenebrionidi italiani (Coleoptera Tenebrionidae). Bollettino della Società entomologica italiana, 133: 43-53.

Ferrer J., 2015. Revisión del género Leptoderis Billberg, 1820 y comentarios sobre el origen, composición, anatomía y necrofagia de la Tribu Elenophorini (Coleoptera, Tenebrionidae). Boletín de la Sociedad Entomológica Aragonesa, 57: 19-38.

Ferrer J., 2018. Contribución al conocimiento del Genero Pachychila Eschscholtz, 1831 y descripción de dos especies nuevas de la Península Ibérica y una de Italia (Coleoptera, Tenebrionidae, Pimeliinae). Boletín de la Sociedad Entomológica Aragonesa, 62: 35-54.

Ferrer J., Martínez Fernández J.C. \& Castro Tovar A., 2008. Aportación al conocimiento del genero Akis Herbst, 1799 (Coleoptera; Tenebrionidae, Pimeliinae). Boletín de la Sociedad Entomológica Aragonesa, 43: 153-172.

Ferro G., 1971. Su alcuni reperti inediti di Idrofilidi nelle regioni meridionali italiane (Coleoptera). Bollettino della Società entomologica italiana, 103: 150-152.

Ferro G., 1974. Reperti inediti di Idrofilidi nelle regioni meridionali italiane. II contributo. Bollettino della Società entomologica italiana, Genova, 106: 22-23.

Ferro G., 1976. Diagnosi preliminare di una nuova specie di Hydrobiini. Rivista di Idrobiologia Istituto Idrobiologico Pesc. G.B. Grassi Università di Perugia, 15: 433-435.

Ferro G., 1979. Ricerche coleotterologiche sul litorale ionico della Puglia, Lucania e Calabria, Campagne 19561957-1958. XVIII. Coleoptera Palpicornia. Bollettino della Società entomologica italiana, 111: 25-33.

Ferro G., 1990. Due nuovi Ochthebius (Hymenodes) Paleartici (Coleoptera Hydraenidae). XXIII. Contributo alla conoscenza degli Hydraenidae. Bulletin et Annales de la Societe Royale Belge d'Entomologie, 126: 51-54.

Fery H., 1991. Revision der "minutissimus-Gruppe" der Gattung Bidessus Sharp (Coleoptera: Dytiscidae). Entomologica Basiliensia, 14: 57-91.

Fery H. \& Nilsson A.N., 1993. A revision of the Agabus chalconatus- and erichsoni-groups (Coleoptera: Dytiscidae), with a proposed phylogeny. Entomologica Scandinavica, 24: 79-108.

Fiori A., 1894. Alcune nuove specie e varietà di Staphylinidae. Il Naturalista siciliano, 4: 86-100.

Fiori A., 1903. Note di Corologia e di Caccia. Nuove indicazioni topografiche. Rivista Coleotterologica italiana, 1: 198-205.

Fiori A., 1904. Note di Corologia e di Caccia. Nuove indicazioni topografiche. Rivista Coleotterologica italiana, 2: 131-136. 
Fiori A., 1914a. Le Cantharis di Sicilia confrontate con quelle di altri paesi. Rivista Coleotterologica italiana, 12: $1-18$.

Fiori A., 1914b. Descrizione di alcune specie di Coleotteri mirmecofili del Gargano, Sicilia e Cirenaica. Rivista Coleotterologica italiana, 12: 105-120.

Fiori A., 1915a. Appunti sulla fauna coleotterologica dell'Italia Meridionale e della Sicilia. Rivista Coleotterologica italiana, 12 (1914): 166-191.

Fiori A., 1915b. Nuove specie italiane dei generi Malthinus e Malthodes. Rivista Coleotterologica italiana, 13: 45-56.

Fiori A., 1915c. Appunti sulla fauna coleotterologica del1'Italia Meridionale e della Sicilia. Rivista Coleotterologica italiana, 13: 57-84.

Fiori G., 1948. I Byrrhus L. s. str. italiani. Bollettino dell'Istituto di Entomologia della Università di Bologna, 17: 1-21.

Fiori G., 1951. Alcuni appunti sui Byrrhus L. s. str. Europei. II contributo alla conoscenza della famiglia Byrrhidae. Bollettino dell'Istituto di Entomologia della Università di Bologna, 18: 293-304.

Fiori G., 1953. I Seminolus Muls. et Rey. III contributo alla conoscenza della famiglia Byrrhidae. Redia, 38: 85-110.

Flach K., 1907. Beiträge zur Käferfauna Kalabriens. Deutsche Entomologische Zeitschrift, 52: 15-17.

Focarile A., 1949. I Contributo alla conoscenza dei Trechini paleartici (Coleoptera: Carabidae). Bollettino della Società entomologica italiana, 79: 71-79.

Focarile A., 1959. Ricerche coleotterologiche sul litorale ionico di Puglia, Lucania e Calabria. Campagna 1956-1957-1958. I. Notizie introduttive, Coleoptera Carabidae. Memorie della Società entomologica italiana, 38: 17-109.

Focarile A., 1960a. Ricerche coleotterologiche sul litorale ionico della Puglia, Lucania e Calabria, Campagne 1956-1957-1958. III. Coleoptera Haliplidae, Dytiscidae, Gyrinidae. Memorie della Società entomologica italiana, 39: 41-114.

Focarile A., 1960b. Ricerche coleotterologiche sul litorale ionico della Puglia, Lucania e Calabria, Campagne 1956-1957-1958. V. Coleoptera Helodidae. Bollettino della Società entomologica italiana, 90: 112-113.

Focarile A., 1961. Revisione dei coleotteri Helodidae conservati nel Museo Civico di Storia naturale di Milano. 1. Genere Cyphon Payk. Atti della Società italiana di Scienze naturali e del Museo civico di Storia naturale di Milano, 100: 257-268.

Focarile A., 1964. Ricerche coleotterologiche sul litorale ionico della Puglia, Lucania e Calabria, Campagne 1956-1957-1958. X. Coleoptera Staphylinidae. Bollettino della Società entomologica italiana, 94: 4970 .
Fogato W., 1978. Note sui Luperus bicolori italiani e descrizione di L. leonardii n. sp. Memorie della Società entomologica italiana, 57: 46-64.

Francia F., 1986. I Polydrosus del sottogenere Thomsoneonymus Desbюchers. Memorie della Società entomologica italiana, 64 (1985): 53-72.

Franciscolo M.E., 1956. Ricerche Zoologiche sul Massiccio del Pollino. XVIII. Coleoptera. 8. Mordellidae, Scraptiidae. Annuario dell'Istituto e Museo di Zoologia della Università di Napoli, 8: 1-7.

Franciscolo M.E., 1961. Haliplidae, Dytiscidae, Gyrinidae dei Monti Picentini e dell'Aspromonte. Memorie del Museo Civico di Storia Naturale di Verona, 9: 155-172.

Franciscolo M.E., 1964. Haliplidae, Dytiscidae, Gyrinidae della Sila e dei Monti Siculi. Memorie del Museo Civico di Storia Naturale di Verona, 12: 173220.

Franciscolo M.E., 1997. Fauna d'Italia. XXXV. Coleoptera Lucanidae. Ed. Calderini, Bologna, XI+ $228 \mathrm{pp}$.

Franzini G., 2019. Contribution to the knowledge of the genus Clanoptilus Motschulsky, 1854 in Italy (Coleoptera: Cleroidea, Malachiidae). Fragmenta entomologica, 51: 201-216.

Gardini G., 1979. Nuovi dati sui Tenebrionidi dell'Arcipelago Toscano. Bollettino del Museo Civico di Storia Naturale di Verona, 6: 73-77.

Gardini G., 2005. Insecta Coleoptera Tenebrionidae. In: Ruffo S. \& Stoch F. (Eds.), Checklist e distribuzione della Fauna italiana. Memorie del Museo Civico di Storia Naturale di Verona, 2 serie, Sezione Scienze della Vita, 16: 217-218 + CD-ROM.

Gardini G., 2010. Boromorphus italicus n. sp. dell'Italia meridionale (Coleoptera, Tenebrionidae). Doriana, supp. Annali del Museo civico di Storia naturale "G. Doria" di Genova, 8 (368): 1-7.

Gentile E. \& Chiesa A., 1975. Revisione dei Laccobius Paleartici (Col. Hydrophilidae). Memorie della Società entomologica italiana, 54: 5-187.

Gerecke R., 1990. Ein Beitrag zur Kenntnis der Dryopidae und Elmidae in Fliessgewassern und Quellen Suditaliens. Lauterbornia, 5: 27-41.

Gerini F., 1955. Ricerche Zoologiche sul Massiccio del Pollino. XV. Coleoptera. 5. Buprestidae. Annuario dell'Istituto e Museo di Zoologia della Università di Napoli, 7: 1-7.

Gimmel M.L., Bocakova M., Gunter N.L. \& Leschen R.A.B., 2019. Comprehensive phylogeny of the Cleroidea (Coleoptera: Cucujiformia). Systematic Entomology, 44: 1-32. http://doi:10.1111/syen.12 338

Gimmel M.L., Leschen R.A.B. \& Esser J., 2019. Revised type species designations for Cryptophylus Reitter, 1874 and Pteryngium Reitter, 1887 (Coleoptera: Cu- 
cujoidea: Erotylidae, Cryptophagidae). The Coleopterists Bulletin, 73: 528-530. http://doi.org/10.1649/ 0010-065X-73.3.528

Giusto C., 1987. Note su Apion (Synapion) perrauderi Desbrochers, 1884 ed Apion (Synapion) falzonii Schatzmayr, 1922. Bollettino della Società entomologica italiana, 119: 45-50.

Giusto C., 1993. Aspidapion (s. str.) caprai n. sp. dell'Italia centro-meridionale (Coleoptera: Apionidae: Apioninae). Memorie della Società entomologica italiana, 71 (1992): 551-558.

Giusto C., 1997. Revisione del genere Oryxolaemus Alonso-Zarazaga, 1990. (Coleoptera: Apionidae: Apionine). Bollettino del Museo Regionale di Scienze naturali di Torino, 15: 313-353.

Gobbi G., 1970. Contributo alla conoscenza dei coleotteri Buprestidi d'Italia. Bollettino dell'Associazione romana di entomologia, 25: 35-45.

Gobbi G., 1971a. Ricerche entomologiche dell'A.R.D.E sull'Appennino Lucano e sul Massiccio del Pollino. Bollettino dell'Associazione romana di entomologia, 26: 27-29.

Gobbi G., 1971b. I Buprestidi dell'appennino lucano. Bollettino dell'Associazione romana di entomologia, 26: 33-64.

Gobbi G., 1973a. Interessanti reperti coleotterologici sull'appennino lucano. Bollettino dell'Associazione romana di entomologia, 28: 33-41.

Gobbi G., 1973b. Aggiunte inedite di Paolo Luigioni al catalogo "I Coleotteri d'Italia". Bollettino della Società entomologica italiana, 105: 29-35.

Gobbi G., 1974. Note geonemiche ed ecologiche su alcuni Buprestidi italiani. Bollettino della Società entomologica italiana, 106: 112-119.

Gobbi G., 1981. Interessanti reperti di Buprestidi italiani e diagnosi di Anthaxia liae n. sp. Bollettino dell'Associazione romana di entomologia, Roma, 36: 33-41.

Gobbi G., 1983. Appunti su Coleotteri Cleroidei predatori xilofagi (Coleoptera Trogositidae, Cleridae, Melyridae). Bollettino dell'Associazione romana di entomologia, 38: 51-62.

Gobbi G., 1993. Nuovi reperti di Buprestidi nell'Italia meridionale (Coleoptera). Bollettino dell'Associazione romana di entomologia, 47 (1992): 75-79.

Gobbi G., 1994. Interessanti reperti di Cerambycidae italiani, specialmente delle regioni centro-meridionali. Bollettino dell'Associazione romana di entomologia, 48 (1993): 83-101.

Gobbi G., 1996. Due notevoli reperti coleotterologici nel Parco Nazionale del Pollino (Coleoptera Trogossitidae, Melandryidae). Bollettino dell'Associazione romana di entomologia, 50 (1995): 65-66.

Gobbi G., 2003. Nuovi reperti di Coleotteri nel biotopo di Policoro (Matera) ed in altre aree protette della Lucania (Coleoptera). Annali del Museo civico di Storia naturale di Ferrara, 5 (2002): 41-50.
Gobbi G., 2011. Il contributo dell'Associazione Romana di Entomologia (ARDE) alle ricerche faunistiche in Lucania. Bollettino dell'Associazione romana di entomologia, 66: 89-95.

Gobbi G., 2018. Ricerche entomologiche nel biotopo di Policoro (Matera). 2. I Coleotteri Buprestidi (Coleoptera, Buprestidae). Bollettino dell'Associazione romana di entomologia, 73: 39-64.

Gobbi G. \& Piattella E., 2008. Ricerche entomologiche nel biotopo di Policoro (Matera). 1. Nota introduttiva. I Coleotteri Lamellicorni. Bollettino dell'Associazione romana di entomologia, 63 (2007): 3-41.

Grandi G., 1956. Campagna di ricerche dell'Istituto di Entomologia Agraria dell'Università di Bologna alla "Foresta Umbra" (Gargano). Accademia Italiana di Scienze Forestali: 405-418.

Gridelli E., 1949a. Il problema delle specie a diffusione transadriatica. La Ricerca Scientifica, 19: 654-665.

Gridelli E., 1949b. Raccolte faunistiche compiute nel Gargano da A. Ghigi e F.P. Pomini. IX. Coleotteri. Acta Pontificia Academia Scientiarum, 13: 145-196.

Gridelli E., 1950. Il problema delle specie a diffusione transadriatica con particolare riguardo ai Coleotteri. Memorie di Biogeografia adriatica, 1: 7-299.

Gridelli E., 1956a. Ricerche Zoologiche sul Massiccio del Pollino. XXI. Coleoptera. 11. Staphylinidae. Annuario dell'Istituto e Museo di Zoologia della Università di Napoli, VIII (5): 1-29.

Gridelli E., 1956b. Ricerche Zoologiche sul Massiccio del Pollino. XXIII. Coleoptera 13. Tenebrionidae. Annuario dell' Istituto e Museo di Zoologia della Università di Napoli, VIII (9): 1-6.

Grimm R., 1985. Zur kenntnis der Tenebrioniden aus Suditalien. Stuttgarter Beiträge zur Naturkunde, Ser. A, 379: 1-32.

Gudenzi I. \& Neri P., 1988. Ocydromus fuliginosus (Netolitzkt) buona specie (Coleoptera, Carabidae). Bollettino del Museo Civico di Storia Naturale di Verona, 14 (1987): 531-538.

Hájek J. \& Fery H., 2019. Catalogue of Palearctic Gyrinidae (Coleoptera). Internet version 2019-01-01. http://www.waterbeetles.eu/documents/PAL CAT G yrinidae_2019.pdf

Háva J., 2015a. World Catalogue of Insects. Volume 13. Dermestidae (Coleoptera). Leiden/Boston, Brill, xxvi +419 pp.

Háva J., 2015b. Review of Dermestidae (Coleoptera: Bostrichiformia) deposited in the Museo Civico di Scienze Naturali Trieste. Studies and Reports, Taxonomical Series, 11: 247-266.

Háva J. \& Nardi G., 2007. Notes on some Attagenus from Italy (Insecta, Coleoptera: Dermestidae). Aldrovandia, 3: 121-124.

Holdhaus K., 1911. Über die Coleopteren und Molluskenfauna des Monte Gargano. Denkschriften der 
Mathematisch-Naturwissenschaftlichen Klasse der Kaiserlichen Akademie der Wissenschaften Wien, 87: 431-465.

Izzillo F., Liberto A., Baiocchi D. \& Crovato P., 2002. Buprestis humeralis nuovo per l'Italia ed altri interessanti reperti di Buprestidi dalle regioni centro meridionali e Sardegna (Coleoptera, Buprestidae). Bollettino dell'Associazione romana di entomologia, 57: 11-25.

Izzillo F. \& Sparacio I., 2011. A new subspecies of Perotis lugubris Fabricius, 1777 from Southern Italy (Coleoptera, Buprestidae). Biodiversity Journal, 2: 153-159.

Jäch M.A., 1990. Revision of the Palearctic species of the genus Ochthebius Leach. V. The subgenus Asiobates (Coleoptera: Hydraenidae). Koleopterologische Rundschau, 60: 37-105.

Jäch M.A., 1991a. Revision of the Palearctic species of the genus Ochthebius Leach. VII. The subgenus Enicocerus Stephens (Coleoptera: Hydraenidae). Elytron, 5: 139-158.

Jäch M.A., 1991b. Revision of the Palearctic species of the genus Ochthebius Leach. XI. The subgenus Calobius Wollaston, 1854 (Insecta: Coleoptera: Hydraenidae). Reichenbachia, 30: 33-45.

Jäch M.A., 1993. Taxonomic revision of the Palearctic species of the genus Limnebius Leach, 1815 (Coleoptera: Hydraenidae). Koleopterologische Rundschau, 63: 99-187.

Jelínek J., Audisio P., Hájek J., Baviera C., Moncoutier B., Barnouin T., Brustel H., Genç H., \& Leschen R.A.B., 2016. Epuraea imperialis (Reitter, 1877), new invasive species of Nitidulidae (Coleoptera) in Europe, with a checklist of sap beetles introduced to Europe and Mediterranean areas. Atti della Reale Accademia Peloritana dei Pericolanti, Classe di Scienze Fisiche, Matematiche e Naturali, 94: 1-24. http://doi: 10.1478/AAPP.942A4

Kapp A., 2005. Die palaarktischen Arten der Gattung Taxicera Mulsant \& Rey. 1873 und Discerota Mulsant \& Rey, 1873 (Staphylinidae, Aleocharinae, Subtribus Taxicerina). Linzer biologische Beiträge, 37/2:1277-1323.

Kapp A., 2019. Revision der westpaläarktischen Arten der Gattungen Oligota Mannerheim, 1830 und Holobus Solier, 1849 (Coleoptera, Staphylinidae, Aleocharinae, Hypocyphtini). Linzer biologische Beiträge, 51(1): 587-698.

Klausnitzer B., 1987. Neue Arten aus der Helodes minuta-Gruppe. Entomologische Nachrichten und Berichte, 31: 247-252.

Klausnitzer B., 2009. Insecta: Coleoptera: Scirtidae. In: Suswasserfauna von Mitteleuropa. Begrundet von A. Brauer, herausgegeben von P. Zwick. Band 20/17. Heidelberg: Spektrum Akademischer Verlag, 326 pp.
Koch C., 1933a. Risultati scientifici delle cacce entomologiche di S.A.S. il Principe Alessandro Della Torre e Tasso in Italia. 1. Anthophagus. Bollettino della Società entomologica italiana, 65: 27-30.

Koch C., 1933b. Risultati scientifici delle cacce entomologiche di S.A.S. il Principe Alessandro Della Torre e Tasso in Italia. II. Anthicus. Bollettino della Società entomologica italiana, 65: 149-159.

Koch C., 1937. Appunti sugli Staphilinidi italiani. III. Bollettino della Società entomologica italiana, 69: 83-88.

Koch C., 1938. Appunti sugli Staphilinidi italiani. IV. Bollettino della Società entomologica italiana, 70: 135-141.

Konstantinov A.S., Baselga A., Grebennikov V.V., Prena J. \& Lingafelter S.W., 2011. Revision of the Palearctic Chaetocnema species (Coleoptera: Chrysomelidae: Galerucinae: Alticini). Pensoft Publishers, Series Faunistica, 95, 363 pp.

Košt’al M. \& Caldara R., 2019. Revision of Palaearctic species of the genus Cionus Clairville (Coleoptera: Curculionidae: Cionini). Zootaxa, 4631: 1-144. https://doi.org/10.11646/zootaxa.4631.1.1

Kundrata R., Baena M. \& Bocak L., 2015. Classification of Omalisidae based on molecular data and morphology, with description of Paradrilinae subfam. nov. (Coleoptera: Elateroidea). Zootaxa 3915 (3): 413422. http://dx.doi.org/10.11646/zootaxa.3915.3.6

Kundrata R. \& Bocak L., 2011. The phylogeny and limits of Elateridae (Insecta, Coleoptera): is there a common tendency of click beetles to soft-bodiedness and neoteny? Zoologica Scripta, 40: 364-378. http://doi: 10.1111/j.1463-6409.2011.00476.x

Kundrata R., Bocakova M. \& Bocak L., 2013. The phylogenetic position of Artematopodidae (Coleoptera: Elateroidea), with description of the first two Eurypogon species from China. Contributions to Zoology, 82: 199-208.

Kundrata R., Bocakova M. \& Bocak L., 2014. The comprehensive phylogeny of the superfamily Elateroidea (Coleoptera: Elateriformia). Molecular Phylogenetics and Evolution, 76: 162-171. http://dx.doi.org/10. 1016/j.ympev.2014.03.012

Kundrata R., Gunter N.L., Janosikova D. \& Bocak L., 2018. Molecular evidence for the subfamilial status of Tetralobinae (Coleoptera: Elateridae), with comments on parallel evolution of some phenotypic characters. Arthropod Systematics \& Phylogeny, 76: 137-145. https://www.senckenberg.de/wp-content/ uploads/2019/07/08_asp_76-1_kundrata_137-145. pdf

Kundrata R., Jäch M.A. \& Bocak L., 2016. Molecular phylogeny of the Byrrhoidea-Buprestoidea complex (Coleoptera, Elateriformia). Zoologica Scripta, 46: 150-164. https://doi:10.1111/zsc. 12196 
Kundrata R., Kubaczkova M., Prosvirov A.S., Douglas H.B., Fojtikova A., Costa C., Bousquet Y., AlonsoZarazaga M.A. \& Bouchard P., 2019. World catalogue of the genus-group names in Elateridae (Insecta, Coleoptera). Part I: Agrypninae, Campyloxeninae, Hemiopinae, Lissominae, Oestodinae, Parablacinae, Physodactylinae, Pityobiinae, Subprotelaterinae, Tetralobinae. ZooKeys 839: 83-154. https://doi:10.3897/zookeys.839.33279

La Greca M., 1967. Considerazioni sulla origine del popolamento faunistico pugliese. Archivio Botanico e Biogeografico Italiano, 43, 4^ serie, 11: 297-320.

Leo P., 1982. Gli Pseudoseriscius italiani del gruppo helvolus (Kuster). Natura. Società italiana Scienze naturali, Museo civico di Storia naturale e Acquario civico di Milano, 73: 97-107.

Leo P., 1985. Segnalazioni faunistiche italiane. 54. Dichillus minutus (Solier) (Coleoptera Tenebrionidae). Bollettino della Società entomologica italiana, Genova, 117: 64.

Leo P., 2008. Osservazioni su Dichillus corsicus e descrizione di tre nuove specie del Mediterraneo occidentale (Coleopera, Tenebrionidae). Annali del Museo civico di Storia naturale "G. Doria" di Genova, 99: 603-627.

Leonardi C., 1971. Considerazioni sulle Psylliodes del gruppo napi e descrizione di una nuova specie (Coleoptera Chrysomelidae). Atti della Società italiana di Scienze naturali e del Museo civico di Storia naturale di Milano, 112: 485-533.

Leonardi C., 1972. La "Psylliodes wachsmanni" Csiki specie distinta e suo inquadramento nel gruppo della "Psylliodes picina". Atti del Museo Civico di Storia naturale di Trieste, 28: 137-146.

Leonardi C., 1975. Le Psylliodes appenniniche del Museo Civico di Storia naturale di Verona. Bollettino del Museo Civico di Storia Naturale di Verona, 2: 5190.

Leonardi C., 1976. Ricerche coleotterologiche sul litorale ionico della Puglia, Lucania e Calabria, Campagne 1956-1957-1958. XVII. Coleoptera Chrysomelidae, Alticinae. Atti della Società italiana di Scienze naturali e del Museo civico di Storia naturale di Milano, 117: 46-58.

Leonardi C., 2007. Dati inediti sul genere Psylliodes Latreille, con descrizione di quattro nuove specie mediterranee (Coleoptera Chrysomelidae). Atti della Società italiana di Scienze naturali e del Museo civico di Storia naturale di Milano, 148: 161-240.

Leonardi C., 2014. Indagine critica su Psylliodes laevifrons Kutschera con descrizione di due nuove specie (Coleoptera Chrysomelidae). Atti della Società italiana di Scienze naturali e del Museo civico di Storia naturale di Milano, 154: 81-114.

Leonardi C. \& Sassi D., 1997. I Crisomelidi (Coleoptera
Chrysomelidae) del Monte Barro (Italia, Lombardia, Lecco). Memorie della Società italiana di Scienze naturali e del Museo civico di Storia naturale di Milano, 27: 189-227.

Leonardi C. \& Sassi D., 2001. Studio critico sulle specie di Cryptocephalus del gruppo hypochaeridis (Linné, 1758) e sulle forme ad esse attribuite (Coleoptera Chrysomelidae). Atti della Società italiana di Scienze naturali e del Museo civico di Storia naturale di Milano, 142: 3-96.

Leseigneur L., 1967a. Ricerche coleotterologiche sul litorale ionico della Puglia, Lucania e Calabria, Campagne 1956-1957-1958. XII. Coleoptera Elateridae, Throscidae, Melasidae. Bollettino della Società entomologica italiana, 97: 116-123.

Leseigneur L., 1967b. Ricerche coleotterologiche sul litorale ionico della Puglia, Lucania e Calabria, Campagne 1956-1957-1958. XIII. Coleoptera Cerambycidae. Bollettino della Società entomologica italiana, 97: 123-129.

Leseigneur L., 1969. Contribution a l'etude du genre Adrastus Eschsch. Bollettino della Società entomologica italiana, 99-101: 86-95.

Leseigneur L., 1996. Trixagus atticus Reitter et T. minutus Rey, deux especes meconnues d'Europe occidentale presentes en France (Coleoptera Throscidae). Bulletin mensuel de la Societe Linneenne de Lyon, 65: 181-192.

Leseigneur L., 1997. Rehabilitation de Trixagus gracilis Wollaston (Coleoptera Throscidae). Bulletin de la Société entomologique de France, 102: 137-142.

Leseigneur L., 2005a. Description de Trixagus meybohmi n. sp. et note sur la morphologie des Trixagus du groupe carinifrons (Coleoptera, Throscidae). Bulletin de la Société entomologique de France, 110: 89-96.

Leseigneur L., 2005b. Trixagus angelinii n. sp. (Coleoptera, Throscidae). Bulletin de la Société entomologique de France, 110: 181-184.

Liberti G., 1978. Revisione delle specie italiane del genere Danacaea, primo gruppo. Memorie della Società entomologica italiana, 57: 29-45.

Liberti G., 1981. Description of a new Danacaea from Calabria (Italy) (Col., Dasytidae). Entomologica Basiliensia, 6: 420-421.

Liberti G., 1984. III Contributo alla conoscenza del Genere Danacaea. Revisione delle specie italiane del $4^{\circ}$ gruppo. Atti della Società italiana di Scienze naturali e del Museo civico di Storia naturale di Milano, 125: $159-179$.

Liberti G., 1985a. IV contributo alla conoscenza del genere Danacaea Cast. (Col., Dasytidae). Descrizione del sottogenere nuovo Allodanacaea e revisione delle specie italiane. Annali del Museo civico di Storia naturale "G. Doria" di Genova, 85: 333362 . 
Liberti G., 1985b. V Contributo alla conoscenza del genere Danacaea (Col., Dasytidae). Revisione delle specie italiane del II e III gruppo. Entomologica Basiliensia, 13: 279-302.

Liberti G., 1995. Revisione delle specie italiane del genere Aplocnemus. Memorie della Società entomologica italiana, 73 (1994): 153-194.

Liberti G., 2004. Il genere Dasytes Paykull in Italia. Revisione e catalogo topografico, sinonimico e bibliografico delle specie italiane (Coleoptera, Dasytidae). Annali del Museo civico di Storia naturale "G. Doria" di Genova, 96: 253-340.

Liberti G., 2007. Descrizione di tre nuove specie di Danacaea subg. Allodanacaea Liberti della fauna italiana (Coleoptera, Cleroidea, Dasytidae). Doriana, Supplemento agli Annali del Museo civico di Storia naturale "G. Doria" di Genova, 8 (355): 1-10.

Liberti G., 2012. A contribution to the knowledge of the european Trichoceble Thomson, 1859 (Coleoptera, Cleroidea, Dasytidae). Annali del Museo civico di Storia naturale "G. Doria" di Genova, 104: 191-252.

Liberti G., 2015. Le specie di Malthodes Kiesenwetter, 1852 d'Italia nord-occidentale (Coleoptera, Cantharidae). Annali del Museo civico di Storia naturale "G. Doria" di Genova, 107: 1-151.

Liberti G., 2016. Le specie di Malthodes Kiesenwetter, 1852 delle Alpi (Coleoptera, Cantharidae) (Malthodes del Nord-Est: $3^{\circ}$ contributo alla conoscenza del genere Malthodes in Italia). Annali del Museo civico di Storia naturale "G. Doria" di Genova, 108: 89198.

Liberti G., 2017. Le specie appenniniche di Malthodes Kiesenwetter, 1852 (Coleoptera, Cantharidae). Annali del Museo civico di Storia naturale "G. Doria" di Genova, 110: 33-164.

Liberti G. \& Constantin R., 2009. The Enicopus Stephens, 1830 species East of the Iberian Peninsula: E. ater (Fabricius, 1787) and E. pilosus (Scopoli, 1763) (Coleoptera, Dasytidae). Annali del Museo civico di Storia naturale "G. Doria" di Genova, 100: 293-321.

Liberti G. \& Plonski I.S., 2019. The Psilothrix Küster, 1850 of the group viridicoerulea Geoffroy, 1785 (Coleoptera: Melyridae: Dasytinae). Zeitschrift der Arbeitsgemeinschaft Österreichischer Entomologen, 71: $153-170$.

Liberti G. \& Poggi R., 2018. I Malthodes (Coleoptera, Cantharidae) della collezione di Agostino Dodero, conservata nel Museo Civico di Storia Naturale "G. Doria" di Genova. Annali del Museo civico di Storia naturale "G. Doria" di Genova, 111: 241-303.

Liberti G. \& Zinetti F., 2009. Nota su alcuni Aplocnemus italiani nuovi o poco noti, con descrizione di Aplocnemus etruscus n. sp. (Coleoptera, Dasytidae). Bollettino della Società entomologica italiana, 141: 45-53.
Liberto A., 1993. Osservazioni sistematiche ed ecologiche su Anostirus (Parastirus) cerrutii Binaghi (Coleoptera, Elateridae). Bollettino dell'Associazione romana di entomologia, 47 (1992): 81-85.

Liberto A. \& Gigli M., 2003. Agrilus viscivorus Bily, un buprestide nuovo per la fauna italiana (Coleoptera, Buprestidae). Bollettino dell'Associazione romana di entomologia, 58: 47-51.

Löbl I., 1970. Revision der palaarktischen Arten der Gattungen Scaphisoma Leach und Caryscapha Ganglbauer der Tribus Scaphisomini. Revue suisse Zoologie, 77: 727-799.

Löbl I. \& Löbl D. (eds.), 2015. Catalogue of Palaearctic Coleoptera, Volume 2. Revised and updated edition. Hydrophiloidea-Staphylinoidea. Brill, Leiden, Boston, XXV + 1702 pp.

Löbl I. \& Lobl D. (Eds.), 2016. Catalogue of Palaearctic Coleoptera, Volume 3. Revised and updated edition. Scarabaeoidea-Scirtoidea-Dascilloidea-BuprestoideaByrrhoidea. Brill, Leiden, Boston, XXVIII + 983 pp.

Löbl I. \& Lobl D. (Eds.), 2017. Catalogue of Palaearctic Coleoptera. Volume 1. Revised and updated edition. Archostemata-Myxophaga-Adephaga. Brill, Leiden, Boston, XXXIV + 1443 pp.

Löbl I. \& Smetana A. (Eds.). 2007. Catalogue of Palaearctic Coleoptera. Volume 4. ElateroideaDerodontoidea-Bostrichoidea-LymexyloideaCleroidea-Cucujoidea. Apollo Books, Stenstrup, 935 pp.

Löbl I. \& Smetana A. (Eds.). 2008. Catalogue of Palaearctic Coleoptera. Volume 5. Tenebrionoidea. Apollo Books, Stenstrup, 670 pp.

Löbl I. \& Smetana A. (Eds.) 2010. Catalogue of Palaearctic Coleoptera. Volume 6. Chrysomeloidea. Apollo Books, Stenstrup, 924 pp.

Lohse A., 1991. Ernobius angelinii n. sp., eine neue Anobiidae aus Italien (Coleoptera Anobiidae). Acta Coleopterologica, 7: 49-51.

Longo S., Russo A. \& Palmieri V., 1991. Sulla diffusione in Italia di Coccotrypes dactyliperda e Dactylotrypes longicollis (Coleoptera: Scolytidae). Atti XVI Congresso nazionale italiano di Entomologia, Bari-Martina Franca (TA) 23/28 settembre 1991: 711-715.

Luigioni P., 1929. I Coleotteri d'Italia. Catalogo sinonimico-topografico-bibliografico. Memorie Pontificia Accademia delle Scieze, "Nuovi Lincei", Roma, ser. 2, 13: 1-1160.

Magistretti M., 1953. Le specie italiane del genere $M y$ labris F. Annali del Museo civico di Storia naturale "G. Doria" di Genova, 62: 30-54.

Magistretti M., 1955. Ricerche Zoologiche sul Massiccio del Pollino. XI. Coleoptera 1. Carabidae. Annuario dell'Istituto e Museo di Zoologia della Università di Napoli, 8: 1-30.

Magistretti M., 1956. Ricerche Zoologiche sul Massiccio 
del Pollino. XIII. Coleoptera 3. Oedemeridae, Meloidae, Alleculidae. Annuario dell'Istituto e Museo di Zoologia della Università di Napoli, V7: 1-6.

Magistretti M., 1960a. Coleotteri Cicindelidi e Carabidi dell'Aspromonte. Memorie del Museo Civico di Storia Naturale di Verona, 8: 111-158.

Magistretti M., 1960b. Coleotteri Edemeridi, Pitidi, Aderidi, Meloidi, Serropalpidi, Lagriidi, Alleculidi dell'Aspromonte. Memorie del Museo Civico di Storia Naturale di Verona, 8: 159-167.

Magistretti M., 1962a. Coleotteri Cicindelidi e Carabidi della Sila. Memorie del Museo Civico di Storia Naturale di Verona 10: 121-194.

Magistretti M., 1962b. Coleotteri Odemeridi, Pitidi, Pirocroidi, Meloidi, Lagriidi, Alleculidi della Sila. Memorie del Museo Civico di Storia Naturale di Verona 10: 195-205.

Magistretti M., 1962c. Ricerche coleotterologiche sul litorale ionico della Puglia, Lucania e Calabria; Campagne 1956-1957-1958. IX, Coleoptera Oedemeridae, Pyrochroidae, Meloidae, Lagriidae, Alleculidae. Bollettino della Società entomologica italiana, 92: 83-92.

Magistretti M., 1965. Coleoptera. Cicindelidae, Carabidae. Catalogo topografico. Fauna d'Italia, VIII. Ed. Calderini, Bologna, 512 pp.

Magistretti M., 1966. Coleotteri Cicindelidi e Carabldi della Puglia. Memorie della Società entomologica italiana, Genova, 45: 5-110.

Magistretti M., 1967. Catalogo degli Oedemeridae italiani. Memorie della Società entomologica italiana, 46: $181-200$.

Magistretti M., 1969. Catalogo topografico dei Coleoptera Cicindelidae e Carabidae d'Italia. I Supplemento. Memorie della Società entomologica italiana, 47: 177-217.

Magistretti M. \& Ruffo S., 1961. Considerazioni sulla diffusione nell'Italia appenninica di alcuni generi di Coleotteri Carabidi e Chrysomelidi. Atti Accademia Nazionale Italiana di Entomologia, Rendiconti, 8: 137-179.

Magistretti M. \& Ruffo S., 1969. Quindici anni di ricerche del Museo Civico di Storia naturale di Verona sulla fauna appenninica (1954-1969). Memorie della Società entomologica italiana, 48 , fasc. 3 : 385-401.

Magnano L., 1957a. Ricerche Zoologiche sul Massiccio del Pollino. XXIV. Coleoptera. 14. Curculionidae. Annuario dell'Istituto e Museo di Zoologia della Università di Napoli, 9: 1-53.

Magnano L., 1957b. Contributi alla conoscenza dei Coleotteri Curculionidi - IV. Appunti per servire alla migliore conoscenza della distribuzione geografica dei Curculionidi italiani. Bollettino della Società entomologica italiana, 87: 138-141.
Magnano L., 1964. I Coleotteri Curculionidi dell'Aspromonte. Memorie del Museo Civico di Storia Naturale di Verona, 8: 107-148.

Magnano L., 1986. Attuali conoscenze sui Limatogaster Apfelbeck (1898) e del gruppo Dialonedus Reitter (1913) (Coleoptera Curculionidae Otiorhynchinae). Frustula entomologica, n.s., 7-8 (20-21): 647-666.

Magnano L., 1993a. Note sugli Otiorhynchus del subg. Arammichnus. 3. Le specie dei gruppi lubricus, hellenicus e torretassoi. Fragmenta entomologica, 24 (1992): 219-242.

Magnano L., 1993b. Note sugli Otiorhynchus del subg. Arammichnus. 4. Le specie dei gruppi setosulus e umbilicatoides (Coleoptera Curculionidae). Fragmenta entomologica, 25: 129-154.

Magnano L., 1996. Note sugli Otiorhynchus del subg. Arammichnus Gozis. 5. Le specie dei gruppi villosus, ferrarii e juvencus (Coleoptera Curculionidae). Fragmenta entomologica, 27: 377-398.

Magnano L., 1998. Otiorhynchus (Prodeminus) angelinii n. sp. dell'Italia meridionale e note sulle specie del sottogenere Prodeminius (Coleoptera, Curculionidae). Bollettino della Società entomologica italiana, 130: $155-158$.

Magnano L., 1999a. Note sugli Otiorhynchus del sottogenere Podoropelmus Reitter, 1912 (Coleoptera Curculionidae). Bollettino del Museo Civico di Storia Naturale di Verona, 23: 247-307.

Magnano L., 1999b. Note sugli Otiorhynchus del subg. Arammichnus Gozis. 6. Le specie dei gruppi concavirostris, velutinus, mandibularis, calabrensis e championi (Coleoptera Curculionidae). Fragmenta entomologica, 31: 117-172.

Magnano L., Osella G.B. \& Abbazzi P., 2008. Una nuova specie italiana di Mesagroicus Schöenherr e ridescrizione delle specie paleartiche occidentali del genere. Bollettino dell'Associazione romana di entomologia, 63: 63-81.

Magrini P., 1984. Trechus angelae n. sp. dell'Appennino Calabro-Lucano. Redia, 67: 247-252.

Magrini P., 1987. Nebria pennisii, nuova specie del Monte Sirino (Coleoptera, Carabidae). Sezione Fiorentina C.A.I., Notiziario n. 2: 3 pp.

Magrini P., 1990. Note su alcuni Anillus italiani con descrizione di tre nuove specie (Coleoptera, Carabidae, Anillini). Redia, 76: 71-82.

Magrini P., 1993. Note su alcuni Trechus italiani (Coleoptera, Carabidae). Redia, 73: 275-282.

Magrini P., 1997. Premiere revision des Duvalius s. str. Italiens (première partie) (Coleoptera, Carabidae, Trechinae). Les Comptes-rendus du L.E.F.H.E., 2: 202-292.

Magrini P., 1999. Descrizione del maschio di Nebria pennisii Magrini, 1987 e considerazioni sulla sua validità specifica (Coleoptera: Carabidae: Nebriinae). Acta Entomologica Slovenica, 7: 25-30. 
Magrini P., 2014. Revisione delle specie italiane del genere Anillus Jacquelin Du Val, 1851 di Italia e Corsica, con descrizione di una nuova specie (Coleoptera, Carabidae, Bembidiini, Anillina). Annali del Museo civico di Storia naturale "G. Doria" di Genova, 106: 133-171.

Magrini P. \& Casale A., 2015. Revisione del genere Rhegmatobius Jeannel, 1937, con descrizione di una nuova specie e una nuova sinonimia (Coleoptera, Carabidae, Bembidiini, Anillina). Annali del Museo civico di Storia naturale "G. Doria" di Genova, 107 (2014): 285-325.

Magrini P., Meoli C. \& Abbazzi P., 2005. Tre nuove specie italiane di Otiorhynchus del sottogenere Lixorrhynchus Reitter, 1914 e note su O. (Lixorrhynchus) camaldulensis (Rottenberg, 1870) (Coleoptera, Curculionidae). Annali del Museo civico di Storia naturale "G. Doria" di Genova, 96 (2004): 215-239.

Magrini P. \& Sciaky R., 1995. Note sui Rhegmathobius italiani, con descrizione di $R$. solarii n. $\mathrm{sp}$. (Coleoptera Carabidae Anillini). Redia, 78: 235-241.

Magrini P. \& Vanni S., 1990. Ridescrizione di Duvalius caoduroi Pace, 1986 e considerazioni sulle sue affinità sistematiche (Coleoptera, Carabidae, Trechinae). Redia, 73: 181-185.

Marcuzzi G., 1962. Studi ecologici e faunistici sui Tenebrionidi della Puglia. Memorie di Biogeografia Adriatica, 6: 1-79.

Marcuzzi G., 1970. Contributo alla conoscenza dei Tenebrionidi delle Tremiti (XV Contributo alla conoscenza dei Tenebrionidi). Bollettino della Società entomologica italiana, 102: 35-38.

Marcuzzi G. \& Turchetto Lafisca M., 1977. Ricerche sui Coleotteri della Puglia raccolti da G. Marcuzzi (196063). I elenco delle specie. Quaderni di Ecologia Animale, 9: 1-186.

Marek O., 1961. Eine gebirgsausbeute von Nitiduliden aus den Apenninen. Memorie del Museo Civico di Storia Naturale di Verona, 9: 1-4.

Mariani G., 1958. Revisione delle specie italiane di Geotrupes subgen. Trypocopris. Memorie della Società entomologica italiana, 38: 23-43.

Mariani G., 1959. Ricerche coleotterologiche sul litorale ionico della Puglia, Lucania e Calabria. Campagne 1956-1957-1958. II. Lamellicornia (ScarabaeidaeLucanidae). Memorie della Società entomologica italiana, fasc. spec., 38: 143-184.

Mariani G., 1969. Sugli Aphodius del sottogenere Agrilinus Muls, della Regione Italiana. Bollettino della Società entomologica italiana, 99-101: 171-192.

Mariani G., 1971. Zoogeografia degli Scarabeidi Laparosticti orofili dell'Appennino centrale (Coleoptera Scarabaeoidea). Biogeographia, Lavori Società italiana di Biogeografia, Bologna, (N. S.), 2: 225-270.
Mascagni A., 1995. Nuovi dati su alcuni coleotteri italiani (Limnichidae, Dryopidae, Elmidae et Georissidae). Bollettino della Società entomologica italiana, 127: $122-126$.

Mascagni A., 2005a. Insecta Coleoptera Georissidae. In: Ruffo S. \& Stoch F. (Eds.), Checklist e distribuzione della Fauna italiana. Memorie del Museo Civico di Storia Naturale di Verona, 2 serie, Sezione Scienze della Vita, 16: 171-172 + CD-ROM.

Mascagni A., 2005b. Insecta Coleoptera Dryopoidea. In: Ruffo S. \& Stoch F. (Eds.), Checklist e distribuzione della Fauna italiana. Memorie del Museo Civico di Storia Naturale di Verona, 2 serie, Sezione Scienze della Vita, 16: 199-200 + CD-ROM.

Mascagni A., 2014. The variegated mud-loving beetles of Europe (first part) (Coleoptera: Heteroceridae). Onychium, 10 (2013): 78-118.

Masutti L., 1964. Considerazioni preliminari sui Coleotteri Scolitidi della foresta di Campigna e notizie su alcune specie reperibili lungo la catena appenninica. Memorie della Società entomologica italiana, 43: 172-183.

Masutti L., 1968. Coleotteri Scolitidi dei Pini d'altitudine in alcuni biotopi delle Alpi e sul Massiccio del M. Pollino. Archivio Botanico e Biogeografico Italiano, Forlì, 44: 213-223.

Mazzei A., Audisio P., Vigna Taglianti A. \& Brandmayr P., 2019. Geographical distribution and conservation status of the threatened saproxylic beetles Rhysodes sulcatus (Fabricius, 1787), Clinidium canaliculatum (O.G. Costa, 1839) and Omoglymmius germari (Ganglbauer, 1891) in Italy (Coleoptera: Rhysodidae). Fragmenta entomologica, 51: 89-96.

Mazzei A., Bonacci T. \& Brandmayr P., 2012. La diversita funzionale della "Guild" di Coleotteri Carabidi lungo la successione ecologica secondaria nel bioma delle sclerofille in Calabria. Quaderno di Studi e Notizie di Storia naturale della Romagna, 36: 79-87.

Mazzei A., Bonacci T., Brandmayr P. \& Dutto M., 2015. Gnorimus variabilis (Linnaeus, 1758) in Calabria (Italia Meridionale) (Coleoptera Cetoniidae). Il Naturalista siciliano, 39: 51-57.

Mazzei A., Bonacci T., Contarini E. \& Brandmayr P., 2011. Coleotteri saproxilobionti del Parco Nazionale della Sila (Calabria) (Insecta Coleoptera). Quaderno di Studi e Notizie di Storia naturale della Romagna, 32: 81-93.

Mazzei A., Bonacci T., Contarini E., Zetto T. \& Brandmayr P., 2011. Rediscovering the "umbrella species" candidate Cucujus cinnaberinus (Scopoli, 1763) in Southern Italy (Coleoptera Cucujidae), and notes on bionomy. Italian Journal of Zoology, 78: 264-270. https://doi:10.1080/24750263.2018.1449906

Mazzei A., Bonacci T., Gangale C., Luzzi G., Pizzolotto R. \& Brandmayr P., 2014. La carabidofauna delle al- 
nete della Sila: influenza dei fattori di antropizzazione. XXIV Congresso Nazionale Italiano di Entomologia, Orosei (Sardegna), 9-14 giugno 2014, poster.

Mazzei A., Bonacci T., Gangale C., Pizzolotto R. \& Brandmayr P., 2015. Functional species traits of carabid beetles living in two riparian alder forests of the Sila plateau subject to different disturbance factors (Coleoptera: Carabidae). Fragmenta entomologica, 47: 37-44.

Mazzei A., Bonacci T., Horakb J. \& Brandmayr P., 2018. The role of topography, stand and habitat features for management and biodiversity of a prominent forest hotspot of the Mediterranean Basin: Saproxylic beetles as possible indicators. Forest Ecology and Management, 410: 66-75. https://doi.org/10.1016/j. foreco.2017.12.039

Mazzei A., Bonacci T., Laudati M., Luzzi G., Dutto M. \& Brandmayr P., 2014. Osservazioni sul ritrovamento di Osmoderma italicum Sparacio, 2000 (Coleoptera Scarabaeidae Cetoniinae) nel Parco Nazionale della Sila (Calabria, Italia) e revisione della presenza storica nell'Italia Meridionale. Il Naturalista siciliano, 38: 331-337.

Mazzei A. \& Brandmayr P., 2016. I coleotteri del Parco nazionale della Sila. Specie saproxilobionti di maggior interesse comunitario, faunistico e conservazionistico. Parco Nazionale della Sila, serie "Parco", Nr. $14,190 \mathrm{pp}$

Mazzei A. \& Brandmayr P., 2018. Agonum tulliae sp. n. from the Sila National Park (Calabria, southern Italy) (Coleoptera: Carabidae: Platynini). Journal of Entomological and Acarological Research, 49: 48-53. https://doi:10.4081/jear.2017.6677

Mazzei A., Brandmayr P., Contarini E. \& Luzzi G., 2016. I Coleotteri del Parco Nazionale della Sila. Ente Parco Nazionale della Sila, 190 pp

Mazzei A., Gangale C., Laurito M., Luzzi G., Menguzzato G., Pizzolotto R., Scalise C., Uzunov D. \& Brandmayr P., 2017. I Coleotteri Carabidi (Coleoptera, Carabidae) come indicatori di passati interventi selvicolturali in foreste vetuste del Parco Nazionale della Sila (Calabria, Italia). Forest, 14: 162-174. https://doi: 10.3832/efor2351-014

McKenna D.D., Farrell B.D., Caterino M.S., Farnum C.W., Hawks D.C., Maddison D.R., Seago A.E., Short A.E.Z., Newton A.F. \& Thayer M.K., 2014. Phylogeny and evolution of Staphyliniformia and Scarabaeiformia: forest litter as a stepping stone for diversification of nonphytophagous beetles. Systematic Entomology, 40: 35-60. https://doi:10.1111/syen. 12093

Meggiolaro G., 1960. Ricerche coleotterologiche sul litorale ionico della Puglia, Lucania e Calabria, Campagne 1956-1957-1958. IV. Coleoptera Pselaphidae.
Bollettino della Società entomologica italiana, 90: 80-94.

Meggiolaro G., 1966a. Descrizione di alcuni nuovi Pselaphidae paleartici e note sinonimiche. Bollettino della Società entomologica italiana, 96: 150-161.

Meggiolaro G., 1966b. Su alcuni euplectini paleartici. Bollettino della Società entomologica italiana, 96: 163-168.

Meggiolaro G., 1967. Studi sugli Pselaphidae dell'Appennino centro-meridionale. Fragmenta entomologica, 5: 133-151.

Meregalli M., 1985. Revisione del genere Plintus Germar. Memorie del Museo Civico di Storia Naturale di Verona, 2 serie, 5: 1-133.

Migliaccio E. \& Zampetti M.F., 1988. Megabruchidius dorsalis e Acanthoscelidis pallidipennis, specie nuove per la fauna italiana. Bollettino dell'Associazione romana di entomologia, 43: 63-69.

Miksic R., 1959. Ricerche Zoologiche sul Massiccio del Pollino. XXIX. Coleoptera. 15. Beitrag zur Kenntnis der Lamellicornis-Fauna der Apenninen. Annuario dell'Istituto e Museo di Zoologia della Università di Napoli, 11: 1-24.

Miksic R., 1961. Beitrag zur kenntnis der LamellicornisFauna der Apenninen. IV. Aspromonte. Memorie del Museo Civico di Storia Naturale di Verona, 9: 5-24.

Minelli A. \& Vittorelli S., 1976. Il Rassenkreis Timarcha tenebricosa (F.). Bollettino del Museo Civico di Storia Naturale di Verona, 3: 1-33.

Mochi O., Terzani F. \& Bartolozzi L., 2019. Contributo alla conoscenza della distribuzione italiana di Amorphocephala coronata (Germar, 1817) (Insecta: Coleoptera: Brentidae). Quaderno di Studi e Notizie di Storia naturale della Romagna, 49: 137-140.

Monzini V. \& Angelini F., 1997. Nuovi dati geonemici su Carabidi dell'Italia meridionale. Bollettino della Società entomologica italiana, Genova, 129: 39-50.

Monzini V. \& Romano V.A., 2001. I Coleotteri Carabidi del fiume Basento (Potenza): nuovi dati per l'Italia meridionale e per la regione Basilicata. Bollettino della Società entomologica italiana, 133: 27-35.

Moscardini C., 1962. Tre nuove specie appenniniche del genere Cantharis del gruppo tristis Fabr. Memorie del Museo Civico di Storia Naturale di Verona, 10: 105-119.

Moscardini C., 1964. Osservazioni sulle Cantharis pellucida Fabr., C. pellucida rauterbergi Reitt. e $C$. baudii Fiori. Bollettino della Società entomologica italiana, 94: 167-171.

Moscardini C., 1965. Osservazioni sulle Cantharis nigricans Mull., alpestris Fiori e sulle specie affini. Bollettino della Società entomologica italiana, 95: 112-123.

Moscardini C., 1967. Cantharis puncticollis Levrat, C. erichsoni Bach e descrizione di una nuova specie. 
Bollettino della Società entomologica italiana, 97: 28-33.

Moscardini C., 1968. I Coleotteri Cantaridini della Regione Appenninica (Col. Cantharidae). Memorie del Museo Civico di Storia Naturale di Verona, 16: 4996.

Müller G., 1955. Ricerche Zoologiche sul Massiccio del Pollino. XVI. Coleoptera. 6. Histeridae. Annuario dell'Istituto e Museo di Zoologia della Università di Napoli, 7: 1-7.

Müller G., 1960. Ricerche coleotterologiche sul litorale ionico della Puglia, Lucania e Calabria. Campagne 1956-1957-1958. VI. Coleoptera Histeridae. Bollettino della Società entomologica italiana, 90: 136140.

Muscarella C., Sparacio I., Liberto A. \& Nardi G., 2013. The genus Lichenophanes Lesne, 1899 in Italy (Coleoptera Bostrichidae) and short considerations on the saproxylophagous beetle-fauna of Nebrodi Mountains (Sicily). Biodiversity Journal, 4: 451-466.

Mutinelli F., Federico G., Carlin S., Montarsi F. \& Audisio P., 2016. Preliminary investigation on other Nitidulidae beetles species occurring on rotten fruit in Reggio Calabria province (south-western Italy) infested with small hive beetle (Aethina tumida). Journal of Apicultural Research, 54: 1-3. http://dx.doi. org/10.1080/00218839.2016.1142733

Nadein K., 2009. Revision of the genus Mniophila Stephens, 1831 (Coleoptera: Chrysomelidae). Beitrage zur Entomologie, 59: 103-131.

Nardi G., 1994. Note su alcuni Coleotteri rinvenuti sui Monti Lepini e nelle aree limitrofe (Lazio) (Coleoptera Histeridae, Bothrideridae, Tenebrionidae, Curculionidae). Quaderni Museo storia Naturale di Patrica, 6: 99-117.

Nardi G., 2007. Nomenclatorial and faunistic notes on some world Aderidae (Coleoptera). Zootaxa 1481: 21-34.

Nardi G. \& Biscaccianti A.B., 2017. New Italian records of Lichenophanes varius (Illiger, 1801) (Coleoptera, Bostrichidae). Nature Conservation, 19: 219-229. https://doi: 10.3897/natureconservation.19.12449

Nardi G. \& Hava J., 2013. Italian Dermestidae: notes on some species and an updated checklist (Coleoptera). ZooKeys 360: 45-81. https://doi: 10.3897/zookeys. 360.6023

Nardi G. \& Zahradnik P., 1998. Nuovi dati geonemici su Anobidi di Spagna, Italia e Transcaucasia (Coleoptera, Anobiidae). Bollettino dell'Associazione romana di entomologia, 53: 33-40.

Neri P., 1982. Un nuovo Ocydromus dell'Appennino. (Coleoptera, Carabidae). Bollettino del Museo Civico di Storia Naturale di Verona, 8: 157-163.

Neri P., Bonavita P., Gudenzi I., Magrini P. \& Toledano L., 2011. Bembidiina della fauna italo-corsa: chiavi di identificazione (Insecta Coleoptera Carabidae). Quaderno di Studi e Notizie di Storia naturale della Romagna, 33: 1-183.

Neri P. \& Gudenzi I., 2011. Descrizione di Bembidion (Philochtus) demartini n. sp. dell'Appennino Meridionale (Insecta Coleoptera Carabidae). Quaderno di Studi e Notizie di Storia naturale della Romagna, 32: 95-104.

Neri P. \& Magrini P., 2011. Note concernenti i Bembidion appartenenti al sottogenere Lymnaeum Stephens, 1828 (Insecta Coleoptera Carabidae). Quaderno di Studi e Notizie di Storia naturale della Romagna, 31 (2010): 135-154.

Neri P. \& Pavesi M., 1989. Ocydromus (Peryphiolus) lucifugus n. sp. dell'Italia meridionale (Coleoptera, Carabidae, Bembidiinae). Atti della Società italiana di Scienze naturali e del Museo civico di Storia naturale di Milano, 130: 217-227.

Nilsson A.N. \& Hájek J., 2019. Catalogue of Palearctic Dytiscidae (Coleoptera). Internet version 2019-01-01 (http://www.waterbeetles.eu/documents/PAL_CAT_ Dytiscidae_2019.pdf).

Norbiato M. \& Bartolozzi L., 2017. Contributo alla conoscenza geonemica degli Antribidi italiani (Insecta: Coleoptera: Curculionoidea: Anthribidae: Anthribinae, Choraginae). Quaderno di Studi e Notizie di Storia naturale della Romagna, 45: 125-154.

Nyholm T., 1948. Studien über die familie Helodidae arten. III: Neue Palaarktische arten der Gattung Cyphon Payk. Arkiv för Zoologi, 41: 1-7.

Olmi M., 1972. The paleartic species of the genus Dyops Olivier (Col. Dryopidae). Bollettino Museo di Zoologia Università di Torino, 5: 69-132.

Olmi M., 1976. Revisione delle specie italiane del genere Limnius Illiger con note sulle altre specie paleartiche. Bollettino del Museo civico di Storia naturale di Venezia, 28: 1-15.

Olmi M., 1977. Coleoptera. Dryopidae, Elminthidae. Fauna d'Italia, XII. Ed. Calderini, Bologna, 272 pp.

Osella G.B., 1967. Revisione delle specie italiane del genere Dichotrachelus Stierlin. Memorie del Museo Civico di Storia Naturale di Verona, 15: 349-445.

Osella G.B., 1977. Revisione della sottofamiglia Raymondionyminae (Coleoptera, Curculionidae). Memorie del Museo Civico di Storia Naturale di Verona, 1: $1-162$.

Osella G.B., 1981a. Il genere Styphlidius Pececke, 1936. Bollettino del Museo Civico di Storia Naturale di Verona, 7 (1980): 57-77.

Osella G.B., 1981b. Ruffodytes servadeii nuova specie di Erirrhininae del Gargano. Memorie della Società entomologica italiana, 60: 273-277.

Osella G.B. \& Andretti A., 1996. Pseudostyphlus pillumus (Gillenhal, 1836): prima segnalazione per la fauna italiana (Coleoptera Curculionidae: Notarinae). Redia, Firenze, 79: 11-25. 
Osella G.B. \& Bellò C., 2010. Revisione di Minyops Schoenherr, 1823 e Paraminyops nov. gen. (Coleoptera, Curculionidae, Molytinae). Memorie del Museo Civico di Storia Naturale di Verona, 19: 3133.

Osella G.B., Biondi S., Di Marco C., Magnano L. \& Zuppa A.M., 2005. Insecta Coleoptera Curculionoidea. In: Ruffo S. \& Stoch F. (Eds.), Checklist e distribuzione della Fauna italiana. Memorie del Museo Civico di Storia Naturale di Verona, 2 serie, Sezione Scienze della Vita, 16: 231-234 + CD-ROM.

Osella G.B. \& Lodos N., 1979. Hoplopteridius lutosus (Frivaldsky): brevi note sistematiche (Coleoptera: Curculionidae-Hylobiinae). Türkiye Bitki Koruma Dergisi, Izmir, 3: 161-170.

Osella G.B. \& Magnano L., 1986. I Coleotteri Attelabidi e Curculionidi a diffusione transadriatica. Biogeographia, Lavori Società italiana di Biogeografia, Bologna, 10 (1984): 701-792.

Osella G.B., Pannunzio G. \& Zanetti A., 2009. Il popolamento ad Artropodi dei muschi igropetrici del Parco Nazionale del Gran Sasso d'Italia e Monti della Laga. Bollettino del Museo Civico di Storia Naturale di Verona, Botanica Zoologia, 33: 3-26.

Osella G.B. \& Zuppa A.M., 1994a. Gli Otiorhynchus Germar, 1824 del Monte Nerone e montagne viciniori (Appennino umbro-marchigiano). Biogeographia, Lavori della Società italiana di Biogeografia, Bologna, 17 (1993): 367-397.

Osella G.B. \& Zuppa A.M., 1994b. Anthribidae, Apionidae, Attelabidae e Curculionidae del Monte Nerone (e territori viciniori) (Appennino umbromarchigiano) (Coleoptera Curculionidae). Biogeographia, Lavori della Società italiana di Biogeografia, Bologna, 17 (1993): 399-426.

Osella G.B., Zuppa A. \& Altea T., 1994. Il popolamento a Curculionoidea delle Montagne Gemelle dei Fiori e di Campli (Marche-Abruzzo). Rivista Museo Scienze naturali “E. Caffi” di Bergamo, 16: 45-78.

Otero J.C., 2002. Morphometric study of the species Hypocoprus latridioides Motschulsky, 1839 and $H$. quadricollis Reitter, 1877 (Coleoptera: Atomariinae). Entomologica Fennica, 13: 139-145.

Otero J.C., 2011. Coleoptera, Monotomidae, Cryptophagidae. In: Fauna Iberica, vol. 35, Ramos M.A. et al. (Eds.). Museo Nacional de Ciencias Naturales. CSIC. Madrid, 365 pp.

Otero J.C., 2012. Telmatophilus Heer, 1841 (Coleoptera: Cryptophagidae) of western Palaearctic region. Entomologica Fennica, 23: 113-120.

Otero J.C., 2013. Cryptophaginae (Coleoptera) de la Región Paleártica occidental. Asociacion Europaea de Coleopterologia, Barcelona, Coleopterological Monographs, 4: 7-295.

Otero J.C. \& Angelini F., 1981. Su alcuni Cryptophagus italiani nuovi o poco conosciuti (Coleoptera, Cryptophagidae). Bollettino della Società entomologica italiana, 113: 96-100

Otero J.C. \& Angelini F., 1984. Contributo alla conoscenza dei Cryptophagidae italiani. Entomologica, 19: 81-96.

Otero J.C. \& Angelini F., 1996. Segnalazione di dodici specie di Cryptophagidae nuove per la fauna italiana. Bollettino della Società entomologica italiana, 128: 125-136.

Otero J.C. \& López M.J., 2016. Coleoptera Latridiidae. In: Ramos M.A. et al. (Eds.), Fauna Iberica, vol. 42, Museo Nacional de Ciencias Naturales. CSIC. Madrid, 288 pp.

Pace R., 1974a. Tre nuove specie di Pselaphidae dell'Italia meridionale. Bollettino del Museo Civico di Storia Naturale di Verona, 1: 121-134.

Pace R., 1974b. Otto nuove specie di Leptotyphlinae dell'Italia centro-meridionale (Coleoptera Staphylinidae). Bollettino del Museo Civico di Storia Naturale di Verona, 1: 135-166.

Pace R., 1977a. Dodici nuove specie di Leptotyphlinae dell'Italia peninsulare (Coleoptera Staphylinidae). Bollettino del Museo Civico di Storia Naturale di Verona, 4: 155-201.

Pace R., 1977b. Nuove specie di Geostiba Thomson dell'Italia e della Iugoslavia. Nouvelle Revue Entomologie, 7: 299-305.

Pace R., 1977c. Quindici nuove specie di Stafilinidi ipogei dell'Italia centro-meridionale. Redia, 60: 125177.

Pace R., 1977d. Nuove specie di Scydmaenidae della Toscana e della Puglia (Coleoptera). Redia, 60: 211219.

Pace R., 1977e. Studio sul genere Geostiba Thomson con descrizione di nuove specie italiane. Fragmenta entomologica, 13: 183-229.

Pace R., 1978. Leptusa Kr. nuove o poco note del Museo Civico di Storia naturale di Genova. Annali del Museo civico di Storia naturale "G. Doria" di Genova, 82: 295-322.

Pace R., 1979. Una nuova specie di Vulda della fauna d'Italia (Coleoptera Staphylinidae). Nouvelle Revue Entomologie, 9: 107-109.

Pace R., 1985. Descrizione di Allotyphlus sipontinus nuova specie del Gargano e di Metrotyphlus viti nuova specie della Liguria. Società Veneziana di Scienze Naturali. Lavori, 10: 59-63.

Pace R., 1986. Descrizione di Duvalius caoduroi, nuova specie della Grotta di Trecchina (Lucania). Bollettino del Museo Civico di Storia Naturale di Verona, 12: 149-151.

Pace R., 1989. Monografia del genere Leptusa Kraatz. Memorie del Museo Civico di Storia Naturale di Verona, 8: 1-307. 
Pace R., 1996. Coleoptera. Staphylinidae Leptotyphlinae. Fauna d'Italia, XXXIV. Ed. Calderini, Bologna, 328 pp.

Pace R., 2002. Nuove specie del genere Geostiba Thomson (Coleoptera, Staphylinidae). Bollettino del Museo Civico di Storia Naturale di Verona, 26: 3-25.

Pace R. \& Zanetti A., 1977. I Philonthus (sensu lato) appenninici del Museo Civico di Storia naturale di Verona. Bollettino del Museo Civico di Storia Naturale di Verona, 4: 287-305.

Paganetti-Hummler G., 1910. Beitrag zur Kenntnis der Halticinenfauna Mittel- und Süditaliens. Zeitschrift für Wissenschaftliche Insektenbiologie, 6: 169-171.

Palm T., 1939. En exkursionsdag på Monte Pollino. Entomologbladet, 3: 58-69.

Palmeri V. \& Campolo O., 2006. Sulla presenza di Phoracantha recurva Newman e Phoracantha semipunctata F. (Coleoptera Cerambycidae) in Calabria. Bollettino di Zoologia Agraria e Bachicoltura, 38: 251-254.

Palmeri V., Scirtò G., Malacrinò A., Laudani F. \& Campolo O., 2015. A new pest for European honey bees: first report of Aethina tumida Murray (Coleoptera: Nitidulidae) in Europe. Apidologie, 46: 527-529. https://doi:10.1007/s13592-014-0343-9

Pan A. \& Bologna M.A., 2014. Taxonomy, Bionomics and Faunistics of the Nominate Subgenus of $M y$ labris Fabricius, 1775, with the description of five new species (Coleoptera: Meloidae: Mylabrini). Zootaxa 3806: 1-78. http://dx.doi.org/10.11646/ zootaxa.3806.1.1

Pantaleoni R.A., 2012. Achille Costa (1823-1898), Entomologo Naturalista Esploratore: tratteggio biografico. Il Naturalista siciliano, 36: 3-17.

Pardo Alcaide A., 1961. Ricerche Zoologiche sul Massiccio del Pollino. XXXI. Coleoptera 16. Lycidae y Malachiidae de los Montes Pollino y Aspromonte (Italia meridionale). Annuario dell'Istituto e Museo di Zoologia della Università di Napoli, 17: 1-8.

Pardo Alcaide A., 1967. Notas sobre Malachiidae del Mediterraneo Occidental (Col.) V. Memorie del Museo Civico di Storia Naturale di Verona, 15: 97 104.

Pasqual C. \& Angelini F., 2001. Malachiini dell'Italia meridionale e della Sicilia (Coleoptera, Melyridae). Bollettino del Museo Civico di Storia Naturale di Verona, Botanica Zoologia, 25: 101-126.

Pederzani F., 1999. Hydroporus (Sternoporus) jurjurensis Régimbart, 1895 nel Massiccio del Pollino. Quaderno di Studi e Notizie di Storia naturale della Romagna, 11, suppl.: 3-4.

Pederzani F. \& Rocchi S., 2005. Due interessanti Hydroporus Clairville del Parco Nazionale d'Abruzzo: Hydroporus (Sternoporus) apenninus n. sp. e Hydroporus (Hydroporus) sanfilippoi Ghidini, 1958
(Coleoptera Dytiscidae). Atti Accademia Roveretana degli Agiati, ser. 8, 5B: 317-324.

Pedroni G., 2004. Nuovi dati geonemici ed ecologico-biologici sugli Anthribidae italiani (Coleoptera Curculionoidea). Bollettino del Museo Civico di Storia Naturale di Verona, Botanica Zoologia, 28: 39-50.

Pedroni G., 2012. Le specie italiane del gruppo di Leiosoma scrobiferum con descrizione di sei specie nuove (Coleoptera, Curculionidae, Molytini). Bollettino del Museo Civico di Storia Naturale di Verona, 36: 73-90.

Pesarini C., 1964. Appunti per una migliore conoscenza delle specie circummediterranee del genere Sphenophorus Schoenherr (I Contributo alla conoscenza dei Coleotteri Curculionidi). Bollettino della Società entomologica italiana, 94: 159-164.

Pesarini C., 1972. Ricerche coleotterologiche sul litorale ionico della Puglia, Lucania e Calabria, Campagne 1956-1957-1958. XV. Coleoptera Curculionidae. Bollettino della Società entomologica italiana, 104: 75-85.

Pesarini C., 1974. Note sulla geonemia di alcune specie italiane di Curculionidi della sottofamiglia Cleoninae (XIX Contributo alla conoscenza dei Coleoptera Curculionidae). Bollettino della Società entomologica italiana, 106: 170-174.

Pesarini C., 1975. Su alcuni Curculionidi paleartici nuovi o poco conosciuti (XVII Contributo alla conoscenza dei Coleotteri Curculionidi). Memorie della Società entomologica italiana, 53: 39-55.

Penati F. \& Vienna P., 2005. Insecta Coleoptera Histeridae. In: Ruffo S. \& Stoch F. (Eds.), Checklist e distribuzione della Fauna italiana. Memorie del Museo Civico di Storia Naturale di Verona, 2 serie, Sezione Scienze della Vita, 16: 173-175 + CD-ROM.

Petagna V., 1787. Specimen Insectorum Ulterioris Calabriae. Varentrapp et Werner. Francofurti et Magontiae apud Varrentrapp et Wenner: I-VI, 1-46.

Petagna L., Terrone G. \& Tenore M., 1827. Viaggio in alcuni luoghi della Basilicata e della Calabria Citeriore effettuato nel 1826. Tipografia Francese, Napoli, 152 pp. https://books.google.it/books?id=VIqeX5dl MkMC\&hl=it\&source=gbs_book_other_versions

Pierotti H., 1974. Gli Aphodius italiani del sottogenere Melinopterus Mulsant. Bollettino della Società entomologica italiana, 106: 24-37.

Pierotti H., 1976. Ritrovamento dell'Aphodius ragusae Reitt. in Basilicata e in Sicilia. Società Veneziana di Scienze Naturali. Lavori, 1: 37-41.

Pierotti H., 1977. Contributo alla conoscenza degli Aphodius della Calabria e del Pollino. Bollettino della Società entomologica italiana, 109: 173-198.

Pierotti H., 1980. Deux neuveaux Rhyssemus italiens (Col. Aphodiidae). L'Entomologiste, 36: 22-25.

Pierotti H., 2011a. Peritelini nuovi o interessanti della fauna w-mediterranea. XIII. Una nuova Pseudomeira 
Stierlin, 1881, dell'Italia meridionale (Coleoptera, Curculionidae, Entiminae). Bulletin de la Société entomologique de France, 116: 15-16.

Pierotti H., 2011b. Contributi al riordinamento sistematico dei Peritelini W-paleartici. VIII. Revisione delle specie europee del genere Heteromeira Solari, 1955 (Coleoptera, Curculionidae, Entiminae). Bulletin de la Société entomologique de France, 116: 195-239.

Pierotti H. \& Bellò C., 1994. Peritelini nuovi o interessanti della fauna tirrenica. $3^{\circ}$ contributo alla conoscenza della Tribu Peritelini. Il Naturalista siciliano, 18: 107-122.

Pilon N., 1998. Atlante faunistico degli Staphylinini italiani con note sinonimiche (Coleoptera). Memorie della Società entomologica italiana, 76: 61-129.

Pilon N., 2005. Insecta Coleoptera Staphylinidae Staphylininae. In: Ruffo S. \& Stoch F. (Eds.), Checklist e distribuzione della Fauna italiana. Memorie del Museo Civico di Storia Naturale di Verona, 2 serie, Sezione Scienze della Vita, 16: 187-188 + CD-ROM.

Pittino R., 1978. Revisione del genere Psammodius Fallen, 1: le specie paleartiche del gruppo nocturnus. Bollettino della Società entomologica italiana, 110: 106-137.

Pittino R., 1979. Note su alcuni Coleotteri Scarabaeoidea Laparosticta della fauna italiana. Bollettino dell'Associazione romana di entomologia, 34: 32-41.

Pittino R., 2006. New or noteworthy records of Western Palaearctic species of the genus Ochodaeus (Coleoptera, Ochodaeidae). Fragmenta entomologica, 38: 75-81.

Pittino R. \& Mariani G., 1993. Aphodius (Agrilinus) convexus Erichson: a misinterpreted valid species from the Western Paleartic fauna. Bollettino della Società entomologica italiana, 125: 131-142.

Platia G., 1983. Nuovi dati sulla distribuzione italiana di alcune specie del genere Ampedus Dejean. Giornale italiano di Entomologia, 1: 141-144.

Platia G., 1985. Descrizione di due nuove specie di Elateridi paleartici. Bollettino della Società entomologica italiana, 117: 93-96.

Platia G., 1994. Fauna d'Italia. XXXIII. Coleoptera Elateridae. Ed. Calderini, Bologna, 429 pp.

Platia G., 2005. Insecta Coleoptera Elateridae. In: Ruffo S. \& Stoch F. (Eds.), Checklist e distribuzione della Fauna italiana. Memorie del Museo Civico di Storia Naturale di Verona, 2 serie, Sezione Scienze della Vita, 16: 201-203 + CD-ROM.

Platia G. \& Bartolozzi L., 1988. Contributo alla conoscenza degli Elateridi della Calabria. Redia, 71: 61-97.

Platia G. \& Gudenzi I., 1985. Descrizione di un nuovo Cidnopus Thomson della fauna europea. Bollettino del Museo Civico di Storia Naturale di Verona, 10 (1983): 21-29.
Platia G. \& Gudenzi I., 1986. Note sistematiche, ecologiche e geonemiche sull'Agriotes aequalis Schwarz con descrizione della larva (Coleoptera Elateridae). Bollettino della Società entomologica italiana, 118: 43-48.

Platia G. \& Gudenzi I., 1998. Descrizione di una nuova specie di Athous Eschscholtz, 1829 e della femmina di Athous pedemontanus Platia, 1988, del Piemonte, con nuovi dati geonemici su alcuni Elateridi della fauna italiana. Rivista Piemontese di Storia Naturale, 19: 267-273.

Platia G. \& Gudenzi I., 2005. Description of eleven new species of click-beetles of the Palearctic region, a case of teratology and new records of some species of the Italian fauna (Insecta Coleoptera Elateridae). Quaderno di Studi e Notizie di Storia naturale della Romagna, 21: 109-127.

Platia G. \& Sama G., 1981. Nuovi dati geonemici su Coleotteri Carabidi italiani. Bollettino dell'Associazione romana di entomologia, 36: 23-32.

Poggi R., 1971. Brevi note corologiche su due Scopaeus dell'Italia meridionale. Bollettino della Società entomologica italiana, 103: 41-42.

Poggi R., 1983. Note di caccia. V. Reperti di specie italiane rare o poco note. Bollettino della Società entomologica italiana, 115: 156-160.

Poggi R., 1984. Note su alcuni Pselaphidae paleartici, con descrizione di Bryaxis pescaroloi n. sp. delle Alpi Pennine. Bollettino della Società entomologica italiana, 116: 89-95.

Poggi R., 1985. Caratteri diagnostici e distribuzione delle specie italiane del genere Diodesma Latreille. Annali del Museo civico di Storia naturale "G. Doria" di Genova, 85: 363-374.

Poggi R., 1994. Appunti sinonimici su Tychobythinus gularis (Dod.) e T. anellii (Kar.) (Coleoptera Pselaphidae). Bollettino della Società entomologica italiana, 126: 141-144.

Poggi R., 1995. Note su Derodontus raffrayi Gouvelle e D. macularis (Fuss) (Coleoptera Derodontidae). Bollettino della Società entomologica italiana, 126 (1994): 249-254.

Poggi R., 2009. Agostino Dodero (1864-1937). Appunti per una biografia - In: Bottelli F. \& Giachino P.M. (Eds.), Contributo alle conoscenze naturalistiche della Valle Oropa (Biella, Italia). Memorie Associazione naturalistica piemontese, 11: 75-82.

Poggi R. \& Sabella G., 2005. Insecta Coleoptera Staphylinidae Pselaphinae. In: Ruffo S. \& Stoch F. (Eds.), Checklist e distribuzione della Fauna italiana. Memorie del Museo Civico di Storia Naturale di Verona, 2 serie, Sezione Scienze della Vita, 16: 183$184+$ CD-ROM.

Przewoźny M., 2019. Catalogue of Palearctic Hydrophiloidea (Coleoptera). Internet version 2019-01- 
01 (http://www.waterbeetles.eu/documents/PAL CAT_Hydrophiloidea_2019.pdf).

Puppin O., 1974. Dictyoptera aurora caprai n. subsp. ed illustrazioni di altri Dictyopterini. Bollettino della Società entomologica italiana, 106: 40-46.

Puthz V., 1986. Uber drei italienische Vertreter der Gattung Stenus Latr. Bollettino del Museo Civico di Storia Naturale di Verona, 12 (1985): 419-429.

Ranius T., Aguado L.O., Antonsson K., Audisio P., Ballerio A., Carpaneto G.M., Chobot K., Gjurašin B., Hanssen O., Huijbregts H., Lakatos F., Martin O., Neculiseanu Z., Nikitsky N.B., Paill W., Pirnat A., Rizun V., Ruicănescu A., Stegner J., Süda I., Szwałko P., Tamutis V., Telnov D., Tsinkevich V., Versteirt V., Vignon V., Vögeli M. \& Zach P., 2005. Osmoderma eremita (Coleoptera, Scarabaeidae, Cetoniinae) in Europe. Animal Biodiversity and Conservation, 28: $1-44$.

Rapuzzi P. \& Arcorace L., 2018. Purpuricenus (s. str.) coccineus Breit, 1917: a distinct species endemic from Calabria, Italy (Coleoptera Cerambycidae Cerambycinae Purpuricenini). Biodiversity Journal, 9: 89-94.

Rapuzzi P. \& Sama G., 2014. Descriptions of nine new species of Longhorn Beetles (Coleoptera, Cerambycidae). Munis Entomology and Zoology Journal, 9: $1-16$.

Ratti E., 2000. Note faunistiche ed ecologiche sui $\mathrm{Cu}-$ cuidi italiani (Coleoptera Cucujidae). Bollettino del Museo civico di Storia naturale di Venezia, 50: 103 129.

Ratti E., 2005. Insecta Coleoptera Cucujidae. In: Ruffo S. \& Stoch F. (eds.), Checklist e distribuzione della Fauna italiana. Memorie del Museo Civico di Storia Naturale di Verona, 2 serie, Sezione Scienze della Vita, 16: 211-213 + CD-ROM.

Ratti E., 2007. I coleotteri Silvanidi in Italia (Coleoptera Cucujoidea Silvanidae). Bollettino del Museo civico di Storia naturale di Venezia, 58: 83-137.

Ravizza C., 1972. I Pogonus dei litorali italiani. Bollettino del Museo civico di Storia naturale di Venezia, 22-23: 7-65.

Ravizza C., 1973. Cicindelidi e Carabidi raccolti sul Massiccio del Sirino (Appennino Lucano). Memorie del Museo Civico di Storia Naturale di Verona, 20: 55-69.

Regalin R., 1978. Dati geonemici su alcuni alticini italiani. Bollettino della Società entomologica italiana, 110: 202-204.

Regalin R., 1980. Le specie italiane del genere Labidostomis Redtenbacher. Memorie della Società entomologica italiana, 59: 37-48.

Reibnitz J., Graf R. \& Coray A., 2013. Verzeichnis der Ciidae (Coleoptera) der Schweiz mit Angaben zur Nomenklatur und Ökologi. Checklist of the Ciidae
(Coleoptera) of Switzerland, with comments on nomenclature and ecology. Mitteilungen der Schweizerischen Entomologischen Gesellschaft, Bulletin de la Société Entomologique Suisse, 86: 63-88.

Robertson J.A., Ślipiński A., Moulton M., Shockley F.W., Giorgi A., Lord N.P., McKenna D.D., Tomaszewska W., Forrester J., Miller K.B., Whiting M.F. \& McHugh J.V., 2015. Phylogeny and classification of Cucujoidea and the recognition of a new superfamily Coccinelloidea (Coleoptera: Cucujiformia). Systematic Entomology, 40: 745-778. https://doi:10.1111/ syen. 12138

Rocchi S., 2000. Segnalazione di nuovi reperti di Ditiscidi in Italia (Insecta Coleoptera Dytiscidae). Quaderno di Studi e Notizie di Storia naturale della Romagna, 13, suppl.: 11-16.

Rocchi S., 2002. Reperti inediti di Hydrophiloidea in Italia (Insecta Coleoptera Helophoridae, Hydrochidae, Hydrophilidae). Quaderno di Studi e Notizie di Storia naturale della Romagna, 16, suppl.: 43-48.

Rocchi S., 2005a. Insecta Coleoptera Hydroadephaga. In: Ruffo S. \& Stoch F. (Eds.), Checklist e distribuzione della Fauna italiana. Memorie del Museo Civico di Storia Naturale di Verona, 2 serie, Sezione Scienze della Vita, 16: 165-166 + CD-ROM.

Rocchi S., 2005b. Insecta Coleoptera Hydrophiloidea. In: Ruffo S. \& Stoch F. (Eds.), Checklist e distribuzione della Fauna italiana. Memorie del Museo Civico di Storia Naturale di Verona, 2 serie, Sezione Scienze della Vita, 16: 167-168 + CD-ROM.

Rocchi S., 2005c. Nuovi dati geonemici su alcuni Coleotteri Idroadefagi italiani (Coleoptera Haliplidae, Gyrinidae, Dytiscidae). Onychium, 3: 17-20.

Rocchi S., 2007. Il genere Oreodytes Seidlitz, 1887 negli Appennini (Coleoptera, Dytiscidae). Onychium, 5: 29-33.

Romano M., 2006. La ricerca entomologica in Sicilia: protagonisti, cultori e collezioni a cavallo di tre secoli. Il Naturalista siciliano, 30: 151-226.

Rössner E. \& Fery H., 2014. Aphodius (Biralus) mahunkaorum (Ádám, 1983) in Europa (Coleoptera: Scarabaeidae, Aphodiinae). Entomologische Zeitschrift, 124: 113-122.

Rössner E. \& Krell F.T., 2009. Identität und taxonomischer Status von Amphimallon ochraceum (Knoch, 1801) und A. fallenii (Gyllenhal, 1817) sowie weiterer mit $A$. solstitiale (Linnaeus, 1758) verwandter Taxa (Coleoptera: Scarabaeidae: Melolonthinae). Vernate Fortsetzung der Veröffentlichungen des Naturkundemuseum Erfurt, Erfurt, 27: 221-261.

Roubal J., 1932. Addition à liste des Coléoptères de Monte Gargano. Bollettino della Società entomologica italiana, 64: 66.

Rücker W.H., 1998. Una nuova specie italiana di Latridiidae: Dienerella angelinii nov. sp. (Coleoptera). Do- 
riana, supp. Annali del Museo civico di Storia naturale "G. Doria" di Genova, 7 (306): 1-7.

Rücker W.H., 2006. Revision von Corticaria crenulata (Gyllenhal, 1827) und Corticaria olympiaca Reitter, 1875 mit Beschreibung vier neuer Arten (Coleoptera: Latridiidae). Entomologische Zeitschrift, 116: 277 281

Rücker W.H., 2018. Latridiidae und Merophysiidae der West-Paläarktis. Wolfgang H. Rücker Selbstverlag, Neuwied, 676 pp.

Rücker W.H. \& Jonson C., 2007. Revision of Melanophthalma taurica (Mannerheim, 1844) species-group and description of three new species. (Coleoptera: Latridiidae). Latridiidae, 5: 11-24.

Rücker W.H. \& Poggi R., 2013. Descrizione di Dienerella polyhymnia, nuova specie mediterranea di Latridiidae, con note su Dienerella elongata (Curtis, 1839) (Coleoptera). Annali del Museo civico di Storia naturale "G. Doria" di Genova, 105: 447-458.

Ruffo S., 1957. Sulle ricerche faunistiche nell'Appennino. Bollettino della Società entomologica italiana, 87: 64-67.

Ruffo S., 1964a. Contributi alla conoscenza della distribuzione dei Coleotteri Crisomelidi nella Regione Appenninica. I. Orsodacnini, Donaciini, Criocerini. Memorie del Museo Civico di Storia Naturale di Verona, 12: 41-96.

Ruffo S., 1964b. Contributo alla conoscenza della distribuzione dei Coleotteri Crisomelidi nella Regione Appenninica. 2. Alticini: generi Sphaeroderma, Argopus. Memorie del Museo Civico di Storia Naturale di Verona, 12: 97-106.

Ruffo S. \& Vigna Taglianti A., 1988. Appunti per una storia delle ricerche entomologiche nell'Appennino centromeridionale. Atti del XV Congresso Nazionale Italiano di Entomologia, L'Aquila, 13-17 giugno 1988: 7-30.

Ruzzier E., 2013. Taxonomic and faunistic notes on Italian Mordellidae (Coleoptera Tenebionoidea) with redescription of Falsopseudotomoxia agyropleura (Franciscolo, 1942) n. comb. Bollettino della Società entomologica italiana, 143: 103-115.

Sabatinelli G., 1976. Note su alcuni Scaraboidea floricoli dell'Italia Meridionale e descrizione di Amphimallon pseudomajale n. sp. Bollettino dell'Associazione romana di entomologia, 31: 35-46.

Sabella G., 1997. Brachygluta angelinii, a new species from Southern Italy (Insecta, Coleoptera, Pselaphidae). Spixiana, 20: 137-140.

Sabella G. \& Angelini F., 2001. Gli Pselaphidi dell'Aspromonte (Coleoptera, Staphylinidae, Pselaphinae). Bollettino del Museo Civico di Storia Naturale di Verona, Botanica Zoologia, 25: 9-61.

Sabella G. \& Poggi R., 1997. Revisione dei Tychus Leach del gruppo florentinus Reitt. con descrizione di nuove specie. Annali del Museo civico Storia naturale "G. Doria" di Genova, 91: 383-409.

Salamanna G., 1968. Osservazini sugli Scarabeidi delle Isole Tremiti. Atti VII Congresso Nazionale di Entomologia, Verona (1967): 134-135.

Salfi M., 1951. Ricerche faunistiche sul Massiccio del Pollino. La Ricerca Scientifica, 21: 79-84.

Salfi M., 1963. Viaggi di naturalisti in Calabria. Annuario dell'Istituto e Museo di Zoologia della Università di Napoli, 15: 1-22.

Sama G., 1979. Note ecologiche e geonemiche su alcuni Buprestidi italiani. Bollettino dell'Associazione romana di entomologia, 34: 16-17.

Sama G., 1981. Materiali per una fauna dei Cerambycidae d'Italia. Annali del Museo civico Storia naturale "G. Doria" di Genova, 83: 473-522.

Sama G., 1988. Fauna d'Italia. XXVI. Coleoptera Cerambycidae. Catalogo topografico e sinonimico. Ed. Calderini, Bologna, 216 pp.

Sama G., 2005. Insecta Coleoptera Cerambycidae. In: Ruffo S. \& Stoch F. (Eds.), Checklist e distribuzione della Fauna italiana. Memorie del Museo Civico di Storia Naturale di Verona, 2 serie, Sezione Scienze della Vita, 16: 219-222 + CD-ROM.

Sama G. \& Rapuzzi P., 2011. Una nuova Checklist dei Cerambycidae d'Italia (Insecta Coleoptera Cerambycidae). Quaderno di Studi e Notizie di Storia naturale della Romagna, 32: 121-164.

Sanfilippo N., 1955. Ricerche Zoologiche sul Massiccio del Pollino. XVII. Coleoptera 7. Dytiscidae, Gyrinidae. Annuario dell'Istituto e Museo di Zoologia della Università di Napoli, 7: 1-4.

Sassi D., 1994. Le Cassidine appenniniche del Museo di Storia naturale di Verona (Coleoptera Chrysomelidae). Bollettino del Museo Civico di Storia Naturale di Verona, 18 (1991): 53-90.

Sassi D., 2001. Nuove specie del genere Cryptocephalus vicine a Cryptocephalus marginellus (Coleoptera Chrysomelidae). Memorie della Società entomologica italiana, 80: 107-138.

Sassi D., 2005. Insecta Coleoptera Chrysomelidae Cryptocephalinae. In: Ruffo S. \& Stoch F. (Eds.), Checklist e distribuzione della Fauna italiana. Memorie del Museo Civico di Storia Naturale di Verona, 2 serie, Sezione Scienze della Vita, 16: 223-225 + CDROM.

Sassi D. \& Borowiec L., 2006. Cassida inopinata, a new species from Italy and Balkan Region (Coleoptera: Chrysomelidae: Cassidinae). Genus, 17: 545-560.

Schaefer L., 1965. Une nouvelle espece d'Acmaeodera decouverte en Italie (Coleoptera Buprestidae). Bollettino dell'Associazione romana di entomologia, 20: 36-38.

Schatzmayr A., 1926. Le Oedemera italiane. Memorie della Società entomologica italiana, 2: 131-151. 
Schatzmayr A., 1941. Coleotteri raccolti nella Grande Sila dal Dott. E. Moltoni. Atti della Società italiana di Scienze naturali e del Museo civico di Storia naturale di Milano: 49-102.

Schatzmayr A., 1943. Elateridi raccolti dal Dott. E. Moltoni nella Grande Sila (Calabria). Natura, 34: 138-139.

Schawaller W., 2002. Evidence of two species of Eledona Latreille and Eledonoprius Reitter in Europe (Coleoptera: Tenebrionidae). Entomologische Blätter, Berlin, 98: 31-34

Scheerpeltz O., 1956. Ricerche Zoologiche sul Massiccio del Pollino. XXII. Coleoptera 12. Neue Kurzflugler aus Süditalien (Col., Staphylinidae). Annuario dell'Istituto e Museo di Zoologia della Università di Napoli, 8: 1-33.

Scheerpeltz O., 1961. Wissenschaftliche ergebnisse der Zoologischen Explorationen des Gebietes von Aspromonte im südlichsten Calabrien in den Jahren 1957/58 durch Prof. S. Ruffo und Dr. M. Magistretti. Col. Staphylinidae. Memorie del Museo Civico di Storia Naturale di Verona 9: 115-154.

Schimmel R., Tarnawski D., Han T. \& Platia G., 2015. Monograph of the new tribe Selatosomini from China (Elateridae: Denticollinae) Part I: Genera Pristilophus Latreille, 1834 stat. nov., Selatosomus Stephens, 1830, Warchalowskia (Tarnawski, 1995) stat. nov., and Sinophotistus gen. nov. Polish Entomological Society, Monographs, 11, Poznañ, 328 pp.

Schülke M., 1996. A new subspecies of Tachinus marginellus (Fabricius) fron Italy (Coleoptera Staphylinidae). Il Naturalista siciliano, 20: 99-107.

Sciaky R., 1979. Gli Anisodactylus italiani con riferimento alle altre specie mediterranee. Memorie della Società entomologica italiana, 57 (1978): 3-18.

Sciaky R., 1984. Revisione dei Pterostichus italiani affini a cristatus Duf. Bollettino del Museo Civico di Storia Naturale di Verona, 11: 149-170.

Sciaky R., 1987. Revisione delle specie paleartiche occidentali del genere Ophonus Dejean, 1821. Memorie della Società entomologica italiana, 65 (1986): 29 120.

Sciaky R., 1991. Revisione dei Philorhizus della Regione Paleartica con descrizione di quattro nuovi taxa. (XXIII Contributo alla conoscenza dei Coleoptera Carabidae). Memorie della Società entomologica italiana, 69 (1990): 53-78.

Sciaky R. \& Facchini S., 1999. Quattro specie di Carabidi nuove o confermate per la fauna italiana (Coleoptera, Carabidae). Bollettino dell'Associazione romana di entomologia, 54: 59-63.

Sciaky R. \& Pavesi M., 1986. Nuovi dati geonemici su Carabidae italiani. Atti della Società italiana di Scienze naturali e del Museo civico di Storia naturale di Milano, 127: 13-26.
Scupola A., 2000. Revisione della tribu Cossyphini Latreille, 1802. Parte I. Introduzione e genere Cossyphus Olivier, 1791 (Coleoptera Tenebrionidae). Atti del Museo Civico di Storia naturale di Trieste, 48: 185-249.

Seago A.E., Giorgi J.A., Li J. \& Ślipiński S.A., 2011. Phylogeny, classification and evolution of ladybird beetles (Coleoptera: Coccinellidae) based on simultaneous analysis of molecular and morphological data. Molecular Phylogenetics and Evolution, 60: 137-151. https://doi.org/10.1016/j.ympev.2011.03. 015

Simonetta G., 1989. Contributo alla conoscenza dei Cerambicidi della Calabria. Bollettino dell'Associazione romana di entomologia, 43 (1988): 49-53.

Smetana A., 1954. Ein Beitrag zur Kenntniss der italienischen Philonthus und Gabrius-Arten. Entomologisk Tidskrift, 75: 39-43.

Solari F., 1932. Tre nuove specie di Curculionidi mediterranei. Bollettino della Società entomologica italiana, 64: 160-164.

Sörensson M. \& Delgado J.A., 2019. Unveiling the smallest - systematics, classification and a new subfamily of featherwing beetles based on larval morphology (Coleoptera: Ptiliidae). Invertebrate Systematics, 33: 757-806. https://doi.org/10.1071/ IS19007

Sparacio I., 2000. Osservazioni sulle Osmoderma Le Peletier et Audinet-Serville europee con descrizione di una nuova specie dell'Italia meridionale (Coleoptera Cetoniidae). Il Naturalista siciliano, 24: 225-239.

Sparacio I., 2006. Una nuova specie del genere $A c$ maeoderella Cobos, 1955 dell'Italia Meridionale (Coleoptera Buprestidae). Il Naturalista siciliano, 30: 35-42.

Sparacio I., 2009. Note tassonomiche su Cetoniidae italiani (Coleoptera Scarabaeoidea Cetoniidae). Il Naturalista siciliano, 33: 157-165.

Švec Z. \& Angelini F., 1995. The Italian species of the family Phalacridae with the description of Olibrus demarzoi sp. n. (Coleoptera). Bollettino della Società entomologica italiana, 127: 199-212.

Szawaryn K., Bocak L., Ślipiński A., Escalona H.E. \& Tomaszewska W., 2015. Phylogeny and evolution of phytophagous ladybird beetles (Coleoptera: Coccinellidae: Epilachnini), with recognition of new genera. Systematic Entomology, 40: 547-569. https:// doi:10.1111/syen.12121

Tamanini L., 1955. Ricerche Zoologiche sul Massiccio del Pollino. XIV. Coleoptera. 4. Catopidae, Liodidae, Scaphidiidae, Silphidae. Annuario dell'Istituto e Museo di Zoologia della Università di Napoli, 6: 119.

Tamanini L., 1969. Gli Scaphidiidae del Museo Civico di Storia naturale di Verona e descrizюne di una 
nuova specie. Memorie del Museo Civico di Storia Naturale di Verona, 16: 483-490.

Tassi F., 1962a. Coleotteri Buprestidi dell'Aspromonte e della Sila. Memorie del Museo Civico di Storia Naturale di Verona, 10: 211-231.

Tassi F., 1962b. Su alcuni Buprestidi italiani specialmente delle Regioni centro-meridionali. Bollettino della Società entomologica italiana, 92: 53-57.

Tassi F., 1966a. Ricerche Zoologiche sul Massiccio del Pollino. XXXII. Coleoptera 17. Cerambycidae. Annuario dell'Istituto e Museo di Zoologia della Università di Napoli, 17: 1-65.

Tassi F., 1966b. Su alcuni Buprestidi italiani nuovi o particolarmente interessanti. Bollettino della Società entomologica italiana, 96: 17-27.

Tassi F., 1966c. Su alcuni Coleotteri Buprestidi dell'Italia meridionale ed insulare. Accademia Gioenia di Catania, 8: 610-625.

Tassi F., 1967. Ricerche coleotterologiche sul litorale ionico della Puglia, Lucania e Calabria, Campagne 1956-1957-1958. XIV. Coleoptera Buprestidae. Bollettino della Società entomologica italiana, 97: 135152.

Tassi F., 1970. Alcuni reperti interessanti dai Monti della Laga. Bollettino dell'Associazione romana di entomologia, 25: 101-106.

Terzani F. \& Ceccolini F., 2015. Il genere Mycterus Schellenberg, 1798 in Italia (Insecta Coleoptera Mycteridae). Quaderno di Studi e Notizie di Storia naturale della Romagna, 41: 109-115.

Terzani F., Ceccolini F., Hàjek J. \& Cianferoni F., 2017. Taxonomic review of the genus Dascillus Latreille, 1797 in the Western Palaearctic (Coleoptera: Dascillidae). Zootaxa 4282 (2): 255-268. https://doi.org/ 10.11646/zootaxa.4282.2.2

Toledo M. \& Rocchi S., 2017. Reperti inediti di Coleotteri acquatici in Italia (Coleoptera: Hydroscaphidae, Gyrinidae, Dytiscidae, Helophoridae, Hydrophilidae, Hydraenidae, Limnichidae, Erirhinidae). Onychium, 13: $63-74$

Tomaszewska W. \& Szawaryn K., 2016. Epilachnini (Coleoptera: Coccinellidae). A Revision of the World Genera. Journal of Insect Science, 16: 1-91. https:// doi:10.1093/jisesa/iew082

Tosevski I., Caldara R., Jovic J., Baviera C., HernandezVera G., Gassmann A. \& Emerson B.C., 2014. Revision of Mecinus heydenii species complex (Curculionidae): integrative taxonomy reveals multiple species exhibiting host specialization. Zoologica Scripta, 43: 34-51. https://doi:10.1111/zsc.12037

Toskina I.N., 2012. Some new palaearctic species of the genus Stagetus Wollaston, 1861 (Coleoptera: Ptinidae: Dorcatominae). Bulletin of the Moscow Society of Naturalists. Ser. Biol., 117: 16-28. - Russian: Тоскина И. Н., 2012. Несколько новых палеаркти- ческих видов рода Stagetus Wollaston,1861 (Coleoptera: Ptinidae: Dorcatominae). Бюл. Москов. об-ва испытателей природы. Отд.биол., 117: 1628.

Trizzino M., Carnevali L., De Felici S. \& Audisio P., 2013. A revision of Hydraena species of the "Haenydra" lineage (Coleoptera, Hydraenidae). Zootaxa 3607 (1): 1-173. http://dx.doi.org/10.11646/zootaxa. 3607.1.1

Vanni S. \& Magrini P., 1995. Ridescrizione di Duvalius silvestrii (Gestro, 1896) e ulteriori note su Duvalius caoduroi Pace, 1986 (Coleoptera Carabidae Trechinae). Redia, Firenze, 78 (1): 141-147.

Vasilikopoulos A., Balke M., Beutel R.G., Donath A., Podsiadlowski L., Pflug J.M., Waterhouse R.M., Meusemann K., Peters R.S., Escalona H.E., Mayer C., Liu S., Hendrich L., Alarie Y., Bilton D.T., Jia F., Zhou X., Maddison D.R., Niehuis O., Misof B., 2019. Phylogenomics of the superfamily Dytiscoidea (Coleoptera: Adephaga) with an evaluation of phylogenetic conflict and systematic error. Molecular Phylogenetics and Evolution, 135: 270-285. https://doi. org/10.1016/j.ympev.2019.02.022

Vienna P., 1971. Gli Histeridae del Museo Civico di Storta naturale di Verona. Memorie del Museo Civico di Storia Naturale di Verona, 19: 267-301.

Vienna P., 1978. Elenco critico degli Histeridae di Puglia e considerazioni sulla transadriaticita di alcune entità. Società Veneziana di Scienze Naturali. Lavori, 3: 4954

Vienna P., 1980. Fauna d'Italia. Coleoptera Histeridae. XVI. Ed. Calderini, Bologna, 386 pp.

Vienna P., 1999. Sulla presenza in Italia di Saprinus lautus Erichson, 1839 (Coleoptera, Histeridae). Società Veneziana di Scienze Naturali. Lavori, 24: 121-122.

Vigna Taglianti A., 1967. Note su alcuni Carabidae (Col.) della Regione Pugliese. Bollettino dell'Associazione romana di entomologia, 22: 50-51.

Vigna Taglianti A., 2005. Checklist e corotipi delle specie di Carabidae della fauna italiana. Appendice B, pp. 186-225. In: Brandmayr P., Zetto T. \& Pizzolotto R. (Eds.), I Coleotteri Carabidi per la valutazione ambientale e la conservazione della biodiversità. Manuale operativo: APAt, Manuali e Linee Guida, 34: 240 pp.

Vigna Taglianti A. \& Bonavita P., 1995. Nuovi dati geonemici su Carabidi italiani. Bollettino dell'Associazione romana di entomologia, 49 (1994): 137-149.

Vigna Taglianti A., Spettoli R., Brandmayr P. \& Algieri M.C., 2001. Note tassonomiche e corologiche su Carabus granulatus in Italia con descrizione di una nuova sottospecie di Calabria (Coleoptera Carabidae). Memorie della Società entomologica italiana, 80: 65-86.

Vit S., 1985. Quelques elements de la faune coleopterologique resistant a la desctruction de l'an- 
cienne foret de Pantano de Policoro (Basilicata). Annali del Museo civico di Storia naturale "G. Doria" di Genova, 85: 307-331.

Vit S., 1985. Etude de la morphologie des espèces paléarctiques du genre Eucinetus Germar et quelques remarques sur son utilisation taxonomique (Coleoptera: Eucinetidae). Revue Suisse Zoologie, 92: 421-460.

Vomero V., 1968, Nuovi reperti di Coleotteri Carabidi in Lucania. Bollettino dell'Associazione romana di entomologia, 23: 25-29.

Wilson C., 2001. Aphodius pedellus (Degeer), a species distinct from $A$. fimetarius (Linnaeus) (Coleoptera: Aphodiidae). Tijdschrift voor Entomologie, 144: 37 143.

Wilson C. \& Angus R.B., 2004. A Chromosomal Analysis of the West European Species of Aphodius Illiger, subgenus Aphodius s. str. (Coleoptera: Aphodiidae). Tijdschrift voor Entomologie, 147: 259-264.

Wittmer W., 1935. Risultati scientifici delle cacce entomologiche di S.A.S. il Principe Alessandro Della Torre e Tasso in Italia. IV. Malacodermata von M.te Pollino (Lucania). Bollettino della Società entomologica italiana, Genova, 67: 72-76.

Wittmer W., 1974. Zur Kenntnis der Gattung Malthinus Latr. II (Col. Cantharidae) (60. Beitrag zur Kenntnis der palaarktischen Cantharidae). Entomologischen arbeiten aus dem Museum G. Frey, 25: 358-427.

Wrase D.W. \& Magrini P., 2012. Taxonomic revision of the mediterranean species of the genus Amblystomus Erichson, 1837, with description of a new species (Coleoptera, Carabidae, Harpalini, Amblystomina). Annali del Museo civico di Storia naturale "G. Doria" di Genova, 104: 1-77.

Zahradník P., 2013. Beetles of the family Ptinidae of Central Europe. Akademie České republiky, Praha, 349 pp., 60 pls.

Zampetti M.F., 1979. Note sulla geonemia di alcuni Coleotteri Clavicorni. Bollettino della Società entomologica italiana, 111: 96-97.

Zampetti M.F., 1982. Posizione sistematica di alcune specie appartenenti al genere Bruchidius Schilsky. Bollettino del Museo Civico di Storia Naturale di Verona, 8: 383-410.

Zampetti M.F. \& Ricci M.S., 2012. Guida ai Coleotteri Bruchidi della Fauna Italiana. Darwin Edizioni, Roma, $430 \mathrm{pp}$.

Zanetti A., 1971. Un nuovo Stilicus (Col., Staphylinidae) dell'Italia centromeridionale. Bollettino del Museo Civico di Storia Naturale di Verona, 4: 650-653.

Zanetti A., 1977. Due nuove specie di Stafilinidi dell'Appennino. Memorie del Museo Civico di Storia Naturale di Verona 4: 307-315.

Zanetti A., 1978. Note sulle specie italiane del genere Acrolocha Thoms. con descrizione di una nuova specie (Col. Staphylinidae). Bollettino del Museo Civico di Storia Naturale di Verona, 5: 547-553.

Zanetti A., 1980a. Contributo alla conoscenza delle specie italiane del genere Cryptobium Mannh. con note sulle specie europee e del Caucaso. Società Veneziana di Scienze Naturali. Lavori, Venezia, 5: 31-41.

Zanetti A., 1980b. Note su Eusphalerum italicum (Koch) e sulle specie vicine, con descrizione di quattro nuove entità (Coleoptera: Staphylinidae). Annali del Museo civico di Storia naturale "G. Doria" di Genova, 83: 159-173.

Zanetti A., 1982. Materiali per una revisione degli Eusphalerum italiani e centro europei con descrizione di nuove sottospecie e note sinonimiche. Memorie del Museo Civico di Storia Naturale di Verona, 8: 97156.

Zanetti A., 1983. Descrizione di Osellia calabra gen. n. sp. dell'Italia Meridionale. Bollettino del Museo Civico di Storia Naturale di Verona, 9: 423-436.

Zanetti A., 1987. Fauna d'Italia. Coleoptera Staphylinidae. Omaliini. Ed. Calderini, Bologna, vol. XXV, $\mathrm{XII}+472 \mathrm{pp}$.

Zanetti A., 1991. Osservazioni su alcuni Quedius (Raphirus) del gruppo nemoralis Baudi, con descrizione di due nuove specie (Coleoptera, Staphylinidae). Animalia, 18: 125-139.

Zanetti A., 2004. Eusphalerum martinae n. sp. from southern Italy (Coleoptera Staphylinidae: Omaliinae). Bollettino del Museo Regionale di Scienze naturali di Torino, 21: 487-494.

Zanetti A., 2005a. Insecta Coleoptera Staphylinidae. In: Ruffo S. \& Stoch F. (Eds.), Checklist e distribuzione della Fauna italiana. Memorie del Museo Civico di Storia Naturale di Verona, 2 serie, Sezione Scienze della Vita, 16: $181+$ CD-ROM.

Zanetti A., 2005b. Insecta Coleoptera Staphylinidae Omaliinae. In: Ruffo S. \& Stoch F. (Eds.), Checklist e distribuzione della Fauna italiana. Memorie del Museo Civico di Storia Naturale di Verona, 2 serie, Sezione Scienze della Vita, 16: 185-186 + CDROM.

Zanetti A., 2011a. New synonymies in the genus Liogluta Thomson, 1858, with notes on Italian species (Coleoptera, Staphylinidae, Aleocharinae). Bollettino del Museo Civico di Storia Naturale di Verona, 35, Botanica Zoologia: 147-154.

Zanetti A., 2011b. Contribution to the knowledge of Staphylinidae from southern Sardinia (Coleoptera), pp. 331-352. In: Nardi G., Whitmore D., Bardiani M., Birtele D., Mason F., Spada L. \& Cerretti P. (Eds.), Biodiversity of Marganai and Montimannu (Sardinia). Research in the framework of the ICP Forests network. Conservazione Habitat Invertebrati, 5. Cierre Edizioni, Sommacampagna, Verona. 
Zanetti A., 2012. New species and new synonymies in the Mediterranean Omaliinae (Coleoptera: Staphylinidae). Bollettino del Museo Civico di Storia Naturale di Verona, Botanica Zoologia, 36: 55-66.

Zanetti A. \& Pace R., 2005. Insecta Coleoptera Staphylinidae Aleocarinae (genere Leptusa Kraatz, 1859). In: Ruffo S. \& Stoch F. (Eds.), Checklist e distribuzione della Fauna italiana. Memorie del Museo Civico di Storia Naturale di Verona, 2 serie, Sezione Scienze della Vita, 16: 189-190 + CD-ROM.

Zanetti A. \& Tagliapietra A., 2005. Studi sulle taxocenosi a Staphylininae in boschi di latifoglie italiani (Coleoptera, Staphylinidae). Studi trentini di Scienze naturali, Acta biologica, 81 (2004): 207-231.

Zanetti A., Sette A., Poggi R. \& Tagliapietra A., 2016. Biodiversity of Staphylinidae (Coleoptera) in the Province of Verona (Veneto, Northern Italy). Memorie della Società entomologica italiana, 93: 3-237.

Zhang S.-Q., Che L.-H., Li Y., Liang D., Pang H., Ślipiński A. \& Zhang P., 2018. Evolutionary history of Coleoptera revealed by extensive sampling of genus and species. Nature Communications, 9 (205): 1-11. https://doi:10.1038/s41467-017-02644-4

Zoia S., 1987. Appunti su Catopidi italiani con descrizione di una nuova specie. Bollettino della Società entomologica italiana, 119: 117-122.

Zoia S., 1989. Sulla presenza in Italia di Catops kirby (Spence, 1815) e Catops dorni Reitter, 1913 (Coleoptera, Cholevidae). Bollettino dell'Associazione romana di entomologia, 44: 61-66.
Zoia S., 1990. Note sui Namadeus italiani con descrizione di Namadeus italicus n. sp. (Coleoptera, Cholevidae). Bollettino del Museo Regionale di Scienze naturali di Torino, 8: 233-242.

Zoia S., 2014. The Mediterranean Colaspidea (Coleoptera Chrysomelidae Eumolpinae). Entomologia, 2 (159): 1-34. https://doi.org/10.4081/entomologia.2014.159

Zoia S. \& Latella L., 2005. Insecta Coleoptera Cholevidae e Platypsyllidae. In: Ruffo S. \& Stoch F. (Eds.), Checklist e distribuzione della Fauna italiana. Memorie del Museo Civico di Storia Naturale di Verona, 2.serie, Sezione Scienze della Vita 16: 177-180+ CD-ROM.

Zombori L., 1984. The Symphyta of the Dodero Collection. 3. The list of species (Hymenoptera). Bollettino della Società entomologica italiana, 116: 105-120.

Zunino M., 1972. Revisione delle specie paleartiche del genere Onthophagus (Coleoptera, Scarabaeoidea). I. Il sottogenere Euonthophagus Balth. Bollettino del Museo di Zoologia Univiversità di Torino, 1: 1-28.

Zuppa A.M. \& Osella G.B., 1999. Revisione del genere Aparopion Hampe, 1861 (Coleoptera, Curculionidae, Molytinae). Bollettino del Museo Civico di Storia Naturale di Verona, 23: 1-49.

Zyła D. \& Solodovnikov A., 2019. Multilocus phylogeny defines a new classification of Staphylininae (Coleoptera, Staphylinidae), a rove beetle group with high lineage diversity. Systematic Entomology, 44: 1-14. https://doi.org/10. 1111/syen.12382 